Sapere l'Europa, sapere d'Europa 5 年ISSN 2610.9247

\title{
Tortura e migrazioni \\ Torture and Migration
}

a cura di

Fabio Perocco

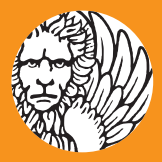

Edizioni

Ca'Foscari 

Tortura e migrazioni | Torture and Migration

\section{Sapere l'Europa. Sapere d'Europa}

5

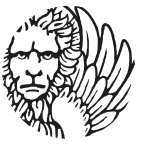

Edizioni

Ca'Foscari 


\title{
Sapere l'Europa. Sapere d'Europa
}

\author{
Direttore | General Editor \\ Lauso Zagato (Università Ca' Foscari Venezia, Italia)
}

\section{Comitato scientifico | Advisory Board}

Antonio Arantes (UNICAMP, Brasile)

Pietro Clemente (già Università degli Studi di Firenze, Italia)

Giovanni Luigi Fontana (Università degli Studi di Padova, Italia)

Giuseppe Goisis (già Università Ca' Foscari Venezia, Italia)

Maria Laura Picchio Forlati (già Università degli Studi di Padova, Italia)

Girolamo Sciullo (Università di Bologna, Italia)

Tullio Scovazzi (Università degli Studi di Milano-Bicocca, Italia)

\section{Comitato di redazione | Editorial Board}

Maria Luisa Ciminelli (già Università Ca' Foscari Venezia, Italia)

Roberta Dreon (Università Ca' Foscari Venezia, Italia)

Marco Giampieretti (Università degli Studi di Padova)

Giovanna Pasini (Consulente Culturale)

Simona Pinton (già Università Ca' Foscari Venezia, Italia)

Stefania Tesser (Università Ca' Foscari Venezia, Italia)

\section{Direzione e redazione | Head Office}

Dipartimento di Filosofia e Beni Culturali

Palazzo Malcanton Marcorà

Dorsoduro 3484/D

30123 Venezia

In questa collana sono usciti i volumi:

Il patrimonio culturale immateriale. Venezia e il Veneto come patrimonio europeo, a cura di Maria Laura Picchio Forlati (2014) Trasformazioni e crisi della cittadinanza sociale, a cura di Dino Costantini, Fabio Perocco, Lauso Zagato (2014) Citizens of Europe. Cultures and Rights, a cura di Lauso Zagato, Marilena Vecco (2015)

Cultural Heritage. Scenarios 2015-2017, a cura di Simona Pinton, Lauso Zagato (2017)

$\begin{array}{rr}\text { e-ISSN } & 2610-9247 \\ \text { ISSN } & 2611-0040\end{array}$

URL http://edizionicafoscari.unive.it/it/edizioni/collane/sapere-leuropa-sapere-deuropa/ 


\section{Tortura e migrazioni Torture and Migration}

a cura di Fabio Perocco

Venezia

Edizioni Ca' Foscari - Digital Publishing 2019 
Tortura e migrazioni | Torture and Migration

Fabio Perocco (a cura di)

(C) 2019 Fabio Perocco per il testo

(c) 2019 Edizioni Ca' Foscari - Digital Publishing per la presente edizione

(ㄷ) (1)

Quest'opera è distribuita con Licenza Creative Commons Attribuzione4.0 Internazionale This work is licensed under a Creative Commons Attribution 4.0 International License

Qualunque parte di questa pubblicazione può essere riprodotta, memorizzata in un sistema di recupero dati o trasmessa in qualsiasi forma o con qualsiasi mezzo, elettronico o meccanico, senza autorizzazione, a condizione che se ne citi la fonte.

Any part of this publication may be reproduced, stored in a retrieval system, or transmitted in any form or by any means without permission provided that the source is fully credited.

Edizioni Ca' Foscari - Digital Publishing Università Ca' Foscari Venezia | Dorsoduro 3246, 30123 Venezia http://edizionicafoscari.unive.it|ecf@unive.it

1a edizione dicembre 2019

ISBN 978-88-6969-358-8 [ebook]

ISBN 978-88-6969-359-5 [print]

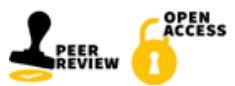

Certificazione scientifica delle Opere pubblicate da Edizioni Ca' Foscari - Digital Publishing: tutti i saggi pubblicati hanno ottenuto il parere favorevole da parte di valutatori esperti della materia, attraverso un processo di revisione doppiamente anonima sotto la responsabilità del Comitato scientifico della collana. La valutazione è stata condotta in aderenza ai criteri scientifici ed editoriali di Edizioni Ca' Foscari.

Scientific certification of the works published by Edizioni Ca' Foscari- Digital Publishing: all essays published in this volume have received a favourableopinion bysubjectmatter experts, through a double anonymous peer review process under the responsibility of the Scientific Committee of the series. The evaluations were conducted in adherence to the scientific and editorial criteria established by Edizioni Ca' Foscari.

Tortura e migrazioni | Torture and Migration / A cura di Fabio Perocco - 1. ed.

- Venezia: Edizioni Ca' Foscari - Digital Publishing, 2019. - 432 p.; $23 \mathrm{~cm}$. - (Sapere l'Europa. Sapere d'Europa; 5). - ISBN 978-88-6969-359-5.

URL http://edizionicafoscari.unive.it/it/edizioni/libri/978-88-6969-359-5/

DOI http://doi.org/10.30687/978-88-6969-358-8 
Tortura e migrazioni | Torture and Migration

a cura di Fabio Perocco

\section{Sommario}

Tortura e migrazioni

Fabio Perocco

PARTE I QUESTIONI DI FONDO

Tortura e razzismo

Teoria sociale, analisi storica, evidenze empiriche

Iside Gjergji

Sur le rapport torture et migration Une réflexion philosophique/politique

Marie-Claire Caloz-Tschopp

Delocalizzazione della tortura e 'tortura di Stato'

Tra accordi di riammissione, esternalizzazione delle frontiere e chiusura dei porti

Alessandra Algostino

PARTE II UN FENOMENO GLOBALE

Vulnerable to the System: Migration and Torture in Spain

Olga Jubany, Martina Pasqualetto, Alèxia Rué

Violences systémiques dans les centres fermés

L'expérience des femmes sans-papiers en Belgique

Nouria Ouali

Torture and the UK's 'War on Asylum'

Medical Power and the Culture of Disbelief

Monish Bhatia, Jon Burnett

Dreams and Nightmares. The Legal Legacy that Authorized Civil Detention Centers in the US 
La politica di tolleranza zero dell'Amministrazione Trump alla frontiera con il Messico

La pratica di separazione delle famiglie e di detenzione dei bambini

Rosemarie Brisciana

Tortura e immigrazione in America Latina e in Brasile nel contesto della crisi e della crescita dei partiti di estrema destra

Karina Quintanilha, Patricia Villen

Migración y tortura en la Argentina contemporánea Juliana Carpinetti

Controllo delle frontiere e violazione dei diritti umani lungo la rotta balcanica

Francesca Cucchi

Tortura e violenzecontro gli immigrati in Marocco

Rabi Ouenniche, Zineb Saaid

The Story of Yaser Abdulla

Darfuri Asylum Seeker Who Was Tortured in the Sinai Desert Only to Face Imprisonment in Israel and a Life Without Refugee Status

Mya Guarnieri Jaradat

Libia: dalle torture dei profughi nelle carceri libiche alla loro criminalizzazione in Europa

Marco Omizzolo

Immigrazione via mare

Non-refoulement, legge 110/2017, Memorandum d'Intesa

Italia-Libia e rischio di tortura

Monica Gazzola

La vulnerabilità degli immigrati nel sistema di accoglienza extra-ordinem dei richiedenti asilo 
PARTE III CONDIZIONI DI SALUTE, DISAGIO PSICHICO,

TUTELA ASSISTENZIALE

Migration and Torture: Building a Map of Knowledge

Pau Pérez-Sales

Immigrazione e salute mentale

Violenza, psicotraumatologia, politiche per la salute

e tutela assistenziale

Marco Mazzetti, Salvatore Geraci

Tortura e disagio psichico tra i rifugiati e i richiedenti asilo Presa in carico e intervento psico-sociale

Michela Nosè

Da vittima a testimone: il percorso di rinascita del sopravvissuto alla tortura

Carlo Bracci, Angelo Coppola 



\title{
Tortura e migrazioni
}

\section{Fabio Perocco}

Università Ca' Foscari Venezia, Italia

\begin{abstract}
As a social relationship of submission whose scope goes beyond those directly affected, torture is still an ongoing practice, widespread everywhere, and this is also due to several processes typical of the neo-liberal era - starting from the policies aimed at the security armoring of society. The essay, which examines the causes and dimensions of torture, inhuman and degrading treatment of migrants, shows how this global phenomenon today has a close link with the worsening conditions of migration, the global war on immigrants, the tightening of migration policies, the stigmatization of immigrants, the rise of institutional racism, the illegalization of migrations, all elements that favor the production of contexts, environments and situations permeable to torture.
\end{abstract}

Keywords Torture. Migration. Racism. Neo-liberalism. Violence.

Sommario 1 Un rapporto sociale di sottomissione. - 2 Un divieto assoluto a favore di un bene assoluto? - 3 I fattori di permanenza della tortura. - 4 Guerra agli immigrati e tortura. -5 Conclusione.

\section{Un rapporto sociale di sottomissione}

Nonostante le conquiste compiute dall'umanità nel corso del tempo, la tortura è ancora un fenomeno diffuso in tutto il mondo e un fatto di grande attualità. Ė, come il razzismo, più viva che mai, non è un ferrovecchio che riguarda il passato. È, come la violenza sulle donne, un fenomeno che interessa i quattro angoli del pianeta e non soltanto qualche Paese 'sotto-sviluppato'.

Gli eventi che negli anni scorsi hanno destato più scalpore e che sono finiti sotto i riflettori (Abu Ghraib, G8 di Genova, Guantanamo) sono solo la punta di un iceberg, ma soprattutto non sono casi eccezionali: la tortura è una pratica ordinaria, sistematica, quotidiana, non è il frutto di schegge impazzite, di mele marce, di malfunzionamenti accidentali degli apparati statali o del sistema. È parte integrante del sistema. 
La tortura - che non è da considerare un evento insolito, un fatto anomalo, isolato, compiuto da mostri o pazzi criminali - va colta infatti nella logica della sua produzione sociale (Gjergji 2019) in quanto elemento strutturale del sistema sociale. Vanno colte le radici sociali sistemiche della tortura come rapporto sociale di sottomissione, la cui portata va al di là delle persone direttamente colpite. La società e le circostanze la producono e la normalizzano: essa non sussiste in maniera isolata e casuale, essa è presente all'interno di determinati contesti, di determinati rapporti sociali, di particolari narrazioni, così che in ciascun contesto storico-politico-sociale-culturale è presente uno specifico potenziale di tortura che fa diventare alcuni individui o gruppi ritenuti ostili, nemici, dei soggetti torturabili (Jubany, Pasqualetto, Rué in questo volume).

Rispetto al passato le pratiche di tortura sono mutate, ma i significati e gli obiettivi sono rimasti grossomodo gli stessi. In quanto «distruzione deliberata della personalità e della dignità della vittima attraverso l'inflizione di gravi sofferenze fisiche o psichiche» (Lalatta Costerbosa 2016, 9), la tortura annulla e disumanizza la persona, ne distrugge la dignità e l'identità, la riduce ad oggetto. A «bestia umana», a «sotto-uomo», (Sartre 1958, 17). Nel suo spettacolo teatrale Morti senza tomba del 1946, Sartre dipinge le vittime di tortura come dei morti senza tomba, che hanno perso l'uso della parola e che sono spezzati dentro: persone vive fisicamente ma morte dentro, perché con la tortura, dirà nel saggio introduttivo al volume $L a$ question di Henri Alleg, «si lascerà vivere il corpo ma si ucciderà lo spirito» (Sartre 1958, 19).

Forma di violenza intenzionale estrema finalizzata alla sottomissione totale del soggetto, la tortura è inscritta in rapporti sociali di produzione, di classe, di razza, disuguali, è l'espressione di rapporti di potere diseguali che determinano l'umiliazione del soggetto, la sua demolizione fisica e psichica, per lasciarlo ammutolito e senza speranza nel presente e nel futuro. La tortura, infatti, non termina con la fine dei supplizi: essa produce traumi profondissimi sulla mente, sul cervello, sul corpo, che sono continuamente rivissuti e riaperti dal ricordo insistente e invadente del trauma stesso (van der Kolk 2015). La questione non riguarda soltanto la tortura e il trauma avvenuti nel passato, ma anche e soprattutto il ricordo continuo delle torture subite e il ritorno ossessionante del trauma, l'incessante irruzione del passato traumatico nel presente che compromette e paralizza il futuro. Nell'annientare l'umanità del soggetto, la tortura distrugge i progetti e le speranze di autonomia, di libertà, di solidarietà, sia del torturato sia di chi gli sta intorno, tanto del soggetto singolo quanto del soggetto collettivo.

Che si tratti di tortura giudiziaria (per castigare, per strappare informazioni) o che si tratti di tortura politica (per annichilire il nemico, l'oppositore politico, il ribelle), la tortura ha un'ampia portata 
sociale che va al di là di coloro che ne sono direttamente feriti: essa mira a limitare la libertà di pensiero e di azione del torturato e del suo entourage, a mettere in riga la vittima di tortura e la sua cerchia sociale, ad ammonire l'intera società e in particolare i gruppi considerati recalcitranti all'ordine sociale, ad avvertire chi si mette contro la classe dominante, chi infrange le regole dell'ordine costituito (Scott 1999). Modalità estrema di affermazione e di consolidamento del potere della classe dominante attraverso la produzione di terrore, di paura (Lalatta Costerbosa 2016), la tortura è spesso parte del processo di costituzione e di mantenimento del dominio sociale, dello sfruttamento materiale di una classe sociale su un'altra classe, dell'imposizione di una data visione del mondo da parte di un gruppo sociale dominante (una classe, una casta) su un altro gruppo qualificato come oppositore, delinquente, sovversivo, nemico, brigante. Praticata sui singoli individui, per distruggerne l'io, l'identità, la personalità, la tortura instilla terrore nell'entourage del torturato e nella società, per imporre il silenzio sociale, per fiaccare la resistenza sociale delle popolazioni e dei gruppi dominati, delle classi subalterne. E, sottolinea Butler (2009), parte della missione «civilizzatrice» perseguita dalle classi dominanti e messa in atto dalle istituzioni o dai soggetti che rappresentano il potere costituito; elemento integrante del dominio e del controllo sugli individui, sui gruppi, sulle classi, definiti e considerati pericolosi, devianti, criminali, banditi, sovversivi. Pratica normale e normalizzata rivolta contro i soggetti reputati nocivi, temibili, recalcitranti verso l'ordine economico e politico dominante, è una modalità di svilimento, di sottomissione, di inferiorizzazione, individuale e collettiva, tramite l'affermazione della superiorità del torturatore sul torturato - torturatore che non è, però, soltanto il singolo aguzzino che mette in pratica la tortura, è anche il soggettivo collettivo (una classe sociale, un gruppo dominante) che crea le condizioni favorevoli alla tortura e che direttamente o indirettamente ne arma la mano. Nella storia e nella geografia sono presenti moltissimi casi di tortura come risultato di un continuum di pratiche violente provenienti dalle istituzioni statali, da centri di potere, per sottomettere e dominare soggetti considerati e definiti minacciosi, riottosi; qui, per ragioni di spazio, mi limito a ricordare il caso dei Rom nell'Europa moderna e contemporanea, popolazione contro la quale le politiche discriminatorie e persecutorie hanno cinque secoli di vita. Questo accanimento, osserva Di Noia:

è stato parte integrante della lotta al vagabondaggio che il capitalismo nascente scatenò in Europa a partire dalla fine del XV secolo. Ma all'interno di questo secolare processo di proletarizzazione della forza-lavoro vi è indubbiamente un filone specificatamente antizigano [...] Costretti in schiavitù, deportati, marchiati a fuoco e mutilati, incatenati, massacrati, giustiziati, sterilizzati, privati 
dei propri figli, i Rom sono però sopravvissuti alla 'civiltà' europea fondendosi con il resto delle classi popolari o arroccandosi sempre più in attività economiche secondarie, continuamente erose dallo sviluppo capitalistico [...] Così, come per i contadini europei scacciati dalle campagne e ridotti al vagabondaggio, gli stati dell'Europa moderna hanno imposto ai Rom di scegliere tra la schiavitù salariata, la marginalizzazione sociale e lo sterminio. In altre parole, il capitalismo, distruggendone le tradizionali basi di esisten$z a$, ha chiuso loro la strada del passato e ha aperto, invece, la strada al declino e all'etnocidio. (Di Noia 2016, 24-7)

Un esempio di tortura e pratiche inumane ai danni degli immigrati come parte del sistema (e del sistema politico democratico) - tanto per fare un esempio legato all'oggetto del volume - è quello degli Stati Uniti, rispetto ai quali Moss, Parks e Lori dimostrano che i trattamenti degradanti riservati agli immigrati provenienti dalla Frontiera Sud e internati nei centri di detenzione sono il risultato di un sistema legale, di un corpus giuridico, di una giurisprudenza, fondati su una logica che unisce colonialismo, nazionalità e sovranità. Un sistema basato sulla whiteness come soggetto di diritto, i cui principi di base assumono che il soggetto legittimo sia il bianco, mentre la qualificazione dei non-bianchi come 'altri, alieni, estranei' (ossia inferiori) ne impedisce o ne limita sia l'ingresso sia la parità nella tutela legale. Tali associazioni, osservano gli autori, sono profondamente radicate nella coscienza culturale degli Stati Uniti e hanno influenzato negativamente la politica migratoria di qualsiasi amministrazione avvicendatasi nel tempo, tanto che la stessa Amministrazione Trump non ha inventato nulla, ha soltanto inasprito in maniera estrema una politica migratoria già di per sé punitiva e restrittiva e ha fatto di questo inasprimento un perno della politica nazionale. Il sistema di disumanizzazione, confinamento, punizione ed espulsione degli immigrati attualmente presente negli Stati Uniti non è un'invenzione dell'Amministrazione Trump, è frutto di un sistema giuridico dalle radici profonde. $\mathrm{Su}$ questa linea, Ouali, riferendosi al Belgio (ma la sua osservazione può essere estesa alla gran parte del mondo) sottolinea che il regime di violenza inflitto ai sans-papiers e ai loro figli all'interno dei centri di detenzione costituisce una forma di tortura, che i trattamenti inumani e degradanti che si verificano nei centri rappresentano delle forme specifiche di tortura proprie dei centri di detenzione. Eppure la normativa internazionale stabilisce il divieto assoluto di tortura e di trattamenti inumani o degradanti: ma allora come stanno le cose? 


\section{$2 \quad$ Un divieto assoluto a favore di un bene assoluto?}

L'art. 5 della Dichiarazione universale dei diritti umani del 1948 dichiara che «nessun individuo potrà essere sottoposto a tortura 0 a trattamento o a punizioni crudeli, inumani o degradanti». La Convenzione contro la tortura ed altre pene o trattamenti crudeli, inumani o degradanti del 1984, entrata in vigore nel 1987, stabilisce il divieto assoluto di tortura, senza eccezioni. L'art. 3 della Convenzione di Ginevra relativa al trattamento dei prigionieri di guerra vieta la tortura. L'art. 7 del Convenzione internazionale sui diritti civili e politici del 1966 dichiara che nessuno può essere sottoposto a tortura o trattamenti degradanti, neppure in caso di pericolo pubblico eccezionale che minacci l'esistenza della nazione (art. 4). L'art. 3 della Convenzione europea per la salvaguardia dei diritti dell'uomo e delle libertà fondamentali (CEDU) del 1950 stabilisce che «nessuno può essere sottoposto a tortura né a pene o trattamenti inumani o degradanti», neppure in caso di guerra o in caso di altro pericolo pubblico che minacci la vita della nazione (art. 15). L'art. 5 della Carta africana dei diritti dell'uomo e dei popoli del 1981 dichiara che «ogni individuo ha diritto al rispetto della dignità inerente alla persona umana e al riconoscimento della sua personalità giuridica. Qualsiasi forma di sfruttamento e di svilimento dell'uomo, specialmente la schiavitù, la tratta delle persone, la tortura fisica o morale, e le pene o i trattamenti crudeli, inumani o degradanti sono interdetti». L'art. 5 della Convenzione americana sui diritti umani del 1969 stabilisce che «nessuno deve essere sottoposto a tortura $o$ a pene $o$ trattamenti crudeli, inumani o degradanti. Tutti coloro che sono privati della libertà vengono trattati con il rispetto dovuto alla dignità inerente di persona umana».

Considerando la tortura nell'ambito del fenomeno migratorio, recentemente il Global Compact for Migration (GCM), approvato il 19 dicembre 2018, menziona la tortura nel capitolo relativo ai rientri e alle riammissioni (Perocco 2019a). Durante i negoziati per il GCM, l'Europa ha sottolineato la responsabilità dei Paesi di partenza in materia di ritorni e riammissioni, nonché l'obbligo legale per gli Stati di riprendersi i propri cittadini, facendo inserire questo punto nell'Objective 21 «Cooperate in facilitating safe and dignified return and readmission, as well as sustainable reintegration»; tuttavia, dato che le riammissioni e i rientri possono comportare il rischio di tortura, nell'Objective 21 è stato previsto che: «we commit to facilitate and cooperate for safe and dignified return and to guarantee due process, individual assessment and effective remedy, by upholding the prohibition of collective expulsion and of returning migrants when there is a real and foreseeable risk of death, torture, and other cruel, inhuman, and degrading treatment or punishment, or other irreparable harm, in accordance with our obligations under international human rights law» (United Nations 2018a). 
Nel Global Compact on Refugees (GCR), approvato il 17 dicembre 2018, la tortura e i trattamenti inumani hanno ampio spazio, si sollecita a considerare le vittime di tortura come beneficiari specifici delle politiche pubbliche per i rifugiati. Agli Stati è richiesta una maggiore capacità di affrontare le esigenze specifiche dei sopravvissuti alla tortura, vengono indicate disposizioni specifiche in materia di sostegno alle vittime. Nel capitolo «Reception» (section «Addressing Specific Needs»), il GCR prevede che: «the capacity to address specific needs is a particular challenge, requiring additional resources and targeted assistance. Persons with specific needs include: children, including those who are unaccompanied or separated; women at risk; survivors of torture, trauma, trafficking in persons, sexual and genderbased violence, sexual exploitation and abuse or harmful practices» (United Nations 2018b).

Inoltre esso sollecita gli Stati a prevedere «mechanisms for identification, screening and referral of those with specific needs to appropriate and accessible processes and procedures». Nel capitolo «Meeting needs and supporting communities» (section «Health»), il GCR chiede agli Stati «to contribute with resources and expertise to expand and enhance the quality of national health systems to facilitate access by refugees and host communities, including [...] survivors of trafficking in persons, torture, trauma or violence, sexual and gender-based violence» (United Nations 2018b). È da ricordare, inoltre, che nel febbraio 2018 il Comitato ONU contro la tortura (CAT) ha emanato delle nuove linee-guida sui diritti dei richiedenti asilo che ne rafforzano la protezione dalla tortura a seguito dei rimpatri (United Nations 2018c).

Come si vede il diritto internazionale - recepito dal diritto interno di molti Stati del mondo - considera la tortura un reato di gravità assoluta e specifica, stabilendo nei suoi confronti un divieto assoluto che non può essere sottoposto a deroghe, eccezioni, sospensioni. Il divieto di tortura è perentorio e inderogabile perché la tortura, oltre a danneggiare l'integrità fisica e psichica, annienta la dignità umana, la quale costituisce un bene assoluto, intangibile, inviolabile, alla base dell'umanità e dei diritti umani:

la dignità umana non è mai negoziabile nell'interesse dello Stato. La tortura non è mai ammissibile nel nome dello Stato. È vietata in ogni luogo e circostanza [...]. È il fondamento giuridico del diritto e dei diritti [...]. La protezione della dignità umana, essendo essa a fondamento di tutti i diritti umani, è prevalente rispetto a qualsivoglia esigenza di giustizia o di sicurezza. (Gonnella 2013, 26-7, 93)

Tale concezione della tortura come elemento assolutamente negativo (è da ricordare che fino a pochi secoli fa in Europa essa era parte del codice penale) è frutto di molteplici elementi e forze sociali. Tra 
questi c'è il movimento storico delle classi popolari, che, restando all'Occidente, nel corso dei secoli si sono scrollate di dosso con grande fatica e molti conflitti lo statuto di 'cosa', di oggetto senza diritti, imposto dalle classi dominanti e dal potere assoluto (imperiale, feudale, religioso, etc.). È stato un lungo processo storico, complicato, per nulla lineare, che nel tempo ha visto l'acquisizione graduale di diritti civili, politici, sociali, la conquista progressiva (ancorché incompleta) del diritto ad avere diritti; un processo in cui le classi popolari e la nascente borghesia urbana hanno posto le questioni dell'universalità della dignità umana, dell'uguaglianza, delle radici sociali (non naturali) della disuguaglianza. Tale acquisizione è avvenuta con le lotte politiche, il conflitto sociale, la resistenza quotidiana, non si è trattato di un conferimento spontaneo e gratuito da parte delle classi dominanti e degli apparati statali, anzi. Si pensi, ad esempio, all'imponente fenomeno della caccia alle streghe organizzato dagli Stati europei e dalle classi dominanti per spazzare via le credenze popolari, soprattutto quelle del mondo rurale, e imporre una nuova visione del mondo funzionale al capitalismo e alla società borghese nascenti (Levack 2008). O, altro esempio, alla feroce repressione nell'evo moderno delle jacqueries e dei movimenti di protesta sociale a sfondo più o meno religioso in quanto recalcitranti alla schiavitù salariata imposta dalla nascente economia capitalistica.

C'è stato poi l'anti-colonialismo, con i movimenti di liberazione dei popoli oppressi, che ha denunciato e combattuto lo schiavismo e il colonialismo, il razzismo e la tortura come elementi strutturali del sistema coloniale. Il sistema schiavistico di sfruttamento dei neri e degli indios prevedeva nella morte per sfinimento, si reggeva sulla violenza organizzata e sul terrore quotidiano, con la tortura ad elemento costitutivo di tale sistema - sia nella fase del commercio degli schiavi (slave economy) sia nella fase della produzione agricola nelle piantagioni (plantation economy). La tortura ha costituito un fattore strutturale del sistema schiavistico e del razzismo il quale sosteneva tale sistema; la tratta degli schiavi, la schiavitù degli indios e dei neri nelle colonie - che erano ritenuti bestie umane, sotto-uomini - equivaleva ad una lunga agonia quotidiana collettiva (Ferro 2003; Stannard 2001), ad un inferno di massa:

La vita di uno schiavo consisteva in una lunga tortura. Cominciava con la sua cattura da parte dei razziatori e finiva con la morte in una piantagione [...] Una volta sbarcati sulle isole e venduti come manodopera, gli schiavi andavano incontro a persecuzioni ancora peggiori, per il numero e per il grado. In precedenza, per quanto terribile, il trattamento loro riservato evitava le mutilazioni e la flagellazione nelle sue forme più diaboliche, per la buona e semplice ragione che uno schiavo storpio o con la schiena piena di cicatrici e di segni non rimarginabili non sarebbe costato quan- 
to uno schiavo sano. Ma quando un colono aveva acquistato uno schiavo, in molti casi non era mosso alla clemenza da simili motivi. Era più probabile che lo facesse lavorare fino allo sfinimento o alla morte e, nel frattempo, cercava di ricavare dal suo acquisto sino all'ultima briciola di lavoro, torturandolo e sottoponendolo a castighi. (Scott 1999, 143-5)

C'è inoltre il movimento di pensiero contro la tortura sviluppatosi nei secoli scorsi, soprattutto in Europa, che ha preparato il terreno alla sua abolizione (ancorché formale). È un movimento, sottolinea Lalatta Costerbosa (2016), che è iniziato nel Cinquecento con le riflessioni e le prese di posizione di Juan Luís Vives, Michel de Montaigne, Erasmo da Rotterdam, Tommaso Moro, i quali giudicano la tortura una pratica contro l'umanità e una pratica inutile dal punto di vista giudiziarioinvestigativo. Si è rafforzato nel Seicento, grazie soprattutto all'opera di Friedrich Spee von Langenfeld, e si è consolidato nel Settecento con i lavori di Christian Thomasius, Montesquieu, Cesare Beccaria, Wilhelm von Humboldt, Pietro Verri, Joseph von Sonnenfels, Gaetano Filangieri, i quali, nel gettare le basi giuridiche e filosofiche dell'abolizionismo, mettono in luce il duplice significato giudiziario della tortura (punizione, pena; modalità per estorcere informazioni e confessioni) e il duplice significato politico della tortura (assoggettamento e distruzione del nemico; modalità di governo attraverso la paura).

Infine, ma non meno importante, sono da considerare gli orrori della Seconda guerra mondiale, le atrocità del nazi-fascismo, i campi di concentramento, lo sterminio degli ebrei, dei Rom, degli oppositori politici e in generale degli Asozialen, ovvero quell'insieme di eventi del primo Novecento che rappresenteranno un punto di svolta fondamentale nella messa al bando della tortura. Eppure la tortura resta un fenomeno permanente, più vivo che mai. Come mai?

\section{I fattori di permanenza della tortura}

Nonostante le legislazioni internazionali, regionali, nazionali, nonostante la ricca tradizione giuridica e filosofica, nonostante l'abisso della Seconda guerra mondiale, oggi nel mondo la tortura è un fenomeno ampiamente diffuso, una pratica ordinaria e organizzata «la pratica della tortura è diffusissima, anche se gli Stati non lo ammettono. Secondo Amnesty International, su 192 Stati, per ben 132 è provato che la esercitano più o meno sistematicamente. Altri Stati lo fanno con tecniche più o meno raffinate per dissimulare gli effetti della tortura» (Cassese 2011, 143-4).

Nel 2015 erano 122 i Paesi in cui si è praticata la tortura (Noury 2016, 113-14), negli ultimi cinque anni sono stati censiti 144 Pae- 
si in cui è stata praticata la tortura, ${ }^{1}$ in pratica un fenomeno globale che interessa i quattro angoli del pianeta, che coinvolge la gran parte dei Paesi che hanno firmato le varie convenzioni in materia, e che ha fatto parlare di una crisi globale della tortura. Nel mondo sono più di cento le aziende produttrici di strumenti di tortura: una vera e propria industria, quanto mai florida, che - insieme all'altrettanto fiorente industria della sicurezza - produce una molteplicità di congegni elettrici, sostanze chimiche, spray, schiume, per il sistema della tortura. Sistema che opera in maniera accurata, precisa, scrupolosa (anche nell'auto-occultamento), che si avvale di diverse figure 'esperte' tra cui il personale medico. Spesso i medici sono un elemento permanente e centrale del sistema-tortura, come dimostrano in questo volume Bathia e Burnett, o per l'Italia le inchieste sulla caserma Bolzaneto (G8-2001) e il caso Cucchi. La tortura è «condotta in modo serio e rigoroso da 'professionisti' del settore [...] ci sono scuole internazionali per torturatori, ci sono medici che assistono alle sevizie con compiti precisi: identificare i punti deboli su cui si possono concentrare gli abusi, tenere sotto controllo la vittima affinché non muoia durante la tortura, far rinvenire la vittima svenuta, curare la vittima in modo che possa essere sottoposta a nuove sedute [...] il $60 \%$ delle persone entrate in cura presso il Centro per la riabilitazione delle vittima della tortura di Copenhagen è stato torturato in presenza di medici» (Scaglione 1999, 4-5).

Le pratiche di tortura non solo non sono casi rari, non solo sono strutturali, sono anche fenomeni strutturati e strutturanti.

Alcuni fattori, strettamente legati tra di loro, hanno contribuito a mantenere viva la tortura. Mi riferisco innanzitutto all'inarrestabile ascesa del pensiero sicuritario, della questione sicurezza, delle politiche di sicurezza, nell'ambito della globalizzazione dell'ideologia neo-liberista e delle politiche neo-liberiste (Bigo 1998, 2005; Wacquant 1999). Un'ascesa che ha costituito un processo pluridecennale, profondo, multiforme, giunto allo stadio dell'«ossessione della sicurezza» (Klinenberg 2001), e che è parte integrante del modello di gestione della società neo-liberista - contraddistinta dalla crescita strutturale delle disuguaglianze, dall'allargamento dell'esclusione sociale, dalla globalizzazione della polarizzazione sociale interna nei singoli Paesi, dalla demolizione del welfare state e dei diritti sociali (Perocco 2018a). Una società in cui quello della sicurezza - ma sarebbe meglio dire l'insicurezza sociale globale - è un elemento pervasivo della vita quotidiana, uno strumento di governo della società

1 Tanto che Amnesty International ormai parla di una vera e propria «crisi della tortura», stante la sua imperterrita diffusione e acutizzazione: https://www.amnesty. org/en/get-involved/stop-torture/ (2019-11-10). 
e di comunicazione. L'impoverimento e l'insicurezza sociale di massa prodotti negli ultimi decenni dalle classi dominanti e dagli Stati (Gallino 2012, 2015), cioè dall'alto, hanno generato un diffuso bisogno antisociale di sicurezza (armata) che viene presentata come la (falsa) soluzione alla produzione istituzionale di insicurezza sociale (vera). Attraverso una propaganda martellante e una potenza di fuoco senza precedenti, questo 'bisogno' è stato convogliato dall'alto verso il basso della società, contro i poveri, gli immigrati, la micro-criminalità (dimenticando la macro-criminalità che la controlla e la organizza), e si è concretizzato in una miriade di provvedimenti criminalizzanti di chi sta peggio nella (falsa) idea che l'insicurezza provenga dal basso. Utilizzando la formula 'è la gente che ce lo chiede perché si sente insicura', laddove è resa insicura dalla precarizzazione strutturale della vita sociale, questo regime di verità si è incarnato in un complesso di politiche sicuritarie nazionali e locali, prassi di controllo sociale, fenomeni di militarizzazione della vita quotidiana, che in qualche modo favoriscono le pratiche di tortura. Il paradigma della sicurezza, fondato sulla produzione istituzionale di insicurezza sociale e sulla costruzione sociale del problema pubblico della sicurezza, ha facilitato l'acquiescenza verso la tortura e i trattamenti inumani e degradanti.

Mi riferisco inoltre alle politiche della paura, al discorso e alla cultura della paura (Furedi 1997, 2018; Glassner 1999; Skoll 2010; Wodack 2015), la cui diffusione è stato un processo organizzato e sistematico, che ha sostenuto la produzione sociale di insicurezza e la securitarizzazione della società, con gli Stati e i professionisti della paura quali principali protagonisti (Bigo 1998). Insieme all'aumento della violenza, che pervade strutturalmente la vita della società contemporanea, la globalizzazione della paura ha sostenuto il mantenimento e la produzione di contesti e ambienti favorevoli alla tortura, ha alimentato la formazione di situazioni eccezionali, emergenziali (il terrorismo dell'emergenza), in cui prospera anche e proprio la tortura. La filosofia della paura, armatura ideologica delle politiche di sicurezza neoliberali, ha avuto un ruolo importante nel «restituire spazio pubblico alla pratica della tortura [...]. In questo rapporto dialettico tra paura, sicurezza e libertà ha trovato spazio la rileggitimazione della tortura, quale pratica investigativa e punitiva» (Gonnella 2013, 101-2).

Mi riferisco, poi, all'eclissi dello stato sociale e alla parallela ascesa dello stato penale (Wacquant 2002), all'interno di un profondo processo di trasformazione del ruolo e della forma dello stato avvenuto negli ultimi decenni. Col rafforzamento dei meccanismi di discriminazione, segregazione, esclusione, lo Stato ha avuto nell'era neo-liberista un ruolo di primo piano nella creazione di disuguaglianze, di disuguaglianze di Stato; in particolare ha costituito uno dei protagonisti principali - insieme al mercato e ai mass-media - del dupli- 
ce processo di produzione sociale dell'esclusione e criminalizzazione dell'esclusione socialmente prodotta. Wacquant (2009) ha sottolineato lo stretto rapporto tra violenza di Stato e ascesa dello Stato penale nel contesto della crescente criminalizzazione dei poveri, cosicché per coloro che vivono ai margini della società il pericolo di essere sottoposti a tortura è persistente e fa parte dell'apparato di controllo sulla loro realtà quotidiana. La trasformazione dello Stato da welfare a warfare via workfare, insieme alle derive autoritarie e ai processi di fascistizzazione che interessano lo Stato e la società di diversi Paesi del mondo, ha facilitato la presenza di climi permeabili, disponibili, aperti alla tortura (giudiziaria e politica), come emerge anche da diversi saggi contenuti in questo volume.

Un altro fattore da tener presente è l'insieme di teorie (filosofiche, politiche, giuridiche) e di discorsi pubblici che, in nome della stato di necessità, dell'eccezione, dell'emergenza, della sicurezza nazionale, della difesa della democrazia e della libertà, giustificano la tortura derogando ai principi fondamentali, ai diritti umani, allo stato di diritto. Tali teorie, affermatesi soprattutto negli Stati Uniti dopo l'11 settembre, e corrive alle legislazioni anti-terrorismo, affermano l'ammissibilità della tortura nell'interesse dello Stato in casi eccezionali. Per Dershowitz (2003, 2011), che in linea generale condanna la tortura, essa può essere utilizzata in situazioni particolari, circoscritte, eccezionali, mediante una "tortura leggera», che usa una certa «pressione fisica» (salvo che, sostiene l'autore, le dichiarazioni/confessioni rilasciate sotto tortura debbono essere suffragate da prove concrete altrimenti da sole hanno scarso valore). Il quale afferma che, poiché la tortura è diffusa in tutto il mondo nonostante i divieti e le Convezioni, si dovrebbe codificarne e regolamentarne l'uso, facendola diventare una pratica che avviene alla luce del sole, a tutela sia del torturato onde evitare che sia soggetto ad arbitrio (cioè ad una tortura 'inumana', non regolamentata) sia delle figure incaricate a torturare. Tali riflessioni, fatte proprie da alcuni Stati per giustificare le pratiche di tortura, aprono dei problemi enormi, tra cui le questioni relative all'ammissibilità dello stato di necessità come fondamento giuridico della liceità della tortura e all'individuazione di una 'tortura leggera', 'umana', che provochi poca sofferenza e causi pochissimi danni. Quanto al primo punto, il ricorso al concetto di 'stato di necessità' rende relativo un divieto che invece è (dovrebbe essere) assoluto e fa sì che la definizione della situazione su cui decidere (se ricorrere o no alla tortura) sia basata su elementi soggettivi; a questo riguardo Cassese osserva che lo stato di necessità «è una piccola porta attraverso la quale può passare ogni arbitrio [...] opera come circostanza che elimina la responsabilità del torturatore» (2011, 145-7). Quanto al secondo punto, il dibattito sulla giusta sofferenza da infliggere al 
torturato è semplicemente ridicolo, perché da un lato il concetto di 'tortura umana' è inconcepibile (essendo la tortura disumana per definizione e un divieto assoluto) e dall'altro lato (qualora si decidesse di far diventare relativo il divieto) è praticamente impossibile (oltreché impensabile) stabilire in maniera oggettiva una soglia della sofferenza: quale sarebbe una giusta sofferenza? Chi e come lo decide? In base a quali criteri ed elementi? Così come sono ridicoli, grotteschi, i vari escamotage e camuffamenti linguistici che sono stati adottati in giro per il mondo per umanizzare, normalizzare, con linguaggio tecnico-burocratico, la tortura: «interrogatori coercitivi», «interrogatori di salvezza», «interrogatorio coattivo e forzoso», «cooperazione violentemente imposta per la salvezza di vite umane» (Lalatta Costerbosa 2016).

Per Jacobs, si dovrebbe distinguere, all'interno di un unico e comune sistema di diritto penale, un «diritto penale del cittadino» (Bürgerstrafrecht) da un «diritto penale del nemico» (Feindstrafrecht), in cui il nemico è colui che attenta all'ordine costituito e che per tale ragione dovrebbe godere di un riconoscimento limitato, condizionato, dei diritti umani fondamentali. A causa della sua attitudine e della sua condotta il nemico dovrebbe essere estromesso «dal Diritto, non prestando pertanto la garanzia cognitiva minima necessaria per il trattamento alla stregua di persona [...]. Nella misura in cui gli vengono tolti dei diritti, egli non viene trattato come persona in diritto» (2007a, 17; 2007b, 118). Questa compressione delle garanzie sostanziali e processuali dello stato di diritto dovrebbe sostanziarsi in un diritto penale speciale che punisce anche comportamenti potenzialmente pericolosi o lontani nel tempo («il trattamento del nemico, che è immediatamente bloccato già nello stadio precedente e che si combatte in quanto pericoloso» [2007a, 19]), che prevede delle pene differenti rispetto al cittadino e che consente la 'tortura legale' nel caso in cui più vite umane siano in pericolo; la tortura è intesa come strumento da usare nei confronti di soggetti «rispetto ai quali non è più vigente la presunzione di comportamento fedele al diritto» $(2007 \mathrm{~b}, 125)$. Tale negazione dell'universalismo dei diritti fondamentali, però, avverte Jacobs, dovrebbe essere applicata raramente e temporaneamente, per casi eccezionali, in uno spettro più ampio del diritto di polizia.

Ora, queste teorie, che fanno parte dell'armatura ideologica della guerra infinita, dell'esportazione della democrazia a suon di cannonate, della war on terror, sono la componente filosofico-giuridica del bagaglio ideologico della globalizzazione neo-liberista a regime d'accumulazione finanziaria, del rilancio dell'aggressione neo-coloniale verso i Paesi del Sud del mondo per la nuova ripartizione del mercato mondiale. Rimasticate e divulgate da gazzettieri e opinionisti vari, queste teorie hanno facilitato la creazione di ambiti e di climi favorevoli alla tortura. Diversi saggi contenuti in questo volume evi- 
denziano come l'eccezionalismo e l'arbitrio, che sono elementi strutturalmente caratteristici dell'immigrazione, si combinino con l'eccezionalismo e l'arbitrio del diritto speciale del nemico. ${ }^{2}$

Mi riferisco, infine, alle pessime condizioni di vita e di detenzione nelle carceri di gran parte del mondo. Nonostante alcuni e timidi passi in avanti avvenuti negli ultimi decenni, non c'è stato un reale e concreto processo di apertura della società verso il carcere e di apertura del carcere verso la società, così come non c'è stata un vera svolta nelle condizioni nelle carceri e delle carceri. Anzi, spesso c'è stata una ricomposizione del sistema carcerario intorno ad una visione carcero-centrica, intorno ad una prospettiva ultra-sicuritaria e meramente punitiva della carcerazione, che si è tradotta in diversi Paesi in un duplice fenomeno di ipercarcerazione (Wacquant 2013) e di aggravamento della condizione carceraria (sovraffollamento, degrado edilizio e degli ambienti di vita, peggioramento della qualità della vita) testimoniata per il contesto italiano anche dall'aumento dei suicidi in carcere (Antigone 2019). Facendo riferimento al Brasile, dove il fenomeno dello 'tsunami carcerario' è uno "strumento di controllo sociale in un Paese che presenta uno dei peggiori indici di disuguaglianza al mondo», Quintanila e Villen evidenziano che il «sistema che struttura l'incarcerazione di massa in Brasile costituisce il terreno più fertile per le pratiche di tortura» e che in questo contesto gli immigrati sono «tra le vittime principali di questo processo, principalmente le donne non brasiliane e nere, accusate di essere mulas del traffico internazionale di droga», intanto che «la combinazione imprigionamento/espulsione [è] un fattore aggiuntivo di esposizione alla tortura».

Come fattori di permanenza delle pratiche di tortura sono poi da considerare da un lato la guerra agli immigrati e dall'altro lato l'acutizzazione del razzismo che nell'ultimo decennio hanno interessato diversi contesti del mondo. Questi due elementi sono oggetto di discussione del prossimo paragrafo.

\section{Guerra agli immigrati e tortura}

Il rapporto tortura/migrazioni ha interessato e attraversato i secoli scorsi, non è certo una novità di oggi: la tratta dei neri, ad esempio, è un fatto storico di grande ampiezza e di terribili sofferenze. Ma non si può non sottolineare che oggi il binomio tortura/migrazioni è più vivo che mai, seppur in forme diverse rispetto al passato. Questa per-

2 I saggi di Carpinetti e di Quintanilha e Villen mettono in luce, però, come negli anni Settanta e Ottanta in Argentina e in Brasile il diritto penale del nemico fosse già una realtà consolidata ben prima di tali teorizzazioni, così come lo era in Italia (Gonnella 2013, 90). 
sistenza è dovuta a diversi motivi, tra cui i caratteri strutturali delle condizioni della migrazione e delle politiche migratorie nell'ambito del capitalismo neo-liberista.

Se il sistema di sfruttamento dell'immigrazione in tale contesto ha favorito la formazione negli ultimi decenni di situazioni, condizioni, ambienti e climi favorevoli alla tortura nei confronti degli immigrati, la fase di guerra agli emigranti e agli immigrati apertasi negli ultimi dieci anni ha alimentato, moltiplicato e peggiorato tali situazioni. Questa fase, che possiamo definire 'trumpiana' nonostante inizi prima di Trump, è il risultato della maturazione dopo il 2008 di tendenze ed elementi già presenti nel decennio scorso: la globalizzazione di politiche migratorie restrittive, selettive e repressive; la dilatazione e l'inasprimento della propaganda anti-immigrati; la generalizzazione delle discriminazioni istituzionali con gli Stati di mezzo mondo che fanno a gara nel peggiorare le condizioni della migrazione e le condizioni di vita degli immigrati. Militarizzazione delle politiche migratorie, securitarizzazione dei confini, esternalizzazione delle frontiere, nuovi dispositivi di deterrenza, illegalizzazione delle migrazioni, clandestinizzazione (anche sociale) degli immigrati, concezione della migrazione come crimine, ${ }^{3}$ allargamento dell'economia sommersa e del grave sfruttamento lavorativo, appalto dei movimenti migratori alle organizzazioni criminali, incarcerazione nei centri di detenzione come prassi ordinaria di 'accoglienza', privazione della libertà, respingimenti, riammissioni (legali e illegali), rappresentazione degli immigrati come invasori, scrocconi e delinquenti, pandemia della sicurezza nazionale: sono tutti elementi che hanno favorito la formazione di un terreno ricettivo e permeabile alle pratiche di tortura e ai trattamenti inumani e degradanti (da parte di pubblici ufficiali e non ${ }^{4}$ ). I saggi di questo volume mettono in luce che dal Regno Unito ai Balcani, dal Brasile all'Italia, dalla Spagna a Israele, dagli Stati Uniti al Marocco, la guerra agli emigranti e agli immigrati ha creato ovunque condizioni e contesti permeabili a pratiche di tortura, trattamenti inumani e degradanti, ${ }^{5}$ così che il fenomeno della tortura degli immigrati si presenta come un fenomeno globale - che Pau Pérez-Sales analizza in maniera sistematica individuandone i vari aspetti e le diverse problematiche.

Considerando il contesto europeo, la guerra dell'Europa agli emigranti del Sud del mondo è la guerra contro i poveri dei continenti di colore, resi poveri dal colonialismo di ieri e dal neo-colonialismo di oggi.

3 Palidda 2000.

4 Lo ha sottolineato anche il relatore speciale delle Nazioni Unite sulla Tortura, secondo il quale le politiche migratorie repressive possono essere causa di torture e trattamenti degradanti (United Nations, HRC 2018).

5 Ad esempio i saggi di Brisciana, Guarnieri Jaradat, Ouenniche e Saaid. 
Guerra a cui partecipano, con modalità differenti, i singoli Paesi europei, il super-stato Unione Europea, ampi settori dei mass-media, numerosi partiti politici, organismi sovra-statali o semi-statali. Attraverso una miriade di regolamenti, protocolli, accordi, circolari, leggi, memorandum e trattati (pubblici e segreti), all'accordo di Schengen è stato dato un volto oltremodo ringhioso, fissando i tratti e gli strumenti di una politica migratoria effettivamente restrittiva del movimento migratorio (specie nei confronti degli immigrati 'economici'). Il percorso è iniziato nel 2006 con il Processo di Rabat per arrivare all'odierna chiusura semi-totale attraverso il Processo di Khartoum (2014), gli accordi di Malta (2015), il trattato con la Turchia (2016), il memorandum Italia-Libia (2017) e successivi patti particolari. Il rafforzamento dei confini, la chiusura rigidissima, la militarizzazione delle frontiere, lo spostamento delle frontiere nei Paesi di partenza o di transito, l'esternalizzazione dei controlli e delle frontiere in Africa, i campi di detenzione per emigranti nei Paesi di origine o di transito, i respingimenti individuali e collettivi in mare e via terra, la privatizzazione del diritto internazionale e della 'governance' delle migrazioni, ha prodotto un deciso peggioramento del percorso migratorio e delle condizioni della migrazione, nonché l'ulteriore illegalizzazione degli immigrati. Questo processo, schiettamente autoritario, ha inciso sul rapporto tra gli Stati e le popolazioni immigrate, tra i soggetti operanti nel settore dell'immigrazione e le popolazioni immigrate «legittimando trattamenti tipici di relazioni schiavistiche o di sudditanza, e non certo rapporti di cittadinanza» (Basso, Perocco, in corso di stampa).

Alla guerra contro gli emigranti i mass-media hanno dato un contributo enorme. In maniera ossessiva e martellante li hanno rappresentati come invasori provenienti da mondi retrogradi e malati, locuste voraci del 'nostro' welfare, fannulloni dotati dei più bassi istinti animali, importatori di criminalità, fissando un immaginario totalmente negativo che ha alimentato nei loro confronti avversione e rifiuto. I mass-media hanno creato un immaginario di forte e profonda estraneità tanto che nel linguaggio comune si è radicato il concetto dell'incontro con 'l'altro', ${ }^{6}$ hanno incoraggiato politiche ultra-restrittive e punitive preparando il terreno a violenze, aggressioni, omicidi, torture.

Alla guerra agli emigranti hanno partecipato anche numerosi partiti, i quali, in nome della difesa degli interessi nazionali, del lavoro degli autoctoni, della cultura locale, hanno derubricato l'immigrazione ad una questione militare e di sicurezza nazionale da affidare alle marine militari e alle polizie di frontiera, hanno vellicato la massa delle popolazioni autoctone con la convinzione di occupare una posizione privilegiata nella scala sociale e di godere di un trattamento privilegiato da parte dello Stato, hanno sollecitato le popolazioni 
locali a tenere la 'giusta distanza' e a vigilare attentamente sui rifugiati. Non è mancato il contributo di intellettuali e studiosi, i quali hanno contribuito alla legittimazione o alla costruzione del discorso pubblico sugli emigranti come radicalmente diversi (il fantomatico 'altro'), impermeabili all'integrazione, da tenere fuori dall'immacolato territorio europeo o da isolare nell'emarginazione.

Neppure le popolazioni immigrate residenti da tempo in Europa sono rimaste al di fuori di questo attacco. Un diluvio di leggi, circolari, regolamenti, provvedimenti delle autorità nazionali e locali, le ha penalizzate nei più vari ambiti della vita sociale (soggiorno, residenza, lavoro, sanità, accesso ai servizi, scuola, abitazione, autonomia privata), limitandone i diritti e precarizzandone la condizione lavorativa e sociale in un contesto di peggioramento generale delle condizioni delle classi popolari. Le politiche di integrazione sono rimaste ad un livello sempre più simbolico, mentre nei fatti sono prevalse, sul piano nazionale e/o locale, politiche di esclusione, pratiche e processi di inferiorizzazione e di marginalizzazione.

Contemporaneamente il discorso pubblico ha perso ogni freno nel raffigurare le popolazioni immigrate come una minaccia globale, un pericolo epocale da cui difendersi sotto ogni aspetto: il lavoro, la casa, l'andamento scolastico, il welfare, la vivibilità dei quartieri, la salute pubblica, la sicurezza individuale e nazionale, le comunità locali, il patrimonio genetico, la cultura, l'identità (nazionale, religiosa), i valori occidentali. In questa guerra di parole, la gran parte dei mass-media (o meglio: l'industria mediatica del disprezzo) ha coniato molteplici stereotipi inferiorizzanti costruiti ad hoc per stigmatizzare volta a volta gli immigrati arabo-musulmani, cinesi, africani, albanesi, polacchi, romeni, i Rom, le donne immigrate, le seconde generazioni, gli alunni stranieri, i quartieri ad alta concentrazione di immigrati, legittimando ogni forma di sopruso. Le ricadute di tale discorso si sono abbattute sull'intero mondo dell'immigrazione, ma anche sull'intera classe lavoratrice perché si incitano autoctoni e immigrati alla competizione più sfrenata e alla reciproca ostilità.

Questa furia anti-immigrati ha voluto e vuole impedire o rallentare il processo di radicamento sociale delle popolazioni immigrate, poiché esso rende meno docile, meno flessibile, meno disponibile la forza-lavoro straniera. La stabilizzazione e l'inserimento sociale aumentano il valore sociale delle popolazioni immigrate, accrescono le aspettative e la resistenza delle lavoratrici e dei lavoratori immigrati, i quali vogliono veder riconosciuta la propria dignità e il proprio diritto ad un trattamento da esseri umani a tutti gli effetti, non da strumenti animati di lavoro. Per tali ragioni governi, istituzioni internazionali come il FMI, imprese, puntano sempre più sulle migrazioni tempora- 
nee e circolari (Perocco 2018b), ${ }^{7}$ sull'impiego, cioè, di lavoratori temporanei, senza famiglia, con pochi legami con la società di arrivo e scarse conoscenze del territorio, che auto-comprimano i propri bisogni di socialità dando per scontato il carattere provvisorio della propria presenza, ma siano in possesso della risorsa più preziosa (per le imprese e i Paesi di 'accoglienza'): la disponibilità assoluta della propria capacità di lavoro per un tempo delimitato. In questo sforzo di allargare al massimo le migrazioni circolari e ridurre quelle definitive, limitandole - se possibile - solo alle persone dotate di alte qualifiche professionali, si è diffusa la falsa idea secondo cui le migrazioni circolari sarebbero vantaggiose allo stesso modo per i Paesi d'arrivo, i Paesi di partenza e gli immigrati. Sul piano teorico questo processo di ampliamento delle migrazioni temporanee poggia sul paradigma impalpabile e fluido della mobilità, che viene incaricato di sostituire quello assai più concreto e solido di 'immigrazione'. Gjergji (2016) ha evidenziato da un lato che la combinazione tra migrazioni circolari e accordi bilaterali implica un 'modello migratorio' in cui i lavoratori immigrati - rigorosamente selezionati - si muovono seguendo i ritmi dei cicli di produzione e le fluttuazioni di breve periodo del mercato del lavoro, e dall'altro lato che le politiche a sostegno delle migrazioni temporanee oltrepassano le tradizionali politiche migratorie dei Paesi europei e dell'Unione Europea ma allo stesso tempo integrano le politiche restrittive e repressive degli Stati europei istituzionalizzando la precarietà dei lavoratori immigrati e facendo della migrazione temporanea l'unico canale di ingresso regolare.

Questo molteplice processo di precarizzazione, irregolarizzazione e atomizzazione delle migrazioni ha intensificato la mercificazione della forza-lavoro straniera, ha estremizzato la naturale condizione di merce che ha la forza-lavoro nell'economia capitalistica, avvicinandola verosimilmente alla condizione di strumento di lavoro animato di lavoro, allo statuto di cosa; sennonché questo processo di mercificazione estrema della forza-lavoro straniera è un ulteriore elemento che ha favorito la formazione di contesti e ambienti disponibili a pratiche degradanti.

Non è un mistero e non è una esagerazione affermare che le politiche migratorie degli Stati europei e dell'Unione europea contribuiscono ad alimentare - direttamente o indirettamente - la tortura ai danni degli immigrati. In generale mi riferisco, come detto in precedenza, a quel complesso di elementi che generano, favoriscono o sostengono la formazione di condizioni permeabili alla tortura: la militarizzazione delle politiche migratorie, la criminalizzazione delle migrazioni, la clandestinizzazione degli immigrati (resi 'clandestini'), la

7 Stagionali, a contratto, spontanee o regolate. 
privatizzazione della governance delle migrazioni, la delega dei movimenti migratori alle organizzazioni criminali. In particolare mi riferisco, ad esempio, alla delocalizzazione delle frontiere europee in Africa, che ha prodotto la creazione di campi di detenzione nei Paesi di partenza e di transito (specie nei Paesi nord-africani, ma non solo) e che è avvenuta all'interno di un processo d'estensione (all'Africa, alla Turchia, ai Paesi dell'Europa del sud-est) dell'hotspot approach dell’UE inaugurato nel 2015. La diffusione dei campi di detenzione in diversi Paesi africani (soprattutto, anche se non solo, in quelli costieri) li ha fatti diventare dei grandi hotspot a cielo aperto. In Libia, i campi di detenzione sono gestiti da forze di polizia libiche, da militari libici, dalla guardia costiera libica, da milizie libiche, ma sono stati voluti e sono organizzati dai Paesi europei. Nel centri libici gli aguzzini sono persone del posto (o a volte sono degli emigranti), ma l'Europa ha dato un contributo fondamentale alla formazione del contesto (le forze di polizia libiche, che vendono i detenuti alle organizzazioni criminali i quali in alcuni casi sono stati ridotti in schiavitù, sono addestrate e rifornite dai Paesi europei, in primis dall'Italia ${ }^{8}$ ).

Il triplice processo di precarizzazione, militarizzazione ed esternalizzazione delle politiche migratorie si è basato prevalentemente sulla stipula di accordi, più o meno segreti, finalizzati al controllo e al contenimento dei movimenti migratori, all'ammissione selettiva, all'espulsione, alla riammissione e al rimpatrio forzato o semi-forzato degli emigranti. In questo ambito, il memorandum d'intesa ItaliaLibia, mai passato dal Parlamento, ha subappaltato alla guardia costiera libica e alle milizie locali una serie di funzioni di controllo e gestione (tra cui la delega alla Libia dell'attività di respingimento in mare) con cui sono stati aggirati la Convenzione di Ginevra sullo status dei rifugiati e il principio di non-refoulement. A questo proposito, Algostino, nel sottolineare che il divieto di tortura «cede a fronte delle politiche di chiusura ed esternalizzazione delle frontiere», avverte che gli accordi di riammissione, sebbene contengano dei richiami formali alla tutela dei diritti fondamentali, quando sono «conclusi con Stati come la Libia, violano il divieto di tortura e di trattamenti inumani o degradanti infrangendo il principio di non-refoulement». Tali violazioni possono avvenire anche in caso di rimpatrio indiretto o «quando si demandano a Stati terzi controlli, identificazioni, trattenimenti [...] gli accordi di riammissione, nella loro ansia di facilitare i rimpatri, possono condurre all'introduzione anche nei Paesi dai quali vengono effettuati i rimpatri, di modalità di identificazione,

8 Tanto che il 14 novembre 2017 l'Alto commissario dell'ONU per i diritti umani ha definito «disumana» la collaborazione tra l'UE e la guardia costiera libica: https:// news.un.org/en/story/2017/11/636022-libyas-detention-migrants-outrage-humanity-says-un-human-rights-chief-zeid (2019-11-29). 
trattenimento e rimpatrio che integrano trattamenti inumani o degradanti». Le violazioni avvengono anche con la politica di chiusura dei porti dato che «le condizioni a bordo delle navi condannate a stare in mare per giorni configurano trattamenti inumani o degradanti, di cui è responsabile chi dispone la chiusura dei porti [...]. Impedire l'ingresso nelle acque territoriali viola il divieto di tortura, quantomeno come divieto di trattamento inumano o degradante, sia in relazione alle condizioni del forzato soggiorno, per non dire tout court 'detenzione', sulle navi, sia in relazione alla violazione del dovere di soccorso» (Algostino in questo volume).

Per l'autrice si tratta di una vera e propria delocalizzazione ed esternalizzazione della tortura di stato, che avviene all'interno del processo di trasformazione dello Stato in quale risulta segnato sempre di più da una progressiva involuzione autoritaria.

Jubany, Pasqualetto e Rué sottolineano come i richiedenti asilo, in particolare quelli che sono stati vittime di tortura, non solo subiscano l'inefficienza dei sistemi d'asilo, ma siano anche potenziali vittime della tortura prodotta dall'uso diretto della violenza nell'applicazione di politiche migratorie particolarmente punitive. In un contesto di crescente criminalizzazione delle migrazioni, le violenze dei pubblici ufficiali nell'applicazione delle politiche migratorie vengono considerate come normali, come un risultato dell'uso legittimo della forza a tutela della sovranità nazionale. Anche coloro che hanno ottenuto qualche tipo di protezione internazionale sono comunque soggetti vulnerabili stante il rafforzamento della securitarizzazione delle frontiere. Anche coloro che hanno ottenuto qualche tipo di protezione internazionale sono comunque soggetti vulnerabili a causa del rafforzamento della securitarizzazione delle frontiere, in cui la detenzione e la deportazione sono una costante, esponendoli pertanto a violenze e trattamenti degradanti. Jubany, Pasqualetto e Rué (in questo volume) sottolineano che queste pratiche favoriscono «the recurrence of torture in modern liberal societies in its contemporary forms and its position in mechanisms of control of the socially excluded, among which policies and practices of migration control play a central role». Al contempo osservano che il mancato riconoscimento di queste violenze come violazione del divieto di tortura «contributes to their normalisation and legitimates the exercise of force in the enforcement of migratory policies, targeting undocumented migrants and contravening the protection that the right to claim asylum entails. Western democracies claim to offer international protection against torture to asylum seekers is contradicted by their own practices of migration management».

Anche dall'altro lato dell'Europa, nei Balcani, alle frontiere di Bosnia, Serbia, Croazia, Slovenia, la decentralizzazione dei confini dell'UE lungo questi Paesi e un approccio punitivo verso gli emigranti sono sfociati, evidenzia anche Cucchi, in violenze, trattamenti degradanti, condizioni di vita a dir poco terribili. 
Su questa linea, Caloz-Tschopp mette in luce che la scomparsa è una caratteristica delle politiche migratorie contemporanee e che la tortura rientra nella politica d'annichilimento che è tipica della società capitalistica di cui le politiche della scomparsa sono un paradigma. Le scomparse e le politiche della scomparsa, storicamente e strutturalmente presenti nelle colonie, nelle conquiste imperialistiche, nelle guerre, nelle dittature (ad esempio quelle sud-americane, che hanno prodotto il fenomeno appunto dei desaparecidos), oggi sono visibili nelle migrazioni e nelle politiche migratorie. Le vicende della città di Juarez $\left(\right.$ Messico $\left.{ }^{9}\right)$, i morti nel Mediterraneo, ${ }^{10}$ nei deserti, sono buchi neri in cui sono caduti gli scomparsi delle migrazioni, in cui, scrive l'autrice, «le néant, est le visage ultime de la torture» (il nulla, è l'ultimo volto della tortura); oggi la tortura è riconoscibile nei morti in mare, nei campi (senza andare troppo lontano, quello di Moria a Lesbo, o quello di Vucjak in Bosnia), alle frontiere, nei femminicidi e nelle violenze sulle donne emigranti, nelle sparizioni. Se nel periodo della tratta dei neri le torture avvenivano sulle navi, nelle piantagioni, ed erano un elemento strutturale della dominio e della violenza coloniale, adesso la tortura si riscontra nei centri di detenzione, nelle aree di partenza, nelle zone di confine e di transito, nelle aree di arrivo, ed è parte integrante delle dinamiche migratorie e delle politiche migratorie contemporanee. La tortura e la scomparsa però, ricorda l'autrice, sono anche elementi intrinseci al dominio capitalistico globalizzato, il quale cerca di appropriarsi dello Stato e a cui richiede la servitù degli immigrati. Le scomparse e le politiche della scomparsa possono essere considerate come il paradigma del capitalismo contemporaneo, in cui la violenza strutturale dello Stato è inflitta agli immigrati per reati amministrativi legati al divieto di partenza, d'ingresso, di permanenza. Di più: la tortura e le politiche di tortura non sono soltanto legate alle politiche migratorie e alle politiche della sicurezza, ma anche alle politiche del lavoro, all'economia politica.

Emerge, dunque, come le corresponsabilità dei Paesi europei siano fortissime e dirette. In questo senso sono da criticare gli aguzzini libici e le loro autorità (senza far di tutta l'erba un fascio, demonizzando l'intera popolazione libica, la quale, sulla scia delle vecchie

9 Paese che alla frontiera meridionale negli ultimi anni ha visto un'intensificazione dei sequestri, della violenza e della tortura nei confronti degli emigranti (provenienti soprattutto da El Salvador, Guatemala, Honduras). Cf. Médecins sans frontières, Aumentan los secuestros y la violencia extrema hacia migrantes en la frontera sur de México, https://www.msf.es/actualidad/mexico/aumentan-los-secuestros-y-la-violencia-extrema-migrantes-la-frontera-sur-mexico (2019-11-06).

10 Da segnalare che il 24 ottobre 2019 il Parlamento Europeo ha votato contro una mozione di risoluzione volta a favorire i salvataggi nel Mediterraneo (SAR) da parte delle ONG: http://www.europarl.europa.eu/doceo/document/B-9-2019-0154_EN.html (2019-11-11). 
rappresentazioni coloniali, è dipinta ancora oggi come una popolazione di orchi, cannibali, lupi), così come sono da criticare le controparti europee.

Tutto ciò ha degli effetti anche nei Paesi europei poiché la guerra agli emigranti e agli immigrati determina l'estensione dell'hotspot approach a tutta l'immigrazione presente in Europa. In una sorta di effetto alone, il sospetto, il controllo occhiuto, l'eccezionalismo, calano su tutti gli immigrati, aumentando il rischio di violenze e maltrattamenti nei loro confronti. La detenzione in quanto immigrati, la globalizzazione della detenzione amministrativa come modello di gestione del dissenso e di controllo delle classi popolari, la privazione della libertà come risposta automatica alla migrazione 'irregolare', le espulsioni e i rimpatri forzati (nel proprio Paese o in altri Paesi), la creazione di contesti istituzionali, politici, amministrativi e culturali schiettamente ostili verso gli immigrati, sono tutti elementi che hanno facilitato la tortura, le angherie, i soprusi, le violenze.

Tale situazione non è frutto di irrazionalità o di limiti conoscitivi: questo sistema di trattamento dell'immigrazione mette a disposizione del mercato del lavoro una massa di lavoratori ultra-ricattabili, impauriti, che dopo essere passati sotto le forche caudine sono disposti ad accettare le più misere condizioni. Esso implica e determina la selezione (sociale, professionale, politica) degli immigrati e la loro socializzazione allo sfruttamento: nel percorso migratorio essi vivono condizioni ed esperienze infernali, che li prepara e li socializza all'inferiorità sociale, a ciò che li aspetta nei Paesi di arrivo, ossia l'integrazione nel sotto-proletariato o nello scalino più basso della classe operaia. Ecco emergere dunque la radice della produzione sociale di tortura contro gli immigrati, ecco apparire la 'funzione' della tortura nei confronti delle popolazioni immigrate: essa è un modo per inferiorizzare l'immigrazione, per fiaccarne la forza, la resistenza; è una modalità della dinamica di svalorizzazione dei lavoratori e delle lavoratrici immigrate all'interno del processo globale di svalorizzazione del lavoro.

In tale pedagogia dell'instabilità, la guerra all'immigrazione 'educa' gli immigrati alla precarietà permanente, ma 'educa' anche le popolazioni locali all'ostilità, al disprezzo; questo trattamento abbrutisce in prima istanza gli immigrati, e di riflesso le popolazioni autoctone che si intossicano di razzismo.

\subsection{Ascesa del razzismo e tortura}

Questa nuova fase (che ha luogo in uno stadio iper-liberista del ciclo neo-liberista) è caratterizzata anche da una violenta ripresa del razzismo, il quale non è certo frutto dell'ignoranza o della paura dello 
straniero. Il razzismo produce, legittima e conserva la disuguaglianza: il razzismo è disuguaglianza.

Basso (2000) ha messo in luce che il razzismo rimanda ad un rapporto materiale di sfruttamento tra razze, ad un rapporto sociale di dominazione e sfruttamento di una «razza signora» su altre «razze di colore» che comprende un momento ideologico che naturalizza, giustifica e legittima lo sfruttamento e la dominazione (un rapporto che s'intreccia con la dominazione di classe e l'oppressione di genere). Figlio dello schiavismo, supporto ideologico del sistema coloniale, coevo e organico al capitalismo, il razzismo inferiorizza idealmente chi è già in una condizione materiale di inferiorità e sfruttamento. La bestializzazione fisica, psichica e morale del nero, la disumanizzazione del colonizzato, la dichiarazione di inferiorità naturale dei popoli di colore, la razzializzazione inferiorizzante dei neri, sono state funzionali alla conservazione del rapporto sociale di dominazione e di sfruttamento dell'Europa colonialista sui popoli colonizzati: il colonialismo ha costituito il fondamento storico e materiale del razzismo in quanto rapporto sociale e in quanto ideologia. Dottrina elitista, anti-egualitaria, volontaristica (poiché incita alla lotta contro le razze e le classi inferiori), il razzismo persegue la disuguaglianza tra razze, classi, generi e nazioni, facendosi politica di Stato, politica razziale, Stato razziale, programma e azione di partito. Il razzismo - che è ideologia del colonialismo e dell'imperialismo, ma, osserva l'autore, anche ideologia della divisione internazionale del lavoro, dello sfruttamento dei popoli di colore, dei proletari e delle donne - non è pertanto qualcosa di astratto, un elemento della natura umana, un pregiudizio personale che nasce spontaneamente nella mente: è un rapporto materiale di sfruttamento, un rapporto sociale di dominazione, un elemento strutturale, pervasivo, organico, peculiare, della società moderna. Fenomeno sociale storicamente determinato, socialmente appreso, è radicato in relazioni materiali disuguali, in rapporti e strutture storico-sociali che hanno prodotto, nel tempo, una rappresentazione dei dominati come esseri inferiori di natura (oggi si dice 'per cultura').

$\mathrm{E}$ in questo quadro, teorico e storico, che va collocato il rapporto tra razzismo e tortura: la tortura costituisce un elemento strutturale del razzismo. Essa è la concretizzazione del razzismo sul corpo del colonizzato, dell'oppresso, è uno strumento di cui si avvale il razzismo nel processo di disumanizzazione e sottomissione di colui che è considerato inferiore o insubordinato rispetto allo sfruttamento. La tortura è il marchio a fuoco del razzismo sulla pelle del colonizzato, il segno sul corpo dell'inferiorizzazione. Se per Sartre (1958) la tortura è un sistema, questo sistema a sua volta è parte del sistema razziale, del razzismo come sistema di disuguaglianze tra classi, razze, generi e nazioni. Numerosi saggi di questo volume ri-mettono in evidenza il legame tra razzismo e tortura, in particolare Gjergji 
evidenzia come sia assolutamente necessario esaminare e cogliere a fondo il rapporto razzismo/tortura se si vuol comprendere la relazione tortura/migrazioni (per ridurre, di nuovo, allo stato di bestia umana, di sotto-uomo). Analisi del rapporto tortura/migrazioni che va legata, come sottolinea anche Carpinetti, all'ascesa globale del razzismo nell'era neoliberista.

La violenta ripresa del razzismo avvenuta negli ultimi due decenni in molti Paesi del mondo è consistita prevalentemente in razzismo anti-immigrati, i cui centri irradiatori sono l'Europa e gli Stati Uniti, da dove si è allargato ai quattro angoli del pianeta, diventando un autentico fenomeno globale - insieme all'altrettanto fenomeno globale della disuguaglianza razziale legata all'immigrazione (la disuguaglianza derivante dall'essere immigrati in un Paese straniero, Perocco 2018c).

L'ascesa del razzismo ha avuto come protagonista indiscusso il razzismo di Stato (Basso 2010), il razzismo istituzionale, il quale ha costituito il principale propellente delle discriminazioni razziali (istituzionali e di fatto) e il primo produttore del razzismo popolare che è dilagato in tanti Paesi. In Europa, dalla xenofobia in nome del welfare al razzismo municipale, dal razzismo selettivo delle politiche statali alla caccia all'immigrato dei gruppi di estrema destra, l'ascesa del razzismo è avvenuta secondo molteplici modalità a seconda dei contesti nazionali, degli eventi e delle congiunture, ed ha avuto svariati bersagli: gli immigrati dell'Europa dell'Est, i giovani figli di immigrati, le assistenti familiari, i cinesi, gli alunni di origine straniera. Tuttavia ai primi posti, quanto a intensità, diffusione e sistematicità, ci sono l'islamofobia e il razzismo contro gli immigrati musulmani, la romfobia e il razzismo contro i Rom, il razzismo contro i sans-papiers, oggi in particolare i richiedenti asilo. Rispetto alle varie forme di razzismo che costituiscono il sistema mondiale del razzismo contemporaneo (anti-africano, anti-slavo, anti-cinese, anti-ebraico, ecc.) da almeno vent'anni l'islamofobia è senza dubbio la forma più diffusa e più profonda di razzismo: è la principale e la più alta espressione del razzismo contemporaneo, è la punta di lancia del razzismo dell'era neo-liberista; nel sistema mondiale del razzismo, il razzismo contro gli immigrati musulmani detiene il primato (Perocco 2018d, 2019b).

L'acutizzazione del razzismo anti-immigrati - che mira a contrastare il radicamento sociale delle popolazioni immigrate, a risospingerle in posizioni marginali, a svilirne il valore sociale, a ridurre i cosiddetti costi sociali e politici dell'immigrazione, a rallentare o influenzare la direzione dei processi di trasformazione sociale indotti dall'immigrazione - ha comportato la riduzione dei diritti sociali degli immigrati, ha alimentato l'inasprimento delle politiche migratorie, ha mandato in soffitta il multiculturalismo e addirittura il neo-assimilazionismo a favore dell'esclusione e del rifiuto, ha avuto un ruolo 
fondamentale nella costruzione sociale e politica del razzismo popolare e dei sentimenti di ostilità delle popolazioni autoctone nei confronti delle popolazioni immigrate, favorendo la formazione di condizioni e ambienti permeabili alle pratiche di tortura. Con cui svilire, inferiorizzare, bestializzare, le popolazioni immigrate.

L'aumento del fenomeno della tortura contro gli immigrati è legato all'impennata del razzismo istituzionale nel contesto neo-liberista. La demonizzazione sistematica degli immigrati ha aperto le porte a comportamenti degradanti nei loro confronti, disumanizzando tangibilmente i singoli immigrati che l'hanno subita. Da alcuni decenni, sottolinea Carpinetti, gli immigrati rappresentano un gruppo sociale che è costantemente disumanizzato (diventando un soggetto torturabile), e a questo processo lo Stato ha dato un importantissimo contributo. La gestione razzista dei movimenti migratori non ha comportato soltanto la proliferazione di muri nelle aree di confine e di centri di detenzione, ha anche contribuito in modo decisivo alla normalizzazione delle milizie cittadine contro gli undocumented, alla negazione dell'accoglienza, alla diffusione della violenza con sangue e senza sangue nei confronti delle popolazioni immigrate, alla detenzione dei minori, ai rastrellamenti, ai censimenti 'etnici'.

All'interno del rapporto tortura/migrazioni, risalta il fenomeno, messo in luce da diversi saggi di questo volume, delle molestie, delle violenze sessuali, degli stupri, subiti dalle donne emigranti lungo il percorso migratorio, nei Paesi di transito, nei Paesi di arrivo, ai quattro angoli del pianeta. Per numerosità, per sistematicità, per ampiezza, la violenza sessuale appare costituire una modalità specifica e sistematica di degradazione, disumanizzazione, dominazione, delle donne emigranti.

Geraci e Mazzetti, Nosè, Bracci e Coppola, che esaminano a fondo le molteplici e complesse conseguenze della tortura sulla salute fisica e psichica degli immigrati, dei profughi, dei richiedenti asilo, sottolineano che sono necessarie specifiche modalità di presa in carico e di intervento psico-sociale, ma anche adeguate politiche per la salute e per la tutela assistenziale dato che la migrazione può costituire un determinante importante nei percorsi di cura delle vittime di tortura.

\subsection{L'Italia apripista della guerra agli immigrati}

In questa fase di guerra agli emigranti e agli immigrati l'Italia ha avuto ancora una volta - mi riferisco alla legge 189/2002, che ha rappresentato un modello per l'Europa in quanto a precarizzazione e criminalizzazione degli immigrati (Basso, Perocco 2003) - un ruolo d'apripista o quantomeno di primo piano: ha sostenuto l'inasprimento delle politiche migratorie e la marginalizzazione degli immigrati, ha anticipato tali processi con svariati provvedimenti quali la legge 
94/2009 ('pacchetto sicurezza'), il blocco degli ingressi regolari (con la soppressione di fatto dei decreti-flussi), la circolare 14260/2014 sul lavoro gratuito dei richiedenti asilo, la legge 46/2017, gli accordi Italia-Libia, il bando delle ONG, la legge 132/2018 ('decreto-sicurezza') e la successiva legge 77/2019 ('decreto-sicurezza bis').

La legge 132/2018, un vero e proprio salto di qualità nell'inasprimento delle legislazioni sull'immigrazione con la decretazione della fine di fatto dell'asilo, costituisce la punta di lancia delle normative e delle politiche anti-immigrati e un simbolo della guerra agli emigranti. Tanto che, se la legge 189/2002 era stata definita una buona legge per tempi di guerra, non è esagerato affermare che la legge $132 / 2018$ è una buona legge per tempi di crisi economica strutturale e di caos politico internazionale. Legge che non è certo un fulmine a ciel sereno, un incidente di percorso frutto di irrazionalità o impreparazione: è in stretta continuità con le precedenti legislazioni sull'immigrazione, è l'ennesima tappa (molto aspra) del processo di precarizzazione e inferiorizzazione degli immigrati che ha caratterizzato la storia dell'immigrazione in Italia, è l'estremizzazione di un processo pluridecennale di esclusione e criminalizzazione degli immigrati che oggi è focalizzato soprattutto sui richiedenti asilo e sul sistema di accoglienza.

Con l'adozione di nuove misure schiettamente punitive (l'eliminazione della protezione umanitaria, il peggioramento della detenzione, il restringimento delle garanzie, lo smantellamento del sistema di accoglienza, ecc.), essa estremizza un lungo processo di svalorizzazione e demonizzazione degli immigrati favorendo la formazione o il mantenimento di condizioni, ambienti e climi permeabili alla tortura, ai trattamenti inumani e degradanti, come mettono in luce anche Ferrero, Gazzola e Omizzolo in questo volume. ${ }^{11}$ Cito soltanto alcuni punti previsti da questa legge: con l'allungamento della detenzione, il richiedente protezione internazionale può essere accolto con un caloroso benvenuto di 210 giorni di detenzione amministrativa senza aver commesso nessun reato; il prolungamento del termine del periodo massimo di trattenimento dello straniero in fase di espulsione all'interno dei Centri per il Rimpatrio viene elevato da 90 a 180 giorni; è introdotta la revisione dei luoghi idonei al trattenimento in attesa di espulsione, con cui si prevede la possibilità di

11 Il CAT (Comitato ONU contro la Tortura) nelle sue Osservazioni conclusive sulla situazione italiana ha mosso delle critiche durissime allo stato italiano, tra cui: «insoddisfazione per la legge che ha introdotto il delitto di tortura, le criticità rispetto a molteplici aspetti della politica migratoria contemporanea, le anomalie che circondano l'esercizio della forza pubblica e la repressione dei relativi abusi». Rispetto alla politica migratoria, il CAT ha mosso dei rilevi alquanto critici rispetto al divieto di nonrefoulement, all'accordo Italia-Libia, ai maltrattamenti negli hotspot e nei centri di detenzione, alla detenzione amministrativa degli stranieri (Cancellaro 2018, 301, 303). 
trattenimento temporaneo dello straniero in attesa della definizione del procedimento di convalida dell'accompagnamento alla frontiera in strutture diverse dal Centro per il Rimpatrio «nella disponibilità dell'autorità di pubblica sicurezza o in locali idonei presso l'ufficio di frontiera» (ovvero in non precisate 'strutture indeterminate'); insieme all'«Elenco dei Paesi di origine sicuri», è introdotto il principio dell'«alternativa di fuga interna», con cui si prevede la possibilità di rigettare la domanda di protezione qualora il richiedente possa essere rimpatriato in una zona differente del proprio Paese di origine (considerata sicura) rispetto a quella da cui è fuggito. La legge in parola, come si vede, non solo non introduce fattori protettivi dai trattamenti inumani e degradanti, al contrario immette degli elementi che li possono favorire.

Ma oltre a questi aspetti legati alla legge 132/2018, ci sono poi molti altri elementi, assai diffusi nel Paese, che possono indurre a descrivere l'Italia come una terra di tortura: il lavoro forzato e il grave sfruttamento lavorativo nelle campagne, nelle case, nei cantieri; la segregazione nei campi e nelle baraccopoli; l'agonia sulle navi bloccate in mezzo al mare, i respingimenti, i naufragi (a partire dall'affondamento nel 1997 della nave albanese Kater i Rades da parte di una corvetta italiana); l'espulsione dal sistema dell'accoglienza; la detenzione (a partire dagli albanesi rinchiusi a migliaia nello stadio di Bari nel 1991), la detenzione dei minori; il diritto speciale, il regime legale speciale riservato allo straniero (leggi: meno diritti); le discriminazioni razziali nella vita quotidiana; i raid organizzati, le stragi (Castelvolturno, Rosarno, i circa 80 braccianti polacchi assassinati nelle campagne pugliesi; cf. Leogrande 2008; Borretti 2010), le aggressioni; la denigrazione mediatica, la bestializzazione nei discorsi pubblici.

\section{Conclusione}

L'esame del rapporto tra condizioni della migrazione, politiche migratorie e tortura, non esaurisce l'analisi della questione. È necessario infatti collegare il fenomeno tortura/migrazioni con i processi sociali generali, inquadrarlo all'interno del più ampio contesto sociale, delle dinamiche sociali complessive, anche per evitare il rischio di rappresentare l'immigrazione come una cosa a sé. Quintanilha e Villen, ad esempio, nel sottolineare che in Sud-America gli immigrati in condizioni socioeconomiche vulnerabili sono sempre più esposti a violenze, inclusa la tortura (soprattutto i non bianchi e le donne che attraversano i confini senza visto), evidenziano che ciò avviene in uno «scenario di profonda crisi economica, di attacco violento ai diritti e alle condizioni di lavoro, di ascesa dei partiti di estrema destra e della crescente militarizzazione della regione». Jubany, Pasqualetto e Rué, nell'evidenziare che gli immigrati «stand at the intersection that challenges 
the state's sovereignty by their presence, particularly as poor and racialized populations, while living and working in a situation of legal exclusion», sottolineano che nell'analisi fenomeno tortura/migrazioni nell'Europa di oggi «torture must be understood in a continuum that makes no sharp divide between direct forms of state violence, and other, more subtle forms, in what is frequently referred to as 'structural violence'». Carpinetti, nel sottolineare che il discorso neo-liberista ridefinisce la portata e gli effetti del sistema razzista (il quale integra la premessa che gli individui sono naturalmente disuguali con l'affermazione che alcuni di essi non sono interamente umani), afferma che oggi la disumanizzazione presenta alcune peculiarità rispetto al passato: da un lato essa si consuma quotidianamente sotto gli occhi di tutti, dall'altro lato è il frutto di atti legislativi e/o amministrativi formulati in sistemi democratici, è una disumanizzazione che non richiede la segretezza e l'alterazione dell'ordine istituzionale. La tortura, di nuovo, come parte integrate del sistema.

Nell'esame di questo insieme di nessi è fondamentale sottolineare il ruolo degli immigrati. La marcia degli immigrati dall'Africa, dal Medio Oriente, dal Sud-America, verso l'Europa, verso il Nord-America, è la marcia di inserimento nel mercato mondiale del lavoro, che è alimentata da profondissime cause strutturali che spingono milioni di persone a intraprendere necessariamente la via dell'emigrazione, da umanissimi bisogni sociali di emancipazione sociale e di avere una vita decente. La radicalità e la profondità di queste cause oggettive alla base dell'emigrazione sono sussunte nell'atto soggettivo dell'emigrare, nonostante i muri, i fili spinati, i centri di tortura; sono sintetizzate in un progetto migratorio che è semplicemente un progetto di vita dignitosa, di soddisfazione di bisogni umani. Questa umanità in cammino - la chiama così Salgado $(2000,2016)$ - ha conosciuto, direttamente o indirettamente, individualmente o collettivamente, il colonialismo storico, il neo-colonialismo, il razzismo, la morte apparente data dalla tortura, tuttavia per necessità storica essa fa avanzare la vita vera, che si incarna nella resistenza quotidiana, nella fermezza per una vita decente, nella lotta per una vita normale, che non accetta di farsi schiavizzare né 'lì' e né 'qui'.

Il volume raccoglie alcuni contributi che sono stati presentati al Convegno Tortura e migrazioni organizzato nel giugno 2018 dal Centro Studi Diritti Umani (Cestudir) dell'Università Ca' Foscari Venezia, in collaborazione con il Master sull'Immigrazione dello stesso ateneo, e alcuni contributi di autori che non hanno partecipato al convegno. Un ringraziamento a Pietro Basso, Rossana Cillo, Francesco Della Puppa, Ivana Padoan, Lauso Zagato, per l'aiuto e i suggerimenti.

Come avrà avuto modo di intendere il lettore, nel volume ci si riferisce alla tortura - che necessita di una espansione e revisione concettuale - in senso largo, generico, non in senso stretto, nell'accezio- 
ne strettamente legale del termine. Il volume è suddiviso in tre parti: la prima è dedicata a questioni teorico-storiche ad una ricostruzione generale del fenomeno; la seconda parte prende in esame le torture e i trattamenti degradanti nei confronti degli immigrati in diversi contesti del mondo. In questa sezione prima sono presi in esame alcuni contesti europei (Spagna, Belgio, Regno Unito), poi alcuni contesti americani (Stati Uniti, Brasile, Argentina), nord-africani (Marocco, Libia), asiatici (Israele), infine il contesto balcanico e italiano. Il volume si concentra soprattutto sui punti caldi, sui contesti più noti al pubblico (l'asse Africa-Europa), l'area balcanica, la frontiera Messico-USA, i centri di detenzione in Europa, ma si sa che il fenomeno non è circoscritto soltanto a questi contesti, in Medio-Oriente, in Sud-Africa, etc., è altrettanto presente. È un fenomeno globale che non è prerogativa di qualche area del mondo, riguarda tutti i Paesi - del Sud e del Nord del mondo, i Paesi di partenza, di transito, di arrivo - e pertanto nell'ordine di presentazione dei saggi non si è seguita l'idea dominante che la tortura e i trattamenti degradanti sono tipici degli stati non occidentali poco rispettosi dei diritti fondamentali a causa della 'loro cultura', come sottolinea Ouali:

ces pratiques résulteraient de comportements découlant de certaines traditions culturelles; «l'Occident» se situant en haut de l'échelle du respect des droits humains [...] cette approche culturaliste produit non seulement une polarisation entre les cultures qui persécutent et celles qui protègent, mais également une «normalisation» des persécutions pratiquées dans les pays européens [...] Cette représentation sociale s'accompagne souvent d'une opposition entre les pays «producteurs de réfugiés» et les pays qui les accueillent.

La terza parte affronta gli aspetti sanitari e la tutela della salute (fisica e mentale) delle vittime di tortura, le quali, come indicano Bracci e Coppola nel saggio conclusivo, possono fare un percorso che le trasforma da vittime in testimoni. Dopo la disumanizzazione, la riumanizzazione.

\section{Bibliografia}

Antigone (2019). Il carcere secondo la Costituzione. XV rapporto di Antigone sulle condizioni di detenzione. URL https://www. antigone.it/quindicesimo-rapporto-sulle-condizioni-di-detenzione/wp-content/uploads/2019/06/xv-rapporto-antigone.pdf (2019-09-09).

Basso, Pietro (2000). Razze schiave e razze signore. Milano: Franco Angeli.

Basso, Pietro (2010). Razzismo di stato. Stati Uniti, Europa, Italia. Milano: Franco Angeli. 
Basso, Pietro (2018). «La grande crisi della 'civiltà del denaro'». Basso, Pietro; Chiaretti, Giuliana (a cura di), Le grandi questioni sociali del nostro tempo. Venezia: Edizioni Ca' Foscari, 11-26. DOI http://doi.org/10.30687/978-88-6969273-4/००2.

Basso, Pietro; Perocco, Fabio (a cura di) (2003). Gli immigrati in Europa. Razzismo, disuguaglianze, lotte. Milano: Franco Angeli.

Basso, Pietro; Perocco, Fabio (in corso di stampa). «Immigrazione e trasformazione sociale dell'Europa: una svolta epocale e le sue prospettive». Perspectiva.

Bigo, Didier (1998). «Sécurité et immigration». Cultures \& Conflits, 31-32, 13-38. DOI http://doi.org/10.4000/conflits.537.

Bigo, Didier (2005). «La mondialisation de l'(in)sécurité?». Cultures \& Conflits, 58, 53 10. DOl http://doi.org/10.400๑/conflits.1813.

Borretti, Biagio (2010). «Da Castel Volturno a Rosarno. Il lavoro vivo degli immigrati tra stragi, pogrom, rivolte e razzismo di stato». Basso, Pietro (a cura di), Razzismo di stato. Stati Uniti, Europa, Italia. Milano: Franco Angeli, 493-524.

Butler, Judith (2009). «Sexual Politics, Torture, and Secular Time». Intimate Citizenships: Gender, Sexualities, Politics, 59(1), 17-39.

Cancellaro, Francesca (2018). «Pubblicate le osservazioni del Comitato ONU contro la Tortura sulla situazione italiana». Diritto penale contemporaneo, 1, 301-6.

Cassese, Antonio (2011). L'esperienza del male. Guerra, tortura, genocidio, terrorismo alla sbarra. Bologna: il Mulino.

Dershowitz, Alan (2003). Terrorismo. Capire la minaccia, rispondere alla sfida. Roma: Carocci.

Dershowitz, Alan (2004). «Tortured Reasoning». Sanford, Levinson (ed.), Torture: A Collection. Oxford: Oxford University Press, 257-80.

Di Noia, Luigi (a cura di) (2016). La condizione dei Rom in Italia. Venezia: Edizioni Ca' Foscari. DOI http://doi.org/10.14277/978-88-6969-065-5.

Ferro, Marc (dir.) (2003). Le livre noir du colonialisme. Paris: Laffont.

Furedi, Frank (1997). Culture of Fear. London: Bloomsbury.

Furedi, Frank (2018). How the Fear Works. Culture of Fear in the Twenty-First Century. London: Bloomsbury.

Gallino, Luciano (2012). La lotta di classe dopo la lotta di classe. Laterza: Roma-Bari.

Gallino, Luciano (2015). Il denaro, il debito e la doppia crisi spiegati ai nostri nipoti. Torino: Einaudi.

Gjergji, Iside (2016). Sulla governance delle migrazioni. Milano: Franco Angeli.

Gjergji, Iside (2019). Sociologia della tortura. Venezia: Edizioni Ca' Foscari. DOI http:// doi.org/10.30687/978-88-6969-391-5.

Glassner, Barry (1999). The Culture of Fear. New York: Basic Books.

Gonnella, Patrizio (2013). La tortura in Italia. Roma: DeriveApprodi.

Lalatta Costerbosa, Marina (2016). Il silenzio della tortura. Roma: DeriveApprodi.

Leogrande, Alessandro. (2008). Uomini e caporali. Viaggio tra i nuovi schiavi delle campagne del Sud. Milano: Mondadori.

Levack, Brian (2008). La caccia alle streghe in Europa. Roma-Bari: Laterza.

Jacobs, Günther (2007a). «Diritto penale del nemico?». Donini, Massimo, Papa, Michele (a cura di), Diritto penale del nemico. Un dibattito internazionale. Milano: Giuffré, 6-23.

Jacobs, Günther (2007b). «Diritto penale del nemico». Gamberini, Alessandro, Orlandi, Renzo (a cura di), Delitto politico e diritto penale del nemico. Bologna: Monduzzi, 109-29.

Klinenberg, Eric (2001). «L'obsession sécuritaire». Le Monde diplomatique, février.

Noury, Riccardo (2016). «Amnesty International». Antonazzo, Andrea et al. (a cura di), I segni addosso. Storie di ordinaria tortura. Bologna: Becco Giallo, 113-20.

Palidda, Salvatore (2000). «Le migrazioni come crimine». Basso, Pietro; Perocco, Fabio (a cura di), Immigrazione e trasformazione della società. Milano: Franco Angeli, 63-75. 
Perocco, Fabio (2012). Trasformazioni globali e nuove disuguaglianze. Milano: Franco Angeli.

Perocco, Fabio (2018a). «La crescita strutturale delle disuguaglianze nell'era neoliberista». Basso, Pietro; Chiaretti, Giuliana (a cura di), Le grandi questioni sociali del nostro tempo. Venezia: Edizioni Ca' Foscari, 55-86. DOI http://doi. org/10.30687/978-88-6969-273-4/006.

Perocco, Fabio (2018b). «Precarizzazione strutturale del lavoro e precarizzazione globale delle migrazioni. L'esempio dei lavoratori in distacco intracomunitario». Economia e Società Regionale, 36(3), 132-53.

Perocco, Fabio (2018c). «Immigration and racial inequality». Europolity, 12,(2), 115-34.

Perocco, Fabio (2018d). «Anti-Migrant Islamophobia in Europe. Social Roots, Mechanisms and Actors». REMHU, 26(53), 25-40.

Perocco, Fabio (2019a). «The Potential and Limitations of the Global Compact for Safe, Orderly and Regular Migration: A Comment». Torture, 29(1), 127-32.

Perocco, Fabio (2019b). «Islamofobia». Costellazioni, 9, 97-106.

Salgado, Sebastião (2000). In cammino. Roma: Contrasto

Salgado, Sebastião (2016). Êxodos. Köln: Taschen.

Sartre, Jean-Paul (1958). «Saggio introduttivo». Alleg, Henri, La tortura. 3a ed. Torino: Einaudi, 7-21.

Scott, George (1999). Storia della tortura. Milano: Mondadori.

Scaglione, Daniele (1999). «Prefazione». Solet, Bertrand, Tortura. Testimoni contro il silenzio. Torino: EGA, 3-5.

Skoll, Geoffrey (2010). Social Theory of Fear. Terror, Torture, and Death in a Post-Capitalist World. New York: Palgrave Macmillan.

Stannard, David (2001). Olocausto americano. Torino: Bollati Boringhieri.

United Nations (2018a). Global Compact for Safe, Orderly and Regular Migration. URL https://refugeesmigrants.un.org/sites/default/files/180713_agreed_outcome_global_compact_for_migration. pdf (2019-11-27).

United Nations (2018b). Global Compact on Refugees. A/73/12. URL https://refugeesmigrants.un.org/refugees-compact (2019-11-27).

United Nations (2018c). General Comment No. 4 (2017) on the Implementation of Article 3 of the Convention in the Context of Article 22. URL https://www.ohchr.org/Documents/HRBodies/CAT/CAT-C-GC-4_EN.pdf (2019-11-27).

United Nations; Human Rights Council (2018). Report of the Special Rapporteur on Torture and Other Cruel, Inhuman or Degrading Treatment or Punishment. A/HRC/37/50. URL https://www.ohchr.org/Documents/Issues/ Torture/A_HRC_37_50_EN.pdf (2019-11-27).

van der Kolk, Bessel (2015). Il corpo accusa il colpo. Mente, corpo e cervello nell'elaborazione delle memorie traumatiche. Milano: Cortina.

Wacquant, Loïc (1999). Parola d'ordine: tolleranza zero. La trasformazione dello Stato penale nella società neoliberale. Milano: Feltrinelli.

Wacquant, Loïc. (2002). Simbiosi mortale. Neoliberalismo e politica penale. Verona: Ombre Corte.

Wacquant, Loïc. (2009). Punishing the Poor. The Neoliberal Government of Social Insecurity. Durham: Duke University Press.

Wacquant, Loïc (2013). Iperincarcerazione. Neoliberismo e criminalizzazione della povertà negli Stati Uniti. Verona: Ombre Corte.

Wodak, Ruth (2015). The Politics of Fear. London: Sage. 
Parte I

Questioni di fondo 



\title{
Tortura e razzismo Teoria sociale, analisi storica, evidenze empiriche
}

Iside Gjergji

Universidade de Coimbra, Portugal

\begin{abstract}
Torture and racism share a fundamental tendency: both impose on humans the status of sub-humans; both are linked to each other by an embrace that, at the same time, reveals and conceals. The article analyzes this link by focusing on dehumanizing torture, as in this typology it is simpler to be observed. After a thorough review of the theoretical and historical framework, the paper focuses on the 'Regina Pacis case' which provides tangible insights for general reflection.
\end{abstract}

Keywords Torture. Racism. Detention Centre. Immigration.

Sommario 1 Introduzione. - 2 Il razzismo-violenza. - 3 La tortura come verità estrema del razzismo. - 4 Accogliere e punire. - 5 Conclusioni.

\section{Introduzione}

Scena all'aperto. Deserto. Base militare statunitense in Uzbekistan. Due tende color sabbia e una bandiera americana che sventola. Accanto vi sono due agenti sorridenti e coperti di polvere che, con l'aria di chi ha appena concluso con successo una missione difficile, si avvicinano al telefono satellitare per rispondere a una chiamata proveniente dal Pentagono. La telecamera stacca. Scena al chiuso. Un maggiore dell'esercito americano, in atteggiamento amichevole e rilassato, da dentro il suo ordinato ufficio ordina agli agenti operativi di rapire (con ogni mezzo) un presunto terrorista, tale Tariq Mahani (la telecamera stacca ancora per mostrare, in pochi attimi, il volto di un uomo con 
la barba e gli abiti musulmani). «Credevo non potessimo più fare cose del genere agli esseri umani», esclama con un certo sarcasmo uno degli agenti operativi, al quale ribatte con allegra fermezza l'ufficiale del Pentagono, Jim Tisnewski: «Quella feccia non è essere umano per noi». Il maggiore esce dall'inquadratura e la telecamera resta fissa per svariati secondi sulla bandiera americana appesa al muro del suo ufficio.

Il protagonista della serie tv E-Ring - andata in onda alcuni anni fa mentre infuriavano le guerre Nato in Iraq e Afghanistan - difendeva apertamente il trattamento inumano dei soggetti ritenuti pericolosi dal governo del suo Paese. Il suo ragionamento era semplice e facile da accettare: ai soggetti classificabili come non-umani non può essere accordato un trattamento umano. Pensare il contrario sarebbe illogico. La stessa identità di umano del maggiore - e, insieme alla sua, quella di tutti gli uomini bianchi e occidentali che rappresentava - veniva costruita in contrapposizione alla inumana «feccia» musulmana: essere uomo, per il maggiore del Pentagono, significava anzitutto essere superiore al musulmano, all'arabo, all'uzbeko, ecc. Tale qualificazione lo metteva al riparo da ogni ipotesi di trattamento inumano e degradante, ivi compresa la tortura. Anzi, lo poteva trasformare in eroe: «E nella spettacolarizzazione della tortura il poliziotto che si avventura a torturare il terrorista può persino essere osannato come eroe dall'opinione pubblica televisiva» (Di Cesare 2016, s.p.).

L'imposizione dello status di sotto-uomo è sia premessa che obiettivo principale della tortura: consente la propria giustificazione nel mentre crea le condizioni per un'infinita riproduzione; marchia i corpi (e le anime) di certi gruppi sociali per poterli definire come inferiori, sub-umani e, allo stesso tempo, impone, con la violenza di cui è capace, tale status.

Il razzismo e la tortura condividono una tendenza fondamentale: la riduzione dell'uomo a sotto-uomo. Concepiscono una relazione essenziale con l'inumano. Intrattengono un rapporto ambiguo con la morte. Sono legate l'uno all'altra da un abbraccio che, contemporaneamente, svela e occulta.

Questo lavoro intende analizzare tale legame tenendo a mente il monito di Henri Alleg, autore del celebre volume La Question (1958), contenente il racconto dettagliato delle torture inflittegli dall'esercito francese durante la guerra d'Algeria: «Occorre disfarsi della domanda morale se la tortura debba essere usata o meno. La vera domanda è: perché spingono le persone a torturare delle altre?» (Célérier 2014, 157). ${ }^{1}$

Del resto, si può comprendere la tortura come fenomeno sociale soltanto se la si concepisce come conseguenza del modellamento delle relazioni sociali all'interno di un sistema (specifico) (Mackert

1 Tutte le traduzioni nel presente testo sono state ad opera dall'Autrice. 
2015). Questo approccio consente anche di non concentrarsi su tutte quelle riflessioni circa la «banalità del male» (Arendt 2001), alle quali segue puntuale la domanda: come fa un/a torturatore/torturatrice a essere anche un buon marito/moglie, padre/madre, fratello/ sorella, figlio/figlia,... cittadino/cittadina?

Geoffrey Skoll afferma che «torture serves several purposes» $(2010,83)$ e Christopher Tindale (1996) ne individua perfino delle tipologie: 1) tortura da interrogatorio; 2) tortura come monito/deterrente; 3) tortura disumanizzante, altrimenti definita «tortura terroristica» (Hajjar 2013, 23). Nonostante si condivida l'idea che la tortura non riguardi il bisogno di estrarre informazioni - come giustamente sostiene Elaine Scarry: «confession is not the goal» $(1985,29)$ - ma che, al contrario, abbia sempre come obiettivo la disumanizzazione delle vittime e dei gruppi sociali ai quali esse appartengono, per chiarezza espositiva si esplicita che a essere presa in considerazione in questo lavoro sarà soprattutto la terza tipologia, ovvero la tortura disumanizzante, in quanto si ritiene che più intimo e visibile sia, in questo caso, il legame tra tortura e razzismo.

Quanto accaduto alcuni anni fa nel centro per immigrati 'Regina Pacis' (San Foca-Lecce) ne è un chiaro esempio, ragione per la quale si è considerato utile inserirlo nell'analisi complessiva.

\section{II razzismo-violenza}

Non è facile orientarsi nella giungla delle definizioni di razzismo e tortura. Stratificazioni teoriche, confini disciplinari e orientamenti ideologici rendono il terreno scivoloso, disagevole. Eppure, senza una preliminare chiarezza su questo piano non si è in grado di fare neanche un passo nella direzione data.

La ricognizione non può che partire dal concetto di razzismo. Pierre-André Taguieff (1998), sociologo, filosofo e storico francese, definisce come «modernitario-ristretto» quel corpus di teorie - elaborate tra il XVIII e XIX secolo - che considerano il razzismo un insieme di dottrine, ideologie e comportamenti che legittimano le gerarchie tra gruppi umani e individui sulla base della convinzione che le caratteristiche fisiche e genetiche determinino i tratti psicologici, intellettuali e morali. Dello stesso parere è anche l'antropologo Claude Lévy-Strauss, secondo il quale il razzismo idealtipico è «una dottrina che pretende di vedere, nei caratteri intellettuali e morali che si attribuiscono a un insieme di individui comunque definito, l'effetto necessario di un patrimonio genetico comune» (Lévy-Strauss, Eribon 1990, 207).

All'inizio degli anni Ottanta del secolo scorso si fa strada una nuova definizione di razzismo nell'ambito delle scienze sociali. Questa considera superata la versione 'classica' (fondata sul colore della pel- 
le, sulla forma del cranio, del corpo, ecc.), in quanto non sarebbe più in grado di descrivere il fenomeno sociale dopo la fine del colonialismo storico. In questo periodo, infatti, il 'nuovo razzismo' non cercherebbe più il proprio fondamento nel patrimonio genetico. Per giustificare le gerarchie sociali (ovvero le esclusioni, discriminazioni e inferiorizzazioni) fa leva su altre categorie, come 'cultura' e 'nazione'.

Il primo ad aver identificato gli elementi chiave della mutazione storica del razzismo è stato Martin Barker nel suo libro The New Racism: Conservatives and the Ideology of the Tribe (1982). Gli hanno fatto eco Pierre-André Taguieff ed Étienne Balibar. Il primo individua, nel suo libro La force du préjugé. Essai sur le racisme et ses doubles (Taguieff 1988), l'esistenza di due tipologie di razzismo: quello «tradizionale», fondato essenzialmente sulla genetica e che ha come obiettivo l'inferiorizzazione di gruppi e individui, e quello «differenzialista», ossia il neorazzismo, che non si limita a inferiorizzare, pretende la distruzione delle vittime.

Balibar condivide l'idea di Taguieff e, nel suo importante lavoro (scritto insieme a Immanuel Wallerstein), Race, Nation, Classe. Les identités ambiguës (Balibar 1988), afferma che il neorazzismo, quello dell'era della decolonizzazione, si deve qualificare come «razzismo senza razze». Questa qualificazione deve essere intesa nel duplice significato che Balibar le attribuisce: il primo, dettato dall'insegnamento di Lévy-Strauss (1971), considera la cultura come elemento che può funzionare come 'natura' - «La culture peut elle aussi fonctionner comme un nature» (Balibar 1988, 22) - e il secondo intende evidenziare il fatto che la differenza culturale è ora posta in primo piano nel discorso razzista, relegando l'aspetto biologico-genetico sullo sfondo. Ciò che Balibar vuole sottolineare è il fatto che il neorazzismo può dirsi solo parzialmente 'culturalista' e che la 'natura' non scompare affatto dal suo orizzonte. Balibar, infatti, definisce l'antisemitismo come esempio tipico del razzismo differenzialista.

Anche Michel Wieviorka (1991) ha in seguito spiegato come la forma culturale e biologica del razzismo abbiano sempre marciato insieme, ritenendo irrilevante l'esistenza delle razze biologiche nello studio del fenomeno. Su questo punto, Wieviorka attinge dalla riflessione della sociologa Colette Guillamin (1972), la quale aveva ampiamente spiegato come il vero problema sociologico con le 'razze' stia nel fatto che le 'razze immaginarie' e le 'razze reali' giochino lo stesso ruolo nel processo sociale e, di conseguenza, abbiano una identica funzione sociale.

Tutte queste definizioni, 'tradizionali' e 'nuove' - al di là delle specifiche differenze - sono accomunate dal fatto che il razzismo è concepito come una dottrina, un'ideologia, sia quando è considerato frutto del rapporto con l'alterità, sia quando lo si pensa come prodotto di un particolare sistema sociale e politico (come ad esempio il colonialismo). Ed è qui che si annida il vero problema. 
Pensare il razzismo come una dottrina è tipico di chi conosce il razzismo soltanto come esperienza vissuta da altri; da questa posizione, infatti, è capace di cogliere soltanto la dimensione giustificativa e legittimante (cioè ideologica) del razzismo. Registra soltanto le parole che circondano la situazione, ma non sente la forza d'urto del fenomeno. Chi invece il razzismo lo subisce percepisce anzitutto la violenza, fisica e simbolica. Tale violenza è spesso impastata con delle parole (giustificanti), ma quasi sempre queste parole appartengono a lingue sconosciute. Pertanto, l'aspetto ideologico del razzismo lo si tende a vedere spostato sullo sfondo, lo si osserva in un secondo momento, dopo la violenza.

Dunque, se un dualismo c'è nella definizione di razzismo, questo non appare tanto fondato sulla differenza tra razzismo 'biologico' e 'culturale', quanto sulla posizione che assume colui che lo analizza: ${ }^{2}$ se si pone alle spalle degli eserciti coloniali, dei razzisti, percepirà il razzismo attraverso le parole che lo giustificano; se si pone dinanzi a essi, a fianco dei colonizzati, delle vittime, sentirà addosso, prima di ogni cosa, la violenza. Vedrà nel razzismo la sua dimensione operativa, lo riconoscerà soprattutto come razzismo-operazione.

Gli studiosi concordano quasi tutti sul fatto che il razzismo moderno nasca con il colonialismo, il quale è, a sua volta, alla base della genesi e riproduzione del capitalismo. Sono in tanti ad aver dimostrato come il concetto di razza fosse quasi sconosciuto prima del colonialismo. Autori come Hosea Jaffe (2010), Alfred Crosby (1986), David E. Stannard (2001), Tzvetan Todorov (2014) hanno ampiamente dimostrato con le loro ricerche come sia stato il colonialismo capitalista a far nascere e sviluppare la teoria della razza, la psicologia del pregiudizio razziale legato a fattori genetici e la pratica del razzismo a ogni livello. Anche Wallerstein lo conferma, aggiungendo opportunamente che questo razzismo «non ha niente a che fare con gli 'stranieri'», perché a produrlo è la necessità (strutturale) del capitalismo di creare ovunque gerarchie:

Ciò che intendiamo per razzismo ha poco a che fare con la xenofobia che esisteva in vari sistemi storici precedenti. La xenofobia era, letteralmente, paura dello 'straniero'. Il razzismo interno al capitalismo storico non ha niente a che fare con gli 'stranieri'. Tutto al contrario. Il razzismo è stato il modo con cui vari segmenti di forza-lavoro interni alla stessa struttura economica sono stati costretti a porsi in relazione gli uni agli altri. (Wallerstein 1985, s.p.)

2 Non si tratta soltanto di un chiarimento teorico sulla definizione di razzismo, ma di una rilevante questione metodologica, con la quale bisogna fare i conti ogni volta che si analizza tale fenomeno sociale. 
Per rapinare le terre e le risorse delle colonie nonché per sfruttare al massimo la manodopera locale occorreva creare un sistema che riducesse i colonizzati in sotto-uomini. Per realizzare ciò la sola ideologia sulla gerarchia delle razze non era sufficiente. Jean-Paul Sartre è stato uno dei primi ad aver colto pienamente l'essenza del legame tra razzismo, colonialismo e capitalismo (Gjergji 2018). Nel suo efficace saggio sociologico Le colonialisme est un système (Sartre 1964a), egli spiega il funzionamento e l'intreccio dei tre elementi. Per Sartre il razzismo non è una ideologia, ma una violenza, una violenza complessa con giustificazione embedded:

Il razzismo deve farsi pratica: non è un risveglio contemplativo dei significati incisi nelle cose; è in sé una violenza che si dà la propria giustificazione: una violenza che si presenta come violenza indotta, contro-violenza e legittima difesa. (Sartre 1960, 677)

Se il razzismo è inteso come violenza, la sua fonte primaria è da cercarsi inevitabilmente nello Stato. «Il monopolio dell'uso legittimo della forza fisica» (Weber 1998, 178) e della violenza simbolica (Bourdieu 2013) appartiene infatti allo Stato. Del resto, nelle colonie, l'occupazione, l'espropriazione delle terre, la cacciata dei braccianti, il reclutamento, il lavoro forzato, le istituzioni politico-amministrative, le politiche sanitarie, l'istruzione... fino alla repressione (e alla tortura) sono state tutte operazioni sostenute finanziariamente e realizzate concretamente dagli stati colonizzatori.

Il razzismo è inscritto negli stessi eventi, nelle istituzioni, nella natura degli scambi e della produzione; gli statuti politici e sociali si rafforzano reciprocamente: poiché l'indigeno è un sotto-uomo, la Dichiarazione dei Diritti dell’Uomo non lo riguarda; al contrario, poiché non ha dei diritti, egli è abbandonato senza protezione alle forze inumane della natura, alle 'leggi ferree' dell'economia. (Sartre 1964b, 51)

Il sistema coloniale - continua Sartre - è complesso e si regge sull'ipersfruttamento della forza lavoro indigena: «il sistema [...] poggia, come sapete, sull'ipersfruttamento» (1962, XLII). La sua sopravvivenza dipende dalla riduzione dei colonizzati in sotto-uomini: per sfruttare meglio occorre disumanizzare gli sfruttati. Il razzismo rappresenta l'elemento principale per il raggiungimento di tale obiettivo, perché è teso a spezzare e umiliare i colonizzati/sfruttati, a distruggere il loro coraggio, la loro volontà e intelligenza. Nella sua essenza è una violenza disumanizzante che vuole mantenere la propria vittima tra la vita e la morte, la vuole annullare ma mai del tutto (da qui anche il suo rapporto ambiguo con la morte), perché la vittima deve pur sempre continuare a servire e lavorare, a obbedire agli ordini, ma come una bestia, come uno zombie. 
Da questa prospettiva, cioè dalla prospettiva del colonizzato, le categorizzazioni astratte del razzismo, del tipo 'biologico' o 'culturale', appaiono alquanto irrilevanti se non addirittura fuorvianti:

Il razzismo-operazione è la praxis illuminata da una «teoria» (razzismo «biologico», «sociale», empirico, poco importa) che vuole mantenere le masse allo stato di aggregati molecolari, aumentare con ogni mezzo la «sotto-umanità» del colonizzato (Sartre 1960, 678).

Abdelmalek Sayad (2002) ha utilizzato alcuni strumenti concettuali di Sartre sia per spiegare le cause del fenomeno migratorio internazionale sia per analizzare il razzismo subito dagli immigrati in Europa. La situazione nella quale gli immigrati si trovano è simile a quella vissuta dai colonizzati nelle colonie: vessazioni, umiliazioni, inferiorizzazioni, ipersfruttamento. Si tratta, dunque, di una situazione coloniale costruita non Oltremare, ma nel cuore dell'Europa. Non a caso, infatti, Sartre definisce gli immigrati dei «colonizzati interni», ${ }^{3}$ ovvero dei colonizzati situati all'interno del contesto sociale e politico europeo.

Il razzismo-violenza esercitato nei loro confronti - derivante, ora come allora, dalla solita fonte, cioè da quella che ancora conserva il monopolio della violenza (fisica e simbolica), lo Stato - ha gli obiettivi di sempre: umiliare e spezzare, disumanizzare per meglio sfruttare (Basso 2010).

\section{La tortura come verità estrema del razzismo}

In Occidente, la tortura ha sempre giocato un ruolo importante nella conservazione della supremazia razziale (Garland 2005). Il sistema coloniale e il razzismo-violenza, applicato sia Oltremare che nel territorio metropolitano, hanno creato le condizioni per la creazione di una «classe torturabile» (Roberts 2008, 231).

Gli schiavisti bianchi classificavano gli schiavi africani come 'razza animale', separata e inferiore ai bianchi. Da questi, infatti, potevano essere legalmente trattati come oggetti. I padroni degli schiavi erano liberi di violentare e torturare impunemente i loro schiavi. Nel codice civile dello Stato della Louisiana, ad esempio, si prevedeva espressamente che lo schiavo dovesse considerarsi «interamente soggetto alla volontà del padrone», che poteva punirlo e castigarlo, «anche se non con una violenza inusuale, e senza storpiarlo o mutilarlo o metterlo in pericolo di vita, o portarlo alla morte» (Scott

3 Ancor prima di Sartre è stato Marx $(2009,260)$ a parlare di «colonizzazione interna» all'Europa con riferimento alla condizione dei braccianti e lavoratori irlandesi in Inghilterra. 
1999, s.p.). ${ }^{4}$ La tortura marchiava, atrofizzava i corpi (e le anime) dei neri, li rendeva servili, sottomessi, piegati, il che si traduceva in conferma della loro posizione di inferiorità.

La tortura era parte integrale delle migliaia di linciaggi occorsi negli Stati Uniti dal 1882 al 1940. L'uccisione della vittima era, in questi casi, soltanto una tappa del rituale (Waldrep 2002). Non a caso David Garland li ha definiti «linciaggi di tortura pubblica» (2005, 796), sottolineando come, nonostante le vittime finissero tutte impiccate, raramente morivano a causa dello strangolamento:

Le vittime del linciaggio venivano mutilate mentre erano ancora vive, le orecchie o le dita o gli organi genitali venivano amputati, il corpo pugnalato e tagliato, le viscere tirate fuori dal corpo davanti ai loro occhi. (805)

Dopo il linciaggio, «i cadaveri venivano comunemente tagliati e gli spettatori facevano a gara per accaparrarsi parti dei loro corpi, cercavano tra le ceneri per portarsi a casa un pezzo di osso come souvenir» (Kaufman-Osborne 2006, 29-30). ${ }^{5}$

Non era l'esecuzione finale, la morte dei neri a dare un 'senso' all'evento, ma la loro tortura. Inizialmente, i «linciaggi di tortura pubblica» hanno riguardato soltanto i neri, ma poi la «classe dei torturabili» si è allargata, nutrendosi di altri soggetti appartenenti ad altre 'razze', quelle che l'immigrazione portava nel territorio degli Stati Uniti. Sono noti, infatti, i numerosi linciaggi degli immigrati italiani negli Stati Uniti (Deaglio 2015). Il rituale della tortura pubblica, quindi, riguardava soltanto le «razze inferiori», perché doveva essere, prima di tutto, un chiaro messaggio sulla razza, doveva esplicitare la morfologia della razza dominante.

La tortura segnava un confine invalicabile tra le razze anche nei territori coloniali. L'esperienza coloniale europea appare, in questo senso, come un lungo catalogo degli orrori. Per quanto ancora non sufficienti, gli studi sulle atrocità commesse dagli italiani nelle colonie

4 L'immaginario cinematografico ha ampiamente rappresentato la terribile condizione degli schiavi negli Stati Uniti e, in alcuni casi, come in Django Unchained (film di Quentin Tarantino), anche la loro ribellione, la cui violenza estrema non è che lo specchio di quella subita.

5 Gli spettacoli dei linciaggi erano regolarmente fotografati per consentire ai presenti di conservare la memoria dell'evento e ai non presenti di parteciparvi. Particolarmente significativo appare un esempio riportato da David Garland: «Joe Myers inviò ai suoi genitori, nel maggio 1916, una cartolina che aveva la seguente dicitura 'il cadavere carbonizzato, appena riconoscibile, di Jesse Washington, appeso a un palo di servizio a Robinson, Texas'. Il messaggio diceva: 'Questo è il Barbecue che abbiamo fatto ieri sera, la mia immagine è a sinistra con una croce sopra' ed era firmato 'vostro figlio Joe'» $(2005,794)$. Non sono pochi gli studiosi che hanno stabilito un parallelo tra le fotografie dei linciaggi e quelle delle torture commesse dai soldati americani in Iraq e Afghanistan (Apel 2005) 
sono ora in grado di fornire degli elementi validi per comprendere il ruolo attribuito alla tortura nelle colonie italiane. Le testimonianze dirette, quando ci sono, rendono più facile il compito dell'interprete, perché emerge con chiarezza il ruolo della tortura come un demarcatore razziale. Ciò, per esempio, si percepisce nelle parole di Salem Omram Abu Shabur, il quale così descrive la vita quotidiana della popolazione libica nel campo di concentramento di El-Agheila:

Si vedevano tante torture e impiccagioni. Tutti dovevano assistere alle esecuzioni senza parlare, senza commentare, quasi senza piangere. Lasciavano i corpi appesi per due o tre giorni. (Salerno 2005, 96)

Le torture inflitte alle popolazioni colonizzate sono state innumerevoli ed erano autorizzate dal governo, dallo Stato italiano:

Certo non tutti gli italiani che sono stati nelle colonie italiane d'oltremare hanno premuto il grilletto o hanno praticato la tortura e lo schiavismo. Ma avrebbero potuto farlo, tutti, indistintamente, perché il vertice del regime, come abbiamo visto, non proibiva le violenze, anzi le sollecitava, e garantiva l'impunità. Come ricordava Antonio Dordoni, nel raccontare la strage di Addis Abeba, «il solo rischio che si correva era quello di guadagnarsi una medaglia». (Del Boca 2005, s.p.)

Del resto, gli ordini diretti di Mussolini non lasciano spazio a dubbi. Il terrore doveva regnare sovrano nelle colonie:

Autorizzo ancora una volta V.E. a iniziare e condurre sistematicamente politica del terrore et dello sterminio contro i ribelli et le popolazioni complici stop. Senza la legge del taglione ad decuplo non si sana la piaga in tempo utile. Attendo conferma. (telegramma dell'8 luglio 1936, inviato a Graziani) ${ }^{6}$

Terrore e sfruttamento disumanizzano «e lo sfruttatore usa questa disumanizzazione per sfruttare ancora di più» (Sartre 1964b, 54). In questo senso, la politica della tortura - che «è, alla fin fine, una politica del terrore» (Di Cesare 2016, s.p.) -, praticata a grandi dosi nelle colonie dagli apparati di coercizione degli Stati coloniali, rappresenta la verità estrema del razzismo; la tortura è la manifestazione più crudele e intima della violenza razziale eretta in sistema:

6 Molti dei telegrammi di Mussolini inviati nelle colonie si trovano ora raccolti nel sito web: http://www.criminidiguerra.it. Il sopra citato documento è rintracciabile al seguente indirizzo: http://www.criminidiguerra.it/Telegrammi\%20di\%20Mussolini.shtml (2019-02-13). 
L'obiettivo della tortura non è semplicemente quello di costringere qualcuno a parlare o tradire: serve affinché la vittima definisca se stessa, con le sue grida e la sua sottomissione, come una bestia umana. Davanti agli occhi di tutti e anche ai suoi. Bisogna che questo tradimento la spezzi e sbarazzi per sempre di sé. Colui che cede alla tortura, non lo si è soltanto costretto a parlare; gli si vuole imporre per sempre uno status: quello di sotto-uomo. (Sartre 1964c, 84)

L'odio nei confronti della vittima - che emerge con la tortura - è espressione del razzismo-violenza. Perché sia il razzismo che la tortura hanno un obiettivo comune: distruggere l'uomo. Non vogliono farlo morire, non servirebbe, vogliono semplicemente cancellare le sue qualità umane: il coraggio, la dignità, la volontà, l'intelligenza. In altre parole, quelle stesse qualità che razzisti e torturatori rivendicano per se stessi.

\section{$4 \quad$ Accogliere e punire}

...poi l'ha aperta lui perché stava dentro la carta stagnola, ha tirato una cosa da dentro e mi ha detto 'guarda questa cosa' e mi ha detto 'questa la devi mangiare sennò ti ammazziamo'. Gli ho detto 'io sono musulmano, non mangio il maiale'. Mi ha colpito con il manganello a questa parte, alla parte destra e alla parte sinistra delle gambe, mi ha fatto togliere i pantaloni perché ero anche bagnato, c'era il fango e i pantaloni... sono rimasto con la mutanda vestito io. Dopo mi hanno fatto sdraiare sulla spalla, sulla schiena, uno mi ha preso e mi ha bloccato di questa...ha messo il ginocchio sopra la mano, e un altro mi ha bloccato l'altro braccio e quello che teneva la carne in mano si è seduto sopra di me così ed ho cercato di tirare il braccio per bloccare, per chiudere la bocca; mi ha dato un pugno alla mano e poi mi ha colpito e poi mi ha colpito col manganello che mi ha fatto male, ancora non riesco ad aprirlo completamente, e poi ha cercato di aprire, è riuscito ad aprire con la forza la bocca stringendola [...] è entrato il direttore e mentre ci aveva la mano in tasca così sorrideva, rideva e mi ha detto 'bene, va bene cosi' e ha sputato verso di me, mi ha sputato. (Salem) ${ }^{7}$

Questo non è il racconto delle torture perpetrate ai danni di un musulmano detenuto ad Abu Ghraib. La vittima è sempre un musulma-

7 Tutte le testimonianze riportate sono estratte dagli atti del processo e dalla sentenza di condanna di primo grado. 
no, ficcare in gola la carne di maiale cruda con un manganello è una delle torture ricorrenti nel famigerato carcere irakeno (Greenberg, Dratel 2005), ma il setting è diverso: la stanza delle torture questa volta è collocata nel centro di trattenimento per immigrati 'Regina Pacis' di San Foca (Lecce), distante centinaia di chilometri dal famigerato carcere di Abu Ghraib.

A raccontare le violenze ivi subite davanti a un giudice del Tribunale di Lecce - dove, nel 2003, si celebrò il processo che vide come imputati il direttore del centro, alcuni operatori, medici e carabinieri - non è solo Salem, ma anche altri suoi compagni. Sentiamoli (anche se sono passati degli anni):

...poi un carabiniere se n'è andato e ha portato un pezzo di carne di maiale, a me mi hanno preso in quattro persone e mi hanno fatto ingoiare la carne di maiale con la forza e ridendo in modo un po' ridicolo nei confronti della religione e nel mese di decorrenza del Ramadan che era in quel momento, il mese del digiuno musulmano [...] Era carne cruda, non era cotta. [...] Mi hanno preso due dai piedi, mi hanno bloccato i piedi, uno mi ha bloccato dal torace e le braccia, un altro mi ha costretto di aprire la bocca con la forza e mi ha infilato il pezzo di carne tenendo anche il manganello in mano. [...] Prima me l'ha messa vicino la bocca ma rifiutai di ingoiarla e poi mi ha messo il manganello e me l'ha infilata con la forza. (Mohamed)

...il carabiniere ha preso il pezzo di carne di maiale e mi ha messo il braccio sotto al mento e mi ha spinto in modo di alzare la testa e mi ha infilato la carne di maiale in bocca. Poi ha preso il manganello che lo teneva lungo la gamba, io ho cercato di fare resisten$z a$, di non ingoiare la cosa e con il manganello mi ha spinto il pezzo di carne in bocca. [...] La bocca mi faceva male, soprattutto questa parte e i denti, i denti mi facevano male anche da prima e quando mi hanno spinto la carne così mi hanno fatto ancora più male [...] l'altro carabiniere camminava così, passava davanti a me, si è girato e mi ha dato un colpo in girata coi piedi sulla schiena, sono caduto per terra e l'altro mi ha preso e mi ha sollevato e mi ha messo in piedi. (Anis)

...ci hanno bloccato i carabinieri e poi ci hanno portato nel corridoio vicino alla direzione. Dopodiché è arrivato il direttore, mi ha preso dal ciuffo dei capelli davanti e mi ha sbattuto due volte sul muro la testa di dietro; dopo mi ha girato e mi ha preso dalla parte da dietro e mi ha sbattuto la faccia al muro, dalla parte del sopracciglio qui e mi ha fatto una ferita, una grossa ferita qui al sopracciglio. [...] Dopodiché mi ha rigirato e ha preso il manganello dei carabinieri e mi ha preso dal ciuffo dei capelli davanti e mi ha colpito col manganello sulle labbra, alla bocca, dove mi ha procu- 
rato una ferita che è visibile ancora. Poi mi ha colpito due denti superiori. [...] Dopodiché lui insieme a [...] mi hanno cominciato a colpire sul viso. (Montassar)

Come ulteriore forma di umiliazione, ad alcune delle vittime veniva ripetuta la frase: «dove sta Allah che ti salva e ti protegge adesso?».

L'identità religiosa delle vittime era considerata, inequivocabilmente, l'elemento da colpire, ovvero veniva pensata come la via più veloce per ottenere l'umiliazione dei musulmani. Questo aspetto è particolarmente interessante nella presente riflessione perché disvela il razzismo dei torturatori e il loro odio radicale e indiscriminato nei confronti delle vittime, ma rivela anche - almeno fino a un certo grado - una sorta di preparazione tecnica degli imputati in fatto di tortura. ${ }^{8}$

Inoltre, chi ha letto il libro di Eric Salerno (2005) sulle atrocità italiane in Libia può perfino tracciare una continuità storica nelle modalità di umiliazione dei musulmani, dalle colonie di un secolo fa all'Italia di oggi. Le frasi pronunciate da alcuni militari italiani ai loro prigionieri libici, mentre li facevano precipitare vivi, a uno a uno, dall'aereo, sono sovrapponibili a quelle pronunciate contro i musulmani rinchiusi nel centro 'Regina Pacis':

li hanno fatti salire sugli aeroplani e, in presenza dei loro parenti e congiunti, li hanno lasciati cadere da una altezza di quattrocento metri; ed ogni volta che uno di essi precipitava, erano applausi e battimani e sghignazzi da parte degli ufficiali e dei soldati i quali ad alta voce dicevano di loro: «Venga quel beduino di Maometto vostro profeta, che vi ha ingannati con la guerra santa, a salvarvi dalle nostre mani». (Salerno 2005, 44)

Il primo grado del processo che vide come imputati alcuni operatori del centro 'Regina Pacis' si concluse il 23 gennaio 2004 con la condanna degli imputati (salvo pochi carabinieri assolti) per i reati di violenza privata (art. 610 c.p.) e lesioni personali (art. 582 c.p.). Tale condan-

8 Seymour Hersh, autore di un report sulle torture statunitensi in diversi scenari di guerra, The Taguba Report, in un'intervista rilasciata nel 2004 al Guardian (Whitaker 2004) affermò che il libro dell'antropologo Raphael Patai, The Arab Mind (1973), era considerato la bibbia dei teocon americani e del Pentagono. Ė da lì che questi ultimi ricaverebbero certe idee su come meglio umiliare e disumanizzare gli arabi. Il libro di Patai, in realtà, non è che un'accozzaglia di luoghi comuni razzisti, che nulla hanno a che fare con la scienza e che, essenzialmente, servono a riprodurre il razzismo nei confronti delle popolazioni arabe. Bisogna dire, però, che i torturatori di vari Paesi arricchiscono le loro tecniche di tortura anche vedendo dei bei film, come ad esempio quello di Gillo Pontecorvo, La battaglia d'Algeri. Macmaster $(2004,10)$ segnala che la visione del film di Pontecorvo faceva parte del percorso di addestramento alla tortura dei militari del Pentagono e del Naval College in Argentina (durante gli anni della dittatura). 
na è stata poi confermata nei successivi gradi di giudizio. ${ }^{9} \mathrm{Il}$ racconto delle violenze/torture subìte è stato giudicato attendibile, anche se nelle orecchie delle vittime risuona ancora l'eco dell'arringa del difensore di alcuni imputati, la quale si concluse con una terribile domanda: «Come si può credere alla parola degli stranieri, signor giudice?».

Il reato di tortura è stato introdotto nell'ordinamento italiano nel 2017, motivo per cui le violenze e le umiliazioni perpetrate all'interno del centro 'Regina Pacis' non si poterono giuridicamente qualificare come tali. Ciononostante, le similitudini esistenti tra le violenze riportate nei vari Torture Papers (Greenberg, Dratel 2005) - dall'Afghanistan all'Iraq, passando per Guantanamo - e quelle subite dagli immigrati trattenuti nel centro 'Regina Pacis' inducono ragionevolmente a inquadrare tali violenze come torture. Ciò anche alla luce degli elementi strutturali del nuovo reato di tortura, previsto dall'art. 613 bis del codice penale.

Si tratta dell'unico caso in Italia (e forse anche in Europa) in cui le violenze/torture perpetrate all'interno dei centri per immigrati (in Italia) siano state giudizialmente accertate. In altri casi, le parole degli stranieri non sono state credute e, di conseguenza, non vi sono stati dei processi, oppure, quando questi hanno avuto luogo, si sono conclusi con l'assoluzione degli imputati. Tutto ciò fa del 'caso Regina Pacis' un caso emblematico, da diversi punti di vista.

Il contesto è ciò che deve essere analizzato per primo. La tortura necessita, infatti, di un ambiente particolare, che possieda determinate caratteristiche, in grado di renderla possibile. Tale contesto deve trasmettere disagio alla vittima (Farci, Pezzano 2009), deve toglierle ogni contatto con il mondo esterno, minare alla base ogni sua certezza, «destroy life and its attachment to the world» (Scarry 1985, 28).

Elaine Scarry costruisce un parallelo tra corpo e stanza (ambiente): laddove la stanza è confortevole, il corpo si sente protetto e rilassato, nella torture room invece il corpo si sente profondamente minacciato, ancor prima dell'inizio della tortura (Scarry 1985).

Oltre all'ambiente minaccioso, la tortura necessita di altre condizioni per essere realizzata. Tra queste vi deve essere il controllo assoluto dell'ambiente da parte del torturatore. Senza il dominio materiale e simbolico del contesto, il torturatore non può neanche iniziare a definirsi tale. Quanto alla vittima, questa deve essere privata di ogni contatto con l'esterno, spogliata del sé, dell'identità, introdotta in un ambiente talmente ostile da cancellarle ogni desiderio, salvo uno: quello di morire.

9 Il centro 'Regina Pacis', travolto anche da altri procedimenti giudiziari, oggi non esiste più. Resta ancora in piedi la sua 'carcassa', fatta di mura decadenti, inferriate e cancelli arrugginiti, stanze con letti, tavoli e sedie imbullonati, dove ormai trovano rifugio soltanto gli animali della fauna circostante. 
Ero sdraiata nuda, sempre nuda. Potevano arrivare una, due o tre volte al giorno. Non appena sentivo il rumore dei loro stivali in corridoio, iniziavo a tremare. Dopo, il tempo diventava infinito. I minuti mi sembravano ore e le ore giorni. La parte più difficile è tenere duro nei primi giorni, per abituarsi al dolore. In seguito, ci si stacca mentalmente, un po' come se il corpo galleggiasse. [...]

Durante quei tre mesi avevo un solo obiettivo: suicidarmi, e la più grande sofferenza era volermi uccidere a tutti i costi e non trovare i mezzi per farlo. ${ }^{10}$

Tutti gli elementi necessari per costruire una torture room costituiscono anche i tratti essenziali dei centri di detenzione per immigrati: costruiti come «istituzioni totali» (Goffman 2003) si prestano con facilità a diventare setting di torture:

Un'istituzione totale può essere definita come il luogo di residenza e di lavoro di gruppi di persone che, tagliate fuori dalla società per un considerevole periodo di tempo, si trovano a dividere una situazione comune, trascorrendo parte della loro vita in un regime chiuso e formalmente amministrato. $(2003,29)$

L'aspetto sociologicamente interessante è che i centri di detenzione per immigrati sono ufficialmente annoverati nell'elenco delle istituzioni di accoglienza. ${ }^{11}$ Vale a dire che lo Stato non individua elementi di differenza tra i centri di detenzione e quelli di accoglienza (in senso stretto), non vede dunque dissomiglianze rispetto al ruolo da essi svolto nei processi sociali. Questo è un dato importante, del quale si deve tenere conto nell'analisi complessiva del sistema di accoglienza in Italia, in quanto ne rivela la sua reale funzione sociale.

La sovrapposizione tra centri di detenzione e centri di accoglienza in Italia dipende da molti fattori. In primo luogo dalla confusa gestione dell'accoglienza istituzionalizzata, sempre all'insegna dell'emergenza, in cui si confondono sigle e istituzioni. In secondo luogo, dalla stessa genesi storica ${ }^{12}$ e sociale degli attuali centri

10 Sono le parole di Louisette Ighilahriz (detta Lila) nell'intervista rilasciata a Le Monde (Beaugé 2000) per raccontare le torture subite dall'esercito francese in Algeria.

11 Corte dei Conti (2018). La 'prima accoglienza' degli immigrati: la gestione del fondo per le politiche e i servizi dell'asilo (2013-2016). Deliberazione 7 marzo 2018, $\mathrm{nr}$. 3/2018/G. Il testo è rintracciabile online al seguente indirizzo: http://www. astridonline.it/static/upload/deli/delibera_3_2018_g.pdf (2019-01-21).

12 Oggi è dato per scontato che l'accoglienza non possa che essere fornita dai 'centri di accoglienza'. Gli storici, invece, hanno dimostrato come i centri di accoglienza e l'accoglienza istituzionalizzata (in Europa) abbiano una data di nascita precisa: l'anno 314 d.C., durante il tempo di Costantino (Mollat 1983). Questa istituzione era talmente sconosciuta nella precedente prassi sociale dell'ospitalità che non esisteva neanche una parola latina per definirla. Per lungo tempo, infatti, questa istituzione era de- 
di detenzione: nascono storicamente dalle ceneri di quelli che un tempo erano stati centri di accoglienza (il centro 'Regina Pacis' è, in questo senso, un esempio classico, essendo stato il primo e il più grande centro di accoglienza istituito in Italia nel 1997 e trasformatosi poi, nel 1998, senza modificare nulla nella struttura e nello staff, in centro di trattenimento/detenzione). In terzo luogo, dalla crescente tendenza alla limitazione della libertà personale e all'intensificazione del controllo sugli immigrati da parte di tutti i centri, senza distinzione.

Nel momento dell'inserimento nel sistema di accoglienza, gli immigrati subiscono un processo di istituzionalizzazione: sono sottoposti a un sistema di regole che impongono determinati comportamenti. Tali regole finiscono per creare soggetti vulnerabili e dipendenti.

Appena sbarcate, le persone sono collocate nei cosiddetti 'hotspot', centri chiusi e privi di qualsiasi legittimità giuridica, ${ }^{13}$ uno spazio extra-territoriale nel quale avviene l'identificazione e sono prelevate, anche con la forza, le impronte digitali. ${ }^{14}$ La permanenza o meno in

finita con la parola greca xenodocheion (casa per gli stranieri). L'introduzione dell'accoglienza istituzionalizzata nella società dell'epoca provocò un vero terremoto, poiché oltre a cancellare, con grande velocità, la precedente pratica sociale dell'ospitalità nelle case, finì anche per creare una nuova classe sociale, quella dei poveri, composta essenzialmente da: malati, stranieri, anziani, orfani, mendicanti, poveri (Patlagean 1986). Erano questi i soggetti ospiti delle istituzioni di accoglienza. Rinforzate e diffuse ovunque in Europa, durante il medioevo, queste istituzioni mostrarono sin dall'inizio un legame intimo con il lavoro, a partire dalla separazione tra poveri 'abili' e 'disabili' (al lavoro) al loro interno. Tale legame diventò però la loro principale ragione di esistenza con l'inizio dell'era moderna e della rivoluzione industriale. Le workhouses (case di lavoro) - dove lo sfruttamento del lavoro gratuito o a bassissimo costo degli ospiti, utile al capitalismo nascente, rappresentava un elemento costitutivo - non furono che 'figlie primogenite' dei primi xenodocheion di Costantino (Geremek 1986). Anche oggi, in Italia, il legame tra centri di accoglienza e lavoro a basso costo emerge ogni giorno di più in superficie. Emblematica appare, in questo senso, l'introduzione del cosiddetto «lavoro volontario» - prima con la circolare nr. 14290/ 2014 e poi con la legge 'Minniti-Orlando' - per i richiedenti asilo ospiti dei centri di accoglienza. Sia la circolare che la legge parlano di «base volontaria» del «lavoro socialmente utile» che dovrebbero svolgere i richiedenti asilo. Non è difficile però comprendere e immaginare come la previsione normativa si possa trasformare in una sostanziale imposizione per l'immigrato che si trova all'interno del centro, essendo totalmente dipendente dallo staff che lo gestisce.

13 «L'Approccio Hotspot è una delle misure previste in quella che viene chiamata Agenda Europea sulle Migrazioni, mera comunicazione della Commissione europea al Consiglio e al Parlamento (e pertanto, un 'policy document with non mandatory authority'), non trasposta in nessun atto normativo, che in quanto tale non produce effetti sul piano legislativo». La citazione è tratta dalla relazione di minoranza sull'approccio hotspot nell'ambito del Sistema di identificazione ed accoglienza e presentata nel 2017 alla Commissione parlamentare di inchiesta sul sistema di accoglienza, di identificazione ed espulsione, nonché sulle condizioni di trattamento dei migranti e sulle risorse pubbliche impegnate. La Relazione porta la firma del deputato Erasmo Palazzotto: http://www.meltingpot. org/IMG/pdf/relazione_minoranza_hotspot_palazzotto_2_.pdf (2019-01-02).

14 L'identificazione negli hotspot può avvenire anche con la forza, secondo quanto esplicitamente richiesto dalla Commissione europea. Si legga, a tal proposito, il docu- 
un centro (hotspot, oppure altre tipologie) è a totale discrezione delle istituzioni (ministero, polizia, prefetture, gestori dei centri, ecc.), le quali decidono la durata del soggiorno, le dislocazioni geografiche e le tipologie dei centri. La libertà di movimento negli hotspot e nei centri di detenzione (ora definiti CPR, Centri per il Rimpatrio) è assolutamente vietata e negli altri è fortemente limitata dalle norme giuridiche (art. 5, comma 4 d.lgs. 142/2015), da quelle prefettizie e, infine, anche da quelle stabilite (arbitrariamente) dai gestori dei centri.

La possibilità per gli immigrati di prendersi cura di sé stessi è limitata dalla forte dipendenza economica dalle istituzioni di accoglienza. Il sostentamento economico, infatti, si traduce nella distribuzione quotidiana di 'buoni spesa' del valore di circa tre euro, spesso da spendersi in limitati punti di vendita. L'interazione con il mondo esterno è fortemente mediata (controllata) da numerose figure professionali: operatori, assistenti, educatori, psicologi, traduttori, avvocati. Il rapporto con la popolazione autoctona è limitato anche dalla geografia dei centri, i quali sono, assai spesso, costruiti in zone lontane dai centri abitati o con questi mal collegati.

Il potere all'interno delle istituzioni dell'accoglienza è molto polarizzato: da un lato vi è un piccolo staff che gestisce le vite degli immigrati e dall'altro un gruppo numeroso di soggetti controllati, vulnerabilizzati e infantilizzati. È il tipico contesto da istituzione totale dove «c'è una distinzione fondamentale fra un grande gruppo di persone controllate [...] e un piccolo staff che controlla» (Goffman 2003, 37).

Il modello a cui tutti i centri sembrano conformarsi, sempre di più, è il modello hotspot. Come ha affermato Marc Arno Hartwig, funzionario hotspot in Italia per conto della Commissione dell'Unione europea, gli hotspot rappresentano dei luoghi così come dei concetti. L'idea di fondo è quella di estendere lungo tutta l'esistenza degli immigrati il 'trattamento hotspot'. Non a caso, infatti, la Commissione dell'Unione europea parla di «hotspot mobile», anche se sarebbe più corretto parlare di hotspot diffuso.

Che cosa sia un hotspot e il tipo di trattamento in essi riservato agli immigrati lo possiamo apprendere dal racconto di Djoka, sedicenne sudanese della zona del Darfur, giunto in Italia il 7 giugno 2016 in un porto del Sud e intervistato da Amnesty International. La sua intervista è riportata nel Report intitolato Hotspot Italia:

Appena sbarcato sono stato portato insieme agli altri in un centro. All'inizio mi sono rifiutato di dare le impronte digitali. [...] Dopo tre giorni senza cibo e acqua, mi hanno portato nella «stanza dell'e-

mento della Commissione europea, denominata Relazione sull'attuazione dei punti di crisi in Italia, del 15 dicembre 2015: https://ec.europa.eu/transparency/reg- doc/ rep/1/2015/IT/1-2015-679-IT-F1-1.PDF (2019-02-13). 
lettricità». C'erano tre agenti in divisa e una donna in borghese. A un certo punto è entrato nella stanza anche un uomo senza divisa che parlava arabo... I poliziotti allora mi hanno chiesto di dare le impronte digitali e io mi sono rifiutato. Allora mi hanno dato scosse con il manganello elettrico diverse volte sulla gamba sinistra, poi sulla gamba destra, sul torace e sulla pancia. Ero troppo debole, non riuscivo a fare resistenza e a un certo punto mi hanno preso entrambe le mani e le hanno messe nella macchina. Non riuscivo a oppormi. (Amnesty International 2016)

Le relazioni sociali all'interno delle istituzioni dell'accoglienza sono di tipo gerarchico, fondate su una rigida suddivisione dei ruoli tra staff e immigrati-ospiti. Tale suddivisione crea un «rapporto di sopraffazione e di violenza fra potere e non potere» (Basaglia 2014, s.p.). I gradi in cui questa violenza viene gestita possono essere diversi «a seconda del bisogno che chi detiene il potere ha di velarla e di mascherarla» (Basaglia 2014, s.p.). La violenza e l'esclusione si giustificano sul piano della necessità, come conseguenza le une (i centri di accoglienza in senso stretto) sul piano dell'emergenza oppure della gestione efficace, le altre (hotspot e centri di detenzione) su quello della sicurezza. Queste istituzioni, come ha ampiamente spiegato Franco Basaglia, «possono essere definite come le istituzioni della violenza» (2014, s.p.).

Sono luoghi in cui può nascere facilmente un odio errante, anonimo, un odio radicale. La tortura si nutre di questo odio, perché è «anzitutto il potere di dominare l'altro, di sopraffarlo con il tormento, di sottometterlo con la sofferenza, di soggiogarlo con la vessazione» (Di Cesare 2016, s.p.). Le violenze perpetrate nella torture room del centro 'Regina Pacis' erano figlie di quell'odio.

\section{Conclusioni}

Per gettare luce sull'abbraccio intimo tra tortura e razzismo si potevano prendere in considerazione molti casi, antichi e contemporanei, così come si poteva selezionare lo spazio geografico, dato che tortura e razzismo infestano il globo intero, e non da oggi. Si doveva fare una scelta e questa avrebbe avuto ripercussioni nel metodo e nei risultati finali. La scelta non poteva essere, però, frutto della mera preferenza di chi scrive, doveva essere guidata da un metodo e quello ritenuto adeguato in questo caso è quello idealtipico prospettato da Weber (1997). ${ }^{15}$

15 Trattasi di un approccio utile per l'interpretazione dei fenomeni sociali, in quanto capace di attribuire loro un significato o di rivelarne le tendenze principali: «quale che sia il contenuto di un tipo ideale razionale [...] la sua costruzione ha sempre [...] lo 
Con questa consapevolezza, si è scelto di analizzare il legame tra razzismo e tortura ponendo in rilievo la torture room del centro 'Regina Pacis', in quanto i centri di detenzione per immigrati (o di accoglienza, come significativamente li considera lo Stato) rappresentano emblematicamente le funzioni sociali, manifeste e latenti, delle politiche migratorie degli ultimi anni, in Italia così come in Europa (Perocco 2012, 2018). Sono politiche che si esprimono, quotidianamente, attraverso leggi discriminanti, circolari amministrative (Gjergji 2013) e accordi bilaterali (semi)segreti (Gjergji 2016), espulsioni di massa, impronte digitali, contratti di soggiorno schiavizzanti, fili spinati lungo i confini. In altre parole, sono politiche che contribuiscono alla proliferazione delle torture room nel territorio europeo.

Inoltre, va detto che la scelta, in qualche modo, ha coperto di silenzio le torture room dei centri libici (Veglio 2018) e di molti altri costruiti lungo i percorsi migratori, di cui molto si parla oggi, sia nei documenti delle Nazioni Unite che nei molteplici servizi giornalistici. Di recente se ne è parlato anche nelle sentenze dei Tribunali italiani, che hanno condannato alcuni dei torturatori operanti nei centri libici. In realtà, il silenzio 'imposto' su questi casi è solo apparente. Il centro 'Regina Pacis' è qui considerato l'emblema delle politiche migratorie europee proprio come lo è ogni altro centro costruito in Africa (o altrove) con il supporto politico e finanziario dei Paesi europei (Campesi 2013). Dunque, il centro 'Regina Pacis' è il centro idealtipico, attraverso il quale si può descrivere il fenomeno nelle sue linee più essenziali.

In aggiunta, centrare la riflessione sul 'caso Regina Pacis' ha consentito di evitare anche alcune trappole (razzistiche) costruite dalla narrazione dominante, quella che considera la tortura una peculiarità dei popoli non europei:

Il popolo europeo che tortura è un popolo decaduto, che tradisce la propria storia. Il popolo sottosviluppato che tortura agisce secondo natura, fa il suo lavoro di popolo sottosviluppato. (Fanon 2007, 32)

La tortura disumanizzante, come si è avuto modo di spiegare in questo lavoro, è stata, al contrario, un formidabile strumento di dominio dei colonizzatori europei, uno strumento utilizzato a grandi dosi ed eretto a sistema. Senza il razzismo-violenza tale sistema non sarebbe sopravvissuto. Era l'ingrediente magico', l'elemento che rendeva possibile la sua riproduzione. Ed è ciò che la rende possibile ancora oggi.

scopo di 'comparare' con esso la realtà empirica, e di stabilire il suo contrasto o la sua lontananza da essa oppure il suo relativo accostarsi ad essa, per poterla descrivere e intendere mediante l'attribuzione causale e quindi spiegarla, facendo uso di concetti intelligibili il più possibile univoci» (Weber 1997, 366). 


\section{Bibliografia}

Alleg, Henri (1958). La Question. Paris: Édition de Minuit.

Amnesty International (2016). Hotspot Italia. Come le politiche dell'Unione europea portano a violazioni dei diritti dei rifugiati e migranti. Roma: Amnesty International.

Apel, Dora (2005). «Torture Culture: Lynching Photographs and the Images of Abu Ghraib». Art Journal, 64(2), 88-100. DOI https://doi.org/10.1080/ 00043249.2005 .10791174$.

Arendt, Hannah (2001). La banalità del male. Eichmann a Gerusalemme. Milano: Feltrinelli.

Balibar, Étienne (1988). Race, Nation, Classe. Les identités ambiguës. Paris: La Dècouverte.

Barker, Martin (1982). The New Racism: Conservatives and the Ideology of the Tribe. London: Junktion Books.

Basaglia, Franco (2014). «Le istituzioni della violenza». Basaglia, Franco (a cura di), L'istituzione negata. Milano: Baldini \& Castoldi.

Basso, Pietro (2010). Razzismo di stato. Stati Uniti, Europa, Italia. Milano: Franco Angeli.

Beaugé, Florence (2000). «Torturée par l'armée française en Algérie, 'Lila' recherche l'homme qui l'a sauvée». Le Monde, 20 juin.

Bourdieu, Pierre (2013). Sullo Stato. Corso al Collège de France. Vol. I (1989-1990). Milano: Feltrinelli.

Campesi, Giuseppe (2013). La detenzione amministrativa deglistranieri. Storia, diritto, politica. Roma: Carocci.

Célérier, Patriacia-Pia (2014). «An Interview with Henri Alleg». African Studies Review, 57(2), 149-62.

Crosby, Alfred W. (1986). Ecological Imperialist: The Biological Expansion of Europe, 900-1900. Cambridge: Cambridge University Press.

Deaglio, Enrico (2015). Storia vera e terribile tra Sicilia e America. Palermo: Sellerio Editore.

Del Boca, Angelo (2005). Italiani brava gente? Vicenza: Neri Pozza Editore.

Di Cesare, Donatella (2016). Tortura. Torino: Bollati Boringhieri.

Farci, Manolo, Pezzano, Simona (2009). Blue Lit Stage. Realtà e rappresentazione mediatica della tortura. Milano: Mimesis.

Fanon, Frantz (2007). Scritti politici. L'anno V della rivoluzione algerina, vol. 2. Roma: DeriveApprodi.

Garland, David (2005). «Penal Excess and Surplus Meaning: Public Torture Lynchings in Twentieth-Century America». Law \& Society Review, 39, 793-809.

Geremek, Bronislaw (1986). La pietà e la forca. Storia della miseria e della carità in Europa. Bari-Roma: Laterza.

Gjergji, Iside (2013). Circolari amministrative e immigrazione. Milano: Franco Angeli.

Gjergji, Iside (2016). Sulla governance delle migrazioni. Sociologia dell'underworld del comando globale. Milano: Franco Angeli.

Gjergji, Iside (2018). 'Uccidete Sartre!'. Anticolonialismo e antirazzismo di un revenant. Verona: ombre corte.

Goffman, Erving (2003). Asylums. Le istituzioni totali: i meccanismi dell'esclusione e della violenza. Torino: Einaudi.

Greenberg, Karen J.; Dratel, Joshua L. (2005). The Torture Papers. The Road to Abu Ghraib. Cambridge: Cambridge University Press. 
Guillamin, Colette (1972). L'idéologie raciste. Genèse et langage. The Hague: Mouton.

Hajjar, Lisa (2013). Torture: Sociology of Violence and Human Rights. New York; London: Routledge.

Jaffe, Hosea (2010). Era necessario il capitalismo? Milano: Jaca Book.

Kaufman-Osborne, Timothy V. (2006). "Capital Punishment as Legal Lynching?». Ogletree, Charles J.; Austin, Sarat (eds), From Lynch Mobs to the Killing State. New York; London: New York University Press, 21-54.

Lévy-Strauss, Claude (1971). "Race et Culture». Revue Internationale des Sciences Sociales, 23(4), 647-66.

Lévy-Strauss, Claude; Eribon, Didier (1990). De près et de loin. Paris: Seuil-Odile Jacob.

Macmaster, Neil (2004). «Torture: from Algier to Abu Ghraib». Race \& Class, 46(2), 1-21.

Mackert, Jürgen (2015). «The Secret Society of Torturers: The Social Shaping of Extremely Violent Behaviour». International Journal of Conflict and Violence, 9(1), 106-20.

Marx, Karl (2009). Quaderni antropologici. Appunti da L.H. Morgan e da H.S. Maine. Milano: Unicopli.

Mollat, Michel (1983). I poveri nel medioevo. Bari-Roma: Laterza.

Patai, Raphael (1973). The Arab Mind. Tucson: Recovery Resources Press.

Patlagean, Évelyne (1986). Povertà ed emarginazione a Bisanzio. Bari-Roma: Laterza.

Perocco, Fabio (2012). Trasformazioni globali e nuovo diseguaglianze. Il caso italiano. Milano: Franco Angeli.

Perocco, Fabio (2018). «Anti-Migrant Islamophobia in Europe. Social Roots, Mechanisms and Actors». Revista Interdisciplinar da Mobilidade Umana, 26, 25-40.

Roberts, Dorothy (2008). «Torture and Biopolitics of Race». University of Miami Law Review, 62, 228-47.

Salerno, Eric (2005). Genocidio in Libia. Le atrocità nascoste dell'avventura coloniale italiana (1911-1931). Roma: manifestolibri.

Sartre, Jean-Paul (1960). Critique de la raison dialectique. Tome I. Théories des ensembles pratique. Paris: Gallimard.

Sartre, Jean-Paul (1962). «Prefazione». Fanon, Frantz, I dannati della terra. Torino: Einaudi, XLI-LIX.

Sartre, Jean-Paul (1964a). «Le colonialisme est un système». Situations V. Colonialisme et néo-colonialisme. Paris: Gallimard, 25-48.

Sartre, Jean-Paul (1964b). «'Portrait du colonisé', précédé du 'Portrait du colonisateur'». Situations V. Colonialisme et néo-colonialisme. Paris: Gallimard, 49-56.

Sartre, Jean-Paul (1964c). «Une victoire». Situations V. Colonialisme et néo-colonialisme. Paris: Gallimard, 72-88.

Sayad, Abdelmalek (2002). La doppia assenza. Dalle illusioni dell'emigrato alle sofferenze dell'immigrato. Milano: Raffaello Cortina.

Scarry, Elaine (1985). The Body of Pain. The Making and Unmaking of the World. New York: Oxford University Press.

Scott, George R. (1999). Storia della tortura. Milano: Mondadori.

Skoll, Geoffrey R. (2010). Social Theory of Fear. Terror, Torture and Death in a Post-Capitalist World. New York: Palgrave Macmillan.

Stannard, David E. (2001). Olocausto americano. Torino: Bollati Boringhieri.

Taguieff, Pierre-André (1988). La force du préjugé. Essai sur le racisme et ses doubles. Paris: La Découverte. 
Iside Gjergji

Tortura e razzismo. Teoria sociale, analisi storica, evidenze empiriche

Tindale, Christopher (1996). «The Logic of Torture. A Critical Examination». Social Theory and Practice, 22(3), 349-74.

Todorov, Tzvetan (2014). La conquista dell'America. Torino: Einaudi.

Veglio, Maurizio (2018). L'attualità del male. La Libia dei 'Lager' è verità processuale. Torino: Seb27.

Waldrep, Christopher (2002). The Many Faces of Judge Lynch. Extralegal Violence and Punishment in America. New York: Palgrave Macmillan.

Wallerstein, Immanuel (1985). Il capitalismo storico. Economia, politica e cultura di un sistema mondo. Torino: Einaudi.

Weber, Max (1997). Il metodo delle scienze storico-sociali. Torino: Einaudi.

Weber, Max (1998). Scritti politici. Roma: Donzelli.

Whitaker, Brian (2004). «Its Best Use is as a Doorstop». The Guardian, 24th May. URL https://www.theguardian.com/world/2004/may/24/worlddispatch.usa (2019-02-05).

Wieviorka, Michel (1991). L'espace du racisme. Paris: Le Seuil. 



\title{
Sur le rapport torture et migration Une réflexion philosophique/ politique
}

\author{
Marie-Claire Caloz-Tschopp
}

Université de Gèneve, Suisse

\begin{abstract}
Describing the relationship between torture and migration means examining its unpredictable foundations, its major civilizational challenges. In the relationship between capitalism and torture and torture and migration, a philosophical/political reflection proposes to identify an aporia: what happens to violence going to extremes (Balibar) inscribed in the self-destruction of humanity by itself? Torture, like an octopus extends its tentacles, poses new enigmas to struggles, knowledge, human rights. The general challenge is to radicalize critical work, to learn to think about extremes, to redesign the relationship to violence, to identify new forms of torture and the conditions for struggle and survival. To experience the democratic vertigo rooted in the report on torture and migration in Europe and elsewhere is to invent, on fragile soil, insurrectional democratic policies of counter-violence and civility.
\end{abstract}

Keywords Migration. Torture. Philosophy. Politics. Democracy.

Sommaire 1 Introduction: les énigmes du rapport torture et migration. - 2 Résistances a savoir. L'imaginaire de la guerre, de la torture. - 2.1 Remarques à propos de la torture et la migration. - 2.2 Le nœud central de la torture : la tentative de destruction de la liberté politique. - 2.3 L'enfer de Dante. Travail sur l'imaginaire. - 3 Comment pouvoir penser? Questions de méthode. - 3.1 Questions de méthode. - 4 Les énigmes actuelles de l'histoire. - 4.1 Histoire : technique dévastatrice et torture. - 5 Parcourir une aporie. - 5.1 Parcourir l'aporie de la violence allant aux extrêmes. - 6 Déplacement, horizons, vertige. - 6.1 La liberté politique de se mouvoir. - 6.2 Conclusion : vivre le vertige démocratique transpolitique. 


\title{
1 Introduction : les énigmes du rapport torture et migration
}

\author{
J'entends creuser le silence. \\ (Lorand Gaspar, poète, décédé le 9.10.2019)
}

Dedicace à une exilée héroïne ordinaire : A Kidest, jeune femme érithéenne seule avec un enfant né en Suisse, renvoyée en Grèce de force, menottée. ${ }^{3}$

La violence allant aux extrêmes² (Balibar 2010) et les luttes pour la liberté politique se meuvent dans un contexte d'incertitude planétaire (Europe, Moyen-Orient, Asie, Chine, continent africain, latino-américain). Elles sont traversées par des contradictions, impasses, énigmes insolubles. Dans le contexte actuel où les hégémonies bougent, elles sont difficiles à connaître, vivre, penser, juger. La torture est une des formes de violence extrême dans l'environnement, où se situe la migration prise dans les turbulences de la planète. La neutralité 'scientifique' est impraticable. Nos outils inadaptés. Le déni dangereux. La terreur inouie de la torture structurelle et quotidienne appelle la soumission, l'inertie. La fuite, la résistance, la création sont imprévisibles, fragiles.

La torture a pris de l'ampleur dans la migration ${ }^{3}$ depuis les années 1980. Le simple exemple actuel qu'entre 2 et 3,5 millions de réfugiés syriens, réduits à une arme géopolitique suffit à parler de torture au sens générique et non strictement juridique du terme. Autre exemple. La disparition est une caractéristique structurelle des politiques migratoires qui exige d'élargir la notion de torture, pour l'analyser à la lumière de la violence allant aux extrêmes (au sens de Balibar). Elle ne peut se limiter à être un simple dispositif, un outil technique de la violence d'Etat, des puissants. Elle est une politique destructrice des puissants.

La torture est pratiquée par des responsables politiques, agents d'Etat, multinationales, institutions financières, mafias, etc. Les nouveaux empires, les métamorphoses de la guerre de conquête de nou-

Je remercie Pauline, Graziella, Maria, Sabine, Teresa, Françoise, Silvia, Ahmet, Marcelo, Salomé, Steph pour leur soutien.

1 Pour plus d'informations, voir Droit de rester pour tou.te.s, http://droit-de-rester.blogspot.com/ (2019-11-19).

2 Dorénavant, j'écrirai violence extrême sans guillemets dans le texte. Cela indique que je me réfère aux recherches de Balibar à ce propos.

3 La guerre aux migrants en fuite se structure. D'ici à 2027, le personnel de Frontex passera de 700 à 10.000 fonctionnaires, voir https ://www.sosf.ch/cms/upload/pdf/ SOSF-BULLETIN_3-2019_FR_DEF_A4.pdf (2019-11-19). 
veaux marchés, lisibles dans la migration, nous met au défi, de penser ensemble violence et création, philosophie et politique. Il devient alors possible d'interroger des soubassements du rapport torture et migration, la dialectique entre violence allant aux extrêmes et poussées de lave de la démocratie insurrectionnelle, les difficultés de la philosophie politique, de l'Etat, du droit, des droits de l'homme (DH), du droit international humanitaire (DIH). L'enjeu n'est pas de produire un nouveau concept technique de torture sur la base des faits du terrain de la migration, ni de dénier les politiques de torture et de disparition à échelle continentale des dictatures du XXe siècle face aux opposants politiques. ${ }^{4}$

Le travail sur l'histoire récente, les affects et la pensée conduit à identifier le poids des peurs, des énigmes des savoirs, l'autoritarisme sécuritaire (Insel, à paraître), des mécanismes d'adaptation inconsciente (Amati Sas 2004) qui empêchent de comprendre la torture pour tenter de transformer la démolition en survie, la peur en angoisse, dégager la puissance politique de la création humaine de la liberté politique.

Pourquoi une violence inouïe, banalisée à l'encontre des migrants ? Pour éclairer la question, on peut s'appuyer sur le postulat wébérien de l'assimilation de la violence et du pouvoir (Héritier 1996), sur la distinction opérée par $\mathrm{H}$. Arendt et $\mathrm{S}$. Weil entre force et puissance quand elles pensent la guerre au XXe siècle. Aujourd'hui, la puissance et la force imprévisibles sont lisibles dans les métamorphoses de la guerre, et les luttes des migrants, des solidaires (Caloz-Tschopp 2016a).

A quelles conditions la philosophie politique apporterait-elle des outils pour penser à la fois la force d'Etat et la puissance d'action des migrants, exilés, mouvements sociaux qui constituent le rapport torture et migration? L'enjeu est immense : penser la politique comme une dialectique complexe entre domination guerrière et insurrection démocratique dans un contexte planétaire de dé-civilisation (Bozarslan 2019).

En s'interrogeant sur le rapport torture et migration, la philosophie, la politique sont mises au défi de repérer des apories - des difficultés logiques (du grec aporia, absence de passage, difficulté, embarras) à penser, agir - en interrogeant malaises, inconforts, difficultés à voir, penser, juger, agir. Nous sommes mis au défi de nous réapproprier l'imagination pour penser, juger avec et au-delà des moyens fournis par la tradition de la philosophie politique. En clair de la refonder.

En me situant dans le cadre général historique et actuel de la globalisation que peut expliciter l'analyse du schème guerre, terre, tra-

4 Dans la perspective d'un travail de mémoire, ces politiques peuvent aider à de les repenser à la lumière de l'actualité de la torture. 
vail, capital, je me propose d'intégrer ici le conflit entre la violence et la civilité étudiée par Balibar. ${ }^{5}$ L'enjeu est de réfléchir au rapport torture et migration, en se déplaçant dans un cadre politique, philosophique à créer.

\section{Résistances a savoir. L'imaginaire de la guerre, de la torture}

\subsection{Remarques à propos de la torture et la migration}

A chaque fois qu'on la croise, on est ébahi devant la violence inouie, cruelle, les morts aussi, qui fige, force au silence ou alors à la colère, à la rage, à des morts aussi. Elle est présente dans les discours, les gestes, les outils sous de multiples formes. Ecouter les silences, les cris, les mots, les discours. Commençons par situer les mots torture, migration, sans procéder ici à une analyse sémiologique critique (Fiala 2018) et à un déplacement sémiologique effectué dans un autre travail. ${ }^{6}$ Les travaux sur la genèse de discours décrivant la migration, dénonçant ou légitimant la torture abondent dans la recherche en sciences sociales. Mais Schengen, Dublin, Frontex bouchent l'horizon aux regards. Napoléon et le nucléaire aussi par un autre bout, comme on va le voir.

La torture, cette pieuvre dans les eaux obscures des Etats et des sociétés, a une très vieille et longue histoire. Elle étend ses tentacules en dévastant le monde tout au long des routes, des mers où fuient, sont chassés (Chamayou 2010) les migrants, les esclaves du servage, soldats, prisonniers des guerres, populations déplacées. Les tentacules de la torture guerrière s'étendent partout. Dans la mobilité forcée du travail et l'expulsion, avec celle des biens, des capitaux. Dans la guerre. Personne n'est à l'abri.

La torture est un rapport de pouvoir. La violence d'Etat, de groupes sociaux, d'invidivus exercé avec brutalité, cruauté sur les corps (Ulricksen 1998), est un rapport de longue durée, fait d'humiliation, d'atteinte de la dignité, souffrances, douleur, cruauté, tourments, démolition physique et psychique systématique (Vignar et al. 1989), tentative de destruction de toute espérance. Le franchissement du seuil

5 Dans un autre texte à paraître en 2019, j'aborde d'autres apories qui font système avec celle retenue dans cet article (Caloz-Tschopp, à paraître).

6 Déplacement de migrant, réfugié, requérant d'asile, etc. à exilé en desexil. Dans le cadre du Programme Exil-Desexil du Collège International de philosophie (voir site http://exil-ciph.com) et dans l'essai (Caloz-Tschopp 2019) sur la liberté de se mouvoir, est discuté l'hypothèse exploratoire de considérer l'exil et le desexil comme les conditions matérielles de vie des humains sur la planète aujourd'hui forcée de fuir la destruction et à trouver des moyens de survie. 
de la violence exercée sur le corps, la pensée du torturé est une limite qui le fait basculer dans un autre monde, duquel, parfois, il ne parviendra pas à revenir ou alors, lieu infime où il trouvera des ressources de survie. Les angoisses, maladies, suicides, l'invention de modes de résistance sont autant de traces indélébiles de la torture. Elle a souvent été légitimée par les aveux (inquisition, terrorisme), et la punition, mais son but, "n'est pas de faire parler, mais de faire taire » (Sorini, Branche 2002). La torture cherche à annihiler, détruire l'humanité des torturés, instaurer la soumission totale par la punition à tous les niveaux, sous toutes les formes possibles, faire disparaître tout espoir en l'homme lui-même et aux autres. Son but est l'attaque radicale de la « liberté d'être libre » (Arendt 2019) à la base de la liberté politique de se mouvoir de tout humain, dont les migrants, pris dans le tourbillon du rapport capital-travail et guerre (Caloz-Tschopp 2019).

Les descriptions montrent que la torture tente de détruire les bases d'espoir d'autonomie, liberté, solidarité humaine. Une psychanalyste a montré combien dans les moments ultimes de la torture, apparaissent « deux fronts de la survivance psychique » est dégagé ce qu'elle appelle « l'objet à sauver » (Amati Sas 2016, 69), une relation à l'autre imaginé que le torturé imagine pouvoir sauver, ce qui l'aide à survivre.

La torture est pratiquée sur des individus, mais elle est transindividuelle et transpolitique. Elle vise à installer un instrument politique totalitaire, la terreur (Arendt 1972), sur l'entourage, la société sous la férule de pouvoirs absolus pour figer, éliminer toute résistance à la domination du torturé, de son entourage, de la société. Elle s'inscrit dans une politique de l'annihilation (Veloso Bermedo 2018) dont les politiques de disparition sont devenues le paradigme. La politique de disparition dans les colonies, l'impérialisme, les guerres, durant des dictatures et aujourd'hui dans les politiques migratoires. La ville de Juarez au Mexique, les noyés de méditerranée, les morts des déserts sont des trous noirs des disparus de la migration. Le néant, est le visage ultime de la torture.

En résumé, la migration est un rapport pivot de pouvoir constitué par le schème général guerre-terre-travail-capital où peut s'observer le rapport structurel de violence torture et migration qui fait partie du quotidien. Le capitalisme actuel est constitué par la violence guerrière. La migration est constitutive du capitalisme guerrier, dont la torture est une forme de la violence allant aux extrêmes. La torture se pratique dans les prisons, les lieux du quotidien. La torture permet de saisir les logiques d'exploitation, surexploitation de travailleurs, d'humains superflus (Arendt 1972), la subordination et l'insurrection des migrants et de ceux qui pratiquent l'hospitalité, la solidarité, l'égalité. La généralisation des camps aux frontières internes et externes de l'apartheid (Caloz-Tschopp 2004a). L'augmentation des délits de sauvetage et de solidarité (250 arrêtés de justice 
dans 14 pays de l'UE) en sont des signes flagrants. En matière de migration, vu que les politiques migratoires et du droit d'asile sont le fait du système d'Etat-nation dominant sur la planète, la violence d'Etat mérite une attention spéciale.

La violence allant aux extrêmes, dont la torture est un fait structurel de la globalisation. Une approche phénoménologique décrivant les cas, formes multiples de torture ne peut en faire le tour, en dégager le sens. On peut en lire aussi les traces dans la destruction de la nature. Les politiques de disparition de nihilisme extrême sont l'expression de la non politique poussée aux extrêmes, lisible dans les morts en mer sans sépulture, les camps-prisons, aux frontières, les féminicides, les disparitions, les politiques des dictatures total-libérales. On le constate aussi dans une politique migratoire de l'UE qui se résume en utilitarisme extrême, entre la surexploitation des travailleurs migrants dans le brain-drain (fuite des cerveaux, clandestinité, précarisation extrême et superflus jetables du marché du travail chaotique) et dans les politiques aux frontières militarisées. Pour saisir l'ampleur de la torture banalisée, saisir son sens, on peut postuler que les migrants en mouvement chassés, poursuivis sont la figure par excellence des transformations du rapport capital-travail, de la destruction de la planète, de la guerre et du désir de liberté politique qui se traduit en luttes que nous appelons le desexil. Alors que la torture est inscrite structurellement dans la violence allant aux extrêmes, pourquoi les travaux sur la violence, la torture dans la migration, soulèvent des difficultés, dénis, refus?

\subsection{Le nœud central de la torture : la tentative de destruction de la liberté politique}

Posons d'emblée une thèse exploratoire. La torture est présentée avec un visage utilitariste de protection des populations, mais son but ultime est la soumission à n'importe quel prix. Une approche humanitaire, par des techniques médicales, le care n'en épuise pas le sens. Le pouvoir de violence allant aux extrêmes par la torture, la disparition, est intrinsèque à la domination capitaliste globalisée qui tente de s'imposer en s'appropriant l'Etat. La terreur des nouvelles formes de guerre produit peurs, angoisses, résistances énormes, dénis, refus à prendre acte de sa gravité par les Etats et les sociétés.

Torture, migration, les deux mots sont un rapport structurel. Ils traversent l'histoire de l'humanité, avec une institutionnalisation de l'exploitation dans le capitalisme entre le XVIIIe et le XXe siècle, du travail, des ressources tout en proclamant les droits de l'homme après deux guerres mondiales. Une nouvelle acuité de la violence, depuis les années 1980, tout en proclamant la liberté de circulation, la justice (Schengen), indique la présence institutionnalisée de la tor- 
ture dans les politiques migratoires sur la planète. Il est nécessaire de l'analyser pour transformer l'imaginaire philosophique et politique, la conscience sociale, lutter, critiquer les logiques, durcissements, voire l'abolition des droits (Tafelmacher 2019), créer des nouveaux espaces de protection, de civilité, des droits pour la combattre.

La résistance des migrants, mouvements sociaux à la violence, est une sorte de 'retour du refoulé' du désir de la liberté politique qui crée l'embarras, délégitime la violence d'Etat. Elle provoque de la part de l'Etat des réponses sécuritaires (emprisonnement administratif, camps, expulsions, délits de solidarité).

Sur la torture et la migration les travaux abondent, documentés interdisciplinaires, intercontinentaux. La violence structurelle est appliquée aux migrants, le plus souvent pour des délits administratifs (partir, survivre, s'opposer à l'interdiction d'entrée, aux expulsions). Une punition sans faute. En clair, elle concerne le rapport au système d'Etat exigeant la servitude des migrants. Le langage, les dispositifs, outils de la torture se transforment. Si la torture dispose des critères de la Convention contre la torture et autres peines ou traitements cruels, inhumains et dégradants ${ }^{7}$ de l'ONU de 1984 ancrée dans les droits de l'homme, elle ne parvient pas à être identifiée dans son but politique (soumettre), sa gravité dé-civilisationnelle (Bozarslan 2019) à tendance génocidaire et destructrice observable dans l'environnement, dont fait partie la migration. Elle ne fait pas l'objet de prévention sérieuse, condamnations, sanctions, réparations à la hauteur de la gravité des actes.

Le droit national, international des Etats souverains en matière de migration et de torture a des lacunes, zones d'ombre, trous noirs. Des institutions comme la Commission européenne des droits de l'homme $(\mathrm{CEDH})$, des textes comme la Déclaration des droits de l'homme interdisent aux Etats de pratiquer la torture. Formaliser la torture dans les droits fondamentaux est la tentative pour la nommer, sans aborder de front le nœud central politique du sens de la torture : le déni du désir de liberté politique et la légitimation, l'imposition de la soumission à n'importe quel prix. Jusqu'au néant. Face aux actes de torture actuels, des lacunes juridiques, le vocabulaire diplomatique pour confiner les conflits, la délégation de responsabilité produit l'indignation (ex. non répartition des réfugiés face à l'urgence).

Les tensions entre soumission et insurrection, la manipulation de la haine, aurait-elle pour fonction d'éviter ce que Vidal-Naquet (1972) ${ }^{8}$

7 Dès lors j'utilise le mot Convention.

8 Il a remarqué que le Général Massu a fait l'apologie de la torture fonctionnelle comparable à l'acte médical du chirurgien et du dentiste. Le Général a éludé le fait que la torture, loin de se limiter à une question morale était une question politique en ce qui concerne les rapports de la France à l'Algérie. On sait que les dispositifs et outils des 
a appelé, à propos de la torture coloniale en Algérie, un « retour du refoulé », dans les politiques migratoires alors que la plupart des Etats d'immigration torturent et/ou tolèrent la torture ?

La fuite des migrants, les délits de solidarité, rendent visibles le poids de la violence, de la torture, des crimes, le cynisme des intérêts, les transformations des politiques de disparition grâce à l'agir des migrants, à l'hospitalité des solidaires, des ONG qui s'opposent à l'accès, au tri aux frontières, ouvrent des refuges, visitent les camps, les prisons, sont aux passages des routes d'expulsion, interviennent pour rappeler les droits fondamentaux auprès du Conseil des droits de l'homme de l'ONU, à la CEDH, etc.

\subsection{L'enfer de Dante. Travail sur l'imaginaire}

La torture a un rapport direct à la guerre, à l'apartheid des politiques migratoires, du travail, etc. Où que l'on se trouve dans le monde, le rapport torture et migration est en effet un rapport de violence d'une philosophie d'apartheid (Monnier 2004), de séparation, hiérarchisation, tri, inclusion-expulsion des migrants à de multiples frontières, impliquant la surexploitation et le refus de la pleine participation civique de tous aux affaires publiques, comme l'explique Arendt (2019). ${ }^{9}$ L'apartheid puise ses sources historiques dans des régimes institutionnalisés (nazisme, Afrique du sud). En reproduisant la dualité guerrière ami-ennemi de Carl Schmitt dans les rapports de classe, de sexe, de race, l'apartheid s'inscrit dans la guerre (Caloz-Tschopp 2016a). La violence devient si évidente, si inouie, qu'elle brouille les cartes, fait éclater les repères, y compris celui de l'apartheid des démocraties sécuritaires. Le clivage dans les discours d'Etat qui essentialisent, naturalisent (racisme) l'autre (Sayad, à paraître) est mis en lumière par les récits d'expérience incroyables des migrants et solidaires aux frontières. Ils disent autre chose.

Les saccages, la surexploitation destructrice des humains, de la nature dans un espace globalisé mouvant, les métamorphoses des luttes, de la guerre s'explorent avec des outils construits dans l'histoire des savoirs qui montrent leurs limites. L'invention de démarches, concepts (anthropocène, capitalocène, d'occidentalocène, etc.), mettent en exergue le besoin d'une nouvelle philosophie, théorie politique, épistémologie, méthodologie, d'un usage de concepts en mouvement qui puissent décrire les enchevêtrements complexes entre guerre, terre, travail, capital. Les migrants sont une sorte

guerres de colonisation française (Vietnam, Algérie) ont été exportés, par ex. dans les dictatures latino-américaines, que les exilés ont dû fuir pour sauver leur vie et leur liberté.

9 L'histoire du droit de vote aux immigrés est édifiante à ce propos. 
d'avant-garde sacrifiée aux frontières multiples. Ils sont aux prises avec le mouvement illimité de la valeur du travail traduite en croissance, en destruction, en soumission à n'importe quel prix en déniant à la fois la liberté de se mouvoir et les limites de la planète, dont les migrants en fuite sont un des indicateurs.

Que pouvons-nous percevoir ou non sur les métamorphoses de la guerre et de la paix ${ }^{10}$ dans le rapport torture et migration en avançant le concept de torture de la Convention qui fait partie de l'arsenal des droits de l'homme ? En bref, les catégories de la Convention méritent une approche critique de la souveraineté étatique, de concepts comme celui d'intentionnalité mis en rapport avec l'approche transindividuelle (Balibar 2018) et transpolitique (Caloz-Tschopp 2019). Un tel travail théorique critique est indispensable pour renverser la force de souveraineté de l'Etat en puissance démocratique et réélaborer la question de la responsabilité. Par ailleurs, dans le travail d'identification, description de la violence, est-il suffisant de parler d'abus, dérapages, mauvais traitements, absence d'assistance, de protection, de droits ou alors comment, pourquoi faut-il parler de torture ? Pour repérer quoi ?

Deux faits dans le domaine de la migration aux frontières permettent de concrétiser les transformations de la torture visibles dans les politiques migratoires : le féminicide, les camps. Le féminicide est devenu une pratique courante aux frontières sans être reconnue. Des recherches mettent en lien féminicides et génocides, camps et génocides dans la colonisation en Afrique (Brepohl 2019). Par ailleurs, depuis les années 1980 (Schengen), un seuil a été franchi, avec l'institutionnalisation des camps en Allemagne pour les réfugiés, ${ }^{11}$ puis leur extension en réseaux de prisons dans toute l'Europe et à ses frontières externalisées. Malgré d'âpres luttes, ni l'un ni l'autre ne sont dénoncés, condamnés comme des crimes contre l'humanité. Ces faits s'inscrivent dans les politiques de disparition qui sont en quelque sorte le paradigme du capitalisme actuel.

Dès 1957, R. Antelme, prisonnier à Birkenau et Dachau, avait lancé un avertissement à propos du rapport entre l'exploitation et les camps après la deuxième guerre mondiale : «Il n'y a pas de différence de nature entre le régime normal d'exploitation de l'homme et celui des camps. Le camp est simplement l'image nette de l'enfer plus ou moins

10 Kant réfléchit à la paix, au droit international, à la propriété commune de la terre. Cet aspect n’a pas souvent été relevé (Caloz-Tschopp 2019).

11 Un fait oublié par la mémoire publique mérite d'être rappelé. En 1980, J.-P. Hocke, Commissaire pour les Réfugiés de l'ONU, a publié un numéro spécial du magazine Réfugiés de l'ONU sur la création de camps en Allemagne. Ce pays a menacé de couper les fonds au HCR si ce magazine était diffusé. Et le magazine a été retiré de la diffusion. Le Haut Commissaire pour les Réfugiés de l'ONU a démissionné. Aujourd'hui, il mériterait une autre médaille des Justes ! 
voilé dans lequel vivent encore tant de peuples » (Antelme 1957, 123). Il articulait exploitation et camps, histoire, présent, avenir.

La situation des femmes migrantes 'clandestines', les camps (dortoirs des travailleurs en Chine, camps en Grèce, prisons en Lybie, etc.) sont l'enfer de Dante d'aujourd'hui. Il nous faut articuler le féminicide et les camps-prisons en les situant dans le rapport capital-travail-guerre pour pouvoir dégager d'une part la logique de disparition et d'autre part ce que deviennent les rapports sociaux de classe, de sexe, de race, les luttes dans le marché mondial du travail et du capital et les transformations de la guerre et de la torture.

\section{Comment pouvoir penser ? Questions de méthode}

\subsection{Questions de méthode}

On peut commencer par s'interroger sur ce qu'indique l'émergence de l'usage du mot torture dans les discours sur la migration. Ensuite, on peut se demander ce qu'implique épistémologiquement le transfert de la notion de torture chargée de l'héritage de l'histoire politique la plus ancienne de l'humanité (inquisition, esclavage, colonisation, dictatures, pouvoirs hors Etat, paramilitaires, maffieux, etc.) à la migration. Légitimation cachée de la violence ? Comparer l'incomparable? L'opération analogique entre violence et torture et entre torture et migration se justifie-elle? Je postule que les constats nous amènent à établir un déplacement théorique et pratique pour comprendre et dépasser ces difficultés derrière ces questions.

Comment, à partir des constats de violence allant aux extrêmes, penser le rapport torture et migration aujourd'hui ? Que nous montre la présence de la torture sur les politiques migratoires ? En quel sens, comment et pourquoi pouvons-nous parler de politiques de torture dans le domaine des politiques migratoires ? On peut prendre appui sur la Convention. Mais il faut aller plus loin pour en dégager le sens. L'enjeu est, de penser avec et au-delà des moyens (outils), des styles existants pour s'inscrire dans la dialectique entre destruction et mouvement de création social-historique de la connaissance, de l'art (Sustam 2016), de la liberté politique.

Tout d'abord, alors que l'urgence, l'insoutenable est sur les écrans, on peut s'interroger : les rapports torture et migration, sont-ils une question humanitaire, les victimes suscitant la pitié, la culpabilité, qui se retourne en haine ou une question politique et philosophique incluant les transformations du désir de liberté politique et de la guerre ? Au premier abord, remarquons que la Convention se situe dans le corpus des droits de l'homme (DH) et non dans le celui du droit international humanitaire (DIH); elle situe ainsi la torture comme une atteinte aux droits fondamentaux. 
Situer le rapport torture et migration dans l'humanitaire serait l'inscrire d'emblée dans une vision des migrants comme victimes et non comme sujets de droit, et dans le droit de la guerre (masses) explique un juriste qui distingue les DH et le DIH (Rigaux, à paraître) et oblige à prendre en compte les enjeux du marché et géopolitiques de l'humanitaire.

Comment penser le rapport torture et migration depuis la philosophie politique en intégrant ce postulat ? Faut-il y voir un signe de plus du basculement d'une politique de la paix dans une non politique, l'effacement d'une culture des droits (contraints par le national, l'Etat national souverain sur un territoire, des populations) vers une culture du marché humanitaire et de la guerre, processus qui s'accélère, notamment dans le domaine de la migration depuis les années 1980, alors que les luttes deviennent plus visibles, plus dures (Caloz-Tschopp 2016c)?. L'usure, la fatigue et aussi la désobéissance civique (Caloz-Tschopp, à paraître) sont des indicateurs de la transformation de la violence. Les transformations institutionnelles de ces trente dernières années inciteraient à une telle interprétation. Il est nécessaire de faire un pas de plus pour repérer les implications de la violence allant aux extrêmes, quasi généralisée et banalisée dont parle Balibar (2010). Dès lors avec quels instruments approcher le phénomène de la torture aujourd'hui comme forme de la violence extrême, la torture, sa présence postulée, constatée dans la migration, ses buts?

Percevoir les transformations de la torture forme de violence et ses implications exige de se déplacer, considérer avec d'autres lunettes conceptuelles, théoriques, épistémologiques, méthodologiques la violence faite aux migrants. Une approche du rapport torture et migration implique de développer une philosophie politique mettant en cause la longue histoire de la souveraineté étatique sur les corps, le sol, les armes, les biens, etc., la violence d'Etat légitimée. L'enjeu majeur est l'attaque et le devenir de la politique et de la philosophie sur la planète.

Les Etats pour la plupart, ${ }^{12}$ soumis aux 'interdictions' de principe des textes d'instances européennes (CEDH) de l'ONU (Déclaration des DH), déclarent qu'ils ont aboli la torture tout en la pratiquant en toute impunité dans la migration et ailleurs, en vendant des armes tout en développant des discours dans les guerres « humanitaires » (Brauman 2001, 2018). Etrange paradoxe : au XVIIIe siècle ont émergé les valeurs (liberté, égalité, fraternité) des révolutions libérales limitées aux empires et dans ces empires aux classes dominantes tout en s'appuyant sur la colonisation, alors que la science de l'époque inventait l'idéologie raciste (Guillaumin 2000).

12 Les Etats-Unis, Israël justifient la torture par nécessité de sauvegarde de la sécurité intérieure de l'Etat. 
Le rapport de torture fait partie des politiques du travail, de ce qui est appelé la mobilité des migrants, en déniant le fait qu'elle est forcée, donc violente. C'est un clignotant majeur ${ }^{13}$ des métamorphoses des luttes et de la guerre. Il émerge dans la conscience collective planétaire. Il caractérise l'état du monde où se meuvent les travailleurs hyperprécarisés, pris dans les turbulences des logiques d'appropriation, chasse, pillage, surexploitation, destruction. La violence actuelle est plus difficile à caractériser qu'auparavant, les frontières entre les types de violence, de crimes se brouillent. Les transformations de la géopolitique au Moyen-Orient, la guerre en Syrie, au Yémen en sont des exemples (violence intra-étatique, interétatique, transnationale, diversité des sujets politiques). Qu'en est-il de la torture ? Où, quand, comment, pourquoi faut-il alors repérer le franchissement institutionnalisé du passage de la maltraitance à la torture, de la violence d'Etat au crime d'Etat (Vahabi 2019) dans la migration ?

La question est complexe. Elle renvoie à un déplacement, à un renouvellement de l'épistémologie, positions, choix méthodologiques, outils d'exploration dans le travail de recherche. Les luttes des femmes migrantes 'clandestines' aux frontières et en particulier, dans les camps-prisons aux frontières sont des lieux-clés d'observation, d'action.

La torture fait partie des politiques du travail, de la sûreté après septembre 2001 (Patriot Act, prisons secrètes, Guantanamo), des politiques migratoires, du droit d'asile, mais les mots de « torture et autres peines ou traitements inhumains et dégradants » ne figurent pas en tant qu'interdiction punissable dans les textes (pacte mondial sur les migrations de 2018, lois des pays). L'Etat de droit, le droit existant se heurtent aux limites de la violence d'Etat et aux lacunes des droits.

L'enjeu historique et actuel est de prendre acte de la torture dans la nouvelle conformation chaotique des empires, du marché du travail et du capital globalisés, impliquant la circulation, la mobilité forcée combinée avec la fixation illusoire des populations, en interrogeant ses rapports à l'histoire et à la guerre. Quand on observe Schengen, Dublin, les camps-prisons, Frontex, on ne peut parler de laboratoire d'essai, de cobayes, d'abus, de débordement sécuritaires, etc. Sa présence structurelle indique une transformation de la violence guerrière dans les rapports de pouvoir et les rapports torture/migration. Leur présence structurelle indique une transformation de la violence guerrière dans les rapports de pouvoir et les rapports torture et migration. C'est aussi un des signes de la destruction de l'environnement dans son ensemble ${ }^{14}$ sur la planète.

13 Le statut des femmes, la question du climat en sont d'autres. On verra en quel sens, ils sont liés.

14 La question de ce qui est appelé les 'réfugiés climatiques' exige un changement de paradigme pour être abordé sérieusement. 


\section{$4 \quad$ Les énigmes actuelles de l'histoire}

\subsection{Histoire : technique dévastatrice et torture}

Un arrêt historique peut permettre d'intégrer des interrogations sur la technique et la torture pour comprendre le rapport torture et migration actuel. On peut choisir de s'arrêter à l'histoire récente (fin XIXe-XXe siècle) dans l'impérialisme de la Première Guerre mondiale et ses « effets boomerang » que décrit R. Luxemburg (Caloz-Tschopp 2018), la Seconde Guerre mondiale avec Auschwitz et Hiroshima (Traverso 1997). La disparition d'empires (austro-hongrois, turc, russe), la domination de l'Etat-nation et la montée de nouveaux empires en reconfiguration, l'engagement dans ces guerres, précédées des massacres, génocides coloniaux (Namibie), impériaux (Arménie) a signifié le passage dans l'histoire, où la question de l'intégrité, de la mortalité sont non seulement devenues celle de la torture, de l'assassinat industriel de masse, d'une civilisation de pillage, de destruction, mais l'aporie de l'autoextermination de l'humanité par elle-même sur la planète Terre. Pour la Seconde Guerre mondiale, deux analyses de cas ont fait couler beaucoup d'encre : le procès d'A. Eichmann et le pilote d'Hiroshima. Ces deux analyses de cas non exhaustives ${ }^{15}$ ont situé la destruction dans l'histoire (Auschwitz et Hiroshima). Son élaboration conflictuelle amène une rupture de ce que Castoriadis appelle l'Etre social-historique et une aporie majeure de la pensée sur la violence mise au défi d'intégrer les conquêtes, les colonialismes, l'impérialisme qui ont précédé et accompagne le capitalisme.

Pour continuer la réflexion, depuis aujourd'hui où la crise environnementale menace les soubassements de notre civilisation, et l'existence même (Magnénat 2019), revenons un instant sur un chemin ouvert par une psychanalyste (Silvia Amati Sas) et un philosophe (Günther Anders) s'interrogeant sur les rapports entre science, technique, civilisation qui ont une influence directe aujourd'hui sur l'expansion des dispositifs de la torture, y compris dans la migration, tout en complexifiant les questions de l'intention, l'aliénation, la conscience, la pensée, la responsabilité. Le développement du nucléaire, ses 'accidents' et ses suites, par exemple remet à l'ordre du jour, ces interrogations des années 1950-70.

La lecture successive des deux discours permet d'approfondir des difficultés philosophiques et politiques dans une civilisation technique se développant avec le capitalisme avancé et pouvant traduire aujourd'hui l'intentionnalité, la responsabilité des Etats, agents dans l'ensemble des politiques, y compris dans le rapport torture et mi-

15 Le génocide des Roms est quasi invisible, il a pourtant été planifié dans la 'Solution finale' nazie. 
gration actuel. On peut s'en inspirer pour penser les rapports travail-capital dans le monde, les féminicides, les camps, les décès des migrants en méditerranée, sur les chemins d'exil.

\subsubsection{Imaginer des millions de morts}

Les développements de la science et la technique ont un rapport direct avec la guerre et la torture, en engageant les savoirs, la responsabilité, la culpabilité, mais en les complexifiant. On le voit bien dans la migration. La thèse a été formulée par de nombreux auteurs. Les agents de l'invention de la bombe atomique ont créé une civilisation de mégamorts (Amati Sas 1984), " d'obsolescence de l'homme » (Anders 2011). Relire le discours d'Albert Camus et d'autres discours quelques jours après le bombardement d'Hiroshima permet de mesurer la capacité très limitée de saisir l'impact, les conséquences incalculables. L'autodestruction de l'humanité devient inimaginable, et même non perceptible par l'imagination, les affects, la pensée, la conscience. En clair, l'intentionnalité, supposant les affects, la pensée et la conscience disparaissent comme attitude subjective à la base de la responsabilité, écrivent une psychanalyste et un philosophe, marqués par la guerre totale, les camps d'extermination, la bombe nucléaire et la civilisation technique qui accompagne ces faits historiques. Elle peut conduire au déterminisme, à oublier la liberté politique devant la toute-puissance de la technique, des techniciens, des bureaucrates.

La création scientifique de traumatismes et de situations extrêmes pour manipuler les hommes est le propre de notre civilisation. Commencée dans les laboratoires, elle a abouti à la torture institutionnalisée et aux camps de concentration [...] Le désir de maîtriser la nature propre à la science a abouti au désir de quelques hommes de maîtriser les autres hommes jusqu'à leurs dernières défenses. (Amati Sas 1984, 14)

Il est question de morts et de morts de masse pas quantifiables (alors que la statistique est l'outil par excellence de la migration, de la politique du chiffre des polices). L'Etat qui a lancé les bombes, pour enjoindre le Japon de finir la guerre, et surtout le mettre à genoux devant le nouvel empire vainqueur voulant asseoir sa puissance sur le monde. Les Etats-Unis qui ont lancé la bombe sur Hiroshima et Nagasaki n'ont pas été condamnés pour crime contre l'humanité. Ils ont lancé le Plan Marshall pour contrôler la reconstruction de l'Europe.

Impossible de calculer. Impossible de décrire positivement, le nonEtre, la destruction. Même le concept théologique de 'mal' relayé par le concept du 'mal radical' de Kant et du 'mal politique' traduit 
en « banalité du mal » par Arendt (1966) peut décrire le phénomène mais pas le penser. "Il me fallait comprendre », dira-t-elle à plusieurs reprises. Elle prolongera sa recherche sur la pensée (Arendt 1981) et fera des conférences sur le jugement. La psychanalyste invente le mot de mégamorts en se demandant si c'est une " unité de mesure » ou une « métaphore » (Amati Sas 1984). Elle écrit :

pour nous pencher sur la guerre nucléaire, il faut vraiment forcer notre résistance, car le sujet fait bouger les convictions et les certitudes qui protègent notre joie de vivre. Il s'agit de saisir la signification du choix de la politique nucléaire conçue comme un signe de puissance, la dissuasion par la terreur, et évaluer comment la sécurité est prise en otage par quelques Etats pratiquant l'imprévoyance et l'irresponsabilité qui nous côtoie dans notre monde nucléaire et cela dans un contexte de guerre en mutation, de monde multipolaire. C'est une guerre de quantités inimaginables. Nous avons l'impression de livrer passivement la destinée de chacun aux signes mathématiques et à la concrétude des données statistiques et informatiques ! Les valeurs de l'ère technologique sont des valeurs froides et désaffectées qui conviennent à notre besoin de nous cacher à nous-mêmes notre peur et angoisse de la mort. (Amati Sas 1984, 9)

Imaginer des millions de morts, c'est déjà se consoler en perdant la démesure de la question liée à la destruction. La perspective de la guerre nucléaire est « l'arrêt du projet de vie de l'humanité » et la destruction de l'humanité et de tous les êtres vivants, écrit-elle.

\subsubsection{Percevoir à quoi sert un bidon de Zyklon B}

Dans ces mêmes années, un philosophe exilé d'Europe durant 14 ans, marqué à vie par la guerre de 1914-18, puis par Auschwitz et Hiroshima, a été taraudé entre les années 1950-70 par les thèmes qu'il a appelé l'obsolescence de l'homme (Anders 2010) et aussi un « monde sans hommes »(1950-70), ce qu'il a déclaré dans l'entretien publié sous le titre suivant, après s'être effondré à la nouvelle d'Hiroshima: Et si je suis désespéré que voulez-vous que j’y fasse ? (Anders 2001). Plus tard, après une visite à Hiroshima, il écrira, Hiroshima est partout, traduit aux éditions du Seuil en français en 2008 ! Il a parlé alors de nihilistes actifs. Dans l'entretien, il évoque aussi ses travaux sur la pathologie de la liberté, de Weltfremdheit des Menschen (étrangeté de l'homme au monde), "d'apocalypse sans royaume », de la fin de l'humanité par autodestruction sans signification, sans tribunal ultime, sans rédemption. Il s'est attelé à l'étude des cas du pilote d'Hiroshima, de la guerre du Vietnam, tout en travaillant sur une phi- 
losophie de la technique devenue, pour lui, un monde autosuffisant et décidant de notre existence. Il a polémiqué avec Heidegger, tout en reprenant par un autre bout sa question sur la technique. Sa méthode, expliquée par Roger Pol-Droit, ${ }^{16}$ se résume par l'exagération et l'attention aux traces infimes pour ce qui est dénié, négligé, voilé : la déréalisation du monde, la déshumanisation du quotidien, la marchandisation générale.

En constatant les limites, les dérives de philosophes, tout en travaillant sur leurs œuvres en se déplaçant, Anders va développer sa propre philosophie autonome minoritaire.

Imaginer la signification d'un bidon de Zyklon B, d'un réacteur atomique supprimant des millions de gens, déplace radicalement le travail de pensée et la question de la conscience, de l'intentionnalité, de l'aliénation et de la responsabilité. Nous sommes dans un monde de physiciens, d'ingénieurs telosblind (mot-valise qui combine le mot grec, telos, but, la fin et l'anglais blind, aveugle), écrit-il en s'interrogeant sur les limites de la tradition philosophique et les duplicités de certains philosophes (Anders 2001).

"Même si l'imagination seule reste insuffisante », écrit Anders $(2001,68)$, elle est entraînée de façon consciente, elle saisit (nimmt) infiniment plus de "vérité » (mehr «wahr») que la "perception» (Wahrnehmerung). Pour être à la hauteur de l'empirique. Et aussi paradoxal que cela puisse paraître, il nous faut mobiliser notre imagination. C'est elle la «perception d'aujourd'hui », écrit-il $(2001,68)$. Trace infime, infra de liberté politique?

Sur ce point, il rejoint un philosophe grec exilé, Castoriadis, qui n’a pas comme référence la phénoménologie de Husserl et de Heidegger mais le marxisme, la psychanalyse, l'économie, la Grèce ancienne et travaillera dans les années 1960-89 sur l'imagination radicale et la création humaine, après avoir fondé le groupe Socialisme ou Barbarie.

Pour Anders, ce n'est pas une question morale mais de cognition, car science et conscience sont toujours liées. Ceux qui provoquent des génocides ne sont pas plus mauvais que les générations précédentes, écrit-il. "Dans ma correspondance avec le pilote d'Hiroshima, Eatherly, j'ai forgé le concept de 'coupable sans faute' (schuldlos Schuldigen). Je ne prétends pas que 'l'homme' soit plus mauvais, mais je dis que ses actions, à cause de l'énormité des outils dont il dispose, sont devenues énormes » (Anders 2001, 69). Anders, se référant au massacre de My Lay au Vietnam, ajoute un élément à sa réflexion : celui de "l'assassinat indirect » à l'aide de machines (on pense aux drones). Il n'y a plus intervention directe mais à distance, ce qui amoindrit la perception des faits et leurs conséquences. 
«La situation dans laquelle nous sommes n'est plus celle de la seconde guerre mondiale, quand on bombardait d'en haut, sans voir ce que l'on faisait »; il s'agit d'un troisième type de situation, « où nous imitons nos outils, pour voir enfin, un peu ce que nous faisons » (6869). Pour décrire l'aliénation produite par la technique, Anders parle alors « d'innocentement » (Verharmlosung) et « d'enjolivement » (Verniedlingug) qui est "supraliminaire », c'est-à-dire " les actions trop grandes pour être encore conçues par l'homme » (72) et donc perçus, mémorisés. Trop grand pour être perçus ou s'en rappeler (mémoire). Il y a un décalage entre production et imagination qui oblige à une critique radicale des concepts de la tradition et des facultés humaines (imagination, perception, intentionnalité).

Amati Sas se réfère plus tard à son collègue Bleger (Caloz-Tschopp 2014; Amati Sas 2016) qui n’a pas vécu dans la vieille Europe, mais en Argentine, qui, on peut le penser, a pressenti la dictature et la politique des disparus, quand il interroge les mécanismes d'ambiguité, d'adaptation, d'étrange familiarité, qui rend acceptable la destruction, la pratique l'exil forcé, l'isolement, la terreur politique par la torture.

Un autre psychanalyste latino-américain, Marcelo Vignar, insiste sur la fonction de la terreur dans la torture, concept-clé d'Arendt pour décrire Le système totalitaire (Arendt 1972): terrifier le torturé, son entourage pour immobiliser, soumettre la société. Il insiste sur l'importance de la terreur politique dans les dictatures, sur le fait que le métier d'analyste pour lutter contre la torture n'est pas une technique médicale de 'résilience', mais une relation située dans l'histoire et la politique, avec ses outils spécifiques basées sur le recouvrement de la puissance de l'inconscient, de la parole, de la pensée (Vignar et al. 1989; Vignar 2018). Son approche permet une analyse critique et concrète des outils professionnels et politiques non seulement des médecins, mais de l'ensemble des professions.

Consentement et résistance de survie. Amati Sas s'interroge : « comment est-il possible que nous acceptions l'inacceptable, que nous tolérions n'importe quoi ? Par quel mécanisme psychique prenons-nous les choses très graves comme allant de soi ?» $(2014,14)$. " Pour nous adapter à notre culture technologique de masse, si confondante et envahissante à travers les mass media, une bonne partie de nous doit rester (ou même devenir) ambiguë » (16). Elle avance les notions d'indifférenciation, de noyau agglutiné archaïque, " ensemble d'affects non discriminés et sans organisation ni hiérarchie » (16), à la base de l'ambiguïté, concepts mis en exergue par le psychanalyste José Bleger. Un tel concept permet la circulation de l'angoisse (vulnérabilité psychique et sentiment d'impuissance), mais interroge la solution proposée par les pouvoirs militaires : « ils ne nous posent pas un choix de vie, mais un défi à la survie à travers une froide manipulation » (17). Ils prétendent « offrir la sécurité à travers un système hautement mortifère » (17). 
Les liens entre traumatologie, vulnérabilité, adaptabilité psychique peuvent être aussi élaborés dans le rapport torture et migration. Le mandat analytique implique un travail de renforcement de l'insight, de la symbolisation, l'élaboration du conflit, l'intégration de la personnalité, " C'est-à-dire l'autonomie de la pensée et de la personne » (Amati Sas 2014, 18). Einstein, rappelle-t-elle, face au danger nucléaire en appelait à un "mode de pensée radicalement nouveau » (Einstein, Russell 1955). Après l'interdiction du cannibalisme et de l'inceste, nous sommes mis au défi de créer un nouvel interdit, " une évolution fondamentale, transcendantale », écrit-elle (Amati Sas 2014, 19).

Pour Anders, L'éthique à Nicomaque d'Aristote ne nous aide pas à penser la situation actuelle. Le courage, la consolation, l'espoir non plus. L'aporie à propos de la perception à la base de l'intentionnalité, amène Anders à " conserver le monde avant de le transformer » $(2001,77)$ et à inventer une nouvelle philosophie et une nouvelle langue à partir du constat de l'obsolescence de l'homme. Anders, qui avait suivi les cours d'Husserl et d'Heidegger dans l'université allemande, est devenu très critique vis-à-vis de Heidegger et a milité activement contre l'industrie nucléaire et la guerre du Vietnam (membre du Tribunal Russell).

En résumé, pour ces auteurs, le poids du déterminisme de la civilisation capitaliste et de la technique traduite en obsolescence de l'homme, en guerre 'totale', nucléaire, génocides, terreur, torture institutionnalisée, attaque l'espoir positif en liberté politique, redouble l'exigence d'agir partout où c'est possible (l'espoir, le désespoir, le courage ne sont plus la question pour Anders) tout en inventant une nouvelle philosophie, mais exigent un travail sur les difficultés à penser, à élaborer le conflit politique en intégrant l'histoire du XXe siècle. Pour les deux auteurs, comme pour Einstein, Arendt, les chercheurs de l'Ecole de Francfort (raison instrumentale), etc., la réappropriation, de la pensée de la philosophie, de la politique est fondamentale. Ils montrent que la torture tente de détruire les corps, les affects, la capacité de penser, le désir d'être libre. D'où la reprise de l'imagination chez Anders et le travail sur l'inconscient individuel et social face à la violence d'Etat (Puget 1989) chez les psychanalystes.

Comment penser dans une maison qui brûle se demandent aujourd'hui des chercheurs de la crise environnementale (Magnénat 2019)? Le terme englobe la migration. Comment pouvoir percevoir le rapport torture et migration qui fait partie de l'héritage historique du lien entre violence, pensée, conscience, technique et pouvoir dépasser l'impuissance, l'indifférence, l'apathie pour modifier nos modes de vie et se réapproprier la puissance de la liberté politique ? Dans une perspective phénoménologique, des chercheurs ont décrit les murs, la civilisation militaire des barbelés (Razac 2008) de fortification des frontières, des clôtures d'élevage, renvoyant au quadrillage 
terrestre de la propriété privée interprété en terme de déclin de la souveraineté étatique (Brown 2009). Ces descriptions ont certes saisi les logiques d'apartheid sur le sol, dans les têtes, les affects. Pour ce faire, la réflexion, les luttes sur les frontières, lieux mouvementés, chaotiques multiples de conflits, contradictions dans le rapport travail-capital-guerre, ne doit pas se centrer sur la seule dynamique de l'expulsion, mais sur l'ensemble des logiques, des dispositifs, outils de séparation, d'inclusion/expulsion, ${ }^{17}$ de classement, hiérarchisation, la présence et la nouvelle qualité de la violence qu'indique la torture et la torpeur face à des faits inacceptables.

\section{$5 \quad$ Parcourir une aporie}

\subsection{Parcourir l'aporie de la violence allant aux extrêmes}

Arrêtons-nous à une aporie dégagée par la démarche philosophique de recherche critique qui a un lien direct avec la torture et la migration pour en saisir les enjeux politiques et philosophiques. Elle est une sorte de nœud gordien dans les soubassements de la torture et du rapport torture et migration. Dans le rapport torture et migration, comment pouvoir penser, s'en sortir de la violence allant aux extrêmes, contenu dans la violence d'Etat et le crime d'Etat qui paradoxalement produit un effet anesthésiant?

Il y a la violence des puissants, la violence d'Etat, la violence de la résistance qu'interroge Ahmet Insel (à paraître) en réfléchissant aux grèves de la faim dans les prisons turques et à une politique de la paix. Il y a la violence allant aux extrêmes, dégagée par Balibar quand il relit Clausewitz observant Napoléon, qui, en intégrant l'histoire et le présent, repère une qualité de la modernité capitaliste guerrière qui bouscule profondément notre approche de la politique et de la philosophie.

La violence extrême, avec les urgences et les limites de la planète, se transforme en nouvel impératif philosophique et politique de civilité. Torture et Démocratie ne sont pas compatibles. Nous allons voir que lutter contre la torture implique d'accepter de vivre le vertige démocratique.

L'interrogation sur la violence allant aux extrêmes, revient sur les difficultés, postures d'Amati Sas, Anders, Arendt, Vignar, en les reprenant par un autre bout. Le rapport torture et migration nous montre que qualifier la torture en s'appuyant sur des concepts, des

17 Le texte de Balibar présenté au Groupe de Genève en 1986, Qu'est-ce qu'une frontière?, a ouvert ce chantier de réflexion critique essentiel. Il a été repris par l'auteur dans son livre La crainte des masses (Balibar 1997). 
échelles de classement des crimes et en se contentant des critères de la définition de la torture de l'ONU, ne permet pas de lutter contre la torture. Alors que faire ? Si l'on accepte d'intégrer la torture comme un enchevêtrement complexe d'actes multiples, hétérogènes de la terreur, de la violence extrême aux effets imprévisibles, on ne peut plus se satisfaire d'une approche humanitaire, utilitariste, d'un pensée d'Etat-nation souverain, d'une pensée-calcul, d'une pensée d'échelle avec l'illusion de définir des niveaux et des limites ?

La solution courante de l'aporie se fixe sur des limites (plus jamais ça !), des normes, des critères juridiques, des procédures pour le droit pénal des droits de l'homme. Son approfondissement philosophique sur la politique face au chaos abyssal d'autodestruction des humains par eux-mêmes implique pour Balibar d'aller plus loin, de se déplacer de la tradition philosophique dominante pour poser un pari tragique : le pari tragique de la civilité. Il présente le processus de création politique de civilité, de contre-violence, de re-civilisation pour contenir la dé-civilisation (Bozarslan 2019).

Résumons des lignes de force d'un essai fondamental de Balibar (2010) qui l'amène à penser ensemble la violence et la civilité. Soulignons qu'il aborde la question de la violence extrême dans les développements de la guerre qui change de statut (Clausewitz tirant les leçons des guerres napoléoniennes devenues incontrôlables, imprévisibles), des nouvelles formes d'appropriation de la valeur, de l'exploitation, de la surexploitation du travail et aussi de pratiques d'humains superflus (Caloz-Tschopp 2000), celles et ceux qui ne sont pas intégrables dans la réorganisation globalisée du marché du travail ou alors subissent des formes d'expulsion des rapports de classe, de sexe, de race. A propos de la construction l'Etat national social, de l'Etat tout court, il concerne les expulsés à des titres divers pris dans des processus complexe d'expulsion de conditions matérielles de vie, de l'exercice de la liberté politique en participant aux affaires publiques, du monde précise Arendt (1972).

Face aux débats sur la question sociale (présente en France et aussi ailleurs dans l'UE, dans d'autres continents), l'auteur préconise d'articuler citoyenneté sociale et invention de nouvelles formes de la citoyenneté, qu'il appelle la civilité pour surmonter les contradictions de l'Etat national social et penser l'imprévisibilité de la liberté et de la violence extrême et ses conséquences incalculables. Les dilemmes, écrit-il, sont difficiles à énoncer et à mettre en place (Balibar 2010). Des savants face au nucléaire ont déjà parcouru ces dilemmes de la science. Peut-on s'en sortir de la violence exterminatrice ?

Balibar développe une philosophie qui traduit la phronesis d'Aristote (prudence, autolimitation), non sur la base de la distinction quantité/qualité, ou en philosophie des degrés, des limites (supportables, pour qualifier, classer des crimes dont la torture) mais en développant une philosophie des seuils, située dans la dialectique possible/ 
impossible, transformée en pari tragique de l'action humaine collective et individuelle. On passe ainsi d'une métaphysique déterministe du limité/illimité à une philosophie politique. Une anthropologie de la puissance/impuissance, du possible/impossible humain, rien qu'humain pour agir. Cette approche de la responsabilité ouvre au transindividuel (Balibar 2018) et au transpolitique tout en refusant ainsi une philosophie catastrophiste en ne déniant pas la part de violence dans l'action humaine et les sociétés. Point fondamental. La violence est devenue une aporie dès lors qu'elle met en cause la possibilité de la politique et de la philosophie, écrit-il dans son essai (Balibar 2010). Là se trouve la limite infranchissable du néant. On ne s'en sort pas de la violence. Alors, comment penser aux extrêmes? C'est un pari tragique pour une politique d'anti-violence. C'est le pari tragique irréductible de l'altérité contre celui de la tentation de l'absolu, du jusqu'au bout, abîme où l'extrême violence attire, séduit quand on la subit, on désire s'en défendre en se faisant happer.

\section{Déplacement, horizons, vertige}

\subsection{La liberté politique de se mouvoir}

La fuite des migrants face à la violence destructrice de conditions matérielles de vie et d'horizons, la résistance des solidaires, leur désir de liberté politique ouvre l’horizon incertain pour déplacer le rapport torture et migration et explorer des outils de lutte contre la torture. En d'autres termes, penser le rapport torture et migration implique les luttes de dénonciation, de pénalisation et la création d'espaces de liberté politique de se mouvoir, ce qu'Arendt appelle la liberté d'être libre.

Aborder le pari tragique de la civilité en proposant la liberté politique de se mouvoir, en interrogeant ce que j'appelle le vertige démocratique (Caloz-Tschopp 2019), ne résout pas l'aporie de la violence extrême, mais la parcourt inlassablement depuis le désir de liberté politique, en pensant aux extrêmes, avec et au-delà de nos moyens, de nos outils pour saisir, se réapproprier inlassablement la puissance démocratique. Sur le terrain des rapports torture et migration, cela implique de ne pas se laisser prendre au piège des idéologies 'populistes nationalistes', du marché de l'humanitaire et du marché capitaliste, pour pouvoir, en se déplaçant, se réapproprier la philosophie et la politique et repenser le rapport guerre-terre-travail-capital, les régulations/dérégulations du marché du travail basé sur la violence politique, l'absence de politiques migratoires dominées par le chaos utilitariste.

Un tel déplacement implique d'établir la distinction critique entre liberté de circulation des capitaux, des biens, de la main-d'œuvre qui se 
trouve dans les textes de l'UE et de Schengen, entre mobilité et liberté politique de se mouvoir. Dans les débats en cours sur les politiques migratoires (Pacte migratoire, lois) la mobilité est présentée comme un concept alternatif face à la violence économique et policière qui tend, sans succès à figer, à surexploiter, à jeter les populations dans le néant de la disparition. On peut se demander jusqu'où le concept de mobilité peut répondre à l'impératif de la circulation planétaire de la valeur produite par la force de travail du capitalisme avancé. Suffit-il de rendre mobile les travailleurs migrants pour s'en sortir?

Remarquons que le concept de mobilité, en débat actuellement autour du pacte migratoire (Carlier, Crépeau 2017), et de la politique européenne de migration, fait l'impasse sur la mondialisation de la migration et ses exigences, sur l'aporie des frontières imposées par l'apartheid, l'Etat-nation territorial et souverain. Rappelons que dans la Déclaration des droits de l'homme (art. 13.2) tout individu a le droit de quitter son pays mais pas d'entrer dans un autre pays. Angelopoulos, dans son film, Le pas suspendu de la cigogne, montre ce qui se passe aux frontières, dans les zones de transit, devant les murs, les barbelés. La cigogne aujourd'hui, loin de suspendre son vol, en quadrillant les frontières, se heurte aux barbelés, ne peut plus voler, exercer sa liberté politique de se mouvoir! Les droits de l'homme sont ainsi soumis au principe de souveraineté nationale des Etats, à l'apartheid, à la violence allant aux extrêmes.

La tentative d'inclure le concept de mobilité dans le corpus des droits de l'homme est certes un pas notoire pour dépasser l'idéologie de la force sécuritaire, nationale, identitaire, de tri inclusive/expulsive/jetable. Elle fait l'impasse sur un déplacement radical nécessaire. Arendt avait déjà insisté sur l'importance de distinguer la liberté de circulation (concept économique, utilitariste) de la liberté de mouvement pour pouvoir dégager la liberté politique.

En résumé, en se heurtant à la violence allant aux extrêmes imprévisibles, ses formes dans la torture constituant les politiques migratoires, en parcourant la brèche ouverte par Arendt, j'en suis arrivée à me déplacer radicalement pour considérer la liberté politique de se mouvoir comme l'axe philosophique central pour penser la vie humaine, la migration en reformulant les rapports de migration par le desexil de l'exil. Les conditions matérielles de vie des migrants nous montre l'urgence du déplacement pour reconsidérer l'ensemble du genre humain de la planète. Il devient possible de repenser le rapport torture et migration avec le souffle de la création et l'ouverture d'horizons. Ce n'est pas de mobilité qu'il faut parler, mais de liberté politique de se mouvoir, proposition à inscrire dans une nouvelle approche de l'ensemble de la politique, de la philosophie, de la citoyenneté/civilité. Elle concerne le corps, les pieds des fugitifs qui marchent, qui avec leur tête pensent philosophiquement l'exploitation, la destruction, l'extermination qu'ils fuient. Pour le dire méta- 
phoriquement, aujourd'hui, l'ensemble des humains fuit toutes sortes de formes de torture !

L'essai philosophique publié en 2019 (Caloz-Tschopp 2019) est parti des acquis d'un essai antérieur : L'évidence de l'asile (Caloz-Tschopp 2016c). Il part d'une critique des politiques de droit d'asile, de l'action humanitaire, et la réappropriation de "l'évidence de l'asile », trésor perdu, confiqué à la base d'une «philosophie dys-topique du mouvement ».

Ce deuxième essai a été la découverte, en observant les fuites des migrants, de la liberté politique de se mouvoir, en revisitant l'exil (domination), le desexil (lutte créatrice). Il propose une philosophie du droit de fuite pour échapper à la violence prédatrice, qui est une nouvelle forme de desexil (luttes) pratiquée par des millions de gens sur la planète. Le but est de sortir d'un capitalisme expansionniste sans limites, d'une pensée d'Etat, de police, de guerre, de force, d'état d'urgence (Bigo 2019), d'état d'exception, des catégories territoriales, souverainiste des Etats (nations), de parcourir des références (hospitalité, égalité, solidarité, etc.) de débattre avec des philosophes, chercheurs, résistants et d'imaginer des espaces de transpolitiques démocratiques.

La double démarche a été motivée par le refus d'aller sur le terrain miné de la migration par les populistes nationalistes en choisissant de chercher un espace, une assise philosophique du droit d'avoir des droits d'Arendt, de l'ordre du plus général, s'inscrivant dans la politique et la philosophie du genre humain qualifié par le rapport travail-capital-terre-guerre, par la violence de l'exil dans le capitalisme contemporain à combattre par le desexil. L'appropriation de la liberté politique de se mouvoir est l'impératif du genre humain par excellence du XXIe siècle. Elle m’a été montrée par l'agir de fuite des migrants devant la torture !

Le déplacement dans la recherche d'une alternative est collectif. Il continue. Un article qui met radicalement en cause la fracture entre genre humain et nature, apporte à la philosophie du travail, de la migration de l'exil, du desexil, un autre angle d'attaque, en effectuant un déplacement épistémologique, catégoriel majeur. Elle permet de rejoindre les préoccupations autour du climat et de reprendre des réflexions féministes (sur le corps).

\subsection{Conclusion : vivre le vertige démocratique transpolitique}

Le vertige démocratique est aussi grisant que le vertige en montagne. Il est à la fois transindividuel (Balibar 2018) et transpolitique (Caloz-Tschopp 2019). Les risques sont majeurs. L'horizon est en tension entre la liberté d'être libre et la violence autodestructrice des humains, de la planète, toutes deux imprévisibles. Réélaborer le rapport à la vio- 
lence, à la création démocratique comme un rapport transindividuel et transpolitique, c'est prendre le risque incalculable de ce qu'Arendt nomme la 'liberté d'être libre', Castoriadis 'l'autonomie' (auto-nomos, se donner ses propres lois), Balibar 'le pari tragique'. Le vivre exige de la curiosité, du courage, de l'imagination pour lutter pour la pleine participation active de tout humain à sa propre vie, aux affaires publiques dans une planète finie. Personne ne peut décider pour personne du désir de liberté politique. C'est le socle, l'art du droit d'avoir des droits (Caloz-Tschopp 2000) et l'art politique de fuir toutes les formes nihilistes d'apartheid, de torture, de destruction, de mort.

Les migrants sont la figure du passage de l'essence, de la naturalisation, à la relation, de l'autre ailleurs et en soi. Le rapport torture et migration évoque le bruit de la violence politique poussée aux extrêmes, la terreur, la folie dont parle Toni Morrison dans le refus de l'autre, du tiers, le mépris, l'exploitation, la destruction dont les politiques de disparition seraient le seul horizon imaginable. Angoisse devant la brutalité inouie, la destruction. Il suffit d'écouter les femmes migrantes, les récits d'africains en Europe, de latino-américains creusant les tunnels sous le mur de Trump. Nuit. Absence de rêve. Cauchemars.

Dans la vie, Borges (1965), aveugle, se promène à tâtons « dans les jardins des chemins qui bifurquent ». En clair dans l'histoire, ses bifurcations infinies. Castoriadis explore les carrefours du labyrinthe (1975) de la pensée pour dégager la puissance de l'imagination et la création humaine. Mathieu (1991) pour cerner les contraintes matérielles de la soumission distingue entre céder et consentir en analysant les stratégies de liberté politique des femmes et des minoritaires. Balibar (2010) invite à penser aux extrêmes, tenir un pari tragique et à pratiquer la civilité. Carrefours de découvertes, débats, conflits. Ils nous apprennent à penser aux extrêmes, avec et au-delà des moyens dont nous avons hérité. A vivre ce qu'on peut appeler le vertige démocratique jusque dans ses ultimes désirs, retranchements, pensée, fragilités, imprévisibilité (Caloz-Tschopp 2019, 497-523).

Penser aux extrêmes, penser la torture, la migration avec et au-delà des moyens hérités, c'est avoir l'audace de déplacer le poids des systèmes, concepts, catégories, préjugés, modes de gouvernement autoritaires et pilleurs, inventer une démarche philosophique pour les bousculer, les mettre en mouvement. Spinoza, Deleuze ont ouvert le chemin.

En bref, c'est prendre acte de la difficulté à imaginer, voir, percevoir. C'est penser, réaliser le risque qu'implique le désir révolutionnaire de la liberté politique et la violence au-delà de toute mesure (Amati Sas 2014), son incalculabilité, imprévisibilité et la possibilité/ impossibilité de contenir son mouvement créateur et destructeur par l'agir politique de création auto-organisée d'espaces en mouvement de civilité solidaire aux frontières de l'environnement dont fait partie 
la migration. Les concepts attachés, figés sur l'illusoire souveraineté verticale d'Etat national (ignorance des peuples !) sur le sol, les humains, les armes, les biens, la servitude, le pillage légitimé, cachent le pouvoir de mort de masse, de destruction, éludent la liberté et la responsabilité politiques. Quand la violence annihilatrice devient la règle du jeu planétaire, l'autonomie, la liberté politique, la responsabilité sont transpolitiques, sont un pari tragique.

Par ailleurs, glisser, sans distance critique des discours sur la libre-circulation des capitaux, des biens, de la force de travail, à ceux sur la mobilité ne peut suffire à contenir la violence, de l'appropriation de la valeur du travail, de la surexploitation et de la destruction prédatrice. Etre mobile c'est la règle du capitalisme acculé. Etre mobile ce n'est pas forcément être libre! Ouvrir l'horizon c'est imaginer que la liberté politique de se mouvoir avec son corps, sa tête, ses pieds est constitutif de la condition humaine, de l'invention de la politique, de la civilité à chaque commencement. Elle contient la violence. Elle est la lutte pour la prévention par excellence de la torture. Le pacte sur la migration, la dénonciation, la pénalisation d'actes de torture sont certes nécessaires. Mais des luttes hétérogènes ont lieu pour sortir du capitalisme (ex. lutte contre les multinationales), un droit du travail pour tous, de véritables politiques de la migration, du droit d'asile en inscrivant dans les constitutions l'hospitalité, l'égaliberté, la solidarité, l'échange créée dans les pratiques démocratiques insurrectionnelles.

Repérer une aporie du capitalisme a permis de dégager l'horizon pour penser des énigmes du rapport torture et migration et retrouver la liberté politique de se mouvoir. Je n'évoque pas dans cet article les mesures multiples, dispositifs, outils qui existent mais ne sont pas choisis, appliqués (Caloz-Tschopp 2016c). Le débat est saturé. Inertie. Manque de volonté politique, de courage, d'imagination. Les philosophies de la patate chaude sont le visage de la violence d'Etat. Elles sont une grave irresponsabilité à courte vue.

Retenons que la politique a besoin d'un cadre auto-organisé, ce que nous apprennent les expériences d'invention démocratique horizontale par ex. dans les villes du Kurdistan, les multiples expériences d'auto-organisation, les universités libres, et aussi les failed States, les guerres en ex-Yougoslavie, au Liberia, les zones de maffias en Colombie, les métroples prises entre ordures, pollution, drogues, les politiques sécuritaires des camps, prisons, etc. Eclats dans l'horizon et enfer de Dante. Combien il difficile de parler des gains invisibles dans les luttes et aussi de repenser l'Etat vertical hérité, de ne pas être séduit par la spirale autoritaire, sécuritaire de la force, par la tentation de renvoyer la violence plutôt que de ruser pour l'esquiver, la fuir.

Vouloir remplacer les victimes par des combattantes, la désobéissance civile par la guerre civique, ou face à la violence allant aux extrêmes, penser en terme d'autodéfense ne peuvent suffire à créer 
une politique positive de citoyenneté-civilité, d'antiviolence dont parle Balibar impliquant l'initiative du commencement, le tiers, l'altérité, le conflit, la dispute, le débat, que l'on voit à l'œuvre dans l'ouverture d'espaces en mouvement aux frontières, les Refuges, les Tribunaux d'opinion, le projet d'une Cour internationale de justice par ex.

Penser aux extrêmes, avec et au-delà des moyens, c'est se réapproprier la liberté politique à la base de l'invention démocratique (imaginaire, projet, difficultés, énigmes). Le vertige démocratique ne se limite ni à la démocratie représentative, ni à la démocratie semi-directe (que nous connaissons en Suisse). C'est un mouvement d'autonomie fragile, incertain sans cesse remis sur le métier, face aussi à la logique de la torture sous toutes ses formes. Un des arguments souvent avancé après Platon contre la démocratie est celui des risques de dérapages. Il a été repris sous une autre forme par Spinoza sur le mode « crainte des masses » (autre débat).

J'aimerais insister ici sur un autre risque positif à prendre, celui de la liberté politique dans un temps de violence allant aux extrêmes, sur l'art d'esquiver la destruction, de fuir, ruser, en travaillant sur les ambiguïtés, en développant une double capacité dialectique de déplacement, contournement, report : avoir le courage lucide de durer, de rester ferme, de tenir une position dans la défense du droit d'avoir des droits dans un contexte de torture, de disparition, sans basculer dans une radicalité abstraite, absolue, impliquant le glissement dans l'usage de la violence incontrôlable et devenir prisonnier des effets boomerang destructeurs (Luxemburg) et des dégâts collatéraux incalculables.

Penser aux extrêmes, avec et au-delà des moyens existants c'est faire le pari tragique du possible/impossible, que tout n'est jamais joué, qu'il existe des marges de manœuvre de liberté, d'autonomie. Les évaluations de concepts liés à l'agir (ex. imagination, autonomie, servitude, désobéissance civique, responsabilité), les créations d'outils dans les luttes, vont bien au-delà de ce que décrivent les théories de Hobbes sur l'Etat et la violence souveraine. Nous pouvons imaginer, penser dans ces lieux où l'humour créatif côtoie l'absurde du rouleau compresseur de la force, nous apprend Kafka.

Vivre la torture dans la migration, la comprendre, lutter pour la contenir est un long apprentissage fragile, incertain de déplacement, à la recherche d'un nouveau paradigme politique de l'environnement dont fait partie la migration en mouvement. Elaborer la complexité, l'imprévisibilité la violence et de la liberté, l'autonomie sans dénier la violence destructrice tragique. La liberté politique de se mouvoir à un prix, ce que Arendt a appelé « la liberté d'être libre », Castoriadis « l'autonomie » et Marx « le travail vivant». 


\section{Bibliographie}

Amati Sas, Silvia (1984). « Mégamorts, unité de mesure ou métaphore ?». Bulletin de la revue suisse de psychanalyse, 18, 11-19.

Amati Sas, Silvia (2004). «Traumatic Social Violence : Challenging our Unconscious Adaptation ». International Forum of Psychoanalysis, 13, 51-9.

Amati Sas, Silvia (2016). «Violence sociale extrême : les deux fronts de la survivance psychique ». Amati Sas, Silvia et al. (dirs), Trois concepts pour comprendre José Bleger. Paris : L'Harmattan, 69-83.

Anders, Günther (2001). Et si je suis désespéré que voulez-vous que j'y fasse? Paris : Alia.

Anders, Günther (2011). L'obsolescence de l'homme. Tome II : Sur la destruction de la vie à l'époque de la troisième révolution industrielle. Paris : Fario.

Antelme, Robert (1957). L'espèce humaine. Paris : Gallimard.

Arendt, Hannah (1966). Eichmann à Jérusalem. Rapport sur la banalité du mal. Paris : Gallimard.

Arendt, Hannah (1972). Le système totalitaire. Paris : Points-Essai.

Arendt, Hannah (1981). La Vie de l'esprit. Paris: PUF.

Arendt, Hannah (2019). La liberté d'être libre. Paris : Payot.

Balibar, Etienne (1997). La crainte des masses. Paris: Galilée.

Balibar, Etienne (2010). Violence et civilité. Paris : Galilée.

Balibar, Etienne (2018). Spinoza politique. Le transindividuel. Paris: PUF.

Bigo, Didier (2019). « Les modalités des dispositifs d'état d'urgence ». Cultures \& Conflits, 113, printemps, 7-15. URL http://journals.openedition. org/conflits/20710 (2019-11-19).

Borges, Jorge-Luis (1965). « Le jardin aux sentiers qui bifurquent ». Fictions. Paris : Folio, 91-107.

Bozarslan, Hamit (2019). Crise, violence, dé-civilisation. Paris : éd. CNRS.

Brauman, Rony (2001). Humanitaire : le dilemme. Paris : éd. Textuel.

Brauman, Rony (2018). Guerres humanitaires? Mensonges et intox. Paris : éd. Textuel.

Brepohl, Marion (2019). «Recherche d'historiens, politique de mémoire et colonialité ». Caloz-Tschopp, Marie-Claire et al. (dirs), Vers le desexil. Paris : L'Harmattan, 313-31.

Brown, Wendy (2009). Les murs de séparation et le déclin de la souveraineté étatique. Paris : Prairies ordinaires.

Caloz-Tschopp, Marie-Claire (2000). Les sans-Etat dans la philosophie d'Hannah Arendt. Lausanne : éd. Payot.

Caloz-Tschopp, Marie-Claire (2004a). Les étrangers aux frontières de l'Europe et le spectre des camps. Paris : La Dispute.

Caloz-Tschopp, Marie-Claire (2004b). Le devoir de fidélité à l'Etat entre servitude, liberté, (in)égalité. Paris : L'Harmattan.

Caloz-Tschopp, Marie-Claire (2006). "Clandestinité des femmes migrantes ». Colfen (dir.), Vivre clandestines. Bruxelles : Université des Femmes, 63-113.

Caloz-Tschopp, Marie-Claire (2007). «Philosophie, migration, démocratie et droits de l'homme ». Caloz-Tschopp, Marie-Claire ; Dasen, Pierre (dir). Mondialisation, migration et droits de l'homme. Bruxelles : Bruylant, 76-170.

Caloz-Tschopp, Marie-Claire (dir.) (2014). Ambiguïté, violence et civilité. (Re)lire aujourd'hui José Bleger (1923-1972) à Genève. Paris : L'Harmattan. 
Caloz-Tschopp, Marie-Claire (2016a). «Apartheid en Europe : le défi de la citoyenneté/civilité dans un temps de guerre imprévisible ». Revue Française d'Histoire des Idées Politiques, 43(1), 231-55.

Caloz-Tschopp, Marie-Claire (2016b). Ambiguité, violence et civilité. Paris : L'Harmattan.

Caloz-Tschopp, Marie-Claire (2016c). L'évidence de l'asile. Paris : L'Harmattan.

Caloz-Tschopp, Marie-Claire (2018). « Rosa Luxemburg : la découverte de l'effet boomerang de l'impérialisme et la liberté ». Caloz-Tschopp, Marie-Claire et al. (dirs), Rosa Luxemburg, Antonio Gramsci actuels. Paris : Kimé, 103-39.

Caloz-Tschopp, Marie-Claire (2019). La liberté politique de se mouvoir. Paris : Kimé.

Caloz-Tschopp, Marie-Claire (à paraître). « Changer de logiciel civique ». Choisir, nr. de janvier 2020.

Carlier, Jean-Yves ; Crépeau, François (2017). «De la 'crise' migratoire européenne au pacte mondial sur les migrations: exemple d'un mouvement sans droit?». Annuaire français de droit international, 1, 461-99. URL http://hdl.handle.net/2078.1/210014 (2019-11-19).

Castoriadis, Corneluis (1975). L'institution imaginaire de la société. Paris : Seuil. Chamayou, Grégoire (2010). La chasse à l'homme. Paris : La Fabrique.

Chemillier-Gendreau, Monique (2019). Régression de la démocratie et déchainement de la violence. Paris : éd. Textuel.

Einstein, Albert, Russell, Bertand (1955). The Russell-Einstein Manifesto. London, 9th July.

Fiala, Pierre (2018). «La famille migr-, champ lexical, champ discursif ». Calabrese, Laura ; Veniard, Marie (dirs), Penser les mots, dire la migration. Paris : L'Harmattan, 145-52.

Héritier, Françoise (1996). De la violence. Paris : Odile Jacob.

Guillaumin, Colette (2000). L'idéologie raciste. Paris : Folio.

Insel, Ahmet (à paraître). «Violence, philosophie, politique aujourd'hui ». Repenserl'exil. URL http://exil-ciph.com/revue-en-ligne (2019-11-19).

Magnénat, Luc (2019). La crise environnementale surle divan. Genève : éd. in Press.

Mathieu, Nicole-Claude (1991). L'anatomie politique. Paris : Côté-Femmes.

Monnier, Laurent (2004). «L'apartheid ne sera pas notre passé, il est notre avenir ». Caloz-Tschopp, Marie-Claire (dir.), Le devoir de fidélité à l'Etat entre servitude, liberté, (in)égalité. Paris : l'Harmattan, 207-21.

Puget, Jeanine (dir.) (1989). Violence d'Etat et psychanalyse. Genève : Dunod.

Razac, Olivier (2008). Histoire politique du barbelé. Paris : Champs.

Rigaux, François (à paraître). «Qu'est-ce que l'action humanitaire ?». Repenser l'exil. URL http://exil-ciph.com/revue-en-ligne (2019-11-19).

Sayad, Abdelmalek (à paraître). "L'asile dans l'espace Schengen : la définition de l'Autre (immigré, réfugié) comme enjeu des luttes sociales ». Repenser l'exil. URL http://exil-ciph.com/revue-en-ligne (2019-11-19).

Sayad, Abdelmalek (2013). La découverte de la sociologie en temps de guerre. Paris : Cécile Défaut.

Sorini, Françoise; Branche, Raphaëlle (2002). «La torture aux frontières de l'humain ». Revue internationale des sciences sociales, 174, 591-600.

Sustam, Engin (2016). Art et subalternité kurde. Paris : L'Harmattan.

Tafelmacher, Christophe (2019). « Durcissement du droit, dilemmes de l'avocat ». Caloz-Tschopp, Marie-Claire (dir), Vers le desexil. Paris : L'Harmattan, 357-67.

Traverso, Enzo (1997). L'Histoire déchirée. Paris : Cerf.

Vahabi, Nader (2019). Violence d'Etat, crime d'Etat. Paris : L'Harmattan. 
Marie-Claire Caloz-Tschopp

Sur le rapport torture et migration. Une réflexion philosophique/politique

Veloso Bermedo, Teresa (2018). Franchir le seuil de la douleur extrême. Paris : L'Harmattan.

Vidal-Naquet, Pierre (1972). La torture dans la République (1954-1962). Paris : Ed. de Minuit.

Vignar, Marcelo (2018). Experiencias psicoanaliticas en la actualidad sociocultural. Buenos Aires: Noveduc.

Vignar, Marcelo et al. (1989). Exil et torture. Paris : Denoël.

Ulricksen, Maren (1998). «Le corps comme inscription de la violence sociale ». Caloz-Tschopp, Marie-Claire (dir.). Hannah Arendt, la banalité du mal comme mal politique. Paris : L'Harmattan, 94-8. 



\title{
Delocalizzazione della tortura e 'tortura di Stato' Tra accordi di riammissione, esternalizzazione delle frontiere e chiusura dei porti
}

Alessandra Algostino

Università degli Studi di Torino, Italia

\begin{abstract}
The essay argues how to reject a person in Libya or close ports to migrants, as well as criminalize and obstruct the NGOs which are saving humans in the Mediterranean, violate the prohibition of torture and of inhuman or degrading treatments and constitute a crime against humanity. Responsibility lies with those who personally practice torture, but the European States' governments or the EU executives are certainly not exempt from responsibility.
\end{abstract}

Keywords Torture. Borders. Crimes Against Humanity. Readmission Agreements. Principle of non-refoulement.

Sommario 1 Ill controllo delle frontiere: la Grundnorm delle politiche nazionali ed europee sull'immigrazione. - 2 Gli accordi di riammissione quale icona dell'esternalizzazione delle frontiere. - 3 Rimpatri, delocalizzazione dei controlli e chiusura dei porti: violazione del principio di non-refoulement e trattamenti inumani o degradanti. - 4 Osservazioni conclusive: torture di Stato? 


\title{
1 Il controllo delle frontiere: la Grundnorm delle politiche nazionali ed europee sull'immigrazione
}

\author{
Lasciamo quest'Europa che non la finisce più di par- \\ lare dell'uomo pur massacrandolo dovunque lo in- \\ contra, a tutti gli angoli delle stesse sue strade, a \\ tutti gli angoli del mondo. Sono secoli che l'Europa \\ ha arrestato la progressione degli altri uomini e li ha \\ asserviti ai suoi disegni e alla sua gloria; secoli che \\ in nome d'una pretesa 'avventura spirituale', soffo- \\ ca la quasi totalità dell'umanità... (Fanon 1962, 240)
}

Il tema del controllo delle frontiere oltre che oggetto di un ossessivo marketing politico è, ormai da alcuni anni, il cardine delle politiche nazionali ed europee in materia di immigrazione. I due assi lungo i quali si snodano tali politiche sono il rimpatrio e la riammissione: in entrambi i casi centrale si rivela la cooperazione con Stati terzi.

Restando agli ultimi anni, ${ }^{1}$ esplicita in tal senso è una comunicazione della Commissione europea del 2016, Creazione di un nuovo quadro di partenariato con i Paesi terzi nell'ambito dell'Agenda europea sulla migrazione, dove si ribadisce la centralità di «una politica coerente, credibile ed efficace relativa al rimpatrio» e come fondamentale nei patti con i Paesi terzi sia il funzionamento del sistema di rimpatrio e riammissione, nell'intento di «aumentare in modo specifico e misurabile il numero dei rimpatri e delle riammissioni». ${ }^{2}$

Nel 2017 la Commissione europea adotta quindi due atti dai titoli evocativi: Per una politica dei rimpatri più efficace nell'Unione europea - Un piano d'azione rinnovato ${ }^{3}$ e Raccomandazione per rendere i rimpatri più efficaci nell'attuazione della direttiva 2008/115/CE del Parlamento europeo e del Consiglio. ${ }^{4}$

Quanto ai documenti che si presentano con un respiro più ampio, come l'Agenda europea sulla migrazione, adottata dalla Commissione europea il 13 maggio 2015, «gestire le frontiere» rappresenta uno dei quattro «livelli di azione» individuati, ma attraversa anche gli al-

1 In precedenza, si possono ricordare, soprattutto a partire dal 2005, diversi atti, per lo più di soft law, come il Global Approach to Migration and Mobility, che segnano un mutamento di stile e di intensità delle politiche europee (Gjergji 2016, 70), con l'affidamento di un ruolo viepiù crescente alla cooperazione con Stati terzi, a «partenariati per la mobilità», sulla base dell'assunto che "senza controlli efficaci alle frontiere, senza una riduzione dell'immigrazione irregolare e un'efficace politica di rimpatrio, l'UE non sarà in grado di offrire maggiori opportunità di migrazione legale e mobilità» (Commissione europea, L'approccio globale in materia di migrazione e mobilità, $\operatorname{COM}(2011) 743$ del 18-11-2011, 5).

$2 \operatorname{COM}(2016) 385$ del 07-06-2016.

3 Comunicazione della Commissione al Parlamento europeo e al Consiglio, COM(2017) 200 final, 02-03-2017.

4 Raccomandazione della Commissione, C(2017) 1600 final, 7.3.2017. 
tri tre livelli («ridurre gli incentivi alla migrazione irregolare», «onorare il dovere morale di proteggere: una politica comune europea di asilo forte», «una nuova politica di migrazione legale»). ${ }^{5}$ Ugualmente, il controllo delle frontiere è ricorrente nei vari scenari prospettati nel Libro bianco sul futuro dell'Europa del primo marzo 2017. ${ }^{6}$

Nel 2018 la Commissione europea pubblica una Relazione sullo stato di attuazione dell'agenda europea sulla migrazione, ${ }^{7}$ ed anche in tal caso largo spazio è dedicato al controllo delle frontiere e, in specie, alla collaborazione con i Paesi terzi sul fronte del rimpatrio e della riammissione.

Esplicativi sono alcuni stralci della Relazione relativi alla rotta del Mediterraneo centrale; dopo aver rilevato che «la cooperazione con un certo numero di partner in Africa si è intensificata», si osserva:

Nonostante i disordini politici, la collaborazione con le autorità etiopi sui cinque pilastri della dichiarazione di La Valletta è proseguita regolarmente. Il dialogo sulla migrazione e la cooperazione con la Guinea e la Gambia proseguono in modo regolare, come attestato dal successo riportato dal seminario sulle migliori pratiche da seguire per l'organizzazione delle attività legate ai rimpatri organizzato a marzo dall'Agenzia europea della guardia di frontiera e costiera. Il dialogo sulle migrazioni è stato rilanciato in Costa d'Avorio. Continuano le discussioni con la Nigeria in merito a un pacchetto in materia di migrazione e mobilità riguardante la crescita e gli investimenti, la gestione delle frontiere e l'identificazione digitale, il traffico di migranti e la tratta di esseri umani, nonché la riammissione e il reinserimento. Oltre alla cooperazione operativa in corso, tutti gli ulteriori aiuti al Niger si concentrerebbero su azioni volte ad affrontare il problema delle rotte migratorie emergenti vicino al confine nigeriano [...]. Il Niger, il Ciad, la Libia e il Sudan hanno inoltre raggiunto un nuovo accordo per istituire una cooperazione volta alla difesa delle frontiere comuni e alla lotta alla criminalità organizzata. ${ }^{8}$

Non manca un paragrafo dedicato alla Libia, con la quale «l'UE prosegue il suo operato al fine di migliorare le tremende condizioni in cui vivono molti migranti e rifugiati in Libia»: un operato che contempla interventi di assistenza umanitaria nei centri di trattenimen-

5 Commissione europea, $\operatorname{COM}(2015) 240$ final, 13-05-2015, Bruxelles.

6 Commissione europea, COM2017(2025) del 1 marzo 2017.

7 Comunicazione della Commissione al Parlamento europeo, al Consiglio europeo e al Consiglio, $\operatorname{COM(2018)~} 301$ final, 16-05-2018 Bruxelles.

8 Relazione sullo stato di attuazione dell'agenda europea sulla migrazione, COM(2018) 301 final, 16-05-2018 Bruxelles, 14-15. 
to, evacuazioni verso altri Paesi (fra i quali in primo luogo il Niger), il sostegno alla guardia costiera libica. ${ }^{9}$

Politiche analoghe sono praticate in relazione alla rotta balcanica, così come accordi sulle procedure di rimpatrio e di riammissione sono raggiunti con Paesi asiatici, quali il Pakistan e il Bangladesh. ${ }^{10}$

Il quadro è chiaro: rafforzare, attraverso la cooperazione con Stati terzi, il controllo dei confini, ossia esternalizzare le frontiere. La fortezza Europa, prima di tutto: non rileva il fatto che molti di questi Paesi siano Stati in guerra, autoritari, che non garantiscano i diritti della persona umana, che non tutelino il diritto di asilo.

A livello nazionale si replica lo stesso modello: procedure facilitate per rimpatri e riammissioni e cooperazione con Stati terzi per esternalizzare i controlli e chiudere le frontiere.

È un processo caratterizzato da un'alta informalità, che coniuga cooperazione allo sviluppo e controllo dei movimenti migratori, ${ }^{11}$ le cui linee sono decise in sedi 'ufficiose', quali, in relazione al coinvolgimento dei Paesi africani di origine e di transito dei migranti, il Processo di Khartoum del 2014 o il vertice de La Valletta del 2015.

La cooperazione allo sviluppo, senza misconoscere i vantaggi che essa arreca anche ai Paesi che governano gli aiuti economici e senza scordare come essa, comunque, non compensa l'estrazione e la predazione di ricchezza dai Paesi destinatari degli aiuti, ${ }^{12}$ diviene merce di scambio per ottenere il controllo delle frontiere (in tema, Ferri 2016). ${ }^{13}$ Il (neo)colonialismo assume una declinazione nuova, dai contorni paradossali: i Paesi europei - alcuni di essi - non di rado hanno contribuito, per utilizzare un eufemismo, alla devastazione in termini di povertà e guerre che attraversano il continente africano ed ora pretendono che siano gli Stati di quello stesso continente ad affrontare gli esodi che ciò comporta, esponendo le vittime di disastri economici e ambientali e di conflitti armati ad una ulteriore violazione dei propri diritti.

9 Relazione sullo stato di attuazione dell'agenda europea sulla migrazione, $\mathrm{COM}(2018)$ 301 final, 16-05-2018 Bruxelles, 11-13.

10 Relazione sullo stato di attuazione dell'agenda europea sulla migrazione, $\mathrm{COM}(2018)$ 301 final, 16-05-2018 Bruxelles, rispettivamente 8 e ss., 17.

11 Commissione europea, Creazione di un nuovo quadro di partenariato con i Paesi terzi nell'ambito dell'Agenda europea sulla migrazione, COM(2016) 385 del 07-06-2016.

12 Riferimenti in termini quantitativi si leggono nel report, a cura di alcune organizzazioni non governative, Honest Accounts 2017: How the World Profits from Africa's Wealth. URL https://www.globaljustice.org.uk/sites/default/files/files/resources/honest_accounts_2017_web_final_updated.pdf (2019-09-09).

13 Per un esempio concreto, cf. la creazione (nel vertice euro-africano del novembre 2015 a La Valletta) di un fondo apposito, il Fondo fiduciario dell'UE per l'Africa, anche chiamato 'Trust Fund', gestito dalla Commissione europea, verso il quale sono stati dirottati fondi destinati alla cooperazione e agli aiuti umanitari; si veda Parlamento europeo, Risoluzione sul Fondo fiduciario dell'UE per l'Africa: le implicazioni per lo sviluppo e gli aiuti umanitari, 13-09-2016, P8_TA(2016)0337. 


\section{Gli accordi di riammissione quale icona dell'esternalizzazione delle frontiere}

Fulcro delle politiche di controllo delle frontiere sono gli accordi di riammissione, che mostrano una notevole spregiudicatezza, dato che i partner scelti spesso sono Paesi autoritari o dilaniati da conflitti interni, e testimoniano una abdicazione rispetto al compito di tutela dei diritti, con relativa violazione di norme costituzionali, sovranazionali e internazionali. ${ }^{14}$

Nel primo decennio del secolo in corso (per la precisione, dal 2004 al 2014) risultano adottati 17 accordi di riammissione tra Unione europea e Paesi terzi, ${ }^{15}$ in base alla competenza che l'art. 79, par. 3, TFUE conferisce; ad essi possono aggiungersi oltre trecento accordi conclusi fra Stati membri dell'UE e Stati terzi (Cassarino 2014, 132).

Quanto all'Italia, nell'Archivio dei Trattati internazionali Online del Ministero degli Affari Esteri e della Cooperazione Internazionale, ${ }^{16}$ cercando con la voce 'riammissione', compaiono più di 30 testi, fra accordi, protocolli e intese di attuazione. La forma prevalente pare essere quella semplificata (Marchegiani 2008, 144), con notifica e comunicazione in Gazzetta ufficiale, ${ }^{17}$ senza legge di autorizzazione alla ratifica..$^{18}$

Un quadro completo delle ipotesi di riammissione peraltro deve tener conto anche delle clausole inserite in accordi di associazione e di cooperazione (Vitiello 2016, 13 ss.; Borraccetti 2016, 40 ss.), sulla falsariga dell'art. 13 del c.d. Accordo di Cotonou, Accordo di partenariato tra i membri del gruppo degli Stati dell'Africa, dei Caraibi e del Pacifico, da un lato, e la Comunità europea e i suoi Stati membri, dall'altro, firmato il 23 giugno 2000 (2000/483/CE; primi riferimenti in Cassarino 2016, 21 ss.).

14 Questo paragrafo riprende Algostino, Alessandra, «L'esternalizzazione soft delle frontiere e il naufragio della Costituzione». Costituzionalismo.it, 1, 2017, 139-82.

15 Per l'elenco, nonché il testo degli accordi, si veda la scheda online Return\&readmission, sul sito ufficiale della Commissione europea (https://ec.europa. eu/home-affairs, 2019-10-17), nonché, in lingua italiana, Diritto, immigrazione e cittadinanza, 1-2, 2016, 119 ss.

16 http://itra.esteri.it (2019-10-17).

17 A mo' di esempio, si vedano l'accordo fra Italia e Nigeria, Accordo in materia migratoria (riammissione), Roma, 12 settembre 2000, notificato il 24-04-2006 - 20-022007, comunicato in Gazz. Uff., nr. 180 SO del 04-09-2011; l'accordo fra Italia ed Egitto, Accordo di cooperazione in materia di riammissione, con protocollo esecutivo e allegati, Roma, 9 gennaio 2007, notificato il 24-03-2008 - 26-03-2008, comunicato in Gazz. Uff., nr. 242 SO del 15-10-2008.

18 Vi sono peraltro accordi per cui figura unicamente una nota verbale (è il caso ad esempio dell'Accordo sulla riammissione delle persone che soggiornano senza autorizzazione, Roma, 15-04-2014, concluso con il Kosovo), mentre in relazione ad alcuni, come quello con la Tunisia del 2011, non si è trovata notizia (cercando con le voci «riammissione» e «Tunisia»). 
Tali accordi, ma anche i benefit contemplati nei patti aventi come oggetto principale la riammissione, rendono esplicita la natura dello scambio 'denaro per persone'; uno scambio, invero, spesso doppiamente vincente per i Paesi che cooperano e che quindi godono dei vantaggi che la cooperazione reca (anche) allo Stato che 'fornisce' cooperazione, con il surplus di un controllo dei movimenti migratori semplificato ed esternalizzato. Certo, c'è un prezzo: il naufragio dei diritti e della democrazia, ma per la global economic governance, siamo sicuri sia da ascrivere fra i costi?

Negli ultimi anni - ovvero dal biennio 2015-16 19 -, gli accordi (o le clausole) di riammissione sono sempre più spesso conclusi in sedi para-istituzionali - con interpretazioni finanche paradossali come nel caso dell' 'accordo UE-Turchia' - e attraverso forme iper-semplificate, ovvero soft: agende, partenariati, dichiarazioni, scambi di note, memorandum (Gjergji 2016).

I vantaggi dell'informalità sono molteplici: maggior semplicità nella stipulazione e nella gestione dell'accordo, impossibilità di discussione e critica pubblica (dagli organi parlamentari alle forze politiche e sociali), elusione degli strumenti di controllo predisposti dagli ordinamenti (in primo luogo, giurisdizionali).

Modello di tali accordi ${ }^{20}$ è la Dichiarazione UE-Turchia del 18 marzo 2016 (Favilli 2016), che compare sotto la forma di comunicato stampa sul sito istituzionale del Consiglio europeo. ${ }^{21}$ Invero la forma è così 'informale' da porre dubbi sulla natura di accordo internazionale (Corten 2016; den Heijer, Spijkerboer 2016; Peers 2016). In tal senso però depongono il criterio sostanzialista seguito in ambito UE in materia di fonti e il contenuto dell'accordo, che comporta obblighi giuridici (non riconducibili ad altri atti). Un tipico atto, dunque, di soft law: non ha efficacia giuridica vincolante per la sua forma ma ha effetti giuridici pratici (Snyder 1993; Algostino 2016).

La natura informale dell'accordo contrasta con il diritto comunitario, il quale nelle materie oggetto della Dichiarazione richiede, ai sensi degli artt. 77-78 TFUE, la procedura legislativa ordinaria, ovvero, per la conclusione di un accordo internazionale, di seguire il

19 Il biennio 2015-16 segna una nuova fase delle politiche migratorie dell'Unione europea, non, come già accennato, per un cambio di rotta nel contenuto, quanto per una accelerazione e una de-formalizzazione delle stesse.

20 La natura di 'modello' dell' 'accordo' UE-Turchia è riconosciuta nei vertici politici e nelle comunicazioni della Commissione europea. Per tutti, cf. Commissione europea, Creazione di un nuovo quadro di partenariato con i paesi terzi nell'ambito dell'agenda europea sulla migrazione, $\operatorname{COM(2016)~} 385$ del 07-06-2016, laddove, a proposito della Dichiarazione UE-Turchia, si afferma: «i suoi elementi possono ispirare la cooperazione con gli altri paesi terzi strategici e indicare le principali leve da attivare».

21 http://www.consilium.europa.eu/it/press/press-releases/2016/03/18-euturkey-statement/ (2019-10-17). 
procedimento di cui all'art. 218 TFUE, con la partecipazione, stante la materia, del Parlamento europeo.

Per inciso, compare qui un must degli accordi soft: il ruolo, sostanzialmente esclusivo, degli organi esecutivi nella loro stipulazione. Ora, nella consapevolezza che le relazioni internazionali sono tradizionalmente terreno dell'esecutivo, l'estromissione pressoché totale del legislativo segna un passo oltre; un passo coerente, peraltro, con la crescente concentrazione di potere negli esecutivi, sia che agiscano nell'ambito del government sia in quanto operino nello spazio duttile e promiscuo della governance.

Quanto al contenuto della Dichiarazione, esso è tipico degli accordi di riammissione: rendere più easy il rimpatrio, ${ }^{22}$ con tutto ciò che ne consegue in termini di impatto sul diritto di asilo, sul rispetto dei diritti umani e, in particolare, sul divieto di tortura.

La scelta senza alcuna remora della Turchia come partner e come Stato sicuro esemplifica quindi come sia considerata irrilevante la natura democratica o meno del Paese con il quale si conclude l'accordo, ovvero il rischio che migranti e profughi subiscano torture o trattamenti inumani o degradanti.

La natura soft concede l'irresponsabilità, a fronte di un contenuto in contrasto con norme della stessa Unione europea, come risulta dalla pronuncia, invero paradossale, del Tribunale dell'Unione europea.

Il Tribunale viene adito, a norma dell'art. 263 TFUE, da due cittadini pakistani e un cittadino afgano, richiedenti asilo in Grecia, che temono, in ragione dell'accordo, di essere rinviati in Turchia. ${ }^{23}$ I ricorsi sono rigettati per incompetenza. Nel comunicato stampa del 18 marzo 2016 il quale annuncia l'accordo - si legge nell'ordinanza del Tribunale - sono utilizzate in maniera inappropriata le espressioni «membri del Consiglio europeo» e «Unione europea». Il 17 e il 18 marzo 2016 si sono tenuti in parallelo due incontri distinti: la sessione del Consiglio europeo e un vertice internazionale, ed è nell'ambito di quest'ultimo che i capi di Stato e di Governo, in quanto tali e non in quanto membri del Consiglio europeo, hanno adottato, con i loro omologhi turchi, la Dichiarazione. ${ }^{24}$ Dunque, «indépendamment du point de savoir si el-

22 «La Turchia ha convenuto di accettare il rapido rimpatrio di tutti i migranti non bisognosi di protezione internazionale che hanno compiuto la traversata dalla Turchia alla Grecia e di riaccogliere tutti i migranti irregolari intercettati nelle acque turche»; «Tutti i nuovi migranti irregolari che hanno compiuto la traversata dalla Turchia alle isole greche a decorrere dal 20 marzo 2016 saranno rimpatriati in Turchia, nel pieno rispetto del diritto dell'UE e internazionale, escludendo pertanto qualsiasi forma di espulsione collettiva» (Dichiarazione Unione europea-Turchia del 18 marzo 2016).

23 Cause NF, NG e NM/Consiglio europeo (T-192/16, T-193/16 e T-257/16).

24 Tribunale dell'Unione europea, NF v European Council, causa T-192/16, ordinanza del 28 febbraio 2017, ma di identico tenore sono le ordinanze adottate in relazione agli altri due casi. 
le constitue, comme le soutiennent le Conseil européen, le Conseil et la Commission, une déclaration de nature politique ou, au contraire, comme le soutient le requérant, un acte susceptible de produire des effets juridiques obligatoires, la déclaration UE-Turquie, telle que diffusée au moyen du communiqué de presse $n^{\circ}$ 144/16, ne peut pas être considérée comme un acte adopté par le Conseil européen, ni d'ailleurs par une autre institution, un organe ou un organisme de l'Union, ou comme révélant l'existence d'un tel acte et qui correspondrait à l'acte attaqué», donde la dichiarazione di incompetenza. ${ }^{25}$

Nessun accordo, nessun atto, quindi nessuna violazione del diritto comunitario, né sotto il profilo della procedura, né sotto quello del rispetto del diritto in materia di diritti umani e di protezione internazionale. $\mathrm{E}$ - si può aggiungere - riconoscimento di un estremo margine di manovra agli esecutivi, che assumono in parallelo diverse vesti giuridiche, passando senza soluzione di continuità dall'abito comunitario a quello di negoziatori internazionali a quello di membri di un vertice politico, come si addice all'habitat della governance, 'libera' da procedure e forme (e dalle ristrettezze imposte dai parametri democratici).

Ora, anche ammettendo e non concedendo che la Dichiarazione del 18 marzo 2016 non sia, grazie al rocambolesco sdoppiamento di ruolo dei vertici degli esecutivi, un atto dell'Unione europea, si può ancora osservare, da un lato, come, se si ritiene essa non sia che una mera dichiarazione politica, i respingimenti effettuati in suo nome sono tout court illegittimi. Mentre, dall'altro lato, se la si configura come un accordo internazionale, se pur concluso informalmente, resta che essa dovrebbe comunque rispettare - a prescindere dal diritto nazionale di ciascuno Stato - lo ius cogens, ovvero le «norme proibitive alle quali è vietato derogare» (Carreau, Marrella 2016, 65), cui è ascrivibile, come si dirà a breve, il principio di non-refoulement.

Nella Relazione sullo stato di attuazione dell'agenda europea sulla migrazione ${ }^{26}$ nel 2018, quindi post pronuncia del Tribunale UE, si legge, peraltro, che «la dichiarazione UE-Turchia continua a rivestire un'importanza cruciale» e si vantano i «risultati tangibili» raggiunti, ${ }^{27}$ e, a conferma della 'validità' del modello, si precisa che «sebbene garantire la cooperazione dei paesi terzi sulla riammissione dei propri cittadini resti una sfida per l'UE, notevoli passi avanti sono stati com-

25 Tribunale dell'Unione europea, NF v European Council, par. 71. Avverso la pronuncia è proposto appello da parte dei ricorrenti innanzi alla Corte di Giustizia (I Sez.), che lo rigetta, con una ordinanza di manifesta inammissibilità (causa C-208/17, ord. del 12-09-2018).

26 Relazione sullo stato di attuazione dell'agenda europea sulla migrazione, COM(2018) 301 final, 16-05-2018 Bruxelles.

27 Non mancano nemmeno i report ufficiali sullo stato di applicazione della Dichiarazione; cf., ad esempio, COM(2017) 204 final del 2 marzo 2017, Relazione della Commissione al Parlamento europeo, al Consiglio europeo e al Consiglio, Quinta relazione sui progressi compiuti in merito all'attuazione della dichiarazione UE-Turchia. 
piuti nel 2017 grazie alla conclusione di una serie di accordi pratici» (corsivo aggiunto).

La Dichiarazione è, in sintesi, un atto sfuggente e ambiguo, in un limbo giuridico quanto a responsabilità e ricorsi, che genera concrete violazioni dei diritti umani, in primis di quello che Bobbio considerava - si può quasi chiosare, con 'eccessivo' ottimismo - esempio dei diritti «privilegiati, perché non vengono posti in concorrenza con altri diritti» e non vengono limitati per il verificarsi di casi eccezionali (Bobbio 1990, 11): il divieto di tortura, nel caso di specie, come si vedrà a breve, nella sua declinazione come divieto di refoulement (Amnesty International 2017, 18-20).

L'Italia, invero, aveva precorso il modello della Dichiarazione UETurchia, ad esempio in un accordo di riammissione concluso nel 2011 con la Tunisia. Si tratta di un 'accordo-fantasma': di esso si ha notizia solo perché nella sentenza del 15 dicembre 2016 della Grande camera della Corte europea dei diritti dell'uomo (Corte EDU), caso Khlaifia e altri c. Italia, ricostruendo la normativa relativa alla causa, nell'ambito dei rapporti bilaterali Italia-Tunisia, la Corte cita per l'appunto un accordo concluso il 5 aprile 2011 dal Governo italiano con la Tunisia «in materia di controllo dell'ondata di immigrazione irregolare proveniente da tale paese», il cui testo «non era stato reso pubblico», ma di cui alcuni estratti del verbale della riunione nella quale era stato 'stipulato' erano stati allegati dal Governo italiano nella domanda di rinvio dinanzi alla Grande camera. ${ }^{28}$

Un testo non pubblico, di cui non si conosce il contenuto preciso, eppure anche in tal caso gli effetti sono concreti, come quelli subiti dai ricorrenti nel caso de quo, tre cittadini tunisini oggetto di trattenimento in condizioni inumane e degradanti dapprima a Lampedusa, quindi in una nave attraccata al porto di Palermo, per poi essere rimpatriati in Tunisia, dopo un sommario accertamento della loro identità, in applicazione proprio dell'accordo del 5 aprile 2011.

L'accordo, infatti, secondo quanto è dato conoscere, impegna la Tunisia ad accettare il ritorno immediato dei cittadini tunisini giunti irregolarmente in Italia dopo la conclusione dell'accordo, «attraverso procedure semplificate, che prevedono la semplice identificazione della persona interessata da parte delle autorità consolari tunisine ${ }^{29}$ (rimpatri rapidi e facilitati), oltre che a «rafforzare il controllo delle sue frontiere allo scopo di evitare nuove partenze di clandestini, con l'aiuto di mezzi logistici messi a sua disposizione dalle autorità italiane $»^{30}$ (esternalizzazione delle frontiere).

28 Corte EDU, Grande camera, Khlaifia e altri c. Italia, ricorso nr. 16483/12, sentenza 15 dicembre 2016, par. 37.

29 Corte EDU, Grande camera, Khlaifia e altri c. Italia, par. 38.

30 Corte EDU, Grande camera, Khlaifia e altri c. Italia, par. 37. 
Quanto all'indifferenza per la situazione del Paese con il quale si stipula un accordo, si può citare, restando sempre agli accordi italiani, il «Memorandum d'intesa tra il Dipartimento della Pubblica Sicurezza del Ministero dell'Interno italiano e la Polizia Nazionale del Ministero dell'Interno sudanese per la lotta alla criminalità, gestione delle frontiere e dei flussi migratori ed in materia di rimpatrio», firmato a Roma il 3 agosto 2016.

Il Sudan, per limitarsi a citare un solo dato (e senza considerare la situazione attuale), nell'anno di conclusione dell'accordo, era collocato, nel Democracy Index stilato da The Economist (Intelligence Unit), fra i regimi (stabilmente) autoritari, con un indice di $2.37 \mathrm{su} 10 .^{31}$

Infine, non si può non citare l'accordo con la Libia, il «Memorandum d'intesa sulla cooperazione nel campo dello sviluppo, del contrasto all'immigrazione illegale, al traffico di esseri umani, al contrabbando e sul rafforzamento della sicurezza delle frontiere tra lo Stato della Libia e la Repubblica Italiana», sottoscritto dal Governo di Riconciliazione Nazionale dello Stato di Libia e il Governo della Repubblica italiana il 2 febbraio 2017; un accordo concluso senza alcuno specifico passaggio formale, dunque in forma semplificata, o, meglio, iper-semplificata (con violazione dell'art. 80 Cost.).

Il Governo italiano non reputa rilevante il fatto che la controparte non abbia giurisdizione sull'intero Stato libico. Il Memorandum, infatti, è stato siglato per la Libia dal Governo di unità nazionale libico guidato da Al Serraj, riconosciuto dalle Nazioni Unite, ma che, anche all'epoca, possedeva il controllo solo di una parte del territorio, conteso dal Parlamento di Tobruk e dall'esercito del generale Haftar, oltre che occupato da decine di gruppi armati. Quanto si leggeva, già nel 2017, sul sito Viaggiare sicuri della Farnesina, che scriveva di una «situazione di instabilità e di frammentazione politico-istituzionale che si registra ormai da tempo nel Paese», ${ }^{32}$ non sembra preoccupare il Governo italiano quando conclude l'accordo.

Fulcro dell'accordo è l'esternalizzazione delle frontiere, con l'avvio di iniziative di cooperazione «al fine di arginare i flussi di migranti illegali e affrontare le conseguenze da essi derivanti»: in particolare, si prevede «supporto tecnico e tecnologico agli organismi

31 https://infographics.economist.com/2017/DemocracyIndex/, nonché https:// www.eiu.com/public/topical_report.aspx?campaignid=DemocracyIndex2016 (201910-17); sono numeri che, ferme restando perplessità sulla veridicità di tali rapporti, non lasciano adito a molti dubbi sul carattere autoritario. Si veda anche, fra i vari report sul Paese, Amnesty International: https://www.amnesty.org/en/countries/africa/ sudan/ (2019-10-17)

32 http://www.viaggiaresicuri.it/paesi/dettaglio/libia.html (valido al 04-052017, pubblicato il 11-01-2017); in data 12 settembre 2019, l'invito che si legge sul sito è «a non recarsi in Libia»e, se presenti, «a lasciare temporaneamente il Paese». 
libici incaricati della lotta contro l'immigrazione clandestina», rappresentati dalla guardia di frontiera e dalla guardia costiera, e la «predisposizione dei campi di accoglienza temporanei in Libia, sotto l'esclusivo controllo del Ministero dell'Interno libico, in attesa del rimpatrio o del rientro volontario nei paesi di origine», quale soluzione per i migranti che attraversano la Libia con il progetto di recarsi via mare in Europa.

Nel luglio 2019, in presenza di una conclamata guerra civile, con report e sentenze che descrivono di inenarrabili torture nei centri per migranti, ${ }^{33}$ il Parlamento italiano, a maggioranza, rifinanzia, fra le missioni italiane all'estero, anche quella in Libia, con il sostegno alla guardia costiera libica.

\section{Rimpatri, delocalizzazione dei controlli e chiusura dei porti: violazione del principio di non-refoulement e trattamenti inumani o degradanti}

Gli accordi di riammissione sono suscettibili di collidere sotto più profili con il divieto di tortura, sancito nei principali cataloghi internazionali sui diritti umani (ormai parte dello ius cogens), così come nei patti regionali ${ }^{34}$ e nelle costituzioni, e oggetto di specifiche convenzioni; ${ }^{35}$ nonché, da ultimo, contemplato nello Statuto di Roma della Corte penale internazionale del 1998, che, fra i crimini contro l'umanità, annovera la tortura, se commessa «nell'ambito di un esteso o sistematico attacco contro popolazioni civili».

Eppure, oggi, l'affermazione che «nessun individuo potrà essere sottoposto a tortura o a trattamenti o punizioni crudeli, inumani o degradanti» ${ }^{36}$ cede a fronte delle politiche di chiusura ed esternalizzazione delle frontiere, che violano sotto più profili il divieto di tortura.

33 Oltre le sentenze citate infra, si veda, ex multis, il rapporto Desperate and Dangerous: Report on the human rights situation of migrants and refugees in Libya dell'Alto commissario per i Diritti umani presso le Nazioni Unite del 20 dicembre 2018 URL https:// www.ohchr.org/Documents/Countries/LY/LibyaMigrationReport.pdf (2019-09-09).

34 Convenzione europea per la salvaguardia dei diritti dell'uomo e delle libertà fondamentali (CEDU) del 1950, art. 3; Carta dei diritti fondamentali dell'Unione europea, art. 4; Convenzione Americana sui diritti umani del 1969, art. 5 (c. 2); Carta africana dei diritti dell'uomo e dei popoli del 1981, art. 5.

35 Nell'ambito delle Nazioni Unite, si può ricordare la Convenzione contro la tortura ed altre pene o trattamenti crudeli, inumani o degradanti (1984); a livello continentale, si possono citare la Convenzione europea per la prevenzione della tortura e delle pene o trattamenti crudeli, inumani o degradanti (1987) e la Convenzione inter-americana sulla prevenzione e repressione della tortura (1984).

36 Così la Dichiarazione universale dei diritti umani del 1948, art. 5; similmente, la Convenzione internazionale sui diritti civili e politici del 1966, art. 7. 
Non mancano negli accordi di riammissione formule a salvaguardia dei diritti umani, quali: «pienamente impegnati a promuovere e rispettare i diritti umani», ${ }^{37}$ "le Parti si impegnano ad interpretare ed applicare il presente Memorandum nel rispetto degli obblighi internazionali e degli accordi sui diritti umani di cui i due Paesi siano parte»; ${ }^{38}$ ma la natura degli accordi e dei contraenti mostra come esse non siano che mere interlocuzioni di rito.

In primo luogo, gli accordi di riammissione, quando conclusi con Stati come la Libia, violano il divieto di tortura e di trattamenti inumani o degradanti infrangendo il principio di non-refoulement: «nessuno Stato Contraente espellerà o respingerà, in qualsiasi modo, un rifugiato verso i confini di territori in cui la sua vita o la sua libertà sarebbero minacciate a motivo della sua razza, della sua religione, della sua cittadinanza, della sua appartenenza a un gruppo sociale o delle sue opinioni politiche» (art. 33, par. 1, della Convenzione di Ginevra relativa allo status dei rifugiati del 1951). ${ }^{39}$

Come ha chiarito la Corte EDU, che collega il principio di non-refoulement all'art. 3 CEDU, recante Proibizione della tortura, la ratio della norma è tutelare la vita e la libertà di ciascuna persona, il che comporta che titolare ne è qualsiasi persona umana (a prescindere dal possesso o dalla volontà di ottenere lo status di rifugiato).

Il principio di non-refoulement «ha carattere assoluto ed inderogabile» e senza dubbio la sua operatività non può essere limitata attraverso accordi internazionali bilaterali con Paesi terzi, quali quelli di riammissione (Grosso 2009, XVII).

In questa prospettiva, la Grande Camera della Corte EDU, Hirsi Jamaa e altri c. Italia ${ }^{40}$ condanna la politica dei respingimenti italiani (relativa al 2009), in relazione all'art. 3 CEDU, in quanto con il trasferimento dei ricorrenti, nel caso di specie in Libia, le autorità italiane li hanno esposti «con piena cognizione di causa a trattamenti contrari alla Convenzione», ${ }^{41}$ data l'esistenza di «fonti affidabili» che mostrano come in Libia «tutte le persone entrate nel paese con mez-

37 Memorandum Italia-Sudan del 2016, preambolo; similmente in art. 1: «le Parti concordano che l'obiettivo del presente Memorandum è quello di promuovere e sviluppare la cooperazione di polizia per la prevenzione e il contrasto alla criminalità nelle sue varie forme. Nell'attuazione della cooperazione, le Parti assicurano e promuovono il pieno rispetto dei diritti umani».

38 Memorandum d'intesa Italia-Libia del 2017, art. 5.

39 Il principio di non-refoulement è sancito in numerosi trattati internazionali (ex multis, Convenzione delle Nazioni Unite contro la tortura e altre pene o trattamenti, crudeli, inumani o degradanti, New York, 10 dicembre 1984, art. 3), regionali (si veda, ad esempio, Carta dei diritti fondamentali dell'Unione europea, art. 19) e parte del diritto internazionale consuetudinario, anche nella sua accezione di ius cogens.

40 Corte EDU, Grande camera, Hirsi Jamaa e altri c. Italia, ricorso nr. 27765/09, sentenza 23 febbraio 2012, par. 128.

41 Corte EDU, Grande camera, Hirsi Jamaa e altri c. Italia, parr. 126 e 137. 
zi irregolari erano considerate clandestine, senza alcuna distinzione tra i migranti irregolari e i richiedenti asilo» ed «erano sistematicamente arrestate e detenute in condizioni... inumane». ${ }^{42}$

L'inderogabilità e l'assolutezza operano non solo in relazione al dato formale, ma guardando all'effettività: uno Stato è sicuro e non espone al rischio di subire, in primis, tortura o trattamenti inumani o degradanti, quando garantisce effettivamente che ciò non avvenga; un approccio - quello fondato sulla garanzia concreta - che è una costante nella giurisprudenza della Corte di Strasburgo ${ }^{43}$ ed è alla base della tutela dei diritti nella Costituzione italiana (emblematicamente, cf. art. 3, c. 2).

La Corte EDU, così come la Corte di giustizia UE, hanno precisato, ad esempio, che non esiste una presunzione assoluta di sicurezza nemmeno per gli Stati membri dell'Unione europea. ${ }^{44}$

La violazione del principio di non-refoulement, e del divieto di tortura, sempre nella prospettiva di una tutela effettiva, può darsi anche in caso del rimpatrio indiretto. Lo Stato di (primo) rinvio, cioè, deve fornire sufficiente garanzia in ordine al fatto che non respingerà i migranti verso Paesi nei quali sussista il rischio che siano soggetti a trattamenti vietati dall'art. 3 Conv.: «è lo Stato che procede al respingimento a doversi assicurare che il Paese intermedio offra garanzie sufficienti che permettano di evitare che la persona interessata venga espulsa verso il suo Paese di origine senza valutare il rischio cui va incontro». ${ }^{45}$ Un altro profilo, quello del rimpatrio indiretto, per il quale il giudice di Strasburgo, nella sentenza Hirsi Jamaa $e$ altri c. Italia, ha ritenuto l'Italia colpevole di violazione dell'art. 3 Conv.: «la Corte ritiene che, al momento di trasferire i ricorrenti verso la Libia, le autorità italiane sapevano o dovevano sapere che non esistevano garanzie sufficienti a tutelare gli interessati dal rischio di essere rinviati arbitrariamente nei loro Paesi di origine». ${ }^{46}$

In secondo luogo, una violazione del divieto di tortura - quale emblema delle violazioni dei diritti umani - può avvenire quando si demandano a Stati terzi controlli, identificazioni, trattenimenti. I cen-

42 Corte EDU, Grande camera, Hirsi Jamaa e altri c. Italia, parr. 128 e 125.

43 La Corte EDU da tempo ricorda che i diritti sono sanciti non come «theoretical or illusory but rights that are practical and effective» (Corte EDU, Artico c. Italia, sent. 13 maggio 1980, par. 33).

44 In questo senso, per la giurisprudenza Corte EDU, cf., fra le altre, Grande camera, M.S.S. c. Belgio e Grecia, sentenza del 21 gennaio 2011, ricorso nr. 30696/09; per la giurisprudenza comunitaria, Corte di giustizia dell'Unione europea (Grande sezione), cause riunite C-411/10, N.S. c. Secretary of State for the Home Department, e C-493/10, M.E. e altri c. Refugee Applications Commissioner Minister for Justice, Equality and Law Reforme, sentenza del 21 dicembre 2011.

45 Così, Corte EDU, Grande camera, Hirsi Jamaa e altri c. Italia, par. 147, che riprende una giurisprudenza consolidata.

46 Corte EDU, Grande camera, Hirsi Jamaa e altri c. Italia, par. 156. 
tri per migranti in Libia sono una tragica testimonianza di come non si tratti solo di possibilità ma di conclamate realtà. Quando gli accordi sono conclusi con Stati non democratici o dittature, è (quasi) una certezza il mancato rispetto del divieto di tortura.

Affidare il soccorso in mare alla guardia costiera libica, così come demandare alla Libia la gestione dei migranti, significa, ormai senza poter in alcun modo evocare la scusa del 'non sapere', condannare le persone ad una vita di torture e violenze: in questo senso si può ragionare di tortura delocalizzata, o per interposta persona.

Non si contano i report o le posizioni di organi internazionali che descrivono la drammatica condizione dei migranti in Libia; per restare in Italia, si può ricordare la pronuncia della Corte di assise di Milano che ha dimostrato, in una documentata e cruda sentenza del 2017, senza ombra di dubbio l'esistenza di violenze e torture all'interno dei centri di raccolta dei migranti. ${ }^{47}$

Il Tribunale di Trapani il 3 giugno 2019 ha depositato una sentenza (caso Vos Thalassa) nella quale, dopo aver ricostruito il fondamento nel diritto internazionale del principio di non-refoulement e del divieto di tortura, afferma: il Memorandum Italia-Libia è «privo di validità, atteso che ai sensi dell'art. 53 della Convenzione di Vienna sul diritto dei trattati (del 1969) «è nullo qualsiasi trattato che, al momento della sua conclusione, sia in contrasto con una norma imperativa di diritto internazionale generale»; lo stesso risulta inoltre incompatibile con l'art. 10, c. 1, Cost., data la natura di norma consuetudinaria del principio; oltre che mancante della legge di autorizzazione alla ratifica ex art. 80 Cost., per cui al più sarebbe «un'intesa giuridicamente non vincolante». ${ }^{48}$

In terzo luogo, gli accordi di riammissione, nella loro ansia di facilitare i rimpatri, possono condurre all'introduzione anche nei Paesi dai quali vengono effettuati i rimpatri di modalità di identificazione, trattenimento e rimpatrio che integrano trattamenti inumani o degradanti: si pensi agli hotspot, ${ }^{49}$ ma anche ai Centri di permanenza per il rimpatrio (CPR) (Garante nazionale 2018). Il processo di disumanizzazione del migrante, e di negazione della sua soggettività giuridica (Gjergji 2016, 106), che raggiunge il suo apice con la politica di chiusura dei

47 Corte d'assise di Milano, sent. 10 ottobre 2017 (depositata il 1 dicembre 2017); si veda anche Corte d'Assise di Agrigento, II. Sez., sent. 12 giugno 2018 (depositata il 22 giugno 2019).

48 Tribunale di Trapani, Ufficio del Giudice per le Indagini Preliminari, sent. 23 maggio 2019 (depositata il 3 giugno 2019).

49 Amnesty International 2016; da ultimo, si veda la nota condivisa di Associazione per gli studi giuridici per l'immigrazione, ActionAid, ARCI, Borderline Sicilia, IndieWatch, Medici per i Diritti Umani - MEDU, Sea-Watch, Illegittimo trattenimento nell'hotspot di Messina dei migranti sbarcati dalla Sea-Watch, 10 luglio 2019 (https:// bit.ly/2NoHy48, 2019-10-25) 
porti e di indifferenza nei confronti di quanti muoiono nel tentativo di raggiungere l'Europa, si esprime anche nella creazione di un sub-diritto, fatto di circolari, regolamenti interni, capitolati d'appalto: ${ }^{50}$ un sub-diritto che attesta il naufragio delle istanze di uguaglianza, della centralità della persona umana, alla base del costituzionalismo e della democrazia, e nella cui duttilità e fluidità si insinua con facilità la 'possibilità' che siano praticati trattamenti inumani o degradanti.

In quarto luogo, di tortura o, quantomeno, trattamento inumano o degradante può ragionarsi in relazione alla politica di «chiusura dei porti», politica strettamente correlata all'approccio di esternalizzazione delle frontiere sotteso agli accordi di riammissione, con il trattenimento forzato dei migranti - che porta la magistratura a ipotizzare il sequestro di persona (Zirulia, Cancellaro 2019) ${ }^{51}$ - a bordo delle navi che li hanno salvati.

Per restare agli ultimi anni, si possono ricordare il caso della nave Aquarius (giugno 2018), in navigazione nel Mediterraneo senza porto per giorni; della nave Diciotti della Guardia costiera italiana bloccata nel porto di Catania con 177 migranti a bordo (agosto 2018); delle navi Sea-Watch 3 e Sea-Eye, costrette in mare per rispettivamente 20 e 13 giorni fra dicembre 2018 e gennaio 2019. L'estate del 2019, con l'entrata in vigore del 'decreto sicurezza bis' (d.l. 14 giugno 2019, nr. 53), convertito nel corso dell'estate in legge (l. 8 agosto 2019, nr. 77), vede il blocco di numerose navi, alle quali è notificato il divieto di ingresso, transito o sosta in acque territoriali italiane e che vengono altresì colpite dalle pesanti sanzioni introdotte dal decreto e inasprite dalla legge. Nel giugno 2019 è nuovamente bloccata in mare per 17 giorni la Sea-Watch 3; nel luglio 2019, il veliero Alex della Ong Mediterranea Saving Humans resta senza autorizzazione allo sbarco per giorni; nell'agosto 2019, la nave Ocean Viking, gestita da Sos Mediterranée e Medici senza frontiere, è costretta in mare tra Malta e Lampedusa con 356 persone a bordo per 13 giorni, mentre la Open Arms è senza porto con oltre 100 persone a bordo per 19 giorni, la Eleonore della Ong Lifeline, trascorsi 8 giorni di attesa, forza il divieto di ingresso nelle acque territoriali e di approdo e la nave Mare Jonio, dopo giorni in mare, è colpita dalle sanzioni del decreto.

Le condizioni a bordo delle navi condannate a stare in mare per giorni configurano trattamenti inumani o degradanti, di cui è responsabile chi dispone la chiusura dei porti; limitandosi ad una testimo-

50 Per una riflessione critica sull'uso delle circolari, in materia di immigrazione, cf. Gjergji 2013.

51 Si veda il caso della nave Diciotti della Guardia costiera italiana, ma anche la vicenda della nave Open Arms, dove, oltre il procedimento per sequestro di persona, si ipotizza - con l'atto con il quale si pone fine alla vicenda (Procura della Repubblica presso il Tribunale di Agrigento, Decreto di sequestro preventivo di urgenza, 20 agosto 2019, nr. 3770/2019 R.G. notizie di reato) - il reato di omissione e rifiuto di atti d'ufficio (art. 328, c. 1, c.p.). 
nianza: «a bordo della nave Diciotti le condizioni erano terribili. Era impossibile stare al sole ma c'era solo un tendone. L'ombra non bastava per tutti, e quando pioveva ci bagnavamo. C'erano solo due bagni».52

Il trattamento inumano o degradante consiste nelle condizioni in cui si svolge la forzata permanenza in mare (mancanza di spazi, di servizi igienici, di acqua) ed è quindi aggravato dalle condizioni personali di molti migranti, persone vulnerabili e che «hanno subito traumi importanti»: «in molti hanno subito torture o violenze sessuali in Libia» e «l'attesa dello sbarco, consumata in uno spazio confinato in mezzo al mare non può che peggiorare le loro condizioni». ${ }^{53}$

Nel decreto che il 20 agosto 2019 sblocca la vicenda della nave Open Arms, da 19 giorni costretta in mare, si legge di un «evidente sovraffollamento della nave» e di «pessime condizioni» («i migranti occupavano interamente il ponte della nave adagiati sul pavimento, avevano a disposizione due soli bagni alla turca...») e di «uno stato di esasperazione in capo ai soggetti rimasti per diversi giorni a bordo [...] che ha determinato situazioni sanitarie assai critiche (sul piano fisico e/o psichico dei soggetti interessati)». ${ }^{54}$

In precedenza, sempre in relazione alla nave Open Arms, nel ricorso per l'annullamento del provvedimento dell'1 agosto 2019 (reso dal Ministro dell'Interno, di concerto con il Ministro della Difesa e con il Ministro delle Infrastrutture e dei Trasporti), con cui era stato disposto il divieto di ingresso, transito e sosta della nave nel mare territoriale nazionale, il TAR Lazio aveva già rilevato, quanto al periculum in mora, come la documentazione prodotta (medical report, relazione psicologica, dichiarazione capo missione) prospettasse una "situazione di eccezionale gravità ed urgenza», «tale da giustificare la concessione [...] della richiesta tutela cautelare monocratica, al fine di consentire l'ingresso della nave Open Arms in acque territoriali italiane».55

Il blocco delle navi dà luogo a numerose prese di posizione dei garanti dei diritti, dai giudici ordinari al Garante nazionale dei diritti delle persone detenute o private della libertà personale e all'Autorità garante per l'infanzia e l'adolescenza, dall'Alto Commissariato delle Nazioni Unite per i rifugiati (UNHCR) alla Corte EDU (esemplari, in tal senso, sono gli interventi che riguardano la vicenda del blocco della nave Sea-Watch 3 del gennaio 2019, ricordati in Del Guercio 2019).

52 Testimonianza di B.B., eritreo, 29 anni, in Oxfam Italia, Borderline Sicilia 2019.

53 L. Pigozzi, medico di Medici senza frontiere, in C. Lania, «Quei 356 fantasmi della Ocean Viking senza un approdo», il manifesto, 21 agosto 2019.

54 Procura della Repubblica presso il Tribunale di Agrigento, Decreto di sequestro preventivo di urgenza, 20 agosto 2019, nr. 3770/2019 R.G. notizie di reato.

55 TAR Lazio, Seziona Prima Ter, Decreto cautelare monocratico, 14 agosto 2019, proc. nr. 10780/2019 R.G. 
Impedire l'ingresso nelle acque territoriali viola il divieto di tortura, quantomeno come divieto di trattamento inumano o degradante, sia in relazione alle condizioni del forzato soggiorno, per non dire tout court 'detenzione', sulle navi, sia in relazione alla violazione del dovere di soccorso (volto a tutelare la vita, così come le condizioni, mediche e psicologiche, delle persone salvate in mare); con la possibilità di ipotizzare altresì la commissione del reato di tortura di cui all'art. 613 bis del codice penale. ${ }^{56}$

Come ha rilevato il Tribunale di Agrigento (Ufficio del Giudice per le Indagini Preliminari), ${ }^{57}$ nell'ordinanza, depositata il 2 luglio 2019, nel procedimento contro Carola Rackete, capitana della Sea-Watch 3 , indagata per i reati di resistenza o violenza contro nave da guerra (art. 1100 cod. nav.) e resistenza a pubblico ufficiale (art. 337 c.p.), in relazione alle condotte tenute nella notte del 29 giugno 2019 durante l'ingresso nel porto di Lampedusa: l'ordinamento giuridico italiano, e le norme internazionali che incorpora, sanciscono l'inderogabile obbligo di assicurare il soccorso, quale dovere il quale «non si esaurisce nella mera presa a bordo dei naufraghi, ma nella loro conduzione fino al... porto sicuro» (donde, nel caso di specie, il richiamo all'art. 51 c.p., con esclusione della punibilità in quanto il fatto è stato compiuto nell'adempimento di un dovere imposto da una norma giuridica). ${ }^{58}$

«L'obbligo di salvataggio delle vite in mare costituisce un dovere degli Stati e prevale sulle norme e sugli accordi bilaterali finalizzati al contrasto dell'immigrazione irregolare», ${ }^{59}$ così come sulle direttive ministeriali in tema di porti chiusi (pur se 'coperte' da norme di legge), ${ }^{60}$ in forza degli artt. 10 , c. 1 , e 117, c. 1 , della Costituzione. ${ }^{61}$

56 Zirulia, Stefano; Cancellaro, Francesca (2019). «Divieto di sbarco, perché può essere tortura». il manifesto, 30 agosto. URL https://ilmanifesto.it/open-arms-puoessere-stata-tortura/ (2019-10-16).

57 Ordinanza sulla richiesta di convalida di arresto e di applicazione della misura cautelare, 2 luglio 2019 (N. 3169/19 R.G.N.R.; N. 2592/19 R.G.GIP).

58 In merito alla vicenda della Sea-Watch 3 viene avanzata anche la richiesta di misure provvisorie alla Corte EDU, in relazione agli artt. 2 e 3 della Cedu, ma la Corte respinge la richiesta (25-06-2019).

59 Procura della Repubblica presso il Tribunale di Agrigento, Decreto di sequestro preventivo di urgenza, 20 agosto 2019, nr. 3770/2019 R.G. notizie di reato.

60 L. 8 agosto 2019, nr. 77.

61 «In forza della natura sovraordinata delle fonti convenzionali e normative [...], nessuna idoneità a comprimere gli obblighi gravanti sul capitano della Sea Watch $3[\ldots]$ potevano rivestire le direttive ministeriali in materia di 'porti chiusi'» (Tribunale di Agrigento, Ufficio del Giudice per le Indagini Preliminari, Ordinanza sulla richiesta di convalida di arresto e di applicazione della misura cautelare, 2 luglio 2019 (N. 3169/19 R.G.N.R.; N. 2592/19 R.G.GIP). 
La politica di criminalizzazione della solidarietà (Masera 2019a) ${ }^{62}$ chiude quindi il cerchio di politiche migratorie che uccidono e torturano, punendo testimoni scomodi di una chiusura delle frontiere che causa un vero e proprio genocidio delle popolazioni emigranti.

Si palesano l'appropriazione e la violenza che stanno dietro l'idea di confine (Mezzadra 2018) e l'ipocrisia di 'Stati democratici' che delocalizzano la tortura. Tornano tragicamente attuali le parole che Livio attribuisce a Romolo, quando uccide il fratello Remo, reo di aver scavalcato le mura della neo fondata Roma: «così, d'ora in poi, possa morire chiunque osi scavalcare le mie mura».

\section{Osservazioni conclusive: torture di Stato?}

Respingere una persona in Libia, o impedirle di valicare i confini del Niger (Spagnolo 2018; Olivito 2019) o del Sudan, così come criminalizzare e ostacolare le Ong che operano nel Mediterraneo, chiudere i porti o demandare alle autorità libiche il soccorso in mare, viola il divieto di tortura e trattamenti inumani o degradanti e costituisce un crimine contro l'umanità.

A perpetrarlo sono certamente i soggetti che praticano in prima persona le torture, così come gli Stati che le tollerano, quando non conferiscano loro una qualche forma di 'istituzionalizzazione', ma non sono certo esenti da responsabilità i governi degli Stati europei o gli esecutivi dell'Unione europea, che, nel compiere determinate scelte politiche, non possono non raffigurarsi lo scenario che ne consegue: come ha affermato la Corte EDU in relazione alla situazione libica, «le autorità italiane sapevano o dovevano sapere». ${ }^{63}$

Si prospetta, per riprendere quanto affermato dal Garante nazionale dei diritti delle persone detenute o private della libertà personale, Mauro Palma, con riferimento al blocco in mare della nave Mare Jonio (agosto 2019), la violazione della Convenzione europea dei diritti dell'uomo e della Convenzione di Ginevra sullo status dei rifugiati del 1951, con conseguenti profili di responsabilità in sede internazionale per l'Italia. ${ }^{64}$

62 Da ultimo, cf. le misure, chiaramente dirette a colpire le attività di salvataggio in mare da parte delle Ong, di cui alla l. 8 agosto 2019, nr. 77 (che ha convertito in legge il d.l. 14 giugno 2019, nr. 53), con un inasprimento delle sanzioni - già di assai dubbia legittimità costituzionale - previste nel decreto (in merito, si vedano, in primis, le osservazioni contenute nella Lettera di accompagnamento alla promulgazione della legge del Presidente della Repubblica, indirizzata ai Presidenti del Senato della Repubblica e della Camera dei Deputati e al Presidente del Consiglio dei Ministri, Roma, 8 agosto 2019).

63 Corte EDU, Grande camera, Hirsi Jamaa e altri c. Italia, cit. ante.

64 Appello del Garante nazionale per la situazione della nave Mare Jonio, 30 agosto 2019, comunicato stampa (http://www.garantenazionaleprivatiliberta.it, 2019-10-25). 
A ciò si può aggiungere che

le politiche e le prassi dell'UE e dei suoi Stati membri sull'asilo e sull'immigrazione costituiscono nel loro insieme una totale negazione dei diritti fondamentali delle persone e dei migranti e integrano dei veri e proprio crimini contro l'umanità, che, anche quando non sono personalmente addebitabili a specifici autori secondo le condivise regole garantistiche penali, devono definirsi come 'crimini di sistema'. ${ }^{65}$

In questa prospettiva, è stato recentemente presentato (giugno 2019) un esposto alla Corte penale internazionale che accusa l'Unione europea e gli Stati membri di crimini contro l'umanità per le politiche che hanno reso la rotta mediterranea la via migratoria più letale al mondo e orchestrato trasferimenti forzati nei campi di detenzione in Libia, simili a campi di concentramento, dove vengono compiuti atroci crimini.

Coloro che, esternalizzando le frontiere, delocalizzano e subappaltano tortura e trattamenti inumani o degradanti, sono co-responsabili; così come responsabili sono coloro che adottano i provvedimenti di chiusura dei porti, condannando a trattamenti inumani o degradanti i naufraghi. Chi, restando alle politiche italiane, stipula il Memorandum d'intesa del 2017 con la Libia (ministro Minniti, governo Gentiloni), chi chiude i porti e criminalizza i salvataggi in mare (ministro Salvini, governo Conte), chi continua a votare il rifinanziamento alla Guardia costiera libica (la maggioranza dei parlamentari, XVIII legislatura), si rende complice, per limitarsi al caso più eclatante, dei crimini commessi nei centri per migranti in Libia, per non parlare delle responsabilità dei morti in mare.

Non solo: a essere revocata in dubbio è la natura democratica di Stati che adottano politiche e stringono accordi, i quali di fatto (ma i diritti esistono in quanto effettivi) contemplano la tortura o sono diretta causa di trattamenti inumani o degradanti.

La 'tortura di Stato' non è certo una novità - Genova 2001 docet; può - deve - essere fermata e punita nelle aule di tribunale, ma occorre anche una forte mobilitazione sociale, se del caso con una disobbedienza, nel nome dei diritti, che renda pratica diffusa atti di testimonianza, come quelli delle capitane e dei capitani coraggiosi delle navi delle Ong, così come dei migranti della Vos Thalassa, che si ribellano di fronte all'ordine rivolto dalle autorità italiane al comandante della nave di riportarli in Libia (Masera 2019b).

65 Tribunale Permanente dei Popoli, Sessione sulla violazione dei diritti delle persone migranti e rifugiate (2017-2019), Atto conclusivo, Parlamento Europeo, Bruxelles, 9 aprile 2019. 
La tortura, delocalizzata e non, nei confronti dei migranti, è indice di un'involuzione autoritaria dello Stato, che, in coerenza con i dogmi di una governance mondiale segnata dall'egemonia di un modello e di una classe sociale, conduce ad un conflitto (di classe) contro chi, anche solo con la sua esistenza, mostra le diseguaglianze e la violenza del sistema neoliberista.

Nel contempo i migranti, così come le persone che vivono ai margini della società, ma anche coloro che esprimono dissenso, costituiscono un comodo nemico contro il quale canalizzare la rabbia sociale, creando una fittizia comunanza di intenti fra chi sta al vertice e chi alla base della piramide (le masse atomizzate) per evitare che la diseguaglianza esploda verso l'alto. ${ }^{66} \mathrm{Si}$ crea un nemico e si inibisce la creazione di una coscienza - di classe (per utilizzare un termine non a caso ostracizzato) - in grado di unire coloro che, in luoghi diversi, subiscono gli effetti del neoliberismo.

Si sperimentano operazioni di disumanizzazione; ritornano gli hebetes di De Vitoria (1539): i migranti come esseri non pienamente umani, nei confronti dei quali, svelando tragicamente le ambiguità che accompagnano la proclamazione dei diritti universali e la loro sottomissione agli interessi economici o alle velleità sovraniste, torture e trattamenti inumani o degradanti assumono un peso differente.

\section{Bibliografia}

Algostino, Alessandra (2016). «La soft law comunitaria e il diritto statale: conflitto fra ordinamenti o fine del conflitto democratico?». Costituzionalismo.it, 3, 255-85. URL http://www.costituzionalismo.it/articoli/593/ (2019-10-17).

Amnesty International (2016). Hotspot Italia: come le politiche dell'Unione europea portano a violazioni dei diritti di rifugiati e migranti. URL https://www.

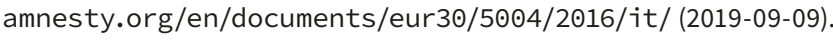

Amnesty International (2017). A Blueprint for Despair. Human Rights Impact of the EU-Turkey Deal. URL https://www.amnesty.org/en/documents/ eur25/5664/2017/en/ (2019-09-09).

Bobbio, Norberto (1990). L'età dei diritti. Torino: Einaudi.

Borraccetti, Marco (2016). «L'Italia e i rimpatri: brevi ricognizione degli accordi di riammissione». Diritto, immigrazione e cittadinanza, 1-2, 33-58.

Carreau, Dominique; Marrella, Fabrizio (2016). Diritto internazionale. Milano: Giuffrè.

Cassarino, Jean Pierre (2014). «A Reappraisal of the EU's Expanding Readmission System». The International Spectator, 49(4), 130-45.

66 Emblematici in tal senso sono i vari 'decreti sicurezza', come, per restare agli ultimi, il c.d. pacchetto Minniti (d.l. 13 del 2017, convertito in l. 46 del 2017, e d.l. 14 del 2017, convertito in l. 48 del 2017) e i decreti salviniani (d.l. 113 del 2018, convertito in l. 132 del 2018, e d.l. 53 del 2019, convertito in l. 77 del 2019). 
Cassarino, Jean Pierre (2016). «La centralità periferica dell'art. 13 dell'accordo di Cotonou». Diritto, immigrazione e cittadinanza, 1-2, 21-32.

Corten, Olivier (2016). «Accord politique ou juridique: quelle est la nature du 'machin' conclu entre l'UE et la Turquie en matière d'asile?». EU Immigration and Asylum Law and Policy, 10 June. URL https://bit.ly/20X8wkq (2019-09-09).

De Vitoria, Francisco (1539). Relectio de Indis. La questione degli Indios. Ed. latino-italiano, curata da A. Lamacchia e ripresa dalla edizione critica bilingue di: Pereña, L.; Perez Prendes, J.M., Relectio de Indis o libertad de los Indios. Madrid: Consejo superior de investigaciones cientificas, 1967.

Del Guercio, Adele (2019). «Il caso della 'Sea-Watch 3' tra obblighi di diritto del mare, diritti umani e tutela dell'infanzia». Diritti umani e diritto internazionale, 2, 331-62.

Den Heijer, Maarten; Spijkerboer, Thomas (2016). «Is the EU-Turkey Refugee and Migration Deal a Treaty?». EU Law Analysis, URL http://eulawanalysis. blogspot.it (2019-09-09).

Fanon, Frantz (1962). I dannati della terra. Torino: Einaudi.

Favilli, Chiara (2016). «La cooperazione UE-Turchia per contenere il flusso dei migranti e richiedenti asilo: obiettivo riuscito?». Diritti umani e diritto internazionale, 2, 405-26.

Ferri, Federico (2016). «Convergenza delle politiche migratorie e di cooperazione allo sviluppo dell'Unione europea e accordi con Stati terzi». Diritto, immigrazione e cittadinanza, 3-4, 39-69.

Garante nazionale 2018 = Garante nazionale dei diritti delle persone detenute o private della libertà personale (2018). Rapporto sulle visite tematiche effettuate nei Centri di Permanenza peril Rimpatrio (CPR) in Italia (febbraio-marzo 2018). Roma. URL https://bit.ly/3302mnt (2019-10-17).

Gjergji, Iside (2013). Circolari amministrative e immigrazione. Milano: Franco Angeli.

Gjergji, Iside (2016). Sulla governance delle migrazioni. Sociologia dell'underworld del comando globale. Milano: Franco Angeli.

Grosso, Enrico (2009). «l respingimenti in mare: quando i Governi tentano di sottrarsi alla fatica di prendere i diritti sul serio». Diritto Pubblico Comparato ed Europeo, 3, 15-23.

Marchegiani, Maura (2008). «L'accordo di riammissione quale strumento in De Vitoria, Francisco (1539). Relectio de Indis. La questione degli Indios tegrato di cooperazione tra Stati per il contrasto all'immigrazione irregolare». Palmisano, Giuseppe (a cura di), Il contrasto al traffico di migranti nel diritto internazionale, comunitario e interno. Milano: Giuffrè, 113-50.

Masera, Luca (2019a). «La criminalizzazione delle ONG e il valore della solidarietà in uno Stato democratico». Federalismi.it, 2, 18-43.

Masera, Luca (2019b). «La legittima difesa dei migranti e l'illegittimità dei respingimenti verso la Libia (caso Vos-Thalassa)». Diritto Penale Contemporaneo. URL https://bit.ly/2MmDals (2019-09-09).

Mezzadra, Sandro (2010). «Metamorfosi di un solco. Terra e confini». Parolechiave, 2, 9-28.

Olivito, Elisa (2019). "Accordi in forma (semi)semplificata, istanze di accesso civico e vulnus alla Costituzione». Rubrica "Diritti senza confini". URL https://bit.ly/2NsNNUG (2019-09-09).

Oxfam Italia, Borderline Sicilia (2019). Accordo Italia-Libia: scacco ai diritti umani in 4 mosse. URL https://www.oxfamitalia.org/wp-content/uplo- 
ads/2019/01/SCACCO_AI_DIRITTI_UMANI_IN_4_MOSSE_DEF..pdf (2019-09-09).

Peers, Steve (2016). «The Draft EU/Turkey Deal on Migration and Refugees: is It Legal?». EU Law Analysis, 16 March URL http://eulawanalysis. blogspot.com/2016/03/the-draft-euturkey-deal-on-migration.html (2019-09-09).

Snyder, Francis (1993). «The Effectiveness of European Community Law: Institutions, Processes, Tools and Techniques». The Modern Law Review, 56(1), 19-54. DOI https://doi.org/10.1111/j.1468-2230.1993.tb02852.x.

Spagnolo, Andrea (2018). «Di intese segrete e alibi parlamentari: tra la decisione del TAR sull'accordo col Niger e il Global Compact sulle migrazioni». SIDIBlog, 5 dicembre. URL https://bit.ly/2psXN6N (2019-09-09).

Vitiello, Daniela (2016). «L'azione esterna dell'Unione europea in materia di immigrazione e asilo: linee di tendenza e proposte per il futuro». Diritto, immigrazione e cittadinanza, 3-4, 9-38.

Zirulia, Stefano; Cancellaro, Francesca (2019). "Caso Diciotti: il Tribunale dei ministri qualifica le condotte del ministro Salvini come sequestro di persona aggravato e trasmette al Senato la domanda di autorizzazione a procedere». Diritto Penale Contemporaneo. URL https://bit.ly/2qozaZv (2019-09-09). 
Parte II

Un fenomeno globale 



\title{
Vulnerable to the System: Migration and Torture in Spain
}

Olga Jubany

Universitat de Barcelona, Espanya

Martina Pasqualetto

Universitat de Barcelona, Espanya

Alèxia Rué

Universitat de Barcelona, Espanya

\begin{abstract}
Grounded on the results of an ethnographic investigation on asylum seekers as victims of torture in Spain, this chapter analyses the governmental devices that define the reality of migrants as victims of torture. Stemming from a critical review of the concept of torture, in relation to migration in contemporary European societies, the chapter puts forward the double vulnerability to which migrants are exposed, as unprotected victims of torture in their countries of origin and as potential victims of torture on arrival to Europe. The paper argues that the precarious legal status and the failure of systems victim protection, combined with meritocratic approaches to migrant incorporation, not only fail to ensure full protection of victims of torture but, moreover, it exposes these victims to further situations of violence and exclusion.
\end{abstract}

Keywords Torture. State violence. Asylum seekers. Immigration. Spain.

Summary 1 Torture, Migration and State Violence. - 2 Spain and the [un]protection against Torture. - 3 Asylum-Seeking Victims of Torture. - 4 Torture and the Enforcement of Migration Policies. -5 Conclusion: The Double Vulnerability. 


\section{Torture, Migration and State Violence}

Torture is an age-old phenomenon, which has been present in Western societies for centuries, and was revealed as particularly prevalent throughout the 20th century. The understandings, interpretations and different forms torture has taken have been the subject of complex debates, both from academic, political and legal approaches. It is not until relatively recently, in the last decades, however, that the connection between torture and migration started to become evident and, as such, turn into the focus of study, largely as a response to its growing global perceptibility and its multiplicity in practices.

While the purpose of this chapter is not to provide an epistemological review of torture and migration, but rather to advance an indepth contemporary analysis of this phenomenon in a current setting, it is necessary to begin with a brief consideration of the key underpinnings of the concept, in order to offer an accurate examination. This implies going beyond common perceptions of torture, its victims, perpetrators and the societies where it takes place, to provide solid grounds for the discussion of the intertwining between the two complex realities that torture and migration presuppose.

Despite common understandings by most public and political debates of torture and inhuman or cruel degrading treatments as exceptional deviant acts (Tulloch 2005) with the media presenting torture as a barbarous aberration alien to democratic states, ${ }^{1}$ it is critical to understand that, from an analytical perspective, torture cannot be considered as an exceptional act in our society, as torturers cannot simply be dismissed as monsters. Rather torture is understood as an exercise practiced as part of work duties, by ordinary people, because, as Arendt (1964) vindicated, far from being monstrous, evil is often banal. It is, as Zimbardo (2002) explored, the society and the circumstances that provoke and eventually normalise these acts - such as that endorsed by the so-called Global North's 'war on terror'.

Thus, whilst from a legal point of view torture has tended to be studied as a form of exceptional state violence, from a social and cultural analytical viewpoint this has to be understood as both a criminal and a deviant act, yet consistent in Western societies as part of much less exceptional forms of deviance, as the case of torture and migration illustrates. From this understanding, torture cannot be considered solely as an exceptional event in the hands of 'folk devils', but is rather to be understood within the logic of its social production

1 There is a relatively developing trend in media and popular debate in this regard, shown by measures such as "extraordinary rendition" in the USA, which implies flying terrorist suspects to countries where allegedly they can be tortured outside Western legal jurisdiction. 
(Cohen 1972). It is crucial to consider that the practice of torture does not take place in isolation, but under the influence of certain narratives, sites and times where particular subjects are construed as dehumanised (Bauman 2008). These frameworks denote the potential for torture as inherent in the power relations that determine the dehumanisation of the disempowered as the "torturable subjects" (Mendiola 2014). Such [re]construction of the disempowered as 'the other' assents to their identification with whatever "questions the imagined security, peace, order and rule of law of Western democratic societies" (Mendiola 2014, 2018).

In a context of increasing criminalisation of the poor, for those living at the edges of society the persistent and latent danger of being subjected to torture is part of the apparatus of control over their daily reality (Wacquant 2009). Torture becomes not only a means of punishment but also "part of the civilizing mission" (Butler 2009, 16). State violence, non-exceptional but normalised, is practiced against this 'other' as a mode of disciplining them into the prevailing social order and asserting the moral superiority of the torturer over the tortured as guardian of this social order. Torture becomes thus not the only but the most visible outcome of a continuum of violent state practices aiming to control and discipline populations that are regarded as criminal and as the threatening 'other'.

Arguably one of the most evident imprints of 'othering' processes in the current Global North is manifested by the production and normalisation of social spaces and boundaries between 'us' and 'them'. This is nowadays epitomised by the exploitation of modern migration categories, most of which leave people stateless and thus rightless (Arendt 1973).

Migrants and asylum seekers are at the core of the socially excluded, being constantly [re]constructed as the 'alien others', through discourses of sovereignty and national security, as the flagged values of modern societies. They stand at the intersection that challenges the state's sovereignty by their presence, particularly as poor and racialized populations, while living and working in a situation of legal exclusion. Thus, in analysing the link between migration and torture in contemporary Europe, torture must be understood in a continuum that makes no sharp divide between direct forms of state violence, and other, more subtle forms, in what is frequently referred to as "structural violence" (Galtung 1969). It is arguable that, following Parry $(2010,17)$, "[t]he use of these forms of violence by modern states as a way of regulating populations is far more significant than whether 'torture' is the particular form of violence used".

Within this continuum, the states' disciplinary practices that create the narratives and sites of torture extend to other spaces and milieus, including spaces of protection. In terms of the different debates that address this topic, most accounts of torture and migration con- 
centrate on exploring the experience of those who have suffered violence in their countries of origin and during transit, and seek protection in the 'modern liberal democracies', allegedly free of torture. The core of this literature addresses the mental health impact of torture among asylum seekers ${ }^{2}$ and the consequences this has for their asylum applications and incorporation at countries of settlement (Daniel, Knudsen 1995; Haoussou 2017; McColl, Bhui, Jones 2012; Oomen 2007). There have also been a number of studies tackling the lack of protection for victims of torture in countries of arrival regarding the absence of social safeguards, poverty and destitution, ${ }^{3}$ and problems and considerations with reference to health care (Vannotti, Bodenmann 2003). Still, there is a lack of ethnographic approaches that address the mechanisms that underpin the absence of protection and neglect that asylum seekers suffer in Europe.

On the other hand, while there is a solid body of literature regarding violence suffered by migrants and asylum seekers in Europe, especially concerning violence at borders, detention and deportation, ${ }^{4}$ this issue is rarely addressed in regard to the prohibition of torture (Morales 2016; Sanggaran, Zion 2016).

Grounded on the results of an ethnographic investigation of asylum seekers as victims of torture in Spain, ${ }^{5}$ this chapter addresses both these issues to analyse the governmental devices that define the reality of migrants as victims of torture. The empirical investigation included two consecutive research projects that took place over a period of four years (2015-19). The arguments presented in this chapter are thus built on an exhaustive examination of documentation, with a particular focus on reports and official files related to the prevalence of torture against migrants in Spain, as well as an extensive ethnographic research. This involved over 50 in-depth interviews with key actors in civil society organisations and public officers working for asylum seekers and victims of torture. The results of this research reveal how asylum seekers, especially those who have been victims of torture, are not only subject to the violent

2 See, for instance, Kalt et al. 2013; Mazzetti 2008; Schubert, Punamäki 2011; Steel et al. 2009, amongst others.

3 See Allsopp, Sigona, Phillimore 2014; Bloch, Schuster 2005; Davies, Isakjee, Dhesi 2017; Menjívar, Abrego 2012, amongst others.

4 See Alpes et al. 2015; De Genova 2016; Morales 2016; Mountz, Loyd 2014; Schuster 2005, to name a few.

5 The chapter is based on two consecutive ethnographic investigations about the support for victims of crime and the reception system for asylum seekers and refugees in Spain, as well as on the analysis of reports and official documents on the prevalence of torture against migrants in Spain. Including both research projects, more than 50 in-depth interviews were conducted with key actors in civil society organisations and public officers working for asylum seekers and victims of torture. 
consequences of inaction and neglect of a failing asylum system, but are also potential victims of torture by the direct use of violence in the enforcement of migration policies.

The case of Spain stands as paradigmatic in the exploration of migration and torture within Europe. This is not only because Spain has become one of the 'gates of Europe' but also for its central role in the development of border control technologies and strategies in the EU (Andersson 2014). In addition, since the beginning of the 21st century, with the exception of the peak years of the economic crisis, Spain has been consistently among the top immigration receiving countries in the EU, experiencing, in recent years, a sharp increase in the number of asylum applications, many of which are made at ports of entry. In this regard, condemnatory reports and measures by Human Rights international bodies against Spain for its practices at borders, in detention and deportation are testimony of the pervasiveness in Spain of all the modern forms of torture against migrants.

This chapter argues that the precarity of asylum seekers' legal status and the failure of the systems of social and health protection for victims, combined with meritocratic approaches to migrant incorporation, expose these victims to further situations of violence and abandonment and can lead them to chronic situations of exclusion and violence. Whilst this might be applicable to migrant victims of torture in general, the case of asylum-seeking victims of torture is especially representative due to the right of protection they enjoy as asylum claimants and the specific need of protection that all asylum regulations recognise for victims of torture.

The chapter begins by offering an overview of the context of torture in Spain: its legal framework, its limitations, and its violations. Grounded on ethnographic evidence, it later discusses the double vulnerability that asylum seekers face in Spain, as potential victims of torture and as unprotected victims of torture. Finally, building on the analysis of this double vulnerability, the paper sheds light onto the mechanisms that underpin the particular types of violence that arise in the enforcement of migration policies.

\section{Spain and the [un]protection against Torture}

Understanding the current settings and progress of the legal and social background of torture in Spain is key to recognise how modern forms of torture have developed and taken a central place in the enforcement of migration policies in this country. After a 40-year dictatorship, in which the most evident practices of torture were a legitimate state tool (Acosta Bono, del Río Sánchez, Valcuende del Río 2008), starting from the democratic transition in the late seventies, Spain has ratified all international legislation against torture and 
has adapted relevant national legislation geared to prevent and punish these acts. The Spanish Constitution prohibits torture in art. 15, stating that "Everyone has the right to life and physical and moral integrity, and under no circumstances may be subjected to torture or to inhuman or degrading punishment or treatment".

Further, in 1984, Spain ratified the UN Convention against Torture and Other Cruel, Inhuman or Degrading Treatment or Punishment, and, in 2005, the 2002 UN's Optional Protocol. Thus, at face value, Spain adopts a clear approach against torture as it has ratified all international covenants and their amendments, as well as integrated them into its national law. However, when looking closely into how Spain has transposed these responsibilities and principles, as well as into the practices of torture in its different expressions, it becomes evident that Spanish legislation for the prevention and reparation of torture does not offer all the guarantees recognised in international law (Rights International Spain 2017).

Considering the recent historical trajectory of Spain, with a background of a long lasting dictatorship, a major problem in the transpositions of rules and interpretation of torture stems from the definition of the concept of torture included in the Spanish legislation, and particularly in the current Spanish Penal Code. This, for instance, does not recognise that torture can be committed by "any person exercising public functions" other than public officers, and it does not recognise "intimidation" as torture. Also, it fails to consider torture as a crime against the international community (Amnesty International 2015, 8) and it distinguishes between "severe" and "light" types of torture (Rights International Spain 2017, 1). A further illustration of this flaw, linked to the conceptualisation of torture, is seen by the authorisation of incommunicado detentions, a situation of deprivation of liberty in which the detainee has no possibility to access an attorney, an independent physician or family members. ${ }^{6}$ This lack of development of the national legislation for the prevention of torture can, and often does, lead to cases where torture is not condemned because it is not recognised as such. Also, and crucially, this contributes to a normalisation of behaviours and punishments that legitimises the excessive use of violence by public officials in the exercise of their duty.

However, this underdevelopment and lack of revision of the concept in the national legislation is not the only factor that hinders the possibilities of reporting and recognising torture and inhuman

6 Whilst international laws do not explicitly prohibit incommunicado detention, there is a general agreement among human rights bodies in the international community (Human Rights Watch 2005) that this can lead to severe human rights violations, including torture, and that it could be constitutive of torture in itself. 
treatment in Spain. As the empirical evidence reveals, public bodies and private organisations investigating allegations of such acts face barriers to their inquiries, mainly in accessing existing evidentiary documentation and information on the cases, as well as delays, which severely curtail their capacity to provide documentary proof for these allegations:

We also face difficulties when investigating torture complaints because when we ask public bodies for information, well, finally they are the ones who decide and filter what they tell you. So, some things are out of our reach; we don't get them. We cannot take the civil servants statements nor watch the images. When we finally ask for the images, well, they have already been erased [due to programmed erasure], so there is a difficulty in proving torture. (SG-I-1)

The strongest illustration of this is that the European Court of Human Rights (ECHR) has condemned Spain for not investigating duly torture allegations on nine different occasions. ${ }^{8}$ This systematic lack of investigation relates to an invisibility of this practice by which, as Rivera Beiras puts it, "public authorities consistently deny the existence of torture [...] due to the lack of condemnatory rulings" (in Bergalli, Rivera Beiras 2006, 73). Yet, beyond this condemnation, this situation also raises the question of whether such a lack of condemnatory rulings responds to a denial of the existence of torture rather than to a scarcity of cases, as is the concern of many professionals in the field:

The system is perhaps not ready to acknowledge its own violation of human rights, because it would imply paying compensations and changing surveillance and confinement structures. But if there are 200 reports per year it is hard to imagine they are all made up or exaggerated, you can easily see this is a recurrent and structural issue, and that there would be 2,000 reports if people were aware of the possibility of reporting. (EX/B/TS/FN)

7 All interviews have been codified to guarantee the anonymity of the interviewees. Own codes are included in reference to each quote for access and data management purposes.

8 The nine cases are: Martinez Sala and others v. Spain; San Argimiro Isasa v. Spain; Beristain Ukar v. Spain; B.S. v. Spain; Otamendi Egiguren v. Spain; Etxebarria Caballero v. Spain; Ataun Rojo v. Spain; Arratibel Garciandia v. Spain; Berotegui Martinez v. Spain; Portu Juanenea and Sarasola Yarzabal v. Spain (Source: HUDOC, European Court of Human Rights Data Base). In all cases Spain was condemned for not duly investigating torture claims, except in Portu Juanenea and Sarasola Yarzabal v. Spain, where the court condemned Spain for inhuman and degrading treatment. In B.S. v. Spain, the court condemned Spain for not duly investigating on the grounds that it should have considered the victim's ethnicity, gender and migration status. 
In this regard, in addition to the already mentioned sentences of the ECHR for not duly investigating torture cases, Spanish government bodies have repeatedly nullified the rulings condemning public servants for torture or inhuman or degrading treatment. Moreover, Spanish institutions have not only pardoned but also decorated or promoted the offenders of torture (Bergalli, Rivera Beiras 2006), projecting a clear message of impunity to both torturers and the victims, often perceived as a form of institutional violence.

I believe there should be independent mechanisms [to assessment] institutional violence, complaints against the police shouldn't be managed by the same public authorities [...] from experience we can say that public bodies rarely accept that they have done a wrong. So, people see this and think: 'this was a clear case and the culprit has not been sentenced', or 'they have been sentenced but later they have been pardoned'. You are conveying a clear message of impunity. (SG-I-1)

Thus, even when reporting is possible, the outcome is rarely positive for the victim, as Spain's approach to torture has been characterised by the impunity of perpetrators.

\section{Asylum-Seeking Victims of Torture}

This general lack of visibility and accountability of torture within the Spanish context leads to an insufficient protection of victims who have to obtain a recognition of their victimisation to be able to access support services:

Socially, it is an irrelevant issue, that is, it is uninteresting or invisible... so there are not enough mechanisms or resources [...]. If someone has been victim of the police in Spain, the public health service has to acknowledge that their 'blood relative' has done something... has committed a crime, really, [...] so, it's difficult. $(\mathrm{EX} / \mathrm{B} / \mathrm{TS} / 1 / \mathrm{FN})$

This has particular consequences for migrants, who often face additional barriers for reporting, including the fear of being arrested and deported, particularly if undocumented. Their lack of legal protection enables situations of abuse and defencelessness against the law, which in turn lead to further impunity of perpetrators and fewer reporting: "We have had cases of police beating some of the girls, sex workers, so we have had to figure out how to follow-up on this so they continue reporting, how to set a precedent so they don't feel it goes unpunished" (AH/B/C/1/FN). 
Human rights organisations have raised concerns about possible victims and witnesses being deported before they could give testimony after having raised a complaint for having suffered torture. ${ }^{9}$ Particularly, in the case of asylum seekers who have been previously victims of torture and police violence in their countries or origin, reporting becomes especially challenging. As this social worker from a migrants support organisation explained, they encounter many challenges including: "fear, their [previous] relationships with the authorities, the fear that nothing will change, that reporting will have consequences, the time they will lose on this... these are very long and taxing processes" (AH/B/C/1/FN).

However, in Spain, the asylum system assumes, by definition, that asylum seekers may have been victims of severe violations of human rights, including torture, for which there are specific provisions to provide health support and social protection to asylum seekers. Most of this protection is provided through the State's "System for the integration and autonomy of claimants and beneficiaries of international protection". Yet, this programme presents a series of shortcomings that contribute to the vulnerability of victims of torture and potentially drive them to further situations of exclusion and violence. These can be seen in its access criteria; in the behavioural and administrative requirements; and in the meritocratic logic in social care; by which the Spanish foreigners' law increases the vulnerability of victims of torture instead of protecting them. At the core of these are barriers of access to adequate mental healthcare for victims of torture.

This is particularly relevant because, as mentioned earlier, in recent years Spain has experienced a sharp increase in the number of asylum claims. ${ }^{10}$ Still, despite the large prevalence of victims of torture among asylum seekers - considering victimisation in origin, transit and destination (Vannotti, Bodenmann 2003) - the increase in the availability of specialised protection services for asylum-seeking victims of torture has not kept up with this rise in applications. Currently, most mental health and social protections for asylum seekers, including those regarding the specific care for victims of torture, are provided within the State's asylum reception programme.

However, this programme is characterised by an abandonment of asylum seekers due to the saturation of the system (Garcés-Mas-

9 Sònia Calvó, "Un intern denuncia agressions al CIE de Barcelona per part de la Policia Nacional", Catalunya Plural, 13 de febrer 2018. URL http://catalunyaplural.cat/ca/ intern-denuncia-agressions-cie-barcelona-part-policia-nacional/ (2019-08-01).

10 Whereas from 1994 to 2014 the number of international protection applicants was consistently below 10,000 , since the generalised rise in the number of asylum seekers in Europe in the 2015-16 period and the worsening of the Venezuelan crisis, the number of applications has surged in Spain. While, in 2014, there were only 5,947 asylum applications, in 2018 there were 54,065. 
careñas 2019). In general, there is an alarming lack of accommodation and the few available slots are destined to those who are classified as "extremely vulnerable":

To have access to certain services, which are really scarce, really limited, you need not only be vulnerable but have a series of additional issues. $100 \%$ of those who come here are vulnerable. But beyond being in a situation of social and economic vulnerability, you need to have additional issues which are the ones that give you priority access to certain resources. Children, physical or mental health conditions, etc. (Coordinator of Emergency Services for Migrants, NGO) (CR/B/C-SA/1/F0)

Despite the fact that under all legal frameworks and protocols victims of torture are recognized as especially vulnerable, the detection of vulnerability often responds to a criterion of urgency, although according to the professionals working with victims of torture on a daily basis, most cases of torture are frequently invisible: "They will not take you in unless you are visibly about to lose an arm... no, really, unless something very visible is about to happen to you when you come through the door" (AH/B/TS/1/FN).

This implies that mental health issues are rarely considered, unless claimants have documentary proof, or unless the frontline worker is able to identify them in the frame of the one-hour first social screening interview, often mediated by an interpreter. This absence of detection often leaves victims of torture without access to emergency shelter accommodation, leading to further situations of vulnerability such as the appearance and worsening of physical and mental health disorders or drug addictions:

As they go through the first screening they might look perfectly fine, they have just arrived, so the social worker there does not identify any issues and is not obliged to give them a full medical check. Then they get here, and they have a very visible mental health issue or a substance abuse problem or tuberculosis [...] because they have been living in the streets for six months before being assigned here. (Social Worker, NGO in Asylum Reception) (CE/B/TS/1/F1)

Such deficiency in the identification of victims of torture is a common concern among organisations that provide legal, social and health care for victims. Irídia - a main organisation defending human, civil and political rights in Spain - has denounced the lack of training within public institutions, such as the Forensic Medicine Institute, in assessing these cases (Irídia 2017). On these lines, the ethnography shows how the scarce preparation of professionals can lead to 
the credibility of the victim being questioned and their symptomatology wrongly assessed, leading to situations of exclusion from access to basic services:

The worst is that the clinical presentation or the psychosomatic reactions of victims of torture or of traumatic processes are so unknown that many people are taken for something they are not. That is, if a person has suffered torture and goes to a public service and is not treated as they would have expected... they will most likely have a reaction of distrust, lack of control or lack of empathy or whatever, which will make other people regard them as... as something they are not. (Psychologist, specialised NGO) (EX/B/P/1/FN)

Credibility is a recurrent barrier that asylum-seeking victims of torture face and is not only questioned due to insufficient training but also because of professionals' expectations. In the context of asylum screening, cases of rape and torture are where "[t]he fragility of a concept of credibility is most evident [...], where officers may deny alleged events could have taken place, usually because of pre-attached labels" (Jubany 2017, 195).

At the same time, the asylum reception programme demands a high performance by asylum seekers. In this regard it is interesting how all social workers refer to how the bureaucratic maze and nearimpossible requirements to obtain social benefits put a lot of pressure on asylum claimants, which is taxing for those who are experiencing effects on their health due to having suffered torture:

Adding stress to a person who is already stressed or who comes with a traumatic process, who sees that all they are doing administratively is not working, they lead people to self-exclude which is frustrating for the professionals who support them, so the user is, like, abandoned, because there's nothing you can do, right? They have to leave. They have to leave the centre and there's no other centre to go to. There are shelters, of course, but well, anyway, everything is precarious and temporary. (Psychologist, NGO) $(\mathrm{EX} / \mathrm{B} / \mathrm{P} / 1 / \mathrm{FN})$

Although the asylum reception programme considers extensions for vulnerable cases, professionals consider that this is clearly not enough for people who are experiencing the symptomatology of torture or are in a recovery process from having suffered severe trauma. In addition, there is a scarcity of resources independent to the asylum programme for referrals for those who have exhausted asylum reception without achieving the expected degree of autonomy. Furthermore, and as previously mentioned, many of these cases are not properly identified or recognised, and extensions are often diffi- 
cult to obtain due to different and changing criteria of assessment of vulnerability, which may exclude victims of torture.

This lack of resources and attention to the specific needs of asylum seekers in situations of vulnerability leads to a system that mainly supports those who are able to pull through the system by their own means, whilst it further burdens those who struggle to get through.

While this is especially obvious in the case of asylum seekers and refugees, it can also be applied to other migrant victims of torture or to those who have suffered severe trauma of other kinds, such as rape, but who have not entered the system of international protection. In fact, asylum seekers are perceived as being well supported by a reception programme that anticipates the specific vulnerability of victims of torture and plans the referral of these victims to specialised services although, as has been shown, this is not working adequately. However, the high number of negative final decisions in asylum cases ${ }^{11}$ means that most of these asylum seekers will later become undocumented, which places a particular toll on their mental health: "[the rejection] is terrible, for everything it implies, losing your job, having your bank account blocked, everything. It's... and... the lack of recognition, not being a person with the right to be protected. This is something that, emotionally, is really hard" (FC/B/TS/1/FN).

The general malfunctioning of the mental health services in Spain adds to the insufficiency of referral mechanisms for asylum seekers beyond the reception programme and other migrants who have access to generalist health services: "[t]he mental health public network, in general, is terrible. So referring this type of profiles [migrants] is complicated, because there are no resources" (BCN/SA/1/FN).

This deficiency of resources, together with the inadequate identification of victims of torture and a meritocratic social services approach in asylum seekers' reception can lead to situations of chronic exclusion:

I had this kid, he'd been here since 2014 [...] he had his papers and all, but he was still homeless so [his social worker said to me]: 'This kid's been here since 2014, he should have done his bit'. And I was like... precisely because he's been here since 2014 and he continues to be in the street, he has a vulnerability. I can clearly see it, why can't you? I needed their authorisation to act on it. But no, [for them it was] just the opposite; if you've been here since 2014, [...] you've had your chance, you should have made the best of it. (Social Worker, NGO) (AH/B/TS/1/FN) 
Such policies of inaction, that subject asylum-seeking victims of torture to abandonment and neglect despite the mandate of protection towards them, are part of a wider logic and narrative of governmentality of migration that are also at the core of practices of overt violence in the enforcement of policies of border control.

\section{Torture and the Enforcement of Migration Policies}

As previously discussed, migrant victims of torture are not only made vulnerable by the system due to a lack of protection and neglect but also by being made into 'torturable subjects'. In a context of increased criminalisation of migration, violent acts by public officials in the enforcement of migratory policies are normalised and understood as a legitimate use of force in the protection of the state's sovereignty (Bigo 2014). Even those migrants who have been legally recognised as asylum seekers, and therefore are subject to the specific protection that this category entails, are vulnerable to the state's effort to enforce border securitisation.

This is particularly obvious in the practices of border security, where detention and deportation are constantly present as a form of violence and degrading treatment. In recent years, migrants have consistently been among the main complainants in allegations of torture in Spain, as the reports by the Coordinadora para la Prevención de la Tortura ${ }^{12}$ show. The number of torture allegations by migrants or their representatives has increased almost every year, often exceeding half the complaints registered. ${ }^{13}$ These complaints relate, in the most part, to violence in border crossing, including the practice of pushbacks, and situations of confinement, especially in detention centres.

Spain's Southern border has become one of the main 'gates of Europe' and a central site in the development of border control technologies and strategies in the EU (Andersson 2014). Every other day, subSaharan migrants attempt crossing the Spanish-Moroccan border at the enclaves of Ceuta and Melilla by jumping over the threatening fences that separate the two countries. These fences are protected

12 The Coordinadora para la prevención de la tortura is the most prominent civil society organisation for the prevention of torture in Spain and publishes a yearly report aggregating data from all known cases that fall within the parameters of torture as defined by article 1 of the United Nations Convention against Torture.

13 In 2014, complaints made by migrants were 37\% of the total, in 2015 a 50\%, in 2016 a $54 \%$. In 2017 , the number of complaints filed by migrants represented a $28 \%$ due to the large number of complaints for police violence in relation to the referendum in Catalonia (Coordinadora de la Prevención de la Tortura: http://www.prevenciontortura. org/wp-content/uploads/2018/06/INFORME-CPDT-2017.pdf, 2019-11-07). 
with technological gadgets, barbed wire and guarded by armed police. Occasionally, images of everyday violence are leaked, showing the police trying to pull migrants from the fence with blows from batons and carrying them back to Morocco across the doors that were installed to facilitate these pushbacks. This practice is against the right of claiming asylum, as has been condemned by various international bodies, including the European Court of Human Rights (N.D. y N.T. vs España, 03-10-201744). Despite this, these practices not only continue to be in place, but further deterrence mechanisms aiming at producing physical harm, such as concertina wire which have repeatedly caused serious injuries to refugees and migrants trying to jump the fences, have been introduced.

Similarly, pushbacks are common across the Mediterranean Sea. Migrants detected on their way towards Gibraltar or Canary Islands have been returned due to the collaboration between Spanish and the Moroccan and Mauritanian police forces (Andersson 2014). However, as in the Tarajal case, ${ }^{15}$ where fifteen people died after the police used antiriot equipment to stop them from swimming around the Spanish-Moroccan border (Sánchez 2018), courts often dismiss such cases arguing lack of evidence or that the police were "acting under their obligation as border custodians, which compels them to prevent anyone from entering illegally in Spain". ${ }^{16}$ Yet the majority of torture complaints are dismissed and, when a trial is held, they often end in acquittal, or, if condemned, perpetrators have been repeatedly pardoned and later even promoted and decorated (Bergalli, Rivera Beiras 2006).

This is despite the fact that several United Nations human rights mechanisms, such as the Human Rights Committee and the Committee against Torture, have expressed their rejection of the application of such Amnesty Law that pardons torturers in Spain and further NGOs, such as Rights International Spain, have protested against these decisions on several occasions, urging Spain to ensure the nonapplicability of statutory limitations to torture.

14 https://www.abogacia.es/wp-content/uploads/2017/10/TEDH-STC.pdf (2019-11-07).

15 In February 2014, about 200 persons tried to cross the Spanish border at Ceuta by swimming around the breakwater. Spanish police tried to deter them from swimming to shore by shooting rubber bullets and gas grenades. Fifteen were killed in the incident. The case was dismissed twice by the local Court in Ceuta, the judge arguing that the police were acting under their obligation as border custodians. In neither occasion could any of the migrants who survived the incident testify, as they were sent back to Morocco through pushbacks or deported later. The case was finally reopened at the third attempt and it is now still pending resolution (Sánchez 2018).

16 Europa Press, "Archivan la causa de las 15 muertes en El Tarajal por falta de pruebas sobre un uso 'inadecuado' del material antidisturbios", El Mundo, 15 de octubre 2015. URL https://www.elmundo.es/sociedad/2015/10/15/561f9524e2704e6e518b 45e2.html (2019-07-23). 
A further interpretation of modern forms of torture refers to a definition of torture that relates to the deprivation of freedom without trial and the conditions migrant suffer in detention centres (CIE, Centros de Internamiento de Extranjeros). These centres were first established in 1985 across the Spanish territory, and are the only case under Spanish law that allows for the deprivation of freedom of movement due to an administrative sanction (Solanes Corella 2016, 38). Yet, only a small part of those detained in CIEs end up being deported. Most people are set free after the maximum time of detention is reached, leaving them in a legal limbo that prevents them from being deported as well as from regularising (Servicio Jesuita a Migrantes 2016). These centres are characterised by a lack of transparency regarding their internal conditions and proceedings. The number of inmate is not made public, nor are any other aspects of their detention, despite there being an obligation to do so under Spanish law (Escamilla 2016, 13). The UN Human Rights Committee raised concerns about the prevalence of these situations in Spain in a 2015 report denouncing "the recurrent use of deprivation of liberty to migrants in an irregular situation" and the repeated complaints of mistreatment filed by inmates against the police forces. ${ }^{17}$ The scarce control over these institutions and the fact that recently arrived migrants are sent frequently to CIEs often implies contravening their right to claim asylum.

These practices expose the recurrence of torture in modern liberal societies in its contemporary forms and its position in mechanisms of control of the socially excluded, among which policies and practices of migration control play a central role. The absence of recognition of such violent events as breaches of the prohibition of torture contributes to their normalisation and legitimates the exercise of force in the enforcement of migratory policies, targeting undocumented migrants and contravening the protection that the right to claim asylum entails. Western democracies claim to offer international protection against torture to asylum seekers is contradicted by their own practices of migration management. On the ground, this protection is only recognised if torture is perpetrated by a 'folk devil' or a 'threatening other' despite the many gaps in these protection mechanisms. In the meantime, as the empirical evidence shows, torture practiced in a so-called modern democratic state like Spain is still disregarded, normalised and legitimated by the lack of recognition and protection against it.

17 Comité para la Prevención de la Tortura del Consejo de Europa (2015). Informe al Gobierno español sobre la visita a España del Comité europeo para la prevención de la tortura, del 14 al 18 de julio de 2014. [CPT/Inf (2015) 19]. URL http://www.cpt.coe. int/documents/esp/2015-19-inf-eng.pdf (2019-11-07). 


\section{Conclusion: The Double Vulnerability}

Migration control policies have come to merge high levels of active involvement of the state in politics of inaction (Davies, Isakjee, Dhesi 2017) with violent consequences for migrants. This combination of migration policies of intensive state presence in areas like border control, with the politics of inaction in other areas like refugee reception, have exposed asylum-seeking victims of torture to a double vulnerability. On the one hand migrants are exposed to insufficient legal and social safeguards as victims of torture and to the deficiency of investment and resources in all mechanisms for the protection for asylum-seeking victims of torture, despite all legal provisions to this effect. On the other, they are subject to the potential violence of the enforcement of migration policies, including that of neglect and inaction.

The enforcement of migration control policies exposes migrants to specific forms of state violence that contravene the prohibition of torture. Borders, detention and deportation have been identified as the main sites where serious violations of human rights occur in relation to migration and where modern forms of torture are exposed. Within this, the case of asylum seekers is especially paradigmatic due to the specific mandate of protection towards them. Yet, because of the weak juridical status of asylum seekers, which leaves them at the fringe of the political community, states are not fully committed, nor concerned, in providing the protection that national and international regulations anticipate. This leads to a political abandonment that has violent consequences for asylum seekers, especially for those victims of torture or inhuman and cruel degrading treatments. Asylum seekers who have been victims of torture, either in origin, transit or arrival, not only experience a lack of legal protection but are also made vulnerable to protracted situations of exclusion by the enforcement of migratory policies, even by those policies designed for their protection and the promotion of their inclusion (Freedman 2019), such as asylum reception programmes.

There is nothing exceptional about this abandonment of victims of torture but rather the denial of its existence. The Spanish State's approach to torture has been one of impunity that has led to the neglect of victims, to which migrant victims are made especially vulnerable. Strong legal protection is not enough in a context of impunity founded on narratives that construe migrants as criminals and legitimise the violent enforcement of migration policies. As the empirical evidence put forward in this chapter has shown, we must look at what happens on the ground to expose modern forms of torture and their prevalence, before we can refer to the states of Europe today as modern democracies. 


\section{Bibliography}

Acosta Bono, Gonzalo; del Río Sánchez, Angel; Valcuende del Río, Jose Maria (2008). La recuperación de la memoria histórica: una perspectiva transversal desde las ciencias sociales. Sevilla: Fundación Centro de Estudios Andaluces.

Allsopp, Jennifer; Sigona, Nando; Phillimore, Jenny (2014). "Poverty among Refugees and Asylum Seekers in the UK: An Evidence and Policy Review". IRiS Working Paper Series, 1. URL https://www.birmingham.ac.uk/ Documents/college-social-sciences/social-policy/iris/2014/ working-paper-series/IRIS-WP-1-2014.pdf (2019-10-28).

Alpes, Maybritt Jill; Blondel, Charlotte; Preiss, Nausicaa; Sayos Monras, Meritxell (2015). "Débouté du droit d'asile: la triple peine". Plein Droit, 107(4), 23-5. DOI https://doi.org/10.3917/pld.107.0023.

Amnesty International (2015). Informe para el comité contra la tortura de las Naciones Unidas. London: Amnesty International Publication. URL https:// www. amnesty.org/es/documents/eur41/1350/2015/es/ (2019-11-07).

Andersson, Ruben (2014). Illegality, Inc.: Clandestine Migration and the Business of Bordering Europe. Berkeley (CA): University of California Press.

Arendt, Hannah (1964). Eichmann in Jerusalem: A Report on the Banality of Evil. New York: Viking Press.

Arendt, Hannah (1973). The Origins of Totalitarianism. Boston: Houghton Mifflin Harcourt.

Bauman, Zigmunt (2008). Modernity and the Holocaust. Cambridge: Polity Press.

Bergalli, Roberto; Rivera Beiras, Iñaki (2006). Torturas y Abuso de Poder. Rubí: Anthropos.

Bigo, Didier (2014). "The (in)securitization Practices of the Three Universes of EU Border Control: Military/Navy-Border Guards/Police-Database Analysts". Security Dialogue, 45(3), 209-25. DOI https://doi. org/10.1177/0967010614530459.

Bloch, Alice; Schuster, Lisa (2005). "At the Extremes of Exclusion: Deportation, Detention and Dispersal”. Ethnic and Racial Studies, 28(3), 491-512. DOI https://doi.org/10.1080/0141987042000337858.

Butler, Judith (2009). "Sexual Politics, Torture, and Secular Time". Intimate Citizenships: Gender, Sexualities, Politics, 59(1), 17-39. DOI https://doi. org/10.1111/j.1468-4446.2007.00176.x.

Cohen, Stanley (1972). Folk Devils and Moral Panics. Oxfordshire: Routledge.

Daniel, Valentine; Knudsen, John (1995). Mistrusting Refugees. Berkeley: University California Press.

Davies, Thom; Isakjee, Arshaad; Dhesi, Surindar (2017). "Violent Inaction: The Necropolitical Experience of Refugees in Europe". Antipode, 49(5), 1263-84. DOI https://doi.org/10.1111/anti.12325.

De Genova, Nicholas (2016). "Detention, Deportation, and Waiting: Toward a Theory of Migrant Detainability". Global Detention Project Working Paper, 18. URL https://www.globaldetentionproject.org/detentiondeportation-waiting-toward-theory-migrant-detainabilitygdp-working-paper-no-18 (2019-10-28).

Escamilla, Margarita Martínez (2016). "Centros de Internamiento para Extranjeros. Estado de la cuestión y perspectivas de futuro". Revista Electrónica de Ciencia Penal y Criminología, 18, art. 23. URL http://criminet.ugr. es/recpc/18/recpc18-23.pdf (2019-10-28). 
Olga Jubany, Martina Pasqualetto, Alèxia Rué Vulnerable to the System: Migration and Torture in Spain

Freedman, Jane (2019). “The Uses and Abuses of 'vulnerability' in EU Asylum and Refugee Protection: Protecting Women or Reducing Autonomy?". Papeles del CEIC, 1(3), 1-15.

Galtung, Johan (1969). "Violence, Peace and Peace Research". Journal of Peace Research, 6(3), 167-91. URL http://www.jstor.org/stable/422690 (2019-10-28).

Garcés-Mascareñas, Blanca (2019). "SER O NO SER: deficiencias del sistema estatal de acogida". Notes internacionals CIBOD, 214. URL https://bit. ly/36fFxie (2019-10-28).

Haoussou, Kolbassia (2017). "Perspective - The Long Journey to Rehabilitation for Torture Survivors". Torture: Journal on Rehabilitation of Torture Victims and Prevention of Torture, 27(1). DOI https://doi.org/10.7146/torture.v27i1.26537.

Human Rights Watch (2005). Setting an Example? Counter-Terrorism Measures in Spain. URL https://www.hrw.org/report/2005/01/26/setting-example/counter-terrorism-measures-spain (2019-10-28).

Irídia (2017). SAIDAVI 2017. Informe Sobre Violència Institucional. Barcelona. URL http://iridia.cat/wp-content/uploads/Informe-SAIDAVI-2017.pdf (2019-10-28).

Jubany, Olga (2017). Screening Asylum in a Culture of Disbelief: Truths, Denials and Skeptical Borders. Cham: Springer.

Kalt, Anne; Hossain, Mazeda; Kiss, Ligia; Zimmerman, Cathy (2013). "Asylum Seekers, Violence and Health: A Systematic Review of Research in High-income Host Countries". American Journal of Public Health, 103(3), 30-42. DOI https://doi.org/10.2105/AJPH.2012.301136.

Mazzetti, Marco (2008). "Trauma and Migration: A Transactional Analytic Approach toward Refugees and TortureVictims". Transactional Analytic Journal, 38(4), 285-302. DOI https://doi.org/10.1177/036215370803800404.

McColl, Helen; Bhui, Kamaldeep; Jones, Edgar (2012). "The Role of Doctors in Investigation, Prevention and Treatment of Torture". Journal of the Royal Society of Medicine, Supplement, 105(11), 464-71. DOI https://dx.doi. org/10.1258\%2Fjrsm.2012.120100.

Mendiola, Ignacio (2014). Habitar lo inhabitable. La práctica político-punitiva de la tortura. Barcelona: Edicions Bellaterra.

Menjívar, Cecilia; Abrego, Leisy (2012). "Legal Violence: Immigration Law and the Lives of Central American Immigrants". American Journal of Sociology, 117(15), 1380-421. DOI https://doi.org/10.1086/663575.

Morales, Katelin (2016). "Australia's Guantanamo Bay: How Australian Migration Laws Violate the United Nations Convention Against Torture". American University International Law Review, 31(2), 327-50. URL http://digitalcommons.wcl.american.edu/auilr/vol31/iss2/5 (2019-10-28).

Mountz, Alison; Loyd, Jenna (2014). "Constructing the Mediterranean Region: Obscuring Violence in the Bordering of Europe's Migration 'Crises'”. ACME: An International E-Journal for Critical Geographies, 13(2), 173-95. URL https://acme-journal.org/index.php/acme/article/view/1003 (2019-10-28).

Oomen, Janus (2007). "Torture Narratives and the Burden of Giving Evidence in the Dutch Asylum Procedure". Intervention, 5(3), 250-5. URL https:// www.interventionjournal.com/sites/default/files/oomen.pdf (2019-10-28). 
Parry, John (2010). Understanding Torture: Law, Violence, and Political Identity. Ann Arbor (MI): University of Michigan Press. DOI https://doi. org/10.3998/mpub.155927.

Rights International Spain (2017). Principales lagunas en la lucha contra la tortura en España, 26 de junio. URL https://tbinternet.ohchr.org/Treaties/CAT/Shared\%20Documents/ESP/INT_CAT_ICS_ESP_27995_S. pdf (2019-10-28).

Sánchez, Gabriela (2018). "La Justicia ordena reabrir el caso de las muertes de Ceuta". El Diario, 31 de agosto. URL https://www.eldiario. es/desalambre/Justicia-ordena-reapertura-muertes-Ceuta_O_809469180.html (2019-10-28)

Sanggaran, John-Paul; Zion, Deborah (2016). "Is Australia Engaged in Torturing Asylum Seekers? A Cautionary Tale for Europe". Journal of Medical Ethics, 42(7), 420-3. DOI https://doi.org/10.1136/medethics-2015-103326.

Schubert, Carla; Punamäki, Raija-Leena (2011). "Mental Health Among Torture Survivors: Cultural Background, Refugee Status and Gender". Nordic Journal of Psychiatry, 65(3), 175-82. DOI https://doi.org/10.3109/080 39488.2010 .514943$.

Schuster, Liza (2005). "A Sledgehammer to Crack a Nut: Deportation, Detention and Dispersal in Europe". Social Policy and Administration, 39(6), 606-21. DOI https://doi.org/10.1111/j.1467-9515.2005.00459.x.

Servicio Jesuita a Migrantes (2016). Vulnerables vulnerabilizados. Informe anual 2015. URL https://sjme.org/gallery/vulnerables-vulnerabilizados-informe-cie-2015 (2019-10-28).

Solanes Corella, Ángeles (2016). "Un análisis crítico de los Centros de Internamiento de Extranjeros en España”. Revista Telemática de Filosofía Del Derecho, 19, 37-76. URL http://hdl.handle.net/10550/58558 (2019-10-28).

Steel, Zachary; Chey, Tien; Silove, Derrick; Mamane, Claire; Bryant, Richard; Van Ommeren, Mark (2009). "Association of Torture and Other Potentially Traumatic Events With Mental Health Outcomes Among Populations Exposed to Mass Conflict and Displacement: A Systematic Review and Meta-Analysis". JAMA The Journal of the American Medical Association, 302(5), 537-49. DOI https://doi.org/10.1001/jama.2009.1132.

Tulloch, John (2008). "Normalising the Unthinkable". Ethical Space: The International Journal of Communication Ethics, 2(4), 25-32. URL http://eprints. lincoln.ac.uk/1135/ (2019-10-28).

Vannotti, Marco; Bodenmann, Patrick (2003). "Migration et Violence". Revue Médicale Suisse, 1, 23315. URL https://www.revmed.ch/RMS/2003/RMS2455/23315 (2019-10-28).

Wacquant, Loic (2009). Punishing the Poor: The Neoliberal Government of Social Insecurity. Durham: Duke University Press.

Zimbardo, Philip George (2002) Violence Workers: Police Torturers and Murderers Reconstruct Brazilian Atrocities. Berkeley (CA): University of California Press. 



\title{
Violences systémiques dans les centres fermés L'expérience des femmes sans-papiers en Belgique
}

\section{Nouria Ouali}

Université Libre de Bruxelles, Belgique

\begin{abstract}
The essay examines one type of state-sponsored violence against undocumented migrants (especially women and children), which have been implemented since the late 1980s to control migration flows in Belgium. These policies are based on two pillars: the detention of undocumented migrants and their forced eviction. The examination of these practices reveals the systemic violence of this policy and the many violations of the fundamental rights. The author raises the question of the status of the regime of violence inflicted on undocumented migrants and their children in administrative detention centers: is it a case of abuse or torture? Regarding the detrimental effects on the mental and physical health of migrants (and their children), as powerful as those resulting from torture, the author suggests that, in some case, abuses designated by the courts could be called torture.
\end{abstract}

Keywords Undocumented migrant women. Administrative detention centers. Abuse. Torture. Belgium.

Sommaire 1 Introduction. - 2 La création des centres fermés. - 3 La politique d'enfermement. - 3.1 La violence organisationnelle. - 3.2 La violence des agents. - 3.3 La violence sur les enfants. - 4 La pratique des expulsions forcées. - 4.1 Les expulsions collectives. - 4.2 Les expulsions individuelles. - 5 Du juridique au politique. 


\section{Introduction}

Dans la représentation sociale dominante en Europe, les pratiques de tortures et de mauvais traitements humains sont généralement associées aux États non occidentaux peu respectueux des libertés et des droits fondamentaux. Ces pratiques résulteraient de comportements découlant de certaines traditions culturelles ; 'l'Occident' se situant en haut de l'échelle du respect des droits humains. Pour Freedman et Valluy, cette approche culturaliste produit non seulement une polarisation entre les cultures ${ }^{1}$ qui persécutent et celles qui protègent, mais également une "normalisation » des persécutions pratiquées dans les pays européens. En outre, elle masque les effets systémiques des rapports de pouvoir, notamment patriarcal, qui génèrent précisément ces oppressions dans divers contextes socioculturels (Freedman, Valluy 2007).

Cette représentation sociale s'accompagne souvent d'une opposition entre les pays "producteurs de réfugiés » et les pays qui les accueillent et d'une polarisation négative et positive de leur image clairement contestée ${ }^{2}$ depuis la décennie 1990 (Fassin et al. 1997). En effet, certaines ONG en Europe (Ligue des Droits de l'Homme et Amnesty International notamment) dénoncent les traitements violents exercés sur les migrants et les migrantes. Pour la première fois, en 2009, Amnesty International-Belgique se préoccupe de l'ampleur des violations des droits fondamentaux des migrants en Belgique et en Europe :

On a constaté la persistance de violations des droits humains liées à l'arrestation, à la détention et à l'expulsion d'étrangers, même lorsque ces derniers demandaient à bénéficier d'une protection internationale. Dans certains pays, on refusait à certaines personnes la possibilité d'effectuer une demande d'asile. Dans d'autres, le niveau de protection accordé aux demandeurs d'asile irakiens était réduit, et certains ont même été expulsés. (Amnesty International 2009, 61)

1 C'est également ce que souligne Makaremi, en s'inspirant de Talal Asad, qui observe dans cette opposition une justification différente fondée sur la culture : «[1]a violence exercée par les 'nations civilisées', souvent étiquetée comme opération de sécurité, intervention humanitaire ou dommage collatéral, ne semble relever d'aucune culture ou rapport à la violence particuliers, tandis que le rapport à la violence des 'nations non-civilisées' requiert des grilles de compréhension spécifiques mobilisant le concept de culture » (Makaremi 2016, 15).

2 «Cette image d'Epinal est fausse : les pays riches sont aujourd'hui au plus bas du classement de l'hospitalité internationale (en nombre de réfugiés accueillis rapporté au nombre d'habitants et plus encore au revenu par habitant)» (Freedman, Valluy 2007, 20). Ce constat établit est toujours valable en 2019. 
Ces pratiques s'inscrivent dans les nouvelles politiques migratoires et d'asile restrictives élaborées dans les années 1990 lorsque les pays membres de l'Union européenne décident de se concerter pour renforcer les contrôles aux frontières et se "protéger » de populations qualifiées d'«indésirables » à travers deux principaux instruments : les accords de Schengen (1985, entrés en vigueur en 1995) et la Convention de Dublin (1990).

Ce chapitre propose d'examiner une des formes de la violence d'État exercée sur les migrants sans-papiers, en particulier sur les femmes et les enfants, depuis l'instauration de ces nouvelles politiques qui ont réduit l'accès des migrants au territoire, institué les centres fermés pour les sans-papiers et mobilisé une rhétorique criminalisante qui permet de justifier les mauvais traitements. Ces politiques de contrôle reposent sur deux piliers : l'enfermement des migrants sans-papiers dans des centres fermés définis comme des lieux « de privation de liberté pour les personnes en séjour irrégulier en attente de leur expulsion » (CIRÉ 2019, 10) et leur expulsion forcée du pays. ${ }^{3}$

La question que l'article soulève porte sur la qualification du régime de violence infligé aux sans-papiers et à leurs enfants dans les centres fermés : relève-t-il du mauvais traitement ou s'apparente-t-il, dans certaines de ses formes, à de la torture ? Sur le plan juridique, le droit international établit une distinction de degré de gravité entre les deux sur base du niveau de douleur/souffrance infligée et de l'intentionnalité de l'acte : la torture vise à obtenir des aveux, punir, faire pression ou intimider alors que les mauvais traitements (cruels, inhumains, humiliants, dégradants qui font outrage à la dignité de la personne) n'ont aucun but spécifique et présente un degré élevé de souffrance/douleur ou d'humiliation/dégradation. Cette qualification juridique de la violence a une visée opératoire : rétablir la justice par la réparation de l'acte subit au regard des droits fondamentaux en hiérarchisant les catégories de victimes (légitimes et illégitimes) (Calzolaio, Colombo, Makaemi 2016). En sciences sociales, la violence est un objet complexe tant sur le plan épistémologique que méthodologique : c'est une construction sociale qui, pour certains, renvoie à un processus descriptif d'un champ d'expériences et des circonstances structurantes qui exige un examen éthique et politique de « la position de celui qui qualifie l'acte de violence » (Naepels 2006, 489). Pour d'autres, c'est un concept normatif et évaluatif qui produit une « disqualification » ou une « dénonciation » (Lavergne, Perdoncin, 2010).

S’agissant de la violence d'État exercée dans les centres fermés, je propose de l'examiner, d'une part, la norme d'intentionnalité des

3 L'article ne traite pas des violences subies par les femmes au cours de leur périple migratoire. A ce propos, voyez l'ouvrage de Laacher (2007) et le nrr. 2-3 de la Revue européenne des migrations internationale (2017). 
politiques migratoires qui, dans l'approche juridique, permet précisément d'établir une frontière entre mauvais traitement et torture et, d'autre part, les formes violentes d'enfermement et d'expulsion et leurs impacts sur les enfants et les migrantes sans-papiers. Cette double approche vise identifier l'intentionnalité des acteurs qui définissent les politiques migratoires sur base des desseins explicites ${ }^{4}$ pour décourager les migrants 'indésirables' à s'installer en Belgique et les pratiques répétitives de contrôle, de rétention et de refoulement violentes et technicisées dans les centres fermés. Elle vise également à révéler les souffrances et les traumatismes physiques et psychologiques ${ }^{5}$ connus par l'État et ses agents à partir de témoignages de migrants et d'experts qui, dans bien des cas, s'apparentent à certains traumatismes résultant de la torture.

La première section brosse brièvement le contexte politique et l'architecture institutionnelle des centres fermés élaborée dès la fin des années 1980. La deuxième section décrit la violence de l'enfermement et ses conséquences sur les femmes et les enfants. La troisième section aborde les pratiques d'expulsion sous leur versant collectif et individuel qui ont, notamment, conduit au meurtre de Sémira Adamu le 22 septembre 1998. Ces pratiques d'enfermement et d'expulsion sont illustrées à partir de deux cas emblématiques : celui d'une fillette de cinq ans et celui d'une femme de vingt-deux ans, toutes deux arrêtées à l'aéroport de Bruxelles alors qu'elles transitaient l'une vers le Canada et l'autre vers l’Angleterre.

$\mathrm{Au}$ regard des stratégies politiques, de la légitimation des pratiques et des conséquences tragiques sur les migrants, l'article pose la question de la re-qualification de ce que les juges de la $\mathrm{CEDH}^{6}$ dé-

4 Un des gendarmes inculpés du meurtre de Sémira Adamu déclarait que 3.500 expulsions étaient pratiquées chaque année et que le Ministère de l'intérieur exerçait des pressions pour atteindre l'objectif : " [i]l y a un tableau de pourcentage de réussite des rapatriements qui était affiché chaque jour. Si le chiffre était trop bas, le colonel X nous réprimandait. Via les membres du cabinet des affaires intérieures nous avons senti la pression pour faire partir Sémira soutenue par le Collectif contre les expulsions. Si cette expulsion ne réussissait pas, cela pouvait laisser croire que le Collectif était capable de saper la politique des étrangers » (https://ccle.collectifs. net/Resume-de-la-deuxieme-journee-d.html, 2019-11-11). En 1996, les autorités belges avaient fixé un quota de 15.000 expulsions de candidats réfugiés politiques par an (Carbocci et al. 1998, 9).

5 Conséquences désormais reconnues dans les rapports 2009 et 2018 d'Amnesty international qui concluait pour l'Europe : « En de multiples occasions, les États n'ont pas assumé les responsabilités qui étaient les leurs en termes de protection des personnes réfugiées et migrantes. Les femmes et les filles ont cette année encore été victimes de violations des droits humains et d'abus institutionnalisés, y compris d'actes de torture et d'autres mauvais traitements. Les violences liées au genre étaient toujours très répandues » (Amnesty Internation 2018a, 51).

6 Les huit jugements de la Cour Européenne des Droits de l'Homme (CEDH) à l'encontre de l'État belge pour « violations extrêmement graves » des droits fondamentaux 
signent le plus souvent « de mauvais traitements » en formes spécifiques de torture générées dans les centres fermés afin de porter davantage le débat sur le plan politique et sur ces formes de gouvernementalité que sur la dimension morale ou réparatrice.

L'article a mobilisé plusieurs sources : une littérature scientifique peu développée sur les centres fermés, les documents des ONG de défense des droits fondamentaux (telles que la Coordination et Initiatives pour Réfugiés et Étrangers, CIRÉ ; la Coordination contre les Rafles, les Expulsions et pour la Régularisation, CRER) ou institutionnels (Délégation générale aux droits de l'enfant ; Médiateur fédéral), mais également la presse écrite, l'échange de courriers entre des sans-papiers et des citoyens, et des récits d'expériences de sans-papiers.

\section{La création des centres fermés}

Le tournant des politiques migratoires restrictives et plus répressives s'amorce en Belgique à la fin des années 1980 lors des réformes successives de la première loi (15 décembre 1980) qui régule l'accès au territoire, le séjour, l'établissement et l'éloignement des étrangers. Ces réformes établissent les nouveaux contours de la politique migratoire motivée par la «volonté politique de frein à l'immigration, de renforcement des contrôles, de limitation des droits des migrants et de durcissement de leurs conditions de séjour et d'éloignement » (Perrouty 2003, 108). La loi du 15 juillet 1996 qui intègre les accords internationaux (Schengen et Dublin) conclus à l'échelle européenne supprime l'aide sociale aux sans-papiers, oblige les demandeurs d'asile à résider dans des « centres ouverts » pour recevoir cette aide sociale, et prolonge jusqu'à huit mois la détention en centre fermé des demandeurs d'asile déboutés et des sans-papiers avant leur expulsion. ${ }^{7}$

Dès le début des années 1990, la détention et l'expulsion des sans-papiers devient la «clé de voûte » de la politique migratoire belge et du mode de gouvernementalité (Foucault 2001²) que l’État

des migrants et manquement à ses devoirs de protection des femmes et des enfants, reposent sur la notion de «mauvais traitement » (Van Kiersbilck 2007).

7 Cette législation suscitera notamment la création, en mars 1998, de la Coordination contre les Rafles, les Expulsions et pour la Régularisation (CRER), qui joue un rôle essentiel dans la stratégie de mobilisation des passagers et des pilotes d'avion pour refuser l'expulsion des migrants qui résistent à cette expulsion (Perrouty 2003). La Coordination et Initiatives pour Réfugiés et Étrangers (CIRÉ) est un réseau d'ONG qui, depuis 1954, lutte aussi contre l'enfermement et l'expulsion des étrangers en Belgique et à l'échelle européenne.

8 Pour Foucault, il s'agit d'une forme de pouvoir exercée sur une population, fondée sur un ensemble d'institutions, de procédures, de savoirs, de stratégies et de techniques (sécurité) pour canaliser les conduites des citoyens. 
impose aux migrants sous couvert d'un impératif de sécurité. Le ministre de l'Intérieur rédige une directive qui légitime le durcissement des comportements à l'égard des migrants sans-papiers sous la forme d'un «manuel interne à l'usage des gendarmes [qui] autorise le recours à des techniques coercitives, comme le coussin, pour procéder aux expulsions difficiles »(CIRÉ et al. 2006, 59). L'usage de ce coussin vise à empêcher les personnes de crier et d'alerter les passagers de l'avion qui protestent et bloquent ainsi le décollage de l'appareil. Cette technique provoquera, la mort par asphyxie de Sémira Adamu lors de sa sixième tentative d'expulsion.

Le contrôle et la maîtrise des « flux sauvages » de migrants en séjour irrégulier (Laacher 2007) s’appuie notamment sur les centres fermés destinés à la détention administrative des migrants sans-papiers en vue de leur expulsion. Le premier centre en Belgique, dénommé centre de transit, fut créé hors de tout cadre légal en 1988 sur le site de l'aéroport militaire de Bruxelles (le Centre 127) à Melsbroek pour y retenir 60 personnes. En 1993, la loi Tobback fournira le cadre légal pour la détention et l'expulsion des sans-papiers mais elle est immédiatement critiquée puis abrogée en 2001 en raison du régime pénitentiaire qu'elle imposait aux migrants n'ayant commis aucun délit, ni fait l'objet de la moindre condamnation. Malgré le changement de la loi, les ONG constatent, en 2019, que le régime carcéral est toujours en vigueur dans ces centres :

Le fonctionnement, l'organisation voire l'architecture des centres fermés sont clairement carcéraux (deux d'entre eux sont d'ailleurs d'anciennes prisons). Les conditions de détention sont fixées par un Arrêté Royal, mais chaque centre a son propre règlement d'ordre intérieur. Ils ont en commun un fonctionnement très sécuritaire, un régime de vie en groupe, des possibilités de sanctions allant jusqu'à la mise en cachot, un droit de visite strictement contrôlé (voire inexistant aux INAD). (CIRÉ 2019, 28)

Entre 1994 et 1999, la capacité des cinq nouveaux centres créés atteint près de 650 places. Ils sont désignés "Centre pour illégaux » (1994 Merksplas : 165 places ; 1995 Bruges : 112 places dont 40 réservées aux femmes ; 1999 Vottem : 160 places $^{9}$ ), « Centre de rapatriement» (1994 Centre 127bis de Steenokerzeel : 120 places), «Centre pour inadmissibles » à l'aéroport de Bruxelles (Centre INAD : 30 places) ou encore « Centre de transit » (2012 Centre Caricole et Centre 127bis regroupés : 90 places). Ces centres sont gérés par l'Office des Étrangers qui est l'administration sous la tutelle du

9 Ce centre possède une aile spécialisée destinée aux détenus 'difficiles' qui peuvent faire l'objet d'un isolement de $22 \mathrm{~h}$ sur $24 \mathrm{~h}$. 
Secrétariat d'État à l'Asile et à la Migration.

En mars 2016, les attentats qui ont touché Bruxelles contribuent à renforcer et à légitimer la politique d'enfermement des sans-papiers en l'incluant dans la lutte contre la criminalité et le terrorisme. En 2017, le Premier ministre du gouvernement fédéral décide alors de doubler la capacité de détention en planifiant la construction de trois nouveaux centres fermés : en 2019, le Centre de Holsbeek (50 places) réservé aux femmes; en 2020, le Centre de Zandvliet (144 places) et en 2021, le Centre de Jumet (200 places). L'ensemble du dispositif portera à terme à 1.066 le nombre de places sur les huit sites existant ou programmés. ${ }^{10}$ Enfin, en 2018, malgré les multiples condamnations de la Belgique par la Cour Européenne des Droits de l'Homme $(\mathrm{CEDH})$ pour l'enfermement des enfants, le ministre de l'Asile et de la Migration parachève le dispositif en instituant les « Unités de détention pour les familles » (Centre 127bis : 34 places) afin d'y enfermer les enfants et leurs parents; ce qui souleva une vague de protestation des ONG et des campagnes de sensibilisation. ${ }^{11}$

\section{La politique d'enfermement}

Je ne savais pas qu'en Belgique, les personnes n'ayant commis aucun délit pouvaient se retrouver enfermées [...] Il faut être dans ma peau pour ressentir ce que je ressens. Je vis le cauchemar, le calvaire et la pire époque de ma vie [...] ma liberté, c'est tout ce que j’ai de plus cher. (Ntumba 1999, lettre du 12 novembre 1999)

Selon les rapports des ONG et les témoignages des visiteurs ${ }^{12}$ et des migrantes elles-mêmes, la détention des sans-papiers est marquée par de multiples formes de violence tant dans l'organisation institutionnelle des centres que dans les rapports sociaux quotidiens aux agents chargés de la surveillance ou de leur expulsion.

10 En 2008, la dépense annuelle pour la gestion des six centres fermés et l'organisation des expulsions s'élevait à de plus de 25 millions d'euros (CIRÉ 2009b).

11 La campagne « On n'enferme pas un enfant. Point !» soutenue par trois cents trente associations et la création du collectif NotinMyName en 2018.

12 Le CRER avait organisé avec des citoyens résidant en Belgique une chaîne de solidarité consistant à parrainer un ou plusieurs migrants enfermés. Il s'agissait de soutenir les détenus par des visites, du courrier, l'achat de cartes de téléphone ou la sensibilisation des passagers des vols contre l'expulsion des sans-papiers. 


\subsection{La violence organisationnelle}

La violence institutionnelle résulte du mode d'organisation de l'espace et des mouvements imposé aux sans-papiers qui correspond au régime carcéral : barbelés et hautes grilles, miradors ou tours de contrôle panoramiques, présence de policiers, haut-parleurs, cellules d'isolement, portes blindées, etc. Deux de ces centres fermés sont même d'anciennes prisons, celui situé à Bruges accueillait des femmes. Par exemple, dans le centre fermé de Melsbroek [fig. 1] les sorties sont interdites, les déplacements à l'intérieur du centre sont limités et la proximité de la piste d'atterrissage expose jour et nuit les détenus à un vacarme insoutenable. ${ }^{13}$ Certains évoquent le « sentiment d'être traité comme un criminel » qui les oppresse alors qu'ils n’ont commis aucun délit (ASSFAM et al. 2018, 18; cf. aussi Médiateur fédéral 2009; CIRÉ 2009a ; CIRÉ et al. 2006).

Qu'est-ce que je peux bien faire ! Je n’y peux rien [...] Je ne suis pas une délinquante, pas voleuse et encore moins prostituée, juste une demandeuse d'asile. (Ntumba 1999, lettre du 24 octobre 1999)

Le centre de Steenokkerzeel (Centre 127bis) pratique la surveillance des lieux par caméra, la fouille des chambres, la séparation d'espaces sans possibilité de communication entre elles, le déplacement des détenus d'une aile à l'autre en cas d'indiscipline ou d'échec de leur expulsion, la privation de communication téléphonique, l'injection forcée de produits calmants non prévue dans les moyens de contrainte autorisés par la législation, ou encore la mise en cellule d'isolement.

A Bruges, les témoignages révèlent que des agents confisquent les produits cosmétiques et les médicaments, la douche est limitée, les chambres sont fermées à clef de $9 \mathrm{~h}$ à $21 \mathrm{~h}$ et les détenus sont parfois privés de nourriture et de matelas dans les cellules d'isolement.

La douche se fait trois fois par semaine, le mardi, jeudi et dimanche pendant 10 minutes vers $16 \mathrm{~h}$. Même si nous sommes des réfugiées, nous avons quand même droit à une hygiène comme toutes les autres femmes? Une femme naturellement a des petits problèmes que l'univers entier connaît. D'où les 3 fois par semaine ne suffisent pas. Dans les dortoirs nous avons quatre lavabos et seulement de l'eau froide. (Ntumba 1999, 5)

Les conditions de détention sont régulées par un arrêté royal (AR) mais chaque centre définit son propre règlement intérieur. L'AR pré-

13 La CEDH a condamné la France pour avoir exposé un jeune enfant durant 7 jours à des nuisances sonores importantes qui ont provoqué des séquelles émotionnelles graves. 


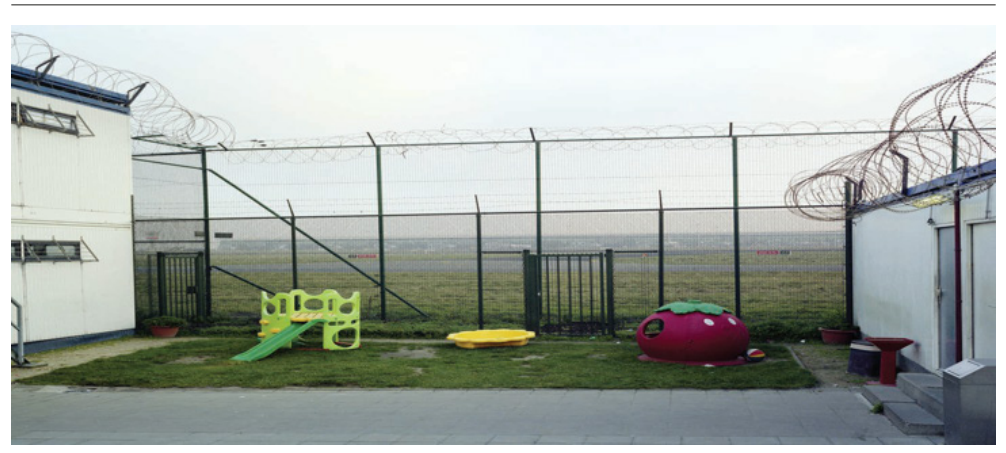

Figure 1 Centre fermé 127 Melsbroek (aéroport de Zaventem). @ Nick Hannes

voit l'interdiction des visites, des mesures coercitives de neutralisation (menottes aux poignets et chevilles, camisole de force) et, à Bruges par exemple, le transfert d'une personne « vers une prison à régime sévère ». Après une crise de nerf, Nancy Ntumba subira ce régime pendant dix jours.

C'est très dur de savoir qu'on est libéré alors qu'on vit toujours enfermé dans le même endroit [...] Plus les jours passaient, plus la haine grandissait en moi. Je ne supportais plus d'être commandée. [Après avoir touché une gardienne] ils m’ont emmenée au cachot où il y a du béton et une toilette métallique. Il était verrouillé avec trois crochets, ça fait très peur, je tremblais [...] Il faisait froid, je me suis endormie sur le béton sans matelas, sans draps. Et à la fin de la punition, je devais prendre une douche et rejoindre pour une semaine la section $\mathrm{Z}$ dans l'aile médical où on est soumis à un régime sévère. (5-6)

La promiscuité dans les centres fermés est une autre source de violence car les espaces d'intimités sont quasi inexistants : selon les témoignages des détenues, les chambres peuvent accueillir de 15 à 20 personnes dans un espace réduit, mal aérée et souvent surchauffé. La vie quotidienne se déroule dans des espaces collectifs confinés et bruyants (pleurs, postes de TV ou radios, activités diverses d'adultes et d'enfants) qui n'offrent aucun espace serein. Les femmes évoquent aussi le viol de leur intimité constamment violée par les fouilles répétitives corporelles ou de leurs effets personnels avant et après une expulsion, après des révoltes ou lors de réunions entre agents qui échangent des informations sur l'état de santé des migrants. 


\subsection{La violence des agents}

Les conditions de vie dans les centres fermés et les rapports sociaux avec les agents chargés du contrôle des détenus sont une source de violence et de souffrance pour les migrants. Celles-ci entraînent divers modes de réactions individuelles (crise de désespoir, dépression, grève de la faim, suicide) ou collectives (grève de la faim, émeute) qui, à leur tour, déclenchent la réaction souvent brutale des agents (sanctions) sur leurs conditions de vie. ${ }^{14}$

Andrew Crosby (2018) a observé qu'au départ, les politiques au sein des centres fermés étaient plus ou moins autonomes avec des règles et des règlements intérieurs variables. Cette autonomie a provoqué des tensions entre les agents en raison de leur conception des relations établies avec les sans-papiers, les uns adoptent une approche sécuritaire alors d'autres développent une approche « humanitaire »:

Leur gestion va un peu dans toutes les directions, sauf leur dysfonctionnement. En effet, il n'existait pas d'arrêté royal qui stipulait les droits et obligations du personnel des centres fermés. Le régime de détention variait donc selon l'équipe de sécurité. Les équipes dures étaient très strictes, elles punissaient arbitrairement les gens car rien ne stipulait qui pouvait mettre qui en cellule d'isolement. Vu le manque de coordination centrale, ces équipes furent longtemps hégémoniques. (Crosby 2018, 16)

Les émeutes récurrentes se déclenchent souvent après des tentatives d'expulsion réussies ou ratées, d'une tentative de suicide, d'un décès par suicide ou accidentels, ou du déclenchement de grèves de la faim. En réaction, les gestionnaires des centres font appel à la police antiémeute qui, selon les témoignages des sans-papiers, agissent avec une grande brutalité (violence physique, usage des chiens, neutralisation physique sur le sol, imposition de menottes, etc.). Les personnes désignées comme les meneurs font l'objet de représailles tels que la mise en cellule d'isolement, le transfert vers d'autres centres fermés, la privation de sortie et d'usage du téléphone, des intimidations, ${ }^{15}$ des brimades et des humiliations.

Ces conditions de vie difficiles dans les centres fermés ont été corroborés, notamment, par des médecins. Le Dr. Ginette Marchant, médecin au centre fermé de Vottem, a démissionné 11 mois après son en-

14 Les ONG ont listé la chronologie des événements tragiques (ex : suicides) qui jalonnent la vie dans les centres fermés et provoquent des révoltes. Voir notamment les rapports d'Amnesty International (2018b), et du Collectif contre les Raffles, les Expulsions et pour la Régularisation (CRER 2015).

15 Selon Nancy, le directeur du Centre de Bruges l'aurait menacée de ne jamais être libérée parce qu'elle conversait avec « des militants des droits de l'homme ». 
trée en fonction pour atteinte à l'indépendance médicale et à l'éthique de la profession. Elle dénonçait les injonctions faites aux agents du Ministère de l'intérieur (assistantes sociales, psychologues, médecins et agents de surveillance) de convaincre les détenus de se laisser rapatrier. Sur le plan médical, elle ne pouvait prodiguer que les soins de base (aide médicale urgente) et le secret médical n'était pas respecté (visite médicale en présence d'une tierce personne, dévoilement de l'état de santé dans les réunions). Enfin, elle a constaté l'imposition de l'isolement médical à des fins punitives (Vanpaeschen 2000) et l'injection forcée de calmant avant une expulsion. Le CIRÉ (2009b, 33) a établi des constats similaires.

Le ressenti de la violence institutionnelle s'accentue dans l'écart entre le non-respect récurrent des règles de droit de la part des institutions et de ses agents et l'état de droit déclaré. Ainsi, la prolongation indéterminée de la durée de détention après les expulsions ratées au-delà de la durée maximale provoque de l'incompréhension voire un état de décompensation psychique sérieux :

Mon avocat a introduit un recours à Bruges et le juge a décidé de me libérer [...] Tu ne peux pas imaginer ma joie lorsque le juge a prononcé le verdict. J'étais très contente, j’ai pleuré de joie. Mais arrivé ici [au centre fermé de Bruges] l'assistant social m'informe que le procureur du Roi avait fait appel contre ma mise en liberté [...] J'étais morte ce jour-là, oui c'est comme si on m'avait crucifiée. J'ai pleuré comme une folle [...] C'est à ce moment que j’ai senti que tout s'écroulait. J'ai pris les chaises et j'ai commencé à les lancer, j'avais perdu le contrôle. C'est ainsi qu'on m'isola pour une journée. Je n'arrive plus à dormir, ma tête est toujours chaude, on a brisé mon cœur. (Ntumba 1999, lettre du 10 août 1999)

Les multiples plaintes déposées auprès de la direction des centres contre les mauvais traitements de la part d'agents de surveillance ou de gendarmes lors des expulsions restent sans suite et accentuent le sentiment d'injustice et d'isolement :

Les violences sont le plus souvent commises sans témoins, dans les locaux de la police de l'aéroport, ou lors du retour vers le centre fermé après une tentative d'expulsion 'ratée'. Le détenu violenté a le droit de porter plainte mais peu de plaintes sont effectivement déposées, et parmi elles, peu aboutissent. Il arrive régulièrement que les médecins des centres refusent d’attester des violences subies. (CIRÉ 2009b, 62)

Avant sa mort, Sémira Adamu signalait qu'elle était l'objet d'une surveillance par une personne en permanence à ses côtés. Après son évasion du Centre 127bis, elle ne pouvait plus téléphoner, elle se sentait seule, n'avait plus droit à des visites et les migrantes avec qui elle 
avait établi des liens dans ce centre ont été déplacées. Selon le témoignage de sa marraine ${ }^{16}$ (médecin, professeure d'université et membre $\mathrm{du}$ Collectif contre les expulsions), le soutien du Collectif contre les expulsions a entraîné des brimades de la part des agents du centre.

\subsection{La violence sur les enfants}

Bien que l'enfermement des enfants soit interdit par les conventions internationales, cette pratique est encore répandue en Europe : selon l'UNICEF, seul l'Irlande interdit d'enfermer les mineurs. La CEDH a condamné la Belgique à de multiples reprises pour avoir détenus 2.341 enfants avec leurs parents dans les centres entre 2004 et 2008. Le gouvernement y mettra momentanément fin en $2008^{17}$ mais il recommencera en dépit des rapports médicaux et psychologiques accablants publiés depuis 1999 (Ligue des droits de l’Homme 2019), qui révèlent les conséquences délétères sur l'état de santé physique et mentale des enfants et sur leur développement futur. ${ }^{18}$ En effet, en 2007, deux psychologues ont rédigé des rapports qui attestaient d'une souffrance et d'une détérioration de l'état psychologique notamment d'Angelica, une jeune équatorienne de onze ans détenue avec sa mère durant un mois. Elle présentait des signes de dépression, de tristesse, d’anxiété, des difficultés à respirer, avait des cauchemars, des maux de tête, des vertiges liés au souvenir de son arrestation et de la menace de son renvoi au pays d'origine.

Le haut degré de déshumanisation de la politique d'enfermement des migrants et la brutalité avec laquelle elle traite les enfants s'incarnent dans le cas de Tabitha Kaniki Mitunga, une jeune congolaise âgée de cinq ans. En août 2002, elle transit à l'aéroport de Bruxelles en compagnie de son oncle maternel (résidant légal aux Pays-Bas) pour se rendre au Canada où elle doit rejoindre sa mère réfugiée reconnue dans ce pays. La police des frontières estime qu'elle ne possédait pas les documents de voyage et de séjour nécessaires et décide d'enfermer cette enfant dans le « centre fermé 127» en vue de son ex-

16 Lise Thiry, témoignage au procès des gendarmes, 10 septembre 2003: https:// ccle.collectifs.net/Temoignage-de-Lise-Thiry-marraine (2019-11-06).

17 Le gouvernement avait alors créé « les maisons de retour » pour héberger les familles en attente d'expulsion : 27 unités pour 169 places sous forme d'appartements ou de studios. Selon l'analyse du CIRÉ, 2.180 enfants ont été placés dans ces maisons entre 2008 et 2016 réparties en 27 unités d'habitation sur le territoire ; en 2016, 39\% des familles ont quitté le territoire, $28 \%$ ont été libérées et 33\% ont disparu dans la nature (CIRÉ 2018, 3).

18 Voir la liste des dommages infligés et la descente aux enfers des personnes soumises à l'enfermement, établie à partir des observations du Dr. Christine Dormal dans le Centre 127bis. Elle a été publiée par la Coordination contre les Rafles, les Expulsions et pour la Régularisation (CRER 2009). 
pulsion vers le Congo. La demande de reconnaissance de la qualité de réfugiée, souvent recommandée aux migrants en transit arrêtés à la frontière ${ }^{19}$ et non justifiée dans le cas d'espèce, est immédiatement introduite mais jugée irrecevable par le Commissariat aux Réfugiés et aux Apatrides. Ce dernier souligne cependant le jeune âge de l'enfant et son droit au regroupement familial garanti par la Convention des droits de l'enfant. L'avocat entame alors des démarches auprès des autorités canadiennes et demande à l'Office des Étrangers de confier l'enfant à une institution d'accueil en attendant d'obtenir le regroupement familial, ce qui ne sera pas accepté puisque Tabitha sera enfermée seule pendant deux mois. L'avocat entame les démarches pour sa libération qui sera ordonnée le 16 octobre 2002 par le tribunal mais, le lendemain, l'Office des Étrangers expulse l'enfant vers la République démocratique du Congo où aucun membre de sa famille ne l'attend. Après plusieurs heures d'attentes à l'aéroport, elle est finalement prise en charge (et hébergée) par une fonctionnaire de l'Agence nationale de renseignement du Congo. Face au scandale, ${ }^{20}$ le Premier ministre contacte le gouvernement canadien qui accorde à Tabitha le droit de rejoindre sa maman qu'elle réalisera une semaine après son expulsion (Van Keirsbilck 2007).

En 2006, la CEDH reconnaîtra la responsabilité des autorités belges qui « avaient ou auraient dû avoir connaissance de ces mauvais traitements [...] [et qui] ne pouvaient ignorer les conséquences psychologiques graves [de cette décision]. A ses yeux, pareille détention fait preuve d'un manque d'humanité et atteint le seuil requis pour être qualifiée de traitement inhumain » (Journal des Droits de Jeunes 2006). Elle condamne la Belgique pour atteinte à la fois au respect de la vie privée et familiale et à la liberté et au droit à un recours effectif (Lelièvre 2006, 227-8; Van Keirsbilck 2007).

En dépit de cette nouvelle condamnation, cette politique se poursuit : en 2014, le gouvernement développe une nouvelle rhétorique annonçant une pratique d'enfermement « plus humaine » des enfants avec leur famille. ${ }^{21}$ Le Secrétaire d'État à la Migration et à l'Asile

19 Les migrants sans-papiers en transit arrêtés indiquaient qu'ils n'avaient nullement l'intention de demander l'asile en Belgique, mais les agents de contrôle aux frontières leur recommandent de l'introduire pour éviter l'expulsion et obtenir leur libération du centre fermé. Ces demandes sont exceptionnellement acceptées.

20 L'affaire suscite des réactions d'indignation et des actions collectives notamment du barreau des avocats francophones et germanophones et de 15 associations qui portent plainte auprès du Conseil d'État (nr. 244.190 du 4 avril 2019) lequel suspend l'arrêté royal permettant l'enfermement des enfants au Centre 127 en raison de l'atteinte à leur vie privée et familiale et à leur exposition à des nuisances sonores graves.

21 Dans le cadre du renforcement de sa politique « de retour de qualité, humaine et durable, conformément au principe du retour volontaire si possible, forcé si nécessaire [...] Les centres fermés seront étendus [...] Le projet d'y aménager des lieux d’hébergement (Centre 127bis) est réalisé afin que des places appropriées soient prévues pour cer- 
crée, en juin 2018, les « Unités de détention pour les familles » ${ }^{22}$ présentées comme des espaces désormais adaptés aux besoins des enfants, ce que démentent les ONG (CIRÉ 2018). Le 14 août 2018, une mère Rom originaire de Serbie et ses quatre enfants âgés de un an à six ans présents en Belgique depuis dix ans, sont détenus 54 jours (au lieu d'un mois) puis expulsés le 24 septembre 2018 (Rasson 2019). ${ }^{23}$ Trois autres familles avec des enfants en bas âges subiront le même sort en septembre et octobre 2018.

\section{$4 \quad$ La pratique des expulsions forcées}

La Belgique détient un lourd passif en matière d'expulsions forcées des migrantes sans-papiers dont le cas tristement célèbre de Sémira Adamu, qui a succombé par étouffement le 22 septembre $1998 .{ }^{24}$ Lors du procès des gendarmes qui l'ont asphyxiée, le film de sa mort a été diffusé : cette séquence de 11 minutes $^{25}$ devait apporter la preuve de l'usage de la force légitime et nécessaire pour maîtriser une victime « violente » qui résistait à son expulsion. Or le film montre une femme très calme, les mains menottées dans le dos, les pieds entravés et deux des trois gendarmes présents qui s'appuient lourdement sur le dos de la victime tout en discutant et ignorant les signes évident de l'asphyxie.

Selon le compte rendu du procès, les gendarmes incriminés décrivent surtout les consignes et les points de contrôle à respecter selon la circulaire et ne portent aucune attention à l'état de la personne qu'ils devaient maîtriser : " Nous avons fait notre travail comme il faut »; «Tout était normal dans la procédure personne parmi mes collègues n'est intervenu »; "J'ai entendu qu'elle respirait, j’ai contrôlé ce que je devais contrôler ». L'un des gendarmes affirmera

tains groupes cibles vulnérables, par exemple, les familles avec enfants, de sorte qu'ils ne doivent plus aller dans le réseau d'accueil » (Accord de gouvernement fédéral, 10-10 2014, 158-60)

22 Les enfants sont autorisés à fréquenter une école et les adultes à sortir pour consulter un avocat, un médecin ou faire quelques courses. Un adulte doit cependant toujours être présent dans la maison pour éviter l'évasion. La Commissaire aux droits de l'Homme du Conseil de l'Europe a demandé au Secrétaire d'État d'abandonner ce projet dans un courrier daté du 5 juin 2018 (Le Soir, 14 juin 2018).

23 La visite des délégués généraux aux droits de l'enfant avec une pédiatre dans ces unités familiales en août 2018 révèle l'état « déplorable » des enfants mais n’a pas mis fin à cette pratique malgré l'existence d'alternatives plus respectueuses des droits humains et moins coûteuses (IDC 2015).

24 Le meurtre de Sémira Adamu entraîne la démission du ministre de l’Intérieur qui avait apporté son soutien aux gendarmes, la suspension provisoire des expulsions qui reprendront très rapidement, et la publication d'une directive ministérielle supprimant l'usage de techniques de contraintes qui obstruent les voies respiratoires.

25 https://ccle.collectifs.net/Les-onze-dernieres-minutes-de.html (2019-11-06) 
qu'il subissait des pressions de la part de la hiérarchie pour atteindre l'objectif chiffré : " dans les couloirs on parlait et on disait 'les trente expulsions doivent réussir ou nous aurons des misères'. Une pression certaine venait d'en haut ». Le responsable du rapatriement inculpé confirme l'ordre d'atteindre l'expulsion de 23 personnes « parce que les centres [fermés] étaient pleins ». ${ }^{26}$ Leur avocat construira leur défense essentiellement sur « l'obéissance aux ordres » et sur l'engagement volontaire des gendarmes affectés à cette " tâche d'expulsion ». Au final, deux des ex-gendarmes bénéficient d'une suspension du prononcé et le troisième qui avait déjà commis des violences sur les sans-papiers lors d'autres expulsions écope d'un sursis. ${ }^{27}$

Le bilan de cette politique vingt ans après le décès de Sémira Adamu révèle que la violence des expulsions et les violations des droits fondamentaux des migrants restent la règle ; le projet de $1 \mathrm{li}^{28}$ autorisant à la police d'intervenir en force dans le domicile privé des citoyens ou dans les associations pour débusquer les sans-papiers (Arnould 2019) et le plan du gouvernement qui prévoit un régime cellulaire annoncent le durcissement du régime de détention des sans-papiers.

\subsection{Les expulsions collectives}

La Belgique pratique non seulement les expulsions individuelles, mais également les expulsions collectives qui lui a aussi valu une condamnation de la CEDH en février 2002, notamment, pour la violation de la Convention européenne des droits de l'homme, les conditions de détention des Roms et le caractère collectif de l'expulsion (Carlier 2002).

La première expulsion collective de grande ampleur que le gouvernement belge a organisée fut mise en œuvre en octobre 1999 pour 74 Roms, dont de nombreux d'enfants, expulsés vers la Slovaquie malgré l'arrêt suspensif de la CEDH rendu une heure avant le décollage de l'avion. Pour arrêter les sans-papiers, la police de la ville de Gand (Flandre) convoque, fin septembre 1999, plusieurs dizaines de ces familles Rom, dont la famille Conka, au motif de « compléter le dossier relatif à la demande d'asile ». Au commissariat, ils reçoivent un ordre de quitter le territoire et sont directement emmenés au centre

26 https://ccle.collectifs.net/Resume-de-la-deuxieme-journee-d.html (2019-11-06).

27 Le gendarme qui a filmé l'expulsion a bénéficié d'un non-lieu puisque durant l'opération il dit avoir demandé à plusieurs reprises si la victime respirait encore alors que la non-assistance en personne en danger aurait pu être retenue. Quant à leur supérieur, ils sont acquittés (Perrouty 2003).

28 En août 2018, la presse annonçait que le gouvernement fédéral aurait abandonné ce projet qui a suscité de nombreuses critiques notamment de la magistrature et des associations d'avocats. 
fermé 127 bis en vue de leur rapatriement. ${ }^{29}$ De surcroit, les policiers inscrivent un numéro au stylo bille sur leur main pour marquer les esprits par une symbolique forte.

Cette pratique d'expulsion collective fut théorisée par les experts de la "Commission chargée de l'évaluation des instructions en matière d'éloignement » instituée après la mort de Sémira Adamu. Présidée par un professeur de philosophie à la retraite de l'Université de Gand, le rapport du 21 janvier 1999 suggère en cas d'échec d'une expulsion forcée d'affréter un avion spécial et de l'organiser à l'échelle européenne :

par exemple du type avion d'affaires, qui permettrait de rapatrier un nombre restreint de personnes sous surveillance suffisante et d'éviter la répression de cris et autres types de provocation, étant donné qu'il n'y a pas de public [...] Cette procédure pourrait d'ailleurs être appliquée en concertation et en collaboration avec d'autres pays européens, de sorte que les accords pourraient être conclus plus facilement avec les pays de retour pour les droits d'atterrissage nécessaires. (Commission Vermeersch I, 1999, 23) ${ }^{30}$

Dans la nuit du 13 au 14 mars 1999, le gouvernement met en pratique cette proposition avec l'expulsion collective de migrants vers le Cameroun accompagnés de douze gendarmes et d'un médecin pour un coût de 50.000 euro. La presse révélera une autre expulsion collective de 45 Albanais, en février 2000, dans un Airbus A310 affrété par l'armée belge (Wibault, Van Meulder, Liebman 2003). La pratique s'est banalisée et peu d'informations existent actuellement sur ces expulsions.

29 En février 2002, la CEDH condamne la Belgique pour trois violations de la Convention européenne des droits de l'homme : les conditions de détention des Roms, le caractère collectif de l'expulsion et l'absence de recours effectifs (Carlier 2002).

30 Ce rapport suscita de vives critiques d'un groupe inter-universitaire, Asile et expulsion, qui condamne une telle collaboration : "Nous condamnons la complicité de ceux qui, parmi nos collègues, ont cru bon de légitimer la politique belge d'asile et d'expulsion, en participant activement à la commission créée par le ministre de l'intérieur [...] Ce rapport jette une ombre inacceptable sur ce que peut ou devrait représenter la participation des universitaires et des intellectuels aux débats de société [...] [Le plus choquant] est que certains universitaires soient sollicités pour aboutir à des fins à propos desquelles ils n'ont aucune compétence d'expertise (types de menottes, etc.) et pour soutenir des recommandations d'ordre technique qui ne nécessitent absolument pas leur avis » (cité par Wibault, Van Meulder, Liebman 2003, 131). En janvier 2005, la Commission Vermeersch II formulera 34 recommandations en vue d'une politique d'éloignement « humaine et effective » (Commission Vermeersch II 2005). 


\subsection{Les expulsions individuelles}

Bien que l'Europe ait 'délocalisé' une partie de sa politique de rétention et d'expulsion à certains pays-tiers (Maroc, Tunisie, Libye) contre rétribution financière et soutien politique aux régimes non démocratiques, les États comme la Belgique maintiennent cette pratique qui revêt une fonction symbolique forte au regard du discours sur « la gestion efficace » des mouvements migratoires (Ouali, Chichah 2005).

L'examen de l'expérience de Nancy Ntumba Kabongo, sur laquelle je propose à présent de nous attarder, est emblématique de la violence exercée sur les sans-papiers à la fois en raison des tentatives répétées d'expulsion avant l'expulsion effective ou la possible régularisation, et du rituel stressant et humiliant, avant et après ces tentatives, d'isolement, de fouille et de pressions morales. J'ai suivi le cas de Nancy au cours de l'année 1999 à travers des visites rendues au centre fermé de Bruges, qui m'ont très vite été interdites, des contacts téléphoniques et une correspondance pendant les dix mois et demi de sa détention. L'analyse de sa trajectoire montre, d'une part, l'imbroglio administratif et les multiples entraves déployées par les institutions et ses agents auxquels doivent faire face les personnes les plus vulnérables pour rester en Belgique, et, d'autre part, les conséquences délétères à très long termes de cette politique d'expulsion sur le plan de la santé physique et mentale des sans-papiers.

Nancy Ntumba est née le 10 août 1976 en République démocratique du Congo et est arrivée en Belgique le 14 février 1999 à $9 \mathrm{~h}$ en transit ${ }^{31}$ vers l'Angleterre où elle envisageait de demander l'asile. Son arrestation a lieu dans la zone de transit de l'aéroport de Zaventem (Bruxelles) après le contrôle de son passeport car les agents de la police des frontières l'accusent de voyager sous une fausse identité. Elle est mise à l'écart durant plusieurs heures puis ces agents lui proposent soit de retourner en Afrique du Sud d'où elle venait, soit de demander l'asile en Belgique. L'un d'eux lui affirme qu'elle recevra une aide, ce qui la persuade d'introduire sa demande en Belgique. Elle est emmenée au Centre fermé 127 (Melsbroeck) situé à côté de l'aéroport et obtient dès le lendemain un entretien avec un agent de l'Office des Étrangers qui rejette sa demande d'asile: "J'étais abattue et confuse. Je ne savais plus quoi faire. Tout d'un coup tout a changé et je devais rentrer vers là d'où j'avais fui » (Ntumba 1999, 1).

Le 16 février 1999, son avocat introduit un recours urgent auprès du Commissariat général aux réfugiés et aux apatrides qui est égale-

31 Le témoignage du Dr. Marchant évoque la détresse qu'elle a constatée chez les personnes en transit vers un autre pays pour rejoindre leur famille, qui ont été arrêtées à l'aéroport de Bruxelles pour absence de document (visa) ou sur soupçon de détention de faux papiers. Elle souligne que ces situations touchaient davantage les hommes et les femmes noires et qu'elles ont provoqué de nombreuses tentatives de suicide (Vanpaeschen 2000). 
ment rejeté le $1^{\mathrm{er}}$ mars 1999. Nancy est alors déplacée au Centre fermé de Bruges pour femmes qui se situe à $100 \mathrm{~km}$ de Bruxelles. Le contact initial avec les agents du centre produit un premier traumatisme : «C'était très étrange, j'avais très peur. Tous ces agents en uniforme pour contrôler les femmes ont fait la fouille de mes affaires, retiré les objets dangereux et ensuite fait la fouille corporelle. L'agent porte des gants médicaux : j'étais en soutien et sous-vêtement et j'avais trop peur. Je sanglotais car je ne comprenais rien, j'étais perdue » $(1999,1-2)$.

La conséquence de son transfert à Bruges est double : les contacts avec son avocat, les ONG et les citoyens visiteurs qui la soutiennent sont plus difficiles car la plupart sont localisés à Bruxelles. Ensuite, les procédures judiciaires pour sa libération se font en néerlandais, alors que Nancy est francophone, et une grande partie de celle-ci lui échappe.

Arrivée le $1^{\text {er }}$ mars 1999 au Centre, Nancy fait rapidement l'objet d'une première tentative d'expulsion non forcée vers l'Afrique du Sud, ${ }^{32}$ soit le 3 mars. Comme elle refuse de partir, elle est ramenée au Centre. Son avocat introduit un recours à la chambre du Conseil de Bruxelles pour sa libération, ce qui lui est refusé. Un autre recours est introduit à la chambre du Conseil de Bruges, le 18 mai 1999, qui est aussi négatif. Son enfermement au Centre est chaque fois prolongé de deux mois puisque la loi autorise, depuis 1996, une détention jusque 8 mois, mais la mise à zéro fréquente du compteur suite à une expulsion ratée rend, dans les faits, la détention indéterminée.

Le 8 juin, elle fait l'objet d'une deuxième tentative d'expulsion à laquelle elle résiste :

J'ai été à l'aéroport pour un rapatriement, je n'avais rien compris. J'ai été torturée moralement pour commencer et puis on m’a ligotée et amenée à bord de l'appareil [...] Les policiers m’ont demandé d'aller m'asseoir sur le dernier siège. Comme mes deux bras étaient ligotés à l'arrière, je me suis retournée afin que tous les passagers voient mes bras. Un dernier passage est arrivé et a demandé ce qui se passait, c'est alors que l'on m'a fait descendre de l'appareil. [...] J’ai beaucoup pleuré ce jour-là parce que j'étais traitée comme une criminelle. (1999, lettre du 21 juin 1999)

Les procédures pour demander la libération de Nancy se succèdent et reçoivent systématiquement un avis négatif jusqu'au jugement du 30 juillet 1999, où le juge de la chambre du Conseil de Bruges décide de la libérer. De retour au Centre, un agent lui annonce que le Procureur du Roi a fait appel de la décision de libération, mais le 12 août 1999 le tribunal la confirme. Elle sera encore suivie d'un appel : 
C'était la $2^{\text {e }}$ fois que j'étais libérée, j'étais contente et je sautais de joie. Arrivée au centre j’attendais qu'on me dise de faire mes bagages. Je gardais le secret pour moi-même etj'avais peur qu'on me dise encore que le procureur avait fait appel [...] Le lendemain, on m’annonce que l'appel a été déposé et que je dois introduire une nouvelle procédure. Le pire c'est que le compteur des mois d'enfermement reprend à zéro. J'étais folle et très malade [...]. C'était très difficile. $(1999,4)$

Le 9 juillet 1999, Nancy est conduite à l'aéroport de Bruxelles pour une troisième tentative d'expulsion à laquelle elle résiste et le 4 octobre 1999 elle revient pour une quatrième tentative. Enfin, la cinquième tentative d'expulsion se déroule le 9 novembre 1999: elle échouera à la faveur de la visite du directeur du Centre pour l'égalité des chances et la lutte contre le racisme présent à l'aéroport et qui demande de s'entretenir avec Nancy :

Je fus mise dans une petite cellule trop sale et j'attendais l'heure $\mathrm{H}$ [de l'expulsion]. Monsieur Cornil était à l'aéroport pour une mission concernant les centres fermés. J'étais donc sauvée et j'ai pu m’entretenir avec lui pendant quelques minutes. C'était une chance pour moi qu'il soit là car j'étais sûre qu'il voulaient me forcer. $(1999,7)$

Avant et après chaque retour d'une expulsion avortée, les détenues et leurs bagages subissent une fouille approfondie et humiliante. Par ailleurs, les agents et la direction considèrent Nancy comme une « meneuse » et une " forte tête » qui crée du désordre dans le centre. En conséquence de quoi elle est l'objet de plus en plus de pressions notamment parce qu'elle est très soutenue par des militants du Collectif contre les expulsions, elle reçoit des visites de citoyens et de parlementaires bruxellois, et que son cas fait l'objet de plusieurs articles de presse. Après la $5^{\mathrm{e}}$ tentative d'expulsion, les gendarmes qui l'accompagnent cherchent à l'impressionner : "Ils ont commencé à m'intimider avec la même chanson : que c'était un ordre du ministre, que je devais partir et que si je ne partais pas aujourd'hui comme une femme libre, la prochaine fois, je partirai entourée de deux policiers » $(1999,8)$.

A son retour au Centre, Nancy ne supporte plus sa détention et les fouilles corporelles : elle pleure, crie et perd le contrôle de ses émotions. La situation est d'autant plus pénible pour Nancy que des femmes du Centre avec qui elle a établi des liens sont libérées et qu'elle y restera au total dix mois et demi. On lui interdit de parler mais elle continue à s'exprimer en lingala (sa langue maternelle), elle est alors maîtrisée physiquement par plusieurs gardiens (attache en plastique aux pieds, étouffement): « j’ai cru que j’allais mourir. Je criais, je pleurais. Quelle souffrance. J’aurai préféré la mort plutôt que la vie » $(1999,8)$. 
Elle est alors placée en isolement et. à 23h, le directeur adjoint lui rend visite et fait pression sur elle :

Encore toi Nancy! Tu es en train d'exciter tout le groupe. Je sais que tu as de l'influence dans le groupe parce que tu parles avec Ecolo et les hommes politiques. Mais les associations et tout le monde te donnent de faux espoirs. Tu es en fin de procédure et tu dois partir. Tu n'as pas le droit de parler ici [...] Il m'avait promis que s'il se passait encore quelque chose, je serais envoyée en 'section $\mathrm{Z}$ ' [section d'isolement] jusqu'au prochain rapatriement. $(1999,8)$

Finalement, le 29 décembre 1999 le Ministère de l'intérieur libère Nancy du centre avec un ordre de quitter le territoire dans les cinq jours : «[s]elon le Gouvernement, cette remise en liberté fut le résultat d'une mesure générale de grâce de fin d'année » (CEDH 2005). Elle migre au Royaume-Uni comme elle le souhaitait en février 1999 et le 11 septembre 2002 elle obtient une autorisation de séjour de quatre année et par la suite sa régularisation définitive.

\section{Du juridique au politique}

La mise en perspective des pratiques d'enfermement et d'expulsion déployées depuis la fin des années 1980 pour maîtriser les mouvements migratoires en Belgique nous renvoient toute la violence systémique de cette politique à l'égard des migrants sans-papiers. Les violations systématiques des droits fondamentaux ; l'imposition d'un régime carcéral ; les dures conditions de vie ; les pressions multiples et les intimidations ; l'énergie physique et mentale investie pour faire face à la profusion de procédures administratives et judiciaires pour obtenir l'asile, la libération du centre fermé ou empêcher l'expulsion ; la prolongation indéterminée de la durée d'enfermement et les tentatives répétées d'expulsion révèlent la logique d'un système qui est un véritable calvaire visant à décourager tout migrant à venir et à s'installer en Belgique.

Cette politique menée depuis plus de trente ans, aussi bien par les sociaux-démocrates que par les nationalistes flamands proche de l'extrême droite, repose sur la prétendue 'menace' que représente l'étranger pour nos emplois et notre sécurité sociale et sur un discours idéologique puissant : "nous ne pouvons accueillir toute la misère du monde » et la rhétorique de la maîtrise " démocratique et humaine des flux migratoires » qui sert à justifier et banaliser à leur expulsion comme solution à nos problèmes (Ouali, Chichah 2005, 25). 
Le caractère construit et intentionnel de ce que les juges de la CEDH désignent le plus souvent comme des «mauvais traitements » à travers, d'une part, une organisation institutionnelle et la légitimation des pratiques violentes des agents, et, d'autre part, leurs conséquences indélébiles sur la santé mentale et physique des migrants (et leurs enfants) aussi puissantes que celles qui résultent de la torture, ne suggèreraient-ils pas de re-qualifier et reconnaître ces traitements inhumains comme des formes spécifiques de torture propres aux centres fermés ? L'enjeu de cette re-qualification réside dans la nécessité de déplacer le débat moral et juridique vers le politique afin de questionner les formes de gouvernementalité appliquées aux migrants sans-papiers plutôt que de se limiter à la dimension réparatrice individualisée. Poser les questions sous l'angle politique est la condition sine qua non pour saisir la logique du régime d'exception imposé aux migrants et du discours de criminalisation des sans-papiers et pour éliminer une politique indigne d'une société fondée sur l'égalité et sur les droits fondamentaux des citoyens.

\section{Bibliographie}

Amnesty International (2009). La situation des droits humains dans le monde. Rapport 2009. URL https://www.amnesty.org/fr/documents/ pol10/001/2009/fr/ (2019-11-06).

Amnesty International (2018a). La situation des droits humains dans le monde. Rapport 2017/18. https://www. amnesty.org/fr/documents/ pol10/670๑/2018/fr/ (2019-11-06).

Amnesty International (2018b). Rapport annuel 2018 Belgique. URL https:// www.amnesty.be/infos/rapports-annuels/rapport-annuel-2018/ europe-et-asie-centrale/article/belgique (2019-11-06).

Arnould, Juliette (2019). «Visites domiciliaires: la société civile à la rescousse des droits fondamentaux». Ligue des Droits de l'Homme 2019, $22-6$.

ASSFAM; Forum réfugiés - Cosi; France terre d'asile; La Cimade; Ordre de Malte France; Solidarité Mayotte (2018). Centres et locaux de rétention administrative. Bruxelles.

Calzolaio, Chiara; Colombo, Pamela; Makaremi, Chowra (2016).«Ethnographies politiques de la violence». Cultures et Conflits, 3-4(103-104), 7-13. URL https://www.cairn.info/revue-cultures-et-conflits-20163-page-7.htm (2019-11-06).

Commission Vermeersch I (1999). Commission chargée de l'évaluation des instructions en matière d'éloignement. Rapport final, 21 janvier 1999.

Commission Vermeersch II (2005). Fondements d'une politique humaine et efficace d'éloignement. Rapport final de la Commission chargée de l'évaluation des instructions en matière d'éloignement (II), 31 janvier 2005.

Carbocci, Marco et al. (1998). Les Barbelés de la honte. Bruxelles: Éditions Luc Pire.

Carlier, Jean-Yves (2002). «L'expulsion collective des Tsiganes (Affaire Conka contre Belgique)». L'Année sociale, 2002, 64-70.

CIRÉ (2009a). Les impacts médicaux et psychologiques de la détention en centres fermés. Bruxelles. URL https://www.cire.be/wp-content/ 
uploads/2011/10/impacts-psychologiques-medicaux-detention-centre-ferme.pdf (2019-11-06).

CIRÉ (2009b). Tu veux savoir si j'ai assez souffert pour pouvoir rester dans ton pays..?. Guide pédagogique sur les centres fermés et les expulsions. Bruxelles. URL https://www.cire.be/wp-content/uploads/2011/10/illegal-cahier-peda.pdf (2019-11-06).

CIRÉ (2018). "Si mineur. Les enfants migrants au quotidien», suppl. 6, Ligueur. URL https://www.cire.be/si-mineurs-les-enfants-migrantsau-quotidien/ (2019-11-08)

CIRÉ (2019). Les Centres fermés. Bruxelles. URL https://www.cire.be/lescentres-fermes/ (2019-11-06).

CIRÉ; Jesuit Refugee Service; MRAX; Ligue des droits de l'homme; Vluchtelingenwerk Vlaanderen (2006). Centres fermés pour étrangers: État des lieux. Bruxelles.

CEDH (2005). Décision sur la recevabilité de la requête no 52467/99 présentée par Nancy NTUMBA KABONGO contre la Belgique. Strasbourg, 2 juin.

CRER, Collectif contre les Rafles, les Expulsions et pour la Régularisation (2009). Les carnets noirs des centres fermés, 3, mai. URL https://theowl. hotglue.me/Refugees_CarnetsNoirs\#Refugees_CarnetsNoirs. head.13221697662 (2019-11-06).

CRER, Collectif contre les Raffles, les Expulsions et pour la Régularisation (2015). «Victimes de tentatives d'expulsions par voie aérienne en Europe (1991-2014)». Les Carnets noirs des centres fermés en Belgique, 14. URL https://theowl.hotglue.me/Refugees_CarnetsNoirs\#Refugees_CarnetsNoirs.head.132218015547 (2019-11-06).

Crosby, Andrew (2018). «Les centres fermés post Sémira Adamu». Agenda interculturel, 340, 14-17.

Fassin, Didier et al. (1997). Les lois de l'hospitalité. Les politiques de l'immigration à l'épreuve des sans-papiers. Paris: La Découverte.

Foucault, Michel (2001) «La gouvernementalité». Foucault, Michel, Dits et écrits I/ 1976-1988. Paris: Gallimard, 635-57.

Freedman, Jane; Valluy, Jérome (dirs) (2007). Persécutions des femmes. Savoirs, mobilisations et protections. Vulaines sur Seine: Editions du Croquant.

IDC, International Detention Coalition (2015). There are alternatives. An handbook for preventing unnecessary immigration detention (Revised Edition). Melbourne: International Detention Coalition. URL https://idcoalition.org/wp-content/uploads/2016/01/There-Are-Alternatives-2015.pdf (2019-11-08).

Journal des Droits des Jeunes (2006). «Droit des étrangers». Journal des Droits des Jeunes, 259, 52-63. DOI https://doi.org/10.3917/jdj.259.0052.

Lavergne, Cécile; Perdoncin, Antonio (2010). «Éditorial. La violence à l'épreuve de la description». Tracés, 19, 5-25. DOI https://doi.org/10.400९/ traces.4878.

Laacher, Smaïn (2007). Le peuple des clandestins. Paris: Calman-Lévy.

Lelièvre, Claude (2006). On garde l'espoir. Rapport annuel du Délégué général de la Communauté française aux droits de l'enfant. Bruxelles. URL https:// bit.ly/2qmIyN3 (2019-11-06).

Ligue des Droits de l'Homme (2019). État des droits humains en Belgique. Rapport 2018. Bruxelles: Ligue des Droits de l'Homme. URL http://www. liguedh. be/wp-content/uploads/2019/O2/EDH_2018_Web.pdf (2019-11-06). 
Makaremi, Chowra (2016). «'Etats d'urgence ethnographies'. Approche empirique de la violence politique». Cultures et Conflits, 3-4(103-104), 15-34. URL https://www.cairn.info/revue-cultures-et-conflits-20163-page-15.htm?contenu=article (2019-11-06).

Médiateur fédéral (2009). Investigation sur le fonctionnement des centres fermés gérés par l'Office des Étrangers. Bruxelles: Médiateur fédéral. URL http:// www.federaalombudsman.be/sites/default/files/auditCF2008-FR_๑.pdf (2019-11-06).

Naepels, Michel (2006). «Quatre questions sur la violence». L'Homme, 1-2(177178), 487-95. DOI https://doi.org/10.400९/lhomme. 21787.

Ntumba, Nancy (1999). Mon séjour en Belgique. Bruxelles: Collectif de Résistance aux Centres Fermés et aux Expulsions.

Ouali, Nouria; Chichah, Souhail (2005). «Mellila et Ceuta: la délocalisation de la honte». La Revue nouvelle, 11, 17-21.

Perrouty, Pierre-Arnaud (2003). «Un système de rouages: l'affaire 'Sémira Adamu'». L'année sociale, 2003, 107-15.

Rasson, Anne-Catherine (2019). «Un jour sombre de l'histoire belge: la détention des enfants migrants». Ligue des Droits de l'Homme 2019, 4-7.

Revue européenne des migrations internationales (2017). Dire la violence des frontières. Mises en mots de la migration vers l'Europe, 33(2-3). URL https:// www.cairn.info/revue-europeenne-des-migrations-internationales-2017-2.htm (2019-11-06).

Van Keirsbilck, Benoït (2007). «L'affaire Tabitha: chronique d'une condamnation annoncée». L'Année sociale, 2007, 77-95.

Vanpaeschen, Laurence (2000). «Centre fermé de Vottem: un médecin démissionne et accuse». Journal du Mardi, 29 février.

Wibault, Tristan; Van Meulder, Griet; Liebman, Daniel (2003). «Été afghan à Ixelles. Chronique d'une expulsion collective entravée». L'Année sociale, 2003, 116-33. 



\title{
Torture and the UK's 'War on Asylum' \\ Medical Power and the Culture of Disbelief
}

\author{
Monish Bhatia \\ Birkbeck, University of London, UK \\ Jon Burnett \\ Swansea University, UK
}

\begin{abstract}
This chapter explores medical power in the UK's 'war on asylum', examining how medical expertise has been undermined in the asylum process when this expertise is utilised to add weight to asylum seekers' claims to have experienced torture. It examines how there have been attempts to narrow the definition of torture in ways which exclude people from the protections to which torture survivors are entitled. It explores the extent to which medical power has been complicit in riding roughshod over existing safeguards to prevent further harm to those who have experienced torture, and also, crucially, how this form of power has been and continues to be challenged.
\end{abstract}

Keywords Immigration detention. Torture. Asylum seekers. Border control. Medical power.

Summary 1 Introduction. - 2 A 'War' on Asylum? - 2.1 Tropes of Invasion. - 3 Torture, Health Needs and the 'War' on Asylum. - 3.1 The Culture of Disbelief. - 4 Torture and the UK 'Detention Estate'. - 4.1 Failures of Rule 35 in Identifying Torture Victims. - 4.2 Failures of Adults at Risk Assessment. - 5 Detention, Re-Traumatisation and Repatriation Medicine. -6 Conclusion. 


\section{Introduction}

When the now 'iconic' images of shackled, humiliated and dehumanised detainees in the Abu Ghraib prison complex in Iraq were broadcast globally, in the mid-2000s, the relationship between medical power and torture in the 'war on terror' was also thrust sharply into focus. Graphic images of coalition troops photographing and posing in front of hooded, naked prisoners forced into a 'human pyramid', and of people made to wear animal collars, indicated a regime in which degradation had a defining role. The photograph of a soldier gloating over the corpse of a man who had died as a result of torture was just one picture of a network of interrogation camps in which detention by coalition forces could be fatal. Yet if there were any expectations that the presence of medical personnel may have checked this violence, these were shattered by the fact that clinicians - in some cases at least - were integral to its practice. "It is now beyond doubt that Armed Forces physicians, psychologists, and medics were active and passive partners in the systematic neglect and abuse of war on terror prisoners", wrote Steven Miles in 2009 (Miles 2009, X). And as he continued, this involved providing interrogators "with medical information to use in setting the nature and degree of physical and psychological abuse during interrogations". It involved monitoring "interrogations to devise ways to break prisoners down or to keep them alive" (2009, X). It involved pathologists holding back death certificates and autopsy reports in order to minimise the number of fatalities or cover up torture-related deaths as deaths by natural causes (Ibid). Procedures including "cramped confinement, dietary manipulation, sleep deprivation, and waterboarding" were among the practices that were "at times [...] legally sanctioned due to medical supervision" in the context of the "war on terror", according to Hoffman (2011, 1535). He continued to suggest that doctors are not just important to "modern torture methods", they are "irreplaceable".

The role of medical power in the practice of torture has been subjected to sustained critique in the context of the "war on terror". However, what follows examines the relationship between medical power and torture in the context of what has been depicted - metaphorically - as another (although to some extents related) 'war': the 'war' on asylum. According to the UNHCR (UN, UNHCR 2017, 3), between 5 and 35 per cent of those asylum seekers who have been granted refugee status have survived torture. And focusing on the UK as a case study, this chapter examines the institutional and legal structures prohibiting torture and inhuman and degrading treatment, particularly as they apply to those subject to immigration control in this context. But further, it also examines the ideological and political conditions within which claims by those seeking asylum that they have been subjected to torture prior to arrival can be (and 
have been) ignored, downplayed and denied. It examines how medical expertise has frequently been undermined in the asylum process when this expertise is utilised to add weight to asylum seekers' claims to have experienced torture. It examines how there have been attempts to narrow the definition of torture in ways which exclude people from the protections to which torture survivors are entitled. But it also explores the ways in which segments of the medical profession have been complicit in riding roughshod over existing safeguards to prevent further harm to those who have experienced torture, thus potentially compounding its effects. In particular, it examines claims that in certain contexts clinicians have administered dangerous 'care' in order to ensure the removal of people from the UK, despite them claiming that they - or their family members - face serious harm and persecution on arrival as a result of this.

In a historical discussion of medical involvement in torture, Giovanni Maio $(2001,1609)$ has noted that from its earliest incarnations one of the features of torture has been its use as an "oppressive instrument used in the preservation of power". Furthermore, whilst methods of torture have certainly "developed", and continue to do so, he argues, this "function" of torture is "especially relevant today" (1609). This chapter argues that the (mis)treatment of those in the UK who say they have been tortured preserves and is bound up with a particular manifestation of state power: the aims, rationale and dictates of immigration control. Its claims are perhaps much more mundane than the forms of direct medical complicity in torture alluded to above. But they are nonetheless important. For it is argued that the acts of omission and commission documented in this chapter expose the tensions between the rights of certain 'categories' of migrants to be afforded adequate clinical care on the one hand, and the goals and aims of immigration control itself on the other. This poses profound questions about the functions of clinical care and the ethical duties, responsibilities and obligations of clinicians, it is suggested. But as this chapter also crucially explores, this is a form of power that many within the medical profession have historically challenged, and continue to do so.

\section{A 'War' on Asylum?}

Metaphors of a 'war' on asylum in the UK are, of course, open to accusations of hyperbole. There has never been a direct declaration of 'war' on asylum by British governments (as aggressive and vitriolic as much rhetoric certainly has been). And given that neither has there ever been its formal instigation - at least in its narrowest sense - why do scholars refer to a 'war on asylum' as a term at all (see for example, Burnett 2015; Kundnani 2007; Philo, Briant, Donald 2013; Web- 
ber 2006)? Indeed, more to the point, is this even a helpful metaphor to do so? We argue it is; and the reason for this is that we suggest it goes at least some way to contextualising the (mis)treatment of those who have been (or claim to have been) subject to torture or indeed may face it. As such, before examining the infrastructural framework with regard to torture and the asylum system, the following briefly examines the extent to which an ideological construct of a 'war on asylum' has punctured understandings of such issues in the UK. Indeed, without wanting to be reductive - and certainly without wanting to suggest that this is not contested -, it nonetheless argues that such discourses cannot be ignored when examining policy frameworks with regard to the treatment of asylum seekers, not least with regard to torture.

\subsection{Tropes of Invasion}

It is well established that dominant discourses, portrayals and representations of those subject to immigration control in the UK have frequently been underpinned by a language of threat, combat and violence. Perhaps the most high-profile example of this in recent years is the infamous Sun article by the columnist Katie Hopkins, in 2015, in which she stated that "What we need are gunships sending these boats back to their own country!" with reference to migrants attempting to reach Europe by the Mediterranean Sea. According to Hopkins, "these migrants" are "like cockroaches [...] built to survive a nuclear bomb"; and she continued to suggest that "Once gunships have driven them back to their shores", their "boats need to be confiscated and burned on a huge bonfire". ${ }^{1}$

Such was the level of vitriol in this column that it prompted an intervention by the United Nations High Commissioner for Human Rights, Zeid Ra'ad Al Hussein. As he suggested, the depiction of people as "cockroaches" echoed language used by the Nazis in the 1930s and also prior to the genocide in Rwanda in the 1990s. However, from his perspective, although particularly venomous, this column was nonetheless symptomatic of "decades of sustained and unrestrained anti-foreigner abuse, misinformation and distortion" by segments of the British media. ${ }^{2}$ Indeed, since the 1990s, at least, an "asylum invasion complex" has intensified in certain media discourses in the UK, according to Tyler (2013). And as Kundnani $(2001,46)$ has docu-

1 Katie Hopkins, “Rescue Boats? I'd Use Gunships to Stop Migrants!”, The Sun, 17 April 2015.

2 https://www.ohchr.org/EN/NewsEvents/Pages/DisplayNews.aspx?NewsID=15885 \&LangID=E (2019-11-07). 
mented in a powerful analysis of this process, one newspaper editor in the late 1990s depicted asylum seekers as the "scum of the earth" who were "targeting our beloved coastline", before appealing to his readers to "clear the backdraft of a nation's human sewage". Whilst in turn, content analysis of media coverage in 2008 highlighted how asylum seekers were frequently depicted as a "threat", a danger and as harbingers of criminality (Khan 2012, 68).

Now of course, this is not to underplay the extent to which narratives are resisted. Not all media is the same, and there have been attempts to counter such tropes (for earlier discussion with regard to this, Smart et al. 2005). However, when set against this backdrop, a British newspaper cartoon appearing to liken Muslim migrants to vermin in $2015,^{3}$ for example, or headlines calling for the army to resolve the Calais "illegal immigrant crisis", ${ }^{4}$ ought not to be seen as isolated events. Rather, they are part of a pattern of coverage about which significant concerns have been expressed over many decades (Smith, Deacon 2018). In Philo, Briant and Donald's (2013, 33) content analysis of media coverage of asylum seekers and refugees in 2011, for instance, they noted repeated references to natural disasters when discussing migration, including references to being "swamped", of "soaring" numbers of people, of "waves", of "masses" and of people "flooding in". Meanwhile, in 2015, a report prepared for the UNHCR examining press coverage of the "migration crisis" in five EU states (Sweden, Germany, Italy, Britain and Spain) noted that "coverage in the United Kingdom was the most negative". Although some UK coverage was "sympathetic", it suggested, the "right-wing press in the United Kingdom expressed a hostility towards refugees and migrants which was unique" (Berry, Garcia-Blanco, Moore 2015, 10).

In such a context, then, the point is not just that Hopkins' column above is indicative of long-standing hostile outlook with regards to migration. So too is her demand for a violent response. Perhaps this has been at its most transparent with regard to migrants in Calais, France, who in at least some cases may have wanted to travel to the UK. Calais occupies a particular place in discourses around irregular migration to the UK, and is frequently held up as a 'weak point' in British immigration control. As such, when the 'jungle' camp in Calais was demolished by French authorities in 2016, this was presented by some segments of the British media as a victory in an ongoing war of attrition. Indeed, as Bhatia (2018) has noted, this was championed as "The battle of Calais" by one paper, which continued to de-

3 Aubery Allegretti, "Daily Mail Cartoon Comparing Refugees To Rats Is 'Islamophobic', Richard Burgon MP Claims", The Huffington Post UK, 19 November 2015. URL https://bit.ly/2BX7iok (2019-10-30).

4 James Slack et al., "Calais: Send in the Army”, Daily Mail, 20 July 2015. 
scribe the destruction of destitute migrants' accommodation and in some cases possessions as a victory over "invaders", as well as "illegals, transgressors and security threats".

Moreover, while such 'invasion' metaphors have certainly been resisted, it is also important to recognise that in some contexts they have not just been confined to media discourses, but have also been both reproduced and took their lead from political and establishment figures. Writing in the Daily Mail in 2001 about his time as Deputy Prime Minster between 1996-97, for example, Michael Heseltine noted that he came to three "stark conclusions" about asylum seekers. "The first is that a very large number [...] are cheats, quite deliberately making bogus claims and false allegations in order to get into this country", he suggested. The second "was that the demands on scarce housing and medical care made by dishonest 'economic migrants' (were) likely to stretch the patience of voters". Whilst the third was that "the problem of phoney asylum seekers was likely to grow as the impression spread that this country was a soft touch". ${ }^{5}$ Meanwhile, speaking to the Home Affairs Select Committee in 2006 about suggestions that the Home Office was not "fit for purpose", former Home Secretary Jack Straw suggested that the "fundamental problem" was not "the quality of the staff". It was "the nature of the individuals it has to deal with [...] They are dysfunctional individuals many of them - criminals, asylum seekers, people who do not wish to be subject to social control" (cited in Mulvey 2010, 445). As above, the issue here is not just that some political figures, in some particular contexts, have sometimes made political capital from articulating asylum seekers as a threat. Nor, in fact, is it that there has been (and continues to be) a certain level of symbiosis between media and elite discourses around asylum and irregular migration. The issue is that if the metaphor of a 'war' on asylum has some conceptual relevance, it is because in both discursive and in practical ways there is a body of evidence to suggest that individuals' rights and access to human rights have been suspended or disregarded with regard to policy in this context - including with regard to the treatment of torture. It is to this that this chapter next turns.

5 Cited in John Grayson, "The Shameful 'Go Home' Campaign", IRR News, 22 August 2013. URL http://www.irr.org.uk/news/the-shameful-go-home-campaign/\#_edn8 (2019-10-30). 
In the UK, torture is prohibited under section 134(1) of the Criminal Justice Act 1988, which defines it as the infliction of severe pain or suffering by a public official in the "performance or purported performance of his public duties". Further, the right to be free from torture is embodied in a range of international human rights instruments including the Universal Declaration of Human Rights (UDHR) (1948), the International Covenant on Civil and Political Rights (ICCPR) (1976) and the Convention against Torture and Other Cruel, Inhuman or Degrading Treatment or Punishment (CAT) (1987) (for discussion, Redress 2018, 7). Article 3 of the Human Rights Act 1998 protects an individual from mental or physical torture, inhuman and degrading treatment or punishment, and deportation/extradition to a place where there is a real risk of facing any of the above. It also obliges states to intervene to stop these rights being breached, to not rely on evidence obtained through torture and also to investigate allegations of these rights being breached (Liberty 2010). As such, whilst the above is certainly not exhaustive, it nonetheless outlines certain key principles regarding the prohibition of torture or degrading and inhuman treatment; and within these, the right to be free from torture is absolute. However, in the context of the 'war on asylum', there have been concerns repeatedly raised that these protections have been undermined in a range of interrelated ways, of which several are necessary to examine briefly here.

\subsection{The Culture of Disbelief}

First, a widely-cited 'culture of disbelief' among at least some of those involved in decision-making processes with regard to asylum cases has intensified, eroding protections against torture in the process. In 2010, for example, a case worker in one UK Border Agency locale alleged that staff involved in deciding asylum claims humiliated, degraded and tricked applicants whilst simultaneously taking pride in refusing applications. It was claimed that there was a toy gorilla in the office - colloquially known as a 'grant monkey' - which was put in the decks of those who accepted claims as a badge of shame. Moreover, the whistleblower suggested that applicants were denied basic safeguards (such as interviews being recorded), with one senior employer allegedly going as far as stating "If it was up to me I'd take them all outside and shoot them". These claims (aside from the presence of the stuffed toy) were denied by the UK Border Agency after an investigation (UK Border Agency 2010). But there can nonetheless be little doubt that over the last few decades an increasing proportion of claims have been initially refused, even in the Home Office's 
terms, wrongly. For example, between 1984 and 2017, the proportion of claims initially refused almost trebled, from around 23 per cent to 68 per cent. Yet in 2017, some 37 per cent of asylum appeals against negative decisions were allowed (Blinder 2019; Sturge 2019, 7). In turn, these appeal rates must be set against a concerted attack on the right to appeal, which has significantly undermined the ability to engage with the appeals process at all. ${ }^{6}$

Second, this culture of disbelief has fundamental implications with regard to those who claim to have survived torture; for even when clinicians provide medico-legal reports suggesting that applicants may have been subject to torture, these claims have nonetheless frequently overridden by caseworkers. This was made clear in the starkest terms in 2016, when Freedom from Torture (set up in 1985 as the Medical Foundation for the Care of Victims of Torture) examined the case files of a sample of 50 asylum seekers that it worked with, who said they had experienced torture and whose claims had initially been refused. According to their data, in 74 per cent of these cases, caseworkers substituted their own opinion for that of the clinicians with regard to the cause of injuries. In 54 per cent of the cases, the worker demonstrated a poor understanding of the Istanbul Protocol with regard to torture claims. ${ }^{7}$ And in every single case, the caseworker applied the wrong standard of proof with regard to torture (Freedom from Torture 2016, 14-15). To put it differently, suggestions that applicants had experienced torture by those with medical expertise were routinely, and erroneously, overridden by those without. What is more, this is not just confined to caseworkers in initial claims. When Tamil asylum seeker 'KV' appealed against a refusal in 2011, for example, he submitted medical opinion stating that scarring from having heated metal rods applied to the skin were "highly consistent" with his account of torture. However, the Asylum Tribunal dismissed this appeal, and the Court of Appeal went as far as saying that the clinician had "trespassed" into the role of decision maker. It was consequently left to the Supreme Court, in KV (Sri Lanka) v Secretary of State for the Home Department (2019), to rule that the lower courts had overruled the clinician wrongly, and that it was in fact part of a clinician's role. ${ }^{8}$

6 Jo Wilding, "Revealed: Legal Advice for Asylum Seekers Disappearing Due to Legal Aid Cuts", The Conversation, 15 November 2017. URL https://theconversation. com/revealed-legal-advice-for-asylum-seekers-disappearing-due-to-legalaid-cuts-86897 (2019-10-30).

7 Under the Istanbul Protocol, which was adopted by the United Nations in 2000, physicians should indicate whether lesions are "not consistent" with the trauma described, "consistent with", "highly consistent", "typical of" or "diagnostic of".

$8 \mathrm{KV}$ (Sri Lanka) (Appellant) v Secretary of State for the Home Department (Respondent) (2019), Supreme Court, UKSC 10, On appeal from: [2017] EWCA Civ 119. URL https://www.supremecourt.uk/cases/docs/uksc-2017-0124-judgment.pdf (2019-10-30). 
Indeed, whilst there may well be between 5 and 35 per cent of refugees who have been subjected to torture, there is in all likelihood a further, unsubstantiated number of people who have been subjected to torture but not been granted some form of protection. Some, no doubt, have been returned to countries from where they initially fled: a fact that was not lost on the UN Committee Against Torture in June 2019 in a damning report which noted how the UK failed to publish any data regarding the number of people removed who have said they face torture in the destination country (UN Committee Against Torture 2019, 10-12). When "credible medical evidence of past torture" is "arbitrarily rejected", it stated, this results in the "arbitrary denial of asylum claims made by victims of past torture" (2019, 10-12). Others, however, are frequently forced into complete destitution - joining the hundreds of thousands of people denied access to many mainstream services, unable to access housing and like all those in the asylum process, not allowed to work. It is somewhat ironic, to say the least, that those who have survived torture are pushed into this situation. For when Section 55 of the Nationality, Immigration and Asylum Act 2002 introduced provisions to make asylum seekers who had not claimed asylum as soon as "reasonably practical" after they had arrived in the UK to be made destitute, the High Court ultimately ruled that it violated Article 3 of the Human Rights Act as inhuman and degrading treatment (Webber 2014, 96-100). With regard to 'refused' asylum seekers though, the practice has only since intensified.

\section{Torture and the UK 'Detention Estate'}

What the above indicates then is that the culture of disbelief cited does not just manifest itself through decision-makers within the asylum system, it is also frequently given legitimacy and structured within distinct policy measures which enable it to flourish. Perhaps the clearest manifestation of this has been with regard to immigration detention. Around 24,700 people entered immigration detention in the UK in 2018. And in the Detention Centre Rules (which came into force in April 2001 and were amended most recently - at the time of writing - in 2018) there are explicit provisions to guard against the detention of torture survivors (Detention Centre Rules $2001^{\circ}$ ). In particular, Rule 35 of these rules operates as a mechanism through which doctors in Immigration Removal Centres $\left(\mathrm{IRC}^{10}\right)$ must report on detainees whose health may be "injuriously affected" by either continued detention or the conditions of detention, along with those suspected

9 http://www.legislation.gov.uk/uksi/2001/238/contents/made (2019-11-07).

10 We use the terms IRC and detention interchangeably. 
of having "suicidal ideations". The Rule 35 report is passed on via a Home Office team at detention, to a casework unit/caseworker managing the individuals' case. The report is in theory supposed to ensure that detainees at risk are brought to the attention "of those with direct responsibility for authorising, maintaining and reviewing detention" (Home Office 2019, 20). Whilst in turn, this report can - and indeed in many cases should - trigger the individual in question's release. As such, although torture is certainly not the only "category" covered in this schema (for example, it also covers having been a victim of sexual or gender-based violence and human trafficking, among other things; Home Office 2019, 20), given the above-mentioned number of detainees who potentially may have been tortured it is a key safeguard with regard to significant human rights abuses.

\subsection{Failures of Rule 35 in Identifying Torture Victims}

Whilst the principle behind Rule 35 is quite clear, its operation is far opaquer - at best. In 2012, the charity Medical Justice documented in detail the extent to which Rule 35 is overridden, ignored and ultimately frequently ineffective in its own stated terms, analysing the medical records of 50 immigration detainees who should theoretically have been covered under its remit (Tsangarides 2012). First and foremost, the report identified a lack of scrutiny, monitoring and accountability within the Rule 35 process, and this resulted in vulnerable individuals being routinely detained. Second, it uncovered that the definition of 'torture' was not adequately offered within the Rule 35 guidance, and neither was it clear as to how caseworkers should interpret and respond to the information contained within the report. Third, the majority of healthcare teams in IRCs were contracted out to private healthcare providers (although these teams nonetheless had to comply with Home Office's Detention Services Operating Manual; Home Office 2005). Despite the importance of the guidance, authorities consistently failed to conduct internal audit of the functioning of the Rule 35 process. Furthermore, the healthcare providers were not subjected to robust clinical accountability, and the safety and quality of their care was not scrutinised by independent medical bodies. Since its creation, Rule 35 has been severely criticised. For instance, over a decade ago, the Joint Commission on $\mathrm{Hu}-$ man Rights highlighted the disjoint between the policy and practice - the report stated:

We are not satisfied that the quality of healthcare currently provided to asylum seekers in detention is fully compliant with international human rights obligations, in particular the rights to freedom from inhuman and degrading treatment and to the enjoyment 


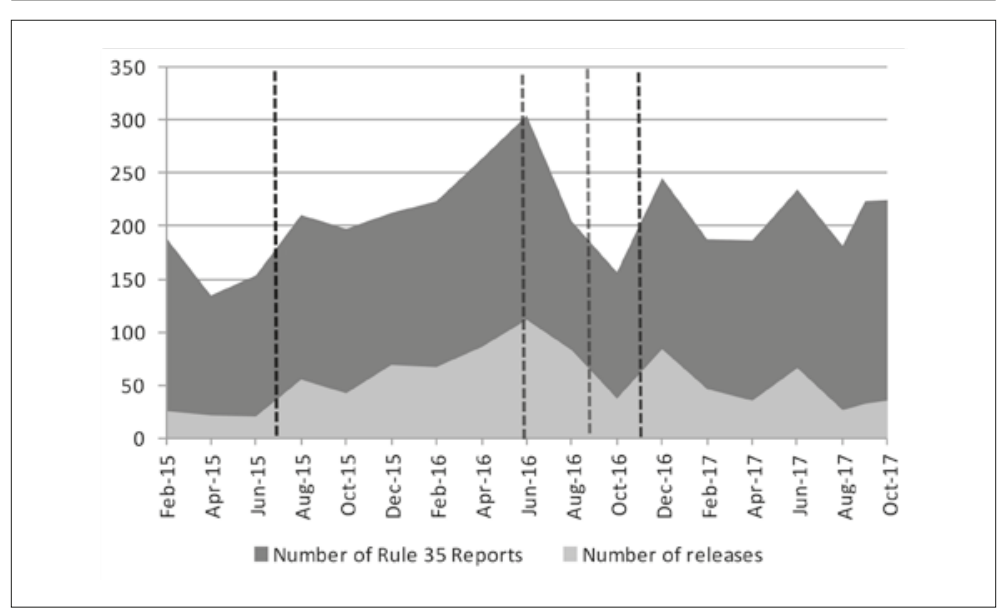

Figure 1 Total Rule 35 Reports vs. Number of Releases (source: Shaw 2018, 38)

of the highest attainable standard of physical and mental health. We are particularly concerned about gaps in care for people with HIV and with mental health problems. It is not clear that procedures for identifying and supporting torture victims work in practice. We recommend that the Department of Health establish a policy for supervising the health services that are available in detention centres, and that the standard of services should be monitored. (Joint Commission on Human Rights 2007, 101)

The above recommendations appear to have been largely ignored by the Home Office, and to this date there continues to be a system wide failure in identifying and protecting vulnerable subjects. More recently, in 2018 (and after previously highlighting inconsistencies within the Rule 35 process in 2016), a report compiled by Stephen Shaw, the former Prisons and Probation Service Ombudsman, once again expressed a strong concern and lack of confidence in the Rule 35 mechanism (Shaw 2016). It alluded to the fact that assessment was routinely rejected by the Home Office for minor inconsistencies. Furthermore, Shaw $(2016,49)$ identified that clinical staff were not competent enough to interpret "what constituted torture". Of course (and as mentioned earlier), these also indicate a widely prevalent culture of disbelief and denial, and Shaw argued for a significant cultural shift in casework areas. The above figure shows the number of Rule 35 reports completed versus small numbers of individuals released. 
The fig. 1 also directs the attention to the extent of neglect and abandonment of vulnerable people. There are a number of individuals who do not appear in the above figure, as they slip through the Rule 35 assessment altogether. This may be due to medical practitioners not being able to identify torture, assessments being conducted by an unqualified person, or the production of report consisting of descriptive and inconclusive information. In Bhatia's (2014), a voluntary sector psychiatrist explained:

I must have seen over 600 people in detention over last 5 years... 150 had medical 'care' which was disastrous, and individuals needed urgent medical attention. All the rest were almost an exception i.e. wrongfully detained... very recently I attended two torture cases: in one case, the Rule 35 report mentioned "no signs of torture" and I counted 15. The other one at Yarls Wood [IRC] stated "no scars" and I counted 30 scars, 30! [emphasis added by the interviewee]. On many occasions detention authorities have taken weeks to respond to the external [charity sector] doctor's reports, causing further damage to the detainee's health. They just don't seem to recognise what's going on... I will give you another recent example: I attended a torture victim, who was 'tasered' [shot by taser gun] in her country of origin. The detention centre nurse, with the help of an interpreter, had written over 4 pages worth of notes and not mentioned about any of the scars and concluded that "she was not tortured". Well, to start with, it should have been a doctor examining the detainee according to the detention rules - which describes that "medical practitioner shall..." which by the legal definition is a doctor on the medical register and not a nurse. (interview with Dr Aaron in Bhatia 2014, 184)

There have been several proposed amendments to immigration and asylum legislation in order to counteract the above systemic failures and culture of disbelief, of which two will be briefly outlined here: the first asking for exemption of torture victims from detention, and the second for a requirement to detail specific action taken for each Rule 35 Report. However, both these amendments were rejected and instead a decision was taken by Home Office to improve the operational guidance (Tsangarides 2012). As noted throughout this section, this has clearly not been sufficient. Moreover, in another recent report released in 2018, the Chief Inspector of Prison sampled ten Rule 35 assessments, out of which nine had detailed the evidence of torture. Nevertheless, the Home Office maintained detention of all but one of these examples. Needless to say, this amounts to the unlawful detention of torture victims, and causes the re-traumatisation of survivors (see § 5). According to figures released under the Freedom of Information Act, the Home Office were challenged in the Court on 
numerous occasions and paid nearly £10million in compensation for wrongful detention between 2011 and 2013. ${ }^{11}$ This figure increased to approximately £16million between 2013 and 2017. ${ }^{12}$

\subsection{Failures of Adults at Risk Assessment}

The 2016 Shaw Report found that "many practices and processes associated with detention are in urgent need of reform" (Shaw 2016, 91). The Conservative government publicly accepted the broad thrust of the report and subsequently introduced an "Adults at Risk" policy. The policy sets out a framework for identifying different levels of vulnerability by using a series of risk categories. Under the policy, the Rule 35 report is completed by medical practitioners and sent to the Home Office workers, who then need to conduct an assessment based on the report and release individuals. Whilst on the surface this appeared to be a streamlined and robust process, the approach was filled with flaws and often failed to identify vulnerable people altogether. In 2018, the charity Bail for Immigration Detainees (BID) released a report based on 30 detainees' casework files, analysing the success of this policy in identifying and protecting individuals (BID 2018). Eighty per cent of their sample were defined as being "at risk" by a medical practitioner. The diagnosed conditions and vulnerabilities were most commonly Post-Traumatic Stress Disorder, depression and suicidal tendencies, with two thirds recorded as being torture victims. However, the Level 3 (high risk) category was rarely designated even to the most vulnerable of clients (which in part explains the disparity in release of vulnerable individuals even if their Rule 35 is completed, highlighted in fig. 1). Only one detainee was designated as a Level 3 risk and subsequently released. BID further stated that Home Office decision makers (who are not medically qualified) were conducting the Adult at Risk assessment. Historically, the individual health concerns are considered secondary, when compared to the immigration control imperatives. The Home Office is yet to release accurate figures of how many 'at-risk' individuals were removed from the United Kingdom - in the 12 month period before and after the implementation of the Adults at Risk policy. As such, this begs the question: how many of these individuals were victims of torture, or individuals experiencing active suicidal ideation and serious mental distress at the time of removal?

11 “Compensation Paid Out for Unlawful Detention from 2011 to 2013", Home Office, 22 October 2014. URL https://bit.ly/32Zn47h (2019-10-30).

12 May Bulman, "More than $£ 500 m$ Spent on UK Immigration Detention Over Four Years", The Independent, 5 February 2018. URL https://bit.ly/2pg8EBl (2019-10-30). 
The other major issue with the policy was that it significantly narrowed the definition of torture. The Home Office drew upon the United Nations Convention Against Torture (UNCAT) definition, which was previously rejected in the case of $\mathrm{R}$ (on the application of EO, RA, CE, OE and RAN) v Secretary of State for the Home Department [2013] EWHC 1236 (Admin). ${ }^{13}$ The UNCAT definition was restrictive and it excluded categories of victims of non-state severe ill-treatment who are equally vulnerable to harm in detention as victims of state torture. In 2017, a case was bought against the Home Office by seven individuals affected by the policy. The Home Office was challenged for its use of this restrictive definition on the basis that it carried unacceptable risks for individuals who were unsuitable for detention, but would not be identified or protected. As such the consequences could be significantly detrimental. A judgement was passed in favour of the seven individuals affected by the policy that lacked "rational or evidence base", ${ }^{14}$ and the narrowing of definition was deemed unlawful.

\section{Detention, Re-Traumatisation and Repatriation Medicine}

The detention centre was the second torture that I had... the first was in DRC and was physical, the second one was psychological.

My time in detention was a nightmare... I found myself having the worst flash backs [of my time in] prison in Cameroon... It was the same event repeating itself twice in detention... I am traumatised... When I see uniformed people I get so frightened. My health is getting worse. My time in detention is something I won't wish my enemy to experience. The whole atmosphere is one of panic.

I was so depressed in detention... it reminded me of torture in Cameroon, they beat me and caused nerve injury to me.

I am traumatized from torture from my country and now feel I am being punished again. (Research Participant; Tsangarides 2012, 46)

No [any other] human being should be treated like that, you suffer in the hands of those who you think will offer support and keep you safe. There are so many victims of torture in there but the

13 https://www.landmarkchambers.co.uk/resources/cases/r-on-the-application-of-eo-ra-ce-oe-and-ran-v-secretary-of-state-for-the-home-department-2013-ewhc-1236-admin (2019-10-30).

14 "High Court Rules Government Redefinition of Torture in Immigration Detention Policy is Unlawful". Medical Justice, 10 October 2017. URL http://www.medicaljustice.org.uk/high-court-rules-government-redefinition-of-torture-in-immigration-detention-policy-is-unlawful (2019-10-30). 
system does not even have time to detect that from the detainees. People do suffer from their origin country and flee to face another torture. Detention in my point of view, it is a torture itself. (Research Participant; Tsangarides 2012, 46)

The UK continues to project itself as a beacon of human rights and refugee protection and having appropriates safeguards. However, as evidenced throughout this chapter, the migration control regime and treatment of at-risk people suggests otherwise. Detention is one of the most contentious areas, not only due to the fact that UK is the only European region to subject people to indefinite confinement - but also because detention is inherently unhealthy (Bosworth 2016) and historically the procedures and practices to safeguard vulnerable people have in many cases miserably failed. For torture victims, the very process of detention can result in re-traumatisation and the exacerbation of mental distress, and as such it is little surprise that individuals experiencing treatment in confinement as describe a "second torture".

Torture is a reason why Rule 35 is triggered; however, as Bhui argues (quoted in BID 2018, 24), torture is also one of the few reasons why it could be considered. He draws cases to explain that, 60 people who had been considered to be at risk of self-harm, of whom half had been considered to be at high level of risk and placed under constant supervision by the staff, were not offered Rule 35 assessment on suicidal ideation. Indeed, the treatment of victims of torture and those experiencing severe mental distress in IRCs indicates a sheer lack of willingness to protect people from further harm. In Bhatia's research (2014), it was noted that medical negligence and denial of care had serious repercussions. The individuals were not processed correctly and medical staff did not take prompt actions - thereby, amplifying individual suffering - as the following psychiatrist argued:

Detainee was suffering from a serious underlying medical condition; however, s/he continued being detained. I'll tell you what was most shocking - S/he was on anti-epileptic medication which ran out. S/he was then transferred from prison to Yarls Wood [IRC], where the nurse is supposed to see them within 2 hours - which eventually happened. The nurse then mentions in the report that "detainee CLAIMS to be epileptic". They made no effort to obtain the medical file from the prison, which they should have done, nor did they provide anti-epileptic medication, which they should have done... She ended up having four fits attacks in that month, before they recognised that she might as well be epileptic. The individual was eventually released and received refugee status, and then filed a case for wrongful detention with the help of her lawyer... medical negligence in the detention centre was extra ordinary... (interview with Dr Aaron in Bhatia 2014, 188) 
There are also cases where the severity of individual condition is known to the health practitioners, and yet they are not treated adequately and appropriately, and given medication only to somewhat 'manage' their condition. This is done so as to continue detention, even if it has a disastrous health outcome for the person. As a charity manager mentions in Bhatia's research (2014):

Over last 3 months we had two cases, both suffering acute psychosis and one of them almost turned like a vegetable. His condition was that bad. [Individual was] on average 3 years in detention, because they couldn't deport him to Zimbabwe. He was refused bail over 5-6 times and his condition turned very bad.... [continues] the other man was kept in detention for 2 years. The detention centre staff knew that he was suffering from psychosis for over a year and his condition turned so bad that he could not even give us instructions. We had to eventually close his file for some months. And then he gave us a call again at some point asking for help. We eventually arranged for Human Rights solicitors, and bailed him last month... Surprisingly we had a medical report from the Home Office stating that he is schizophrenic - then why was he detained? (interview with Dr Aaron in Bhatia 2014, 187)

It is in this context that Burnett (2010) has questioned whether medical care within the detention estate can be conceptualised as a suboptimal form of repatriation medicine. Here, the role of medical expertise is not so much treatment as the facilitation of immigration control. Immigration controls have constantly attempted to reduce asylum seekers from ontological beings to ontic beings, a mere object of security, power and control. And in this regard, at least in some cases, medical power and expertise within the detention has been mobilised in attempts to remove people from the UK. In 2010, for example, research conducted on the experiences of 141 children and young people in the detention estate identified that at least 50 were facing removal "without being adequately protected, [that] were administered with the wrong drugs prior to removal, or were removed without being adequately immunised" (Burnett 2010, 26). As this research documented, some children were being given inappropriate malarial prophylaxis - known to have potentially dangerous side-effects - in order to ensure that they could be removed on certain flights. Indeed, according to one parent, her child was left coughing up blood as a result.

This is not to suggest that these children and young people had necessarily all arrived in the UK as a result of the experience or threat of torture. Indeed, while some had faced threats prior to arrival, many had been born in the UK itself. It is to say, however, that in some cases their parents said they had experienced torture prior to arrival in the UK; and in all cases said they feared for their life if 
they were to be returned. At the very least, this raised questions that remain of central relevance about the role of medical power and the ethical duties of clinicians in the context of immigration and asylum policy, not least with regard to torture.

\section{Conclusion}

As the sociologist Zygmunt Bauman argued, "[o]ne is tempted to say that were there no immigrants knocking at the doors, they would have to be invented [...]. Indeed, they provide governments with an ideal 'deviant other'" (Bauman 2013, 56). This chapter has examined how the creation of a "deviant other" in the context of a metaphorical war on asylum has had significant material impacts. Central to its focus has been the undermining of provisions against torture in a myriad of ways, but with specific reference to medical power. It is no secret that a significant proportion of those seeking asylum have experienced torture, and yet it is clear that provisions to protect against this have frequently been undermined. That this can be traced to both the individual actions of caseworkers and clinicians as well as broader political drives and policy documents indicates something about the ways in which institutional practices are located within broader structural frameworks. To put this in another way, measures to combat this must organise on both micro and micro levels at once.

The consequences of not doing so are clear. The damage done to those who have survived torture in the context of this 'war on asylum' has time and time again proven to be catastrophic. It has intensified the harms of torture and compounded its effects. Yet whilst this chapter has argued that medical power is a key terrain upon which such damage can be wrought, it is also essential to acknowledge that it is also a key terrain upon which it is being resisted. It is within such networks, in conjunction with those from migrant communities and those who have sought asylum, that attempts to uphold protections against torture are being connected to attempts to resist the state power embedded in the 'war on asylum' itself.

\section{Bibliography}

Bauman, Zigmunt (2013). Wasted Lives: Modernity and its Outcasts. New Jersey: John Wiley \& Sons.

Berry, Mike; Garcia-Blanco, Iñaki; Moore, Kerry (2015). Press Coverage of the Refugee and Migrant Crisis in the EU: A Content Analysis of Five European Countries. Cardiff: Cardiff School of Journalism, Media and Cultural Studies.

BID, Bail for Immigration Detainees (2018). Adults at Risk: The Ongoing Struggle for Vulnerable Adults in Detention. London: Bail for Immigration Detainees. 
Blinder, Scott (2019). "Migration to the UK: Asylum and Refugees". Migration Observatory, 4 January. URL https://migrationobservatory.ox.ac.uk/ resources/briefings/migration-to-the-uk-asylum (2019-10-30).

Bhatia, Monish (2014). Resisting 'Bare-Life'?: Impacts of Policies and Procedures on Asylum Seekers and 'Illegal' Migrants [Doctoral Dissertation]. Huddersfield: University of Huddersfield.

Bhatia, Monish (2018). "Social Death: The (White) Racial Framing of the Calais 'Jungle' and 'Illegal' Migrants in the British Tabloids and Right-Wing Press". Bhatia, Monish et al. (eds), Media, Crime and Racism. London: Palgrave Macmillan, 181-212.

Bosworth, Mary (2016). "The Impact of Immigration Detention on Mental Health: A Literature Review". Review into the Welfare in Detention of Vulnerable Persons, Cm 9186. London: HSMO. URL https://ssrn.com/abstract=2732892 (2019-10-30).

Burnett, Jon (2010). "Repatriation Medicine". Criminal Justice Matters, 82(1), 26 8. DOI https://doi.org/10.1080/09627251.2010.525929.

Burnett, Jon (2015). "The War on Welfare and the War on Asylum". Race \& Class, 57(2), 96-100. DOI https://doi.org/10.1177\%2F0306396815595919.

Freedom from Torture (2016). Proving Torture. Demanding the Impossible. Home Office Mistreatment of Expert Medical Evidence. London: Freedom from Torture. URL https://www.freedomfromtorture.org/sites/default/ files/2019-04/proving_torture_a4_final.pdf (2019-10-30).

Hoffman, Steven (2011). "Ending Medical Complicity in State-Sponsored Torture". the Lancet, 378(9802), 1535-7. DOI https://doi.org/10.1016/ S०140-6736(11)60816-7.

Home Office (2001). Detention Centre Rules 2001: Statutory Instrument 238. London: The Stationary Office.

Home Office (2005). Detention Services Operating Standards Manual. London: Home Office.

Home Office (2019). Adults at Risk in Immigration Detention: Version 5.0. London: Home Office.

Joint Committee on Human Rights (2007). The Treatment of Asylum Seekers, Tenth Session 2006-2007. London: Joint Committee on Human Rights. URL http://www.publications.parliament.uk/pa/jt200607/jtselect/jtrights/81/81i.pdf (2019-10-30).

Khan, Amadu Wurie (2012). "UK Media's Pathology of the Asylum Seeker \& the (mis)Representation of Asylum as a Humanitarian Issue", in "The 1951 UN Refugee Convention - 60 Years On", spec. issue, eSharp, 54-86. URL https://www.gla.ac.uk/media/Media_237469_smxx.pdf (2019-10-30).

Kundnani, Arun (2001). "In a Foreign Land: The New Popular Racism". Race \& Class, 43(2), 41-60. DOI https://doi.org/10.1177/0306396801432004.

Kundnani, Arun (2007). The End of Tolerance? Racism in 21st Century Britain. London: Pluto Press.

Liberty (2010). A Parliamentarian's Guide to the Human Rights Act. London: The National Council for Civil Liberties. URL https://www. refworld.org/ pdfid/4ed640552. pdf (2019-10-30).

Maio, Giovanni (2001). "History of Medical Involvement in Torture - Then and Now". The Lancet, 357(9268), 1609-11. DOI https://doi.org/10.1016/ S0140-6736(00) $04729-2$.

Miles, Steven (2009). Oath Betrayed: America's Torture Doctors. 2nd ed. Berkley: University of California Press. 
Mulvey, Gareth (2010). "When Policy Creates Politics: The Problematizing of Immigration and the Consequences for Refugee Integration in the UK". Journal of Refugee Studies, 23(4), 437-62. DOl https://doi.org/10.1093/jrs/feq045.

Philo, Greg; Briant, Emma, Donald, Pauline (2013). "The Role of the Press in the War on Asylum". Race \& Class, 55(2), 28-41. DOI https://doi. org/10.1177/0306396813497873.

Redress (2018). The Convention against Torture and Other Cruel, Inhuman or Degrading Treatment or Punishment: A Guide to Reporting to the Committee against Torture. London: Redress.

Shaw, Stephen (2016). Review into the Welfare in Detention of Vulnerable Persons: $\mathrm{Cm}$ 9186. London: Crown Copyright.

Shaw, Stephen (2018). Assessment of Government Progress in Implementing the Report on the Welfare in Detention of Vulnerable Persons: Cm 9661. London: Crown Copyright.

Smart, Kate; Grimshaw, Roger; McDowell, Christopher; Crosland, Beth (2005). Reporting Asylum: The UK Press and Effectiveness of the PCC Guidelines. London: ICAR. URL https://www.crimeandjustice.org.uk/sites/ crimeandjustice.org.uk/files/ReportingAsylum.pdf (2019-10-30).

Smith, David; Deacon, David (2018). "Immigration and the British News Media: Continuity or Change?". Sociology Compass, 12, 1-13. DOI https://doi. org/10.1111/soc4.12618.

Sturge, Georgina (2019). Asylum Statistics: Number SN01403. London: House of Commons Library.

Tyler, Imogen (2013). Revolting Subjects: Social Abjection and Resistance in Neoliberal Britain. London: Zed Books.

Tsangarides, Natasha (2012). "The Second Torture": The Immigration Detention of Torture Survivors. London: Medical Justice. URL http://www.medicaljustice.org.uk/wp-content/uploads/2016/03/the-secondtorture-full-version.pdf (2019-10-30).

UK Border Agency (2010). An Investigation into the Allegations Made by Louise Perrett About Her Experiences Working for the UK Border Agency. London: UK Border Agency.

UN Committee Against Torture (2019). Concluding Observations on the Sixth Periodic Report of the United Kingdom of Great Britain and Northern Ireland: $C A T / C / G B R / C O / 6$. Geneva: United Nations.

UN; UNHCR (2017). Torture Victims in the Context of Migration: Identification, Redress and Rehabilitation = Report on the Third Annual Expert Workshop (Geneva, 26-27 April 2017). URL https://www.ohchr.org/Documents/Issues/ Torture/UNVFVT/UNVFVT_ExpertWorkshop2017.pdf (2019-11-07).

Webber, Frances (2006). Border Wars and Asylum Crimes. London: Statewatch. Webber, Frances (2014). Borderline Justice: The Fight for Refugee and Migrant Rights. London: Pluto Press. 



\title{
Dreams and Nightmares. The Legal Legacy that Authorized Civil Detention Centers in the US
}

\author{
Aaron Moss \\ Federal Public Defender Office, Northern District of West Virginia, USA \\ Steve Parks \\ University of Virginia, USA \\ Lori Shorr \\ Temple University, Philadelphia, USA
}

\begin{abstract}
Astudy of the juridical and legislative creation of the United States immigration framework premised on a concept of the nation-state as protective of the whitemale of property, with a case-study of its enactment through the policies of the Trump Administration. The essay concludes with a consideration of alternative governance structures to protect the political rights of citizens/non-citizens of a political state.
\end{abstract}

Keywords Refugee. Asylum. Political rights. Human rights. Nation. Nation-State.

Summary 1 Introduction. - 2 The Dreams of the "Good Refugee". - 3 The Dreamwork of Nation-States. -4 The Nightmare of the Refugee in the Nation-State. - 5 The False Dream of International Human Rights. -6 Beyond Dreams and Nightmares. 


\section{Introduction}

[T] he exemplary moment of sovereignty is the act of deportation. (Hannah Arendt)

The photographs and stories have gone viral, sparking outrage over an inhumane immigration system operating in the United States.

South and Central American refugees, packed in cages, covered in sheets made of metallic foil.

Migrant children lying on concrete floors or looking out from behind iron-mesh enclosures asking for their parents.

The Justice Department insisting it is not required to provide soap, toothbrushes, or adequate bedding to children in immigration custody.

A mural of President Trump plastered to the wall of an immigration detention center that reads, "[s]ometimes losing a battle you find a new way to win the war".

These moments make up pieces in the mosaic of American immigration. Collectively, they tell a story that enables a particular cultural narrative on the current meaning of the United States. They tell the story of a sovereign entity born from a bygone era, beset with structural racism, and at battle with its sense of self. A national identity established by the state and its borders and historically premised on whiteness that embedded in its political, legal, and judicial discourses the non-citizen as 'alien', an alien who is excluded first through denial of rights then through deportation from the body of the nation. In the context of immigration, this exclusion of rights means ignoring the U.S. Constitutions' Eighth Amendment and diminishing due process protections of the Fifth and Fourteenth Amendment. It makes for an ill-conceived immigration system. It creates humanitarian crises.

The non-citizen as "alien" ${ }^{1}$ is a linguistically deviant device that dominates the immigration process (Cunningham-Parmeter 2011). It establishes a rhetorical framework around immigration that dehumanizes the migrant, refugee, or asylum seeker to distract from the constitutional crisis produced once found in the United States. Culturally, the word 'alien' is grounded in "the Latin words alienus and alius, which mean 'of or belonging to another person or place', 'hostile', 'strange', and 'other'” (Cunningham-Parmeter 2011, 1570; Skeat 1968). Thus,

1 See 8 U.S.C. $§ 1101(a)(3)$ : “[t]he term alien means any person not a citizen or national of the United States". 
when the law speaks of aliens, it speaks of "dangerous others who are marked by their strangeness" (Cunningham-Parmeter 2011, 1570). The United States Congress created the 'alien' to diminish the non-citizen. ${ }^{2}$ Courts, then, took the term 'alien' and twisted it into something nonhuman. Non-Citizen aliens have been described like hunted and caught animals, "who succumb to the lure" (Michigan Department of State Police v. Sitz, 1990). Aliens have been described as inanimate objects, "imported into this country" (United States v. Brignoni-Ponce, 1975). Aliens have been described like extraterrestrial body snatchers, who undertake a "silent invasion" (United States v. Ortiz, 1975).

We will argue that the 'alien' has not so much invaded our land but has been produced by a governmental state that imagines itself the protector of the 'nation-state'. That linkage of governmental state/nation-state enables an understanding of the current political moment in the United States. Through this conceptual framework, we can articulate that, while popular to image the Trump Administration's immigration policy as an aberrant exception in United States jurisprudence, the inhumane treatment of non-citizens took root in the nation's imagination more than a century ago. It is the result of a carefully crafted body of legislative law and judicial interpretation founded in a logic that interlinks coloniality, nationality, and self-sovereignty; a system premised on whiteness as the subject of law and the non-white peoples as the 'other', the outsider to be denied entry and equal access to legal protections. In this way, the 'stateless' individual, the 'non-citizen', the 'refugee', the 'alien', are all bricks in a border wall written into the system. To create a political context that provides such individuals with rights and legal recourse, we will argue means breaking down fundamental connections between the state and nationalism; it means imagining a different legislative framework. It is to dream of a state moving beyond nationalism, beyond the current nightmare.

To draw out the contours of the current immigration system, this article begins by tracing the story of Liliana Velásquez, whose memoir Dreams and Nightmares (Velásquez 2016), traces her journey from Guatemala to the United States. While personally harrowing, Liliana represents her story as a triumph of how a "model" and "humane" refugee process might produce future citizens. Within her narrative, children pulled away from parents, placed in cages for months, seems an aberration. This article, however, seeks to demonstrate the common legal framework that produces these more common than not moments. In doing so, it exposes the fundamental linkage of the state with white nationalism, denying any current or previous state actor a claim of innocence or ignorance with regard to the current humanitarian crisis. Here, the article draws upon critical race theory within 
the United States to, ultimately, critique the Westphalian nation-state system. The article concludes by positing 'critical regionalism' as an alternative framework to the racially homogenous nation from which the state might govern, a framework informed by a vernacular sense of political agency. The overarching hope is to begin a conversation on how to secure fundamental human rights of all individuals.

\section{The Dreams of the "Good Refugee"}

Liliana could have ended up in a cage. Instead, she received a green card. Liliana Velásquez left her home in Villaflor, Guatemala, alone, at the age of 14, to escape the mental and physical abuse occurring within her family. She sought refuge in the United States. That same year, in 2012, there were 10,146 unaccompanied children making a similar journey. In 2013, the number of unaccompanied minors doubled to $20,715 .^{3}$ This surge in children seeking status in the United States relates to the ongoing economic and political crises that mark modern Central and South America. Crises which often result from neo-liberal policies enacted by global corporate entities that are endorsed by both Democratic and Republican legislators in the United States (Harvey 2005; Mignolo 2013). Exploiting the Americas for the benefit the United States has always been a bipartisan effort.

Liliana's individual journey, then, must be understood within a context where global forces both motivated her journey, then, reframed her identity as an 'unaccompanied minor' and 'alien'. These classifications are deeply intertwined with the cultural conscious of the United States. The language is deliberate and describes a political motivation. A political motivation to 'blame the victim' for needing to seek status in the United States over the turmoil in their own country and without regard for the catalyst. Donald Trump's 2015 presidential campaign captured the political moment: "When Mexico sends its people, they're not sending their best [...] They're sending people that have lots of problems, and they're bringing those problems with us. They're bringing drugs. They're bringing crime. They're rapists. And some, I assume, are good people". ${ }^{4}$

3 According to Factcheck.org, a project of the Anneberg Public Policy Center, "[t]he surge in unaccompanied children from Central America is largely due to increased violent crime in the 'northern triangle' (Guatemala, Honduras, and El Salvador). A July 3 report by the nonpartisan Congressional Research Service says 48 percent of apprehended children 'said they had experienced serious harm or had been threatened by organized criminal groups or state actors, and more than 20 percent had been subject to domestic abuse'. Honduras has the highest murder rate in the world" (Shapiro 2014). Also see the June 2018 Council on Foreign Relations report, "Central America's Violent Northern Triangle” (Labrador, Renwick 2018).

4 “Here's Donald Trump's Presidential Announcement Speech", Time, 16 June 2015 URL https://time.com/3923128/donald-trump-announcement-speech (2019-07-28). 
Luckily, Liliana was understood as one of the "good people" by those involved in the immigration system at that time.

As noted in her memoir, Liliana begins her journey by trying to escape family abuse by herself. She then joins up with a small cadre of individuals attempting to reach the United States. Both collectively and individually, these people are robbed at gunpoint, exploited by corrupt police/army officers, and consistently threatened with sexual abuse. Finally, Liliana crosses over into the United States and is immediately captured by federal agents, who she describes as dressed like soldiers; 'soldiers' who handcuff her and force her to march for an hour to reach the Border Control car, before taking her to an immigration detention center in Tucson, Arizona. There, she witnesses, "children, mothers, men, women - some sleeping on floors some sitting up, some covered in plastic to protect them from the rain" (Velásquez, 2016, 130). She faces the chilling reality of "so many people who were going to be deported to their country" (103).

Importantly, these experiences occur during the Obama Administration. Of course, it should also be noted that the Obama Administration established an in-country refugee/parole program, Central American Minors, as part of the United States' Refugee Admissions Program:

The Central American Minors (CAM) Refugee/Parole Program aims to provide a "safe, legal, and orderly alternative to the dangerous journey" that many unaccompanied children have taken to the United States. It allows certain parents who are lawfully present in the United States to request refugee resettlement for their children who are still residing in their countries of origin. Children who are found to be ineligible for refugee status but are at risk of harm can be considered for parole, which allows individuals to be lawfully present in the United States temporarily. (Meyer et al. 2016, 9)

It also attempted to support economic growth within such countries through economic aid, linked to enhanced border and security requirements. While seemingly more humane than the Trump Administration - who ended the domestic abuse allowance that ultimately authorizes Liliana's ability to remain -, both Administration's premise their work on 'securing the border', a framework which will be discussed further below. Thus, the evils of immigration under the Trump Administration are not simply aberrations. They are just the latest symptoms of the same old ill-conceived immigration system.

Unlike the children who remained in the cages of Tucson, Liliana continues deeper into the heartland of the United States immigration system. As her journey progresses, she begins to fashion a narrative where the system's ability to work rests not so much in legal avenues; but instead, on the individual humanity of those directly involved in 
her case. For instance, Lilian is transported to Phoenix, a trip she describes as being marked by kindly immigration officers who provide her with an influx of snacks. In Phoenix, she faces placement in a new form of immigration detention. A program known as the House of Dreams. Inside the House of Dreams, she finds caring individuals who assuage here fears and provide her medical treatment. She even gains access to a lawyer, despite that fact that she has no constitutional right to counsel. Her lawyer then spends months working to have Liliana reunited with her brothers in North Carolina. When that fails, she finds placement in a foster family from Philadelphia. Up to this point, each moment of the process seems designed to create a safe and caring environment for Liliana. She experiences a system that appears to act in her best interest.

Indeed, it is only in Philadelphia, when placed in a North Philadelphia home, where she encounters direct mistreatment - the foster family refuses to share food with her, forces her to provide childcare, and limits her movement outside of the home. Lilian is saved from this situation when her social worker - who had previously recommended not creating an issue about her living conditions - breaks with standard practice and ensures access to La Puerta Abierta, a center which provides mental health services for non-citizens. Here Liliana meets Layla, a psychologist at the center, who takes up a special form of advocacy. Layla successfully petitions the court to allow Liliana to join her family. Through her commitment to hard work, to never giving up (as expressed in her memoir), Lilian is able to secure a green card, finish high school, and enroll in college. She now travels across Philadelphia and the United States, sharing her story with grade school and college classes. Considered within her self-defined history, Liliana's nightmare has turned into the American Dream.

\section{The Dreamwork of Nation-States}

We start with pictures and stories for several reasons: first, nations and nation-states are built upon their origin stories, stories which make clear who founded a country and, thus, who are rightful citizens. These stories are, as they say, "written by the winners of history". Where such stories start, what events are included or omitted, what is emphasized, and who are cast as heroes, all act to undergird the status quo. These stories, when left unexamined, authorize the mistreatment and even torture of those portrayed as the outsider or intruder by the nation-state. Consider the power of the story just told - a story which ended with a refugee using her experience to gain access to college, to speaking engagements. Liliana's story exists within the narrative of the "good refugee" within the current public discourse of the United States. For as noted at the outset, Lil- 
iana describes a journey through a Central America replete with abusive family member, rapists, bad cops. Indeed, it is through the exceptional few, such as a kind coyote, that the cultural/political geography is established. In the United States, by contrast, Liliana describes a terrain full of kind committed individuals, with the notable exception of the foster family who acts as proof of 'the general rule'.

Essentially, through Liliana, we learn, the refugee/immigration system 'works' for those dedicated to working hard, not giving up, and believing in traditional notions of family, home, and education. By the end of her memoir, she has transformed the image of those left caged in Tucson. What once represented images of abuse, punishment, or torture, now represent an acknowledgement, even if reluctant, that some individuals need to be deemed inadmissible, detained, and deported back to their country of origin, perhaps even back into the violence they (like Liliana) sought to escape. That is, there is a need for a border, a 'wall', that can protect current inhabitants from countries that do send "their worst". Thus, the narrative of the nation-state (and its relationship to the state) is important for how it reframes the chaotic and diffuse reality of existing bodies spread across a multiple terrains into a set of concept-metaphors that create a stable identity, a habitus called 'citizen' who is then granted the benefit of particular unalienable rights

And the dream work of the United States, the desire which informs the citizen habitus, as with all states, is to create a homogeneous nation. As Judith Butler writes, reflecting on the work of Hannah Arendt:

Arendt argues that the nation-state, as a form, that is, as a state formation, is bound up, as if structurally, with the recurrent expulsion of national minorities. In other words, the nation-state assumes that the nation expresses a certain national identity, is founded through the concerted consensus of a nation, and that a certain correspondence exists between that state and the nation. The nation, in this view, is singular and homogeneous, or, at least, it becomes so in order to comply with the requirements of the state. (Butler, Spivak 2011, 30-1)

Historically this exclusion from the United States' nation-state has been enacted upon Indigenous populations already present in North America and African populations brought to this continent, both of whom are seen as illegitimate inhabitants. In a post-Civil Rights Era, there is a popular narrative that such legal/political exclusions have been removed; whiteness decentered. That equality under the law now exists. Critical race theory, however, has been audacious in its critique of the very foundations of the United States' legal system, to its commitment or lack thereof, for the equal protections of all of 
its citizens. Focusing primarily on how the legal system impacts descendants of formerly enslaved African communities, the work of critical race theory scholars meticulously articulates how the most basic assumptions and tenets of the United States' legal and legislative systems assume the legitimate subject of these structures continue to be white. That, in fact, the popular frameworks of 'whiteness' are constructed in part through the power of legal discourses to invest/ disinvest humanity in particular populations.

Recently the paradigms of critical race theory have been extended to articulate the racially exclusionary immigration laws, which also are understood as reinforcing the subordination of domestic minority groups. In equal parts, it recognizes that the Trump Administration shares fair blame for the egregious and inhumane treatment of non-citizens; but also, the Trump Administration is not the first to enact racially exclusive immigration policy nor attempt to justify human rights abuse (Johnson 2002). For the overarching concern about these abuses does not start or stop with the Trump Administration. The concern centers on the legal and legislative rulings that long ago created a rhetorical comparison to conflate two concepts into one legal identity that defined all non-citizens, be they: refugees, asylum seekers, migrants, or criminals, as the same 'alien' entity not worthy of protection. Those dehumanizing associations are deeply engrained into the cultural conscious of the United States and do more to distort notions of justice in the immigration system than any one administration.

Here, the concept of 'statelessness' can be an important lever of analysis. Critical race theory deconstructs 'statelessness' as a racially informed concept. It is a powerful explanatory legal concept used by lawyers and critical theorists alike. 'Statelessness' means, "a person who is not considered as a national by any Sate under the operation of its law;" it is "a person who is not only homeless but productionless" (UN 1954 Article 1). Provided that in the United States the concept of the 'nation', itself, is premised on 'whiteness', then what rights remain to empower or protect those crossing without documentation from Central or South America, from non-European heritage nations? Through a consideration of the 'stateless' individual, we can ask what is the governmental paradigm which requires just and humane treatment of these individuals?

Critical race theory invokes the strategy of the 'counter-story' as a way to begin to argue for a different form of governance. In this regard, critical race theory shares a concern with decoloniality, which, in response to coloniality's imposition of the white male of property as the subject of rights, rights infused with global capitalist values, attempts to learn from indigenous forms of knowledge/communal structures. (The very values which, as discussed above, produced the political/economic crises driving immigration in Central America). For conceptual schools, counter-stories call into question the 
legitimacy of now existing legal/political prejudices. Indeed, Delgado, one of the first critical race theory theorists, highlights how language helps shape human perception. With words, humans comprehend meaning in the world that surrounds them. He writes: "Stories, parables, chronicles, and narratives are powerful means for destroying mindset - the bundle of presuppositions, received wisdoms, and shared understandings against a background of which legal and political discourse takes place" (Delgado 1989, 3).

Our first rendering of Liliana's memoir was to read it within her own terms as a successful story of a child who crossed borders and cultures to gain certain citizenship rights (a green card). As just discussed, this story rested upon a larger governance structure premised on a coloniality that premised 'white male' subjectivity as both the subject of rights and national identity. Returning to Liliana, now framed as 'stateless' within the workings of a historically determined juridical-political system, we want to articulate the system in which such habitus of citizenship rights are offered or denied. And in doing so, we hope to ultimately provide a space to frame a counter-narrative to the legitimacy of the nation-state as 'protector of human rights'.

\section{The Nightmare of the Refugee in the Nation-State}

By the time Donald Trump took office, Liliana already had lived in the United States for four years. During this time, the immediate crisis of rising rates of unaccompanied minors crossing the border lessened. From 2014 to 2016, the numbers dropped from 51,000 to 18,500 (Kandel 2017). This drop correlates with an approximately twenty-year trend. To be certain, in 2000, the monthly average of unaccompanied minors crossing the border equaled 71,000 to 220,000 . Yet by 2018, those numbers had dropped to between 20,000 to 40,000 . In that time, the reasons for crossing into the United States also changed: what started as primarily economic reasons increasingly turned into reasons concerning political hardship. Despite the numbers, there is an exigency to continuing to produce the stateless as a means to reaffirm the legitimacy of the nation-state proper. There will be so long as the nation-state remains. For, as Judith Butler argues, "[t]he state derives its legitimacy from the nation, which means that those inhabitants who do not qualify for 'national belonging' are regarded as 'illegitimate' inhabitants" (Butler, Spivak 2011, 30-1). All "subsequent status that confers statelessness on any number of people becomes the means by which they are at once discursively constituted within a field of power and juridically deprived" (2011, 31). Thus, despite the numbers, there is an exigency to continuing to produce the stateless as a means to reaffirm the legitimacy of the nation-state proper. This need is particularly urgent today at a time when 1) the 
free circulation of global capitalism has called the sovereignty of the nation-state into question; 2) the failed neo-liberal projects in the Global South has caused new massive global migrations; and 3) all of this has ignited the fear of disenfranchisement in those workers displaced in the Global North.

Undoubtedly, the Trump Administration has taken to this task with great vigor. Intentionally implementing policies and arguing agendas designed to incentivize would-be refugees and migrants to stay in their home countries. These tactics include, but are certainly not limited to, the utilization of facial recognition software to sweep civilian databases, the militarization of ICE (United States Immigration and Customs Enforcement), coordination with local law enforcement, pretextual traffic stops, and increased reliance on criminal statute. Consequently, approximately 39,000 people are now being held in immigration detention centers. Around 2,000 are children. Many of these children are being separated from their parents through the utilization of 8 U.S.C. $§ 1325$, which makes it is a federal crime to improperly enter the United States. ${ }^{5}$ Use of this statute means the initiation of criminal proceedings. That process all but guarantees the separation of families. For the person charged with violating 8 U.S.C. § 1325 will be transferred from ICE custody into the custody of the United States Marshal Service (USMS) to stand trial. Whereas ICE custody takes place at immigration detention centers, USMS custody typically takes place at regional jails. This process can take years to complete. In the meantime, the children are left in immigration detention centers, where, as noted above, they live in tents or cages without simple necessities for health, hygiene, and safety. ${ }^{6}$ In these conditions, people die. Indeed, under the Trump Administration at least three minors have died while being held in immigration detention centers. As a result, Sanctuary Cities (which refuse to support ICE activities) as well as consistent protest have emerged across the United States.

Throughout, those residing in the United States have learned two important lessons about its immigration system.

First, it is a system of dehumanization, confinement, aggressive policing, punishment, and deportation. It is a system of contradictions and competing rhetoric where non-citizens found in the United States are subjected to torturous psychological, emotional, and phys-

5 See 8 U.S.C.A. § 1325: “[a]ny alien who (1) enters or attempts to enter the United States at any time or place other than as designated by immigration officers, or (2) eludes examination or inspection by immigration officers, or (3) attempts to enter or obtains entry to the United States by a willfully false or misleading representation or the willful concealment of a material fact, shall, for the first commission of any such offense, be fined under Title 18 or imprisoned not more than 6 months, or both, and, for a subsequent commission of any such offense, be fined under Title 18, or imprisoned not more than 2 years, or both".

6 For example, the Trump Administration defines soap and toothpaste as non-necessities. 
ical abuse while detained pending deportation proceedings. Similar to what happened to Liliana along her journal, pistols may be held to the heads of refugees. Cement floors may act as their beds. Death may come to them before deportation does.

Second, no matter the severity of the treatment, it likely will not be found to violate the legal standards of punishment or deprivation under the United States Constitution. These individuals will not be accorded the constitutional safeguards relating to cruel and unusual punishment or the deprivation of life and liberty. It looks like punishment. It feels like deprivation. It may well be torture. But it is overwhelmingly likely to be determined constitutional.

While the Trump Administration has made their "tough on immigrants" stance a central tenet of policy, Trump did not invent this system. It is allowed within the fabric of the legal system and supported by the legislative intent of immigration policies. In part, the ability to treat non-citizens with such cruelty is due to the fact that for more than a century the United States has defined the deportation process, including detention, as a civil process exercised by the power of Congress. ${ }^{7}$ This Congressional power is rooted within notions of white supremacy, xenophobia, and the Supreme Court's 1893 decision in Fong Yue Ting. There, the Court upheld a provision in the Chinese Exclusion Act of 1892, which required a Chinese person, "claiming the privilege of remaining in the United States, to prove the fact of his residence here at the time of the passage of the act by at least one credible white witness" (Fong Yue Ting v. United States, 1893; emphasis added). Here, we see the resonance of white-vouching for immigrants echoed in Layla's endorsement of Liliana.

Within this racist framework, the Supreme Court accepted the maximum that this nation had an absolute and unqualified right, a "power, as inherent in sovereignty, and essential to self-preservation, to forbid the entrance of foreigners within its dominions, or to admit them only in such cases and upon such conditions as it may see fit to prescribe" (Fong You Ting v United States, 1893). That power falls to Congress. It is "recognized that the determination of a selective and exclusionary immigration policy was for the Congress and not for the Judiciary" (Harisiades v. Shaughnessy, 1952). There it has remained. No matter how crude, cruel, xenophobic, and racist, the responsibility of immigration laws reside in Congress, even when "such determination may be deemed to offend American traditions and may, as has been the case, jeopardize peace" (Harisiades v. Shaughnessy, 1952). Witness the cruelty of a system that would create conditions where crimination Comm., 525 U.S. 471, 491, 119 S.Ct. 936, 142 L.Ed.2d 940 (1999). 
a father and daughter die on a riverbank attempting to cross into the United States from El Salvador to escape violence.

Although Congress repealed the Chinese Exclusion Act in 1943, from the time of Fong Yue Ting, the absolute power of Congress to exercise "deportation, however severe its consequences, has been consistently classified as a civil rather than a criminal procedure" (Harisiades $v$. Shaughnessy, 1952). As an original proposal, this doctrine is highly debatable in light of the close association that exists between criminal convictions and deportation (Harisiades v. Shaughnessy, 1952). Nevertheless, the United States Supreme Court has considered the matter closed for many years. To be certain, in 1913, the Court held it thoroughly established "that Congress has power to order the deportation of aliens whose presence in the country it deems hurtful. The determination by facts that might constitute a crime under local law is not a conviction of crime, nor is the deportation a punishment; it is simply a refusal by the government to harbor persons whom it does not want" (Bugajewitz v. Adams, 1913). While it may be the case that alignment of local penal law with the policy of Congress is a jurisprudential coincidence, it is far from coincidence that both are accomplished under the logic of state-building which employs the exclusion of individuals based upon race/ethnicity as part of its creation of the modern nation-state.

What is neither coincidence nor accident, however, is the inhumane treatment of non-citizen migrants in the United States, which has arisen in the wake of Fong Yue Ting and its progeny. For the century-old legal fiction that makes deportation a civil matter also makes the Eighth Amendment's prohibition against cruel and unusual punishment inapplicable to the detention of people pending deportation hearings because, again, "deportation is not a punishment for crime" (Ingraham v. Wright, 1977). Deportation is civil, albeit in name only, and there is no Eighth Amendment concept of punishment in a civil proceeding: "Excessive bail shall not be required, nor excessive fines imposed, nor cruel or unusual punishment inflicted" (emphasis added).

What constitutional protections may remain exist in due process. For no one in the United States, no matter her origin, can be deprived of life, liberty, or property without due process of law. Whatever other rights denied by virtue of status, the Fifth Amendment and Fourteenth Amendment demand due process protections against deprivations of life or liberty at the hands of state or federal officials. The due process clause may not invalidate all inflictions of severe hardship faced by non-citizen migrants (Harisiades v. Shaughnessy, 1952). However, certain conditions or restrictions will implicate Fifth and Fourteenth Amendment concepts of punishment, which may trigger the recognition of constitutional protections and relief for those who suffered.

Still barring exceptional circumstances, the due process clause has little power to protect non-citizen refugees and migrants, who unlike Liliana do not encounter the humane bureaucrat or find the concern cit- 
izen advocate. Their constitutional protections remain limited by that absolute and unqualified but "overriding concern that the United States, as a sovereign, maintain its right to self-determination" (Lynch v. Cannatella, 1987). The due process clause has even less power to prevent the level of inhumane treatment required to bring a cognizable claim of "punishment" in civil proceeding. ${ }^{8}$ Acts of "gross physical abuse" or "malicious infliction of cruel treatment" may constitute punishment. ${ }^{9}$ However, these determinations must be made by finders of fact - a judge or a jury - in a civil trial, which is cost prohibitive and to which no right to free counsel attaches. Legal standards like these further distinguish Liliana's story because she was provided access to legal advice while in custody. More often, such legal standards make for more legal fictions that further shield human rights violations from coming to light and being litigated in courts of law. There is a very thin constitutional protection against inhumane treatment for non-citizen migrants in the United States. The poorer the person the thinner the protection.

\section{The False Dream of International Human Rights}

A thicker line of protection potentially exists under international standards. The United Nations' Universal Declaration of Human Rights includes "the equal and inalienable rights and fundamental freedoms of each human being" (U.N. General Assembly Resolution 1948). Its Convention Against Torture and Other Cruel, Inhuman or Degrading Treatment or Punishment recognizes "the equal and inalienable rights of all members of the human family" as "the foundation of freedom, justice and peace in the world" and derives those rights from "the inherent dignity of the human person" (U.N. 1984, Art. 16). In the same spirit, the International Covenant on Civil and Political Rights decrees, "[a]ll persons deprived of their liberty shall be treated with humanity and with respect for the inherent dignity of the human person" (U.N. 1966, Art. 10).

The Declaration, however, is premised on a Westphalian nation-state system put in after World War II. Under this system, the concept of justice is defined in terms of internal citizenship rights. As Nancy Fraser writes, "Subtending the lion's share of social struggle in the postwar era, this view channeled claims for justice into the domestic political arenas of territorial states. The effect, notwithstanding lip-service to international human rights and to anti-imperialist solidarity, was to truncate the scope of justice, marginalizing, if not wholly obscuring, cross-border injustices" (Fraser 2013, 214). Thus,

8 See generally Bell v. Wolfish, 441 U.S. 520 (1979).

9 See Medina v. O'Neill, 838 F.2d 800, 801 (5th Cir. 1988). 
while the United States is party to these international prohibitions against cruel, inhuman, or degrading treatment, its participation is subject to reservations and declarations. Those make it "bound by the cruel, inhuman, or degrading treatment prohibitions", but "only to the extent that those words mimic the cruel and unusual treatment or punishment prohibited by the Fifth, Eighth, and/or Fourteenth Amendments" (Budhrani 2012, 804; emphasis added). Consequently, the limited protections afforded to non-citizen migrants under the United States Constitution narrowly adheres to, if not violates, the norms established under international human rights law.

To make matters worse, the United States has declared these international prohibitions "non-self-executing". This means that no private right to action exists in the United States to assert these international prohibitions against cruel, inhuman, or degrading treatment, absent express congressional legislation: "[w]hile treaties 'may comprise international commitments [...] they are not domestic law unless Congress has either enacted implementing statues or the treaty itself conveys an intention that it be self-executing and is ratified on these terms" (Budhrani 2012, 804). No such legislation exists. On the contrary, history makes clear that Congress will control, detain, and exclude those it has deemed undesirable, however severe its consequences. The dream of the United Nations is no match for this American nightmare.

Here, it seems important to return to Liliana's memoir, the narrative which establishes the very humanity which many of the United States border agents, bureaucrats, and advocates seem to recognize. It is important, that is, to realize this entire legal system is premised on taking away that very humanity from Liliana. Unlike the personal narrative of her memoir, this narrative focuses on her statelessness. In this narrative, the moment Liliana arrived in the United states, she stopped being a child. She started being an 'alien'. She was caught, not as a child seeking refuge and reunification with her family, but, instead, as an alien unlawfully present in the United States.

Immediately upon arrest, she was subject to immigration detention. The Immigration and Nationality Act of 1952 establishes that "an alien may be arrested and detained pending a decision on whether the alien is to be removed from the United States (8 U.S.C. \$1226(a)". Her confinement in the program known as the House of Dreams was a form of immigration detention. Immigration detention is considered civil detention. Civil detention exists outside the Eighth Amendment's prohibitions against cruel and unusual punishment. In the absence of the Eighth Amendment, civil detention comes with a decreased adherence to the standards that control constitutional conditions of confinement. During the four months she remained detained at the House of Dreams, it could have easily become the House of Nightmares. Liana was, in essence, an alien at the mercy of her captors.

Her story could have been the following: 


\section{$\begin{array}{lllll}\text { J } & \text { A } & \text { C } & \text { A } & \text { (An Infant) }\end{array}$}

I, J $\quad$ O A $\quad$ A $\quad \mathbf{M}_{\mathbf{1}} \quad$, declare under penalty of perjury that the following is true and correct to the best of my knowledge and recollection.

1. I came from Moncagua in San Miguel, El Salvador. My birthday is

I am 16 years old. I lived in my grandfather's home with my 20 -years-old fiancé, $\mathrm{J} A$ C . Mi , my 20-year-old sister, L A Al R and our one-year-old daughter, J A C. A Our daughter's date of birth is

2. My fiancé, daughter, sister, and I had to leave El Salvador because our grandfather saw the gang kill our neighbor. They threatened to kill him and so we all had to leave. $\mathrm{He}$ is old so he went into hiding and we came to the United States because we have family here. My fiance's uncle, D. $\mathrm{Cl}_{1} \cdot \mathrm{M}$ 。 , lives in Virginia and wants to sponsor us. His telephone number is

3. My fiancé and I crossed with our baby into the United States at Port Juarez and had to cross the river by foot. It was very deep so I held our baby and my fiancé held onto me to keep us above the water. Two hours after we crossed, we met Border Patrol and they took us to a very cold house. We slept on mats on the floor and gave us aluminum blankets. They took away our baby's diapers, baby formula, and all of our belongings. Our clothes were still wet and we were very cold and so we got sick. My fiancé, our daughter, and I got sick from being so cold. We were there for about ten hours.

4. After that they took us to a place with a tent. There was also a structure with a roof, but no walls. They put us in a cell together. Until this point, our family was kept together, but here they came and took our daughter and me out of the cell and separated my fiancé from us. We were all very upset. Our baby was crying. I was crying. My fiancé was crying. We asked the guards why they were taking our family apart and they yelled at us. They were very ugly and mean to us. They yelled at him in front of everyone to sit down and stop asking questions. We have not seen him since.

5. They made us all sit in lines and all face the same way. If we tried to shift positions or turn around and look the other way, they would yell at us and tell us not to turn around. There were about 30 children there. They also were made to sit on the ground lined up and all face the same way. They made us do that for like five hours. Our oneyear-old tried to stand up and take some steps and the guards told me to make my baby sit down and stay still. They got mad at me because I was having trouble making my baby, who is a toddler, sit still for so long. My back was hurting me from sitting there on the ground with my legs folded and my baby in my lap trying to hold her still. 
6. After that, they brought us here to Clint. It was about a 40-minute drive. We stay in a room with 45 other children. There are ten bunkbeds. We sleep two children per mattress so four children sleep in each bed. The other children sleep on thin mats on the concrete floor. The way the beds are assigned is that the child who leaves gives their bed to someone else. A girl left the facility and so she designated that bed for my daughter and me. Until then, my baby and I slept on a cement wall. There was no mat so my baby and I slept directly on the cement. We had two blankets (one each) and so I put one underneath us and the other one on top of us.

7. I have been in the U.S. for six days and I have never been offered a shower or been able to brush my teeth. There is no soap here and our clothes are dirty. They have never been washed. My daughter is sick and so am I.

8. In the morning, they give us Jello, oatmeal, and silver pouches of fruit punch. At lunch, they give us instant soup, another pouch of fruit punch, and a cookie. At dinner, they give us a bean burrito, Jello, and a silver pouch of fruit punch. There is nothing else in the burrito. No rice or cheese. They give my daughter formula, but otherwise we get no milk. We get no fruits or vegetables.

9. My baby asks for her dad all of the time. She frequently looks for him and wonders where her dad is. We both miss him very much and don't understand why they broke up our family.

I, J $\quad \mathbf{O} \quad \mathbf{A} \quad \cdot \mathbf{M} \quad$, swear under penalty of perjury that the above declaration is true and complete to the best of my abilities. This declaration was provided in Spanish, a language in which I am fluent, and was read back to me in Spanish.

June 18, 2019

$\begin{array}{llll}\mathbf{J} & \mathbf{O} & \mathbf{A} & \mathbf{M}\end{array}$ 
And here, then, is the final rendering of Liliana's story. The successful conclusion of her dream should not blind us to the nightmares faced by others. Liliana might have suffered the fate of Hernández Vasquez and two other minors who died while in federal custody. She might have suffered the fate of Óscar Alberto Martínez Ramírez and his daughter, Angie Valeria, who died on a riverbank attempting to reach the United States. That is, her success is deeply enmeshed within a legal system premised on excluding, debasing, and abusing those most in need of political asylum.

\section{Beyond Dreams and Nightmares}

We began this essay with a series of images and stories initially framed through the words of Hannah Arendt: "[t]he exemplary moment of sovereignty is the act of deportation" (quoted in Butler, Spivak 2011, 102). In doing so, we hoped to demonstrate how the United States is enacting a crisis of its sovereignty. And as intimated in the essay, we see the roots of this crisis in the results of a neo-liberal economics that has created a global political and economic crisis. Which is to say that as a push for the open borders of global trade have been implemented, the resulting poverty (and consequent political oppression to maintain order/privilege) has produced as an equally global refugee/immigration crisis. In the context of the United States, the refugee/asylum seeking populations, in our opinion, then, are simply asking the perpetuator of their strife to recognize their responsibility.

What is occurring, however, is exactly the opposite. Clinging onto historic connections between the state and nation-state, we see a form of governance (legislative and juridical) which doubles down on a white-supremacist power structure, a structure that works to enact political borders which deny culpability and 'blame the victim' for their status. The rare 'good immigrant' might be allowed to enter, but only as an alibi for the exclusion of the unfit 'alien' invading the nation - an 'alien' for whom there is not political vehicle to claim restitution for harm or political refuge. Here we are reminded of Foucault's argument that the ultimate act of sovereignty was to punish the very body of its subject through torture or imprisonment. Here we note again the detention centers where daily adult and children refugee/asylum seekers have their dignity and humanity denied in the name of a 'national identity' in which they will not be allowed to participate.

For if the pressures of global capitalism has struck a mortal blow to the nation-state and we are now witnessing its demise, these images and story of refugees - both the dreams (Lilliana) and the nightmares (caged-children) - are pushing us to ask some hard questions 
about our country, our waning nation-state, our government, and our definition of citizenship and to come up with some solutions on how to bring our rhetoric of 'equality for all' in line with this new global reality. Which is to say, this political destabilization requires us to consider alternative concepts of political/human rights which move beyond the limitations of nation-state structures - structures which as noted repeatedly above do not act in the interest of the dispossessed. We need an alternative model to the reactionary politics of the Trump Administration (as well as nascent and overt nationalist leaders in Europe and the Mena region).

In their long interview, published as a book Who Sings the NationState?, critical theorists Judith Butler and Gayatri Spivak (2011) investigate many of the themes of this article: the limits of the concept of the nation-state, its diminishment under global capitalism, the rise of nationalism, the legal dispossession of US immigrants to political agency, and the examination of some nascent geo-political formations that might take the place of the ailing concept of nation-state.

Spivak, in particular, suggests the concept of "critical regionalism" as a way to "go over and under nationalism, but keeps [sic] the abstract structures of something like a state. This allows for constitutional redress against the mere vigilance and data-basing of human rights, or public interest litigation in the interest of a public that cannot act for itself" (Butler, Spivak 2011, 94). That is, the state is something we need in order to address issues of "redistribution, welfare, and constitutionality" (90). Yet unlike the nation-state, premised on homogeneous concepts of the citizen, this type of critical regionalism-informed state formation would include heterogeneous publics and cultures, would include the sovereignty rights and democratic principles of self-determination and self-regulation to a variety of populations, and adhere to a "post-national understanding of what human rights might be" $(2011,106)$.

Of course, in the current moment of crisis, it is difficult to imagine what such a formation might entail. Yet in the struggle of Indigenous populations against the Dakota pipeline in the United States; Indonesian farmers against the global corporations who attempt to take their farmland; and Columbian women creating new forms of community in response to gang-led violence, we recognize the seeds of alternative concepts of political rights being enmeshed within restrictive nation-state concepts. These struggles of Indigenous populations are even more complicated when they transverse national borders, as is the case for the current refugees, Indigenous people of the Americas, who are now held captive in Detention Centers on the very land that only 200 years ago was part of a different nation; 500 year ago was populated by the Caddo and Apache nations. Through such examples, invoking these different historical geographically-located legacies, we can begin to see how expanded publics within a homo- 
geneous 'nation' might gain political recognition for populations too often excluded from power within a geography whose history transcends that nation-state's particular history and borders. Decolonial struggles, perhaps, begin to offer us a multi-versality from which to articulate space of political agency and human rights for those too often defined as stateless, those seeking a right to dignity that should not be reliant on national boundaries.

Still, we recognize that the very idea of a new form of governance not predicated on the nation-state/nationalism may seem to be a far off 'solution' to the foundational causes of the detainment centers at our border. And in the current moment, much of our energy must be placed in addressing the immediate rights of children in detention centers, adults being deported back to dangerous contexts, individuals suffering deprivation and death in their struggle to gain political agency. Yet as we make these arguments, we would argue, we must build the framework from which a future can be built. We must endlessly strive to articulate a future for which there is seemingly no model, but which in its utopian promises mobilizes individuals and collectives to build a governance which fulfils not only current needs but our future hopes. As Judith Butler tells us: "[t]he declaring does not make it so, but it is part of the discursive process of beginning something new; it is an inducement, an incantation, a solicitation" (Butler, Spivak 2011, 95). We must work in the present for increased rights, then, for those most oppressed, but also work to solicit and enact a future where such rights are always already accorded to every individual.

\section{Bibliography}

8 U.S.C. § 1226(a). Apprehension and Detention of Aliens. URL https://www. govinfo.gov/app/details/USCODE-2015-title8/USCODE-2015-title8-chap12-subchapII-partIV-sec1226 (2019-07-28).

8 U.S.C.A. § 1325. Improper Entry by Alien. URL https://www.govinfo.gov/ app/details/USCODE-2015-title8/USCODE-2015-title8-chap12subchapII-partVIII-sec1325 (2019-07-28).

Bell v. Wolfish (1979). 441 U.S. 520. URL https://supreme.justia.com/cases/federal/us/441/520 (2019-07-28).

Budhrani, Anshu (2012). "Regardless of My Status, I Am A Human Being: Immigrant Detainees and Recourse to the Alien Tort Statute". University of Pennsylvania Journal of Constitutional Law, 14, 781-803. URL http://scholarship.law.upenn.edu/cgi/viewcontent.cgi?article=1050\&context=jcl (2019-07-28).

Bugajewitz v. Adams (1913). 228 U.S. 585, 591, 33 S. Ct. 607, 608, 57 L. Ed. 978. URL https://supreme.justia.com/cases/federal/us/228/585 (2019-07-28). 
Butler, Judith; Spivak, Gayatri Chakravorty (2011). Who Sings the Nation-State? Language, Politics, Belonging. Salt Lake City: Seagull Books.

Case 2:85-CV-04544-DMG-AGR (2019). Document 569-2 Filed 06/26/19 page 94 ID. Redacted version of document proposed to be filed under seal 03/23/2019 \#2874. URL http://www.publiccounsel.org/tools/assets/files/1015.pdf (2019-07-28).

Cunningham-Parmeter, Keith (2011). "Alien Language: Immigration Metaphors and the Jurisprudence of Otherness". Fordham Law Review, 79, 1545-68.

Delgado, Richard (1989). "Storytelling for Oppositionists and Others: A Plea for Narrative". Michigan Law Review, 87(8), 2411-41.

Shapiro, Lauren (2014). "Surge of 'Unaccompanied Children'”. Factcheck, 18 July. URL https://www.factcheck.org/2014/07/surge-of-unaccompanied-children (2019-07-28).

Fraser, Nancy (2013). Fortunes of Feminism: From State-Managed Capitalism to Neoliberal Crisis. London: Verso.

Fong Yue Ting v. United States (1893). 149 U.S. 698, 729, 13 S. Ct. 1016, 1028, 37 L. Ed. 905. URL https://supreme.justia.com/cases/federal/ us/149/698 (2019-07-28).

Harisiades v. Shaughnessy (1952). 342 U.S. 580, 596, 72 S. Ct. 512, 522, 96 L. Ed. 586. URL https://www.loc.gov/item/usrep342580 (2019-07-28).

Harvey, David (2005). A Brief History of Neoliberalism. Oxford: Oxford University Press.

Hinds v. Lynch (2015). 790 F.3d 259, 264 (1st Circuit). URL https://casetext. com/case/hinds-v-lynch-2 (2019-07-28).

Ingraham v. Wright (1977). 430 U.S. 651, 667-68. URL https://supreme.justia.com/cases/federal/us/430/651 (2019-07-28).

Johnson, Kevin R. (2002). "Race and the Immigration Laws: The Need for Critical Inquiry". Valdes, Francisco et al. (eds), Crossroads, Directions, and a New Critical Race Theory. Philadelphia: Temple University Press, 187-98.

Kandel, William A. (2017). Unaccompanied Alien Children: An Overview. Congressional Research Service. URL https://fas.org/sgp/crs/homesec/ R43599.pdf (2019-07-28).

Labrador, Rocio Cara; Renwick, Danielle (2018). "Central America's Violent Northern Triangle". Council on Foreign Relations, June 26. URL https:// www.cfr.org/backgrounder/central-americas-violent-northern-triangle (2019-11-21).

Lynch v. Cannatella (1987). 810 F.2d 1363, 1373 (5th Circuit). URL https://casetext.com/case/lynch-v-cannatella (2019-07-28).

Medina v. O'Neill (1988). 838 F.2d 800, 801 (5th Circuit). URL https://casetext.com/case/medina-v-oneill (2019-07-28).

Meyer, Peter et al. (2016). Unaccompanied Children from Central America: Foreign Policy Considerations. Congressional Research Service. URL https:// fas.org/sgp/crs/homesec/R43702.pdf (2019-07-28).

Michigan Department of State Police v. Sitz (1990). 496 U.S. 444, 471 n.15. URL https://supreme.justia.com/cases/federal/us/496/444 (2019-07-28).

Mignolo, Walter (2013). Local Geographies/Global Designs: Coloniality, Subaltern Knowledges, and Border Thinking. Princeton: Princeton University Press.

Reno v. Am.-Arab Anti-Discrimination Comm. (1999). 525 U.S. 471, 491, 119 S.Ct. 936, 142 L.Ed.2d 940. URL https://supreme.justia.com/cases/federal/us/525/471 (2019-07-28). 
Skeat, Walter W. (1968). An Etymological Dictionary of the English Language. 13 ed. Boston: Harvard University Press.

Velásquez, Liliana (2016). Dreams and Nightmares: I Fled Alone to the United States When I was Fourteen. Philadelphia: New City Community Press.

United Nations (1954). Convention relating to the Status of Stateless Persons. Adopted September 28, 1954.

United Nations (1966). International Covenant on Civil and Political Rights. Adopted Dec. 19, 1966, 999 U.N.T.S. 171, 176.

United Nations (1984). The Convention against Torture and Other Cruel, Inhuman or Degrading Treatment or Punishment. Adopted Dec. 10, 1984, S. Treaty Doc. No. 100-20, 1465 U.N.T.S. 113, 116.

United Nation General Assembly Resolution (1948). The United Nations'Universal Declaration of Human Rights, 217A (III), U.N. Doc. A/810, at 71. Dec. 10.

United States v. Ortiz (1975). 422 U.S. 891, 904 (Burger, C.J., concurring). URL https://supreme.justia.com/cases/federal/us/422/891 (2019-07-28).

United States v. Brignoni-Ponce (1975). 422 U.S. 873, 902 (Burger, C.J., concurring). URL https://supreme.justia.com/cases/federal/us/422/873 (2019-07-28). 



\title{
La politica di tolleranza zero
} dell'Amministrazione Trump alla frontiera con il Messico La pratica di separazione delle famiglie e di detenzione dei bambini

\section{Rosemarie Brisciana}

Università Ca' Foscari Venezia, Italia

\begin{abstract}
This study, which analyzes the 'zero tolerance' immigration policy of Donald Trump's Administration on the Mexican border, focuses on the practice of the separation of families and the detention of children in often inhuman conditions. Through an analysis of the criminalisation of asylum seekers without due process, it highlights the probable violations of American laws and Constitutional amendments, as well as international human rights conventions, not to mention the lasting psychological trauma for both parents and children.
\end{abstract}

Keywords Immigration. United States. Custody. Children. Asylum seekers.

Sommario 1 Introduzione. - 2 La tensione crescente tra le forze anti-immigrazione e pro-immigrazione in America Latina e in Brasile. - 3 Tortura e continuità della violenza di Stato in Brasile. - 4 Conclusione. 


\section{1 "Can He Do that?». Le basi sociali del sostegno alla politica migratoria dell'Amministrazione Trump}

Di fronte ai tweet offensivi e altre dichiarazioni xenofobe di Donald Trump, di fronte alle immagini di bambini strappati ai genitori alla frontiera USA-Messico, ci si può chiedere come mai la nazione che si fa paladina dei principi di rispetto dei diritti umani e di democrazia possa sostenere una politica migratoria che da più parti è stata definita come disumana. ${ }^{1}$ La politica di tolleranza zero verso l'immigrazione dal confine sud si combina ad altri aspetti critici come la soppressione della politica di Obama che permetteva ai minorenni entrati senza documenti sul territorio statunitense di rimanerci (i Dreamers), la deportazione degli adulti nel Paese di origine senza i figli e i nipoti che rimangono da soli negli USA, il divieto d'ingresso ai cittadini provenienti da Paesi a maggioranza musulmana che dà luogo anche in questo caso a situazioni di separazione di famiglie. ${ }^{2}$ Per capire l'origine del sostegno alla politica di tolleranza zero da parte della maggioranza Repubblicana della popolazione statunitense, si devono innanzitutto analizzare i fattori che hanno reso possibile la presidenza Trump e il peso del Partito Repubblicano nel potere legislativo e nel potere giudiziario.

Secondo le proiezioni dell'Agenzia statunitense del Censimento (Colby, Ortman 2015), entro il 2045 la popolazione «bianca» - essenzialmente di origine europea, non ispanica - diventerà minoranza («any group other than non-Hispanic White alone»). Questo fenomeno sembra essere alla base di un timore tra la popolazione bianca di perdere la propria posizione dominante nella società, una sorta di white cultural anxiety di fronte alla prospettiva di dover condividere il sogno americano con stranieri 'non-bianchi' o 'meno bianchi'. Questo timore sembra essere particolarmente forte nei sobborghi agiati come quello descritto nella soap opera Desperate Housewives, popolati da ex-tute blu, ovvero famiglie che appartenevano alla lower middle class o alla classe operaia e che hanno conosciuto una certa mobilità ascendente, con il conseguente abbandono delle periferie urbane per la classica casa con giardino e garage con due macchine. Ma questa ascesa sociale conteneva in sé la paura di perdere lo status acquisito, una paura che Barbara Ehrenreich ha esaminato nel suo libro Fear of Falling. The Inner Life of the Middle Class (1989). Come hanno evidenziato gli eventi del 2008, si può scendere, o precipitare,

1 A questo proposito il Washington Post ha pubblicato una serie di podcast, molto critica su Donald Trump, intitolata «Can He Do that?».

2 È noto il caso di una mamma yemenita che, recatasi in visita al suo Paese di origine, non è potuta rientrare negli USA quando si è gravemente ammalato suo figlio, di padre americano. C'è voluto un anno di tempo per ottenere i documenti necessari, e questo solo con l'aiuto dei media. A quel punto, però, il figlio di due anni era già deceduto. 
e perdere tutto. E ora, questa classe sociale teme l'arrivo di persone in forte stato di bisogno, che 'graverebbero' sui programmi di welfare e sui fondi pubblici, pesando sulle tasse degli americani. Inoltre questa classe sociale teme che l'insediamento di nuovi immigrati nei propri quartieri faccia crollare il valore immobiliare della zona.

Questa classe è sensibile alla politica di Trump, un imprenditore simbolo del self-made man, che è fautore di un discorso che produce, alimenta e strumentalizza tale timore. Secondo un sondaggio del Washington Post/ABC del 18 giugno 2018, ben il 75\% dei protestanti evangelici bianchi afferma che la repressione dell'immigrazione undocumented, così come realizzata dall'Amministrazione Trump, è un fatto positivo, a differenza del $46 \%$ del totale della popolazione statunitense. Nonostante condannino, almeno tiepidamente, la separazione delle famiglie, gli evangelici non condividono il liberalismo sociale americano che considerano una minaccia per i loro valori 'cristiani'.

La Destra Cristiana - che copre una varietà di tendenze e sigle religiose, dagli evangelici agli integralisti, passando per le congregazioni più moderate - è uno dei pilastri della base elettorale di Donald Trump, lo vede in senso puritano come un self-made man ed è una forte sostenitrice della sua politica migratoria di tolleranza zero. Benché cento pastori abbiano pubblicato una lettera aperta a Trump chiedendogli di annullare il Muslim Ban, secondo il sondaggio Fact Tank realizzato nel maggio 2018 dal Pew Research Institute, il 51\% degli statunitensi sostiene che il Paese ha il dovere di accogliere gli emigranti mentre il 68\% degli evangelici bianchi sostiene che il Paese non ha nessuna responsabilità in tal senso. È una percentuale vicina a quella degli evangelici che approvano quasi totalmente la politica di tolleranza zero. ${ }^{3}$

I motivi alla base di questa posizione anti-immigrati sono molteplici e hanno radici sociali, politiche e culturali. Tra queste, c'è anche la seguente (in gran parte costruita ovviamente): secondo uno studio del Public Religion Research Institute, realizzato con la rivista politica The Atlantic nel settembre del 2017, circa il 65\% della classe lavoratrice bianca crede che la cultura americana si stia «deteriorando», il 68\% teme la perdita dell'«identità americana», il 62\% considera gli immigrati una minaccia alla «cultura americana». E più della metà ritiene che la discriminazione contro i bianchi sia tanto forte quanto quella che colpisce le minoranze (Cox, Lienesch, Jones 2017). Con il discorso della carovana degli emigranti 'pronta ad invadere il Paese'

3 https://www.vox.com/2018/10/30/18035336/white-evangelicals-immigrationnationalism-christianity-refugee-honduras-migrant; https://www.pewresearch.org/fact-tank/2018/05/24/republicans-turn-more-negative-toward-refugees-as-number-admitted-to-u-s-plummets/ (2019-06-13). 
e la proposta (anticostituzionale) di abbandonare lo ius soli, ${ }^{4}$ Trump ha alimentato e sfruttato in modo ancora più insistente questa white cultural anxiety nei giorni precedenti le elezioni legislative di metà mandato nel novembre 2018.

La destra repubblicana è fortemente influenzata dal principio puritano di self reliance - la capacità individuale di auto-realizzarsi (dono di Dio) - in termini di soddisfazione e di prosperità, senza aiuti esterni. Questo principio, che coincide con quello puritano-protestante dell'etica del lavoro, è alla base degli atteggiamenti pro-armi (per l'autodifesa) e anti-welfare (contro il sistema previdenziale, che favorirebbe la dipendenza, contraria alla legge di Dio) così diffusi nel Paese. Secondo tale visione è la comunità cristiana che assicura la propria sopravvivenza e l'eventuale prosperità, frutto di quest'etica, rappresenta la benedizione di Dio, e forse, un segno premonitore della salvezza; la coesione della comunità risiede nel conformarsi a strette norme di comportamento per proteggerla da ogni pericolo percepito come proveniente da fuori, tuttavia la solidarietà si deve fermare ai confini della comunità escludendo quelli che non condividono gli stessi valori. Secondo Diana Butler Bass, ${ }^{5}$ la destra cristiana reinterpreta le parole di Gesù Cristo, che predicava di accogliere e aiutare i poveri, mettendo in atto un «aiuto selettivo» destinato solo ai cristiani della stessa comunità di pensiero, dando assistenza ai soli «meritevoli» che non mettono in pericolo l'identità bianca della comunità (nonostante gli emigranti ispanici siano pure loro, per la maggior parte, cristiani). Questa visione comunitaria «bianca», politicizzata e «nativista», mette in campo un «nazionalismo cristiano» contro gli immigrati, rinforzato dall'arrivo di Trump e dalla destra repubblicana. ${ }^{6}$

4 Il diritto alla cittadinanza statunitense, sancito nel Quattordicesimo Emendamento della Costituzione e attribuito a tutti quelli che nascono sul territorio statunitense, anche se da genitori entrati 'illegalmente' negli Stati Uniti.

5 https://www.vox.com/2018/10/30/18035336/white-evangelicals-immigrationnationalism-christianity-refugee-honduras-migrant (2019-06-13).

6 Questo atteggiamento, spiega Butler Bass, ignora l'epistola dell'apostolo Paolo ai Galati: «Non c'è più giudeo né greco; non c'è più schiavo né libero; non c'è più uomo né donna, poiché tutti voi siete uno in Cristo Gesù» $(\mathrm{Gal} 3,28)$. Altri brani della Bibbia sono stati sfruttati, in modo errato, da consiglieri dell'Amministrazione Trump: ad esempio Paula White, pastore e sostenitrice della Destra Cristiana, ritiene che quando è fuggito in Egitto Gesù non abbia infranto nessuna legge perché il suo ingresso in Egitto non era illegale e quindi era senza peccato (in caso contrario non sarebbe stato il Messia); con questa interpretazione molto particolare, White suggerisce che gli emigranti sono dei peccatori. 


\section{La tortura nel Paese di origine e durante il viaggio: il problema della violenza nel Triangolo Nord (El Salvador, Honduras, Guatemala)}

Negli ultimi anni, soprattutto dal 2011, la presenza di potenti organizzazioni criminali nel Triangolo Nord dell'America Centrale è fortemente aumentata, e ciò ha avuto molte conseguenze negative sulla vita quotidiana della popolazione, soprattutto quella povera delle zone rurali. Se prima le organizzazioni criminali gareggiavano per conquistare spazi nel mercato della droga, raggruppandosi nei cartelli, oggi le loro attività si sono diversificate e tra queste spicca la pratica dell'estorsione ai danni delle comunità locali mediante un alto livello di violenza e brutalità. In Salvador, dove circa un terzo della popolazione guadagna meno di cinque dollari al giorno, MS-13 - una delle principali organizzazioni criminali, che spartisce il controllo del territorio con Barrio 18, un gruppo criminale altrettanto violento - pratica estorsioni in quasi tutte le 262 municipalità del Paese. I bambini che vivono in queste aree sono spesso obbligati ad aderire alle gang, o sono vittime di stupri, rapimenti e di altri orrori se rifiutano o se i loro genitori si rifiutano di cooperare.

A San Salvador, capitale del Paese, le gang controllano la distribuzione dei beni di consumo e accumulano denaro minacciando i commercianti. Nelle aree rurali bruciano le piantagioni da zucchero se i contadini non accettano di pagare. In Honduras, per le donne la situazione è ancora più critica: Médecins sans Frontières (2017) ha segnalato numerosi casi di stupro e di uso della violenza sessuale come strumento di intimidazione e controllo; nel Paese viene uccisa una donna ogni sedici ore, portando il tasso di femminicidio al livello più alto del mondo. La forza di queste organizzazioni criminali, che operano in diversi Paesi dell'America Centrale, negli Stati Uniti e in Canada, non trova un freno da parte dei governi locali, paralizzati dalla corruzione.

In Guatemala, dove la situazione sembra essere solo un po' meno grave, alcune autorità dello Stato e l'ex presidente Montt sono stati processati e riconosciuti colpevoli di violazione dei diritti umani per il massacro di quasi duemila persone, in gran parte indigeni - ma anche giornalisti e difensori dei diritti umani. Per l'anno 2017 il Congresso degli Stati Uniti ha votato un'assistenza di 655 milioni di dollari per il Piano dell'Alleanza per la Prosperità nel Triangolo Nord, un'iniziativa regionale che dovrebbe durare cinque anni per ridurre le cause dell'emigrazione da questa regione - violenza, land-grabbing, conflitti locali, corruzione - condizionandola al rispetto dei diritti umani. Secondo il Dipartimento di Stato americano, le istituzioni guatemalteche non sarebbero in grado di perseguire questi crimini, di contrastare le attività delle grandi organizzazioni criminali e di controllare la facile disponibilità di armi, ma risulta evidente l'i- 
pocrisia statunitense sulle questioni dei diritti umani e della facile disponibilità di armi.

Il sopracitato rapporto di Médecins sans Frontières (2017) descrive un cammino degli emigranti all'insegna della sofferenza estrema: rapimenti, violenza fisica e sessuale, estorsioni, ricatti in cambio di rifugio o di cibo, violenze compiute dalle autorità dei Paesi attraversati oppure dalle organizzazioni criminali. MSF ha costruito ambulatori lungo i percorsi della migrazione, lungo le ferrovie, soprattutto in alcune aree del Messico come il Chiapas, Tabasco, Hidalgo, Veracruz e Città del Messico. Molti degli emigranti soccorsi hanno vissuto episodi di violenza sessuale. Durante il tragitto circa sei donne su dieci vengono stuprate, e a volte vendute ai trafficanti del sesso.

Un rapporto di Amnesty International (2019) evidenzia che si tratta di un viaggio pericolosissimo. Gli emigranti salgono sui tetti delle carrozze dei treni merci nei punti dove i treni avanzano più lentamente, rischiando di cadere sotto le ruote del treno. Tra la frontiera guatemalteca e Veracruz, sul litorale del golfo del Messico, in un viaggio di 900 chilometri, le gang salgono sul treno e richiedono un pedaggio di 100 dollari per ogni stazione attraversata, minacciando di buttare giù dal tetto del treno gli emigranti - bambini compresi - o di uccidere i parenti rimasti al Paese di origine se questi non inviano agli emigranti il denaro necessario per il loro riscatto. Secondo la Commissione dei Diritti Umani messicana citata nel rapporto, ogni anno vengono rapinate circa 20.000 persone, con un guadagno per le gang di circa 50 milioni di dollari annui. Durante le soste del treno, la polizia locale e altre autorità come gli ufficiali dell'immigrazione estorcono denaro per il passaggio, arrivando a rubare anche le scarpe agli emigranti per impedire loro di proseguire. Le autorità messicane, che non sono in grado di fermare il maltrattamento degli emigranti in transito, accusano quelle degli Stati Uniti di maltrattamento degli emigranti messicani.

\subsection{Il fenomeno della Carovana}

La carovana del 12 ottobre 2018, la più grande conosciuta finora, si è formata in buona parte attraverso post, messaggi sui social e sulle piattaforme messaggistiche, scambi privati e campagne organizzate da attivisti o associazioni come Pueblo Sin Fronteras. Circa duemila persone sono partite da San Pedro Sula nell'Honduras viaggiando a piedi verso il Nord, al ritmo di quaranta chilometri al giorno, per un totale di duemila chilometri fino ad arrivare alla frontiera Messico-Stati Uniti. Non era la prima volta che una carovana partiva dall'Honduras, ma quella del 12 ottobre 2018 è stata la più grande $e$ nel tragitto è cresciuta - anche per l'attenzione dei media. Le carovane garantiscono agli emigranti una certa sicurezza data dal nume- 
ro delle persone, soprattutto quando si attraversano posti pericolosi. Le autorità del Guatemala e del Messico hanno cercato di fermare le carovane ai propri confini, ma quasi tutte le persone hanno trovato il modo di attraversarli. La carovana del 12 ottobre si è presentata al mondo come un convoglio di protesta, contro le difficoltà di sopravvivenza, contro le pesanti condizioni di vita, contro le disuguaglianze e la precarietà, contro i politici incapaci di fornire soluzioni. La carovana era composta da donne e uomini, anziani con nipoti, adolescenti e bambini, persone in fuga dalla violenza e dall'estorsione.

Trump non ha esitato a sfruttare questa situazione nei giorni precedenti le elezioni legislative di metà mandato. Ha presentato la carovana come un'accozzaglia di avanzi di galera, potenziali terroristi, mediorientali pericolosi, nemici occulti, aspiranti criminali - una vera 'invasione' che necessitava di almeno 15.000 militari alla frontiera. Il 25 novembre 2018, e ancora l'1 gennaio 2019, i militari statunitensi hanno spruzzato gas lacrimogeni alla frontiera col Messico contro una folla di emigranti (bambini compresi) ammassata sul lato messicano al valico di San Ysidro, folla che, secondo le autorità statunitensi, avrebbe cercato di forzare il blocco. Trump ha negato la presenza di bambini, ma le foto e le testimonianze dimostrano il contrario. In un comunicato stampa, l'Agenzia delle Dogane e per la Protezione delle Frontiere (U.S. Customs and Border Patrol) ha sostenuto che gli emigranti avrebbero cercato di scavalcare o di passare sotto la recinzione posta tra Tijuana e San Diego, e lanciato pietre. L'agenzia ha poi dichiarato di aver visto alcuni emigranti sollevare dei bambini piccoli sopra il filo spinato, mettendoli in pericolo. Con il pretesto di proteggere i bambini, le forze dell'ordine statunitensi hanno utilizzato lacrimogeni e spray al peperoncino oltre il confine, violando l'integrità territoriale del Messico. ${ }^{7}$ Gli emigranti, stanchi dopo duemila chilometri di tortura, hanno protestato contro il trattamento riservato loro dalle autorità statunitensi. ${ }^{8}$

7 https://www.nytimes.com/2019/01/01/world/americas/migrants-border-tear-gas.html (2019-05-11).

8 Viste la tossicità e la dispersione incontrollata del gas, questo uso sarebbe stato di dubbia legalità anche se gli emigranti fossero nel territorio statunitense, e soprattutto se non avessero presentato un pericolo imminente per la sicurezza del Paese (https:// www.nytimes.com/2018/11/28/world/americas/tear-gas-border.html?action=clic k\&module=RelatedCoverage\&pgtype=Article\&region=Footer, 2019-05-11). 


\section{La tortura dell'arrivo alla frontiera e della custodia: da un inferno all'altro}

Per riprendere i fatti ormai conosciuti, nel marzo 2017 l'ex-capo del Dipartimento della Sicurezza Interna (Homeland Security), John Kelly, ha confermato pubblicamente che il suo dipartimento stava considerando diversi mezzi per scoraggiare e contrastare gli arrivi di emigranti sul territorio statunitense, soprattutto alla frontiera col Messico. La politica migratoria di tolleranza zero di Trump - in realtà un giro di vite ad una ventennale politica migratoria repressiva (Chacón 2010a, 2010b) - è iniziata nell'ottobre del 2017, ma si è intensificata nel maggio 2018 con l'incremento degli arrivi.

All'inizio di aprile 2018, Donald Trump e il suo Procuratore dell'epoca, Jeff Sessions, hanno annunciato l'incriminazione degli emigranti per ingresso illegale negli Stati Uniti, scavalcando il potere giudiziario. Le famiglie di emigranti, private del diritto ad «una procedura pronta ed equa» (due process), sono state trattate come dei criminali ancor prima di essere processate. Per regolamento, i figli di genitori incriminati non possono essere incarcerati con loro, né rimanere in custodia dell'Agenzia di Protezione delle Frontiere del Dipartimento dell'Immigrazione per più di settantadue ore. Pertanto, dopo questa scadenza, è stata avviata la procedura di separazione e i minorenni sono stati affidati a delle agenzie governative (il Dipartimento della Sicurezza Interna, Homeland Security, o il Dipartimento della Salute e delle Risorse Umane, Health and Human Resources), ma a causa di una comunicazione inefficiente tra queste agenzie molti bambini sono finiti a migliaia di chilometri dai genitori. È così che l'amministrazione ha 'fabbricato' una massa di minorenni non-accompagnati.

Il Procuratore Sessions non si è accontentato di sviluppare questa politica, ha persino tentato di giustificarla citando la Bibbia: «L'ingresso illegale negli Stati Uniti è un crimine. Le persone che violano la legge della nostra nazione sono soggette a procedimento giudiziario. Vorrei citarvi l'apostolo Paolo e il suo chiaro e saggio invito contenuto in Romani capitolo 13 di obbedire alle leggi del Governo perché Dio le ha ordinate al fine di garantire l'ordine: 'Ogni persona sia sottomessa alle autorità superiori; perché non vi è autorità se non da Dio; e le autorità che esistono, sono stabilite da Dio'» (trad. dell'Autrice)..$^{9}$ La condanna di leader religiosi e docenti di teologia è stata immediata, in forte disaccordo con ciò che considerano un'er-

9 È il primo versetto del capitolo 13 della Lettera di Paolo ai Romani, abusivamente citato nel passato da simpatizzanti fascisti e schiavisti americani: https://www.nbcnews.com/politics/immigration/sessions-cites-bible-defense-breaking-families-blames-migrant-parents-n883296 (2019-05-11). 
rata interpretazione del Nuovo Testamento per giustificare azioni governative incoerenti con l'insegnamento cristiano e in violazione dello spirito del Vangelo. ${ }^{10}$

Sempre secondo il Procuratore Sessions, i bambini sarebbero stati «contrabbandati» dai genitori e dai parenti o, peggio, da trafficanti che fingevano di essere parenti dei minori, con l'obiettivo di entrare negli USA. È stata così ufficialmente applicata la politica di tolleranza zero verso le famiglie emigranti, anche se già dall'ottobre 2017 circa 700 bambini erano stati separati dai genitori (secondo un rapporto della Sicurezza Interna, citato nel rapporto di un sotto-comitato del Senato del 26 aprile $2018^{11}$ ). Questo rapporto segnala che ad aprile 2018 erano andate perdute le tracce di circa 1.500 bambini dopo l'affidamento a parenti o ad altri sponsor. Motivo, o pretesto, questo per un ulteriore indurimento della politica di tolleranza zero e la custodia dei bambini in luoghi 'adatti' e sorvegliati. Nella confusione di agenzie e dipartimenti che non comunicano tra di loro e che non condividono le stesse banche dati, le tracce perdute di questi bambini mettono in luce che questa procedura oltre ad essere illegale è anche inefficace.

Il 20 giugno 2018, sotto la pressione dell'opinione pubblica e di alcuni politici del proprio partito, Trump ha firmato un decreto presidenziale che ha messo fine alla pratica di separazione delle famiglie. $\mathrm{Ma}$, secondo l'agenzia di stampa Associated Press, 81 bambini sono stati tolti ai genitori anche dopo il decreto del 20 giugno per motivi poco chiari. ${ }^{12}$ Sebbene la separazione sia stata eseguita in nome del benessere e della protezione del bambino in caso di maltrattamento, i metodi applicati alla frontiera con il Messico si sono rivelati arbitrari poiché spesso sono dipesi dalla decisione dell'ufficiale di turno. Inoltre, il decreto non menzionava come dovevano essere riunite le famiglie e non specificava esso andava applicato ai circa 2.700 bambini già in custodia.

Il 27 giugno 2018, il giudice federale della California Dana Sabrow ha dichiarato illegale la separazione delle famiglie e ha ordinato la riunificazione dei genitori con i loro bambini fino a cinque anni d'età entro 14 giorni e con i bambini con più di cinque anni entro 30 giorni. Ha stabilito inoltre l'obbligo del contatto telefonico entro 10 giorni tra genitori e bambini che non avevano più avuto contatti. Secondo la sentenza, «la pratica della separazione delle famiglie è stata applicata senza l'implementazione di un sistema efficace per tenere tracce dei bambini separati dai genitori, di mezzi di comunicazione

10 https://www.apnews.com/dcb05d02726e4d4c9b6de2a31944ba81 (2019-05-11).

11 https://www.hsgac.senate.gov/imo/media/doc/Wagner\%20Testimony.pdf (2019-05-11).

12 https://www.apnews.com/dcb05d02726e4d4c9b6de2a31944ba81 (2019-05-11). 
tra bambini e genitori dopo la separazione, e di un piano per la riunificazione delle famiglie quando i genitori hanno scontato la pena e sono tornati alla custodia dei Servizi dell'Immigrazione [...] una politica che smentisce la governance misurata e ordinata, che è fondamentale per il concetto di processo equo e giusto, sancito dalla nostra Costituzione» (trad. dell'Autrice). ${ }^{13}$

Tuttavia l'ordine del giudice Sabrow non ha specificato se la procedura giudiziaria nei confronti degli adulti considerati 'illegali' dovesse essere annullata. La questione è stata portata davanti al tribunale dall'American Civil Liberties Union, un'associazione storica per la difesa dei diritti civili garantiti dalla Costituzione che, in questo caso, parlava a nome degli adulti separati dai bambini. ${ }^{14}$

Nell'ottobre del 2018 un'altra sentenza del giudice Sabrow ha ampliato la portata della sentenza di giugno per includere tutti i bambini separati, cioè anche quelli affidati dopo la detenzione ad altri servizi o alle tutele di sponsor (parenti, amici di fiducia, famiglie affidatarie), obbligando l'Amministrazione Trump a rivedere la cifra iniziale dei bambini separati dalla famiglia. ${ }^{15}$ Questa cifra può avvicinarsi a 2.800 bambini, ma potrebbe anche includerne mille in più se si fa partire il calcolo dal 2017, con gli arrivi avvenuti prima del censimento dei dipartimenti della Sicurezza Interna e della Salute. Dunque, il numero totale dei bambini separati alla frontiera resta ancora ufficialmente sconosciuto. ${ }^{16}$

Nel febbraio 2019 è stato decretato lo stato di 'emergenza nazionale' per fermare 'la crisi umanitaria al confine' con il Messico. Il 15 febbraio Donald Trump ha dichiarato che «tutti sanno che il muro serve per fermare il crimine e l'invasione dei trafficanti». ${ }^{17} \mathrm{Nel}$ solo mese di marzo 2019 le autorità statunitensi di frontiera hanno arrestato circa 100.000 emigranti in entrata dal Messico; ma, dato che le

13 https://www.nytimes.com/2018/06/26/us/politics/family-separations-congress-states.html (2019-05-11). Diciassette Stati hanno intentato cause contro l'Amministrazione Trump contro questa pratica di separazione.

14 Tra questi adulti c'era una donna congolese entrata dal Messico e separata dalla sua bambina di sette anni alla frontiera presso un designated point of entry (un valico con controllo dei documenti). La madre era detenuta in California, mentre la bambina era stata trasferita a Chicago. Le autorità, evocando un dubbio sulla parentela, hanno imposto accertamenti tramite l'analisi del DNA, che sono stati eseguiti dopo quattro mesi di attesa. Confermato che le due erano madre e figlia, ci sono voluti altri quattro mesi per riunirle.

15 https://www.vox.com/policy-and-politics/2019/1/17/18186773/familieschildren-separated-trump-thousands; https://www.vox.com/2019/3/11/18240136/ family-separation-news-lawsuit-reunite (2019-05-11).

16 https://www.theatlantic.com/entertainment/archive/2019/01/the-trumpadministrations-careless-accounting-on-family-separation/580807/s; https:// oig.hhs.gov/oei/reports/oei-BL-18-00511.pdf (2019-05-11).

17 https://www.nbcnews.com/politics/donald-trump/fact-checking-trump-sspeech-declaring-national-emergency-border-wall-n972116 (2019-11-07). 
capacità di 'accoglienza' si sono esaurite, alcuni arrestati sono stati lasciati liberi di proseguire il loro cammino nell'attesa dell'udienza in tribunale alla quale probabilmente non si presenteranno mai. ${ }^{18}$ In pratica è stata riproposta proprio la situazione che aveva motivato la politica di tolleranza zero in opposizione alla precedente politica del catch and release (beccare e rilasciare) di Obama tanto criticata dall'Amministrazione Trump.

\section{La tortura del soggiorno: lo scandalo delle gabbie e della detenzione dei bambini}

La politica zero tolerance ha prodotto una forma di tortura particolarmente crudele: la detenzione di bambini in luoghi non adatti, perfino in gabbie (il termine preferito dalle autorità è 'recinzione'), spesso affollate, in alcuni ex-magazzini della catena di ipermercati Walmart del Sud Texas e altrove, appositamente riconvertiti in 'centri d'accoglienza'. Centri che ospitano anche adulti soli e famiglie in attesa di procedura e gestiti dall'U.S. Border Patrol.

Nel giugno 2018 un gruppo di parlamentari accompagnato da giornalisti ha ottenuto l'autorizzazione a visitare uno di questi magazzini, chiamato Ursula. I giornalisti non erano autorizzati a parlare con i 'detenuti' né a scattare fotografie. Le scene di bambini piccolissimi, curati da quelli più grandi, traumatizzati, hanno indotto un rappresentante del Congresso, Peter Welch, scioccato alla vista di bambini sdraiati per terra con coperte di alluminio, bottiglie d'acqua e patatine, a pubblicare dei tweet: «Lo chiamano processing facility [struttura per migranti in attesa di procedura] ma non è nient'altro che una prigione. Ho visto delle gabbie, dei bambini seduti su panchine in metallo, che fissavano il muro con lo sguardo vuoto» (trad. dell'Autrice). Il senatore Jeff Merkley, respinto una prima volta quando ha tentato di visitare una di queste strutture, ha aggiunto: «Questi bambini sono già traumatizzati». ${ }^{19}$

18 https://www.reuters.com/article/us-usa-immigration-border/morethan-1000๑९-migrants-encountered-at-u-s-southern-border-in-march-dataidUSKCN1RL2KY (2019-11-07).

19 https://www.theguardian.com/us-news/2018/jun/17/separation-borderchildren-cages-south-texas-warehouse-holding-facility (2019-11-07). 
Rosemarie Brisciana

La politica di tolleranza zero dell'Amministrazione Trump alla frontiera con il Messico

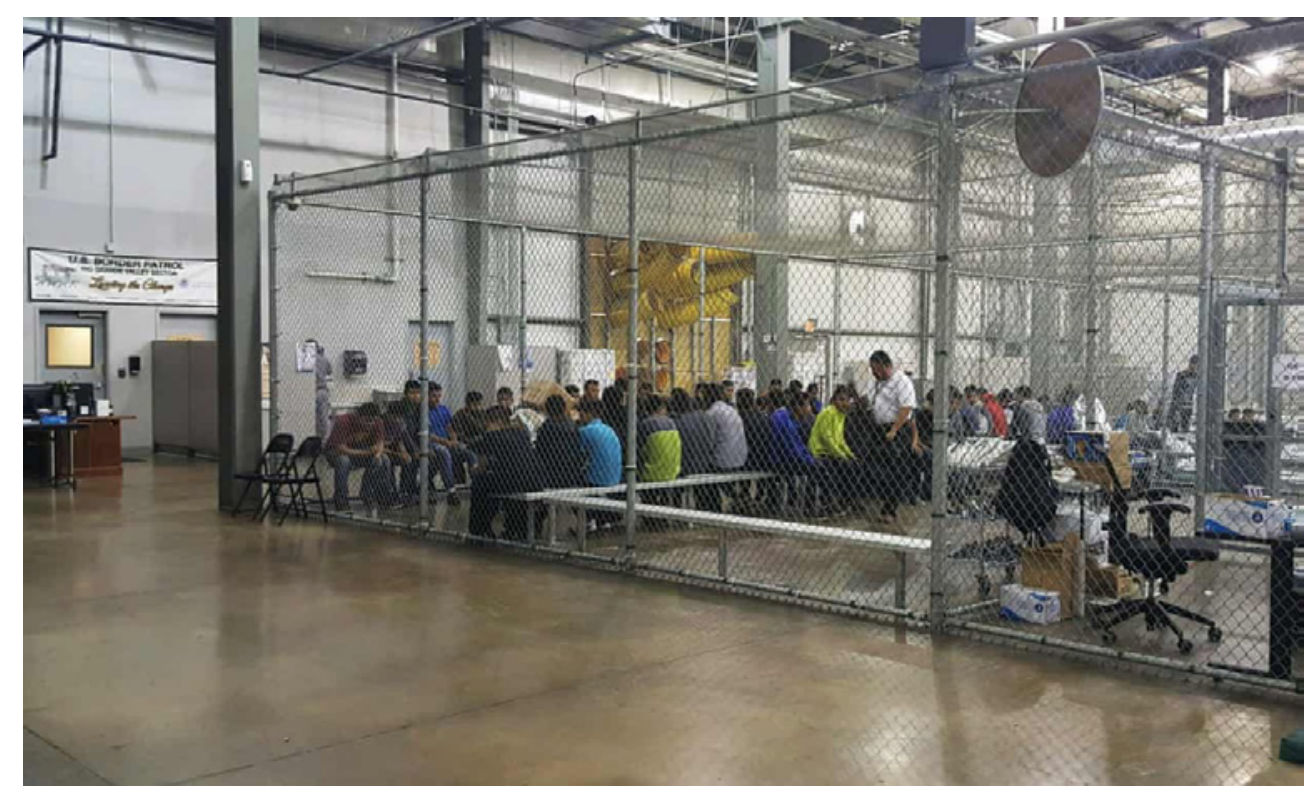

Figura 1 Foto U.S. Customs and Border Protection, pubblicata da The BBC, 18-06-2018. https://www.bbc.com/news/world-us-canada-44518942

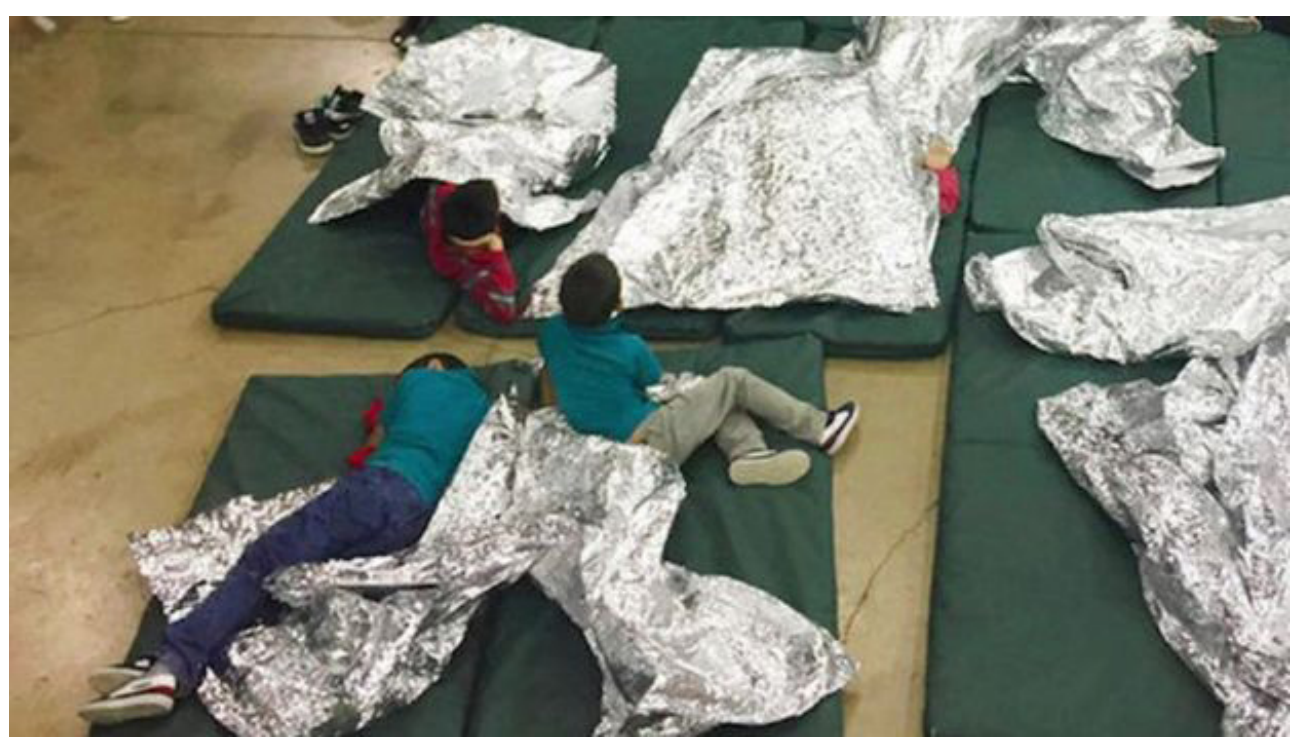

Figura 2 Foto del 17-06-2018, U.S. Customs and Border Protection, McAllen (Texas), pubblicata dal Huffington Post, 22-06-2019 
I parlamentari e i giornalisti ammessi a visitare un altro centro in Texas, chiamato Casa Padre, hanno riferito che i ragazzi sono chiusi all'interno della struttura per ventidue ore al giorno, con due ore soltanto all'aria aperta. I bambini ci rimangono settantadue ore prima di essere trasferiti in altre strutture, lontani dai genitori; ma, visto l'alto numero di bambini separati, la scadenza viene rispettata con difficoltà. Gli agenti sono convinti di compiere una missione di dissuasione conforme ai valori degli Stati Uniti: un'impiegata ha detto ai giornalisti in visita che la loro presenza non era rassicurante per i bambini perché «si sentiranno come animali dello zoo, guardati da tutti». Altra ironia: su di un muro dell'edificio campeggia il ritratto del Presidente Trump con la scritta in inglese e in spagnolo «A volte si perde la battaglia per poter vincere la guerra». ${ }^{20}$

Allo scandalo si sono aggiunte le tragedie. Due bambini sono morti nell'arco di qualche giorno, nel dicembre 2018, mentre erano sotto la custodia dei dipartimenti di Protezione delle Frontiere e di Sicurezza Interna: una bambina di sette anni è arrivata disidratata alla struttura di accoglienza, un bambino di otto anni è morto per una malattia non diagnosticata in tempo. Secondo l'Accademia Americana di Pediatria, i decessi erano evitabili. La segretaria alla Sicurezza Interna dell'epoca, Kirstjen Nielsen, ${ }^{21}$ mentre dichiarava che la storia di questi decessi era davvero sconvolgente, da buon soldato di Trump ha incriminato le famiglie dei bambini deceduti per aver tentato un viaggio lungo e pericoloso con bambini piccoli.

Il 6 marzo 2019 Kirstjen Nielsen è stata interrogata davanti al Congresso sulla politica di tolleranza zero, sui decessi e sulla detenzione nelle gabbie. Ha risposto che le 'gabbie' in realtà sono solo delle recinzioni in filo di ferro, concepite per garantire la sicurezza dei bambini. Ma il senatore Jeff Merkley ha rifiutato questo giro di paro-

20 https://www.theguardian.com/us-news/2018/jun/17/separation-borderchildren-cages-south-texas-warehouse-holding-facility (2019-11-07).

21 Kirstjen Nielsen si è dimessa nell'aprile del 2019 in seguito alla pressante richiesta di Trump di essere più intransigente sull'immigrazione. Le sue dimissioni sono arrivate dopo un vertice tenutosi il 29 marzo 2019 tra lei e le autorità dei tre Paesi del Triangolo Nord, il cui scopo era di trovare delle soluzioni regionali all'emigrazione verso gli USA. L'accordo prevedeva forme di collaborazione per reprimere l'attività delle organizzazioni criminali e il traffico di esseri umani, la condivisione di informazioni e il rafforzamento delle frontiere. Nonostante sia tutta da verificare la capacità di questo accordo di rimuovere alla radice il problema della vulnerabilità delle popolazioni povere, Kirstjen Nielsen ha dichiarato che l'incontro era stato molto positivo. Tuttavia Donald Trump, immediatamente dopo, tramite un tweet, ha dichiarato che questi Paesi non fanno nulla per risolvere il problema, e ha minacciato di tagliare l'assistenza finanziaria e di chiudere completamente la frontiera. Per Kirstjen Nielsen è stata una grande umiliazione pubblica, non soltanto per le dichiarazioni di Trump, ma anche perché la politica di tolleranza zero che doveva difendere si è dimostrata fallimentare con l'aumento continuo degli arrivi alla frontiera Sud (https://www.dhs.gov/news/2019/03/28/secretary-nielsensigns-historic-regional-compact-central-america-stem-irregular, 2019-05-11). 
le: «Ci sono andato. Le ho viste. Sono gabbie!». Sui decessi, Kirstjen Nielsen ha detto che conveniva aspettare i rapporti forensi. Alla domanda di quanti bambini fossero ancora in detenzione, ha dovuto ammettere che non lo sapeva. ${ }^{22}$

Il 6 marzo 2019, poco prima della testimonianza di Kirstjen Nielson al Congresso, per la prima volta l'Agenzia dell'Immigrazione ha ammesso che un centro di detenzione privato, CoreCivic, ospitava 17 bambini sotto l'età di due anni, di cui 9 sotto l'età di un anno (uno di cinque mesi!). A seguito del decesso di un bambino di 19 mesi che era stato rilasciato da poco, le associazioni di difesa dei diritti degli immigrati hanno ricevuto segnalazioni di condizioni igieniche disastrose, insufficienza di cibo e assenza di frequenti controlli medici. Dodici neonati sono stati rilasciati in custodia a parenti o amici di fiducia delle famiglie, ma l'Amministrazione non ha risposto alle domande di chiarimento sulle condizioni di queste detenzioni. I bambini sarebbero arrivati con una carovana di 2.000 emigranti bloccata alla frontiera e sono stati separati dalle famiglie come segnale di avvertimento ai nuovi emigranti in arrivo. ${ }^{23}$

Secondo il Texas Civil Rights Project, citando la condanna dell'Amministrazione Trump da parte dell'Inter-American Commission on $\mathrm{Hu}$ man Rights, dal 28 giugno 2018, termine ufficiale delle separazioni, fino a fine febbraio 2019 circa 245 genitori e circa 1.500 altri parenti adulti 'non-genitori' (questa dicitura può includere anche fratelli, quando uno è minorenne e l'altro maggiorenne) sarebbero stati separati dai bambini che accompagnavano. ${ }^{24}$ Queste separazioni non sono state motivate adeguatamente e - come detto in precedenza - spesso sono state decise in maniera arbitraria dall'ufficiale di turno alla frontiera.

\subsection{Lo scandalo del profitto}

Nel frattempo è emerso il business legato all'affidamento dei bambini dati in custodia dall'agenzia governativa Health and Human Resources a ditte private tramite appalto pubblico. Si tratta, tra le altre, dell'organizzazione no profit Southwest Key che ha sede nel Texas, ad Austin, fondata da un educatore di origine ispanica. Southwest Key e altre aziende private sono specializzate nell'accompagnamento e nella 'rieducazione' di minorenni in attesa di giudizio o condannati in

22 https://www.vox.com/policy-and-politics/2019/3/6/18253396/nielsen-cages-family-separations-house-homelandsecurity-committee (2019-05-11).

23 https://www.cbsnews.com/news/immigrant-children-detained-12-babies-released-from-ice-custody-detention-center-dilley-texas-2019-03-๑4/ (2019-0511).

24 https://texascivilrightsproject.org/families (2019-05-11). 
diversi territori degli Stati Uniti. Benché Southwest Key si vanti sul proprio sito web di essere stata riconosciuta nel 2017 dal Consiglio per i Diritti Umani delle Nazioni Unite come fornitrice di un servizio d'eccellenza nell'affidamento di minori non accompagnati, secondo un rapporto di Craig Harris questa organizzazione avrebbe incassato almeno 458 milioni di dollari nel 2018 per custodire bambini non accompagnati in applicazione della politica di tolleranza zero. ${ }^{25}$ L'amministratore delegato di Southwest Key, Juan Sanchez ha tratto notevoli benefici economici: circa 1,47 milioni di dollari di compenso. ${ }^{26}$

L'organizzazione ha in custodia circa 1.600 bambini in 26 strutture di cui 8 nello Stato dell'Arizona, dove vengono ospitati bambini provenienti dal Guatemala. La media di un soggiorno è di 52 giorni. Il contratto triennale 2017-19 ha portato a Southwest Key 990 milioni di dollari. Ovviamente, più prolungato è il soggiorno di un bambino, più soldi si guadagnano. Ai bambini sono garantite sei ore di scuola al giorno, sedute di orientamento e tempo libero. Ma secondo l'Economic Research Institute, insieme all'American Friends' Service Committee - organizzazioni indipendenti che monitorano le attività delle prigioni private -, l'importo dell'appalto è troppo elevato e trasforma i bambini in beni di consumo. Il problema di fondo, sempre secondo Craig Harris, è che la politica di Donald Trump è un'occasione d'oro per le aziende private del business penitenziario.

Southwest Key e CoreCivic non sono gli unici esempi. Secondo un'indagine del quotidiano Daily Beast, il settore delle organizzazioni private in appalto con l'Agenzia dell'Immigrazione avrebbe incassato per il solo anno 2018 ben 800 milioni di dollari di fondi pubblici (nel 2018 hanno avuto in custodia giornaliera 44.892 immigrati, con previsioni di 52.000 al giorno per il 2019). Il gruppo Geo, coinvolto pienamente nel business della detenzione degli immigrati, avrebbe contribuito generosamente alla campagna elettorale di Trump. ${ }^{27}$

\subsection{Gli effetti psicologici su bambini e adulti}

Anche se riunite, le famiglie - soprattutto i bambini - potranno soffrire comunque gli effetti negativi di questo trattamento. Un funzionario del Governo coinvolto nella politica dell'Amministrazione Trump,

25 https://eu.azcentral.com/story/news/politics/immigration/2018/06/22/ southwest-key-gets-458-million-house-migrant-children/726551002/ (2019-05-18).

26 Nel marzo 2019 Sanchez si è dimesso della sua carica. Contemporaneamente sono state rivelate delle accuse di maltrattamento di bambini in alcuni centri.

27 https://edition.cnn.com/2018/06/14/us/inside-immigrant-children-shelter-brownsville-texas-invs/index.html; https://wapo.st/33m9DP0; https:// www.thedailybeast.com/dollar800-million-in-taxpayer-money-went-to-private-prisons-where-migrants-work-for-pennies (2019-05-19). 
testimoniando davanti al Congresso, ha avvertito il Governo degli effetti traumatizzanti sui bambini: «La politica d'immigrazione americana produce danni per i bambini in molti modi, a cominciare dalle condizioni terribili di vita sotto le tende o nelle celle» (trad. dell'Autrice) ma il suo monito non è stato ascoltato. ${ }^{28}$

Il decreto presidenziale, mettendo un termine alla separazione delle famiglie, non ha messo a tacere le proteste e ancora meno ha messo fine alla criminalizzazione degli emigranti alla frontiera Sud. Come già detto, i 2.700 bambini separati nel giugno 2018 non sono stati riuniti ai loro genitori nel tempo previsto. Rispetto a questa situazione, i pediatri hanno sottolineato che la detenzione, seppur insieme ai genitori e per qualsiasi durata, anche breve, può provocare ai bambini traumi psicologici, di lungo periodo, in certi casi irreversibili. A questo proposito, Coolen Kraft, Presidente dell'Accademia Americana di Pediatria, ha sottolineato che «la continuazione della politica di tolleranza zero metterà ancora più bambini in detenzione. Quelle strutture costituiscono un ambiente dannoso per i bambini, anche per quelli che restano con la propria famiglia» (trad. dell'Autrice). ${ }^{29}$

Secondo l'Accademia Americana di Pediatria, la detenzione di bambini e adulti provoca traumi psicologici, danni a lungo termine alla salute fisica e mentale, con manifestazioni di ansia, depressione e stress post-traumatico. I bambini potrebbero soffrire di problemi emotivi anche in età adulta. Le condizioni stesse della detenzione in alcuni centri sono particolarmente traumatizzanti: servizi igienici aperti, luci accese 24 ore su 24, obbligo di dormire sul pavimento, temperature gelide, insufficienza di cibo di qualità, etc. Anche quando le famiglie non vengono separate, l'Accademia Americana di Pediatria continua a denunciare la politica che prevede l'incriminazione degli emigranti condannandoli a priori senza un giusto processo e tutte le garanzie legali.

Lo stesso parere è stato fornito dall'Associazione Americana degli Psicologi, nel maggio 2018:

La politica dell'Amministrazione di separare i bambini dalle loro famiglie [...] non solo è inutile e crudele, ma è anche una minaccia alla salute mentale e fisica dei bambini [...] La ricerca scientifica dimostra che gli immigrati vivono situazioni di stress legate alle condizioni che li hanno portati a fuggire dai loro Paesi d'origine. Più a lungo i bambini e i genitori vengono separati, maggiori sono i sintomi di ansia e di depressione per i bambini, e anche per i loro genitori. Gli effetti dannosi per i bambini includono stress psichico, difficoltà scolastiche e disturbi nello sviluppo. (trad. dell'Autrice)

28 https://thehill.com/opinion/immigration/400006-they-knew-it-would-cause-lasting-harm-and-still-took-children-from (2019-05-19).

29 https://www.aappublications.org/news/2018/07/24/washington@72418 (2019-05-18). 
Secondo l'UNICEF (2018), la deportazione di bambini verso i Paesi di origine è una procedura crudele poiché con ogni probabilità non hanno più la loro casa e ritornano in situazioni in cui sono ancora più vulnerabili. ${ }^{30}$

\section{$5 \quad$ La sottile linea tra legale e illegale della politica dell'Amministrazione Trump}

La politica di separazione delle famiglie e di detenzione dei bambini dell'Amministrazione Trump si configurerebbe come illegale sia sul piano del diritto internazionale sia sul piano del diritto interno. Oltre a violare la Convenzione di Ginevra sullo status dei rifugiati (che gli Stati Uniti non hanno mai firmato), il Refugee Act statunitense del $1980,{ }^{31}$ la Convenzione internazionale sui diritti civili e politici del $1966^{32}$ (ratificato dagli USA nel 1992), la Costituzione americana, ${ }^{33}$ la convenzione internazionale sui diritti dell'infanzia e dell'adolescenza $a^{34}$

30 https://www.unicef.org/lac/guatemala/en/press-releases/children-returnedcentral-america-and-mexico-heightened-risk-violence-stigma-and (2019-05-18).

31 Il Refugee Act ha aumentato la quota annuale di rifugiati ammessi sul territorio a 5.000, attribuendo al Presidente la possibilità di aumentare la quota per motivi umanitari. Esso dà una definizione di rifugiato come «qualunque persona che per motivi legittimi teme di essere perseguitata nel Paese di origine», la quale secondo l'Harvard Law Review si avvicina a quello del Protocollo del 1967; tuttavia i tribunali statunitensi, dalle corti federali dei singoli Stati alla Corte Suprema, hanno dato molteplici interpretazioni dei termini contenuti nelle convenzioni, a volte differenti da quelle dell'UNHCR, almeno fino a quando le sentenze Immigration and Naturalization Service v. Stevic del 1984 e Cardoza-Fonseca del 1986 hanno fatto chiarezza. Esse stabiliscono che per ottenere l'asilo è necessario dimostrare solo un timore «ben fondato» (well-founded) di poter essere vittima di persecuzione e non «una probabilità evidente» (clear probability), https://www.aclu.org/blog/immigrants-rights/trump-administrationillegally-forcing-asylum-seekers-out-united-states; https://harvardlawreview.org/2018/03/american-courts-and-the-u-n-high-commissioner-for-refugees-a-need-forharmony-in-the-face-of-a-refugee-crisis/; https://www.archivesfoundation.org/documents/refugee-act-1980 (2019-06-16).

32 Prevede all'art. 3 adeguata protezione contro la detenzione arbitraria e all'art. 4 protezione dalle discriminazioni in caso di pericolo pubblico eccezionale.

33 Emendamenti V, VI, VIII e XIV riguardanti il giusto processo. Secondo Fisher i diritti garantiti ai non-statunitensi che si trovano sul territorio nazionale sono gli stessi dei cittadini statunitensi. Nel 1898, la Corte Suprema decise che il termine person, così come figura nel V Emendamento, si applica agli stranieri che vivono negli USA e già nel 1893 considerava che gli stranieri (in questo caso si trattava di operai cinesi) dovevano avere gli stessi diritti alla protezione garantita dalle leggi che avevano i cittadini statunitensi (sentenze U.S. v. Wong Kim Ark 1898, Fong Yue Ting v. U.S. 1893) (https://www.forbes.com/sites/danielfisher/2017/01/30/does-the-constitution-protect-non-citizens-judges-say-yes/\#78d11ca24f1d, 2019-06-16).

34 Garantisce al fanciullo il diritto ad essere curato dai genitori (art. 7), a beneficiare di relazioni familiari senza interferenza di chiunque (art. 8), a non essere separato dalla famiglia (art. 9). L'art. 10 prevede che «ogni domanda presentata da un fanciullo o dai suoi genitori in vista di entrare in uno Stato parte o di lasciarlo ai fini di un ri- 
(non ratificata dagli Stati Uniti che pur avevano partecipato attivamente alla stesura del documento finale e hanno firmato nel 1995), la politica di tolleranza zero viola il Flores Agreement, che è legato ad una sentenza del tribunale della California nel caso Flores c. Reno (1987), la quale impediva di mandare i bambini emigranti in carcere con i loro genitori. La sentenza prevedeva che i bambini venissero ospitati in strutture adeguate e sicure il più rapidamente possibile (entro 20 giorni circa), ma di fatto la separazione delle famiglie era già una pratica comune. Dieci anni dopo, nel 1997, Bill Clinton ha firmato il Flores Agreement che rinforzava tale sentenza e che, oltre alla barriera temporale, fissava determinate norme per la detenzione di minori: sufficienti quantità di cibo e acqua, assistenza sanitaria, servizi igienici e condizioni di temperatura adeguate. Tuttavia, come si è visto, le condizioni di detenzione dei bambini violano l'Accordo Flores (definito dall'ex Segretaria alla sicurezza nazionale, Kirstjen Nielsen, come una «scappatoia legale» che incoraggia l'immigrazione e che impedisce al governo di espellere dal Paese chi non ha il diritto di rimanerci ${ }^{35}$ ).

Nel complesso, nel caso degli emigranti che arrivano dalla frontiera Sud, c'è discriminazione contro persone di determinate origini geografiche - America Centrale -, che sono private dei diritti garantiti dal due process e che subiscono la violazione dei diritti garantiti dal Refugee Act. È vero che l'Amministrazione statunitense può esercitare le proprie prerogative nel determinare le quote (e all'esecutivo di aumentare le quote di persone che arrivano da Paesi in guerra per motivi umanitari), ma non di selezionare l'origine nazionale delle persone da ammettere. Altre sentenze della Corte Suprema, approvate nel periodo che va dal 1890 al 1950, specificarono che in qualsiasi caso il principio del due process è prioritario; una persona di qualunque origine non può essere considerata un criminale senza una procedura giuridica regolare e un'adeguata difesa, anche se priva di documenti.

congiungimento familiare sarà considerata con uno spirito positivo, con umanità e diligenza», l'art. 37 prevede che gli Stati vigilino affinché «nessun fanciullo sia sottoposto a tortura o a pene o trattamenti crudeli, inumani o degradanti. Né la pena capitale né l'imprigionamento a vita senza possibilità di rilascio devono essere decretati per reati commessi da persone di età inferiore a diciotto anni». Nel 2005 la Corte Suprema ha messo termine alla pena di morte dei minori ma è rimasta la condanna dei minorenni all'ergastolo senza possibilità di libertà condizionale. Si veda anche https:// www.globalresearch.ca/indefinite-detention-of-migrants-violates-international-law/5646823 (2019-06-16).

35 https://www.latimes.com/local/lanow/la-me-ln-flores-agreement-trumpimmigration-20180906-story.html (2019-11-07). 


\section{Conclusione}

Gli aspetti della politica di tolleranza zero analizzati in questo saggio illustrano una situazione che, sfruttata a fini politici, giunge ad una sorta di politica della tortura 'ufficializzata', sfidando il potere giudiziario.

La politica di tolleranza zero è stata accompagnata da tagli ai fondi per l'assistenza giuridica ai bambini separati ${ }^{36} \mathrm{e}$ ha avuto conseguenze negative sui bambini, i quali a volte praticano l'automutilazione o parlano di suicidio. ${ }^{37}$ Questi bambini sono in condizioni di custodia paragonabili a quelle di una prigione e spesso non hanno mezzi per comunicare con i genitori e gli assistenti sociali. ${ }^{38}$ Alcuni bambini sono deceduti durante o subito dopo la custodia ${ }^{39}$ o sono stati strappati alle famiglie in maniera brutale: è recente e vergognosa la storia di un neonato di quattro mesi strappato ai genitori rumeni entrati attraverso la frontiera con il Messico. ${ }^{40}$

I giudici federali che hanno condannato e bloccato la politica di separazione delle famiglie hanno rischiato un ricorso in appello da parte dell'Amministrazione sulla base dell'autorità dell'esecutivo, ma probabilmente ciò non si è verificato per motivi politici e per l'impopolarità di questa politica. Il 21 dicembre 2018 la Corte Suprema, con la decisione 5-4, ha respinto la domanda dell'Amministrazione Trump per un nuovo divieto da porre alle richieste d'asilo, ma essa non ha rinunciato completamente a questa pratica e ancora nel 2019 ha tentato di reintrodurla. L'Amministrazione Trump continua a promuovere la politica di separazione delle famiglie come unica soluzione e sta immaginando una nuova misura: mettere le famiglie di fronte alla 'scelta' tra separazione dai figli o incarcerazione familiare. ${ }^{41}$ L'Office for Refugee Resettlement, intervistato, difende la prassi secondo la quale i bambini non possono essere affidati a sponsor di cui non si è accertata una qualsiasi relazione con la famiglia. ${ }^{42} \mathrm{Nel}$ frattempo, convocata da un tribunale federale in California per rispondere delle condizioni di detenzione dei bambini e del mancato rispetto

36 https://wapo.st/2pT53cD (2019-06-16).

37 https://www.washingtonpost.com/outlook/2019/05/20/trump-is-planningcruel-twist-border-policy/?utm_term=.193378bc730e (2019-06-16).

38 https://www.apnews.com/07ac4ee08c8c41a7ba9b19f1762c5115 (2019-06-21).

39 https://www.latimes.com/opinion/op-ed/la-oe-katz-immigrant-concentration-camps-20190609-story.html; https://www.latimes.com/nation/la-na-migrant-child-border-deaths-20190524-story.html (2019-06-16).

40 https://www.nytimes.com/2019/06/14/the-weekly/trump-immigration-border-separation-family.html?smid=fb-nytimes\&smtyp=cur (2019-06-16).

41 https://www.washingtonpost.com/outlook/2019/05/20/trump-is-planningcruel-twist-border-policy/?utm_term=.e8efe624c985 (2019-06-13).

42 https://apnews.com/๑7ac4ee๑8c8c41a7ba9b19f1762c5115 (2019-11-07). 


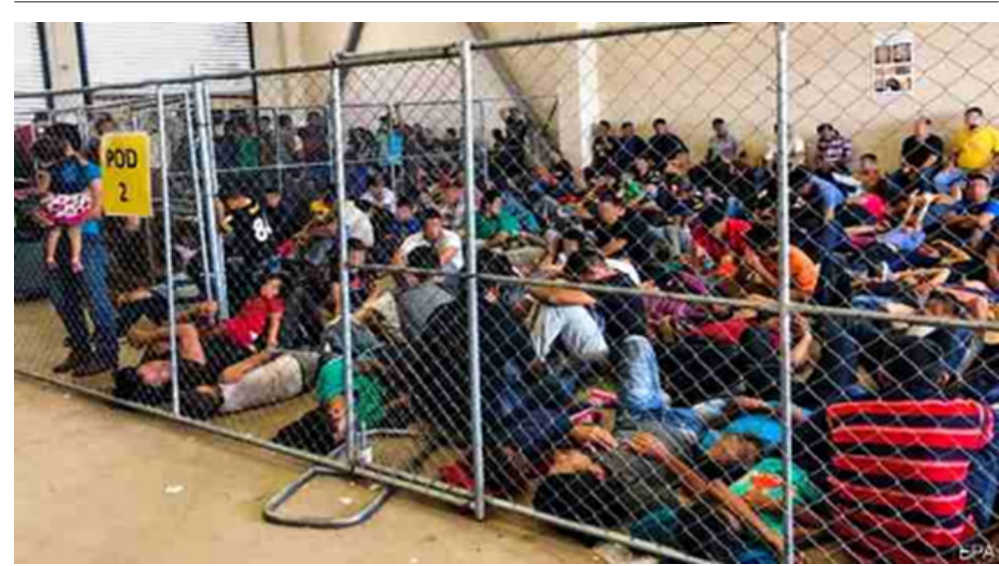

Figura 3 Minori detenuti nei centri di accoglienza. The Economist, 06-06-2019

dell'Accordo Flores, l'Amministrazione Trump ha contestato l'obbligo di fornire prodotti per l'igiene e condizioni normali per dormire, dichiarando che condizioni di vita decenti come sapone e materassi non sono contemplati nell'Accordo Flores. ${ }^{43}$ La situazione è rimasta caotica e scandalosa, come si vede dall'immagine tratta dall'Economist del 6 luglio 2019 [fig. 3].

Visti l'opacità delle comunicazioni e il disprezzo della stampa dimostrato dall'Amministrazione Trump, conviene rendere omaggio sia al lavoro di giornalismo investigativo che ha reso possibili le informazioni e i fatti riassunti in questo articolo sia alle azioni umanitarie in corso e a venire per contrastare una situazione intollerabile di violazione dei diritti umani. ${ }^{44}$

\section{Bibliografia}

Amnesty International (2019). Most Dangerous Journey: What Central American Migrants Face When They Try to Cross the Border. URL https://www.amnestyusa.org/most-dangerous-journey-what-central-ameri-

43 https://www.washingtonpost.com/nation/2019/06/21/detained-migrantchildren-no-toothbrush-soap-sleep/?utm_term=.๑0ac974554f9 (2019-06-30).

44 Si veda il rapporto dell'Ufficio d'ispezione dei dipartimenti del governo (Office of the Inspector General 2018) sulle pratiche della Homeland Security, pubblicato il 27 settembre 2018 e intitolato Special Review. Initial Observations Regarding Family Separation Issues Under the Zero Tolerance Policy, che attesta la situazione caotica provocata dalla politica di tolleranza zero e che contiene la risposta del Dipartimento. 
can-migrants-face-when-they-try-to-cross-the-border (201903-03).

Chacón, Justin Akers (2010a). «La persecuzione degli undocumented e la lotta per i diritti degli immigrati negli Stati Uniti». Basso, Pietro (a cura di), Razzismo di stato. Stati Uniti, Europa, Italia. Milano: FrancoAngeli, 61-100.

Chacón, Justin Akers (2010b). «Vigilanti ai confine con il Messico: la nuova guerra agli emigranti latino-americani». Basso, Pietro (a cura di), Razzismo di stato. Stati Uniti, Europa, Italia. Milano: FrancoAngeli, 101-14.

Colby, Sandra; Ortman, Jennifer M. (2015). Projections of the Size and Composition of the U.S. Population: 2014 to 2060. URL https://www.census. gov/content/dam/Census/library/publications/2015/demo/p251143.pdf (2019-03-03).

Cox, Daniel; Lienesch, Rachel; Jones, Robert P. (2017). «Beyond Economics: Fears of Cultural Displacement Pushed the White Working Class to Trump». PRRI/TheAtlantic, 5 september. URL https://www.prri.org/research/ white-working-class-attitudes-economy-trade-immigrationelection-donald-trump (2019-06-13).

Ehrenreich, Barbara (1989). Fear of Falling. The Inner Life of the Middle Class. New York: Pantheon Books.

Médecins sans Frontières (2017). Forced to Flee Central America's Northern Triangle. A Neglected Humanitarian Crisis. URL https://www. doctorswithoutborders.org/what-we-do/news-stories/research/reportforced-flee-central-americas-northern-triangle (2019-03-03).

UNHCR (2019). Children on the Run. URL https://www.unhcr.org/en-us/ about-us/background/56fc266f4/children-on-the-run-fullreport.html (2019-03-03).

UNICEF (2018). Uprooted in Central America and Mexico. Refugee Children Face a Vicious Cycle of Hardship and Danger. URL https://weshare.unicef. org/Folder/2AMZIFVOSOSO (2019-03-03).

Office of the Inspector General (2018). Special Review. Initial Observations Regarding Family Separation Issues Under the Zero Tolerance Policy. URL https://www.oig.dhs.gov/sites/default/files/assets/2018-10/0IG18-84-Sep18.pdf (2019-11-07). 



\title{
Tortura e immigrazione in America Latina e in Brasile nel contesto della crisi e della crescita dei partiti di estrema destra
}

\author{
Karina Quintanilha \\ Pontifícia Universidade Católica de São Paulo, Brasil \\ Patricia Villen \\ Universidade Estadual de Campinas, Brasil
}

\begin{abstract}
This chapter analyzes the relations between torture and immigration in the current context of crisis and rise of far right-wing parties in Latin America, particularly in Brazil. It considers the changes arising from the global economic crisis - which has been deeply hitting the region since 2012 - and from the hegemony of conservative political forces through a renewed alliance with the military power. These changes have direct repercussions on the immigration policies and legislation, as well as on the treatment of immigrants. Cases of xenophobia, violence and torture against immigrants and refugees from poor countries - especially when they are not white - are constantly registered. Torture is a practice with a long historical tradition in Latin American and was reinforced in the 20th century, during the military dictatorships; now, in this region it finds again a fertile ground to grow. Besides the analysis of the political and ideological-cultural environment characterized by a vindication of torture in Brazil, this chapter shows that the mass imprisonment system is a key-space for this practice, being the State its main protagonist. Immigrants, particularly African black women accused of acting as mulas for international drug trafficking, are increasingly exposed to systematic violations of human rights, including torture.
\end{abstract}

Keywords Torture. Migration. Latin America. Brazil. Economic crisis. Far-right parties.

Sommario 1 Introduzione. - 2 La tensione crescente tra le forze anti-immigrazione e pro-immigrazione in America Latina e in Brasile. - 3 Tortura e continuità della violenza di Stato in Brasile. - 4 Conclusione. 
Quando si evoca la parola 'tortura' in America Latina, la memoria solitamente ci riporta al periodo delle dittature militari che hanno pervaso il continente nella seconda metà del XX secolo; tuttavia sarebbe un grave errore non ricordare che la pratica della tortura è esistita nella regione in altri periodi storici, soprattutto durante il regime coloniale schiavista. ${ }^{1}$

Non vi è dubbio che, seppure a livelli diversi - in alcuni Paesi di più, in altri meno ${ }^{2}$-, le istituzioni e i Paesi latinoamericani che hanno sofferto le dittature in seguito al ritorno alla democrazia hanno generalmente ripudiato, almeno da un punto di vista formale, le violenze di Stato attuate nel precedente periodo storico, tra cui la tortura (principalmente contro i dissidenti politici).

In Brasile, il ritorno alla democrazia è iniziato attraverso una grande mobilitazione popolare nel 1985 ed è stato segnato dall'approvazione di un nuovo patto sociale sancito dai principi democratici distributivi della Costituzione Federale (1988). Da allora, sembrava che gli «anni di piombo» della dittatura militare (1964-85) - segnati da pratiche del terrore e da un'elevata concentrazione di ricchezza e potere - appartenessero a una pagina oscura del passato, ormai superata e ripudiata dalla società civile. Gli studiosi del periodo sostengono, però, che in questa transizione c'è stato un patto con i militari protagonisti della dittatura (Arantes 2010). ${ }^{3}$ Secondo Fernandes (1982), si è verificato

1 La storia coloniale del continente è stata segnata dalla tortura, rivolta principalmente contro gli indigeni e la popolazione nera, durante il periodo della schiavitù. In Brasile, le pratiche di tortura più feroci del periodo coloniale sono state analizzate nel dettaglio da storici e scienziati sociali (Thomson-Deveaux 2018). Oggi, le popolazioni indigene e nere continuano a essere il principale bersaglio della violenza, soprattutto da parte dello Stato e delle sue istituzioni militarizzate. Diversi studi dimostrano che in Brasile la popolazione nera è maggiormente colpita dalla violenza della polizia (Nascimento 1978; Nações Unidas Brasil 2019). Per quanto concerne la popolazione indigena, secondo la relazione della Comissão da Verdade (Commissione per la Verità), «il XX secolo è stato uno dei più violenti della storia dal 1550» (Brighenti 2016, 240). La contesa sulle terre, soprattutto nel processo di espropriazione delle terre indigene, ha acquisito ulteriore spinta dopo il 2014 ed è stata accompagnata dall'uccisione e dalla tortura degli indigeni (Tomazela 2019).

2 In Paesi come Argentina e Uruguay, la memoria del terrore legato alla dittatura militare è stata elaborata in modo migliore rispetto al Brasile. In tal senso, un'iniziativa importante è stata portata avanti dalla Commissione per la Verità, volta a documentare i reati e le atrocità commessi nel periodo dittatoriale. Tuttavia, tale Commissione ha iniziato a essere operativa soltanto nel 2011 e, nell'attuale contesto politico del Paese, non costituisce più una priorità dei governanti. Non è esclusa, addirittura, la possibilità di perseguire penalmente le persone e gli istituti che dirigono la Commissione. Si stanno verificando già dei casi di persecuzione politica contro i movimenti sociali, i partiti politici dell'opposizione (soprattutto di sinistra), i professori e gli studenti che criticano l'attuale governo.

3 Moraes $(2015,2016)$ sottolinea anche la necessità di un'effettiva giustizia di transizione in materia di migrazioni. 
un processo di «transizione lento, graduale e sicuro», che riflette una conciliazione politica dall'alto. In tal senso, la mancata realizzazione di una riforma dell'apparato amministrativo-militare e l'approvazione di una legge di amnistia «generale e illimitata» - che ha garantito l'impunità degli agenti di Stato accusati di crimini contro l'umanità - rispecchiano le lacune di questo processo di ridemocratizzazione.

Oltre alla mancata eliminazione delle vestigia della dittatura dal sistema democratico, negli ultimi anni in Brasile sono stati evocati, senza alcun pudore, segnali contrari a un'interpretazione critica della dittatura. Come spiega Antunes (2018), stiamo vivendo un processo di «controrivoluzione preventiva», una pratica di colpo di stato ricorrente nel sistema del potere politico in America Latina (soprattutto in Brasile), che si è nuovamente manifestata con l'impeachment nel 2016 della presidentessa Dilma Roussef, eletta democraticamente e deposta senza alcuna prova di reato. ${ }^{4}$

Alla fine del 2018, Jair Bolsonaro è stato eletto Presidente della Repubblica e rappresenta il principale simbolo dell'alleanza tra potere politico e potere militare, nonché dell'ascesa dell'estrema destra in America Latina (che trae forte ispirazione dall'esempio degli Stati Uniti e di altri Paesi come l'Italia e l'Ungheria). ${ }^{5}$ La sua elezione, in un certo modo, rispecchia un'alterazione del sentimento del Paese, sulla strada dell'apologia della tortura. Diverse sue dichiarazioni, rese pubbliche dai media, indicano il suo essere d'accordo con la pratica della tortura. ${ }^{6}$ Nonostante una parte della società continui a ripudiare e denunciare la tortura, nonché la sua apologia, il mondo istituzionale non sembra essere turbato da queste dichiarazioni (Della Barba, Wentzel 2016). Una buona parte della popolazione brasiliana - per motivi che non possono essere approfonditi in questa sede - si sente rappresentata dal clamore dell'ordine e della violenza militare come antidoto al malessere sociale provocato dalla profonda crisi economica che pervade il Paese, aggravata dalle scelte politiche e dalla corruzione.

4 In questa occasione, tutti i deputati federali sono stati chiamati a pronunciare il proprio voto palese, a favore o contro la deposizione della presidentessa. Molti di essi, oltre a votare, hanno presentato anche una breve dichiarazione. Jair Bolsonaro, all'epoca ancora deputato, ha votato a favore dell'impeachment, offrendo il proprio voto alla memoria del colonnello Carlos Alberto Brilhante Ustra, che partecipò alla tortura dell'expresidentessa Dilma Roussef durante la dittatura militare (Oliveira 2016). Ustra, noto per essere il responsabile dei metodi di tortura contro i militanti di sinistra, è stato l'unico militare dichiarato torturatore dalla giustizia brasiliana, pur essendo morto nel frattempo, senza scontare alcuna pena.

5 Antunes (2018) spiega che questa forma di potere si è dimostrata la più efficiente non soltanto per dare continuità, bensì anche per rafforzare il funzionamento del sistema neoliberista in Brasile. Non è un caso che il Ministro dell'economia, Paulo Guedes, presenti un percorso fortemente legato agli istituti liberisti nordamericani e ai rappresentanti politici pro Pinochet in Cile (Salém, Hoelever 2018).

6 Cf. https://www.youtube.com/watch?v=G5TiUmiF6ls\&t=12s (2019-05-01). 
È altresì importante sottolineare il movimento ideologico-culturale che cerca di imporsi in Brasile per cambiare il 'senso' critico nei confronti della dittatura militare finora incluso nei programmi di storia della scuola dell'obbligo e della scuola superiore. ${ }^{7}$ La prima richiesta di questo movimento è di proibire il termine dittatura e di sostituirlo con quello di regime. L'idea di fondo è di offrire un trattamento più 'equo' a questo periodo storico, rimuovendo un carico elevato di fattori negativi ad esso associati (tra cui la diffusa corruzione), evidenziando invece i suoi benefici per la società brasiliana. ${ }^{8}$ Nel contesto di questo movimento, anche la tortura viene considerata una pratica non soltanto legittima, ma addirittura necessaria per la società, soprattutto per risolvere i problemi sociali della crisi attuale, che si manifestano principalmente nella crescita della disoccupazione e della violenza, nell'abbassamento dei redditi e nel maggiore indebitamento delle persone. ${ }^{9}$

Diversi fatti, collegati al funzionamento della struttura economica neoliberista in vigore (Antunes 2018), saranno qui esposti per dimostrare la maggiore accettazione della tortura. Indubbiamente, la dittatura è evocata da una parte considerevole dei rappresentanti dello Stato, del sistema economico e dei principali mass-media come un modello esemplare della storia brasiliana, come un modello da perseguire per pensare al futuro della società brasiliana. La relazione tra tortura e immigrazione di conseguenza non è separata da questo contesto.

Ė necessario fare anche la seguente precisazione: una volta ritornata la democrazia, la ricerca sulla tortura è stata poco praticata, a causa di quanto indicato. Per tale motivo, sono scarsi gli studi realizzati su questo tema (cf. Marques de Jesus 2009). In particolare, la

7 La riforma del sistema di istruzione è una priorità dell'attuale governo. Oltre al progetto di privatizzazione totale, è incluso anche un aspetto ideologico per depurare l'istruzione dal marxismo culturale, dalla prospettiva di genere, dalla visione critica della schiavitù e del razzismo, tra le varie tematiche non gradite all'attuale configurazione del potere. L'obiettivo è quello di sostituire queste 'ideologie' con i valori morali 'neutri' di famiglia, Dio e, ovviamente, mercato (Villen, forthcoming). Non è un caso che le stesse riforme dell'istruzione siano in fase di implementazione in altri Paesi governati dall'estrema destra globale (Melegh 2018).

8 In Brasile è presente una retorica molto attiva secondo la quale, durante la dittatura, non vi era corruzione. Tuttavia, diversi fatti storici e studi scientifici dimostrano il contrario e illustrano che la corruzione non soltanto esisteva, bensì era anche resa silenziosa con la forza (Leite 1987).

9 Si sottolinea che tali problemi non si limitano alla crisi attuale, poiché sono strutturali e non sono stati affrontati dai governi precedenti del Partidos dos Trabalhadores (PT), soprattutto se consideriamo gli interessi a lungo termine della classe lavoratrice. La corruzione è stata una delle principali ragioni di scontento della popolazione brasiliana nei confronti del PT. Va sottolineato, tuttavia, che i media presentavano questa pratica come una prerogativa quasi esclusiva del PT, come se fosse meno presente negli altri partiti e non fosse intimamente legata al funzionamento del sistema economico attuale. Per un'analisi delle contraddizioni socio-economiche del Brasile più esplicite nell'attuale contesto di crisi economica e politica, si veda Villen 2017. 
tortura nei confronti degli immigrati è una pratica strategicamente resa invisibile e su cui è difficile fare ricerca. Tuttavia, esistono notizie sui giornali, ricerche sul campo, report di Ong e di istituzioni che si occupano di gruppi sociali specifici - immigrati e rifugiati, indigeni, neri, donne, emigranti interni, vittime del lavoro forzato - che offrono un campo di ricerca da ampliare.

In questo saggio sarà evidenziato che gli immigrati e i rifugiati debbono affrontare uno scenario politico ed economico in cambiamento in America Latina e in Brasile, che si riflette sulle politiche migratorie e sulle loro condizioni di inserimento sociale. Gli immigrati in condizioni socio-economiche vulnerabili sono sempre più esposti a violazioni sistematiche dei diritti umani, inclusa la tortura, soprattutto i non bianchi e le donne, specialmente quando attraversano i confini senza visto. ${ }^{10}$

A tal fine, presenteremo una contestualizzazione delle principali questioni migratorie in America Latina, in particolare in Brasile, nell'attuale scenario di profonda crisi economica, di attacco violento ai diritti e alle condizioni di lavoro, di ascesa dei partiti di estrema destra e della crescente militarizzazione della regione. In un secondo momento, proporremo un'analisi della situazione degli stranieri in Brasile accusati di fungere da mulas (corrieri) ${ }^{11}$ del traffico transnazionale di droga, una parte minoritaria della popolazione straniera che richiede un'attenzione speciale.

Per i motivi che evidenzieremo, il sistema che struttura l'incarcerazione di massa in Brasile costituisce il terreno più fertile per le pratiche di tortura. Il fenomeno dello «tsunami carcerario» (Martins 2018), molto presente su scala globale (Wacquant 2001), diventa sempre più uno strumento di controllo sociale in un Paese che presenta uno dei peggiori indici di disuguaglianza al mondo. ${ }^{12}$ Evidenzieremo come gli immigrati siano tra le vittime principali di questo processo, principalmente le donne non brasiliane e nere, accusate di essere mulas del traffico internazionale di droga, nonché come la combinazione imprigionamento/espulsione sia un fattore aggiuntivo di esposizione alla tortura.

10 Come è noto, i confini tra Paesi rappresentano uno spazio privilegiato per le pratiche di tortura, ma, purtroppo, le ricerche su questo tema in America Latina e soprattutto in Brasile sono limitate.

11 Termine utilizzato per riferirsi a persone (donne e uomini) che svolgono la funzione di corriere nel narcotraffico: «Portano, da un luogo a un altro, piccole quantità di droga in valigie, in altri oggetti o all'interno del proprio corpo» (Bumachar 2016, 41).

12 In Brasile, «sei brasiliani concentrano nelle proprie mani la stessa ricchezza di metà della popolazione più povera», ossia poco più di cento milioni di persone, e il «5\% più ricco [della popolazione brasiliana] detiene la stessa fetta di ricchezza del restante 95\%»! (Rossi 2017). Parallelamente, 165 milioni di brasiliani vivono con un reddito pro capite inferiore a due salari minimi (Oxfam 2017), l'equivalente di 230 euro. 


\section{La tensione crescente tra le forze anti-immigrazione e pro-immigrazione in America Latina e in Brasile}

Dopo la seconda metà del XX secolo, l'America Latina è stata studiata principalmente come continente di emigrazione. Il volume di movimenti in entrata è stato percentualmente basso rispetto a quello delle regioni ricche del pianeta, come l'Europa occidentale, gli Stati Uniti o alcune zone del Medio Oriente. Tuttavia, è indubbio che i movimenti in arrivo non sono scomparsi da un continente che storicamente è stato caratterizzato da arrivi massicci e strutturali di forza lavoro africana, durante il periodo dello schiavismo, e in seguito, di lavoratori 'liberi' europei.

In questo periodo, soprattutto dopo lo scoppio della crisi nei Paesi ricchi (2007-08), accompagnata da politiche migratorie selettive, restrittive e punitive (Basso 2010; Perocco 2011), il continente latinoamericano, come altre regioni del pianeta, ha iniziato a ricevere principalmente immigrati e rifugiati provenienti dai Paesi poveri del Sud del mondo (Baeninger et al. 2018a, 2018b). In America Latina, gli immigrati provengono dal contesto regionale, soprattutto da Colombia, Bolivia, Haiti e Venezuela; tuttavia sono numerosi anche gli immigrati provenienti da diverse regioni africane (Congo, Senegal, Angola, Mozambico), medio-orientali (Siria, Palestina) e asiatiche (Bangladesh, Filippine, Cina).

Questo cambiamento del profilo sociale dell'immigrazione, che non è più formata prevalentemente da lavoratori bianchi europei, considerati «vettori di sviluppo» e portatori di una «cultura più avanzata», ossia «braccianti civilizzatori» (Villen 2018a), si manifesta in tutto il continente latinoamericano. Vi sono molte sfumature di questo cambiamento che potrebbero essere approfondite, in particolare per quanto concerne i bersagli del razzismo e il tipo di inserimento nel mercato del lavoro e nei Paesi di arrivo. La realtà è che questo nuovo profilo di immigrazione presenta condizioni di vita e di lavoro molto precarie e costituisce una miniera d'oro per i partiti di estrema destra, che seguono l'esempio di quanto avvenuto nei Paesi ricchi del Nord del mondo (Basso 2010). Questi partiti, come è noto, si basano su discorsi xenofobi e razzisti per criminalizzare (Quintanilha 2019; Moraes 2015) la presenza nel continente latinoamericano degli immigrati e dei rifugiati provenienti dal Sud del mondo (nonostante la percentuale di questa popolazione sia molto bassa). ${ }^{13}$

13 Le ragioni strutturali che spiegano questo orientamento politico sono state ampiamente analizzate nel libro curato da Basso (2010). Il caso dell'Europa dell'Est è esemplificativo dei fenomeni associati al razzismo di stato (Melegh 2006). 
Ceriani (2017) $)^{14}$ evidenzia che la presenza di questa immigrazione costituisce uno scenario complesso, segnato da crescenti tensioni tra le forze a favore dell'immigrazione (rappresentate da iniziative degli stessi immigrati e delle organizzazioni che li rappresentano), e le forze conservatrici anti-immigrazione che, oggi, hanno preso il sopravvento, soprattutto dopo la manifestazione più accentuata della crisi mondiale nel continente, a partire dal 2012.

Da un lato, Ceriani mette in luce le lotte e le iniziative dei movimenti sociali e degli immigrati in America Latina, che, assieme agli autoctoni e alle organizzazioni di rappresentanza, sono molto attivi e hanno ottenuto vittorie importanti nell'indirizzare le politiche e fare pressioni per l'approvazione di leggi favorevoli all'immigrazione. Le lotte contro il razzismo e la xenofobia, a difesa dei diritti umani e dei diritti dei lavoratori immigrati, costituiscono una parte importante di queste forze. La pressione dei movimenti sociali ha portato all'approvazione di leggi con un orientamento opposto alla criminalizzazione dell'immigrazione in Paesi quali l'Argentina, il Brasile, l'Ecuador, il Guatemala, il Messico. Inoltre, sono stati approvati accordi regionali importanti, ad esempio in ambito del Mercosur (Mercato comune del sud), che hanno alimentato un dibattito sulla costruzione della cittadinanza sudamericana.

Dall'altro lato, l'autore sottolinea che queste iniziative hanno un carattere di «stadio incompiuto» in un contesto globale critico, che si mostra sempre più contrario al diritto alla migrazione. Nei Paesi latinoamericani che hanno operato modifiche legislative favorevoli all'immigrazione, si nota la difficoltà di applicare nella pratica i principi a tutela dei diritti degli immigrati. Parimenti, la propaganda xenofoba e razzista, che associa l'immigrazione alla criminalità (sebbene le statistiche smentiscano tale associazione), ha assunto livelli preoccupanti e si è tradotta nell'applicazione di politiche dure contro gli immigrati. In tal senso è emblematico il caso dell'Ecuador, Paese che fino a poco tempo fa era considerato uno dei più avanzati in America Latina per un trattamento giuridico più aperto nei confronti degli immigrati (Jacques, Linárez, Useche 2019).

Innumerevoli episodi confermano quest'ultima tendenza, quali le deportazioni e le espulsioni in Cile e in Brasile; il tentativo di espulsione degli indios Warao venezuelani in Brasile; ${ }^{15}$ i progetti di legge per creare quote volte alla riduzione degli ingressi; la creazione di un

14 Questa visione dell'autore sulle forze politiche in ambito di immigrazione è stata presentata al Forum internazionale Fontié Ki Kwaze - Fronteiras Cruzadas (2018), tenutosi presso la Escola de Comunicação e Artes dell’Università di São Paulo.

15 Dati di Human Rights Watch dimostrano che nel 2016 la Polizia Federale ha deportato 514 venezuelani di Roraima (nel 2015 sono state deportate 20 persone). Sono frequenti i casi di deportazione individuale e le deportazioni di massa da parte della Polizia Federale: 200 venezuelani sono stati deportati nel settembre 2016, tra cui decine di bambini. Nel dicembre del 2016, la Polizia Federale ha cercato di deportare 450 
dispositivo per controllare l'immigrazione in Argentina, che rende obbligatorio per tutti i funzionari pubblici, principalmente nell'ambito della sanità e dell'istruzione, di verificare la situazione di regolarità degli immigrati prima di fornire servizi; la criminalizzazione dell'entrata (obbligatoriamente) irregolare degli immigrati in Paesi come la Colombia, l'Ecuador, il Perù; i partiti conservatori hanno bloccato molte politiche presentate nell'ambito del Mercosur, volte alla tutela dei diritti degli immigrati; la presenza rilevante delle forze armate ai confini nazionali, che vengono presentate come l'istituzione più adeguata per 'governare' le migrazioni. Infine, non si scarta l'idea che il continente possa ampliare, seguendo l'esempio dell'Europa e degli Stati Uniti, i propri Centri di detenzione per gli immigrati.

Secondo Domenech $(2011,2015)$, l'America Latina presenta un quadro di limitazione dell'immigrazione piuttosto sofisticato. I cambiamenti operati alla legislazione sull'immigrazione in Argentina nel 2011 simboleggiano una serie di idee e di pratiche che, facendo riferimento ai diritti umani come fonte di legittimazione, perseguono la stessa finalità delle politiche migratorie restrittive e punitive del Nord del mondo. Questa affermazione è valida anche nel caso del Brasile, poiché l'asse centrale della politica migratoria, gestita tramite decreti, continua a essere vincolato, come durante la dittatura, alle politiche di sicurezza e di controllo, e ogni giorno vengono presentati nuovi regolamenti che limitano gli ingressi e la regolarizzazione degli immigrati. Inoltre, questa politica repressiva è rafforzata da deportazioni ed espulsioni, attraverso pratiche caratterizzate da scarsa visibilità come si vedrà nelle prossime pagine (Quintanilha 2019).

Nel contesto latinoamericano attualmente la sfida principale riguarda l'esodo venezuelano, per motivi che non possono essere approfonditi in questa sede. Si tratta probabilmente dell'esempio più emblematico di movimenti migratori concepiti e gestiti come 'emergenza' (quando invece sono movimenti strutturali, che aumenteranno in futuro). A causa della crisi economica e politica in Venezuela, sicuramente legata a fattori interni, ma senz'altro alimentata dalla pressione di grandi potenze quali gli Usa, tra il 2014 e il 2018 oltre 2,3 milioni di persone hanno lasciato il Paese. ${ }^{16}$ L'arrivo dei venezuelani ha generato panico sociale nei Paesi di destinazione, ma anche innumerevoli manifestazioni di solidarietà. Molti venezuelani arrivavano affamati e giravano per le strade delle città alla ricerca di aiuto.

16 Le principali destinazioni dei venezuelani in America Latina sono Colombia, Perù, Cile, Argentina, Panama e Brasile. 
La tesi del razzismo di Stato (Basso 2010) è indubbiamente utile per spiegare le nuove tendenze delle politiche migratorie in America Latina per gestire questi movimenti. Il suddetto cambiamento del contesto politico ed economico ha rafforzato le barriere (socio-economiche, culturali, burocratiche e militari) intorno agli immigrati, specie se si considerano gli antichi problemi strutturali dei Paesi ex-colonizzati, accentuati dall'attuale contesto di crisi e dall'applicazione dei piani di aggiustamento strutturale. ${ }^{17}$ Parallelamente, in questo periodo il contesto politico latinoamericano è stato segnato da diversi colpi di stato (Honduras, Paraguay, Brasile), che spiegano il concetto sopracitato di «rivoluzione preventiva» (Antunes 2018). E questo senza considerare la possibile guerra che si sta fomentando in Venezuela per deporre il presidente Nicolas Maduro, con il sostegno dei governi ultra-liberisti di Colombia, Argentina, Cile e Brasile, supporter incondizionati di Donald Trump.

Con l'impeachment della presidentessa Dilma Rousse $\mathrm{f}^{18}$ e l'elezione di Jair Bolsonaro (2018), il Brasile è diventato la seconda principale locomotiva (dopo gli Stati Uniti) di questo processo politico nel continente, che sostiene l'applicazione di politiche ultra-neoliberiste, realizzate in collaborazione con il potere militare e volte alla precarizzazione illimitata del mercato del lavoro (Antunes 2018), nonché a privatizzazioni generalizzate di beni e servizi pubblici.

Il Paese è stato la principale destinazione (insieme all'Argentina) di movimenti immigratori nel primo decennio del XXI secolo. Con la crisi, il Brasile ha una forte probabilità di diventare un Paese di emigrazione (Sassen 2014; Villen 2018b), tuttavia, nonostante i pesanti effetti della crisi mondiale in Brasile, ${ }^{19}$ esso continua a ricevere immigrati e rifugiati da diverse parti del mondo, soprattutto da Venezuela, Haiti e da Paesi africani e asiatici. ${ }^{20}$

17 In Brasile, le riforme del lavoro in fase di approvazione sono state decise senza alcun dialogo con la popolazione e costituiscono un attacco brutale ai diritti dei lavoratori; sono volte alla flessibilizzazione totale del mercato del lavoro, con il fine ultimo di abbassare i salari, eliminare le tutele giuridiche dei lavoratori e fare pressione sui movimenti sindacali (Antunes 2018). Sta per essere approvata anche una riforma volta a distruggere il sistema di previdenza pubblica. I progetti di privatizzazione vanno di pari passo con i notevoli tagli ai servizi pubblici, soprattutto nei settori dell'istruzione e della sanità.

18 Una parte considerevole degli studi sull'impeachment della presidentessa Dilma indica come si sia trattato di un colpo di Stato appoggiato ed esecutato dal Parlamento, con la partecipazione del potere giudiziario e dei media (Löwy 2016; Lucena et al. 2017; Braga 2018; Mascaro 2018). Questi studi denunciano la totale mancanza di rispetto per la democrazia.

19 Si stima che la crisi dal 2015 abbia prodotto 15 milioni di disoccupati e la stessa quantità di sotto-occupati, portando il Paese al secondo posto nella classifica della disoccupazione in America Latina, secondo solo ad Haiti.

20 I dati della Polizia federale del 2017 indicano in 1.232.213 il numero di immigrati regolarmente residenti in Brasile. Gli immigrati sans papier e le presenze di stranieri per breve termine (spesso legati ai circuiti produttivi) non sono contemplati in questo registro. Nel 2017, 10.145 persone avevano lo status di rifugiato, principalmente pro- 
Il quadro dell'immigrazione nel Paese è piuttosto eterogeneo, con diverse nazionalità e settori economici che assorbono la forza lavoro immigrata (Villen 2018a). È possibile individuare un movimento di ingresso legale, ${ }^{21}$ legato a investimenti di capitali e alla richiesta di settori strategici con scarsità di lavoratori altamente qualificati. Pizzaro $(2005,20)$ spiega il collegamento tra questi movimenti e il «mercato globale delle risorse umane qualificate». Questo movimento di lavoratori qualificati riceve un visto temporaneo di residenza, che si inserisce nel contesto della circular migration.

Quello del petrolio è stato il principale settore attrattivo di questi lavoratori wanted and welcome, che hanno maggiore visibilità statistica e riescono a passare per motivi di lavoro le strette maglie dei canali di ingresso. ${ }^{22}$ Questo circuito immigratorio è stato caratterizzato anche dal reclutamento di massa di medici cubani (principalmente donne) per il settore della sanità pubblica attraverso il programma Mais Médicos, realizzato dal Partido dos Trabalhadores (PT) per sopperire ai vuoti nel servizio sanitario delle regioni più remote del Paese e delle periferie delle città. Con la crisi economica, in particolare a causa del declino degli investimenti stranieri e dei tagli alle risorse destinate ai servizi pubblici, questo movimento immigratorio ha subito una significativa diminuzione degli ingressi.

Invece, la massa degli immigrati e dei rifugiati provenienti dai Paesi poveri del Sud del mondo, con un'elevata percentuale di donne e di persone non bianche (Baeninger 2018a, 2018b), non riescono a passare attraverso i canali legali dell'immigrazione, nonostante siano richiesti dal mercato del lavoro brasiliano - soprattutto nei comparti più precari, come il tessile, la macellazione, il lavoro domestico. Questi immigrati ricevono dei titoli di soggiorno provvisori (visti umanitari, richieste di asilo) o sono costretti ad entrare nel Paese senza documenti. Si tratta di movimenti migratori che, come è noto, sono causati dalle profonde disuguaglianze di sviluppo e dai diversi rapporti di forza nell'attuale sistema economico a regime d'accumulazione finanziario (Basso 2003), che nel contesto latinoamericano si manifestano in diverse modalità tra cui la migrazione forzata (Delgado Wise 2016). Ora, nonostante gli effetti della crisi economica e del nuovo scenario politico, questi movimenti sono aumentati.

venienti da Siria, Repubblica Democratica del Congo, Colombia e Palestina. Il numero di richiedenti asilo è decisamente superiore (86.007 nel 2017), provenienti principalmente da Venezuela, Cuba, Haiti e Angola (Quintanilha 2019).

21 Ovviamente, essere immigrati regolari per motivi di lavoro non significa essere immuni ai fattori di precarizzazione del lavoro che si basano sulla mobilità internazionale (Villen 2018a; Perocco 2011).

22 La nazionalità filippina occupa il primo posto tra i lavoratori reclutati. Come spiega Zanin (2007), il funzionamento del settore petrolifero è molto segnato dal lavoro forzato. 
Le politiche migratorie in Brasile, da sempre, presentano tratti selettivi, soprattutto in base a criteri razziali e sulla base dell'idea che l'immigrato bianco europeo sia un vettore di sviluppo e rappresenti una cultura più «avanzata» (Villen 2018a). Nonostante il Brasile sia spesso associato alle immagini di democrazia razziale e di Paese accogliente, le manifestazioni di violenza, razzismo e xenofobia contro gli immigrati e i rifugiati (principalmente i non bianchi provenienti da Paesi poveri) nel contesto della crisi sono aumentate.

La struttura razziale della formazione socio-economica del Brasile, le conseguenze della crisi (la paura della disoccupazione, il peggioramento delle condizioni di vita, l'indebitamento, la riduzione dei servizi pubblici, l'aumento della violenza causato dalla crescita delle disuguaglianze), nonché il razzismo e la xenofobia fomentati dall'alto, da diverse istituzioni (Basso 2010; Perocco 2011), sono fattori che devono essere considerati per capire l'attuale contesto migratorio.

Il disagio e la miseria sociale prodotti nei Paesi poveri dall'attuale sistema economico si concretizzano, anche nel Sud del mondo, nell'immigrazione. Un immigrato in situazione socio-economica vulnerabile normalmente arriva in Brasile per vivere nelle grandi città. Molto spesso si trova senza denaro e senza documenti, non ha alternativa se non vivere per strada o nelle zone periferiche, in condizioni di grave miseria. Per questo motivo nelle città c'è una significativa partecipazione di immigrati ai movimenti per l'occupazione di case..$^{23}$

L'impeachment della presidentessa Dilma Roussef è un segno per comprendere il riorientamento delle politiche migratorie in senso ancor più restrittivo, per cogliere il processo di legittimazione della xenofobia e del razzismo in seno alla società brasiliana. Un caso esemplare in tal senso è stato il processo (nel 2015) contro una docente universitaria di nazionalità italiana, accusata di essere coinvolta con i sindacati e con i movimenti di protesta contro la deposizione di Dilma Roussef (Pereira 2019). La denuncia si basava sui principi della vecchia legge sull'immigrazione 6815/1980, approvata durante la dittatura militare e rimasta in vigore fino alla fine del 2017, che proibiva a qualunque «straniero» di partecipare ad associazioni sindacali e politiche. ${ }^{24}$

23 Il film Era o Hotel Cambridge, uscito nel 2017 e diretto da Eliane Caffé, dipinge la «zona di conflitto» nelle occupazioni a São Paulo, spazi segnati anche oggi dalla relazione degli immigrati e dei rifugiati con i brasiliani. Si tratta di un contesto di lotta per la casa e per un lavoro nella città intrisa dalla repressione della polizia. Un’altra occupazione di case, nota come Leila Khaled (riferimento alla leader palestinese) e la storia di rifugiati palestinesi (che arrivano in Brasile senza conoscere il portoghese e con scarse risorse finanziarie) hanno ispirato lo spettacolo di teatro per ragazzi Cantos do Refugio. Lo spettacolo presenta le barriere dei documenti e della burocrazia, la violenza della polizia, la condizione abitativa precaria dei rifugiati in questa città (Quintanilha 2019).

24 Questo fatto rispecchia anche quanto ci insegna Sayad (1999) sul lavoratore emigrato/immigrato. Quest'ultimo deve infatti vedersi soltanto come lavoratore, non può avere aspirazioni politiche, culturali e religiose che superino il confine del lavoro salariato. 
L'approvazione della nuova legge sull'immigrazione nel 2017, che paradossalmente rappresenta una vittoria della lunga lotta degli immigrati e delle loro organizzazioni di rappresentanza, è un chiaro segnale di questo scenario. I movimenti e i partiti anti-immigrati di estrema destra, spesso dichiaratamente fascisti, sono scesi in piazza per protestare contro il «lassismo» di questa legge, considerata molto permissiva in termini di ingresso e permanenza nel Paese. In questa occasione, gruppi di estrema destra, spesso legati a rappresentanti politici collegati alla campagna presidenziale di Bolsonaro, hanno trovato spazio sui social network per protestare contro la nuova legge, utilizzando argomenti razzisti che cercavano un collegamento (inesistente) tra immigrazione e aumento della criminalità (Quintanilha 2019).

In risposta a queste pressioni, l'allora presidente ad interim Michel Temer ha apposto il veto su 22 articoli della legge già approvata, eliminando gran parte del potenziale di tutela degli immigrati (Quintanilha 2019; Pereira 2019). L’applicazione di questa nuova legge è stata, da allora, il principale terreno di manovre politiche per negare i diritti degli immigrati, per ostacolare la regolarizzazione dei visti e le nuove richieste di ingresso in Brasile (anche per gli immigrati con profili qualificati che possono dimostrare di avere un reddito o un rapporto di lavoro nel Paese).

I confini settentrionali del Brasile, negli stati di Roraima e di Acre, prima utilizzati principalmente dagli haitiani, ora sono diventati la rotta di ingresso principale di venezuelani e di immigrati di altre nazionalità, soprattutto africani. ${ }^{25}$ In questi territori, i venezuelani sono stati oggetto di molti casi di violenza e xenofobia. L'apice è stato raggiunto nell'attacco subito dai venezuelani nella città di Pacaraima, nello stato di Roraima, nell'agosto del 2018. Diverse baracche dove erano alloggiati sono state incendiate e molti sono stati aggrediti fisicamente, come risposta ad un tentativo di rapina con aggressione fisica commesso da due venezuelani nei confronti di un commerciante brasiliano. Una parte della popolazione brasiliana delle zone di confine ad alta concentrazione di venezuelani si è mobilitata, spesso cantando l'inno nazionale, per espellere i venezuelani dalla città, provocando la fuga di molti verso il Venezuela. Soprattutto in questa regione, ma non solo in questa, vi sono stati numerosi casi di violenza contro gli immigrati e i rifugiati. Diversi elementi ci portano a considerare questi fenomeni di razzismo e violenza come molto più ampi, con ripercussioni anche sugli immigrati qualificati e a reddito più elevato, che si inseriscono con più facilità nella società brasiliana.

Il caso dei medici cubani, che analizzeremo in seguito, costituisce un esempio emblematico della xenofobia e dell'odio fomentati dall'alto

25 Si stima che nel 2018 circa 56.000 venezuelani siano entrati in Brasile attraverso quel confine. 
(Ramos 2018); esso raggiunge anche gli immigrati qualificati entrati attraverso i canali legali. Alcuni studi riportano casi di insulti - con frasi del tipo «ci state rubando i posti di accesso all'istruzione» - e aggressione fisica contro studenti provenienti da altri Paesi, soprattutto africani neri.

Le dichiarazioni di Bolsonaro sugli immigrati e sui rifugiati ci aiutano a comprendere come questi fenomeni siano alimentati dall'alto. Già dai primi mesi del suo mandato (2018) è risultato chiaro quanto la sua opinione sugli immigrati e sui rifugiati, resa pubblica in un'intervista del 2015 (quando era ancora deputato federale), si sarebbe tradotta in pratiche discriminatorie e vessatorie. In tale occasione, l'attuale presidente aveva dichiarato che gli immigrati e i rifugiati sono le «scorie del mondo»: «Le scorie del mondo [haitiani, senegalesi, siriani] stanno arrivando in Brasile, come se non avessimo già altri problemi da risolvere» (Azevedo 2015). Nel gennaio del 2019, dopo otto giorni dall'inizio del suo mandato, il governo ha informato l'ONU che il Brasile si sarebbe ritirato dal Global Compact for Migration. Come se il problema più grave del Paese fosse l'immigrazione.

Le sue dichiarazioni hanno avuto degli effetti subito dopo essere stato eletto alla fine dell'ottobre 2018, ancor prima di assumere l'incarico di presidente. In quel momento, quando gli è stato chiesto quali misure politiche avrebbe adottato per governare il Brasile, egli ha indicato come priorità la definizione di nuovi criteri per poter consentire ai medici cubani che lavorano nel servizio pubblico di continuare a esercitare la professione nell'ambito del programma Mais Médicos. Il risultato è stato che il governo cubano, come misura di protezione, ha ritenuto più prudente richiamare questi medici dal Brasile. Si tratta di un caso di 'espulsione di massa', che con motivazioni pretestuose cela la volontà di 'ripulire' il Brasile da tutte le politiche adottate dal $\mathrm{PT}$, specialmente la volontà di evitare che medici con una formazione di alto livello, umanitaria e 'comunista', rappresentino l'esempio di un diverso modello di medicina. Il risultato è stato che oltre 35 milioni di brasiliani in situazione di vulnerabilità socio-economica sono rimasti senza assistenza medica, principalmente nelle regioni più remote e nelle periferie delle città. ${ }^{26}$

In questa nuova fase economica e politica, l'esercito e la Polizia federale sono passati ad occupare un posto centrale nel 'governo' dell'immigrazione. Non è un caso che nei centri destinati ad accogliere i venezuelani nel nord del Brasile siano presenti strutture e corpi militari. Questo contesto di militarizzazione, unito agli effetti della crisi e all'applicazione di politiche neoliberiste che peggiora-

26 Va sottolineato che il livello di gradimento del programma Mais Médicos da parte della popolazione beneficiaria era molto elevato. 
no drasticamente le contraddizioni storicamente presenti nel Paese, è propizio per le pratiche di tortura, per la diffusione di trattamenti inumani e degradanti.

\section{Tortura e continuità della violenza di Stato in Brasile}

Durante la dittatura militare (1964-85), instaurata in un periodo di grande effervescenza nazionale e internazionale dei movimenti progressisti in America Latina, si è consolidata la dottrina della sicurezza nazionale e della lotta ai nemici interni ed esterni, con l'utilizzo della tortura, dell'assassinio, dell'espulsione di stranieri ${ }^{27}$ sospettati di essere dei sovversivi. ${ }^{28}$

Subito dopo il golpe che ha deposto João Goulart nel 1964, nel 1965 il Brasile si è addentrato in una seria questione diplomatica che ha portato all'espulsione, senza prove, di cittadini cinesi per il 'reato di sovversione'. Il 'caso dei nove cinesi' è un esempio famoso di violazione dei diritti umani ad opera dello stato brasiliano contro degli stranieri (Moraes 2016, 138). I cinesi che sono giunti in Brasile tra il 1961 e il 1964 sono stati accusati di essere «agenti pericolosi che comandavano una rete di 191 persone, avevano aghi avvelenati, bombe telecomandate e una lista di personalità da assassinare durante la rivoluzione comunista» (Gaspari 2014). In realtà, si trattava di giornalisti e imprenditori che, nonostante fossero in possesso di un visto regolare, sono stati torturati e hanno subito la confisca dei propri beni.

All'epoca vi era una collaborazione del capitale transnazionale con i militari per creare un clima di terrore contro i 'nemici' sospettati di sovversione; ciò è avvenuto attraverso l'Operazione Condor, ${ }^{29}$ sostenuta dagli Stati Uniti, che utilizzava i metodi di tortura applicati dalla Francia contro la lotta anticoloniale in Algeria. A venticinque anni di distanza da quel periodo di terrore, la pratica della tortura (nonostante sia condannata dalle legislazioni nazionali e internazionali ratificate in Brasile) e la sua apologia da parte degli attuali governanti continuano

27 L'espulsione di stranieri 'indesiderati' era una pratica presente nella storia brasiliana, fin dai tempi dell'impero, ed era rivolta agli africani schiavizzati e ai loro discendenti. Le espulsioni si intensificarono durante il governo di Getulio Vargas (1930-45), e ancora di più in seguito, durante la dittatura militare.

28 Secondo Moraes (2016), l'illegalità dei procedimenti contribuisce alla scarsità di dati relativi a queste misure durante il regime militare.

29 «L'operazione Condor è stata un'alleanza politico-militare, che ebbe luogo negli anni '70 e '80 del XX secolo, tra regimi militari dell'America del sud (Brasile, Argentina, Cile, Bolivia, Paraguay e Uruguay) e gli Stati Uniti, creata con l'obiettivo di coordinare la repressione degli oppositori dei governi dittatoriali, eliminare i leader di sinistra al potere nei paesi del Cono sud, anche attraverso lo scambio di informazioni» (Moraes 2016, 140). 
ad esistere e si nutrono di una rete articolata, sostenuta da interessi geopolitici e dal capitale globale, sotto l'egemonia del Nord del mondo. $\mathrm{Al}$ momento vi sono segnali secondo cui in Brasile si importano i metodi che vengono testati in altre zone del pianeta come Guantanamo o Gaza, per reprimere i «nemici dell'ordine» (Teles 2019). Parallelamente, lo Stato brasiliano esporta le sue forze di guerra per commettere violazioni dei diritti dei cittadini in Paesi stranieri, come nel caso della Minustah (Missione di stabilizzazione delle Nazioni Unite ad Haiti).

La macchina statale della repressione continua ad agire per contenere le mobilitazioni sociali, facendo del Brasile uno dei Paesi più pericolosi per i difensori dell'ambiente e dei diritti umani; si pensi all'esecuzione politica della consigliera Marielle Franco, leader riconosciuta della lotta femminista, di neri e Lgbt, eletta con il partito Socialismo e Liberdade. I dati dell'UNHCR del 2014 indicano che la richiesta di asilo da parte di cittadini brasiliani in altri Paesi corrisponde ai seguenti profili: vittime di tortura o di violenza intenzionale; attivisti in Amazzonia minacciati di morte; paura della persecuzione da parte di poliziotti corrotti, membri di milizie e narcotrafficanti; testimoni di reati commessi da poliziotti (Mello, Donasci 2014). Il rapporto dell'UNHCR del 2018 ha registrato 855 brasiliani rifugiati all'estero e circa 6.800 richiedenti asilo. Questi numeri tendono ad aumentare con i passi indietro prodotti da un contesto caratterizzato dall'ascesa dei movimenti di estrema destra (Villen 2018b).

Come già sottolineato, la tortura, soprattutto nei confronti della popolazione indigena e nera, affonda le sue radici nel passato coloniale, pertanto la violenza di Stato in Brasile ha un colore e una classe, ed è una forma sistematica di controllo e di umiliazione degli 'indesiderati'. I neri e i poveri, allontanati nelle periferie, continuano a essere il principale obiettivo del razzismo e della logica che giustifica e rende naturale l'incarcerazione di massa; questa politica, in modo contraddittorio, ha visto una nuova espansione con il ritorno alla democrazia (Pastoral Carcerária 2018).

Per quanto concerne le violenze e le torture della polizia nei confronti degli immigrati, non ci sono informazioni precise, non esistono dati che uniscano e mettano a sistema le violazioni dei diritti umani, utilizzando come criterio la nazionalità e la condizione migratoria. Ciò nonostante, la relazione tra tortura e immigrazione in Brasile richiede necessariamente la discussione di un punto centrale e urgente: l'impatto dello «tsunami della carcerazione», il cui «contenitore è la Guerra alla droga», due espressioni utilizzate da Carla Benitez Martins (2018) per descrivere la continuità drammatica con l'eredità schiavista brasiliana, oggi espresse dall'aumento sproporzionato dell'incarcerazione dei neri rispetto ai bianchi. Nel 2018, il Brasile ha raggiunto il terzo posto al mondo per popolazione carceraria, la cui stragrande maggioranza è nera, a basso reddito ed è in attesa di giudizio per l'accusa di coinvolgimento nei livelli più bassi del narcotraffico. 
In questo senso, la Pastoral Carcerária, principale istituzione che monitora e denuncia il reato di tortura nel contesto del sistema carcerario brasiliano e delle vittime della violenza della polizia, ricorda che, fin dallo schiavismo, il Brasile è sempre stato un grande territorio di tortura. Secondo il rapporto Tortura ai tempi dell'incarcerazione di massa,

le pratiche di tortura si sono evolute, con nuove tecniche che non lasciano segni, usano armi meno letali, gruppi di intervento che agiscono coperti dall'anonimato, tecniche sofisticate di isolamento e destrutturazione mentale, privazione di diritti e servizi di base, e tante altre forme di imposizione di sofferenza fisica e psicologica acute. (Pastoral Carcerária 2016, 118)

Il rapporto critica la mancanza di misure concrete per estirpare la violazione dei diritti e della dignità umana, nonché l'inefficacia dei meccanismi istituzionali di denuncia e condanna della tortura, come nel caso della legge 12.847 (2013), che ha istituito il Meccanismo nazionale per la prevenzione e la lotta alla tortura (MNPCT) e altri organi correlati. L'allentamento di questo meccanismo, nonché della lotta al lavoro schiavistico, è indicativo della regressione in materia di diritti umani, che va di pari passo con le riforme che peggiorano la tutela dei diritti della classe lavoratrice in tutto il Paese. In tal senso, è importante utilizzare una visione politica della tortura che va oltre i suoi presupposti e confini strettamente giuridici. ${ }^{30} \mathrm{La}$ visione della Pastoral Carcerária sottolinea la necessità di assumere una prospettiva più ampia sulla tortura:

Maltrattamenti e tortura denotano sia aggressioni e sevizie direttamente perpetrate da agenti di stato al fine di infliggere una 'intensa sofferenza' psichica e fisica, sia il funzionamento del sistema carcerario, la cui struttura, di per sé, impone alle persone incarcerate e ai loro familiari (e, in misura minore, anche agli agenti) una quotidianità di perversioni e orrori. (Pastoral Carcerária 2018, 62)

La pubblicazione della Pastoral Carcerária intitolata Lotta anticarceraria nel mondo contemporaneo: uno studio sulle esperienze di riduzione della popolazione carceraria espone dei dati importanti sulla macchina carceraria in Brasile, secondo i quali

oltre 725.000 persone (per la precisione 726.712) sono tenute dalle forze di sicurezza in unità sovraffollate (con un tasso di occupazione del 200\%, nonostante la crescita del lucrativo settore dell'edilizia carceraria), la maggior parte è costituita da giovani neri; la 
popolazione femminile, ancora minoritaria, cresce a un ritmo superiore rispetto a quella maschile; i luoghi per le attività educative e produttive sono infimi, le denunce di maltrattamenti e tortura sono ricorrenti; condizioni psichiche e materiale indegne sono determinanti per la riduzione delle aspettative di vita e condannano a morte migliaia di persone ogni anno. (Pastoral Carcerária 2018, 62)

All'interno di questo quadro, nel 2017 ben 532 persone sono decedute nel solo sistema penitenziario dello Stato di São Paulo (Spechoto 2018). Di questi decessi, 484 sono stati classificati come 'naturali'.

Le poche ricerche esistenti sulle condizioni degli stranieri indicano che il già soffocato dibattito sulle pratiche di tortura contro persone in condizione di privazione della libertà è ancor più ridotto al silenzio quando si tratta di stranieri e neri, sia nei penitenziari, sia nel cosiddetto Conector dell'aeroporto di Guarulhos (São Paulo) - locale di «permanenza provvisoria» estremamente vigilato destinato a immigrati e rifugiati, utilizzato per mantenere isolati coloro ai quali è stato rifiutato l'ingresso in Brasile o è stato impedito di proseguire il viaggio nel caso di voli con scalo nel Paese.

I dati ottenuti attraverso la Legge di accesso alle informazioni rivelano che tra il 2015 e il 2016 sono stati detenuti in queste condizioni circa 2.000 immigrati. I racconti riportano che alcuni sono stati detenuti senza assistenza o informazioni fino a tre mesi. Formalmente, in Brasile non c'è una procedura giudiziaria o amministrativa che renda possibile questo tipo di detenzione, ma la Polizia federale si aggrappa alle falle della legge per portare avanti questa pratica. Come spiega Chiaretti, all'epoca Defensor Público Federal (avvocato d'ufficio a livello federale) che lavorava con gli stranieri a Guarulhos, gli «uomini neri, giovani e provenienti da paesi africani» costituiscono il profilo di straniero inviato sistematicamente al Conector (Ribes 2019).

In Brasile, quasi la metà delle persone incarcerate non ha una condanna definitiva; oltre la metà è detenuta per reati non violenti; oltre il $70 \%$ è privato della libertà a causa di reati contro il patrimonio o per piccolo spaccio; la maggioranza degli arresti è avvenuta in flagranza di reato; la maggioranza assoluta delle condanne per traffico di droga si basa soltanto sulla parola dell'agente di polizia responsabile dell'arresto e della detenzione (Pastoral Carcerária 2018, 62). Trattare queste persone come criminali pericolosi per la società, come avviene nella giustizia penale, per non citare gli stereotipi utilizzati dai mass-media, equivale a cadere nel «macro equivoco della micro criminalità» (Basso 2000).

Di questa popolazione privata della libertà, una percentuale minima è composta da stranieri. I dati dell'indagine nazionale sulle informazioni penitenziarie (Infopen) del 2016 registrano 2.606 cittadini di altre nazionalità (di cui 1.651 uomini), la maggioranza dei quali 
(63\%) concentrata nello Stato di São Paulo, dove la quantità di strutture penitenziarie è passata dalle 21 dei primi anni Ottanta alle 168 attuali. Per le ragioni spiegate in questo saggio, legate all'attuale contesto economico e politico della crisi in Brasile, e per l'aumento delle migrazioni su scala globale, questa popolazione aumenterà in futuro. ${ }^{31}$

Secondo il rapporto annuale del Progetto Straniere dell'Instituto Terra Trabalho e Cidadania (ITTC 2016), che dal 2001 assiste le donne straniere arrestate a São Paulo, circa il 90\% di queste donne è stato arrestato come corriere del traffico internazionale di droga, solitamente colte in flagranza di reato nell'aeroporto di Guarulhos, il principale punto d'imbarco dell'America del Sud. Come le detenute brasiliane, la gran parte di esse proviene da un contesto socio-economico di estrema povertà e disuguaglianza, sono nere, madri sole e capofamiglia a basso reddito, responsabili del mantenimento della famiglia. L'ITTC osserva che queste donne «svolgono ruoli ad alto rischio e non hanno alcun potere nella catena del narcotraffico. Tuttavia, normalmente sono processate penalmente come se fossero grandi narcotrafficanti internazionali. Le difficoltà di comunicazione in prigione sono innumerevoli, tra le quali si sottolinea la mancanza della possibilità di studio» $(2016,23)$.

Lontane dai propri Paesi e dalla rete familiare, queste donne sono maggiormente esposte a violazioni dei diritti e sono ostacolate da diverse barriere nell'accesso alle informazioni sul funzionamento del sistema penale. Tutto questo rende più difficile e rischiosa la possibilità di denunciare le violazioni dei propri diritti, aumentando l'improbabilità di rompere il velo di impunità (Marques de Jesus 2009).

Bumachar (2016) ha condotto una ricerca nel Penitenziario femminile di São Paulo per analizzare le reti di assistenza e resistenza intessute dalle donne straniere in prigione. Lo studio ha documentato due casi di torture nei confronti di donne sudafricane detenute a São Paulo, gettando luce sull'esposizione a pratiche estremamente violente. Queste pratiche sembrano essere sempre più forti e legittime di fronte a una donna straniera che, in generale, ha pochi mezzi in Brasile per denunciare queste violazioni:

È quanto successo, in un episodio, nel Penitenziario femminile di São Paulo a tre sudafricane. Secondo il racconto degli eventi, l'episodio ha avuto inizio quando due agenti penitenziari hanno avvicinato le detenute alla fine dell'orario di lavoro, sostenendo di aver ricevuto una denuncia secondo la quale le tre avevano cocaina. Inviate direttamente alla sezione sanitaria, hanno ricevuto l'ordi-

31 Non dobbiamo ignorare l'esistenza di circa 240 rotte di traffico che usano il territorio brasiliano come spazio di origine, passaggio e destinazione, principalmente nel traffico di donne e bambini (Quintanilha 2019). 
ne di spogliarsi, stendersi sulle barelle, aprire le gambe affinché una delle quattro funzionarie presenti nell'infermeria «infilasse le dita con forza nella vagina di tutte e tre e poi nell'ano, senza lavarsi le mani e con lo stesso guanto. È avvenuto tutto davanti ad altre tre funzionarie, che ci dicevano di stare zitte, di non protestare, perché [il procedimento di esame] era così», mi ha raccontato Juline, una delle tre. (Bumachar 2016, 33)

Lo studio ha registrato anche un altro episodio, in occasione delle rivolte avvenute il giorno della festa della mamma, in cui, dopo che la polizia militare in tenuta antisommossa era entrata nella zona interna del Penitenziario femminile di Santana per porre fine alla manifestazione, una donna sudafricana, che non parlava portoghese, è stata gravemente aggredita.

Aveva perso di vista le sue compagne e, poiché non capiva il portoghese e la sua comprensione della grammatica dipendeva ancora dalla traduzione, era rimasta paralizzata davanti agli ordini incomprensibili della polizia e alle corse, grida, spari di pallottole di gomma e gas lacrimogeni che circondavano la sua disperazione. Il poliziotto, senza conoscere la causa del suo blocco, ha aggredito il suo corpo esile e minuto, ripetendo le parole dell'ordine e passando all'aggressione, finché un agente di sicurezza, vista la scena, ha spiegato gridando al poliziotto che la donna era straniera. Behati è rimasta gravemente ferita, ha perso tutti i denti a causa delle percosse ed è stata portata d'urgenza all'ospedale, altro luogo pubblico dove ha attraversato momenti difficili senza riuscire a comunicare adeguatamente, neppure per assumere i farmaci quotidiani per i suoi problemi respiratori. (Bumachar 2016, 94)

Nel caso degli stranieri, alle punizioni affrontate nelle prigioni si somma una doppia o tripla pena. Dopo aver scontato la pena, non possono tornare al Paese di origine o lasciare il Brasile, finché non si concludono i laboriosi e macchinosi processi amministrativi di espulsione. Se per alcuni il processo di espulsione è l'unico modo per tornare a casa, altri cercano di impedire l'espulsione per garantirsi il diritto di rimanere in Brasile.

Questo è stato il caso della sudafricana Nduduzo G.D., ${ }^{32}$ protagonista di una campagna collettiva contro la sua espulsione, una vera tortura giuridica contro gli stranieri che hanno già scontato la propria pena in Brasile. Per lei, è stato come vivere in una «prigione a

32 Per 3 anni e 6 mesi, la donna è sopravvissuta al confino dell'incarcerazione brasiliana, in cui il numero di donne detenute è cresciuto del 698\% tra il 2000 e il 2016 . 
cielo aperto» 0 avere una «libertà senza libertà». ${ }^{33}$ La campagna intitolata \#NduduzoTemVoz (Nduduzo ha voce) è stata resa possibile grazie alla sua partecipazione a un gruppo teatrale ${ }^{34}$ di São Paulo, che si è mobilitato attraverso una rete di attivisti, difensori dei diritti umani e artisti, ottenendo vittorie in ambito giuridico, nonché sociale e culturale nella resistenza anti-razzista, oltre a costituire un progresso nella sensibilizzazione collettiva verso i processi di incarcerazione ed espulsione degli immigrati che vorrebbero costruire la propria vita in Brasile (Quintanilha 2019).

\section{Conclusione}

Il primo obiettivo di questo saggio è stato quello di offrire un'analisi dell'attuale contesto economico e politico dell'America Latina e del Brasile. Soltanto comprendendo questo contesto, che converge chiaramente con quanto Basso (2010) definisce «razzismo di Stato» e che non è separato dal funzionamento del sistema neoliberista, possiamo comprendere anche il terreno di pratica della tortura subita dagli immigrati.

Abbiamo evidenziato come la tortura nel continente latinoamericano abbia una lunga tradizione storica, di cui i popoli indigeni e neri sono state le vittime principali. Ad oggi, questo profilo sociale è il principale rappresentante della povertà e continua a essere l'obiettivo privilegiato della tortura. Al contempo abbiamo presentato i diversi motivi che spiegano come gli immigrati e i rifugiati del Sud del mondo, che costituiscono il grosso dei movimenti migratori verso il continente e che vivono una condizione socio-economica molto vulnerabile (soprattutto le donne vittime del narcotraffico transnazionale), rappresentino un gruppo sociale sempre più esposto alla tortura, con le carceri come luogo privilegiato di questa pratica.

La tortura, anche se poco esplorata dalla ricerca scientifica dopo il periodo delle dittature militari, continua ad essere una prospettiva analitica molto utile per comprendere le tendenze dei nostri tempi. In Brasile, essa dimostra che la dittatura militare, periodo storico che sembrava ormai sepolto, sta rinascendo dalle proprie

33 Queste dichiarazioni di Nduduzo sono state rese all'evento Diritti dei lavoratori e partecipazione sociale nella nuova legge sulla migrazione, Università Mackenzie, 9 giugno 2018.

34 Dopo aver ottenuto la libertà, Nduduzo racconta di essere riuscita a inserirsi in un progetto di canto dell'Università di São Paulo (USP) e di essere stata invitata a raccontare la storia che l'ha portata in prigione nello spettacolo teatrale Canto inutile e pianto inutile per gli angeli caduti, scritta da Plinio Marcos durante la dittatura e portata in scena in diversi teatri e spazi culturali di São Paulo, sul ciclo di violenza e torture che ruota intorno all'odierna politica di incarcerazione di massa. 
ceneri, con la massima potenza caratteristica dei tempi di crisi, per trasformarsi nel modello da seguire per 'risolvere' i problemi strutturali delle periferie del capitalismo, aggravati dall'attuale crisi economica.

Possiamo affermare che, seguendo la logica del concetto di razzismo di Stato (Basso 2010), anche la tortura si sta costituendo come pratica legittima 'dall'alto verso il basso', a partire dalla rinnovata alleanza tra potere politico e potere militare, dalle scelte politiche che peggiorano la situazione socio-economica della popolazione e generano ancora più violenza e paura, dai cambiamenti legislativi che aprono spazi per questa pratica, ma anche attraverso un processo ideologico-culturale che mira a legittimare, giustificare, fomentare e banalizzare la dittatura (e pertanto della tortura) da parte di coloro che si sentono minacciati dai mali globali prodotti dall'attuale sistema economico.

Parallelamente, i pretesti che il sistema punitivo capitalistico individua per 'appropriarsi' e al contempo 'liberarsi' degli indesiderati sono i più vari e mettono a rischio i diritti umani conquistati nella storia. L'eco crescente di un discorso nazionalista e sicuritario negli apparati statali e mediatici, che cerca di legittimare l'incarcerazione di massa, l'apologia della tortura in prigione («il detenuto deve soffrire») e la banalizzazione della violenza della polizia («il bandito buono è il bandito morto»), dimostra la necessità di pensare alle teorie e alle lotte per l'emancipazione in modo articolato rispetto alla situazione delle persone incarcerate.

L’analisi proposta secondo il concetto di «tsunami carcerario» e dei suoi riflessi sul trattamento degli immigrati e dei rifugiati, soprattutto delle donne che cadono nella trappola del narcotraffico transnazionale, è stato qui un esempio empirico per comprendere la concretezza della tortura subita da questi gruppi sociali. Abbiamo anche ritenuto importante mostrare la presenza di reazioni da parte di questi soggetti, unitamente alle iniziative dei locali, per contrastare le situazioni di sofferenza e di esposizione alla tortura nelle prigioni.

Oggi in America Latina i rapporti tra le forze politiche, anti-immigrazione e pro-immigrazione, propendono per il primo approccio. Purtroppo si tratta di un gioco di forze molto sfavorevole per la popolazione povera e lavoratrice, nazionale e immigrata. La prospettiva di cambiamento di questo quadro è di lungo termine, soprattutto se si considera una ripresa delle lotte per affrontare i problemi strutturali. 


\section{Bibliografia}

Antunes, Ricardo (2018). O privilégio da servidão: o novo proletariado de serviços na era digital. São Paulo: Boitempo.

Arantes, Paulo (2010). «1964, o ano que não terminou». Teles, Edson; Safatle, Vladimir (eds), o que resta da ditadura. São Paulo: Boitempo, 205-36.

Azevedo, Rita (2015). «Bolsonaro chama refugiados de escória do mundo». Exame, 22 jul. URL http://exame.abril.com.br/brasil/bolsonaro-chama-refugiados-de-escoria-do-mundo/ (2019-01-20).

Baeninger, Rosana et al. (eds) (2018a). Atlas Temático Observatório das Migrações em São Paulo. Migrações internacionais. São Paulo: Unicamp; Nepo; Fapesp.

Baeninger, Rosana et al. (eds) (2018b). Atlas Temático Observatório das Migrações em São Paulo. Migração Refugiada. São Paulo: Unicamp; Nepo; Fapesp.

Basso, Pietro (2000). «ll macro equivoco della micro criminalità». Gli argomenti umani: sinistra e innovazione, 4, 79-83.

Basso, Pietro (2003). «Sviluppo diseguale, migrazioni, politiche migratorie». Basso, Pietro; Perocco, Fabio (a cura di), Gli immigrati in Europa: Diseguaglianze, razzismo, lotte. Milano: FrancoAngeli, 82-117.

Basso, Pietro (a cura di) (2010). Razzismo di stato: Stati Uniti, Europa, Italia. Milano: FrancoAngeli.

Braga, Ruy (2018). «A Precarious Hegemony: Neoliberalism, Social Struggles, and the End of Lulismo in Brazil». Globalizations, 16, 201-15.

Brighenti, Clóvis Antônio (2016). "Colonialidade e decolonialidade no ensino de História e Cultura Indígena». De Souza Feltrin, Fábio; Wittman Tombini, Luisa (eds), Protagonismo indígena na história. Tubarão: Copiart, 231-54.

Bumachar, Bruna (2016). Nem dentro, nem fora: a experiência prisional de estrangeiras em São Paulo [PhD Dissertation]. Campinas: Universidade Estadual de Campinas.

Ceriani, Pablo (2017). «Immigration Detention through the Lens of International Human Rights: Lessons from South America». Flynn, Michael; Flynn, Matthew, The Global Detention Project, Working Paper, 23 (september), 1-20.

Delgado Wise, Raul (2016). «Notas sobre la cuestión laboral y migratoria hoy: migración forzada, desarrollo desigual e imperialismo». Revista Theomai, 33, 157-73.

Della Barba, Mariana; Wentzel, Marina (2016). «Discurso de Bolsonaro deixa ativistas "estarrecidos" e leva OAB a pedir sua cassação». BBC Brasil, 20 abril. URL http://www.bbc.com/portuguese/noticias/2016/04/160415_ bolsonaro_ongs_oab_mdb (2019-01-20).

Domenech, Eduardo (2011). «La gobernabilidad migratoria en la Argentina: hacia la instauración de políticas de control con rostro humano». Journal of strategic studies, 34(2), 281-93.

Domenech, Eduardo (2015). «O controle da imigração indesejável: expulsão e expulsabilidade na América do Sul». Ciência e Cultura, 67(2), 25-9.

Gaspari, Elio (2014). "O caso dos nove chineses». O Globo, 2 jul. URL http://oglobo.globo.com/opiniao/o-caso-dos-nove-chineses-13098491 (2019-01-20).

Fernandes, Florestan (1982). A ditadura em questão. São Paulo: T.A. Queiroz. ITTC, Instituto Terra Trabalho Cidadania (2016). Projeto estrangeiras completa 15 anos, 16 nov. URL http://ittc.org.br/projeto-estrangeiras-completa-15-anos/ (2019-01-20). 
Jacques, Ramírez; Linárez, Yoharlis; Useche, Emilio (2019). «Geo)políticas migratorias, inserción laboral y xenofobia. Migrantes venezolanos en Equador». Blouin, Cécile (a cura di), Después de Llegada. Realidades de la migración venezolana. Lima: Themis (PUCP), 1-29.

Leite, Celso Barroso (ed.) (1987). A sociologia da corrupção. Rio de Janeiro: Zahar.

Löwy, Michel (2016). «Da tragédia à farsa: o golpe de 2016 no Brasil». Jinkings, Ivana; Doria, Kim; Cleto, Murilo (eds), Por que gritamos o golpe? São Paulo: Boitempo, 60-5.

Lucena, Carlos; Previtali, Fabiane; Lucena, Lurdes (eds) (2017). A crise da democracia brasileira. Uberlândia: Navegando.

Marques de Jesus, Maria Gorete (2009). O crime de tortura e a Justiça Criminal [Dissertação]. São Paulo: Universidade de São Paulo.

Martins, Carla Benitez (2018). Distribuir e punir? Capitalismo dependente brasileiro, racismo estrutural e encarceramento em massa nos governos do partido dos trabalhadores (2003-2016) [PhD Dissertation]. Goiânia: Universidade Federal de Goiás.

Mascaro, Alysson (2018). Crise e golpe. São Paulo: Boitempo.

Melegh, Attila (2006). On the East-West Slope. Globalization, Nationalism, Racism and Discourses on Central and Eastern Europe. Budapest; New York: CEU Press.

Melegh, Attila (2018). «Moments of Hegemony. Historical Roots of Authoritarian Capitalism and Education Policy: the Case of Hungary». III Global Labour University Conference, Campinas, 1-9.

Mello, Patricia Campos; Donasci, Fernando (2014). «Brasileiros refugiados somam mais de mil». Folha de São Paulo, 22 jan. URL http://www1. folha. uol.com.br/mundo/2014/01/1399789-brasileiros-refugiados-somam-mais-de-mil.shtml (2019-01-20).

Moraes, Ana Luisa Zago (2015). Crimigração: a relação entre política migratória e política criminal no Brasil [PhD Dissertation]. Porto Alegre: Pontifícia Universidade Católica do Rio Grande do Sul.

Moraes, Ana Luisa Zago (2016). «A cidadania como dispositivo de segurança: por uma justiça de transição em matéria de migrações». Revista Direito e Práxis. Rio de Janeiro, 7(4), 96-134.

Nações Unidas Brasil (2019). Campanha Vidas Negras. URL http://vidasnegras.nacoesunidas.org (2019-04-15).

Nascimento, Abdias (1978). O Genocídio do Negro Brasileiro. Rio de Janeiro: Paz e Terra.

Oliveira, André (2016). «Elogio à tortura, dupla moral e enrolados na Justiça em noves votos na Câmara». El Páis Brasil, 20 abril. URL http://brasil. elpais.com/brasil/2016/04/19/politica/1461019293_721277.ht$\mathrm{ml}(2019-10-11)$.

Oxfam (2017). A distância que nos une. Relatório anual da Oxfam - Brasil. URL http://www.oxfam.org.br/sites/default/files/arquivos/ mapa_2017_completo.pdf (2019-04-15).

Pastoral Carcerária (2010). Relatório sobre tortura: uma experiência de monitoramento dos locais de detenção para prevenção da tortura. URL http:// carceraria.org.br/wp-content/uploads/2018/01/relatorio-relatorio_tortura_2010.pdf (2018-12-10).

Pastoral Carcerária (2016). Tortura em tempos de encarceramento em massa. URL http: //carceraria.org.br/wpcontent/uploads/2016/10/Relat\%C3\%B3rio_Tortura_em_Tempos_de_Encarceramento_em_Massa-1.pdf (2018-12-10). 
Pastoral Carcerária (2018). Luta antiprisional no mundo contemporâneo: estudo sobre experiências de redução da população carcerária em outras Nações. URL http://carceraria.org.br/wpcontent/uploads/2018/09/relatorio_luta_antiprisional.pdf (2018-12-10).

Pereira, Tamara Francielle Fernandes (2019). A política migratória nacional e o trabalhador imigrante no Brasil [Dissertação]. Belo Horizonte: Universidade Federal de Minas Gerais.

Perocco, Fabio (2011). «Le discriminazioni razziali nel lavoro: un fenomeno sistematico e multidimensionale». Ferrero, Marco; Perocco, Fabio (a cura di), Razzismo al lavoro. Il sistema della discriminazione sul lavoro, la cornice giuridica e gli strumenti di tutela. Milano: Franco Angeli, 65-86.

Pizarro, Jorge Martínez (2005). Globalizados, pero restringidos: una visión latino-americana del mercado mundial de recursos humanos calificados. Santiago: CELADE.

Quintanilha, Karina Ferreira (2019). Migração forçada no capitalismo contemporâneo: trabalho, direitos e resistências no Brasil [Dissertação]. São Paulo: Universidade Católica de São Paulo.

Ramos, Rogério Macedo (2018). Intolerância e racismo no Brasil. Um estudo sobre o discurso de ódio contra os médicos cubanos no Facebook. Montes Claros: Universidade Estadual de Montes Claros.

Ribes, Felipe (2016). «Dados obtidos via LAI mostram que mais de 2 mil estrangeiros ficaram detidos no aeroporto de Guarulhos». Le Diplomatique Brasil, 6 outubro. URL https://bit.ly/33jgkkz (2019-01-20).

Rossi, Marina (2017). «Seis brasileiros concentram a mesma riqueza que a metade da população mais pobre». El país Brasil, 26 sep. URL http://brasil. elpais.com/brasil/2017/09/22/politica/1506096531_079176. html (2019-01-20).

Salém, Joana; Hoelever, Rejane (2018). «Lições de Pinochet. Brasil novo laboratório da extrema direita». Le Monde Diplomatique Brasil, 5 nov. URL http://diplomatique.org.br/brasil-novo-laboratorio-da-extrema-direita/ (2019-01-20).

Sassen, Saskia (2014). Brutality and Complexity in the Global Economy. Cambridge (MA): Harvard College.

Sayad, Abdelmalek (1999). La double absence: des illusions de l'emigré aux souffrances de l'immigré. Paris: Seuil.

Spechoto, Caio (2018). «Aumenta número de presos mortos nas cadeias de São Paulo». Terra Portal, 6 ago. URL https://bit.ty/20zrMo5 (2019-10-11).

Teles, Vera (2019). «A violência como forma de governo». Le Monde Diplomatique Brasil, 31 jan. URL http://diplomatique.org.br/a-violencia-como-forma-de-governo/ (2019-02-05).

Thomson-Deveaux, Flora (2018). «Nota sobre o calabouço». Revista Piauí, 140, maio. URL http://piaui.folha.uol.com.br/materia/nota-sobre-o-calabouco/ (2019-01-20).

Tomazela, José Maria (2019). «Índio da etnia paresi sofre tortura e perde braço no interior de SP». Estadão, 15 fev. URL https://bit.ly/33LNUpV (2019-01-20).

UNHCR (2018). Tendências Globais: Deslocamentos forçados. URL http://www. unhcr.org/global-trends-2017-media (2019-04-30).

Villen, Patricia (2017). «A crise brasileira e as rachaduras do sistema». Lucena, Carlos; Previtali, Fabiane; Lucena, Lurdes (eds), A crise da democracia brasileira. Navegando: Uberlândia, 109-25. 
Karina Quintanilha, Patricia Villen

Tortura e immigrazione in America Latina e in Brasile

Villen, Patricia (2018a). (In)visiveis globais: imigração e trabalho no Brasil. São Paulo: Alameda.

Villen, Patricia (2018b). «Brasil, país de expulsão? Desemprego e emigração no Brasil». Comciência (Unicamp), 230, novembre. URL http://www.comciencia.br/brasil-pais-de-expulsao-desemprego-e-emigracao-no-brasil/ (2019-01-20).

Villen, Patricia (forthcoming). «International Migration to Brazil and the Crisis of Democracy». Ipek, V.; Akarçay, Ebru (eds), To Democratize or Not: Notes from Tricontinental 2017. Cambridge: Cambridge.

Wacquant, Loïc (2001). As prisões da miséria. Rio de Janeiro: Jorge Zahar, 2001.

Zanin, Valter (2007). I forzati del mare. Roma: Carocci. 



\title{
Migración y tortura en la Argentina contemporánea
}

Juliana Carpinetti

Universidad Nacional de Rosario, Argentina

\begin{abstract}
The article aims to reflect about the linkage between migration and torture in recent Argentinian history. It assumes as work's hypothesis that this link is inseparable from the characteristics that racism acquires as a system within the framework of the configuration of the neoliberal economy and from the political history of Argentina. The analysis is organized around three historical periods: the first begins with the 1976 coup, the second with the December 2001 crisis and the third with the 2015 presidential elections.
\end{abstract}

Keywords Migration. Torture. Argentina. Racism. Neoliberalism.

Sumario 1 Introducción. - 2 El golpe de 1976 y la imposición. - 3 La crisis de 2001 y el desafío a la hegemonía. - 4 Las elecciones de 2015 y la restauración. - 5 Conclusiones: la carrera de la deshumanización neoliberal

\section{Introducción}

Lazzarato (2013) afirma que, en su acepción contemporánea, la economía abarca tanto la producción económica como la producción de las formas de vida y de la subjetividad. En cuanto a la primera de estas dimensiones, el neoliberalismo entiende que el bienestar humano sólo puede lograrse a través de la maximización de las libertades empresariales y que el Estado debe contribuir a este proceso mediante la promoción de un marco institucional apropiado para la proliferación de tales prácticas y a través de la creación de nuevos mercados (Harvey 2008).

Pero este avance del mercado por encima del Estado se encastra en un fenómeno de más amplio espectro, que supone la extensión de la forma 'em- 
presa' a la totalidad del entramado social. Vínculos y actividades sociales diversas, hasta entonces regidas por lógicas diferenciales, son progresivamente invadidos y conquistados por criterios de orden empresarial. El modelo económico - anclado sobre las leyes de la oferta y la demanda, del costo y el beneficio y de la inversión y la competencia - se transforma así «en un modelo de la existencia misma, una forma de relación del individuo consigo mismo, con el tiempo, con su entorno, el futuro, el grupo, la familia» (Foucault 2012, 239).

Esta expansión de la lógica económica por sobre las más diversas esferas sociales convierte a la acumulación de capital en la razón de ser, no sólo ya de la producción económica, sino también de los sujetos y de sus formas de vida. Ludwig von Mises (1968), referente académico de la teoría económica neoliberal, afirma al respecto que dicha acumulación solo resulta posible a través de la competencia, que no es otra cosa que el libre juego de las desigualdades. Sobre esta base, el neoliberalismo despliega una estrategia discursiva centrada en la idea de la natural desigualdad de todos los seres humanos. No se trata de una afirmación explícita, porque «nadie puede pregonar abiertamente la desigualdad como bandera en una sociedad que se dice democrática» (Murillo 2011, 21). Pero tampoco es necesario que sea enunciada explícitamente, para que se instale en la conciencia de los sujetos $(2011,21)$.

Esta nueva construcción discursiva - según la cual los seres humanos son naturalmente libres, racionales y desiguales y esa desigualdad lejos de ser entendida como una desviación a corregir es postulada como una condición deseable en tanto que permite la competencia, la acumulación y el progreso económico - trae aparejada una redefinición de los alcances y los efectos del sistema racista.

Immanuel Wallerstein (1991) sostiene que, si bien la modernidad instala una tensión permanente entre el discurso universal del liberalismo y la realidad permanente del racismo, lo que existe entre estos presuntos opuestos es una relación simbiótica. Esta complementariedad se explica por el hecho de que el sistema racista, ofrece una base no-meritocrática para justificar esa desigualdad. Al legitimarse por estos criterios las limitaciones fácticas de las proclamas universales del liberalismo, estas persistieron como universo de sentidos y permitieron la articulación en torno a ellas de los principales movimientos de resistencia frente a la opresión racista durante el siglo XX. Tal como advierte Hinkelammert, el universal igualitario, precisamente por el hecho de serlo, tiene un efecto particular en favor de los débiles: «los poderosos no lo necesitan, sin embargo, los débiles sí» $(1987,6)$.

En tiempos de hegemonía neoliberal, el racismo viene a complementar la premisa según la cual los individuos son naturalmente desiguales, con el supuesto de que inclusive algunos de ellos no son del todo humanos. De este modo suma a la legitimación de la desigual- 
dad de hecho una legitimación de la desigualdad de derecho. Esto no es otra cosa que la negación, para determinados grupos sociales, de la existencia de derechos inherentes a toda persona humana, anulando de este modo cualquier espacio de reparación.

Esta deshumanización presenta algunas particularidades respecto de las registradas en el pasado. En primer lugar, se consuma cotidianamente y a la vista de todos, sea en el marco de los espacios públicos o al ser amplificadas por los medios masivos de comunicación. En segundo lugar, porque emerge como el reflejo de actos legislativos y/o administrativos formulados en plena vigencia del sistema democrático. Es una deshumanización que, a diferencia del pasado, no requiere ni de la clandestinidad ni de la alteración del orden institucional.

Al repensar estos fenómenos a la luz de los movimientos migratorios contemporáneos, se evidencia que, por un lado, el racismo siempre ha ofrecido una base no-meritocrática para justificar una forma de 'etnificación' de la fuerza de trabajo que ubica a los inmigrantes en la base de la pirámide laboral. Es por ello que Portes y Walton (1981) afirman que el papel social que históricamente se ha reservado al trabajo inmigrante en las sociedades de destino no ha sido el de incrementar la oferta de fuerza de trabajo, sino el de incrementar la oferta de fuerza de trabajo disponible a bajo costo.

Sin embargo, desde hace ya algunos decenios, los inmigrantes representan además un grupo social particularmente sospechado en su humanidad, tarea a la que el Estado ha contribuido enormemente. La gestión racista de los movimientos migratorios no sólo se ha traducido en la proliferación de muros en las áreas de fronteras y de centros de detención de emigrantes en sus zonas adyacentes; sino que además ha contribuido de manera decisiva a la naturalización de prácticas aberrantes como la creación de milicias ciudadanas dedicadas a repeler con armas de fuego el avance de emigrantes irregulares o la negación de ayuda humanitaria a quienes sobreviven a los naufragios de altamar.

El análisis del vínculo que se establece entre migración y tortura resulta indisociable de esta avanzada global del racismo de vertiente neoliberal sobre el conjunto de estos grupos sociales. Desde esta óptica de análisis la tortura, entendida como todo acto en el cual el Estado, a través de sus instituciones y/o funcionarios, intencionadamente y con determinado fin o propósito, infringe a una persona dolores o sufrimientos graves, ya sean físicos o mentales, por fuera de las sanciones legítimas, ${ }^{1}$ no es más que una de las formas en las

1 Esta definición sintetiza los principales elementos detallados en el artículo 1 de la Convención de las Naciones Unidas Contra la Tortura y Otros Tratos o Penas Crueles, Inhumanos o Degradantes de 1984. Argentina ratificó esta convención y su Protocolo Facultativo que, desde la reforma constitucional de 1994, gozan de jerarquía superior a las leyes. También ratificó la Convención Interamericana para prevenir y sancionar la 
que esa deshumanización de la que hablábamos no sólo se consuma sino que además se institucionaliza. Es justamente por ello, que representa una de las formas más acabadas del «racismo de Estado» (Basso 2010).

Los apartados que siguen buscan reflexionar sobre la manera en que estos elementos se articulan en diferentes momentos de la historia argentina contemporánea. Para ello, se organizan en torno a tres períodos históricos: el primero se inicia con el golpe de Estado de 1976 que permitió el ascenso al poder de la última dictadura militar, el segundo con el estallido de la crisis social, política, económica e institucional del año 2001 que marcó en el ámbito nacional el inicio del ciclo político vinculado al 'giro a la izquierda latinoamericano' y el tercero con las elecciones presidenciales del año 2015, que permitieron el triunfo electoral de una fuerza política e ideológica de diferente signo.

\section{$2 \quad$ El golpe de 1976 y la imposición}

Existe un cierto consenso en la literatura académica de situar en el último cuarto del siglo XX el punto de partida para la expansión a escala global de los principales postulados de la economía neoliberal. En América Latina este proceso resulta indisociable de la proliferación de las dictaduras militares que, a lo largo de la década de 1970, ocuparon el poder en los diferentes países del Cono Sur.

Eduardo Duhalde (1999) afirma que estos gobiernos de facto introdujeron en la historia regional un nuevo modelo de Estado de Excepción: el Estado Terrorista. ${ }^{2}$ Este es un Estado militarmente ocupado, asaltado por su brazo militar, que incorpora una doble faz en la actuación de los aparatos coercitivos estatales: una pública y sometida a leyes y otra clandestina, al margen de toda legalidad formal, que encuentra en el crimen y el terror su método fundamental.

El autoproclamado Proceso de Reorganización Nacional argentino que se inicia con el golpe de Estado de 1976, representa el ejem-

tortura y sancionó en el año 2012 la Ley núm. 26.827, que crea el Sistema Nacional de Prevención de la Tortura y Otros Tratos o Penas Crueles, Inhumanos o Degradantes. Estas herramientas determinan el marco jurídico sobre el que se asienta el tratamiento del delito penal de tortura en el país.

2 El autor afirma que la historia latinoamericana ha sido prolífica y abundante en experiencias en las que el Estado Nación adquiere la forma de Estado de Excepción, entendiendo por este último el abandono de la normatividad del Estado de Derecho para adquirir formas excepcionales al margen de la legalidad institucional representada por el modelo tradicional del Estado democrático-parlamentario. Desde esa definición, desarrolla las particularidades que presentaron los regímenes militares de la década de 1970 
plo más acabado del Estado Terrorista. En 1984, tras el retorno de la democracia, la Comisión Nacional sobre la Desaparición de Personas (CONADEP) presentó un informe de más de 50.000 páginas que documentaron que miles de personas habían sido secuestradas y privadas de manera ilegítima de su libertad en aquellos años. La Comisión registró 8.960 personas que hasta ese momento continuaban desaparecidas y dejó sentado que le asistían profundas razones para suponer una cifra aún mayor. Con los años, las denuncias crecieron no sólo en el país sino en el resto del mundo, por lo que los organismos de Derechos Humanos calculan en la actualidad que son 30.000 las personas que aún permanecen desaparecidas (CONADEP 1984).

En la totalidad de las denuncias recibidas por la Comisión, se mencionaron diferentes tipos de torturas y actos de tormento. De esto se deduce que, lejos de acudir a ella de manera esporádica, la tortura fue concebida como una herramienta fundamental del modus operandi del Estado clandestino. Es por ello que fue aplicada de manera sistemática y con idénticos procedimientos en toda la extensión del territorio nacional: las descargas eléctricas en áreas sensibles del cuerpo como tetillas, genitales y/o encías; los golpes propiciados con diferentes objetos; las violaciones y ataques sexuales individuales y colectivos; los cortes y flagelaciones con objetos punzo-cortantes; las inmersiones en recipientes con agua para generar el ahogamiento; el estiramiento de las extremidades con cuerdas y poleas; son sólo algunos ejemplos de los que componen el interminable catálogo del horror que refleja el informe.

Las mismas fueron impartidas sobre un sujeto social de constitución reciente: el subversivo. Bajo esta categoría, delineada en función de ciertos trazos políticos antes que corporales, clasistas, étnicos o culturales, fueron aglutinados todos aquellos que, de manera real o potencial, participaran en organizaciones sociales que dieran muestra de una cierta combatividad. El espectro alcanzó a militantes y dirigentes políticos, sociales y sindicales, referentes religiosos, artistas, periodistas, intelectuales, etc.

Sin lugar a dudas se trató de un proyecto político en el que la deshumanización fue elevada al rango de política de Estado. El Estado Terrorista argentino encarnó la negación del universal que es propia de todo régimen totalitario y propuso, al igual que estos, la solución final para quienes fueran excluidos del mismo. Pero justamente por eso, se trató de una deshumanización que sólo resultó posible en la medida en que fue ejecutada de manera oculta y clandestina, tras la supresión del orden institucional vigente.

En el informe fueron identificados 340 Centros Clandestinos de Detención que, distribuidos en todo el país, fueron concebidos para la propagación del terror y el ejercicio sistemático de la tortura. Aún cuando funcionaran en el corazón de los edificios oficiales de las distintas fuerzas represivas del Estado, los espacios destinados al de- 
sarrollo de estas actividades permanecían ocultos, mientras que un férreo mecanismo de censura impedía su propagación a través de los medios de comunicación. Por último, las circulares administrativas en las que los oficiales amparaban estas acciones emanaban de una autoridad de facto y, por lo tanto, anclada en la más absoluta excepcionalidad. Es por esto que podemos sostener que se trató de una deshumanización originaria. Es decir, una deshumanización que sin ser fruto del neoliberalismo se constituyó en su punto de partida, en tanto resultó determinante para la gestación de sus condiciones de posibilidad.

La política migratoria de la dictadura fue condensada en el decreto con fuerza de ley núm. 22.439/81 titulado Ley General de Migraciones y Fomento de la Inmigración. Esta norma, también conocida como Ley Videla, se distingue por su carácter racista y represivo. El segundo se vislumbra no sólo en el hecho de que concentra la intervención del Estado en materia migratoria en lo concerniente al control y la defensa de sus fronteras nacionales, sino en que involucra al conjunto de la población en la persecución de los migrantes irregulares. Tal como se desprende de su articulado, tanto las autoridades al celebrar actos civiles, como los funcionarios públicos empleados en institutos educativos y sanitarios, como los profesionales independientes al celebrar actos de derecho privado (como por ejemplo en el caso de los escribanos públicos), se veían obligados a informar a la autoridad competente en caso de 'comprobar la ilegalidad de la residencia' del migrante.

Respecto de su contenido racista, la legislación no hizo más que recuperar una premisa de profundo anclaje en el imaginario colectivo cuya formulación se atribuye al escritor mexicano Octavio Paz: «los mexicanos descienden de los aztecas, los peruanos, de los incas, y los argentinos, de los barcos». Basada en el libro del génesis que la Generación del ' 80 construyó como la historia oficial, los orígenes de la nación argentina no deberían rastrearse en los pueblos originarios que ocuparon el continente mucho tiempo antes de la conquista, sino en el siglo XIX con la llegada masiva de inmigrantes de ultramar. Sólo a fuerza del olvido de siglos podía construirse y sostenerse el mito de una nación blanca y europea escindida de su condición indiscutiblemente mestiza y latinoamericana.

Tanto en la Constitución Nacional de 1853, que en su artículo 25 actualmente vigente sostiene que «el Gobierno federal fomentará la inmigración europea»; como en la Ley de Inmigración y Colonización núm. 817 de 1876 (Ley Avellaneda), que en su artículo 12 define como inmigrante a todo extranjero que llegase a la República «en buques a vapor o a vela», lo que subyace es la creencia de que la única inmigración deseable para nuestro país es la inmigración europea. La Ley Videla alimenta este postulado al afirmar en su artículo 2 que el Estado «promoverá» la inmigración de extranjeros «cuyas carac- 
terísticas culturales permitan su adecuada integración en la sociedad argentina». Sociedad a la que definían a su vez como «occidental y cristiana».

En un contexto migratorio como el argentino donde, tras al detenimiento de los flujos de ultramar, los inmigrantes provienen mayoritariamente de los demás países de la región, esto supuso convertir casi a la totalidad de la población extranjera residente en el país en indeseable. Esto permitió, siguiendo el argumento de Wallerstein, un reforzamiento de las jerarquías ya vigentes al interior de la fuerza de trabajo, basadas en lo que Margulis (1999) denomina como la «racialización de las relaciones de clase». El autor alude con este término a los procesos a través de los cuales rasgos de índole corporal, étnico-nacional o cultural han intervenido históricamente en la constitución y reproducción de las relaciones de clase en América Latina, ubicando a mestizos y/o mulatos en una condición de distanciamiento respecto del cuerpo legítimo y en lugares de menor prestigio y jerarquía sociales (1999).

Sin embargo, esto no supuso per se la deshumanización de estos grupos sociales. Por el contrario, la participación en actividades políticas fue el criterio utilizado por las Fuerzas Armadas para distinguir a los inmigrantes deshumanizables de aquellos que no lo eran. La Operación Cóndor ${ }^{3}$ permitió incluso la transnacionalización de esta figura, al posibilitar la persecución, secuestro, tortura y desaparición de los potenciales 'subversivos' a través de las fronteras estatales de los países del Cono Sur.

\section{La crisis de 2001 y el desafío a la hegemonía}

En los años que siguieron al retorno de la democracia, los crímenes perpetrados por las dictaduras fueron radicalmente condenados, al tiempo que fueron divorciados de la ideología que los había dotado de sustento y de sentido. El neoliberalismo fue absuelto de aquellas muertes que habían acompañado su irrupción en la región y esto permitió su consolidación regional una vez restablecida la vía democrática-electoral de la mano del denominado «Consenso de Washington» (Giavedoni, Ginga 2017).

Fue recién con la crisis de diciembre de 2001 que el complejo entramado de resistencias que se había estado tejiendo desde mediados de la década anterior para contener esta envestida ocupó el centro de la escena política nacional. Los saqueos, el estado de sitio, los cacerolazos

3 Se conoce con ese nombre a un plan de coordinación de actividades de inteligencia que celebraron entre sí las cúpulas militares de la región, con el beneplácito del gobierno de los Estados Unidos. 
y finalmente la represión policial que entre el 19 y 20 de diciembre terminó con un total de 36 muertos en todo el país y la renuncia del entonces presidente Fernando De La Rua, fueron el corolario de ese proceso.

Mientras que en los espacios de poder el 'consenso neoliberal' parecía inalterado, ${ }^{4}$ en las calles se estaban produciendo transformaciones sociales y subjetivas que tensionaban esa hegemonía. Paradójicamente, al ritmo de los golpes de las cacerolas vacías exigiendo «que se vayan todos», se refundaba la política en su forma más radical: la igualdad, en su dimensión de operador lógico que hay que suponer, verificar y demostrar, se materializaba cotidianamente en el rechazo de formas de organización jerárquicas y delegativas. Las asambleas barriales y las fábricas 'sin patrón' recuperadas por los trabajadores fueron un ejemplo de estos procesos.

Fue sobre esta base social en estado incandescente que los gobiernos de Néstor y Cristina Kirchner articularon una nueva construcción discursiva. Este viraje - que se tradujo en la reivindicación de la juventud revolucionaria de la década del setenta y, junto con ella, en la legitimación de la política como práctica altruista y necesaria - fue condensado en la siguiente premisa: «la patria es el otro».

Esta afirmación establece una dependencia mutua entre la construcción de la patria y la ampliación de sus fronteras a través de la inclusión de un otro, que no es antagónico sino vulnerable (Landa 2015). Es vulnerable en la medida en que el Estado mantiene con él algunas deudas pendientes y, por el mismo motivo, la intervención estatal aparece como condición y garantía de este proceso de ampliación. El Estado es así definido a través de su capacidad de mediar entre la sociedad y el mercado, reparando el daño y reduciendo las desigualdades de hecho que el segundo introduce en la primera (Yabkowski 2017). Esto supuso la transformación en discurso oficial, de la lógica universalista de la igualdad liberal.

Pero no se trató solamente de un ejercicio retórico, sino de una perspectiva de gestión que fue transversal a las más disímiles problemáticas sociales. Las migraciones no fueron una excepción. La Ley núm. 25.871 sancionada en el año 2003, también conocida como Ley Giustiniani, estableció en su artículo 4 que «el derecho a la migración es esencial e inalienable de la persona y la República Argentina lo garantiza sobre la base de los principios de igualdad y universalidad». Es en este espíritu que asume que de ninguna manera la irregularidad del proceso migratorio puede ser esgrimida como causante

4 Esto quedó demostrado cuando, tras haber derrotado en las urnas en 1999 al candidato del oficialista partido peronista, el nuevo gobierno - sostenido por una alianza electoral celebrada entre el radicalismo y otras fuerzas centro-progresistas -, no sólo no introdujo modificaciones en la política económica implementada hasta entonces, sino que incluso la profundizó al nombrar a Domingo Cavallo como ministro de economía, cargo que había ocupado durante buena parte de la gestión anterior. 
de la privación de derechos fundamentales de la persona, tales como la salud o la educación; y que corresponde al Estado, a través de sus funcionarios y personal dependiente, acompañar a la persona en su proceso de regularización brindándole el asesoramiento y la información que resulten necesarias. Esto supuso la transición del 'Estado Gendarme' de la Ley Videla a un 'Estado Garante', que entiende la propia norma como un instrumento destinado al reconocimiento de derechos (Arias Duval 2012).

Por otra parte, la norma reconoce un trato preferencial para los ciudadanos provenientes de los países que integran el Mercosur y sus Estados Asociados, hecho que también da cuenta del cambio de paradigma que se establece en esta materia. ${ }^{5}$ Mientras que los colectivos de inmigrantes regionales habían sido hasta entonces prácticamente ignorados por las legislaciones precedentes, la nueva norma se propone repensarlos en el marco de los procesos de integración regional vigentes y de la construcción política de la patria latinoamericana (Mármora 2012).

Esta actividad parlamentaria, aún cuando virtuosa, resultó insuficiente para invertir o neutralizar el sistema racista de jerarquización de la fuerza de trabajo. Si bien los migrantes continuaron concentrados en un número relativamente pequeño de ramas de actividad, caracterizadas por ofrecer empleos precarios, informales, mal remunerados y con escasa movilidad social, el impulso de programas de regularización masiva y la apertura de canales permanentes para dichos fines permitieron el mejoramiento de sus condiciones laborales (Baer et al. 2012).

Esta construcción político-discursiva no sólo permitió contener la avanzada del racismo de vertiente neoliberal, sino que además ofició como un paraguas lo suficientemente amplio como para alojar diferentes procesos de 'rehumanización' de aquellas identidades sobre las que históricamente se constituyó la alteridad de la blanquedad argentina. Los inmigrantes, al igual que los afrodescendientes, los pueblos originarios y las disidencias sexuales se vieron contenidos en este esfuerzo a la vez dignificante y legitimador propiciado desde el Estado. Un esfuerzo que no sólo se ancló en una temporalidad presente sino también pasada, al convertir en bandera política la búsqueda de la Memoria, la Verdad y la Justicia por los crímenes cometidos por el terrorismo de Estado.

5 La nueva normativa, que alcanza a la totalidad de los países de América del Sur, permite que los ciudadanos de estos países puedan tramitar en el país una visa de 'residencia precaria' por un plazo de dos años, que les permite permanecer y trabajar en igualdad de condiciones respecto de los nativos. Esta decisión forma parte de los compromisos asumidos por los Estados del bloque en el Acuerdo sobre residencia para nacionales de los estados partes del Mercosur, Bolivia y Chile suscrito en diciembre de 2002 (https://www.mercosur.int/documento/acuerdo-residencia-nacionales-estados-partes-mercosur-bolivia-chile/, 2019-06-03). 
El Comité contra la Tortura, en el cuarto informe periódico aprobado para el país en el año 2004, celebró los esfuerzos realizados «en la lucha contra la impunidad en relación a los crímenes de lesa humanidad cometidos durante la dictadura militar», al tiempo que evaluó positivamente la promulgación de la Ley de Migraciones núm. 25.871 (Comité contra la Tortura 2004). Sin embargo, el mismo documento alertó sobre «las numerosas alegaciones de tortura y malos tratos cometidas de manera generalizada y habitual por parte de las fuerzas y cuerpos de seguridad del Estado»; así como también «la desproporción entre el elevado número de denuncias por actos de tortura y malos tratos y las mínimas condenas dictadas por dichas causas, así como los retrasos injustificables en la investigación de casos de tortura, todo lo cual contribuye a la impunidad existente en esta materia».

Si bien a lo largo del período se registraron algunas decisiones políticas de relevancia con el objeto de revertir estas tendencias, ${ }^{\circ}$ las preocupaciones señaladas por el Comité en el 2004 se repitieron en el informe presentado por el mismo organismo en el 2017 (Comité contra la Tortura 2017). ${ }^{7}$ Este documento alerta sobre la práctica recurrente de la tortura y el maltrato en los centros de detención y en las rutinas del personal penitenciario, a los que identifica como un lastre histórico «de una estructura militarizada y corporativa».

Podemos concluir en que se trató de un proyecto político signado por una fuerte impronta rehumanizadora, que se amparó en el discurso universalista liberal para posicionar al Estado como reparador de desigualdades de hecho y de derecho. Esto resultó insuficiente para erradicar la tortura como práctica recurrente de las fuerzas de seguridad estatal, pero contribuyó a contenerla sobre las jurisdicciones históricas de estas corporaciones, que nunca han sido real objeto de un proceso de democratización.

Los inmigrantes fueron afectados por estos procesos en igual medida que aquellos otros grupos sociales sobre los que pesa el estigma histórico de la alteridad. Por un lado, la implementación de una política migratoria progresista permitió la ampliación de sus derechos laborales contrarrestando de esa manera el patrón aún vigente de racialización de las relaciones de clase. Por el otro, los excesos y arbitrariedades de las fuerzas de seguridad siguieron siendo más frecuentes y violentos, y la justicia más lenta y perezosa, cuando las

6 Pueden mencionarse, al respecto, la modificación de la composición de la Corte Suprema de Justicia de la Nación, a través de la designación de magistrados con reconocidas trayectorias en materia de Derechos Humanos; así como también la creación del Ministerio de Seguridad de la Nación - que hasta entonces había funcionado como una secretaría del Ministerio del Interior - estableciendo entre sus atribuciones la de «supervisar el accionar individual o conjunto de las Fuerzas de Seguridad y Policiales».

7 Este informe abarca el último tramo del período analizado en este apartado y los primeros años del ciclo abordado en el apartado siguiente. 
víctimas se registraron entre las comunidades Quom de la provincia de Formosa, las familias inmigrantes del Parque Indoamericano o el piberío de familias humildes del conurbano bonaerense (CELS 2017).

\section{$4 \quad$ Las elecciones de 2015 y la restauración}

Transcurridas casi dos décadas desde el cambio de milenio, el 'giro a la izquierda' parece haber dejado paso a la 'restauración conservadora' en América Latina. García Delgado (2016) sintetiza los alcances de este proceso para el caso argentino, bajo la afirmación de que tras la victoria de Mauricio Macri, candidato de la Alianza Cambiemos, «el poder vuelve al poder». Es decir que el poder fáctico, el corporativo, el de las empresas más concentradas nacionales y multinacionales, los medios de comunicación y la banca financiera disponen ahora también el poder del Estado.

Cambiemos inaugura una nueva construcción discursiva en la que la política managerial reemplaza al conflicto como lógica de ejercicio del poder. Categorías como 'izquierda' o 'derecha' son entendidas como rémoras del pasado, que resultan anacrónicas para pensar al país en su tiempo presente. Por el contrario, su entrada triunfal en el siglo XXI reclama la transferencia hacia el Estado de los valores del mundo de la empresa. Esta lógica de penetración desde lo privado hacia lo público se pone en evidencia en la selección de gerentes provenientes de compañías multinacionales de primer nivel para ocupar los principales espacios de decisión política. Tal como lo afirma Franco Moccia, uno de los referentes de este espacio político: «Como dice Mauricio: de un buen gestor se puede hacer un buen político. Al revés, es más difícil» (Vommaro 2016, 11).

Tal como lo menciona el autor, en esta nueva construcción, la sociedad es entendida como un conjunto de voluntades con espíritu emprendedor, coordinadas por un team leader que les ofrece las mejores oportunidades de crecimiento personal. De hecho, en su primer discurso como Presidente dirigido a la Asamblea Legislativa, Mauricio Macri sintetizó esta visión: «¿Cuál es el país con el que sueño? Un país que estimula el desarrollo personal y el de tu familia. Un país que te convoca a tu aventura personal». ${ }^{8}$

De modo que, en este nuevo ciclo político, el discurso empresarial neoliberal se convierte en discurso oficial. Contra la lógica universalista propulsada por el liberalismo, la nueva retórica apuesta a una estrategia de distinción como herramienta de construcción y consoli-

8 «Discurso completo de Mauricio Macri ante la Asamblea Legislativa». La Nación, 1 de marzo. URL https://www.lanacion.com.ar/politica/discurso-completo-de-mauricio-macri-ante-la-asamblea-legislativa-nid1875715 (2019-11-08). 
dación de la sociedad de los 'empresarios de sí'. Para ello, la contraofensiva se asienta sobre un discurso dirigido al argentino bienpensante, al 'ciudadano de a pie', al 'laburante', con el objeto de establecer con claridad los elementos que lo distinguen de aquellos otros argentinos de una raigambre inferior, a los que se les atribuye la responsabilidad de los fracasos recurrentes de un país condenado al éxito.

Durante la campaña electoral y en los primeros meses de gestión, la otredad se concentró sobre los 'choriplaneros' y la 'grasa militante'. ${ }^{9}$ Estos dos colectivos sociales, si bien diferentes, comparten el mismo estigma: son las cigarras que intentar vivir sin trabajar, succionando las ubres del Estado. Ubres que, por otro lado, sólo los argentinos bienpensantes, los 'ciudadanos de a pie', los 'laburantes', contribuyen a llenar. El trabajo como valor aparece en esta construcción discursiva como el criterio sobre el que se asienta esa línea divisoria entre nosotros y ellos, por lo cual los primeros deslizan sobre los segundos un único imperativo: «vayan a laburar».

El problema es que, tras casi cuatro años de neoliberalismo recargado, en el país de «la meritocracia al palo», el endeudamiento para la cobertura de gastos corrientes les confirma a las familias bienpensantes argentinas que «por mucho que vayan a laburar» les «sobra mucho mes al final del sueldo». Agotadas las promesas a futuro respecto de la recuperación económica, la respuesta de la alianza gobernante es la de redoblar su promesa simbólica de distinción. Pero para cumplir con este cometido entre la nueva horda de trabajadores ocupados bajo la línea de pobreza, ya no alcanza con que esos otros sean vagos. Ahora es necesario que, además, sean peligrosos.

Este nuevo otro es constituido íntegramente a través de su peligrosidad: el delincuente. No es necesario delinquir para ser un delincuente. Esta categoría condensa en términos prácticos todos los estigmas sociales instalados desde antaño en la conciencia nacional. El delincuente es negro, vago, sucio, pobre, indio, villero, violento, borracho y falopero. Si entre todas esas categorías que responden a fenómenos diferentes puede establecerse una relación de equivalencia, es gracias al racismo de vertiente liberal del que se ha nutrido sistemáticamente el mito de la blanquedad occidental argentina frente a la barbarie mestiza latinoamericana.

Sobre la idea-fuerza de que, a falta de progreso, por lo menos que haya orden, el Estado convierte al 'delincuente' en su enemigo oficial

9 El término 'choriplanero' surge de la fusión de dos palabras: 'choripán' y 'plan social'. Esa combinación se utiliza para referir a quienes, a través políticas clientelares, percibirían algún tipo de ingreso a cambio del cual se comprometerían a participan en manifestaciones y actividades políticas donde regalan choripanes. El concepto de 'grasa militante' fue acuñado por el entonces Ministro de Hacienda y Finanzas Alfonso Prat Gay para referir a quienes participarían de un espacio político con el único objetivo de obtener un puesto de trabajo en el Estado. 
y pone en marcha la deshumanización pública y democrática que define al racismo en su versión neoliberal. Patricia Bullrich, Ministra de Seguridad de la Nación, es quizás la más clara portavoz de este discurso: «¿Quién cuida a la gente?» se preguntó en una entrevista en alusión a la sociedad argentina bienpensante. «Nos enfrentamos contra delincuentes que no tienen ningún problema en matar», mencionó luego para instalar esa otredad. «El Estado es el que realiza las acciones para impedir el delito», sintetizó más tarde en nombre de la investidura que representa. ${ }^{10}$ Así las cosas: el Estado, comprendido en su condición de garante del orden, ya no se ocupa de reparar desigualdades. Su función primordial será la de garantizar que la distancia que separa al 'nosotros' bienpensante de esos 'otros' peligrosos resulte lo más amplia posible. Para cumplir con dicho cometido, será fundamental el auxilio de las fuerzas de seguridad.

Para lograrlo, la Ministra ha sido la impulsora de un nuevo paradigma en materia de seguridad, conocido como Doctrina Chocobar. ${ }^{11}$ Esta última se sustenta sobre la premisa de que, en el marco de un enfrentamiento, las fuerzas de seguridad son inocentes. Esta política ha sido acompañada en el año 2018 por un nuevo reglamento general para el empleo de armas de fuego por parte de los miembros de las fuerzas federales de seguridad, que amplió el horizonte de discrecionalidad de estas últimas respecto del uso de las primeras. ${ }^{12}$

De modo que a la arbitrariedad de las fuerzas represivas y a la complicidad del poder judicial se suma ahora también la impunidad garantizada por los altos mandos del poder político. La propagación de una práctica discursiva y jurídico-administrativa que legitima y legaliza un absoluto laissez faire, laissez passer respecto del accionar de las fuerzas de seguridad es un síntoma claro de que el proceso de deshumanización democrática y visible que reclama el racismo en su vertiente neoliberal, ya está en marcha.

Las consecuencias de esta avanzada están a la vista. Según el último informe anual elaborado por la Coordinadora Contra la Represión Institucional y Policial (CORREPI 2019), en sus tres años de gestión, el de Cambiemos se ha convertido en el gobierno más repre-

10 «Contundente defensa de Patricia Bullrich a Chocobar: 'Cambió la doctrina, la Policía no es culpable en un enfrentamiento'», Infobae, 6 de febrero 2018. URL https:// bit.ly/2VTBdjD (2019-06-21).

11 Luis Chocobar es un policía local de una ciudad del área metropolitana de Buenos Aires que se volvió famoso en diciembre de 2017 tras disparar por la espalda y asesinar a un ladrón mientras intentaba escapar, después de haber apuñalado a un turista para robarle una cámara fotográfica. El policía, que fue procesado por exceso de legítima defensa, fue respaldado por el Presidente y por la Ministra de Seguridad, quienes lo recibieron en la Casa Rosada para demostrarle pública y oficialmente su apoyo.

12 El reglamento las habilita a disparar sobre personas en situación de fuga, a utilizarlas cuando los efectivos se vean superados en número y a disparar sin emitir la voz de alto (Ministerio de Seguridad 2018). 
sor desde la vuelta de la democracia en $1983 .{ }^{13} \mathrm{El}$ documento señala, además, que el aumento del poder de fuego en las calles a partir de la saturación policial y de otras fuerzas hizo crecer como nunca antes los fusilamientos por gatillo fácil; mientras que el uso masivo de las facultades para detener personas arbitrariamente contribuyó a la multiplicación de la población en cárceles y comisarías, con el consiguiente incremento de episodios de torturas y muertes bajo custodia (CORREPI 2019).

Los inmigrantes han sido objeto de un particular esfuerzo de amalgamación con esa nueva alteridad susceptible de recibir todo el peso de la deshumanización del racismo neoliberal: el delincuente. El propio Presidente Macri afirmó en un discurso que: «no podemos permitir que se siga eligiendo a la Argentina como un lugar para venir a delinquir». Días más tarde, agregó la Ministra Bullrich: «Acá vienen ciudadanos paraguayos o peruanos que se terminan matando por el control de la droga. La concentración de extranjeros que cometen delitos de narcotráfico es la preocupación que tiene nuestro país». ${ }^{14}$

Si bien la ley migratoria no ha sido reemplazada, el Poder Ejecutivo ha introducido modificaciones a la norma a través del Decreto de Necesidad y Urgencia (DNU) núm. 70 del 1 o de marzo de 2017, que tiene por objeto primordial acelerar los actos y los plazos administrativos que permitan la expulsión de inmigrantes que «se encontraran involucrados en hechos delictivos y a quienes hubieren ingresado en forma clandestina al territorio nacional, eludiendo el control migratorio».

En términos generales, el decreto propone un endurecimiento de los requisitos de admisión y permanencia previstos en la legislación. Sin embargo, su perversidad es de carácter político antes que jurídico. En los considerandos que acompañan el articulado se establece una vinculación casi directa entre delincuencia, crimen organizado, narcotráfico, seguridad e inmigración. Esto convierte a cualquier inmigrante en potencialmente sospechoso y, al Estado, en el principal encargado de controlar, perseguir y confirmar esa sospecha. En la misma sintonía se inscribe el acuerdo rubricado en agosto de 2016, por el cual la Ciudad de Buenos Aires cede un inmueble al Estado nacional para que sea destinado al alojamiento de las personas infractoras de la ley y de su normativa complementaria. Esto supondría, en la práctica, la instauración de la primera cárcel para inmigrantes del país.

13 Según el informe, frente al promedio de una persona asesinada cada 30 horas por el aparato represivo estatal del conjunto de los 12 años de gobiernos kirchneristas, el macrismo incrementó exponencialmente esta frecuencia hasta registrar un muerto cada 25 horas en 2016, uno cada 23 en 2017 y uno cada 21 en 2018.

14 «Bullrich, Patricia: 'Acá vienen ciudadanos peruanos y paraguayos y se terminan matando por el control de la droga'», La Nación, 24 de enero 2017. URL https://bit. ly/20Ye5z9 (2019-07-03) 


\section{Conclusiones: la carrera de la deshumanización neoliberal}

El Golpe de Estado de 1976 fue condición de posibilidad para el establecimiento y la consolidación del neoliberalismo en Argentina. Para imponer la reconfiguración del Estado, la subjetividad y las formas de vida que dicho proceso reclama, en una sociedad como la argentina - que para la década de 1970 presentaba elevadísimos niveles de movilización social -, fue necesario avanzar sobre la deshumanización de todos aquellos que pudieran oponerse a ella. Fue una deshumanización que acudió a la tortura de manera constante y sistemática, pero que adoptó los mismos trazos que en otros regímenes totalitarios: fue ejercida de manera clandestina, silenciada y en el marco de la alteración del orden institucional democrático. Es por eso que decimos que se trató de una deshumanización originaria, porque sin ser fruto del neoliberalismo se constituyó en su punto de partida.

Esta deshumanización recayó sobre todo aquel sujeto que fuera rotulado con la categoría de 'subversivo'. Es decir, que participara real o potencialmente en actividades políticas. Este mismo criterio fue utilizado para distinguir a los inmigrantes perseguibles de aquellos que no lo eran. Si hubo en Argentina inmigrantes secuestrados, torturados y/o desaparecidos, no fue por ser extranjeros sino por ser subversivos. Sobre los inmigrantes entendidos en tanto que grupo social, lo que prevaleció fue una matriz ideológica arraigada en los postulados históricos del racismo liberal nacional. Si bien esto se tradujo en un reforzamiento de su condición social subalterna, no supuso per se su deshumanización.

Si bien el orden democrático fue restablecido en la década de 1980, fue recién con la crisis de diciembre de 2001 que la hegemonía del orden neoliberal fue discutida y desafiada. Esta fisura social permitió la consolidación de una nueva construcción político-discursiva signada por un fuerte énfasis en la función reparadora del Estado. Esto permitió abrigar diferentes procesos de rehumanización de aquellas identidades sobre las que históricamente se había construido la alteridad argentina. Gracias a esto, los migrantes alcanzaron algunas mejoras en sus condiciones de vida, que coexistieron con la vigencia de una matriz racista de jerarquización de la fuerza de trabajo que los mantuvo en la base de la pirámide social. Por otro lado, estos esfuerzos no alteraron la persistencia generalizada de la tortura y los malos tratos como prácticas habituales de las distintas fuerzas de seguridad que integran el aparato represivo del Estado. Prácticas que fueron ejercidas con mayor intensidad y crudeza sobre aquellos actores sociales históricamente vinculados a la otredad en el imaginario nacional, como por ejemplo ocurre con los inmigrantes regionales.

El triunfo electoral de la Alianza Cambiemos en 2015 significó la conquista del poder del aparato estatal por los sectores más concentrados de la economía nacional y transnacional. Esto supuso la reinstauración del orden neoliberal en su vertiente más ortodoxa, asen- 
tada sobre un patrón de acumulación con eje en la valorización del capital financiero, el endeudamiento externo y la disciplina fiscal.

Como correlato del empeoramiento de las condiciones de vida de la mayor parte de la población, la alianza gobernante impuso una nueva práctica discursiva orientada a la deshumanización de una figura social que condensa todos los estigmas sociales instalados de antaño en la conciencia nacional y establece entre ellas una relación de equivalencia: el delincuente. El Estado constituye la deshumanización de esta figura en política pública de seguridad, garantizando así la dimensión democrática y visible que reclama el racismo en su vertiente neoliberal. Al reforzar la arbitrariedad de las fuerzas represivas, con la impunidad garantizada por los altos mandos del poder político, se multiplican las muertes por gatillo fácil y las denuncia por torturas y muertes bajo custodia.

Esto ha sido acompañado de un enérgico esfuerzo oficial para entretejer un vínculo directo y sin mediaciones entre migración y delincuencia. Producto de este enlace, se multiplican los episodios cotidianos que registran tratos crueles, inhumanos y degradantes, ejercidos por los diferentes poderes y organismos estatales sobre el conjunto de esta población. Uno de los casos que ha cobrado mayor relevancia mediática ha sido el de Vanessa Gómez Cueva. Vanessa es peruana, pero hace más de 15 años que vive en Argentina. En el año 2013 fue condenada a cuatro años de prisión por comercio de estupefacientes. Tras cumplir su condena se recibió de enfermera, profesión de la que trabaja desde entonces y que le permitía sostener económicamente su hogar. El 1o de febrero, la policía buscó a Vanessa en su casa y le pidió que la acompañara a «firmar una notificación». Vanessa llevó a su hijo menor de 2 años con ella y dejó a los otros dos en su casa, uno de 5 años y el otro de 14, todos ellos argentinos. Al llegar fue recluida en una celda sin luz ni agua y desde ahí trasladada al Aeropuerto de Ezeiza, desde donde fue expulsada tres días más tarde junto a su hijo, sin poder despedirse de los otros dos. ${ }^{15}$

Por otra parte, desde hace ya algunos años, la Agrupación Xango viene denunciando el aumento de la violencia y del hostigamiento por parte de las fuerzas de seguridad hacia los ciudadanos senegaleses que trabajan en la venta ambulante en los diferentes barrios de la ciudad de Buenos Aires: revisan sus pertenencias en cualquier lugar, secuestran sus bienes personales, roban su mercadería, entre otras cosas. Si los migrantes reclaman por sus derechos o solicitan actas de su detención, son burlados y detenidos bajo el concepto de resisten-

15 Miguel Pireto Toledo, «El drama de la enfermera peruana que fue deportada y separada de sus hijos llegó a la Comisión Interamericana de Derechos Humanos», Infobae, 15 de mayo 2019. URL https://bit.ly/31o6MU4 (2019-06-21). 
cia a la autoridad. ${ }^{16}$ Massar Ba, referente de la comunidad senegalesa en Argentina, fue uno de los que se animó a denunciar estos atropellos e incluso a organizar una manifestación que reunió a centenares de personas en agosto de 2015, para exigir una reversión de las mismas. El 7 de marzo de 2016, fue encontrado tirado en la calle, con múltiples traumatismos, que le causaron la muerte dos días después. Carlos Álvarez, de la Agrupación Afro Xangó declaró al respecto: «no quiero esbozar la idea de una cacería de brujas ni mucho menos, pero en el último año hubo al menos diez casos de senegaleses muertos por diversas causas que nunca se esclarecieron» (La Vaca 2016).

Estos ejemplos nos permiten esbozar cuanto menos dos conclusiones respecto de esta carrera a la deshumanización neoliberal. La primera es que su cadencia se acelera cuando la superposición de los estigmas sociales lo permiten. Es lo que ocurre en el caso de Vanessa, por mujer y por madre soltera; pero también con la comunidad senegalesa, por negra y clandestina. La segunda es que no parece dispuesta a tolerar los escollos que se presenten en el camino. La deshumanización de derecho, no deja espacio a ningún tipo de reclamo.

No quedan dudas de que este es sólo el comienzo. En un proyecto político que apuesta a la conformación de una «democracia sin demos» (García Delgado 2016), el racismo neoliberal no tiene otro objeto que el de atacar de manera permanente la lógica de los débiles, achicando cada vez más la base de quienes cuentan con la humanidad suficiente para exigir el cumplimiento de derechos universales.

\section{Bibliografía}

Arias Duval, Martín (2012). «Política migratoria y derechos humanos». Griffa, Norberto (comp.), Las migraciones actuales en Argentina. Buenos Aires: Editorial de la Universidad Nacional de Tres de Febrero, 187-96.

Baer, Gladys et al. (2012). «El trabajo de los inmigrantes en una etapa de recuperación del empleo: las políticas migratorias y su situación socio-laboral». Grifa, Norberto (comp.), Las migraciones actuales en Argentina. Buenos Aires: Editorial de la Universidad Nacional de Tres de Febrero, 79-98.

Basso, Pietro (cur.) (2010). Razzismo di stato. Stati Uniti, Europa, Italia. Milano: FrancoAngeli.

CELS, Centro de Estudios Legales y Sociales (2017). El derecho a la protesta social en la Argentina. Buenos Aires: Centro de Estudios Legales y Sociales

CONADEP, Comisión Nacional para la Desaparición de Personas (1984). Nunca más. Informe de la Comisión Nacional sobre la desaparición de personas. Buenos Aires: Eudeba.

16 Anny Ocoró Loango et al., «Migración, afrodescendientes y violencia institucional en Argentina». Márgenes, 2018. URL http://www.unsam.edu.ar/margenes/migracion-afrodescendientes-y-violencia-institucional/ (2019-06-21). 
Comité contra la Tortura (2004). Examen de los informes presentados por los Estados Partes en virtud del artículo 19 de la Convención. Argentina. URL https://bit.ly/2NvKfix (2019-06-11).

Comité contra la Tortura (2017). Observaciones finales sobre los informes periódicos quinto y sexto combinados de la Argentina. URL https://bit. ly/333PixQ (2019-06-11).

CORREPI, Coordinadora Contra la Represión Institucional y Policial (2019). Informe de la situación represiva nacional. URL https://drive.google.com/ drive/folders/1lWq1b2s8jocRvsre9TjNcOfUhIJKMcfZ (2019-07-10).

Duhalde, Eduardo Luis (1999). El Estado Terrorista argentino. Buenos Aires: Eudeba.

Foucault, Michel (2012). Nacimiento de la biopolítica. Madrid: Akal.

García Delgado, Daniel (2016). «La modernización del Estado. El poder vuelve al poder». Estado y Políticas Públicas, 6, 13-17.

Giavedoni, José, Ginga, Luciana (2017). «Neoliberalismo y Violencia: el huevo de la serpiente». Revista Bordes, 6, 19-30.

Harvey, David (2008). «El neoliberalismo como destrucción creativa». Apuntes Del CENES, 27(45), 10-47.

Hinkelammert, Franz (1987). «Frente a la cultura de la postmodernidad: proyecto político y utopía». Pasos, 12, 1-9.

La Vaca (2016). El crimen de Massar Ba no puede quedar impune. URL https:// www.lavaca.org/notas/el-crimen-de-massar-ba-no-puede-quedar-impune/ (2019-06-21).

Landa, Lucía (2015). «La patria es el otro: redefiniendo los sujetos políticos del discurso populista». Identidades, 9, 82-99.

Lazzarato, Mauricio (2013). La fábrica del hombre endeudado. Buenos Aires: Amorrortu.

Margulis, Mario (1999). «La 'racialización' de las relaciones de clase». Margulis, Mario; Urresti, Marcelo, La segregación negada. Cultura y discriminación social. Buenos Aires: Biblos, 37-62.

Mármora, Lelio (2012). «Las políticas de migraciones internacionales en América del Sur». Griffa, Norberto (comp.), Las migraciones actuales en Argentina. Buenos Aires: Editorial de la Universidad Nacional de Tres de Febrero, 207-29.

Ministerio de Seguridad (2018). Reglamento General para el Empleo de Armas de Fuego por parte de los Miembros de las Fuerzas Federales de Seguridad. URL https://bit.ly/2DEllJC (2019-06-21).

Murillo, Susana (2011). «La nueva cuestión social y el arte neoliberal de gobierno». Cátedra Paralela, 8, 9-32.

Pertot, Werner (2018). «Macri se sube a la ola contra los extranjeros». Diario Página 12, 2 de noviembre. URL https://www. pagina12.com.ar/152661macri-se-sube-a-la-ola-contra-los-extranjeros (2019-06-25).

Portes, Alejandro; Walton, John (1981). Labor, Class and the International System. New York: Academic Press.

Vommaro, Gabriel (2016). «Unir a los argentinos: el proyecto de 'país normal' de la nueva centroderecha en Argentina». Nueva Sociedad, 261, 4-12.

Von Mises, Ludwig (1968). La acción humana. Madrid: Sopec.

Wallerstein, Immanuel (1991). «Universalismo, racismo y sexismo, tensiones ideológicas del capitalismo». Wallerstein, Immanuel; Balibar, Etienne. Raza, Nación y Clase. Madrid: Iepala, 49-61.

Yabkowski, Nuria (2017). "Los sentidos del Estado en la identidad kirchnerista». Postdata, 22(1), 489-528. URL http://ref.scielo.org/n269g4 (2019-10-18). 


\title{
Controllo delle frontiere e violazione dei diritti umani lungo la rotta balcanica
}

Francesca Cucchi

Consiglio Italiano Rifugiati

\begin{abstract}
This paper focuses on the possibility of conciliation between one of the main objective of recent European migration policies - the containment of migration movements - and the safeguard of immigrants dictated by European Convention of Human Rights. Furthermore the dissertation will analyze the balance between the right of every State to control their borders together with the right of the European Union to reinforce the control of external borders and the respect of fundamental human rights. In particular, after a brief analysis of the situation over the Balkan route, this paper will illustrate whether and how the guarantees provided in art. 3 ECHR can be applied in relation to the condition of immigrants and the consequent legitimacy of national and European immigration policies implemented along the route.
\end{abstract}

Keywords Art. 3 ECHR. Balkan Route. Fundamental human rights. European migration policies. Inhuman or degrading treatment.

Sommario 1 Introduzione. - 2 La situazione lungo la rotta balcanica. - 2.1 Le associazioni di monitoraggio e tutela. - 3 Controlli dei movimenti migratori. La giurisprudenza della Corte EDU e la tutela assoluta dell'art. 3 CEDU. - 3.1 Proibizione della tortura e il «nessuno» da tutelare. - 3.2 Le condotte vietate dall'art. 3 della Convenzione EDU. 3.3 Responsabilità degli Stati: obblighi positivi e negativi derivanti dal divieto di tortura e trattamenti disumani o degradanti. - 4 Conclusioni. 


\section{Introduzione}

Nell'ultimo decennio l'Unione europea e gli Stati membri hanno adottato un approccio globale all'immigrazione essenzialmente securitario, prevedendo strumenti volti al potenziamento dei controlli alle frontiere e al rafforzamento dell'azione esterna dell'UE e non meccanismi atti a favorire l'integrazione e l'inclusione sociale.

Si sono finanziate politiche di contenimento dei movimenti migratori, di protezione ed esternalizzazione delle frontiere, si sono sottoscritti accordi con i Paesi di transito e di origine, con sempre minor attenzione a soluzioni sostenibili di lungo termine e alla tutela dei diritti umani fondamentali.

Il progressivo sistema di controllo dell'immigrazione ha trovato la sua formalizzazione nell'Agenda Europea sulla Migrazione del 13 maggio 2015, documento programmatico della Commissione Europea, recante una serie di provvedimenti urgenti destinati al rafforzamento delle misure esterne di controllo alle frontiere e interne con la creazione di hotspot, per affrontare l'«emergenza migratoria» determinata dal conflitto siriano e dalla ripresa degli sbarchi lungo la rotta del Mediterraneo centrale (Commissione Europea 2015).

La 'crisi' migratoria balcanica, in particolare, ha determinato un'accelerazione del processo securitario sia perché la rotta europea sud-orientale concentra il consistente movimento migratorio asiatico e quello medio-orientale, sia perché coinvolge direttamente gli Stati, già parte dell'UE come la Croazia, o che, come l'Albania, la Bosnia ed Erzegovina, la Macedonia, il Montenegro, la Serbia, sono interessati alle politiche di allargamento dell'UE e rivestono un ruolo chiave per la sicurezza e lo sviluppo nella regione.

In questo contesto geo-politico l'UE e gli Stati membri si sono trovati a dover risolvere un conflitto tra pretese giuridiche contrastanti, tra il diritto a controllare le frontiere e la tutela dei diritti umani fondamentali. Il risultato di questo difficile bilanciamento è apparso evidente a partire dal 2015-16, quando il progressivo aumento degli arrivi lungo la rotta balcanica ha fatto assumere un ruolo centrale al 'confine' quale unico strumento in grado di bloccare il movimento migratorio; il sistema Schengen della libera circolazione è andato in crisi, gli Stati hanno reintrodotto i controlli alle frontiere interne e hanno innalzato muri e barriere di filo spinato, lasciando migliaia di immigrati bloccati ai confini nazionali in condizioni degradanti.

A marzo 2016, a seguito degli accordi tra UE e Turchia, il Presidente del Consiglio Europeo Donald Tusk ha annunciato la chiusura definitiva della rotta balcanica, rivendicandola come una vittoria dell'UE (Consiglio Europeo 2016a).

Successivamente il Presidente Tusk parlando dell'accordo con la Turchia e della chiusura della rotta balcanica ha aggiunto: «a livello generale qualcuno potrebbe pensare che questo accordo costituisca 
una soluzione miracolosa al problema, ma la realtà è più complessa. Si tratta solamente di un pilastro della strategia globale dell'Unione europea e può funzionare solo se sono attuati anche gli ulteriori pilastri, che comprendono, tra gli altri elementi: rafforzare le frontiere esterne dell'UE, mantenere chiusa la rotta dei Balcani occidentali» (Consiglio Europeo 2016a ${ }^{1}$ ). Ma la rotta balcanica è tutt'altro che chiusa.

\section{La situazione lungo la rotta balcanica}

Nel 2018 Frontex (Agenzia europea delle guardie di frontiera e delle guardie costiere) ha registrato che il numero di emigranti individuati sulla rotta dei Balcani occidentali è sceso al livello più basso dal 2007, con una diminuzione del $60 \%$ rispetto al dato dell'anno precedente, attestandosi a 5.869 persone, provenienti soprattutto dall'Afghanistan e dal Pakistan. Tuttavia i dati di Frontex indicano una pressione costante lungo il percorso secondario che dalla Grecia si snoda attraverso l'Albania, il Montenegro e la Bosnia ed Erzegovina, fino a raggiungere la Croazia e da qui la Slovenia e l'Italia, con gli emigranti che cercano di superare le misure di sicurezza esistenti lungo il percorso principale attraverso la Serbia (Frontex 2018a).

La tendenza è confermata dall'UNHCR e dall'Organizzazione Internazionale delle migrazioni (OIM) che hanno registrato come nel 2018 un maggior numero di rifugiati ed emigranti in viaggio attraverso i Balcani, principalmente dalla Grecia e dalla Bulgaria, ha cercato di entrare in altri Stati membri dell'UE passando attraverso la Bosnia ed Erzegovina, viaggiando verso nord dalla Grecia attraversando l'Albania e il Montenegro (UNHCR 2019; OIM 2018).

Ad agosto 2018, il Ministero dell'Interno ha dichiarato di aver identificato sul confine orientale italiano

oltre 1.000 (1.144) immigrati irregolari provenienti dalla frontiera terrestre rintracciati dalla Polizia di frontiera al lavoro nelle province di Trieste e Gorizia. Sono i numeri che rappresentano una parte [...] di lotta all'immigrazione irregolare svolto nei primi mesi dell'anno dalle questure delle due province del Friuli-Venezia Giulia. A questa attività si aggiunge il potenziamento dei controlli ai confini con la rotta migratoria balcanica, a fronte dell'aumento di migranti rilevato dalla Polizia di frontiera della IV zona di Udine negli ultimi 3 mesi dell'anno, al quale si è accompagnato, in Slovenia, l'incremento di ingressi illegali dalla Bosnia attraverso il confine croato. (Ministero dell'Interno 2018)

1 Se non diversamente indicato, i brani tradotti dall'inglese all'italiano sono a cura dell'Autore. 
Nel report annuale per il Settore Polizia di Frontiera sulle attività di controllo del territorio della Questura di Trieste del 2018, si registrano i rintracci di 1.492 emigranti di cui 300 immediatamente riammessi nella Repubblica di Slovenia. ${ }^{2}$

L'attenzione sulla rotta balcanica sembra essere ancora molto alta nonostante il ridotto numero di arrivi, con un impiego di risorse economiche per il controllo dei confini esterni dell'UE sempre più elevato, come attestato dai finanziamenti riconosciuti alla Croazia dalla Commissione Europea e dalla sorveglianza aerea predisposta da Frontex sulle frontiere esterne dei Balcani occidentali (Frontex 2018b).

A maggio 2018, nel vertice UE-Balcani a Sofia, si è discusso di rafforzamento della cooperazione tra Frontex e gli Stati della regione, di controllo di confini e misure di contrasto all'immigrazione irregolare, ma soprattutto di stanziamenti economici ingenti da destinare a un piano integrato di gestione delle frontiere.

Nelle conclusioni del 13 e 14 dicembre 2018, il Consiglio Europeo, dopo aver constatato che «il numero di attraversamenti illegali delle frontiere è stato ridotto» e che «tale andamento discendente persiste», riconosce che "ciò è dovuto alla politica migratoria esterna dell'Unione e dei suoi Stati membri, che si fonda in particolare sul controllo delle frontiere esterne, la lotta contro i trafficanti e la cooperazione con i paesi di origine e di transito» (Consiglio Europeo 2018).

La contraddizione più evidente è che, nonostante si rivendichi un minor numero di attraversamenti, il tema dell'immigrazione continua a essere affrontato a livello europeo solo come una questione di sicurezza, con ingenti investimenti economici per il controllo della Fortezza Europa (Akkerman 2018).

\subsection{Le associazioni di monitoraggio e tutela}

Ciò che non appare negli incontri e nei report ufficiali è che con la chiusura delle frontiere balcaniche sono nettamente peggiorate le condizioni degli emigranti che provano ad attraversarle.

Al numero di presenze decisamente minimo rispetto a quello del 2015-16 è corrisposto un aumento esponenziale di violenze brutali e sproporzionate alle frontiere dell'UE e una sempre maggiore difficoltà ad accedere alla protezione internazionale.

Il rapporto della FRA (Agenzia Europea dei Diritti Fondamentali) riporta che:

2 «Un 2018 all'insegna del contrasto all'immigrazione illegale», 8 gennaio 2019 URL http://questure.poliziadistato.it/it/Trieste/articolo/13185c3457a3dc ac3993942690 (2019-10-29). 
lungo la rotta dei Balcani occidentali, le accuse di maltrattamento di migranti ad opera della polizia sono aumentate [...] gli sforzi profusi per affrontare in modo più efficace il problema dell'immigrazione illegale ha aggravato i preesistenti rischi di violazione dei diritti fondamentali [...]. Alcune delle violazioni più gravi consistono nel maltrattamento dei migranti che varcano i confini aggirando i controlli alle frontiere. (FRA 2018)

Nei primi dieci mesi del 2018, Amnesty International denuncia la morte di almeno 12 persone nei Balcani occidentali, la maggior parte annegati nel tentativo di attraversare la frontiera tra la Croazia e la Slovenia, e ha rilevato che espulsioni collettive, a volte accompagnate da violenza, si sono verificate regolarmente al confine tra Croazia e Bosnia ed Erzegovina (Amnesty International 2019).

Molte organizzazioni che si occupano da tempo del monitoraggio dei confini sud-orientali dell'Europa confermano una situazione drammatica. Da maggio 2017, le organizzazioni No Name Kitchen, Rigardu, Balkan Info Van e SOS Velika Kladusa hanno raccolto testimonianze di immigrati vittime di respingimenti e di violenze da parte delle autorità di frontiera europee, hanno registrato «un aumento delle pratiche violente che rende il transito legale e sicuro verso l'Europa impossibile» e dichiarano di aver «ricevuto sistematiche segnalazioni da parte di uomini, donne e bambini, di abusi che rimangono spesso nascosti o che vengono negati, e che non portano a nessuna azione penale contro i responsabili se non al proseguimento della violenza alla frontiera» (Balkan Info, No Name Kitchen, Sos Team Kladusa 2018).

A fine dicembre 2018, Save the Children afferma che quasi un terzo dei casi seguiti

ha riguardato violenze inflitte dalla polizia o dalle guardie di frontiera. I casi di violenza sono stati segnalati in particolare dai bambini che viaggiano da soli o separati dalle loro famiglie - in quasi la metà degli oltre 900 casi, i bambini soli hanno testimoniato di guardie di frontiera che hanno usato la forza per respingerli. (Save the Children 2018)

\section{Oxfam International denuncia}

abusi perpetrati dalle autorità statali e incapacità dei Paesi europei di tutelare i diritti umani. Anziché offrire un riparo sicuro, i Paesi lungo la rotta balcanica occidentale hanno negato protezione e le dovute procedure a molti dei nuovi arrivati, rimandandoli indietro verso i Paesi di provenienza o di transito o persino verso Paesi terzi senza offrire loro l'opportunità di chiedere asilo. (Oxfam International 2017) 


\section{Controlli dei movimenti migratori. La giurisprudenza della Corte EDU e la tutela assoluta dell'art. 3 CEDU}

Appare evidente che l'approccio globale europeo a un fenomeno estremamente complesso, come quello migratorio, è essenzialmente volto a garantire il controllo dei confini, senza alcun bilanciamento tra il rispetto dei diritti umani fondamentali e il diritto degli Stati ad affermare e garantire la propria sovranità, tra il diritto dello Stato a controllare le frontiere e il diritto alla libertà e sicurezza personale dell'immigrato (Parisi, Rinoldi 2012; Tria 2014; Centonze, Anastasio 2019; Ciliberto, Roşu 2019).

Da un punto di vista giuridico questo difficile equilibrio fa sorgere in particolare alcune domande: tutte le misure sono legittime per garantire il controllo dell'accesso al territorio? Di quali diritti gode chi è fermato alla frontiera o intercettato in condizione di irregolarità?

La Corte EDU (Corte europea dei diritti dell'uomo) in più occasioni è stata interessata della questione e le politiche sempre più restrittive in materia di immigrazione e asilo adottate a livello europeo e dai singoli Stati sono tra le altre lo sfondo alla sentenza S.F. $e$ altri c. Bulgaria del 7 dicembre 2017 e alla sentenza Ilias e Ahmed c. Ungheria del 14 marzo 2017, che nella presente trattazione si pongono come base di riflessione sulla rilevanza e la portata degli obblighi convenzionali nell'ambito della questione migratoria, che da anni interessa la rotta balcanica.

La sentenza S.F. e altri c. Bulgaria trae origine dal ricorso per violazione dell'art. 3 CEDU (Convenzione europea per la salvaguardia dei diritti dell'uomo e delle libertà fondamentali) di cinque cittadini iracheni, di cui tre minori, che dopo essere entrati irregolarmente in Bulgaria venivano trattenuti, per circa due giorni, nel centro di detenzione della polizia frontaliera a Vidin. Durante il trattenimento i ricorrenti erano costretti a rimanere in una struttura fatiscente e sporca, non adatta per ospitare minori, in condizioni non dignitose, senza la possibilità di accedere ai servizi igienici e privati di cibo e di acqua per almeno ventiquattro ore dopo il loro arrivo.

Nella sentenza Ilias Ilias e Ali Ahmed c. Ungheria la Corte EDU è chiamata a pronunciarsi sul ricorso per la violazione degli articoli 5 , 3, 13 della Convenzione da parte del governo ungherese. I ricorrenti, due cittadini bengalesi che, transitando per il Pakistan, l'Iran e la Turchia, erano entrati nel territorio dell'Unione europea attraverso la Grecia e da qui in Serbia, erano arrivati, infine, nella zona di transito di Röszke, al confine tra Ungheria e Serbia. Lo stesso giorno del loro arrivo avevano presentato domanda di protezione internazionale alle autorità ungheresi e da quel momento non avevano più potuto lasciare la zona di transito. I richiedenti, entrambi analfabeti, venivano sentiti dall'Autorità per l'asilo, che rigettava le domande dei ricorrenti, dichiarandole inammissibili sul fondamento della Serbia come «paese 
terzo sicuro». I richiedenti avevano potuto impugnare la decisione, ma nonostante diversi ricorsi venivano espulsi dal territorio ungherese.

Con queste due sentenze la Corte EDU afferma un principio fondamentale in tema di immigrazione: le difficoltà incontrate dagli Stati membri nel fronteggiare i movimenti migratori non possono esimerli dall'obbligo di tutelare i diritti umani fondamentali e in particolare dal rispetto dell'art. 3 CEDU, stante il carattere assoluto accordato dalla Convenzione al diritto di non essere sottoposti a tortura, trattamenti inumani o degradanti, a cui corrisponde l'obbligo da parte degli Stati di garantire e promuovere condizioni compatibili con il rispetto della dignità umana.

L'art. 3 CEDU, infatti, rientra in quel ristretto novero di disposizioni per le quali l'art. 15 della Convenzione vieta ogni deroga persino «in caso di guerra o di altro pericolo pubblico che minacci la vita della nazione».

Nessun tipo di eccezione può essere prevista, tanto che il divieto opera anche nel caso di contrasto al terrorismo o alla criminalità organizzata; principio affermato già a partire dalla sentenza Chahal c. Regno Unito del 15 novembre 1996, nella quale la Corte si è pronunciata contro l'espulsione di un cittadino straniero per il pericolo di essere sottoposto a tortura, escludendo ogni bilanciamento di diritto anche in caso di pericolo per lo Stato per il sospetto di appartenenza dello stesso ricorrente a un'organizzazione terroristica (Masera 2016).

Nella sentenza Ilias e Ahmed c. Ungheria la Corte EDU riconosce che

The Court has recently summarised the general principles applicable to the treatment of migrants in detention in the judgment of Khlaifia and Others v. Italy. The Court has already had occasion to note that the States which form the external borders of the European Union are currently experiencing considerable difficulties in coping with the increasing influx of migrants and asylum-seekers. That being said, the Court can only reiterate its well-established case-law to the effect that, having regard to the absolute character of Article 3, an increasing influx of migrants cannot absolve a State of its obligations under that provision, which requires that persons deprived of their liberty must be guaranteed conditions that are compatible with respect for their human dignity.

\section{Principio ribadito nella successiva sentenza S.F. c. Bulgaria:}

In any event, in view of the absolute character of Article 3 of the Convention, an increasing influx of migrants cannot absolve a High Contracting State of its obligations under that provision, which requires that people deprived of their liberty be guaranteed conditions compatible with respect for their human dignity. 
Se gli Stati, come ricorda la Corte EDU nella citata sentenza Khlaifia e altri c. Italia del 2015 (Nucera 2017; Perrini 2015), sono autorizzati a limitare la libertà degli immigrati in virtù del loro «diritto inalienabile di controllare l'ingresso e il soggiorno degli stranieri sul proprio territorio (caso Amuur c. Francia, 25 giugno 1996)», devono però esercitare questo diritto «conformemente alle disposizioni della Convenzione europea per la salvaguardia dei diritti dell'uomo e delle libertà fondamentali (Mahdid e Haddar c. Austria nr. 74762/01, 8 dicembre 2005)», in particolare nel rispetto dell'art. 3 CEDU, unanimemente riconosciuto come principio fondamentale delle società democratiche, affermato nelle più recenti Carte sui diritti umani, come la Carta di Nizza, la quale ha adottato la stessa definizione della norma nell'art. 4 . $^{3}$

La sentenza Bouyid c. Belgio del 28 settembre 2015, arriva a riconoscere che «l'articolo 3 della Convenzione sancisce uno dei valori fondamentali delle società democratiche. Infatti, la proibizione della tortura e delle pene o trattamenti inumani o degradanti è un valore di civiltà strettamente legato al rispetto della dignità umana».

\subsection{Proibizione della tortura e il «nessuno» da tutelare}

Affermata la tutela assoluta garantita dall'art. 3 CEDU e riscontrato un notevole peggioramento delle condizioni degli emigranti lungo la rotta balcanica, si può giuridicamente sostenere che, nell'attuazione della politica europea di controllo dei movimenti migratori e di protezione delle frontiere, gli Stati siano responsabili di una continua violazione di diritti umani fondamentali e in particolare del divieto di trattamenti inumani o degradanti?

L'essenzialità della norma convenzionale rende necessario analizzare i fatti ponendo attenzione alle peculiarità dei casi e, in particolare, soffermarsi sugli elementi oggettivi e soggettivi richiesti dalla fattispecie (Danisi, non pubbl.; De Gaetano 2016; Panella 2018; Lobba 2017; Pacini 2011).

Lungo la rotta balcanica, come riportato dai dati di Frontex, sono state registrate persone di varie nazionalità (pakistana, afghana, iraniana), mentre le fonti non governative fanno riferimento genericamente a donne, uomini, minori non accompagnati stranieri.

La Convenzione EDU non prevede una tutela specifica dell'immigrato, ma l'assenza di una disciplina puntuale non implica il mancato

3 Il divieto di tortura è altresì previsto dalla Dichiarazione universale dei diritti umani del 1948, dalla Convenzione di Ginevra relativa al trattamento dei prigionieri di guerra del 1949, dalla Convenzione internazionale sui diritti civili e politici del 1966, dalla Convenzione europea per la prevenzione della tortura e delle pene o trattamenti inumani o degradanti del 1987, dallo Statuto di Roma istitutivo della Corte penale internazionale del 1998 e dalla Carta dei diritti fondamentali dell'Unione europea del 2000. 
riconoscimento di diritti, tanto che l'interpretazione giurisprudenziale ne ha sempre garantito un'adeguata protezione sotto diversi profili. ${ }^{4}$

Nell'ipotesi in esame, i soggetti indicati sono pacificamente riconosciuti come titolari del diritto a non essere sottoposti a maltrattamenti e tortura, stante la genericità del soggetto passivo previsto dall'art. 3 come «nessuno», definizione volta a garantire tutela a ogni persona, a prescindere da fattori quali la cittadinanza o lo status del soggetto.

Elementi particolari, come quelli che si possono riscontrare negli emigranti lungo la rotta balcanica, quali ad esempio l'età, il sesso e la condizione di salute hanno rilievo per affermare la necessità di una maggiore tutela.

Nella sentenza S.F. e altri c. Bulgaria, la Corte EDU presta particolare attenzione alla presenza di minori di età e alla necessità di tenere conto dei loro bisogni specifici, sottolineando che la valutazione circa le condizioni di trattamento debba essere particolarmente stringente alla luce della loro condizione di fragilità, potendo avere la condotta posta in essere un impatto maggiore soprattutto in termini psicologici. ${ }^{5}$

Nell'ipotesi in cui il soggetto passivo sia un richiedente protezione internazionale, come lungo la rotta balcanica, la Corte EDU ha evidenziato, altresì, al fine della determinazione della conformità delle condizioni all'art. 3 CEDU, una vulnerabilità particolare connaturata allo specifico status giuridico. ${ }^{6}$

Nella sentenza M.S.S c. Grecia e Belgio, resa il 22 gennaio 2011, la Corte EDU ha espressamente riconosciuto che se il soggetto passivo è un rifugiato politico «is a member of a particularly underprivileged and vulnerable population group in need of special protection».

\subsection{Le condotte vietate dall'art. 3 CEDU}

In relazione all'elemento oggettivo richiesto, l'art. 3 della Convenzione EDU ha riconosciuto il diritto di ciascun individuo a non subire una violazione dell'integrità fisica e psichica a causa di tortura 0

4 Si riferiscono agli stranieri l'art. 16 CEDU e l'art. 1 Protocollo nr. 7. Diretto specificatamente a tutti gli stranieri, indipendentemente dalla regolarità della loro presenza sul territorio, come norma a tutela esclusiva dei loro diritti, è l'art. 4 del Protocollo 4 della CEDU, che vieta le espulsioni collettive.

5 Principio affermato in numerose sentenze della CEDU, tra le ultime Corte EDU, Sentenza Khan c. Francia, ricorso nr. 12267/16, 28 febbraio 2019. Tutte le sentenze sono reperbili in: https://hudoc.echr.coe.int (2019-11-29).

6 Corte EDU, Khailifa c. Italia, ricorso nr. 16483/12, 15 dicembre 2016; Corte EDU, MSS c. Grecia e Belgio, ricorso nr. 30696/09, 21 gennaio 2011. 
trattamento o pena disumana o degradante. ${ }^{7}$ Tuttavia le associazioni che stanno monitorando la rotta balcanica ci riportano pratiche brutali e manifeste violazioni dei diritti umani da parte delle forze di polizia di Paesi membri dell’UE. Gli operatori di Medici Senza Frontiere segnalano un aumento di pazienti con traumi legati alle violenze subite e in particolare che «nei primi 6 mesi del 2017, il 69\% (86) dei pazienti seguiti da MSF riportano di aver subito violenza diretta. La maggioranza 57\% (71) presentavano lesioni fisiche visibili, compresi i tagli con lame di rasoio e coltelli, percosse severe, privazione di cibo e acqua, privazione sensoriale» (Medici Senza Frontiere 2017).

Human Rights Watch denuncia che in Croazia, Stato dell'UE, firmataria della Convenzione EDU:

la polizia croata picchia ferocemente i richiedenti asilo e li respinge oltre il confine. Secondo le testimonianze dei bambini, alcune guardie di frontiera ai diversi confini della regione dei Balcani occidentali hanno usato spray al pepe su di loro, hanno preso i loro telefoni e li hanno rotti, rubato loro i soldi, li hanno costretti a togliere vestiti e scarpe. Bambini e famiglie hanno riferito di essere, in alcuni casi, tenuti in detenzione senza cibo o acqua. (Human Rights Watch 2018)

Le stesse violazioni vengono riportate da Save the Children:

I respingimenti avvengono in molti modi diversi. Ungheria e Croazia, entrambi Paesi membri UE, hanno usato metodi brutali, per esempio impiegando i cani da attacco e obbligando le persone a spogliarsi completamente pur con temperature glaciali. Brutalità, intimidazione e tattiche subdole da parte delle autorità generano inoltre un clima di paura e diffidenza tra le persone in transito. (Save the Children 2018)

Le associazioni di monitoraggio riferiscono di effetti personali requisiti o dati alle fiamme, violenze fisiche e psicologiche, maltrattamenti, pestaggi (Balkan Info, No Name Kitchen, Sos Team Kladusa 2018).

Sono alcune delle condotte della polizia e delle autorità di frontiera denunciate dagli emigranti in transito attraverso i Paesi balcanici. Si tratta di atti illeciti e condannabili, ma a livello giuridico si può affermare che le condotte poste in essere integrino una violazione dell'art. 3 CEDU?

7 L'estrema sinteticità e genericità della definizione emerge già dai lavori preparatori della Convenzione EDU, dovuta alla necessità di evitare qualsiasi elencazione di condotte per non lasciare prive di tutela alcune situazioni. Questo ha determinato la continua evoluzione interpretativa e giurisprudenziale della Corte EDU (Consiglio d'Europa 1970). 
Sin dalle prime pronunce giurisprudenziali, elemento centrale della previsione convenzionale è considerato il trattamento disumano, che rappresenta la fattispecie principale per valutare se è possibile ricomprendere un caso concreto nella disposizione normativa; per chiarire poi a quale tipologia vietata dalla Convezione sia ascrivibile la condotta nel caso specifico è necessario rilevare la diversa intensità dell'azione e la severità dei maltrattamenti, partendo dal presupposto che la tortura è un trattamento inumano e degradante e il trattamento inumano è necessariamente degradante. ${ }^{8}$

La giurisprudenza europea più recente può essere di supporto per comprendere meglio se i casi denunciati possano essere ricondotti all'art. 3 CEDU.

Nella sentenza Bouyid c. Belgio del 28 settembre 2015, la Corte EDU ha condannato il Belgio per violazione dell'art. 3 CEDU, ritenendo che gli schiaffi inferti dalla polizia a due persone (tra cui uno minore di età) trattenute in un commissariato e, in generale, il ricorso all'uso della forza da parte delle autorità, che non si renda strettamente necessario, possano integrare un trattamento degradante se le condotte vengono percepite come lesive della dignità personale perché poste in essere in condizioni di arbitrarietà e d'impotenza del soggetto passivo.

La Corte EDU nel caso Cestaro c. Italia del 7 aprile 2015, riconosce come tortura le condotte gravi, crudeli e intenzionali ai quali fu sottoposto il ricorrente, che gli provocarono dolori e acute sofferenze, senza alcun nesso tra il suo comportamento e la reazione della polizia.

Con sentenza Bartesaghi Gallo e altri c. Italia del 22 giugno 2017 e con la sentenza Blair e altri c. Italia del 26 ottobre 2017, la Corte ha riconosciuto come violazione dell'art. 3 della CEDU gli atti di violenza subiti in un contesto generale di impiego eccessivo, indiscriminato e manifestamente sproporzionato della forza e che può considerarsi come violazione dell'art. 3 l'essere «testimoni impotenti dell'uso incontrollato della violenza nei confronti delle altre persone» e che alle «offese all'integrità fisica e psicologica individuale si è aggiunto lo stato di angoscia e di stress causato dagli episodi di violenze alle quali hanno assistito».

La Corte EDU ricomprende pacificamente tra le condotte vietate quelle che suscitano sentimenti di paura, di inferiorità e che provocano un'umiliazione della vittima. La valutazione deve basarsi sulle circostanze del singolo caso e sul contesto nel quale si verifica, avendo le autorità statali l'obbligo di vigilare in modo partico-

8 European Commission of Human Rights, Danimarca c. Grecia, ricorso nr. 3321/67, Norvegia c. Grecia, ricorso nr. 3322/67, Svezia c. Grecia, ricorso nr. 3323/67 Olanda c. Grecia, ricorso nr. 3344/67, 5 novembre 1969. URL https://www.echr.coe.int/Documents/Denmark_Norway_Sweden_Netherlands_v_Greece_I.pdf. Corte EDU, Irlanda c. Regno Unito, ricorso $\mathrm{nr}$. 5310/71, 18 gennaio 1978. 
larmente attento al fine di prevenire la violazione convenzionale, assicurando che a ogni persona sotto la sua giurisdizione sia garantita l'integrità fisica e psicologica e condizioni rispettose della dignità umana.

Sulla base dei report internazionali e della giurisprudenza europea, si potrebbe sostenere che quanto accade lungo la rotta balcanica possa rappresentare una violazione dell'art. 3 CEDU, dato che le condotte violente commesse, per la loro severità e per la capacità di provocare sofferenze fisiche e psichiche, possono qualificarsi come trattamenti inumani e degradanti e nei casi più estremi, per il carattere particolarmente grave, come tortura.

\subsection{Responsabilità degli Stati: obblighi positivi e negativi derivanti dal divieto di tortura e trattamenti disumani o degradanti}

L'art. 1 della CEDU stabilisce che ogni persona soggetta alla giurisdizione di uno Stato membro gode dei diritti sanciti nella Convenzione; corollario è la responsabilità dello Stato per gli atti lesivi compiuti dai suoi organi sul proprio territorio.

Il rapporto dell'Agenzia Europea dei Diritti Fondamentali evidenzia come «le segnalazioni di comportamenti abusivi sono aumentate notevolmente nel 2017 in particolare lungo la rotta dei Balcani occidentali» e si registra «violenza da parte della polizia o delle guardie di frontiera» (FRA 2018).

A settembre 2018, Dunja Mijatović, Commissaria per i Diritti Umani del Consiglio d'Europa, in una lettera indirizzata al primo ministro croato mostra preoccupazione per le «espulsioni collettive dalla Croazia verso la Serbia e la Bosnia ed Erzegovina di immigrati irregolari, inclusi potenziali richiedenti asilo» e soprattutto per la violenza delle forze dell'ordine anche «contro donne incinte e minori» (Mijatović 2018). Nelle testimonianze raccolte dalle associazioni non governative gli autori dei maltrattamenti vengono indicati come agenti di polizia e autorità di frontiera.

Medici Senza Frontiere riporta che:

migranti e richiedenti asilo che cercano di attraversare i confini settentrionali della Serbia hanno ripetutamente denunciato le violenze da parte delle guardie di frontiera. Nei primi sei mesi del 2017 le cliniche mobili di MSF hanno trattato 24 casi di danni intenzionali che si sono verificati lungo il confine tra Serbia e Croazia. (Medici Senza Frontiere 2018)

La stragrande maggioranza dei ragazzi curati da MSF indicano le autorità statali come perpetratori (76\%), di cui la maggioran- 
za (92\%) sono stati le forze di frontiera dell'UE: Bulgaria 48\% (30), Ungheria 27\% (17) e Croazia 13\% (8). (Medici Senza Frontiere 2018)

Amnesty International denuncia che «quasi un terzo delle persone incontrate ha subito violenze da parte della polizia croata. Molte di esse hanno raccontato di essere state picchiate e derubate dagli agenti croati» (Amnesty International 2019).

La giurisprudenza europea più recente afferma che non ha alcuna giustificazione il maltrattamento subito da un individuo che si trovi sotto il controllo dello Stato, che ha un dovere di protezione e un obbligo di istruire il proprio personale affinché nessuno sia sottoposto a tortura o ad altro trattamento degradante. ${ }^{9}$ Ha introdotto, inoltre, una presunzione di responsabilità dello Stato per le violazioni previste dall'art. 3 CEDU nell'ipotesi di detenzione e di limitazione della libertà personale, estendendo la tutela nel caso di trattenimento dello straniero nel corso del procedimento di espulsione o di asilo. ${ }^{10}$

La Corte EDU, con una giurisprudenza ormai consolidata, riconoscendo le difficoltà per un soggetto sottoposto alla restrizione della propria libertà di provare i maltrattamenti subiti, afferma che in casi simili spetta allo Stato fornire una giustificazione per le lesioni riportate da chi si trovi sotto la sua giurisdizione. Significativa è la sentenza Pennino c. Italia del 12 ottobre 2017,,11 nella quale la Corte EDU ha ravvisato una responsabilità dello Stato per la violazione dell'art. 3 della Convenzione perché le lesioni all'integrità fisica sono avvenute a danno di un soggetto sottoposto a controllo da parte di agenti statali, ribadendo quanto già affermato nella sentenza Zolotorev $c$. Russia del 19 settembre 2017, nella quale la Corte ha precisato che:

per ciò che concerne la gravità dei maltrattamenti la Corte considera l'intensità degli atti, il fatto che i maltrattamenti subiti sono stati inflitti da un agente nell'esercizio delle sue funzioni e sotto il suo controllo. ${ }^{12}$

9 Corte EDU, Sentenza Davydov e altri c. Ucraina, ricorsi nrr. 17674/02 e 39081/02, 1 luglio 2010.

10 Corte EDU, Sentenza S.D. c. Grecia, ricorso nr. 53541/07, 11 giugno 2009; Corte EDU, Sentenza Abdolkhani e Karimnia c. Turchia, ricorso nr. 30471/08, 22 settembre 2009; Corte EDU, C.D. e altri c. Grecia, ricorsi nnr. 33441/10, 33468/10 e 33476/10, 19 dicembre 2013; Corte EDU, Sentenza F.H. c. Grecia, ricorso nr. 78456/11, 31 luglio 2014; Corte EDU, Sentenza Rahimi c. Grecia, ricorso nr. 8687/08, 5 aprile 2011; Corte EDU, Sentenza Khlaifia e altri c. Italia, ricorso nr. 16483/12, 15 dicembre 2016.

11 Corte EDU, Sentenza Pennino c. Italia, ricorso nr. 21759/15, 12 ottobre 2017.

12 Corte EDU, Sentenza Zolotorev c. Russia, ricorso nr. 13408/07, 19 settembre 2017. 
L'art. 3 della Convenzione prevede per gli Stati aderenti non solo un obbligo negativo, il divieto di infliggere agli individui un trattamento disumano o degradante, ma impone anche una serie di obblighi positivi per garantire un'effettiva prevenzione delle violazioni. ${ }^{13}$

Nella sentenza Saadi c. Italia del 28 febbraio 2008, la Corte ha condannato l'Italia per non aver correttamente valutato il rischio di esporre il ricorrente a tortura nel Paese di destinazione, responsabilità che la giurisprudenza successiva ha esteso all'ipotesi in cui l'espulsione sia verso un Paese intermedio, avendo lo Stato conoscenza che il luogo di destinazione finale della persona allontanata non è il primo Paese di rinvio, ma uno successivo e che lì il rischio di condotte lesive dell'integrità e dignità della persona sia reale e attuale..$^{14}$

Ma vi è di più. Nella sentenza M.S.S. c. Grecia e Belgio del 21 gennaio 2011, la Corte EDU ha condannato la Grecia e il Belgio per la violazione degli artt. 3 e 13 CEDU: e, in particolare, la Grecia, per aver trattenuto il ricorrente, un rifugiato afghano, in condizioni degradanti durante il procedimento di asilo e per averlo lasciato successivamente in condizioni altrettanto degradanti; e il Belgio, per aver violato il principio di non-refoulement, trasferendo il ricorrente in Grecia in applicazione del Regolamento Dublino, pur essendo a conoscenza del rischio che egli avrebbe potuto subire gravi violazioni dei diritti umani. La Corte EDU ha riconosciuto una violazione dell'art. 3, sulla base di gravi «carenze sistemiche» nella gestione e nella procedura di asilo nel Paese di primo ingresso, dichiarando che il trasferimento del richiedente protezione verso il Paese di destinazione avrebbe potuto esporlo a trattamenti inumani e degradanti, mettendo apertamente in discussione la presunzione che gli Stati membri dell'UE non possano essere responsabili di violazione dei diritti fondamentali, affermazione poi confermata in altre pronunce. ${ }^{15}$

L'applicazione erronea del principio di «paese terzo sicuro» e il rischio di respingimento a catena è alla base della violazione dell'art. 3 CEDU riconosciuta nella sentenza Ilias e Ahmed c. Ungheria. La Corte riconosce, infatti, nel caso specifico, che l'espulsione verso la Serbia espone i ricorrenti al rischio di refoulement a catena, poiché «le autorità ungheresi non hanno cercato di garantire che i richiedenti, che sono stati rimpatriati in Serbia, non siano successivamente

13 Principio affermatosi sin da Corte EDU, Sentenza Soering c. Regno Unito, ricorso nr. 14038/88, 7 luglio 1989. Nel 2015 il Consiglio d'Europa ha emanato alcune linee guida in materia di immigrazione per rendere ancora più evidenti gli obblighi gravanti sugli Stati membri (Consiglio d'Europa 2015).

14 Corte EDU, Sentenza Saadi c. Italia, ricorso nr. 37201/06, 28 febbraio 2008; Corte EDU, Sentenza Abdolkhani e Karimnia c. Turchia, ricorso nr. 30471/08, 22 settembre 2009

15 Corte EDU, Sentenza Sharifi c. Italia e Grecia, ricorso nr. 16643/09, 21 ottobre 2014; Corte EDU, Sentenza Tarakhel c. Svizzera, ricorso nr. 29217/12, 4 novembre 2014 
espulsi in Grecia, in particolare a causa delle carenze procedurali e del bassissimo tasso di riconoscimento nell'ex Repubblica Jugoslava di Macedonia».

La circostanza che i ricorrenti non abbiano beneficiato di garanzie efficaci che li avrebbero protetti dall'esposizione ad un rischio reale di essere sottoposti a trattamenti inumani o degradanti contrari all'art. 3 della Convenzione determina una violazione di tale disposizione.

Nonostante le pronunce della Corte EDU riconoscano il principio di non-refoulement come ricompreso nella più ampia tutela dell'art. 3 CEDU (Zorzi Giustiniani 2018), lungo la rotta balcanica si continuano a registrare respingimenti illegittimi. A dicembre 2018, Save the Children fa riferimento a:

più di 1.350 casi di bambini respinti oltre i confini dell'Unione europea tra gennaio e novembre di quest'anno. La maggior parte dei respingimenti segnalati si sono verificati al confine tra Croazia e Serbia, ma i bambini incontrati quest'anno in Serbia hanno segnalato anche respingimenti dai confini di Bulgaria, Macedonia (FYROM), Grecia, Ungheria, Romania, Bosnia ed Erzegovina (BiH) e Slovenia. (Save the Children 2018)

Le associazioni Balkan Info Van, No Name Kitchen e Sos Team Kladusa confermano continue espulsioni sommarie di emigranti ai confini dell'Unione europea, impossibilità di accesso alle procedure di asilo e respingimenti verso i campi temporanei della Serbia e della Bosnia. Tra Bosnia, Croazia e Slovenia, infatti, nel solo mese di dicembre 2018 sono stati documentati 23 respingimenti collettivi vicino a Velika Kladusa. Alcuni hanno dichiarato di essere stati respinti dalla Slovenia e successivamente dalla Croazia fino in Bosnia (Balkan Info, No Name Kitchen, Sos Team Kladusa 2018).

Il report di Amnesty International fa emergere che un numero significativo di persone (67 su 94 intervistate) attualmente bloccate in Bosnia ed Erzegovina sono state vittime di respingimento in particolare dalla Slovenia, ma anche, in alcuni casi, dall'Italia (Amnesty International 2019).

Dalla lettura congiunta degli artt. 1 e 3 CEDU e dai fatti denunciati dalle associazioni di tutela consegue la responsabilità degli Stati europei nei quali le violazioni si verificano, sia perché autori delle condotte sono diretta emanazione dello Stato, sia perché lo Stato non pone in essere provvedimenti a tutela degli immigrati. Chiamati a rispondere, altresì, di violazione dell'art. 3 della Convenzione sono gli Stati che attuano respingimenti ed espulsioni, esponendo il soggetto passivo al pericolo di lesione del diritto. L'aver sottoscritto la Convenzione EDU, infatti, comporta per lo Stato non solo l'obbligo di astenersi dalle condotte vietate, di adottare provvedimenti legislati- 
vi, amministrativi e giudiziari efficaci per impedire che trattamenti inumani e degradanti siano compiuti sotto la propria giurisdizione e svolgere indagini con urgenza nei confronti dei responsabili, ma anche un dovere di controllo preventivo sul rispetto dei diritti umani da parte di un altro Stato, che si concretizza nell'obbligo di valutare la possibile violazione di un diritto.

\section{Conclusioni}

L'approccio sicuritario delle istituzioni europee e degli Stati membri in materia di immigrazione non è recente, ma negli ultimi anni è decisamente prevalso rispetto a un piano di accoglienza e di integrazione.

Con l'intensificarsi dei movimenti migratori, obiettivo primario è diventato quello di controllare i confini e respingere gli emigranti sulla base di una presunta emergenza che ha portato all'adozione di misure come l'accordo tra UE e Turchia, volto principalmente a esternalizzare il controllo delle frontiere e la gestione dell'immigrazione.

I tentativi dell'UE e degli Stati Membri di chiudere la rotta balcanica e la mancata previsione di canali di ingresso legale stanno determinando un'aperta violazione dei diritti umani.

Emigranti e richiedenti asilo vengono respinti ai confini dell'Europa, senza che venga loro data la possibilità di chiedere protezione e di impugnare la decisione di respingimento. Le condizioni in cui si trovano, le violenze subite e i respingimenti illegali integrano una violazione dell'art. 3 CEDU, configurandosi come trattamenti inumani e degradanti e nei casi più gravi come tortura.

Quanto sta accadendo lungo la rotta balcanica non può essere imputato solo agli Stati di confine; tutt'altro che irrilevanti sono le responsabilità europee e dei singoli Stati che respingono a catena gli immigrati fino alle frontiere esterne, fornendo un contributo causale alla violazione dei diritti fondamentali, ignorando report internazionali, trincerandosi dietro al diritto di controllare l'ingresso e il soggiorno nel proprio territorio, senza alcun bilanciamento col rispetto degli obblighi internazionali in materia di tutela dei diritti fondamentali.

Appare evidente che l'unica emergenza alle frontiere dell'Unione europea è quella relativa alla tutela dei diritti umani e che per risolverla è necessario un cambiamento radicale delle politiche europee in materia di immigrazione, potendo solo così far fronte alla drammatica situazione ai confini dell'Unione europea, dove si sta perpetrando la violazione dei diritti fondamentali di migliaia di invisibili «nessuno». 


\section{Bibliografia}

Amnesty International (2019). Pushed to the Edge. Violence and Abuse Against Refugees and Migrants Along the Balkans Route. URL https://www.amnesty.org/download/Documents/EUR0599642019ENGLISH.PDF (2019-05-04).

Akkerman, Mark (2018). Espandendo la fortezza. Chi decide e specula sulle politiche di esternalizzazione delle frontiere UE. URL https://www.tni.org/ en/node/24112 (2019-04-10).

Balkan Info; No Name Kitchen; Sos Team Kladusa (2018). Illegal Pushbacks and Borderline Violence Reports. URL https://www.osservatoriodiritti.it/wp-content/uploads/2019/01/rotta-balcanica-migranti.pdf (2019-01-19).

Centonze, Salvatore; Anastasio, Sara (2019). «Protezione dei migranti: Corte Edu e Corte di giustizia a confronto», in «La Corte di Strasburgo» a cura di Francesco Buffa e Maria Giovanna Civinini, núm. spec., Questione Giustizia, 390-8. URL http://questionegiustizia.it/speciale/pdf/QG-Speciale_2019-1_57.pdf (2019-05-04).

Ciliberto, Giulia; Roşu, Andreea Maria (2019). «Asilo e protezione internazionale nella Cedu», in «La Corte di Strasburgo» a cura di Francesco Buffa e Maria Giovanna Civinini, núm. spec., Questione Giustizia, 381-9. URL http:// questionegiustizia.it/speciale/pdf/QG-Speciale_2019-1_56. pdf (2019-05-04).

Commissione Europea (2015). Comunicazione della Commissione Europea al Parlamento Europeo, al Consiglio, al Comitato Economico e Sociale Europeo e al Comitato delle Regioni. Agenda Europea sulla Migrazione. COM(2015) 240 final. URL https://eur-lex.europa.eu/legal-content/IT/TXT/PDF / ?uri=CELEX:52015DC0240\&from=GA (2019-04-13).

Consiglio d'Europa (1970). Preparatory Work of Article 3 of the European Convention on Human Rights, Memorandum Prepared by the Secretariat of the Commission. DH(56). URL https://www.echr.coe.int/Documents/Library_TravPrep_Table_ENG.pdf (2019-02-02).

Consiglio d'Europa (2015). Protection des Migrants et demandeurs d'asile: principales obligations juridiques des Etats en vertu des Conventions du Conseil de l'Europe. URL https://rm. coe.int/168046e354 (2019-05-04).

Consiglio Europeo (2016a). Osservazioni del Presidente Donald Tusk dopo la riunione dei capi di Stato o di governo dell'UE con la Turchia. URL https://www. consilium.europa.eu/it/press/press-releases/2016/०3/०7/ tusk-remarks-eu-turkey-meeting/ (2019-05-04).

Consiglio Europeo (2016b). Comunicato stampa del 18 marzo 2016. URL https:// www.consilium.europa.eu/it/press/press-releases/2016/03/18/ tusk-remarks-after-euco-turkey/ (2019-04-10).

Consiglio Europeo (2018). Conclusioni del Consiglio Europeo del 13-14 dicembre 2018. URL https://www.consilium.europa.eu/it/press/press-releases/2018/12/14/european-council-conclusions-13-14-december-2018/ (2019-04-10).

Danisi, Carmelo (non pubblicato). Divieto e definizione di tortura nella normativa internazionale dei diritti dell'uomo. URL https://www.diritto.it/ pdf_archive/28401.pdf (2019-02-02).

De Gaetano, Vincent A. (2016). L'immigrazione e la Convenzione Europea dei Diritti dell'Uomo: Breve panoramica della giurisprudenza della CEDU. 
URL http://www.cortedicassazione.it/cassazione/resources/ resources/cms/documents/Relazione_De_Gaetano1.pdf (201904-09).

FRA, European Union Agency for Fundamental Rights (2018). Relazione sui diritti fondamentali 2018. URL https://fra.europa.eu/sites/default/ files/fra_uploads/fra-2018-fundamental-rights-report2018-opinions_it.pdf (2019-01-20).

Frontex (2018a). Migratory Route. URL http://frontex.europa.eu/alongeu-borders/migratory-routes/western-balkan-route (2019-04-10).

Frontex (2018b). Frontex Strengthens Surveillance in Croatia. URL https:// frontex.europa.eu/media-centre/news-release/frontexstrengthens-surveillance-in-croatia-k3u6uv (2019-04-10).

Human Rights Watch (2018). Croatia: Migrants Pushed Back to Bosnia and Herzegovina. URL https://www.hrw.org/news/2018/12/11/croatia-migrants-pushed-back-bosnia-and-herzegovina (2019-03-26).

Lobba, Paolo (2017). «Punire la tortura in Italia. Spunti ricostruttivi a cavallo tra diritti umani e diritto penale internazionale». Diritto Penale Contemporaneo, 10, 181-250. URL https://www.penalecontemporaneo.it/ upload/7559-lobba1017.pdf (2019-05-02).

Masera, Luca (2016). «Il terrorismo e le politiche migratorie: sulle espulsioni dello straniero sospettato di terrorismo», in «Terrorismo internazionale. Politiche della sicurezza. Diritti fondamentali», nr. spec., Questione Giustizia, settembre, 76-90. URL http://questionegiustizia.it/speciale/pdf/QG-Speciale_2016-1_08.pdf (2019-02-17).

Mijatović, Dunja (2018). Lettera al Primo Ministro di Croazia. URL https:// rm.coe.int/letter-to-mr-andrej-plenkovic-prime-ministerof-croatia-concerning-the/16808d7db3 (2019-04-09).

Medici Senza Frontiere (2017). Games of Violence: Unaccompanied Children and Young People Repeatedly Abused by EU Member State Border Authorities. URL https://serbia.bordermonitoring.eu/wp-content/uploads/ sites/11/2017/11/MSF-serbia-games-of-violence-3.10.17.pdf (2019-17-02).

Medici Senza Frontiere (2018). Bosnia: respingimenti, violenze e pessime condizioni umanitarie alla nuova frontiera della rotta balcanica. URL https:// bit.ly/2CqVNPA (2019-04-03).

Ministero dell'Interno (2018). Sicurezza, i numeri delle Questure di Trieste e Gorizia nel contrasto alle attività illecite transfrontaliere. URL https://bit. ly/2pV104f (2019-03-01).

No Name Kitchen (2018). Border Violence on the Balkan Route. URL http:// www. nonamekitchen.org/wp-content/uploads/2019/01/Finished-Border-Violence-on-the-Balkan-Route.pdf (2019-01-19).

Nucera, Gianfranco Gabriele (2017). «Ancora sul caso Khlaifia e a. c. Italia. La pronuncia della Grande Camera della Corte Europea dei Diritti Umani». Ordine internazionale e diritti umani. Osservatorio l'Italia e la CEDU, 1, 119-22. URL http://www.rivistaoidu.net/sites/default/files/4_Italia\%20e\%20CEDU\%201_2017_Nucera.pdf (2019-04-14).

OIM, Organizzazione Internazionale per le Migrazioni (2018). DTM Mediterranean - Western Balkans Overview January - December 2018. URL https:// migration.iom.int/system/tdf/reports/14.\%20WB\%20Brief\%20 2018_Final.pdf?file=1\&type=node\&id=5166 (2019-04-14). 
Oxfam International (2017). A Dangerous Game. The Pushback of Migrants, Including Refugees, at Europe's Borders. URL https://bit.ly/2NQfwgQ (2019-04-10).

Pacini, Marco (2011). Il controllo della Cedu sul rispetto dei diritti umani da parte di Stati terzi. URL https://www.irpa.eu/il-controllo-della-cedu-sul-rispetto-dei-diritti-umani-da-parte-di-stati-terzi/ (2019-03-29).

Panella, Lina (2018). «La giurisprudenza della Corte Europea dei Diritti dell'Uomo e quella della Corte di Giustizia dell'Unione Europea». Ordine internazionale e diritti umani, 3, 276-300. URL http://www.rivistaoidu.net/ sites/default/files/2_Panella.pdf (2019-04-14).

Parisi, Nicoletta; Rinoldi, Dino (2012). «Confini d'Europa, Stato di diritto, diritti dell'uomo. Gerarchia e bilanciamento tra diritti fondamentali con particolare riguardo alla condizione del migrante». Zagato, Lauso; De Vido, Sara (cura di), Il divieto di tortura e altri comportamenti inumani o degradanti nelle migrazioni. Padova: CEDAM, 47-64.

Perrini, Francesca (2015). «Gravi violazioni dei diritti fondamentali dei migranti nel caso Khlaifia e a. c. Italia». Ordine internazionale e diritti umani. Osservatorio l'Italia e la CEDU, 4, 741-3. URL http://www.rivistaoidu.net/sites/default/files/3_CEDU\%20e\%20Italia4_2015.pdf(2019-04-14).

Save the Children (2018). Hundreds of Children Report Police Violence at EU Borders. URL https://www.savethechildren.net/news/hundredschildren-report-police-violence-eu-borders (2019-03-19).

Tria, Lucia (2014). Le politiche dell'Unione europea sull'immigrazione e il controllo delle frontiere. URL http://www.europeanrights.eu/public/ commenti/LUCIA_TRIA_RELAZIONE_POLITICHE_IMMIGRAZIONE.pdf (2019-04-14).

UNHCR(2019).Viaggidisperati. RifugiatiemigrantiinarrivoinEuropaeallesuefrontiere. Gennaio-Dicembre 2018. URL https://data2. unhcr.org/en/documents/download/67715\#_ga=2.243984898.642170523.1548753735126843143.1534746251 (2019-04-09).

Zorzi Giustiniani, Flavia (2018). Divieto di non-refoulement e tortura. Osservazioni in margine al "General Comment" $n$. 4 alla Convenzione ONU contro la tortura e altre pene o trattamenti crudeli, inumani o degradanti del 1984. Focus Human Rights nr. 2. URL https://bit.ly/32P3aMq (2019-03-29). 


\section{Giurisprudenza}

Corte EDU, Sentenza Abdolkhani e Karimnia c. Turchia, ricorso nr. 30471/08, 22 settembre 2009.

Corte EDU, Sentenza Bartesaghi Gallo e altri c. Italia, ricorsi nnr. 12131/13 e 43390/13, 22 giugno 2017.

Corte EDU, Sentenza Blair e altri c. Italia, ricorsi nnr. 1442/14, nr. 21319/14 e nr. $21911 /$ 14, 26 ottobre 2017.

Corte EDU, Sentenza Bouyid c. Belgio, ricorso nr. 23380/09, 28 settembre 2016.

Corte EDU, Sentenza C.D. e altri C. Grecia, ricorsi nnr. 33441/10, 33468/10 e 33476/10, 19 dicembre 2013.

Corte EDU, Sentenza Cestaro c. Italia, ricorso nr. 6884/11, 7 aprile 2015.

Corte EDU, sentenza Chahal c. Regno Unito, ricorso nr. 22414/93, 15 novembre 1996.

Corte EDU, Sentenza Davydov e altric. Ucraina, ricorsi nr. 17674/02 e 39081/02, 1 luglio 2010.

Corte EDU, Sentenza F.H. c. Grecia, ricorso nr. 78456/11, 31 luglio 2014.

Corte EDU, Sentenza Khan c. Francia, rincorso nr. 12267/16, 28 febbraio 2019.

Corte EDU, Sentenza Khlaifia e altric. Italia, ricorso nr. 16483/12, 15 dicembre 2016.

Corte EDU, Sentenza Ilias e Ahmed c. Ungheria, ricorso nr. 472871/15, 14 marzo 2017.

Corte EDU, Sentenza MSS c. Grecia e Belgio, ricorso nr. 30696/09, 21 gennaio 2011.

Corte EDU, Sentenza Pennino c. Italia, ricorso nr. 21759/15, 12 ottobre 2017.

Corte EDU, Sentenza Rahimi c. Grecia, ricorso nr. 8687/08, 5 aprile 2011.

Corte EDU, Sentenza Saadi c. Italia, ricorso nr. 37201/06, 28 febbraio 2008.

Corte EDU, Sentenza S.D. c. Grecia, ricorso nr. 53541/07, 11 giugno 2009.

Corte EDU,Sentenza S.F. e altri c. Bulgaria, ricorso nr. 8138/16, 7 dicembre 2017.

Corte EDU, Sentenza Sharifi contro Italia e Grecia, ricorso nr. 16643/09, 21 ottobre 2014.

Corte EDU, Sentenza Tarakhel contro Svizzera, ricorso nr. 29217/12, 4 novembre 2014.

Corte EDU, Sentenza Zolotorev c. Russia, ricorso nr. 13408/07, 19 settembre 2017.

European Commission of Human Rights, Danimarca c. Grecia, ricorso nr. 3321/67, Norvegia c. Grecia, ricorso nr. 3322/67, Svezia c. Grecia, ricorso nr. 3323/67, Olanda c. Grecia, ricorso nr. 3344/67, 5 novembre 1969. URL https://www.echr.coe.int/Documents/Denmark_Norway_Sweden_ Netherlands_v_Greece_I.pdf (2019-04-14). 


\title{
Tortura e violenze contro gli immigrati in Marocco
}

\author{
Rabi Ouenniche \\ Independent researcher \\ Zineb Saaid \\ Writer
}

\begin{abstract}
In the context of the European 'governance' of international migration, the so-called countries of transit end up being the first line of Europe's borders, acting as laboratories for experimenting with the radical tightening of migration policies and as centers for the selection of immigrants who may reach the European continent. Morocco, that in recent years has also become a country of immigration and transit, is a symbol of this process, in which it plays the role of patroller of the European borders. This essay examines the phenomenon of torture and violence against sub-Saharan migrants in Morocco, focusing on the events of summer of 2018, without forgetting the historical roots of racism in the country and considering the role Morocco aims at playing in the region.
\end{abstract}

Keywords Torture. Violence. Migrants. Morocco. Subsaharans Africans.

Sommario 1 Introduzione. 2 Immigrazione e quadro legale. - 3 Razzismo, violenza e repressione di Stato. -4 Violenze e torture contro gli immigrati di origine subsahariana.

\section{Introduzione}

Nell'ambito delle attuali migrazioni dal Sud al Nord del mondo gli emigranti attraversano diversi Paesi di transito, dove, per tutta una serie di ragioni, sono costretti a rimanere anche per anni; in diversi casi questi Paesi diventano luoghi di stabilizzazione definitiva o luoghi di rimpatrio in maniera spiccia verso i Paesi d'origine. Oggi nell'asse Africa-Europa molti emigranti si trovano bloccati sulla prima linea delle frontiere europee dato che Pae- 
si di transito e di partenza, come la Tunisia, il Marocco, la Libia, costituiscono di fatto un'estensione dei confini dell'Europa. Nell'ultimo decennio la Fortezza Europa attraverso negoziati e accordi bilaterali ha allargato ed esternalizzato le proprie politiche migratorie ai Paesi di partenza e di transito ed espanso i propri confini; questi Paesi rappresentano dei campi di sperimentazione e d'applicazione della 'nuova politica migratoria europea', così come dei laboratori di selezione della forza lavoro, soprattutto per quanto riguarda le competenze professionali e le attitudini politiche degli emigranti (sono preferiti i meno riottosi, i meno organizzati, i più dotati).

Un esempio di tutto ciò è il Marocco, che, con l'ascesa al trono di Mohammed VI nel 1999, ha cambiato registro in tema di politica estera, abbandonando via via il mito dell'Ittihad Al-Maghreb Al-Arabi (Unione del Maghreb Arabo ${ }^{1}$ ) - benché esso sia menzionato nella costituzione del 2011 - e discostandosi leggermente dall'identificazione nella sola matrice storico-religiosa-linguistica arabo-islamica attraverso un orientamento più marcato verso la componente amazigh $^{2}$ storicamente presente nella società marocchina e verso i Paesi dell'Africa subsahariana. ${ }^{3}$ Il riconoscimento del pluralismo culturale, religioso e linguistico va inteso soprattutto come cambio di rotta nell'ambito diplomatico, geo-politico, delle relazioni internazionali, attraverso cui Dar Al-Makhzen (il Palazzo Reale) vuole far diventare il Marocco uno stato leader dell'Africa. ${ }^{4}$ Ma il Marocco è molto attivo anche nella deregolamentazione del mercato del lavoro e nel livellamento verso il basso del costo della forza-lavoro, attraverso cui affronta l'agguerrita concorrenza nel mercato mondiale in un contesto di crisi strutturale del capitalismo.

È questo il contesto in cui sono state definite le linee guida della politica migratoria del Paese, che guarda alla forza-lavoro emigrata all'estero e alla forza-lavoro immigrata presente nel Paese come vantaggio competitivo. Per il Marocco il sistema di controllo, selezione e gestione delle migrazioni deve andare incontro alle esigenze del mercato, del mercato del lavoro; le condizioni e la composizio-

1 Unione politica tra Algeria, Libia, Marocco, Mauritania, Tunisia, siglata nel 1989 con il Trattato di Marrakech. Sul piano concreto questa Unione non ha portato grandi vantaggi ai Paesi firmatari in termini di progresso e di pace; anzi, la questione del Sahara occidentale, che vede contrapporsi Algeria e Marocco, è ancora aperta.

2 Il termine 'berbero', dal greco barbaros, è un termine rifiutato dalla popolazione amazigh perché ritenuto un appellativo dispregiativo.

3 Il preambolo della Costituzione del 2011 sottolinea questa, per così dire, ridefinizione dell'identità del Regno del Marocco: «État musulman souverain, attaché à son unité nationale et à son intégrité territoriale, le Royaume du Maroc entend préserver, dans sa plénitude et sa diversité, son identité nationale une et indivisible. Son unité, forgée par la convergence de ses composantes arabo-islamique, amazighe et saharo-hassanie, s'est nourrie et enrichie de ses affluents africain, andalou, hébraïque et méditerranéen».

4 Nel 2017 il Marocco è rientrato nell’Unione Africana dopo oltre trent'anni di assenza. 
ne dell'emigrazione e dell'immigrazione devono essere subordinate agli interessi nazionali, cioè agli interessi della classe dominante. La borghesia marocchina, come qualsiasi altra classe dominante, è consapevole del nesso inscindibile tra le leggi immanenti del capitale e i movimenti migratori contemporanei, della necessità di avere a disposizione una manodopera immigrata pronta (perché obbligata a causa di una condizione di inferiorità giuridica e sociale) a competere contro quella autoctona al fine di livellare i costi del lavoro vivo e ad abbassare i diritti dei lavoratori (di tutti i lavoratori); come è ben consapevole della necessità di tessere buone relazioni con i Paesi africani al fine di conseguire la leadership regionale. Per questa ragione il tema dell'immigrazione è utilizzato da Dar Al-Makhzen nelle negoziazioni internazionali per avvicinarsi ai Paesi dell'Africa nera, per conquistare il sostegno dei governanti africani, per accreditarsi sulla scena regionale e mondiale. ${ }^{5}$

\section{Immigrazione e quadro legale}

Storicamente Paese di emigrazione, il Marocco è divenuto anche Paese di immigrazione e di transito. Esso rimane un Paese che esporta forza-lavoro: tra il 2010 e il 2015 sono infatti emigrati 450 mila marocchini, ${ }^{6}$ ogni anno circa 600 ingegneri lasciano il Paese, ${ }^{7}$ il numero di cittadini marocchini residenti all'estero si aggira intorno ai

5 In occasione del $30^{\circ}$ summit dell'Unione Africana il re del Marocco ha affermato che: «Je vous soumets un document constituant l'Agenda Africain pour la Migration'. Il a été conçu selon une approche inclusive et participative [...] La migration est un phénomène naturel qui constitue la solution et non pas le problème. Nous devons adopter une perspective positive sur la question de la migration en mettant en avant la logique humaniste de responsabilité partagée et de solidarité [...] la migration ramenée à ses proportions réelles, loin des mythes qui en projettent une image scandaleusement déformée, est un enjeu planétaire et crucial pour Notre continent. Elle mérite une nouvelle approche afro-centrée conciliant le réalisme, la tolérance et la primauté de la raison sur les peurs [...] Cet Agenda doit tendre à faire de la migration un levier de co-développement, un pilier de la Coopération Sud-Sud, et un vecteur de solidarité [...] La création d'un Observatoire Africain de la Migration dont le travail sera basé sur le triptyque 'comprendre, anticiper et agir'. Il aura pour mission de développer l'observation et l'échange d'informations entre les pays africains, afin de favoriser une gestion maîtrisée des flux migratoires. Le Maroc propose d'abriter cet Observatoire» (http://www.maroc.ma/fr/discours-royaux/sm-le-roi-adresse-un-message-au-30e-sommet-de-lunion-africaine-ua, 2019-07-06).

6 I lavoratori marocchini emigrati negli anni 2013, 2014 e 2015 ammontano rispettivamente a 7.930, 8.699 e 8.814. Questi dati, pubblicati nel Bilancio sociale del Ministero del lavoro, si riferiscono a coloro che hanno stipulato un contratto di lavoro prima di partire per l'estero, nella maggior parte dei casi lavoratori qualificati (http://www. emploi.gov.ma/index.php/fr/, 2019-07-06).

7 «Fuite des cerveaux: 600 ingénieurs marocains quittent le pays chaque année», Hespress, 15 janvier 2019. URL https://fr.hespress.com/42094-fuite-des-cerveaux600-ingenieurs-marocains-quittent-le-pays-chaque-annee.html (2019-07-06). 
4,5 milioni su un totale di 35,74 milioni. Tuttavia nell'ultimo decennio il numero degli immigrati è cresciuto: nel 2014 sul territorio nazionale risiedevano circa 84.000 stranieri (Khachani 2019, 171), ${ }^{8}$ in buona parte di origine africana, che si sono progressivamente stabilizzati. ${ }^{9}$ Tra le cause principali di questo cambio di rotta ci sono la scarsità di risorse economiche per affrontare una nuova migrazione verso i Paesi del Nord del mondo, la difficoltà del tragitto che scoraggia a proseguire verso le coste dell'Europa, il radicamento sociale in Marocco, le campagne di regolarizzazione amministrativa. Negli ultimi anni è cresciuto anche il numero dei richiedenti protezione internazionale, i quali provengono principalmente dai Paesi subsahariani e dal Medio Oriente; secondo l'HRC Maroc nel 2018 c'erano 7.455 rifugiati, di cui 5.618 in attesa di una protezione internazionale. ${ }^{10}$

Nel 2003 il Marocco si è dotato di una nuova normativa sull'immigrazione, colmando un vuoto legislativo e abrogando le antecedenti leggi sull'immigrazione (art. 58). ${ }^{11}$ Fino ad allora la legislazione faceva riferimento alla normativa introdotta durante il periodo della colonizzazione francese (Khrouz, Ouardi, Rachidi 2009) e conteneva numerosi tratti coloniali (Belguendouz 2009): il suo obiettivo era infatti il controllo dei movimenti dei cittadini marocchini all'interno del Paese, da e verso le zone francesi e spagnole, e l'organizzazione dell'emigrazione all'estero - prima come carne da macello per le trincee del fronte militare in Europa (Cegarra 1999, 37) poi come forza-lavoro verso la Francia, il Belgio e l'Europa in generale (Bousetta, Gsir, Martiniello 2005).

La legge nr. 2/2003, relativa all'«entrata e al soggiorno degli stranieri nel Regno del Marocco, all'emigrazione e l'immigrazione ir-

8 Nel 2010 il numero degli stranieri soggiornanti si aggirava attorno alle 74 mila unità (Khachani 2011, 6).

9 Circa la metà degli immigrati regolarmente soggiornanti risiede in Marocco mediamente dai 2 ai 4 anni (Mourji et al. 2016, 25), ma una buona parte di coloro che coltivano l'idea di andare in Europa nel corso del tempo si stabilisce definitivamente in Marocco. Significativa è anche la presenza di immigrati senza documenti, in prevalenza persone che hanno fatto ingresso in Marocco con un visto e che sono cadute nell'irregolarità allo scadere dei novanta giorni non avendo avuto la possibilità di rinnovare il titolo di soggiorno. Nel 2018 sono stati fermati circa 70 mila stranieri 'irregolari' (Khachani 2019, 175).

10 «Le nombre de réfugiés au Maroc en hausse de 450\%», L'economiste, 22 novembre 2018. URL https://www.leconomiste.com/article/1036887-le-nombre-de-refugies-au-maroc-en-hausse-de-450 (2019-10-10).

11 A livello di normativa internazionale, nel 1956 il Marocco ha ratificato la Convenzione di Ginevra sullo status dei rifugiati e nel 1971 il relativo protocollo; nel 1993 ha ratificato la Convenzione internazionale sulla protezione dei diritti dei lavoratori migranti e dei membri delle loro famiglie del 1990 (entrata in vigore nel 2003); nel giugno 2019 il Ministro del Lavoro ha depositato gli strumenti per la ratifica di tre convenzioni dell'Organizzazione Internazionale del Lavoro (OIL) tra cui la Convenzione nr. 97/1949 sui lavoratori migranti. 
regolare», è stata redatta ed emanata in un clima di forte tensione a seguito degli attentati di Casablanca del 16 maggio $2003^{12}$ (è stata approvata lo stesso giorno in cui è stata approvata la legge nr. 3/2003 sulla lotta al terrorismo); dà ampio spazio alla dimensione securitaria, ha un approccio repressivo, penalizza l'immigrazione undocumented,$^{13}$ si presenta lacunosa sotto l'aspetto organizzativo (lacune a cui lo stato ha sopperito nel 2010). Nel 2013 il Marocco ha emanato la «Stratégie Nationale d'Immigration et d'Asile» (istituendo anche un Département ministériel chargé des Affaires de la Migration), con cui ha annunciato una nuova politica migratoria, più inclusiva ed efficace; ma, a dispetto dei proclami, prevale un'accoglienza di facciata. Nel novembre del 2013 il governo ha avviato una campagna di regolarizzazione alla scadenza della quale sono state presentate circa 28.000 domande di cui circa 25.000 sono state accettate (Alioua, Ferrié, Reifel 2017); nel 2017, in una nuova campagna di regolarizzazione, sono state presentate 28.400 domande di cui circa 20.000 hanno avuto esito positivo (Politique Nationale d'Immigration et d'Asile 2017).

Quanto ai diritti fondamentali, il Marocco nel 1958 ha ratificato la Convenzione per la prevenzione e la repressione del crimine di genocidio, e nel 1969 ha ratificato la Convenzione sull'eliminazione di ogni forma di discriminazione razziale. Nel 1993 ha ratificato la Convenzione contro la tortura e nel 2014 ha ratificato il relativo Protocollo opzionale che obbliga gli Stati firmatari a istituire un meccanismo nazionale di prevenzione; negli ultimi anni il confronto tra le istituzioni e le associazioni a difesa dei diritti umani sulla configurazione di questo meccanismo di prevenzione è stato molto intenso tuttavia esso non è ancora stato istituito. Il Marocco si è dotato inoltre di disposizioni, costituzionali e di legislazione ordinaria, che vietano la tortura e i comportamenti umilianti e degradanti: il codice

12 Le testate giornalistiche marocchine (sotto la guida di Maghreb Arabe Presse) hanno dato un grande contribuito alla rappresentazione pubblica dell'immigrazione subsahariana come pericolo, piaga, flagello, e alla preparazione dell'"opinione pubblica' per accogliere la nuova legge. Si sono prodigati anche gli esponenti del governo, come, ad esempio, l'allora Ministro degli Interni, il quale ha affermato che: «La lutte contre l'immigration clandestine est l'une des priorités de l'action du gouvernement, qui, conscient du danger qu'elle représente, a mobilisé tous les moyens humains et matériels nécessaires pour lutter contre ce fléau qui a des répercussions négatives aux plan économique et social [...]. Ce programme de lutte contre l'immigration clandestine nécessite d'importants fonds et de ce fait un fardeau pour le budget de l'Etat. Au lieu d'être un pays émetteur d'immigration, le Maroc en devient victime avec ce que cela suppose comme effets négatifs» (Belguendouz 2009, 19).

13 Nel testo della legge la dicitura «minaccia all'ordine pubblico» è utilizzata dieci volte, specialmente negli articoli relativi alla concessione e al ritiro del titolo di soggiorno e all'espulsione (Khrouz, Ouardi, Rachidi 2009, 22). 
penale prevede il reato di tortura, ${ }^{14}$ mentre la Costituzione del 2011 (art. 22) ha consolidato la tutela dell'integrità fisica e morale di qualunque persona e ha ribadito che la tortura è un crimine punito dalla legge. Tuttavia, come si vedrà nelle prossime pagine, molto spesso le normative e le dichiarazioni restano sulla carta, anche perché il Marocco, che ha un'economia fortemente legata a quella europea, funge da gendarme dei confini europei e da controllore dei movimenti migratori secondo i dettami dell'Europa.

$\mathrm{E}$ da ricordare, inoltre, che, nonostante i cambiamenti in ambito normativo, nella storia recente del Marocco ci sono stati diversi casi di uso sistematico della tortura contro gli oppositori politici e, più in generale, attivisti e persone che hanno partecipato a manifestazioni contro la povertà e il carovita. Dopo gli attentati del 16 maggio 2003 a Casablanca, ad esempio, vennero arrestati, torturati e condannati alle massime pene circa mille presunti terroristi (Rollinde 2004). Secondo un'inchiesta dell'Associazione Nazionale di sostegno ai Prigionieri Politici (Annassir) relativa a 300 casi di persone arrestate tra il 2002 e il 2003, vi è stato un utilizzo sistematico e generalizzato della tortura, in tutte le fasi della detenzione, sia prima che dopo il giudizio, che ha incluso un ricorso estremamente diffuso a percosse, waterboarding, scosse elettriche, sospensione per i polsi, stupri, privazione del cibo, dell'acqua e del sonno (Alkarama 2011). ${ }^{15}$

A nemmeno due settimane di distanza dagli attentati del 2003 è stata introdotta la «Loi nr. 3/2003 relative à la lutte contre le terrorisme», che ha incluso una serie di misure repressive che possono contribuire alla moltiplicazione di episodi di tortura. Come ha sottolineato Rollinde, infatti, questa legge

donne aux forces policières le droit de détenir un suspect pendant huit jours, sans qu'il ne puisse contacter un avocat ni aucune

14 L'art. 231-c.1 definisce la tortura come «tout fait qui cause une douleur ou une souffrance aiguë physique ou mentale, commis intentionnellement par un fonctionnaire public ou à son instigation ou avec son consentement exprès ou tacite, infligé à une personne aux fins de l'intimider ou de faire pression sur elle ou de faire pression sur une tierce personne, pour obtenir des renseignements ou des indications ou des aveux, pour la punir pour un acte qu'elle ou une tierce personne a commis ou est soupçonnée d'avoir commis ou lorsqu'une telle douleur ou souffrance est infligée pour tout autre motif fondé sur une forme de discrimination quelle qu'elle soit. Ce terme ne s'étend pas à la douleur ou aux souffrances résultant uniquement de sanctions légales ou occasionnées par ces sanctions ou qui leur sont inhérentes» (http://adala.justice.gov.ma/ production/legislation/fr/Nouveautes/code\%20penal.pdf, 2019-07-07). I commi successivi fino al c.8 fissano le pene per chi la pratica: in base alla gravità dell'atto, le pene vanno da un minimo di cinque anni di reclusione fino ad un massimo di trenta, oltre ad una ammenda che varia da un minimo di 10 mila dirham ad un massimo di 50 mila, a seconda del ruolo (magistrato, funzionario pubblico, agente delle forze dell'ordine).

15 Tra queste 300 persone quasi il $10 \%$ sono state condannate a morte, più del $30 \%$ all'ergastolo e più del 30\% tra 20 e 30 anni di reclusione (Alkarama 2011, 27). 
autre personne, et de perquisitionner maisons et commerces sans mandat. Plus grave encore, la loi inclut dans la définition de terrorisme toute perturbation de l'ordre public, faisant peser une menace permanente sur tous ceux qui refusent le consensus sécuritaire et le politiquement correct, et parmi eux les militants [des associations pour la défense] des droits de l'Homme ou les journalistes (Rollinde 2004, s.p.)

Inoltre, nel 2006 il Regno del Marocco ha aderito al Paternariat TransSaharien contre le terrorisme siglato dagli Stati Uniti e dai Paesi del Nord Africa, servendo di fatto come «base arrière en matière d'information et de tortures de prisonniers, sans véritable statut juridique, au nom de la Global War on Terror (guerre planétaire contre le terrorisme) thématisée par l'administration de George W. Bush» (Bennafla, Seniguer 2011).

La situazione non sembra essere cambiata nell'ultimo decennio. Amnesty International (2015) ha infatti censito 173 casi di tortura e maltrattamenti contro uomini, donne e minori, commessi tra il 2010 e il 2014. Si tratta di casi che hanno riguardato in prevalenza oppositori politici (islamisti, socialisti, militanti favorevoli all'autodeterminazione del Sahara Occidentale) e persone che hanno partecipato a manifestazioni contro la povertà e il carovita organizzate dal Movimento del 20 febbraio, sceso in piazza nel 2011 seguendo l'esempio delle rivolte in Tunisia ed Egitto.

Per quanto riguarda i casi di tortura nei confronti di popolazioni immigrate, al momento della stesura di questo articolo non è stato possibile reperire alcuna fonte o testimonianza. Tuttavia i rapporti redatti da numerose ONG marocchine e da Amnesty International evidenziano come il ricorso alla tortura e ad altri trattamenti degradanti nel corso della detenzione di cittadini marocchini siano pratiche «toujours régulièrement signalés» (Amnesty International $2015,2)$, pratiche che «persistent, voire se sont multipliées» (CMODH 2015,7$)$ e che diventano «systématique[s] et généralisée[s]» in occasione di sommovimenti popolari o di avvenimenti destabilizzanti per il Paese, come gli attentati terroristici (Alkarama 2011, 27). In questo contesto, perciò, è altamente probabile che possano verificarsi episodi di tortura che coinvolgono le popolazioni immigrate dall'Africa Subsahariana, tanto più se consideriamo il ruolo svolto dallo Stato e dalle istituzioni nel garantire un ordine basato sul razzismo, l'inferiorizzazione e la vessazione sistematica delle popolazioni immigrate. 


\section{Razzismo, violenza e repressione di Stato}

In Marocco il razzismo, le discriminazioni razziali, le pratiche inferiorizzanti - partendo dagli alti livelli comprese le istituzioni e le amministrazioni locali, e scendendo al livello dei rapporti quotidiani tra la gente comune - vantano una lunga tradizione.

La situazione degli amazigh ne è un esempio. Gli amazigh, da millenni le popolazioni autoctone, indigene, presenti in una vastissima area del territorio nordafricano, ${ }^{16}$ oggi sono circa 30 milioni, di cui 20 nel solo Marocco (Jacquelin-Andersen 2018, 399). Le autorità marocchine, sulla base del censimento del 2016, stimano che il numero dei parlanti tamazight ${ }^{17}$ si aggiri attorno al $28 \%$ della popolazione, mentre le associazioni amazigh stimano una percentuale che sfiora il 70\%. Il disconoscimento e il disprezzo (hogra) da parte dello Stato e delle classi alte contro gli amazigh sono stati molto forti nel tempo; soltanto dopo un lungo processo (ancora aperto) di lotte e rivendicazioni essi hanno ottenuto un lento ma graduale riconoscimento dell'amazighità'. Ricordiamo due date chiave: nel 2003 è iniziato l'insegnamento 'sperimentale' del tamazight, nel 2011 vi è stata la costituzionalizzazione dell'amazighità' (Mous 2011).

Non possiamo dimenticare il razzismo contro i neri, ossia la popolazione marocchina di pelle nera, che costituisce ancora oggi una questione scottante in tutto il Maghreb. I mass-media, il linguaggio quotidiano, sono intrisi di razzismo nei confronti dei marocchini di pelle nera, ai quali è affibbiato il nomignolo di 'Azzi' o 'Kahlouch' (negro). Ridere dei marocchini neri fa sentire superiori i marocchini 'bianchi', e più vicini ai bianchi e 'civilizzati' occidentali. La seguente testimonianza di una marocchina nera che ha subito razzismo in Francia (Paese di nascita) e in Marocco (Paese di origine della propria famiglia) è quanto mai emblematica:

Je suis marocaine et noire. Je suis née et j'ai grandi en France, mais tous les ans je retourne au Maroc [...] il ne passe pas une année sans que je sois insultée : azzi, kehla [...] Je subis donc un double racisme: en France [...] Mais lorsque je retourne au Maroc, dans mon pays, je me vois également insultée par mes compatriotes! [...] Après avoir été insultée l'été dernier, je me suis rendue au commissariat pour porter plainte, et à ma grande surprise les policiers se sont tout simplement mis à rire, trouvant ma démarche ridicule !!! (Fourest 2007, 136) 
La genesi del razzismo anti-neri affonda le radici nei secoli passati, quando nella società marocchina vigeva la servitù della gleba, che interessava soprattutto i neri, i quali erano obbligati al lavoro domestico e a prestazioni di natura sessuale. ${ }^{18}$

Infine dobbiamo citare gli harratin - marocchini di pelle nera che abitano nelle oasi situate nella parte centrale e orientale del Paese, a ridosso del deserto, discendenti degli schiavi neri - che vivono un vero e proprio apartheid. A costoro è istituzionalmente negato il diritto di usufrutto delle terre cosiddette soulaliyate, le terre di proprietà comune riservate ai soli appartenenti alla confraternita religiosa Zaouïa Nassiria. ${ }^{19}$

Da questa brevissima finestra sui rapporti di dominio 'razziale' e di classe ai danni di alcuni gruppi sociali, si può affermare che il razzismo in Marocco è un fenomeno consolidato, che si è cristallizzato nell'ordinamento giuridico, nel linguaggio quotidiano, nelle relazioni sociali, nei mass-media, non è un fenomeno recente apparso con il fenomeno dell'immigrazione sub-sahariana; è il risultato di pratiche secolari e di politiche segregazioniste perpetrate dalle classi dominanti e dalle istituzioni.

\section{Violenze e torture contro gli immigrati di origine subsahariana}

Le vessazioni registrate contro le popolazioni immigrate interessano prevalentemente quelle di pelle nera di origine subsahariana. Nel 2005 nell'area di Ceuta e Melilla c'è stata una vasta repressione in stile 'caccia all'immigrato' ${ }^{20}$ e da allora questi avvenimenti sono continuati in maniera sempre più intensa. Nel 2013 per un futile motivo (un posto a sedere alla stazione ferroviaria di Rabat) un immigrato senegalese è stato accoltellato a morte da un militare; ${ }^{21}$ nella notte del 29 agosto 2014 a Tangeri un'accozzaglia di autoctoni si è rovesciata in strada con coltelli e machete contro gli immigrati subsahariani: il bilancio è stato di un morto e diversi feriti, nove immigrati

18 Sulla schiavitù si veda N’Diaye 2008; Mehdi Alioua, «Il y a un immense déni maghrébin sur le racisme», Le courrier de l'atlas, 16 de avril 2018. URL https://www.montraykreyol.org/article/mehdi-alioua-il-y-a-un-immense-deni-maghrebin-surle-racisme (2019-08-08).

19 Confraternita composta da marocchini 'bianchi', che si considerano di rango superiore agli harratin.

20 Ben documentata in Migreurop 2007.

21 «Meurtre d'un Sénégalais à Rabat: les actes racistes violents en hausse au Maroc?», Jeune Afrique, 20 août 2013. URL https://www.jeuneafrique.com/169๑41/politique/meurtre-d-un-s-n-galais-rabat-les-actes-racistes-violents-en-hausse-au-maroc/ (2019-06-07). 
aggrediti sono stati arrestati per aver protestato contro il lassismo delle forze dell'ordine nel rispondere alla loro richiesta d'aiuto. ${ }^{22}$ Per strada gli immigrati di pelle nera vengono spinti, insultati, evitati, offesi, anche dai mendicanti marocchini 'doc'. ${ }^{23}$

Nell'estate del 2018 si è registrata un'ondata di violenza da parte dello Stato in seguito al tentativo di centinaia di emigranti subsahariani di scavalcare il 26 luglio le recinzioni che delimitano l'enclave spagnola di Ceuta: 116 persone che erano riuscite a passare sono state ricondotte subito in territorio marocchino; 17 sono state giudicate come colpevoli di immigrazione illegale e violenza contro le forze dell'ordine e condannate a due mesi di reclusione e a un'ammenda di 500 dirham. ${ }^{24}$

Tra agosto e settembre dello stesso anno sono stati arrestati e deportati 6.500 immigrati, ${ }^{25}$ in condizioni molto dure. Un membro di Alarmphone Watch the Med ha riferito che «on cible aujourd'hui de plus en plus les quartiers où résident les migrants qu'on cherche jusque dans leurs maisons sans distinction entre les hommes, les femmes et les enfants. Mêmes les blessés ne sont pas épargnés»; un membro di Mains Solidaires ha raccontato che «nous a révélé que la ville de Tétouan s'est vidée de ses migrants subsahariens. Ils se sont volatilisés du jour au lendemain. On ne voit personne même au niveau du bureau de notre association [...] En fait, ils ont eu peur notamment après les arrestations opérées dans les quartiers de la ville»; ${ }^{26}$ un membro dell'Associazione marocchina per la tutela dei diritti umani ha riportato che:

les opérations de ratissage continuent s'effectuer quotidiennement [...]. Plusieurs témoins nous ont confirmé que ces arrestations se sont soldées par des confiscations de biens et argent [...]. Les migrants vivent aujourd'hui un vrai cauchemar. Ils ont peur, vivent dans l'inquiétude [...]. Ces migrants préfèrent actuellement rester cachés ou se déplacer discrètement. Ils passent la nuit dans d'anciennes maisons ou des grottes et survivent grâce à la générosité de la population locale. Certains d'entre eux n'arrivent plus à dormir. ${ }^{27}$

22 «Racisme au Maroc: à Tanger, un pogrom anti-migrants fait au moins un mort». Jeune Afrique, 1 septembre 2014. URL https://www.jeuneafrique.com/45584/politique/racisme-au-maroc-tanger-un-pogrom-anti-migrants-fait-au-moins-unmort/ (2019-06-07).

23 «A Tanger, des migrants subsahariens racontent: Ici, même les mendiants nous insultent», Le Monde, 10 novembre 2017. URL https://bit.ly/2slIezd (2019-06-07).

24 «Tétouan: Deux mois de prison ferme pour les migrants renvoyés de Ceuta». Yabiladi, 11 septembre 2018. URL https://www.yabiladi.com/articles/details/68814/ tetouan-deux-mois-prison-ferme.html (2019-09-28).

25 Una media di 108 persone al giorno (GADEM 2018, 15).

26 «La chasse à l'homme se poursuit», Libération, 20 août 2018. URL https://www. libe.ma/La-chasse-a-l-homme-se-poursuit_a100783.html (2019-09-28).

27 https://www.libe.ma/La-chasse-a-l-homme-se-poursuit_a100783.html (2019-09-28). 
Questi interventi delle forze dell'ordine non sono stati realizzati in esecuzione di un mandato d'arresto, sono stati effettuati al di fuori di una inchiesta giudiziaria, erano privi di una base giuridica (GADEM 2018). ${ }^{28}$ Gli arrestati non sono stati informati del motivo del loro arresto e in alcuni casi sono state fermate persone che la legge protegge da qualsiasi forma di allontanamento come i rifugiati, i richiedenti asilo, ${ }^{29}$ i minori stranieri non accompagnati, le donne in stato di gravidanza.

Il carattere razzista di queste operazioni di rastrellamento e deportazione ha legittimato l'arresto e il fermo di cittadini stranieri in possesso di un regolare titolo di soggiorno (visa), compreso un turista (ma di pelle nera...); l'azione della polizia si è focalizzata sugli immigrati di pelle nera senza verificare la condizione amministrativa di ciascuno: «J'ai demandé 'pourquoi vous nous refoulez?' Un gendarme a répondu 'L'UE a financé pour qu'on vous refoule et qu'on vous éloigne des frontières du Maroc'. J'ai demandé encore 'Pourquoi arrêter quelqu'un qui a des papiers? Normalement ça l'autorise à rester où il veut' et un gendarme m'a répondu en chuchotant 'Mon ami, ça, c'est parce que vous êtes noirs'» (GADEM 2018, 27).

Molte di queste operazioni sono avvenute di notte: «deux jours avant la fête du mouton, ils sont venus à $6 \mathrm{~h}$ du matin. Nous sommes nombreuses dans la maison. Ils ont tout cassé, il y avait des militaires, qui disaient 'Yallah yallah, sortez!'» (GADEM 2018, 11); «les policiers, masqués, sont arrivés au milieu de la nuit, ont fracassé notre porte et commencé à arrêter ma famille, y compris les femmes et les enfants. De nombreux véhicules se trouvaient dehors, et des policiers faisaient irruption chez nos voisins». ${ }^{30}$ Le abitazioni sono state abbandonate, le forze dell'ordine hanno rubato e distrutto i beni (non è stato redatto un verbale dei beni confiscati e per questo motivo gli immigrati non possono contestare nulla), gli effetti personali sono stati gettati fuori casa. ${ }^{31}$

28 Confermato da Amnesty International, «Maroc. Des milliers de réfugiés et de migrants subsahariens sont visés par une répression illégale continue», 7 septembre 2018. URL https://www.amnesty.org/fr/latest/news/2018/09/morocco-relentless-crackdown-on-thousands-of-sub-saharan-migrants-and-refugees-isunlawful/ (2019-09-28).

29 Amnesty International riferisce di 14 richiedenti asilo e 4 rifugiati trasferiti contro la propria volontà. Cf. https://www.amnesty.org/fr/latest/news/2018/09/morocco-relentless-crackdown-on-thousands-of-sub-saharan-migrants-and-refugees-is-unlawful/ (2019-09-28).

30 https://www.amnesty.org/fr/latest/news/2018/09/morocco-relentlesscrackdown-on-thousands-of-sub-saharan-migrants-and-refugees-is-unlawful/ (2019-09-28).

31 https://www.amnesty.org/fr/latest/news/2018/09/morocco-relentlesscrackdown-on-thousands-of-sub-saharan-migrants-and-refugees-is-unlawful/ (2019-09-28). 
Gli arresti sono avvenuti anche in luoghi pubblici, nei mercati, ospedali, mezzi di trasporto, e sono stati operati anche da agenti in borghese. Gli immigrati hanno cercato rifugio nei boschi e nei cimiteri, dove hanno costruito degli accampamenti di fortuna che sono stati sistematicamente smantellati e bruciati in occasione di qualche retata (Association marocaine des droits humains 2017, 6). Le retate sono state effettuate nelle città del Nord del Paese, vicino ai confini con le enclave spagnole dove gli immigrati s'installano nell'attesa di riuscire a oltrepassare le recinzioni.

Durante i rastrellamenti, che si tratti di attaccare quartieri o accampamenti nelle foreste, gli immigrati sono stati raggruppati e spinti a salire sui bus, sono rimasti seduti per ore fino al termine dell'operazione e poi portati al commissariato per l'identificazione, la fotosegnalazione, con tanto di cartello numerato tra le mani, e la suddivisione tra titolari di un documento di soggiorno e non. Tra la metà di settembre 2018 e la metà ottobre 2018 un gruppo di 142 subsahariani ha trascorso dai tre giorni a un mese nei sottosuoli di un commissariato dove aveva a disposizione un lavabo e una sola toilette (accessibile solo durante il giorno, di notte si usavano bottiglie di plastica), e dove il cibo - generalmente somministrato una sola volta al giorno - consisteva in una porzione di latte, pane e acqua. Prima della deportazione, non sono mancate le violenze fisiche (GADEM 2018).

Durante il trasferimento gli immigrati erano ammanettati, singolarmente o in coppia; ${ }^{32}$ durante le soste alcuni hanno tentano la fuga saltando dai finestrini, qualcuno è riuscito nell'impresa mentre qualcuno ci ha rimesso la vita. ${ }^{33} \mathrm{Il}$ cibo distribuito era scarso, generalmente pane e acqua; per urinare si usava una bottiglia di plastica o la porta in fondo del bus perché il mezzo non doveva fermarsi. Alcuni agenti hanno fatto di queste operazioni uno strumento per 'arrotondare' e hanno chiesto agli immigrati del danaro in cambio della possibilità di scegliere dove scendere: più alta è la somma pagata dall'immigrato, più a Nord egli può scendere dal bus (GADEM 2018). È lo schiavo che paga il proprio riscatto.

Lo scopo di queste operazioni di polizia è di allontanare gli immigrati dalle zone limitrofe ai confini europei e abbandonarli a centinaia di chilometri a Sud del Paese, nelle cosiddette 'terre di nessuno', nel deserto ai confini con l'Algeria (dove la polizia algerina li attende a suon di pallottole per obbligarli a fare marcia indietro) o in lontani paesini

32 «Déplacés de force, le périple de migrants subsahariens dans le sud du Maroc». Les Observateurs, 17 août 2018. URL https://observers.france24.com/fr/20180817maroc-espagne-tanger-migrants-deplaces-police (2019-07-22). Si veda anche il video https://www.youtube.com/watch?v=LstYIw4Q-3o (2019-11-27).

33 È il caso di un maliano sedicenne e di un gambiano morti durante il trasferimento verso il Sud del Marocco. Cf. «Deux migrants meurent pendant leur transfer vers le sud du Maroc», Telquel, 5 septembre 2018. URL https://bit.ly/37A3jWC (2019-07-22). 
del Sud, all'uscita dell'autostrada, lungo una strada in mezzo al nulla. ${ }^{34}$ Senza denaro, si trovano immediatamente nella condizione di dover porgere la mano per raccogliere qualche soldo. Molti hanno perso la casa, ma per la paura di rivivere l'incubo della deportazione alcuni hanno preferito trovare riparo nei cimiteri o nelle foreste; ${ }^{35}$ altri, ritornati al Nord, non sono riusciti a trovare un appartamento in affitto a causa del rifiuto dei proprietari del posto. Lungo la strada per il Nord molti sono stati individuati da agenti in borghese, fatti scendere e condotti in commissariato: è stato detto loro che per i subsahariani è vietato circolare nelle province del Nord, che possono stare solo in quelle del Sud (lontane dai confini con l'Europa), che non possono vivere nei pressi di Tangeri e di Nador (città limitrofe a Ceuta e Melilla).

I rastrellamenti, i raid, le aggressioni, i pogrom, vivere in uno stato di allerta permanente, sono esperienze traumatizzanti che instillano paura nel profondo. ${ }^{36}$ Soltanto camminare per strada è diventata un'azione terribilmente paurosa perché l'immigrato ha il costante timore di incrociare un agente (in divisa o in borghese). Già nel 2010 Médecins sans Frontières aveva registrato diversi disturbi mentali tra gli immigrati subsahariani a causa di condizioni di vita precarie (vivere nelle foreste o nei cimiteri, scarsità d'igiene e acqua potabile, dover mendicare per procurarsi del cibo, mancanza di spazi intimi, etc.). Su 10.500 visite mediche, il 39\% ha evidenziato disturbi d'ansia, il 34\% depressione, il 14\% traumi (Médecins sans Frontières 2013, 11).

Questo trattamento riservato agli immigrati di pelle nera genera e amplifica ininterrottamente la precarietà, distrugge i processi di stabilizzazione e i rapporti coltivati con la popolazione locale, nuoce alle già più o meno fragili relazioni iniettandovi diffidenza, ipocrisia, violenza. Educa all'odio razziale, costruisce gerarchie razziali tra persone appartenenti, in fin dei conti, alla stessa classe sociale, e si abbatte con maggiore violenza sulle donne, più vulnerabili e maggiormente esposte alle violenze sessuali.

Questo trattamento degli immigrati subsahariani affonda le radici in un passato che non è passato e in un presente che vede il Marocco nel ruolo di gendarme in cambio di un po' di 'cooperazione' con l'Occidente nell'attuale 'governance' mondiale delle migrazioni. Nel corso di una riunione svoltasi nel 2018 con alcuni ambasciatori africani per discutere di questi fatti e della decisione del governo marocchi-

34 https://www.amnesty.org/fr/latest/news/2018/09/morocco-relentlesscrackdown-on-thousands-of-sub-saharan-migrants-and-refugees-is-unlawful/ (2019-09-28).

35 «Au Maroc, les migrants se cachent pour éviter les déplacements forcés», La croix, 4 septembre 2018. URL https://www.la-croix.com/Monde/Au-Maroc-migrants-cachent-eviter-deplacements-forces-2018-09-04-1300966251 (2019-07-22).

36 Si veda la testimonianza di una donna a cui sono stati allontanati due figli minori in GADEM 2018, 32. 
no di rimpatriare 1.500 immigrati subsahariani, i funzionari marocchini hanno spiegato che gli spostamenti e i rimpatri sono avvenuti nel rispetto delle leggi nazionali e internazionali e che erano volti a proteggerli da reti criminali coinvolte nella tratta di esseri umani. ${ }^{37}$ In realtà questa crudele dimostrazione di forza contro gli immigrati subsahariani è utile allo stato marocchino per chiedere all'Unione europea maggiori finanziamenti per il controllo dell'immigrazione (leggasi: 'sviluppare il proprio sistema militare') (38 $^{38}$ garantirsi una posizione di forza nei tavoli in cui si discute di accordi commerciali.

\section{Bibliografia}

Alioua, Mehdi; Ferrié, Jean-Noël; Reifel, Helmut (dir.) (2017). La nouvelle politique migratoire marocaine. Rabat: Konrad Adenauer Stiftung, Université Internationale de Rabat. URL https://www.kas.de/c/document_library/get_file?uuid=fd3118d2-365a-113b-06c6-973a65a509c8\&groupId $=252038$ (2019-11-26).

Alkarama (2011). Le Maroc devant de nouveaux défis. Rapport soumis au Comité contre la torture dans le cadre de l'examen du quatrième rapport périodique du Maroc, 14 octobre. URL https://tbinternet.ohchr.org/Treaties/ CAT/Shared\%20Documents/MAR/INT_CAT_NGO_MAR_47_9558_E.pdf (2019-11-26).

Amnesty International (2015). L'ombre de l'impunité. La torture au Maroc et au Sahara Occidental. Rapport. URL https://www.amnesty.org/download/Documents/MDE2914912015FRENCH.PDF (2019-11-26).

Association marocaine des droits humains (2017). Nador, des voies de migration payante de plus en plus meurtrière. URL http://amdh.org.ma/img/ upload/contents/fichiers/95852c08233a13c14a01004a13ac977f. pdf (2019-11-26).

Belguendouz, Abdelkrim (2009). Le Maroc et la migration irrégulière: une analyse sociopolitique. San Domenico di Fiesole (FI): Institut universitaire européen. CARIM Analytic and Synthetic Notes 7. URL https://cadmus.eui. eu/handle/1814/10799 (2019-11-26).

Bennafla, Karine; Seniguer, Haoues (2011). «Le Maroc à l'épreuve du printemps arabe: une contestation désamorcée?». Outre-Terre, 29, 143-58. DOl https://doi.org/10.3917/oute.029.0143.

Bousetta, Hassan; Gsir, Sonia; Martiniello, Marco (éds) (2005). Les migrations marocaines vers la Belgique et l'Union européenne. Bilbao: Université de

37 «Migration: les dessous de la rencontre entre le gouvernement marocain et les ambassadeurs africains», TelQuel, 3 septembre 2018. URL https://telquel. $\mathrm{ma} / 2018 / 09 / 03 / \mathrm{migration}-\mathrm{les}$-dessous-de-la-rencontre-entre-le-gouvernement-marocain-et-les-ambassadeurs-africains_1609115 (2019-09-07).

38 Ad oggi il Marocco ha già in orbita due satelliti attraverso i quali controlla le proprie frontiere: il primo è stato lanciato nel novembre del 2017 in collaborazione con Airbus Defence \& Space e Thales Alenia Space (costo circa 50 milioni di euro); il secondo è stato lanciato in orbita esattamente un anno dopo, sempre in collaborazione con Airbus e al medesimo costo. 
Deusto. URL http://www.deusto-publicaciones.es/ud/openaccess/hnet/ pdfs_hnet/hnet18.pdf (2019-11-26).

Cegarra, Marie (1999). La mémoire confisquée. Les mineurs marocains dans le Nord de la France. Villeneuve-d'Ascq: Presse Universitaires du Septentrion.

CMODH, Coordination Maghrébine des Organisations de Droits Humains (2015). Rapport alternatif de la société civile sur la mise en oeuvre du pacte international relatif aux droits civils et politiques (PIDCP). URL https://novact.org/ wp-content/uploads/2017/11/rapport-parallele-maroc-1-session.pdf (2019-11-26).

Fourest, Caroline (2007). Le choc des préjugés. L'impasse des postures sécuritaires et victimaires. Paris: Calmann-Lévy.

GADEM (2018). Coûts et blessures. Rabat: GADEM. URL https://www.lacimade.org/wp-content/uploads/2018/10/20180927_GADEM_Couts_ et_blessures.pdf (2019-11-26).

Jacquelin-Andersen, Pamela (ed.) (2018). The Indigenous World 2018. Copenhagen: Iwgia. URL https://www.iwgia.org/images/documents/indigenous-world/indigenous-world-2018.pdf (2019-11-26).

Khachani, Mohamed (2011). La question migratoire au Maroc. Données récentes. San Domenico di Fiesole (FI): Institut universitaire européen. CARIM Analytic and Synthetic Notes 71. URL https://cadmus.eui.eu/handle/1814/19877 (2019-11-26).

Khachani, Mohamed (2019). La question migratoire au Maroc. Rabat: Bidaoui.

Khrouz, Nadia; Ouardi, Adila; Rachidi, Hicham (2009). Maroc. Le cadre juridique relatif à la condition des étrangers, au regard de l'interprétation du juge judiciaire et de l'application du pouvoir exécutif. Rabat: GADEM. URL https:// www.lacimade.org/wp-content/uploads/2009/02/ETUDE-GADEMjanv2009.pdf (2019-11-26).

Médecins sans Frontières (2013). Violences, vulnérabilité et migrations: bloqués aux portes de l'Europe. Un Rapport sur les migrants subsahariens en situation irrégulière au Maroc. URL https://www.msf.fr/sites/default/files/ informemarruecos2013_fr_@.pdf (2019-11-26).

Migreurop (2007). Guerre aux migrants. Le livre noir de Ceuta et Melilla. Paris: Syllepse.

Mourji, Fouzi; Ferrié, Jean-Noël; Radi, Saadia; Alioua, Mehdi (2016). Les migrants subsahariens au Maroc, enjeux d'une migration de résidence. Rabat: Konrad Adenauer Stiftung; Université Internationale de Rabat. URL https://www. kas.de/c/document_library/get_file?uuid $=5757725$ d-390b-3cbf1151-999a9653f572\&groupId=252038 (2019-11-26).

Mous, Fatma Aït (2011). «Les enjeux de l'amazighité au Maroc». Confluences Méditerranée, 78(3), 121-31.

N'Diaye, Tidiane (2008). Le génocide voilé. Enquête historique. Paris: Gallimard. Politique Nationale d'Immigration et d'Asile 2017 = Ministère délégué auprès du ministre des Affaires étrangères et de la Coopération internationale, chargé des Marocains résidant à l'étranger et des Affaires de la migration (2017). Politique Nationale d'Immigration et d'Asile. Rapport 2017. URL http://marocainsdumonde.gov.ma/wp-content/uploads/2018/o3/POLITIQUE-NATIONALE_Rapport-2017.pdf (2019-11-26).

Rollinde, Marguerite (2004). «L'alternance démocratique au Maroc: une porte entrouverte». Confluences Méditerranée, 4(51), 57-67. DOI https://doi. org/10.3917/come.051.0057. 



\title{
The Story of Yaser Abdulla Darfuri Asylum Seeker Who Was Tortured in the Sinai Desert Only to Face Imprisonment in Israel and a Life Without Refugee Status
}

Mya Guarnieri Jaradat

writer and independent journalist, international reporting fellow at Columbia University, USA

\begin{abstract}
The 33,000 African asylum seekers currently residing in Israel face a lack of legal status and receive no services from the state. The entire population is under pressure but those who have faced torture in the Sinai desert en route to Israel struggle with additional problems and have nowhere to turn for help. The story of Yaser Abdulla - an asylum seeker from Darfur who was tortured by Bedouin traffickers in the Sinai - illuminates the circumstances that pushed asylum seekers from their home, what they encounter on their way, and the situation they grapple with inside of Israel.
\end{abstract}

Keywords African asylum seekers. Torture. Sinai. Israel.

At the time of writing, in 2019, there are currently some 33,000 asylum seekers in Israel; more than 90 percent are from Eritrea and Sudan.

At the peak of migration to Israel, there were approximately 60,000 asylum seekers in the country. But the Israeli government put this population under tremendous pressure through a number of draconian measures. Upon entering the country, asylum seekers were detained and held in prison, without

The following pages tell the story of Yaser Abdulla and are taken from my book The Unchosen: The Lives of Israel's New Others, published in 2017 by Pluto Press (courtesy of). 
charge or trial, for an arbitrary period ranging from weeks to over a year. When they were released from jail, most asylum seekers received 'conditional release' visas, which must be renewed often and which stipulate that they cannot work legally. This essentially forces African asylum seekers onto the underground economy where they face labor exploitation; not only are asylum seekers unable to work legally, they are not eligible for basic services like healthcare except under emergency circumstances. Those who are suffering from mental health issues stemming from the trauma they experienced in their home countries or on their way to Israel - as in the case with those who were raped and/or tortured in the Sinai desert - do not have access to mental health services.

Israeli officials have also failed to conduct comprehensive reviews of applications for asylum, summarily rejecting most requests. Government officials often claim that asylum seekers are "work migrants". They have also turned public opinion against asylum seekers and have engaged in incitement. In 2012, after an Israeli politician called asylum seekers "cancer in our body", a race riot broke out and dozens were injured. Asylum seekers have also been targeted in numerous acts of violence.

Despite the fact that they do not receive work visas or any sort of benefits from the state, and despite the fact that the wages they receive for working menial jobs on the underground economy are very low, the "Deposit Law" requires asylum seekers' employers to put 20 percent of their pay into an account asylum seekers can only access when they leave the country. Although the law has been poorly enforced, it is one of many ways that the Israeli government has pursued a policy of deterrence to discourage asylum seekers from staying in the country. Beginning in 2013, in an attempt to get asylum seekers to self-deport, Israeli officials told asylum seekers they could choose between "voluntary departure" to a third country - Rwanda or Uganda - or imprisonment. To that end, Holot Detention Center was opened in December of 2013. While the facility was closed in March of 2018, the asylum seekers remaining in Israel are still under stress as they still lack legal status and as politicians like Justice Minister Ayelet Shaked continue to discuss deporting them "voluntarily or by coercion". ${ }^{1}$

So while international law and Israel's obligations as a signatory to different human rights treaties effectively protect asylum seekers from outright deportation to Sudan and Eritrea - at least for now - they live in perpetual legal limbo and their futures remain uncertain.

1 Lee Yaron, "Israeli Justice Minister Seeks New Destination for Deporting Asylum Seekers", Haaretz, 3 April 2019. URL https://www.haaretz.com/israel-news/.premium-israeli-justice-minister-seeks-new-destination-for-deporting-asylumseekers-1.7084343 (2019-10-18). 
As the situation inside Israel devolved, so was the journey to the Jewish state becoming increasingly dangerous. It wasn't just hot returns and Egyptian soldiers. In 2009, human rights organizations had begun to encounter women who had been raped by their Bedouin smugglers in the Sinai; by 2010, Bedouin traffickers had made torture camps in the Sinai, where they held asylum seekers for ransom. Approximately 7,000 victims had entered Israel by 2012, when the newly completed fence on the Egyptian border more or less stopped the flow of Africans into the country.

While many had fallen into their torturers hands on their way to Israel, there were those who had never intended to go to the Jewish state, who had been kidnapped from refugee camps in Eastern Sudan. The Sudanese and Egyptian police and the military of both countries also facilitated these crimes by "hand[ing] victims over to traffickers in police stations, turn[ing] a blind eye at checkpoints, and return[ing] escaped trafficking victims to traffickers", Human Rights Watch reported. ${ }^{2}$ The kidnappers took them to the Sinai where they were held in chains. Victims were forced to call their families so that their loved ones could hear their cries as they were tortured. The Bedouin would then demand ransom, the price for freedom sometimes topping 30,000 USD. Back home, the panicked and poverty-stricken families sold everything; Eritreans the world over pitched in to help their countrymen. But paying didn't always secure release - sometimes the traffickers sold their human goods to other kidnappers who would then hold the victim for yet another ransom. Occasionally, men were sexually assaulted; women and girls were usually gang raped, some arrived in Israel pregnant.

While most victims were Eritrean, some were Sudanese. Yaser Abdulla was among the latter. He'd been held in a Sinai torture camp for two months before arriving in Israel in October of 2011 at the age of 26 .

The events that brought Abdulla to south Tel Aviv, where I interviewed him, began in 1991. Government militias attacked his village in Darfur when he was just six years old. Abdulla and his immediate family fled to Nyala City in south Darfur but his beloved grandfather stayed put. Then the genocide began in 2003. "There was a group of rebels, Darfurian who helped the people", Abdulla said. He joined when he was 19.

2 Gerry Simpson, “'I Wanted to Lie Down and Die': Trafficking and Torture of Eritreans in Sudan and Egypt", Human Rights Watch, 11 February 2014. URL https://www. hrw.org/report/2014/02/11/i-wanted-lie-down-and-die/trafficking-and-torture-eritreans-sudan-and-egypt (2019-10-18). 
He returned, eventually, to Nyala City to study but the Sudanese government caught up with him and he was jailed, for having fought against Bashir's militias. The head of his village stepped in on his behalf and, somehow, persuaded officials that Abdulla was a student, just a student, not a rebel. He was released. Feeling unsafe in Nyala City, Abdulla went on to Khartoum, where he hoped to study law. But he was arrested again; this time he was held for two months; this time it was a human rights organization that came to his aid. And then he moved on again and was arrested again. It was time, he realized, to leave Sudan.

Abdulla made his way to Cairo. But he didn't "see [it] so safe" because of the diplomatic relations between Sudan and Egypt. The Egyptians, he explained, "were deporting people to Sudan". So, Abdulla determined to keep moving. He could go to Libya and from there he could try to make the treacherous journey to Europe. Or, he could go to Israel.

In Cairo, he met a Bedouin who said he could smuggle him through the Sinai to Israel for 3,000 USD. Once they were in the desert, however, the man demanded an additional 8,000 USD, which Abdulla didn't have. He found himself in some sort of a building - he lacked the words to describe it, not because his English was poor but, rather, because language began to fail him as he revisited the experience. He found himself shackled to a dozen other people. Several of those people would die in the coming weeks, while they were attached to each other.

And the torture. "They shock us. They beat us" until Abdulla's forearm broke. "They're pouring, they're pouring by fire", he said, referring to the melted plastic that was dumped onto his body and dripped into his eyes. Other victims were mutilated, their fingers cut off. Their bodies used as ashtrays. They were hung upside down for hours at a time.

"And they didn't give us even water". The only sustenance the captives received, Abdulla recalled, was "at midnight, something like this, they give us yogurt, a small cup of water". Just enough to keep the asylum seekers alive. But a number of them, a number of the people Abdulla was chained to, didn't make it. "A lot of people - they died. There was no water, they died. They died when they tortured them".

"They give you a phone to call them", your family, "to make just one call. My family [was] in a refugee camp then - in Darfur there is a big refugee camp called Zam Zam - and they didn't have the money to pay so I stayed [in Sinai] two months".

Eventually, his family raised the ransom and Abdulla's captors took him to the border. The wounds on his back from the melted plastic were infected; his arm needed to be set - it was healing crooked and was painful. But he made it across. 
Israeli soldiers picked him up and took him to Saharonim prison. He received medical treatment there and, while talking to the other Sudanese, realized an old friend had ended up in south Tel Aviv. Abdulla was released after a month; he and other prisoners were taken to Beer Sheva, where Israeli authorities gave them one-way tickets to the tachana merkazit (the Central Bus Station). "They say, 'You are free to go to the city'... they say 'You are free' and it's amazing to hear that", he recalled.

"And then I come to Central Bus Station and I don't know how to get out of [it]". He laughed at the memory. The tachana merkazit is a cement labyrinth of hallways and ramps and bends and dead ends and stairs and escalators that lead to more hallways and ramps and stairs. It was designed to confuse - in theory, the disoriented consumer, wandering among its many stores, would end up spending more money. But today many storefronts sit empty and the Central Bus Station is just somewhere to get lost.

Once Abdulla found his way out, he went to Levinsky Park. He called his friend from Sudan, who took him in and didn't charge him for rent and food. Abdulla was in no shape to work. He spent the next few months getting regular treatment for his burns at one of the clinics in south Tel Aviv he'd heard about from a human rights worker he met in Levinsky Park.

Healing his mind was another matter. Abdulla was suffering from post-traumatic stress disorder and needed treatment. But, other than the limited counseling services offered by ASSAF and the ARDC, there was almost no help available. This was early 2012; two years later the Israeli $\mathrm{MOH}$ and UNHCR would open a small clinic in Jaffa for asylum seekers suffering from mental health issues. The place can only accommodate a few dozen people a week, however, and it doesn't treat children.

As Abdulla struggled, alone, to recover from what he'd been through in Sinai, his friend gave him "money for alcohol because I couldn't sleep. I could stay for three days without sleeping" so disturbed was he by the memories of torture and death. "So every night, he give me money for one liter of whiskey or vodka and he say, 'This is strong, this will make you go to sleep.' So every night I have one liter of whiskey for like four months".

Realizing that his drinking was out of control, Abdulla quit. After six months in Israel, he felt he was ready to work. "I decided to go look for [a] job by myself. I feel like I'm not completely well but I'm well". He went to Eilat, where many asylum seekers had found work in hotels in the past. But by this time, in 2012, it proved more difficult. And so he moved on, yet again, finding work, eventually, in Netanya; then, later, in Tel Aviv. He also joined a theatre troupe - African Israeli Stage, which included both asylum seekers and Ethiopian Jewish Israelis. Performing, Abdulla explained, keeps him present, 
helping him cope with the trauma of the past as well as the uncertainty of his life in Israel. And when we met in 2015, four years after Abdulla had arrived, his future in Israel was more uncertain than ever - he faced imprisonment under the latest amendment of the Prevention of Infiltration Law.

$* * *$

In the winter of 2015, Yasser Abdulla was facing time in Holot (detention center for African asylum seekers). Despite the August 2015 court ruling, the summons he'd received said he would spend 20 months in the facility. He'd gotten a private lawyer - the NGOs were overworked and, in recent years, asylum seekers in Israel have become increasingly cynical about the human rights organizations. His attorney was fighting the detention on the grounds that Abdulla had been recognized by the UN as a victim of human trafficking.

I asked Abdulla what would happen if he ended up going to Holot.

"It's a prison, eh", he said. "So prison's prison anyways".

I pushed a bit, trying to get him to describe how detention feels - he'd been in jail in Sudan and had spent a month in Saharonim upon his arrival in Israel.

"[Have] you been in prison?" he asked. "It's very bad, I cannot explain to you how prison feels".

He mentioned the discrepancy between the summons he'd received and the High Court ruling, "On paper, they gave me 20 months, but there is [a] new law that they can't hold you more than a year". He shook his head and looked down at the table, where our Styrofoam cups of tea were growing cold. Either way, he said, "It's too much". 


\title{
Libia: dalle torture dei profughi nelle carceri libiche alla loro criminalizzazione in Europa
}

Marco Omizzolo

Eurispes, Amnesty International

\begin{abstract}
The essay analyzes the current state of detention of African refugees in Libyan detention centres, focusing on tortures and inhuman traitments and highlighting their criminalization in Europe.
\end{abstract}

Keywords Libya. Torture. Prison. Violence. Dehumanization. Fortress Europe.

Sommario 1 Introduzione. - 21 movimenti migratori e la trappola libica. - 3 Solo potenziali clandestini da respingere. - 4 La politica dei muri e le stragi di profughi al largo della Libia: dall'inferno agli abissi. - 5 Conclusioni.

\section{Introduzione}

In Libia, la schiavitù, la tortura, il razzismo, la violenza sistematica sono diventate una costante, sono state elevate ad impresa e a governo del territorio. La fragilità, il bisogno, la vulnerabilità, la ricattabilità degli emigranti - in particolare quelli provenienti dalla fascia subsahariana dell'Africa - li spingono nelle mani di trafficanti, mafiosi locali, gendarmi corrotti, sfruttatori e schiavisti che ne fanno patrimonio personale sul quale agire per ottenere potere e profitto. L'intricata situazione politica del Paese, in cui le responsabilità dell'Occidente sono enormi, rende strutturale questo sistema criminale.

Raccogliere le storie di vita e le testimonianze dei profughi torturati nelle carceri libiche è un compito fondamentale, urgente, doveroso, in termini di analisi e di denuncia sociale delle condizioni drammatiche di detenzione e sopravvivenza di migliaia di uomini, donne e bambini. Le interviste che pre- 
sento in questo saggio sono state condotte in prevalenza dagli operatori della cooperativa In Migrazione in collaborazione con alcuni ospiti del centro di accoglienza 'Casa Benvenuto' di Roma (Sprar), grazie alle relazioni da questi ultimi ancora intrattenute coi profughi reclusi nelle carceri libiche e conosciuti durante la loro passata esperienza nel Paese essendo stati anch'essi in stato di detenzione forzata. ${ }^{1}$

Sul piano metodologico, sia per la ricerca 'sul campo' che per l'elaborazione delle interviste, ho fatto riferimento alla multi-sited research, che trova il suo compimento nella riflessione di Marcus (1995) e che si caratterizza per la capacità di configurare locale e globale quali categorie in costante relazione, facendo corrispondere il secondo ad una parte integrante del primo. Lo studio cominciato nel 'sistema mondo' si è evoluto in uno studio del 'sistema mondo', che a partire dalla raccolta di storie di alcuni profughi torturati in Libia, analizza i rapporti di potere che si sviluppano tra dominati e dominanti, colonizzati e colonizzatori, carcerati e carcerieri, 'clandestini' e guardiani, emigranti e aguzzini. Questa metodologia ha permesso di raccogliere la dimensione verbale espressa dagli intervistati con riferimento alla loro condizione di vita nella detenzione forzata, e la dimensione sentimentale che tracima dalle loro parole e testimonianze, a sancire lo stato di gravissima sofferenza e tortura al quale sono stati o sono sottoposti.

Sono state raccolte anche alcune dichiarazioni di rappresentanti istituzionali che si sono occupati della questione libica. Tra le varie, ad esempio, la seguente: «I clandestini vengono accalappiati come cani, messi su furgoncini pick-up e liberati in centri di accoglienza dove i sorveglianti per entrare devono mettere i fazzoletti intorno alla bocca per gli odori nauseabondi [...]. Il centro prevede di ospitare cento persone ma ce ne sono 650, una ammassata sull'altra senza il rispetto di alcuna norma igienica e in condizioni terribili». ${ }^{2}$ Questa testimonianza risale al 2006 ed è dell'ex direttore del Sisde, Prefetto Mario Mori. Nonostante le gravi condizioni degli emigranti e il persistere delle pratiche di tortura, dal 2006 ad oggi tra la Libia e l’Italia sono stati stretti accordi, sono stati sottoscritti impegni economici milionari per la realizzazione di opere pubbliche atte a migliorare l'estrazione e la conduzione del petrolio e del gas, sono stati organizzati incontri istituzionali. ${ }^{3}$ A fronte degli investimenti programma-

1 Una parte di queste interviste sono state usate per redigere il dossier Trappola Libica (In Migrazione 2013).

2 «Immigrati: allarme Sisde, centri in Libia disumani», La Repubblica, 3 febbraio 2006. URL http://ricerca.repubblica.it/repubblica/archivio/repubblica/2006/02/03/ immigrati-allarme-sisde-centri-in-libia-disumani.html (2019-10-12).

3 Riporto quanto previsto dalla relazione finanziaria annuale dell'Eni del 2008: «È stato perfezionato con effetti economici dal $1^{\circ}$ gennaio 2008 l'accordo minerario strategico 
ti, la Libia resta un luogo di detenzione brutale e disumano, ${ }^{4}$ complici l'Europa e Italia.

La morte di Gheddafi nell'ottobre 2011 ha dato il via a una cronica instabilità politica. Le autorità libiche non sono state in grado 0 non hanno voluto porre fine alle violenze e ai soprusi commessi contro i rifugiati. Il vuoto lasciato dallo Stato in seguito alla rivolta del 2011 e il conflitto armato che ne è scaturito, nonché le pressioni imposte dal Daesh, è stato riempito da una moltitudine di gruppi armati spesso in conflitto tra loro, che sono riusciti a prendere possesso di aree molto grandi del territorio libico anche grazie al saccheggio delle armerie. Questi gruppi armati sono penetrati, spesso col silenzio colpevole dell'Unione europea e dei singoli Stati aderenti, anche nelle istituzioni, finendo col condizionarne l'agire sino a tollerare abusi e violenze di varia natura, a partire dalla tratta di esseri umani, sino alle violenze e torture sui profughi. Questo stato drammatico si inserisce in un quadro già precedentemente deteriorato e orientato verso il disconoscimento dei diritti umani. La legge libica, infatti, criminalizza l'ingresso irregolare, il soggiorno o l'uscita dal Paese con una pena detentiva in attesa di espulsione, senza alcuna considerazione di circostanze individuali o esigenze di protezione. Il profugo viene arbitrariamente detenuto per un tempo indefinito. La Libia non ha un sistema di asilo, non ha ratificato la Convenzione del 1951 sullo status dei rifugiati, non riconosce formalmente l'Alto commissariato delle Nazioni Unite per i rifugiati (UNHCR), raramente consente all'agenzia di registrare alcuni richiedenti asilo e rifugiati da un numero limitato di Paesi. Queste sono alcune delle ragioni alla base del fatto che in questo Paese si sia sviluppato un sistema di tratta e maltrattamento così pervasivo e violento.

Alle rivalità tribali e tra clan potentemente armati, si associano milizie autonome consolidatesi dopo il 2011 spesso legate a capi locali presenti in diverse città. A questi si sommano gruppi malavitosi che si spartiscono il mercato del malaffare, a cominciare dal traffico di esseri umani alimentato dalle migliaia di profughi che affluiscono dall'Africa subsahariana, con la speranza, tradita dalle politiche di chiusura dell'Unione europea e, in particolare, dall'Italia, di pro-

definito nell'ottobre 2007 tra Eni e la società petrolifera di Stato NOC che estende, tra l'altro, la durata dei titoli minerari Eni in Libia fino al 2042 per le produzioni a olio e al 2047 per quelle a gas, e individua una serie di iniziative per la valorizzazione dell'ampia base di riserve, in particolare attraverso la realizzazione di importanti progetti gas» (http://civilistico.bilanciinterattivi.com/eni_2009_ita/index.php?id=47, 201910-12). Allo stesso modo, dal trattato di Bengasi emergono le implicazioni di numerose aziende italiane (Impregilo, Finmeccanica, Eni, etc.) in vari settori: dal contrasto all'immigrazione, alla difesa, alla costruzione di infrastrutture (Iacovino 2010).

4 Oltre ai rapporti annuali di Amnesty International, si veda il report UNSMIL, OHCHR 2013. 
curarsi un imbarco per l'Europa. Un malaffare che genera milioni di dollari, come conferma un rapporto dell'ONU del dicembre 2016, nel quale risultano implicati funzionari statali, militari, ufficiali ed agenti di polizia (UNSMIL, OHCHR 2016, 5).

\section{I movimenti migratori e la trappola libica}

Dall'inizio del Duemila, per ragioni che attengono al quadro geopolitico internazionale, la Libia è diventata un'importante destinazione e punto di transito per gli emigranti originari soprattutto della fascia subsahariana e, in parte, asiatici. Nel 2018, la stima dei profughi presenti nel Paese era di 704.142, ossia circa il 12\% della popolazione locale (OIM 2018a, 4). Questo movimento migratorio si è strutturato a partire dal 2014, quando il Paese è diventato, a tutti gli effetti, il principale luogo di transito per le persone che ambivano a raggiungere l'Europa e il principale tragitto preferito dai trafficanti di esseri umani. Secondo l'OIM, nel 2016, 181.436 persone (nel 2017 sono stati 119.247) sono arrivate in Italia attraverso la rotta del Mediterraneo e di queste circa il $90 \%$ ha attraversato la Libia. La rotta del Mediterraneo centrale, oltre ad essere la più percorsa, è anche quella più pericolosa e con il più alto tasso di mortalità: dal 2014 al 2018 il numero di persone decedute in questa tratta è di circa 25.000. Solo nel 2017, un emigrante su trentasei è morto nel tentativo di attraversare il Mediterraneo. Da gennaio ad aprile 2018, a fronte di 15.129 arrivi, sono morte 498 persone (OIM 2018b).

Sempre secondo l'OIM, in Libia ogni anno circa 7.000 emigranti sono vittime di tratta e subiscono violenze di varia natura. La gran parte ha affermato di avere assistito, in prima persona, a omicidi e torture. Alcuni testimoni diretti raccontano di bambini venduti come schiavi. Il traffico di uomini (ticket per i viaggi a terra dal confine sahariano alla costa del Mediterraneo, passaggi in mare, riscatti per essere liberati dai centri di detenzione o dalle carceri improvvisate dei trafficanti, ecc.) corrisponde a circa il 10\% del PIL libico. Si tratta della seconda 'voce' in assoluto del 'bilancio' del Paese dopo quella legata al petrolio. Ė emblematica la testimonianza di Erik (nome di fantasia), giovane eritreo intervistato attraverso una chiamata sul suo cellulare mentre era ancora in stato di detenzione in Libia:

la prigione è sotto il controllo dei militari e noi siamo tornati ad essere prigionieri; ci costringevano ai lavori forzati pulendo carri armati ed armi. Da Kufrah ci hanno portato in aereo a Ganfuda dove sono rimasto quasi due mesi. Ora con un po' di fortuna e molta fatica sono riuscito ad uscire; lavoro in una fattoria di un padrone libico, in attesa di trovare il denaro sufficiente e il momento giusto per cercare di raggiungere mio fratello in Italia. 
Nelle carceri libiche è presente una popolazione reclusa senza motivo, se non per il fatto di essere degli emigranti, degli espiantati, dei profughi, in un Paese governato da milizie criminali, para-statali, agevolate da accordi internazionali di cui l'Italia era ed è protagonista. ${ }^{5}$ Sotto questo aspetto è sempre più evidente la relazione tra trafficanti di uomini e milizie libiche. L'industria delle migrazioni e della tortura è infatti parzialmente controllata in ogni passaggio della sua filiera da gruppi armati.

In Libia gli emigranti, i profughi, non sono tutti uguali. Chi viene dall'Africa subsahariana vive condizioni nettamente più difficili. Se si è donna la propria condizione muta in peggio per il rischio costante di stupri e violenze. I bambini sono a rischio di rapimento, violenze, anche sessuali, vendita clandestina, traffico di organi. Mohammed, giovane eritreo di 19 anni, recluso per un anno in un carcere segreto libico, parla così del suo viaggio nel deserto:

Il viaggio è difficile [...] il deserto è sempre il deserto [...] durante il viaggio ci sono tanti problemi. Per fortuna siamo stati solo 8 giorni, ma ci sono persone che ci sono state anche 20 giorni nel deserto. Non solo hanno perso tempo ma hanno perso anche persone, per la sete [...] se parti in 20 arrivi in 10! È molto difficile perché non puoi credere a nessuno: tutto è stato estremamente rischioso. Qui il discorso è questo: i trafficanti del Sudan non sanno la strada. Loro conoscono solo un altro tramite e ti vendono a un altro sudanese. Il sudanese ti rivende poi a un trafficante libico. Così ci scambiano tra di loro e lì nasce il problema perché quando ti passano da uno all'altro, ognuno vuole avere qualcosa e ti chiedono soldi oltre le tue possibilità e oltre quello che avevi pattuito alla partenza [...] per avere questi soldi a un uomo hanno infilato un tubo di plastica nell'ano, ci sono questo tipo di umiliazioni e torture. Le persone per attraversare il deserto superano queste difficoltà.

5 Il 2 febbraio 2017, ad esempio, il Governo italiano ha firmato un Memorandum d'intesa con il governo di unità nazionale di Tripoli, approvato il giorno successivo dai capi di Stato e di governo nell'incontro informale di Malta. Tale accordo non rispetta i diritti umani né è conforme al diritto internazionale. La Libia non ha mai firmato la Convenzione sui rifugiati del 1951. Sotto questo profilo, l'art. 20 della l. 6/1987 che disciplina l'ingresso, la permanenza e l'uscita degli stranieri dal Paese, stabilisce che chi entra irregolarmente soggiace alla pena del carcere e a una sanzione pecuniaria (MHUB 2015; Global Detention Project 2015). L'art. 6 della l. 19/2010 (per combattere l'immigrazione 'illegale') specifica che chi entra illegalmente nel Paese è condannato alla detenzione con il lavoro forzato ed è espulso al termine del periodo detentivo. Pur non essendo espressamente consentita, la detenzione a tempo indefinito non è esclusa dal citato art. 6 , che non prevede alcun termine massimo di custodia. Il Governo italiano ha varie volte enfatizzato come l'accordo sia stato firmato per porre fine alle morti in mare e ai viaggi della speranza gestiti dai trafficanti di esseri umani, tuttavia il tasso di mortalità nella rotta del Mediterraneo centrale non è variato significativamente nel corso degli ultimi anni: attualmente questa la rotta si conferma come la più pericolosa al mondo. 
Mogos, giovane sudanese di 20 anni che ha trovato posto in un centro di seconda accoglienza a Roma, racconta che «il viaggio è andato male, molto male. Come ti spiego [...] abbiamo viaggiato per dodici giorni, eravamo 50 persone ammassate su un camion». Mohammad, somalo di 32 anni, dice che «gli ostacoli che ci sono in Libia non si possono neanche immaginare. I trafficanti sono più numerosi dei viaggiatori e ogni trafficante pensa solo al suo interesse: prima per esempio prende un accordo con te per 400 dollari e poi ti porta in un appartamento chiuso e ti chiede 800 dollari e non hai scelta. Sei rinchiuso senza poter uscire finché non porti i soldi. Questi trafficanti fanno accordi con i libici».

I profughi intervistati raccontano di loro compagni e compagne di viaggio rimasti nel deserto, che per disperazione si buttano giù dai pick-up sovraffollati, o che ne sono sbalzati fuori o sono costretti dai trafficanti a scendere. Le persone vengono scaricate al confine con il Sudan e il più delle volte consegnate alla controparte libica dell'organizzazione criminale. Yasin, 25 anni, uscito dal carcere libico di Ganfuda, è arrivato a Tripoli dopo essere passato per Benghazi; la sua unica speranza era quella di essere salvato da una nave umanitaria; il rischio era altissimo ma lo ha affrontato sapendo che non aveva alternativa: «meglio che rimanere in questa situazione così pericolosa [...] senza alcuna scelta 0 alternativa [...] perché anche loro [i libici] ci dicono: 'non vogliamo vedere la pelle nera', perché questa è la situazione». Razzismo e rastrellamenti sono una costante. C'è una sorta di 'caccia al profugo' con risvolti drammatici, come racconta Tesfu: «stiamo ancora aspettando per partire. Il problema è che adesso abbiamo paura perché tutte le persone che trovano in strada li portano subito in carcere, ma senza alcun motivo, basta che sei nero e ti portano in carcere. Le carceri sono piene di persone».

A questa pratica si aggiunge quella del prelievo coatto, ossia del rapimento direttamente negli appartamenti dei quartieri di Benghazi e Tripoli, per deportare emigranti, profughi, nelle carceri e nei centri detentivi. Aron, dalla Libia, afferma che

è difficile rintracciare qualcuno in questo Paese, fanno rastrellamenti negli appartamenti, l'altro giorno ne hanno presi 50. Hanno sparato ad A.S. su una mano e non sappiamo dove sia ora. Tripoli è una città pericolosissima, anche se hai il pass dell'UNHCR non sei al sicuro. Anche lì ad Abu Salim [quartiere di Tripoli] c'è una ragazza che è impazzita [...] non ti posso raccontare tutto [...] in questo Paese succedono un sacco di cose [...] abbiamo difficoltà, la situazione è troppo pericolosa.

Su due campi profughi libici è possibile concentrare, sia pure brevemente, l'attenzione. Si tratta dei campi di Tarek al Matar e Tarek al Sika, nei sobborghi di Tripoli. Entrambi sono sotto il controllo del 
Governo e sono considerati 'sicuri' tanto che nel 2018 avrebbero ricevuto la visita di una delegazione dell'Unione europea. Una visita che è stata premessa per le dichiarazioni del ministro dell'Interno italiano circa la sicurezza e l'affidabilità dei campi profughi libici. In realtà sono affollati al punto che nei capannoni-prigione, come risulta dalle testimonianze raccolte, non è possibile muoversi e spesso nemmeno sdraiarsi. Il cibo è pessimo e molto scarso; i servizi igienici pressoché inesistenti e inagibili; non c'è alcuna assistenza medica; viene lesinata ed è di infima qualità persino l'acqua da bere. La giornata è scandita da tormenti terribili e non di rado si muore: per malattia, per denutrizione, per sfinimento. Nell'agosto 2018 a Tarek al Matar hanno perduto la vita almeno tre giovani, un eritreo e due etiopi, nel giro di meno di tre settimane. Alle condizioni inumane di trattamento si aggiunge un senso di assoluta insicurezza. Molti dei detenuti sostengono di non essere stati registrati al momento del loro ingresso nel campo, sicché non risulterebbero nemmeno presenti. «Siamo come dei fantasmi senza nome - hanno raccontato a Mohammad, giovane eritreo ospite nel centro Sprar 'Casa Benvenuto' di Roma - Ci possono far sparire in qualsiasi momento, senza lasciar traccia: basta che dicano di non averci mai visto, citando come prova proprio i registri del campo».

Quanto sia avvertito questo rischio lo dimostrano due episodi denunciati all'inizio di agosto del 2018. Il primo a Tarek al Matar, il secondo a Tarek al Sika. Il caso più grave, anche se il numero dei profughi coinvolti è minore, sarebbe quello di Tarek al Matar, dove è stata segnalata la 'sparizione' di una ventina di giovani, dieci eritrei e dieci somali. A raccontare quanto è accaduto è stato un ragazzo diciottenne, catturato a Homs nel gennaio 2018 da un gruppo di trafficanti e arrivato prima a Tripoli e poi a Tarek al Matar, dopo che la sua famiglia è riuscita a pagare i 12 mila dollari chiesti dai sequestratori per rilasciarlo. Un racconto in 'diretta', fatto attraverso una serie di telefonate protrattesi per oltre un'ora, proprio mentre si svolgevano i fatti. Nella tarda serata, intorno alle 20 (ora italiana) alcune guardie del campo avrebbero cominciato ad ordinare a diversi profughi, in gran parte non registrati, di seguire un libico, arrivato poco prima, per lavorare come braccianti agricoli. Un uomo, raccontano i testimoni, che sembrava in grande familiarità con il personale in servizio nel campo.

La nostra risposta [ha riferito il testimone nelle sue telefonate] $e$ stata un rifiuto in massa: nessuno di noi voleva muoversi dal campo. Avevamo tutti paura di andare con quello sconosciuto. Paura di essere ceduti come lavoratori schiavi o, peggio, di essere venduti, prima o poi, a una banda di trafficanti, come è capitato a me all'inizio dell'anno. Tutti insieme ci siamo rifugiati in una zona appartata del campo per tentare di sottrarci alla consegna forzata e 
urlando la nostra disperazione per sollecitare sostegno e aiuto da altri compagni. Abbiamo resistito per un'ora circa, poi una ventina di noi sono stati prelevati da un gruppo di miliziani del servizio di vigilanza e costretti a seguire quel libico fuori dal campo. Se ne sono dovuti andare così come si trovavano, solo con gli indumenti che avevano indosso. Anzi, credo che li abbiano obbligati a lasciare anche i cellulari. Io sono riuscito a nascondermi. O forse alle guardie bastavano quelli che avevano ormai preso. Così l'ho scampata.

Dalla notte dei fatti nessuno ha saputo più nulla di questi venti ragazzi, alcuni dei quali minorenni, almeno fino alla sera del 5 agosto del 2018, quando uno di loro è stato ricondotto a Tarek al Matar.

Dopo averci presi ci hanno portato con un furgone chiuso in una grossa costruzione in muratura attrezzata come una prigione. Non so dire dove si trovi. Però non deve essere lontana, perché il viaggio è stato breve. Avevano detto che saremmo andati a lavorare. Invece siamo rimasti rinchiusi lì per tutto il tempo, senza poter uscire neanche per pochi minuti. Ne abbiamo dedotto che in realtà volevano venderci. Così ci è sembrato di capire anche origliando i discorsi che facevano tra loro alcuni guardiani. Nel tardo pomeriggio di domenica, poi, sono arrivate due guardie a prendermi. Cercavano proprio me, perché mi hanno chiamato per nome. Mi sono impaurito ma loro hanno detto subito che erano lì per riportarmi a Tarek al Matar. E agli altri hanno assicurato che anche loro dovrebbero tornare tra non molto. E tutto molto strano.

L'episodio di Tarek al Sika, avvenuto tra il 2 e il 3 agosto 2018, seguirebbe lo stesso copione. I profughi interessati, però, in questo caso sarebbero circa 200. I fatti sono stati ricostruiti grazie a testimonianze raccolte attraverso una serie di concitate telefonate fatte durante la notte da alcuni profughi. Nella seconda metà di luglio (probabilmente dopo il 20 del mese), l'intero gruppo è stato trasferito da Tarek al Sika in un'altra prigione, situata nei dintorni di Tripoli: una grossa struttura in muratura, con un cortile circondato da un muro di cinta alto circa due metri, sicuramente non molto lontano perché ci sono arrivati in meno di un'ora di camion. Quasi tutti si trovavano a Tarek al Sika ormai da tempo e buona parte, circa 110, risulterebbero registrati dall'UNHCR nell'ambito di un programma di relocation verso il Niger e da qui, possibilmente, verso l'Europa. I problemi sono iniziati quando, la sera del 2 agosto, sarebbe arrivato al campo un libico sconosciuto ai profughi ma che si sarebbe mosso con estrema disinvoltura e che, presenti le guardie stesse, avrebbe avvicinato gli emigranti, prospettando la possibilità di farli imbarcare entro pochi giorni per l'Italia da un «posto sicuro» e con una organizzazione «affidabile». Per vincere la loro diffidenza, quell'uo- 
mo li avrebbe messi in comunicazione, per telefono, con un eritreo il quale a sua volta, parlando in tigrino, avrebbe confermato che, affidandosi alla persona che li aveva contattati, si sarebbero potuti imbarcare entro pochi giorni. Nessuno dei duecento si è lasciato convincere, anzi molti hanno cominciato a protestare. Tra tutti, ma in particolare tra i novanta che non risulterebbero registrati, era fortissimo il sospetto che si fosse messa in moto un'operazione per cederli come lavoratori-schiavi.

Ci hanno detto [hanno denunciato telefonicamente] di averci trasferito in un campo controllato dal Governo di Tripoli per decongestionare l'affollamento enorme di Tarek al Sika. Ma questo nuovo centro appare fuori controllo. Il personale di guardia non è nemmeno in divisa e non sembra esserci una struttura operativa organizzata. Chiediamo di essere riportati dove eravamo prima, a Tarek al Sika. Certo, lì le condizioni di vita sono durissime, ma almeno è sicuramente una struttura 'ufficiale', gestita da autorità istituzionali. Qui non si capisce bene nemmeno chi siano i nostri carcerieri. É assurdo che quel libico a noi sconosciuto, che sembrava però in grande familiarità con le guardie, sia potuto entrare liberamente e ci abbia messo in contatto con un eritreo che ha parlato come un emissario dei trafficanti di uomini. Lo abbiamo fatto presente a un funzionario arrivato da Tarek al Sika il 3 agosto, dopo la nostra protesta della notte precedente. Ma lui non sembra aver dato peso a quanto denunciavamo. Anzi, dopo aver parlato con alcune guardie, ha ribadito la sua fiducia in quell'uomo, aggiungendo che starebbe addirittura aiutando la polizia a combattere i clan criminali che gestiscono il mercato di esseri umani.

I casi di Tarek al Matar e Tarek al Sika si sono verificati all'indomani del respingimento forzato di 101 persone (tra cui 5 bambini e 5 donne in stato di gravidanza) effettuato dalla nave italiana Asso Ventotto. Trasbordati nel porto di Tripoli su una unità della Guardia Costiera libica, quelle 101 persone sono state sbarcate nella base militare di Abu Sitta. Tutti i profughi che arrivano qui dopo pochi giorni vengono distribuiti nei centri di detenzione della zona, come Tajoura, Ain Zara e, appunto, proprio Tarek al Matar e Tarek al Sika. Per almeno gli ultimi tre governi italiani, però, tutto risulta essere stato sempre regolare. Regolare che profughi salvati da una nave italiana vengano riportati contro la loro volontà in Libia e che quei profughi finiscano in gironi infernali come Tarek al Matar e Tarek al Sika, ma anche Tajoura, Ain Zara, Zuwara, Homs, Gharyan, Bani Walid. Luoghi di detenzione arbitraria e tortura dove decine di persone possono essere fatte sparire senza lasciare traccia. Quella della regolarità di queste procedure è la condizione di una corresponsabilità tra i governi italiani e i trafficanti, torturatori e sfruttatori libici, responsabili di palesi e drammatiche violazioni dei diritti umani e di crimini. 
Infine si può citare, tra le molte storie, quella di Ismael, un ragazzo eritreo scappato dalla carceri libiche insieme alla moglie e ai suoi due bambini, di cui la più piccola nata addirittura in mare. Il loro viaggio è però terminato perché intercettati dalla motovedetta libica (armata e addestrata da vari governi italiani). Ismael e la sua famiglia erano scappati dalla Libia dopo aver sopportato mesi di torture in un hangar dove venivano sistematicamente picchiati e sua moglie stuprata nel tentativo di estorcere denaro ai loro parenti. Il loro respingimento e ritorno in Libia li ha gettati in uno stato di sconforto e depressione molto grave. Secondo le ultime testimonianze raccolte, la moglie di Ismael e la figlia sarebbero state portate prima in un centro di detenzione a Tripoli e poi in un altro nella stessa città dove hanno vissuto condizioni igieniche precarie tanto che, per l'assenza di cibo, acqua potabile e assistenza, la bambina di pochi mesi è deceduta. ${ }^{6}$

\section{Solo potenziali clandestini da respingere}

I casi e le testimonianze di donne e uomini illegalmente detenuti e torturati, nella narrazione corrente vengono ricondotte, anche dal governo italiano, alla categoria dei 'clandestini'. Si tratta di un uso razzista di un lessico costruito per emarginare migliaia di persone. A questo proposito, occorre sottolineare come la costruzione della clandestinità inizi ben lontano dai Paesi d'approdo. Lungi dall'essere una categoria solo giuridica, la figura del 'clandestino' (sia nella percezione dei protagonisti che in quella delle società di transito 0 d'arrivo) si forma tramite un immaginario che caratterizza il viaggio sin dalla decisione di lasciare il proprio Paese, per la necessità di iniziare a muoversi nell'oscurità e affidarsi a intermediari. Condizione di clandestinità che è spesso richiamata anche dal governo italiano quale condizione per i rimpatri forzati, i respingimenti, i rimbalzi delle navi umanitarie verso Paesi europei se non verso addirittura la Libia. Tra le interviste raccolte è particolarmente significativa quella di Ahmed, che dalla Libia ha affermato: «Basta [...] siamo disperati [...] stiamo tutti pensando di andare verso Tripoli e andare via mare [...] Anche se è peggio, ma che facciamo [...] siamo come animali legati un po' con la corda lunga [...] siamo così».

Ahmed è stato contattato nel campo profughi (attualmente dismesso) della Mezzaluna Rossa di Benghazi con almeno 500 'ospiti' tra somali, etiopi ed eritrei. I profughi intervistati nel carcere di Kubz hanno denunciato la presenza di altrettante persone. Coloro che sono stati intervistati in prigione si trovavano invece nel carcere di Gan-

6 Questa storia è stata raccolta ancora una volta per mezzo di una telefonata con un profugo in Libia che si è dichiarato amico e compagno di prigionia di Ismael. 
fuda, a circa dieci chilometri dalla città di Benghazi. Le carceri in questo caso hanno celle in cui i detenuti sono suddivisi per nazionalità. I detenuti intervistati a Ganfuda sono stati arrestati attraverso retate condotte da ronde organizzate dai militari libici. Altri prigionieri in principio erano internati nel centro di detenzione di Kufra, piccolo villaggio di transito lungo la rotta tradizionale del Corno d'Africa, tra Khartoum e le città libiche della costa, nel deserto del Sahara, e successivamente sono stati trasferiti a Ganfuda (carcere dove nel 2009 si è verificata la più grave strage avvenuta nei campi di detenzione libici) a seguito di uno sciopero della fame e di una manifestazione repressa violentemente dai militari libici nell'aprile 2012.

L'accusa, per tutti i detenuti, è di essere dei potenziali clandestini in procinto di raggiungere le coste europee e soprattutto italiane. Molti di loro vengono reclusi per diversi mesi; non hanno un avvocato, non hanno un processo in corso, non c'è un atto amministrativo che certifichi la loro presenza in quel luogo, dormono per terra, senza reti o, nella migliore delle ipotesi, su materassi consumati, su stuoini. Ogni giorno subiscono umiliazioni e vessazioni da parte dei militari. Le condizioni in carcere sono pessime, sia a livello igienico che sanitario (non ci sono né medici né medicine). Se qualcuno si ammala, il destino è l'abbandono e la morte.

Nelle celle di circa 30 metri quadrati sono rinchiuse anche sessanta persone, tenute a pane e acqua distribuiti tre volte al giorno. Ci sono anche minori non accompagnati, come Mogos, fuggito dal campo d'addestramento militare di Sawa, in Eritrea, dove gli studenti trascorrono l'ultimo anno di scuola secondaria. In prigione non c'è speranza. Chi prova a fuggire è braccato e picchiato sotto le piante dei piedi, secondo la pratica della falaqa. Il solo modo di uscire è quello di essere scelti per lavorare da libici locali in cerca di forza lavoro da impiegare in condizione di grave sfruttamento. Sfruttamento lavorativo, detenzione illegale, tortura, sono aspetti di uno sistema che fonda la sua legittimazione sulla violenza organizzata.

I libici in cerca di manodopera pagano il carceriere di turno e riscattano il detenuto per impiegarlo a costo zero (o quasi) nelle proprie aziende, facendo attenzione a scegliere solo persone con il passaporto, in modo da sequestrarlo ed evitare la fuga del lavoratore. Un'altra possibilità è la corruzione dei guardiani da parte dei trafficanti. In alcuni casi questo denaro viene raccolto dal profugo chiamando con il cellulare un familiare residente in Europa, mentre viene torturato dal suo aguzzino e carceriere. Le urla e le grida di dolore convincono chi sta dall'altra parte del telefono ad inviare denaro secondo le modalità richieste dal torturatore.

Le carceri, i campi di detenzione e le strutture adattate a prigioni in questo Paese sono decine: Ganfuda, Majer, Misurata, Zlitan, Abu Salim, Khums, Garabulli, Bin Ulid e Al-Zawiya, Jadida, Fellah, Twaisha, Ain Zarah, etc. I prigionieri sono migliaia e sono rinchiusi senza 
accuse né processi. Peraltro è molto difficile distinguere un centro di detenzione da un luogo di prigionia, un arresto da un rapimento, un poliziotto o un militare da un miliziano. Secondo le stime di Amnesty International, ${ }^{7}$ nel 2013 erano operativi almeno 17 centri di trattenimento in cui vi erano circa 5.000 emigranti a cui si aggiungono le altre diverse migliaia che affollano le carceri comuni e i campi di accoglienza gestiti dai miliziani, stimabili tra le 4.000 e le 6.000 persone. Si parla di poco meno di 30 stabili adibiti a centri detentivi.

La testimonianza di John, ragazzo originario del Senegal e incarcerato a Ganfuda, è emblematica:

Violenza, torture, non ho modo di descrivere quelle cose, ti tirano il cibo in faccia, ti picchiano senza alcun motivo, ti prendono a schiaffi [...] ti minacciano con i fucili e le pistole, qualsiasi libico ora ha fucili o pistole [...] ti minacciano, te le puntano alle tempie. Mi ricordo un giorno, un libico ha picchiato con la sedia un ragazzo e le donne hanno iniziato a urlare e da quel momento è scoppiata una rissa. Poi abbiamo resistito, abbiamo fatto lo sciopero della fame e hanno iniziato a picchiarci e anche a sparare. Quando hanno sparato è stato ferito un ragazzo che si chiama S. [...] ci hanno messo in una stanza in 420 persone. Non ci potevamo neanche sedere per quante persone c'erano [...] tante persone svenivano, perdevamo i sensi [...] dopo qualche giorno ci hanno trasferito in un altro carcere. Alcuni sono andati a Kubz, è molto rigido questo carcere. Io sono stato invece trasferito in una scuola trasformata in un carcere dove sono rimasto per un mese, vicino Sibrata. Poi dopo questa sofferenza così lunga sono riuscito a scappare.

E utile ricordare che a fine dicembre 2017 più di mille giovani eritrei ed etiopi, sequestrati al confine tra il Sudan e la Libia, erano segregati a Berk dai trafficanti da oltre sei mesi in un'enorme prigione. Eludendo la sorveglianza dei miliziani di guardia, alcuni di loro sono riusciti a lanciare una disperata richiesta di aiuto. «Negli ultimi mesi - hanno denunciato - sono morti almeno sei nostri compagni: li hanno uccisi i pestaggi feroci, sistematici, delle guardie, la fame, le ferite infette e le malattie. Il cibo è scarso e cattivo, poca anche l'acqua da bere. E per chi sta male non c’è alcun tipo di cura medica».

Nel 2013 il comitato internazionale della Croce Rossa ha visitato 60 strutture detentive. Su tutti, per condizioni di vita e vessazioni ai danni dei profughi, il campo di Kufra e quello di Sabha sono risultati i più duri. Quest'ultimo 'ospita' al suo interno 1.300 persone. In questi luoghi sono moltissime le denunce di trattamenti crudeli e degra-

7 https://www.amnesty.it/libia-rifugiati-richiedenti-asilo-e-migranti-detenuti-a-tempo-indeterminato-in-condizioni-deplorevoli/ (2019-10-12). 
danti, percosse, stupri e torture. Ahmed, recluso nel centro di detenzione di Sabha, afferma che «la situazione non è vivibile, non c'è neanche il cibo a sufficienza [...] questa situazione è tipo un carcere, non è proprio un campo, è peggio di Ganfuda, non possiamo uscire».

Per i minori, come accennato, la situazione non è meno traumatica. Molti di loro, arrivati al seguito delle loro famiglie o da soli, vengono rinchiusi insieme a tutti gli altri, subendo le stesse violenze. Per avere un'idea di quanti siano i bambini e i ragazzi a passare per la detenzione, basti pensare che solo nel 2011 sono sbarcati a Lampedusa quasi 4.500 minori non accompagnati. Affermano, come le donne che arrivano in numero sempre maggiore, che la fuga e il rischio della morte in mare è meglio dell'incubo di una vita in Libia.

Alle donne non è concessa autonomia e sono soggette a numerose violenze, soprattutto sessuali. «La situazione è drammatica - afferma ancora Tesfu - in carcere ti danno un pane al giorno, solo un pane, poi c'è la tortura [...] ti picchiano in ogni modo possibile [...] se provi a scappare, se fai qualsiasi cosa ti picchiano con il bastone. Le donne vengono stuprate e mandate via».

Il rischio di essere stuprate durante la fuga verso il Mediterraneo e l'Europa è così alto che, prima di avventurarsi in Libia dal Sudan o dal Niger, molte di loro, per evitare una possibile gravidanza, si sottopongono, per almeno tre mesi, a iniezioni di forti dosi di contraccettivi (Drudi 2017). La notizia di questa 'assunzione preventiva'di farmaci (spesso con pesanti conseguenze sulla salute) era filtrata a Roma in alcune confidenze raccolte da medici e operatori sanitari dei centri di accoglienza della Croce Rossa; poi è stata conferma dall'ONU sulla base di una serie di testimonianze di donne di varie nazionalità arrivate dal Sudan. I racconti sono raccapriccianti: episodi di autentica schiavitù, con i carcerieri o i trafficanti che dispongono quando, come e per tutto il tempo che vogliono delle donne scelte. Una ventenne eritrea ha riferito che per oltre un mese una delle guardie l'ha prelevata tutte le notti dal capannone-prigione dove era rinchiusa con le compagne. Anna Lobkowicz, del servizio di assistenza internazionale dell'Ordine di Malta di Berlino, ha raccolto la storia di una siriana, madre di tre bambini di undici, nove e un anno, che «è stata violentata quotidianamente dal trafficante di esseri umani davanti ai suoi figli, è arrivata in Germania al nono mese di gravidanza, ha partorito ed ha detto che quel bimbo non lo voleva tenere».

Una volta fuori dal carcere, si cerca di attraversare il Mediterraneo: si prendono contatti con i trafficanti e si cerca di accumulare o farsi inviare dai familiari i soldi necessari per pagare la traversata.

Una volta usciti da un sistema infernale di tali dimensioni, il percorso è sempre lo stesso: divisi per nazionalità e rinchiusi in appartamenti dove si sopravvive a stento in condizioni terribili. Uscire dall'appartamento non è possibile. È il trafficante che provvede a 
fare la spola e a portare beni di prima necessità. In un secondo momento si viene portati in fattorie dell'entroterra (msahra) nell'attesa delle giuste condizioni ambientali ed economiche per la partenza. Le diverse nazionalità, gestite da diversi trafficanti, si trovano, infine, riunite sulla costa, nei pressi del barcone, con lo scafista che provvede alla traversata.

\section{$4 \quad$ La politica dei muri e le stragi di profughi al largo della Libia: dall'inferno agli abissi}

Le torture praticate in Libia ai profughi reclusi nelle carceri sono la premessa ed una delle ragioni che spingono migliaia di loro a tentare la traversata del Mediterraneo, generalmente organizzata da trafficanti di esseri umani. Molti di questi viaggi si trasformano in tragedie. Stragi che vengono di fatto indotte dalla politica dei muri, dei respingimenti, degli accordi bilaterali e multilaterali (alcuni dei quali vedono l'Italia protagonista), dell'espulsione delle navi delle Ong.

Tutto questo mentre in Europa i discorsi e politiche mirano ad espellere e a recludere gli emigranti. In Italia, sui media e nel dibattito politico trovano spazio le accuse alle navi umanitarie e alle Ong «complici dei trafficanti», così come i discorsi sugli «stranieri invasori». Sono questi gli argomenti alla base della politica dei 'muri'. Muri fisici come quello di Ceuta e Melilla, le due enclave spagnole in Marocco, o quello sulla frontiera dell'Evros, tra Grecia e Turchia; muri politico-legali, che esternalizzano i confini dell'Europa in Africa o in Medio Oriente, il più a sud possibile, sempre più a Sud, affidando alle polizie degli Stati contraenti il lavoro sporco di sorvegliare e impedire agli emigranti di varcarli. Si è cominciato con il Processo di Rabat nel 2006 per giungere alla chiusura pressoché totale di oggi attraverso il Processo di Khartoum (2014), gli accordi di Malta (2015), il trattato con la Turchia (2016), il memorandum con la Libia (2017) e i patti particolari che ne sono seguiti, inclusi quelli con alcune delle principali tribù perché «si prendano cura» dei profughi in arrivo dal Sud. La chiusura dei porti e la guerra alle Ong sono l'ennesimo atto a cui seguono alcune norme italiane a partire dal decreto sicurezza e dal decreto sicurezza bis del governo italiano.

La politica e quasi tutto il sistema di informazione misurano il successo di questo programma unicamente sulla diminuzione degli sbarchi in Europa. Il fatto stesso che arrivino meno persone, secondo questa tesi, dimostrerebbe che il sistema adottato funziona. Nel 2018 in Italia si è registrato un calo degli sbarchi del 77\%, confermando una tendenza iniziata nella seconda metà del 2017. Questa tesi tralascia completamente il fatto che non si tratta semplicemente di numeri e di statistiche, ma di esseri umani, ciascuno con una propria storia e progetto di vita. Si sostiene che il fatto stesso che ne possano par- 
tire di meno fa diminuire anche il numero delle vittime; in realtà la Guardia Costiera libica intercetta i profughi in mare, li riporta indietro e li arresta, rinchiudendoli nei centri di detenzione gestiti dalle milizie, dai trafficanti o dal governo di Tripoli. La stessa Corte Penale Internazionale ha aperto un'istruttoria su tutto il sistema libico di gestione degli emigranti e, in particolare, sulla Guardia Costiera.

«Hanno ucciso otto di noi. E più di venti feriti. Stavano tentando di fuggire dal centro di detenzione di Gharyan. Erano terrorizzati perché poche ore prima una banda di miliziani aveva assaltato il campo e rapito oltre 200 nostri compagni. I poliziotti e i militari di guardia, però, non hanno esitato a sparare per fermarli. Ad altezza d'uomo. Ed è stata una strage». È questa la dichiarazione di Saled, profugo eritreo arrivato in Italia che ha raccontato così la tragedia avvenuta a Gharyan, 94 chilometri a sud est di Tripoli, una prigione controllata dal Governo libico, descritta come un autentico lager. Nel carcere di Gharyan ci sono stati alcuni casi drammatici come la morte per malattia, maltrattamenti, fame, stenti, di cinque ragazzi mentre un sesto, un giovane eritreo, si è suicidato. È iniziato tutto all'alba del 15 maggio del 2018. Verso le cinque, un gruppo di uomini armati ha dato l'assalto al campo. Cercavano giovani da rapire, sia ragazze che ragazzi, in un carcere che ne conteneva oltre 400 «eravamo in tanti - ha riferito Saled. Sono arrivati su alcuni pick-up e anche mezzi più grandi. Tutti ben armati, ma non in divisa militare o in tuta mimetica». Verosimilmente, dunque, uomini alle dipendenze di un'organizzazione di trafficanti di uomini. Le guardie del campo sarebbero state colte di sorpresa. Gli assalitori le avrebbero sopraffatte in pochi minuti per poi tagliare tutte le comunicazioni, distruggendo i computer e devastando gli uffici. Altri miliziani avrebbero contemporaneamente fatto irruzione nei capannoni e nei container adibiti ad alloggi, catturando almeno 200 persone (90 eritrei e oltre 100 somali). Sono riusciti a salvarsi solo i prigionieri che erano nei container più periferici del centro di detenzione. Nella confusione iniziale più di qualcuno dei ragazzi catturati sarebbe riuscito a fuggire.

A Gharyan [ha detto ancora Saled] siamo stati detenuti in condizioni di vita disumane. Dopo l'assalto ci siamo convinti che il campo era anche esposto a ogni genere di razzie e che le guardie non si sarebbero esposte a rischi per difenderci da rapimenti di massa come quello che avevamo vissuto. O che, comunque, non erano in grado di farlo. Abbiamo atteso per un po'. Ci aspettavamo che arrivassero dei funzionari civili del Governo, magari anche dell'UNHCR, per garantirci aiuto e assistenza e, soprattutto, per trasferirci, portarci via da questo posto. Per rassicurarci e darci fiducia, insomma. Invece è stata rinforzata la sorveglianza tutt'intorno al campo. Guardie e militari hanno circondato la struttura per un largo 
raggio. Magari lo avranno fatto per motivi di sicurezza, ma noi ci siamo sentiti ancora di più in trappola. Ci siamo mossi tutti insieme sperando di cogliere di sorpresa i soldati, in modo da riuscire a passare in quanti più possibile. Dopo ciò che avevamo subito, pensavamo che i militari di guardia capissero almeno in parte che cosa ci spingeva. E invece no: hanno cominciato a sparare e ad inseguirci anche con le jeep. Sembra che qualcuno dei nostri sia stato travolto. Molti sono caduti sotto i colpi.

Alla fine sono morti otto giovani (tre eritrei e cinque somali), tutti sui vent'anni, a cui si sommano almeno ventinove feriti, dodici dei quali in condizioni gravi. Solo pochi ce l'hanno fatta a superare indenni il fuoco di sbarramento delle guardie e a dileguarsi, gettandosi tra i cespugli e cercando riparo in qualche anfratto. Poco più di una decina. Verso l'imbrunire, infine, hanno ripreso la fuga. Dopo un giorno e mezzo di marcia, aiutandosi anche con mezzi di fortuna, sono arrivati a Tripoli. Alle porte della città li ha intercettati e fermati una pattuglia della polizia che li ha condotti in un centro di detenzione dell'Agenzia ministeriale anti-immigrazione, in Airport Road. Sono stati loro a ricostruire la strage e il sequestro in massa che l'ha preceduta. Non avevano con sé un cellulare per dare l'allarme, chiedere aiuto e raccontare ma, nel campo dove sono stati portati, alcune ragazze eritree, anch'esse detenute, sono riuscite ad avvicinarli e a parlare con loro a lungo.

\section{Conclusioni}

Sui profughi in Libia si scarica ogni forma di violenza: dagli stupri alle condizioni carcerarie, dai motivi della detenzione alle percosse metodiche e alla tortura, all'uso sistematico dell'internamento. I rapporti di dominio e di potere tra detenuti e carcerieri, il criterio per la detenzione, passano per differenza somatica, la tonalità della pelle, e mirano alla deumanizzazione e all'umiliazione. Il ricorso alla tortura, alla violenza sessuale, mette a tacere la vittima, spogliandola della propria soggettività e facendo parlare il torturatore (diretto e indiretto) e la 'teoria' che lo muove.

Anche il terrore è una forma di tortura. Nelle carceri libiche si spara per svegliare i detenuti, per fare una fila nel momento della distribuzione dei pasti, per spingere le persone ai lavori forzati. I morti nel deserto o in mare rimangono presenze vive e attive nel disagio dei sopravvissuti, non tanto per un senso di colpa (spiegazione più immediata nella nostra tradizione) ma per una mancata pacificazione di quei defunti che non hanno potuto ricevere degna sepoltura, con tutte le implicazioni sociali che questo comporta per i familiari del defunto e il gruppo d'appartenenza. L'idea di un Mediterraneo come 
cimitero nega, sotterrandola, parte della tragedia vissuta da chi nel Mediterraneo ha trovato la morte e dalle loro famiglie. Un dramma nel dramma. Emblematica è la testimonianza di Teklemariam «Ho domandato a me stesso 'cosa ho fatto? dove stavo? dove sono ora?'. Perché so quante sofferenze, fame e sete ho dovuto sopportare, quante persone che conosco sono morte, quanti [...] non ce l'hanno fatta [...] è anche attraverso i loro cadaveri che uno arriva qui». Nella falaqa, ad esempio, è possibile ritrovare uno strumento 'simbolico' per bloccare il movimento di chi tenta la fuga, un monito per gli altri prigionieri, ma anche una concreta deprivazione che fabbrica una discontinuità con la vita precedente e che non assume un nuovo senso se non quello che rimane sul corpo a seguito della violenza e che 'parla la lingua' del torturatore.

In relazione alle torture subite dagli emigranti, è da ricordare che l'articolo 1 del decreto sicurezza, convertito in legge l'1 dicembre 2018 (l. 132/2018), prevede, tra le altre cose, l'abolizione del permesso di soggiorno per motivi umanitari ed ogni riferimento ad esso contenuto nel Testo unico sull'immigrazione (l. 286/98). Prima del 5 ottobre 2018, data di entrata in vigore del citato articolo, la legge italiana prevedeva la possibilità di concedere un permesso di soggiorno per motivi umanitari a tutti gli stranieri arrivati nel territorio italiano che presentavano "seri motivi, in particolare di carattere umanitario o risultanti da obblighi costituzionali o internazionali dello stato italiano», come ad esempio ai minori stranieri non accompagnati fortemente traumatizzati dal viaggio, alle donne con bambino/a che hanno subito torture e/o detenzione in Libia, a coloro cui nel loro Paese non viene garantita la dignità umana attraverso un livello di vita accettabile, ovvero alle persone che fuggivano da emergenze come conflitti, disastri naturali o altri eventi di particolare gravità in Paesi non appartenenti all'Unione europea. È di tutta evidenza che questo provvedimento, insieme ad altri e a diffuse pratiche volte a respingere i profughi verso il Paese di partenza, ossia spesso la Libia, costituisce una delle ragioni per cui migliaia di persone, a volte anche minori, vengono respinti dallo Stato italiano e rigettati nelle mani dei loro carnefici o condannati a vivere in uno stato di clandestinità in Italia, amplificando le condizioni di loro emarginazione e debolezza. Questo tipo di tutela era stata introdotta in Italia nel 1998 ed era regolata dall'art. 5, comma 6 della legge nr. 40/1998, che dava attuazione al cosiddetto «asilo costituzionale», previsto dall'art. 10, comma 3, della Costituzione italiana, ai sensi del quale l'Italia deve riconoscere l'asilo a tutti coloro i quali nei propri Paesi di origine non sono riconosciuti i diritti e le libertà fondamentali riconosciute dalla Costituzione italiana. Non considerare le esperienze vissute dai profughi in Libia a partire dalla tortura subita nelle carceri e non solo ma ispirare la propria azione al solo scopo di respingere donne, uomini e bambini considerandoli possibili cri- 
minali o comunque non titolari di un diritto all'accoglienza, è rendersi complici di torturatori e mafiosi libici. I profughi torturati in Libia, per lo Stato italiano, rimangono totalmente privi di tutela. Accettare questi processi per furore ideologico o ricerca del consenso e di potere significa diventare complici dei carnefici, dei torturatori e degli sfruttatori. A questo tradimento si sommano le persecuzioni per via normativa che sono in corso in Italia nei confronti degli immigrati, dei richiedenti asilo, dei profughi, e di chi si occupa di accoglienza e di solidarietà. La Libia sembra il volto vero riflesso nello specchio di un'Europa e di un'Italia che mirano a riscrivere le regole della convivenza e della democrazia orientandola verso la discriminazione, l'indifferenza e il razzismo.

\section{Bibliografia}

Drudi, Emilio (2017). Fuga per la vita. Macerata: Tempi Moderni-Simple.

Global Detention Project (2015). Immigration Detention in Libya. URL https:// www.globaldetentionproject.org/countries/africa/libya (2019-10-12)

lacovino, Gabriele (2010). «I rapporti bilaterali tra Italia e Libia alla luce del trattato di amicizia». Osservatorio di politica internazionale, 8, s.o. URL https://bit.ly/33kUfBW (2019-05-05).

In Migrazione (2013). 0021 Trappola Libica. Indagine sulle condizioni detentive dei migranti in Libia e il viaggio in mare. Luglio 2013. URL http://www. africanews.it/wp-content/uploads/60_Dossier-LIBIA.pdf (2019-10-12)

Marcus, Georg (1995). «Ethnography in/of the World System: The Emergence of Multi-Sited Ethnography». Annual Review of Anthropology, 24, 95-117.

MHUB, Mixed Migration Hub (2015). Detained Youth: The Fate of Young Migrants, Asylum Seekers and Refugees in Libya Today. URL http://www. mixedmigrationhub.org/2015/02/01/httpwww-mixedmigrationhub-orgresourcesmmtf-noah-research (2019-10-12).

OIM, Organizzazione internazionale per le migrazioni (2018a). Libya's Migrant Report. Round 17: January-February 2018. Flow Monitoring. URL https:// www.iom.int/sites/default/files/dtm/Libya_DTM_201801201802.pdf (2019-10-12).

OIM (2018b). Missing Migrants. URL https://missingmigrants.iom.int/ (2019-10-12)

UNSMIL, United Nations Support Mission in Libya; OHCHR, Office of the United Nations High Commissioner for Human Rights (2013). Torture and Deaths in Detention in Libya. October 2013. URL http://www.ohchr.org/Documents/ Countries/LY/TortureDeathsDetentionLibya.pdf (2019-04-03).

UNSMIL; OHCHR (2016). Detained and Dehumanised. Report on Human Rights Abuses against Migrants in Libya. 13 December 2016. URL https://www. ohchr.org/Documents/Countries/LY/DetainedAndDehumanised_ en.pdf (2019-04-03). 


\title{
Immigrazione via mare Non-refoulement, legge 110/2017, Memorandum d'Intesa Italia-Libia e rischio di tortura
}

\section{Monica Gazzola}

avvocato, membro affiliato del Centro Studi sui Diritti Umani (CESTUDIR)

\begin{abstract}
The essay examines the national and international legal framework which regulates the rescue activities at sea, the principle of non-refoulement in the light of the jurisprudence of the European Court of Human Rights and the introduction of the crime of torture in Italy. In this context, the Memorandum of Understanding between Italy and Libya of 2 February 2017 is - in relation to the situation in Libya - in violation of the obligation to rescue at sea and the principle of non-refoulement.
\end{abstract}

Keywords Non-refoulement. Immigration by sea. Rescue obligation. Torture. Memorandum Italy-Libya.

Sommario 1 Sorveglianza delle frontiere marine, tutela della vita umana e principio di non-refoulement. - 2 L'obbligo di soccorso in mare. - 3 Il principio di non-refoulement. - 4 Il principio di non-refoulement nel Testo Unico Immigrazione e il rischio di tortura. - 5 Il Memorandum d'intesa del Governo italiano con le Forze Governative libiche. 6 L'affidamento delle operazioni SAR alla Guardia Costiera libica: violazione dell'obbligo di soccorso in mare e del principio di non-refoulement. 


\section{Sorveglianza delle frontiere marine, tutela della vita umana e principio di non-refoulement}

L'immigrazione via mare esaspera due nodi principali della materia dell'immigrazione: l'attività di controllo della frontiera e la tutela dei diritti fondamentali delle persone, in primis la tutela del diritto alla vita. Operativamente e giuridicamente, infatti, si caratterizza per l'intreccio tra attività di sorveglianza e attività di salvataggio: un'operazione può iniziare come attività di controllo delle frontiere, per diventare un'operazione di ricerca e soccorso qualche ora dopo, e viceversa.

Tale intreccio si manifesta in tutta evidenza nell'attività del Corpo delle Capitanerie di Porto e Guardia Costiera, che è istituzionalmente preposto al soccorso in mare, come sancito dal D.P.R. 662/1994, ma che è altresì coinvolto nelle operazioni di contrasto all'immigrazione undocumented. Infatti, in base al D.M. 220/2003, le attività di vigilanza e contrasto all'immigrazione via mare sono coordinate dalla Direzione Centrale dell'immigrazione e Polizia di frontiera del Dipartimento della Pubblica Sicurezza del Ministero dell'Interno, e sono condotte da forze della Marina Militare, della Polizia e, appunto, delle Capitanerie di Porto e Guardia Costiera.

Principio cardine del diritto del mare è l'obbligo di soccorso. A questo, nel caso di soccorso di emigranti, si accompagna il c.d. principio di non-refoulement, ossia il divieto di respingimento di persone vittime di torture o persecuzioni.

\section{L'obbligo di soccorso in mare}

L'obbligo di soccorso in mare è un principio consuetudinario di lunga tradizione marittima, positivizzato da strumenti pattizi internazionali ratificati dall'Italia, e codificato nell'ordinamento italiano.

La Convenzione delle Nazioni Unite sul diritto del mare di Montego Bay del 1982 (UNCLOS), ratificata dall’Italia con legge 689/1994, costituisce la fonte primaria e sovraordinata del diritto internazionale del mare. L'art. 98 impone agli Stati di obbligare i comandanti delle navi che battono la propria bandiera nazionale a prestare assistenza a chiunque venga trovato in mare in pericolo di vita, di informare le autorità competenti, di fornire alle persone salvate le prime cure e di trasferirle in un luogo sicuro (place of safety), nonché di creare e curare il funzionamento di un servizio permanente ed efficace di ricerca e salvataggio.

Tali obblighi formano oggetto specifico di due convenzioni elaborate dall'IMO (International Maritime Organization): la La convenzione internazionale per la salvaguardia della vita umana in mare del 1974 (SOLAS), ratificata dall'Italia con legge 313/1980, e la Conven- 
zione internazionale sulla ricerca ed il salvataggio marittimo del 1979 (SAR), ratificata con legge 147/1989. ${ }^{1}$

La Convenzione SAR di Amburgo obbliga gli Stati parte a «garantire che sia prestata assistenza a ogni persona in pericolo in mare [...] senza distinzioni relative alla nazionalità o allo status di tale persona o alle circostanze nelle quali tale persona viene trovata (capitolo 2.1.10)».

Corollario all'obbligo per i Comandanti delle navi di prestare soccorso immediato, è l'obbligo per gli Stati costieri di «promuovere l'istituzione, l'attivazione e il mantenimento adeguato di un servizio effettivo di ricerca e soccorso relativo alla sicurezza in mare (art. 98 co.2)», ossia la c.d. zona SAR (Search and Rescue), vale a dire la zona di 'ricerca e salvataggio' ove vengono assicurate tutte le operazioni dirette a salvare le persone in pericolo.

La medesima Convenzione stabilisce che le Autorità dello Stato costiero competente su una determinata zona SAR che abbiano avuto notizia di persone in pericolo di vita all'interno di detta zona, devono intervenire immediatamente senza tener conto della nazionalità o della condizione giuridica.

Ai sensi della Convenzione di Amburgo e delle successive Linee guida dell'IMO sul trattamento delle persone soccorse in mare adottate con la Risoluzione del Comitato Marittimo per la Sicurezza n. 167-78, qualora l'Autorità nazionale che sarebbe competente secondo la ripartizione delle acque marittime non intervenga in tempi utili, sussiste in capo allo Stato di 'primo contatto' con le persone in pericolo l'obbligo di intervenire e coordinare le operazioni di soccorso anche al di fuori della propria zona SAR.

L'obbligo di prestare soccorso non si esaurisce nel sottrarre i naufraghi al pericolo di perdersi in mare e annegare, ma è adempiuto solamente con lo sbarco in un luogo sicuro. In base alle Linee guida attuative della Convenzione di Amburgo sopra citate, il 'luogo sicuro' è una località dove le operazioni di soccorso si considerano concluse e la sicurezza della vita dei sopravvissuti non è più minacciata e i bisogni umani primari (cibo, alloggio, servizi medici) possono essere soddisfatti. Inoltre è un luogo da dove possa essere organizzato il trasporto dei sopravvissuti verso la loro destinazione successiva o finale.

Lo Stato nella cui regione SAR siano stati recuperati i sopravvissuti, ovvero lo Stato che ha assunto il controllo delle operazioni di salvataggio, ha la responsabilità di garantire loro lo sbarco in luogo sicuro.

Il soccorso in mare è un obbligo sancito anche dall'ordinamento interno italiano, e la sua violazione costituisce reato. L'art. 489 del

1 Convenzione SAR dell'IMO: http://www.imo.org/en/About/Conventions/ListofConventions/Pages/International-Convention-on-Maritime-Search-andRescue-(SAR). aspx (2019-02-28). 
codice della navigazione dispone l'obbligo di assistere le navi in difficoltà: "L'assistenza di nave o aeromobile in mare o in acque interne, che siano in pericolo di perdersi, è obbligatoria, in quanto possibile senza grave rischio della nave soccorritrice, del suo equipaggio e dei suoi passeggeri, quando siano in pericolo persone».

Il successivo art. 490 stabilisce l'obbligo di soccorrere i naufraghi: «il comandante della nave soccorritrice è tenuto, nelle circostanze e nei limiti indicati dall'articolo precedente, a tentarne il salvataggio, ovvero, se ciò non sia possibile, a tentare il salvataggio delle persone che si trovano a bordo. Ė del pari obbligatorio, negli stessi limiti, il tentativo di salvare persone che siano in mare o in acque interne in pericolo di perdersi».

La violazione dell'obbligo di assistenza e di salvataggio è punita dall'art. 1158 del medesimo codice della navigazione quale delitto, in ipotesi sia dolosa che colposa. ${ }^{2}$ L'obbligo di soccorso non è derogato dalla normativa nazionale in tema di favoreggiamento dell'immigrazione 'clandestina' e, anzi, costituisce una specifica discriminante.

L'art. 12 del Testo Unico Immigrazione nel disciplinare il reato di favoreggiamento dell'immigrazione 'clandestina', al secondo comma dispone infatti che: «fermo restando quanto previsto dall'art. 54 c.p., non costituiscono reato le attività di soccorso e assistenza umanitaria prestate in Italia nei confronti degli stranieri in condizioni di bisogno comunque presenti nel territorio dello Stato».

In ogni caso, come lo stesso art. 12 co. 2 del citato Testo Unico rammenta, resta ferma l'applicabilità dell'esimente generale dello stato di necessità di cui all'art. 54 c.p., a prescindere dalla circostanza se la persona soccorsa si trovi o meno nel territorio dello Stato o in acque territoriali, ogniqualvolta tale persona si trovi in una situazione di pericolo attuale per la propria vita o integrità fisica. ${ }^{3}$

2 Art. 1158 cod. nav. - Omissione di assistenza a navi o persone in pericolo: «Il comandante di nave, di galleggiante o di aeromobile nazionale o straniero, che ometta di prestare assistenza ovvero di tentare il salvataggio nei casi in cui ne ha l'obbligo a norma del presente codice, è punito con la reclusione fino a due anni. La pena è della reclusione da uno a sei anni, se dal fatto deriva una lesione personale; da tre a otto anni, se ne deriva la morte. Se il fatto è commesso per colpa, la pena è della reclusione fino a sei mesi».

3 Il 13 giugno 2018 il Giudice per le Indagini Preliminari di Palermo, accogliendo la richiesta di archiviazione avanzata dalla Procura della Repubblica, ha archiviato l'indagine nei confronti del Capitano e degli operatori delle navi di due ONG indagati dal maggio 2017 per il reato di associazione a delinquere finalizzata all'agevolazione dell'immigrazione 'clandestina', concludendo che «avendo l'imbarcazione umanitaria soccorso dei migranti che si trovavano in stato di pericolo, la condotta trova giustificazione nella disciplina dell'art. 51 c.p. per aver adempiuto ad un obbligo imposto da una norma giuridica internazionale». 


\section{Il principio di non-refoulement}

Le linee guida IMO del 2004 sul trattamento delle persone soccorse in mare sanciscono, con riguardo ai rifugiati e richiedenti asilo, il principio di non-refoulement, stabilendo la necessità di evitare lo sbarco di richiedenti asilo e rifugiati soccorsi in mare in quei territori ove la loro vita e la loro libertà sarebbero minacciate.

Il principio di non-refoulement vieta il respingimento, il rimpatrio o il trasferimento di persone vittime di persecuzioni, torture o altri danni gravi. Il divieto si applica non solo ai rifugiati formalmente riconosciuti dalle autorità del Paese ospitante, ma anche ai richiedenti asilo in generale, in quanto il riconoscimento dello status di rifugiato non ha natura costitutiva bensì declaratoria, poiché un individuo diviene rifugiato per il solo fatto di essere in possesso dei requisiti posti dalla Convenzione di Ginevra del 1951, e non in virtù di una formale determinazione. ${ }^{4}$

Il divieto di respingimento è riconosciuto quale norma di diritto internazionale consuetudinario, ed è codificato a livello internazionale ed europeo. Oltre alle già citate linee-guida IMO del 2004, il principio di non-refoulement è stabilito espressamente dall'art. 33 della Convenzione di Ginevra del 1951 sullo status di rifugiato, dall'art. 78 del Trattato sul funzionamento dell'Unione Europea, dall'art. 18 della Carta dei diritti fondamentali dell'Unione europea, che ribadisce altresì il divieto di tortura e trattamenti inumani (art. 4) e vieta il rimpatrio nei Paesi in cui vengono praticati tali trattamenti (art. 19).

La Convenzione europea per la salvaguardia dei diritti dell'uomo e delle libertà fondamentali (CEDU) non riconosce espressamente tale principio, ma è stato desunto per giurisprudenza costante della Corte europea dei diritti dell'uomo (Corte EDU) dall'art. 3, che sancisce il divieto di tortura e di pene o trattamenti inumani o degradanti, dall'art. 2 sul diritto alla vita, e dall'art. 4 prot. 4 che vieta le espulsioni collettive. ${ }^{5}$

L'Italia è stata condannata più volte dalla Corte EDU per violazione del divieto di respingimento. Con la sentenza Sharifi c. Italia e Grecia del 21/10/2014, la Corte ha statuito che non vi può essere un'applicazione automatica di accordi bilaterali, senza verifica delle condizioni, neppure tra Paesi UE, e ha ribadito l'obbligo di immediato esame delle richieste d'asilo e la necessità di assistenza legale.

La sentenza Khalifia c. Italia del 01/09/2015 ha condannato l'Italia per violazione degli artt. 3 e 5 CEDU e dell'art. 4 del Protocollo nr. 4

4 Così Comitato Esecutivo, UNHCR, Conclusione Nr. 6 (XXVIII) - Non-refoulement, 1977: https://www.unhcr.org/excom/exconc/3ae68c43ac/non-refoulement.html (2019-02-28).

5 Tutte le sentenze della Corte EDU citate sono reperibili nel sito della Corte: https:// www.echr.coe.int/Pages/home. aspx?p=caselaw/HUDOC\&c= (2019-02-28). 
CEDU per il trattenimento e poi l'espulsione di emigranti nel settembre 2011 nell'allora Centro di Primo Soccorso ed Accoglienza di Lampedusa, senza avere svolto una verifica individuale.

Particolarmente importante è la sentenza Hirsi c. Italia della Gran Camera del 23/02/2012. ${ }^{6}$ La Corte ha sancito che gli Stati membri devono rispettare il principio di non respingimento non solo durante i controlli di frontiera, ma anche in operazioni di soccorso in alto mare. La sentenza Hirsi ha poi sancito l'applicabilità, quale obbligo assoluto e inderogabile, del principio di non-refoulement anche nelle operazioni di salvataggio in alto mare, precisando che tale principio vieta non solo il rimpatrio della persona nel Paese di origine, ma anche il suo trasferimento in altri Paesi in cui vi sia il rischio di rimpatrio nel Paese d'origine (c.d. respingimento indiretto). La sentenza ha poi precisato che il principio di non-refoulement deve essere garantito dallo Stato che ha il controllo effettivo, diretto o indiretto, delle persone, applicando così una nozione estesa di 'giurisdizione' di cui all'art. 1 CEDU: la nozione di 'giurisdizione' ha una portata più ampia rispetto al concetto di territorio nazionale dello Stato, poiché si applica a tutti gli individui che risultano essere sotto l'autorità o l'effettivo controllo delle autorità dello Stato o di altre persone che agiscono per suo conto. Inoltre, il concetto di giurisdizione si applica a tutte le aree extraterritoriali, appartengano esse ad uno Stato terzo o meno, dove il Paese in questione esercita un controllo effettivo. Infine, la sentenza Hirsi ha ribadito che un respingimento in alto mare senza previa identificazione configura violazione del divieto di espulsioni collettive.

Il Comitato ONU contro la tortura nel General Comment nr. 4 del 2017 traccia delle linee guida tenendo conto del principio di non-refoulement. Il Comitato stabilisce l'obbligo per lo Stato di procedere alla valutazione della sussistenza di motivi di rischio di tortura nello Stato verso cui si vorrebbe respingere la persona, sottolineando che il divieto di respingimento ha carattere assoluto al pari del divieto di tortura. Pertanto, gli Stati hanno l'obbligo di procedere a esami individuali e di assicurare ai richiedenti le garanzie proprie di un procedimento equo, prevedendo il diritto di appello avverso le decisioni negative. ${ }^{7}$

6 Il 6 maggio 2009 circa 200 persone, su tre barche dirette in Italia, vennero intercettate da motovedette italiane, in acque internazionali approssimativamente a trentacinque miglia marine a sud di Lampedusa, ossia all'interno della zona SAR di Malta. Gli occupanti vennero trasferiti a bordo delle navi italiane e ricondotti a Tripoli. Non venne effettuato alcun accertamento sulla loro identità e sulla loro eventuale condizione di richiedenti asilo. Una volta arrivati al porto di Tripoli, dopo dieci ore di navigazione, gli emigranti furono consegnati alle autorità libiche.

7 General Comment nr.4 del 2017 sull'attuazione dell'art. 3 della Convenzione contro la tortura del 1984, che sostituisce il General Comment nr. 1 del 1997: https://www. ohchr.org/Documents/HRBodies/CAT/CAT-C-GC-4_EN.pdf (2019-02-28). 


\section{$4 \quad$ Il principio di non-refoulement nel Testo Unico Immigrazione e il rischio di tortura}

Passando dal contesto internazionale ed europeo a contesto italiano, il Testo Unico sull'Immigrazione del 1998 aveva previsto all'art. 19 il divieto di espulsione e respingimento di persone «verso uno Stato in cui lo straniero possa essere oggetto di persecuzione per motivi di razza, sesso, lingua, cittadinanza, religione, opinioni politiche, condizioni sociali o personali, ovvero possa rischiare di essere rinviato verso un altro Stato nel quale non sia protetto dalla persecuzione», ossia esclusivamente in favore di persone vittime di persecuzioni e, quindi, in relazione alla sussistenza dello status (sostanziale o dichiarato) di rifugiato.

La Legge nr. 110/2017 che, recependo la Convenzione ONU sulla tortura del 1987, ha finalmente introdotto il reato di tortura nel nostro ordinamento, all'art. 3 ha modificato l'art. 19 del testo Unico Immigrazione, inserendo il comma 1.1: «Non sono ammessi il respingimento o l'espulsione o l'estradizione di una persona verso uno Stato qualora esistano fondati motivi di ritenere che essa rischi di essere sottoposta a tortura. Nella valutazione di tali motivi si tiene conto anche dell'esistenza, in tale Stato, di violazioni sistematiche e gravi di diritti umani».

Cosicché oggi anche nella legislazione interna italiana è espressamente sancito il divieto di respingimento o il trasferimento verso uno Stato ove vi sia il rischio che la persona possa essere sottoposta a tortura, a prescindere dalla titolarità (sostanziale o formale) dello status di rifugiato.

\section{Il Memorandum d'intesa del Governo italiano con le Forze Governative libiche}

Nello stesso periodo in cui il Parlamento italiano emanava la legge sulla tortura e introduceva il principio di non-refoulement nel Testo Unico sull'Immigrazione anche per il caso di pericolo di essere sottoposti a tortura, il Governo italiano stipulava il 2 febbraio 2017 un accordo con rappresentanti del Governo libico, riguardante il controllo dell'immigrazione.

La cooperazione italo-libica in tema di controllo dell'immigrazione risale all'Accordo per la collaborazione nella lotta al terrorismo, alla criminalità organizzata, al traffico illegale di sostanze stupefacenti e all'immigrazione 'clandestina' firmato a Roma il 13 dicembre 2000, reso operativo nel 2007 con due protocolli esecutivi firmati a Tripoli il 29 dicembre 2007.

Successivamente, il 30 agosto 2008 venne siglato a Bengasi il Trattato di Amicizia, Partenariato e Cooperazione, ratificato in Italia con legge 7/2009, che toccava un largo ventaglio di attività di coopera- 
zione italo-libica, e che all'art. 19, rubricato 'Collaborazione nella lotta al terrorismo, alla criminalità organizzata, al traffico di stupefacenti, all'immigrazione clandestina' prevedeva: «Le due Parti intensificano la collaborazione in atto nella lotta al terrorismo, alla criminalità organizzata, al traffico di stupefacenti e all'immigrazione clandestina, in conformità a quanto previsto dall'Accordo firmato a Roma il 13/12/2000 e dalle successive intese tecniche, tra cui, in particolare, per quanto concerne la lotta all'immigrazione clandestina, i Protocolli di cooperazione firmati a Tripoli il 29 dicembre 2007». ${ }^{8}$

Il Memorandum d'intesa del 2 febbraio 2017 si occupa esclusivamente della lotta all'immigrazione undocumented. Nei consideranda le Parti dichiarano: "Riaffermando la ferma determinazione di cooperare per individuare soluzioni urgenti alla questione dei migranti clandestini che attraversano la Libia per recarsi in Europa via mare, attraverso la predisposizione dei campi di accoglienza temporanei in Libia, sotto l'esclusivo controllo del Ministero dell'interno libico, in attesa del rimpatrio o del rientro volontario nei paesi d'origine, lavorando al tempo stesso affinché i paesi di origine accettino i propri cittadini ovvero sottoscrivendo con questi paesi accordi in merito».

Nello specifico, il testo prevede che la Parte italiana si impegna a fornire supporto tecnico e tecnologico agli organismi libici incaricati della lotta contro l'immigrazione 'irregolare'. Tali organismi sono rappresentati dalla Guardia di frontiera e dalla Guardia Costiera del Ministero della Difesa e dagli organi e dipartimenti competenti presso il Ministero dell'Interno (art. $1 \mathrm{~b}$ ). Inoltre, ai sensi dell'articolo 2, le Parti si impegnano all'adeguamento e al finanziamento dei centri di accoglienza già attivi, attingendo ai finanziamenti disponibili da parte italiana e a finanziamenti dell'Unione Europea. In cambio, il Governo italiano si impegna a finanziare la cooperazione per lo sviluppo in infrastrutture, sanità, trasporti, energie rinnovabili e formazione (art. 1 b). ${ }^{9}$ Il 28 luglio 2017 il Consiglio dei Ministri ha deliberato la partecipazione dell'Italia alla missione internazionale in supporto alla Guardia Costiera libica con l'obiettivo di «fornire supporto alle forze di sicurezza libiche per le attività di controllo e contrasto all'immigrazione illegale e al traffico di esseri umani».

8 Trattato di Amicizia, Partenariato e Cooperazione firmato a Bengasi il 30 agosto 2008: http://www.camera.it/_dati/leg16/Lavori/schedela/apritelecomando_wai. asp?codice=16pdl0017390 (2019-02-28).

9 Memorandum di intesa tra Italia e Libia firmato a Roma il 2 febbraio 2017: http:// www.governo.it/sites/governo.it/files/Libia.pdf (2019-02-28). 


\section{L'affidamento delle operazioni SAR alla Guardia Costiera libica: violazione dell'obbligo di soccorso in mare e del principio di non-refoulement}

Appare evidente che gli accordi italo-libici costituiscono una forma di esternalizzazione del controllo delle frontiere, in questo caso delle frontiere marine.

Esternalizzazione delle frontiere che, già costituendo di per sé uno strumento in generale gravemente critico sotto il profilo della vigilanza sulla salvaguardia dei diritti fondamentali, nel caso di specie, con l'individuazione della Libia quale partner dell'accordo, appare violare sia nei termini dell'accordo, sia nella sua attuazione, norme internazionali e nazionali in materia di diritti umani fondamentali.

Sotto un profilo strettamente semantico, ma assai significativo, il Memorandum parla esclusivamente di «immigrazione clandestina», senza mai affrontare il tema del soccorso e della gestione degli emigranti che potrebbero essere titolari del diritto di asilo.

In merito all'affidamento alla Libia del controllo di una zona SAR, appare assai incerta l'effettiva capacità di gestione delle operazioni di salvataggio da parte della Guardia Costiera libica. La Libia fino al mese di luglio del 2017 non aveva mai né individuato né comunicato la propria zona SAR. Il 10 agosto 2017, immediatamente a ridosso degli accordi con l'Italia sulla esternalizzazione delle frontiere e sollecitato dall'Italia, il Governo di riconciliazione nazionale di Tripoli ha notificato la costituzione di una zona SAR libica all'organismo competente, ossia all'IMO. Ma dopo le difficoltà riscontrate dall'IMO sull'affidabilità della Guardia Costiera libica come interlocutore, in mancanza perfino di un Governo unitario cui fare riferimento, e in ragione delle ripetute segnalazioni di abusi perpetrati dalla stessa Guardia Costiera libica, il Governo libico nel successivo mese di dicembre ha ritirato la comunicazione. Dal sito dell'IMO risulta intervenuta successivamente una nuova comunicazione di zona SAR dal Governo libico alla fine del mese di giugno del 2018. ${ }^{10}$

La previsione, contenuta nel Memorandum, di affidare il soccorso e la gestione degli emigranti alla Guardia Costiera libica e, addirittura, di stabilire la riconsegna ai libici di quelli soccorsi da altre imbarcazioni, si pone in palese violazione del principio di non-refoulement con riferimento, in particolare, al pericolo di tortura, poiché i porti della Libia non possono assolutamente considerarsi un place of safety ai sensi della Convenzione di Amburgo, delle indicazioni dell'IMO

10 Le informazioni sulle zone SAR comunicate all'IMO sono reperibili nella directory Global SAR Plan del sito web dell'IMO https://gisis.imo.org/Public/Default. aspx (2019-02-28). 
e dell'UNCHR, nonché alla luce della giurisprudenza costante della CEDU, in particolare dalla sentenza Hirsi del 2012.

Non solo la Libia non ha mai ratificato la Convenzione ONU sui rifugiati e, quindi, non ha assunto alcun vincolo pattizio sui principi fondamentali di trattamento e garanzie umanitarie, ma sono altresì certe e conclamate le violazioni dei diritti fondamentali commesse dalla Guardia Costiera libica e dai responsabili governativi nei centri di detenzione per emigranti. La Corte d'Assise di Appello di Milano, con sentenza del 10 ottobre 2017, ha riconosciuto l'esistenza di pesantissime violazioni dei diritti umani nei campi di detenzione in Libia. ${ }^{11}$ Sono stati documentati episodi di minacce ed aggressioni da parte della Guardia Costiera libica nei confronti di navi di ONG. ${ }^{12}$ Il report del Segretario Generale delle Nazioni Unite del 4 aprile 2017 ha accusato la Guardia Costiera libica di «condotta spregiudicata e violenta» nel corso di salvataggi e intercettazioni in mare, confermando le torture e le efferatezze commesse nei campi di detenzione documentati ampiamente da Amnesty International. ${ }^{13}$ La drammatica situazione in cui si trovano gli emigranti, intercettati dalla Guardia Costiera libica e trattenuti nei centri di detenzione, è stata sempre evidenziata nei successivi rapporti. ${ }^{14}$

Il Comitato ONU contro la tortura nel General Comment nr. 4 del 2017 sopra citato ha rilevato come la gestione degli emigranti in Libia sia in mano a gruppi paramilitari, e ha dichiarato che il Memorandum del 3 febbraio 2017 «è stato sottoscritto a dispetto delle notizie di sistematiche e generalizzate violazioni dei diritti umani poste in essere contro i migranti nei centri di detenzione, integrando così una politica di respingimento sistematico dei migranti diretti in Italia». Secondo il Comitato, alcune fonti rivelerebbero che alcuni di questi gruppi armati sarebbero finanziati dal Governo libico per trattenere gli emigranti in Libia.

11 La sentenza è pubblicata in https://www.penalecontemporaneo.it/d/5976-unacondanna-della-corte-d-assise-di-milano-svela-gli-orrori-dei-centri-diraccolta-e-transito-dei (2019-02-28). Il processo su fatti accaduti in Libia si è tenuto in Italia su richiesta del Ministero della Giustizia data la gravità dei fatti in giudizio e le condizioni di insicurezza e il livello di violenze riscontrato in Libia.

12 Si veda l'interrogazione parlamentare del 23 marzo 2018 relativa all'aggressione subita dalla nave Open Arms dell'organizzazione non governativa catalana ProActiva da parte della Guardia Costiera libica nel corso di un evento SAR in mare a settantatre miglia dalla costa libica: https://www.senato.it/japp/bgt/showdoc/18/Sindisp/๑/1066811/index.html (2019-02-28).

13 Report del Segretario António Guterres del 4 aprile 2017: https://unsmil.unmissions.org/report-secretary-general-antónio-guterres-united-nations-support-mission-libya (2019-02-28).

14 Si veda da ultimo il report del 7 gennaio 2019: https://unsmil.unmissions.org/ sites/default/files/sg_report_on_unsmil_s_2019_19e.pdf (2019-02-28). 
La Procura della Corte Penale Internazionale ha avviato ad aprile del 2018 un'indagine sui crimini contro l'umanità commessi in Libia, a seguito di una serie di segnalazioni circostanziate che documentano l'uso di forza eccessiva e illegale da parte dei funzionari del dipartimento libico per contrastare l'immigrazione 'irregolare'. ${ }^{15}$ La Libia non ha ratificato lo Statuto di Roma e quindi non è parte del Tribunale Penale Internazionale, ma le autorità libiche potrebbero essere messe in stato d'accusa a seguito di risoluzione del Consiglio di Sicurezza ONU.

Il Parlamento Europeo, nella Raccomandazione del 30 maggio 2018, ha ribadito «il suo pieno sostegno al mandato della Corte Penale Internazionale riguardo alla violazione dei diritti umani in atto in Libia» (punto 1.a septies). ${ }^{16}$ Da tutto ciò consegue che l'approvazione e l'applicazione del Memorandum tra Italia e Libia del 2017 pare porsi in contrasto con la normativa internazionale e nazionale e con la giurisprudenza della Corte EDU in tema di immigrazione via mare.

Nel caso in cui l'Autorità italiana preposta, ricevuta notizia di una richiesta di soccorso, anziché procedere all'attivazione delle operazioni di salvataggio e recupero dei naufraghi, deleghi la Guardia Costiera libica, ovvero se, dopo aver proceduto al salvataggio, consegni i naufraghi alle Autorità libiche, appaiono configurabili gravi violazioni dell'obbligo di soccorso in mare e del divieto di respingimento. ${ }^{17}$

Da ultimo, non può sfuggire che potrebbe configurarsi un coinvolgimento nell'indagine avviata dalla Procura della Corte Penale Internazionale contro le Autorità libiche anche di esponenti del Governo italiano - che è Stato parte e, quindi, soggetto alla giurisdizione della Corte -, qualora si evidenziassero elementi di attribuzione della responsabilità penale sotto forma dell'agevolazione alla commissione di crimini positivizzati dallo Statuto di Roma. ${ }^{18}$

15 Si veda il report pubblicato in https://www.icc-cpi.int/iccdocs/otp/otp-unsclib-11-2017-ENG.pdf (2019-02-28).

16 Raccomandazione del Parlamento Europeo del 30 maggio 2018 al Consiglio, alla Commissione e al vicepresidente della Commissione/alto rappresentante dell'Unione per gli affari esteri e la politica di sicurezza sulla Libia: http://www.europarl.euro-

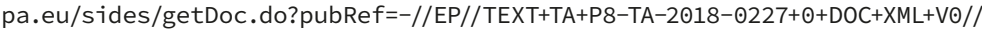
IT (2019-02-28).

17 Il 3 maggio 2018 è stato presentato un ricorso alla Corte EDU contro l'Italia da parte dei 17 superstiti del naufragio del 6 novembre 2017, con l'accusa di avere operato un «respingimento per procura».

18 Ipotesi prevista dall'art. 25(3) dello Statuto di Roma. 



\title{
La vulnerabilità degli immigrati nel sistema di accoglienza extra-ordinem dei richiedenti asilo
}

\section{Marco Ferrero}

Università Ca’ Foscari Venezia, Italia

\begin{abstract}
In the existing Italian asylum system we can witness a huge discrepancy between what is prescribed by the law and what happens in practice in the hosting structures for asylum seekers. Responsibilities for the creation and development of such a twisted system rely on all different political parties, which have either consciously strived to hinder the proper application of the norms, or so much insisted in the depiction of reception as an ethical obligation as to blur the binding juridical sources of the duty to reception itself. This has led to continuous violations of the fundamental rights of asylum seekers, especially of vulnerable ones, which will be briefly enumerated in this writing.
\end{abstract}

Keywords Unaccompanied minors. Asylum seekers. Human rights. International protection.

Sommario 1 Il sistema di accoglienza extra-ordinem dei richiedenti asilo in Italia. 2 L'Accoglienza dignitosa imposta dalla Direttiva UE 33/2013, in particolare con riferimento ai soggetti vulnerabili. - 3 I diritti umani sistematicamente violati nel sistema extra-ordinem dell'accoglienza dei minori richiedenti asilo: artt. 3, 8 e 13 CEDU. 


\section{Il sistema di accoglienza extra-ordinem dei richiedenti asilo in Italia}

Il Sistema di Accoglienza italiano, c.d. SPRAR (Sistema di Protezione dei Richiedenti Asilo e dei Rifugiati $\left.{ }^{1}\right)$, dovrebbe consistere nell'attuazione del Sistema Europeo Uniforme dell'Asilo (CEDAS). Il condizionale è d'obbligo, stante il fatto che, come è noto, solo una minima parte dei richiedenti protezione internazionale è 'accolto' dai comuni facenti parte di tale rete di accoglienza, coordinata dal Ministero dell'Interno. ${ }^{2}$ La ragione di tale disfunzione è eminentemente politica: l'adesione al Sistema di accoglienza SPRAR, infatti, non è mai stata resa obbligatoria, nemmeno nel pieno dello stato di emergenza dichiarato all'epoca della c.d. Emergenza Nord Africa dall'allora ministro dell'Interno Maroni. L'ex ministro preferì allo strumento giuridico della Protezione temporanea (prevista nel nostro ordinamento dall'art. 20 TUI) - più adeguato a gestire l'arrivo delle migliaia di giovani delle primavere arabe -, quello 'ordinario' della Protezione Internazionale, con i conseguenti oneri di tutela giuridica e di accoglienza. La conseguenza di tale decisione fu quella di dover arginare il numero di contenziosi giudiziari inevitabilmente prodottisi accondiscendendo al rilascio generalizzato di un permesso umanitario: proprio quello che l'attuale Ministro dell'Interno e Vice-Premier, del medesimo partito, ha deciso di abrogare.

Ma l'incapacità di costituire un sistema di accoglienza diffusa proporzionato alla popolazione residente e omogeneamente incardinato su tutto il territorio nazionale è stata trasversale e ha riguardato non solo i governi di centrodestra - poco inclini a costringere i propri sindaci a una politica necessaria e tuttavia resa impopolare da una serrata ed efficace propaganda politica -, ma anche quelli di centrosinistra, oramai da anni alla rincorsa di un'ampia fetta di elettorato in fuga, sempre più attratto dalla destra populista, capace di produrre una vera e propria egemonia culturale sul tema immigrazione.

Che la questione abbia natura eminentemente politica è reso evidente dal fatto che l'obbligatorietà dell'adesione al sistema SPRAR trova addirittura copertura a livello costituzionale nell'art. 118 Cost., il quale prevede che le funzioni amministrative spettino a tutti i Comuni: tra queste, certamente vi rientra l'accoglienza dei richiedenti

1 Oggi SIPROIMI (Sistema di protezione per titolari di protezione internazionale e per minori stranieri non accompagnati) dopo le modifiche apportate al sistema dalla l. 132/2018, di conversione del d.l. 113/2018, c.d. decreto sicurezza.

2 A fronte della presentazione di 123.600 richieste d'asilo nel 2016, 130.119 nel 2017 e 54.473 nel 2018, i posti nel sistema SPRAR di accoglienza 'ordinario' ammontano a soli 35.831, di cui 3.500 per minori. Fonte: https://www.sprar.it/i-numeri-dellosprar. Cf. http://www.libertaciviliimmigrazione.dlci.interno.gov.it/it/documentazione/statistica/i-numeri-dellasilo (2019-10-14). 
protezione internazionale. Lo Stato, quindi, avrebbe il potere di imporre il sistema SPRAR, finanziandolo integralmente, come prevede la stessa Costituzione all'art. 119.

L'effetto di cinque lustri di propaganda continua, dapprima sulle reti pressoché unificate dei governi berlusconiani, in pieno e mai risolto conflitto di interessi, che ha visto la Lega Nord tenere costantemente la guida del Ministero chiave di quell'unità nazionale tanto contestata nella prima fase di ascesa della Lega di Bossi dei primi anni Novanta, ${ }^{3}$ quanto fondamentale per costruire la politica escludente e criminalizzante nei confronti del soggetto reso nemico pubblico numero uno (Ferrero, Perocco 2011), è che l'immigrato è costretto alla clandestinità da politiche migratorie tuttora basate sulla finzione dell'incontro della domanda e dell'offerta che dovrebbe realizzarsi nei Paesi d'origine degli immigrati, pur in assenza di qualsivoglia politica di reclutamento della manodopera all'estero. ${ }^{4}$

Si è assistito, in sostanza, a un quarto di secolo di preparazione all'introduzione del razzismo istituzionale condiviso nella società italiana, all'insegna di una retorica del 'prima gli italiani' ormai penetrata trasversalmente nei gangli delle istituzioni, degli apparati amministrativi, della società, persino tra quelli che per mandato istituzionale dovrebbero essere gli attori dell'inclusione e invece oggi, spesso inconsapevolmente, diventano i principali agenti di discriminazione ed esclusione: i Servizi Sociali e il Privato-sociale, anch'essi immersi nel discorso pubblico razzista e nazionalista, che dagli schermi televisivi dei tardi anni Novanta si è propagato in forma amplificata (oggigiorno si direbbe 'virale') sui social network. Non a caso, il contenzioso giudiziario in materia di discriminazione ha come principali protagonisti proprio le agenzie del Welfare State (INPS in primis) e gli Enti Locali (Baracchi, Guariso 2017).

Ma la definitiva rottura dell'ultimo argine a una deriva sempre più soggettiva e opinabile del confronto sulle politiche migratorie è stata resa possibile proprio ad opera di quel governo che a parole ha sempre tentato di 'tenere duro' sul fronte di valori di civiltà non negoziabili.

3 Ancora oggi la prospettiva secessionista della lega Nord non è del tutto abbandonata (nonostante la penetrazione della Lega di Salvini al Sud, con la costituzione a fini elettorali di un'apposita organizzazione parallela), essendo ancora ben scolpita nell'art. 1 dello Statuto del partito approvato dal Consiglio federale nel 2015. Cf. https://www. leganord.org/il-movimento/statuto-lega-nord-padania (2019-10-14).

4 Sarà utile ricordare che la prima timida sperimentazione della possibilità di ingresso legale per ricerca di lavoro, corredata da una garanzia fideiussoria delle eventuali spese di accoglienza, da una previa ospitalità e da una polizza assicurativa per eventuali spese mediche, fu abolita proprio dalla Legge Bossi-Fini del 2002, insieme all'azzeramento dei fondi per il finanziamento della Cooperazione allo sviluppo, con buona pace del tanto sbandierato 'aiutiamoli a casa loro', che da anni accompagna il simulacro di dibattito pubblico sul tema, messo in scena quotidianamente. 
L'affermazione per cui salvare vite umane sarebbe un 'dovere morale', infatti, lungi dal contrastare la politica razzista, le ha fornito l'ultimo assist per chiudere il cerchio iniziatosi a disegnare venticinque anni or sono, ${ }^{5}$ legittimando una visione secondo cui sono obblighi meramente etici, e non strettamente giuridici, quelli che devono guidare l'azione pubblica in materia accoglienza dei richiedenti asilo.

Svendere principi giuridici di rango internazionale e costituzionale per affermare la propria superiore levatura morale, alla ricerca di consenso presso la parte 'migliore' del proprio elettorato, oltre a non aver giovato al Presidente del Consiglio che su quel terreno si era avventurato, ha definitivamente spostato sul piano dell'etica un tema (accogliamoli/respingiamoli, ovvero 'aiutiamoli a casa loro') che prima di essere politico è giuridico e di civiltà democratica.

In tal modo è stata eliminata ogni residua resistenza - in un'epoca di forte relativismo etico - alla definitiva obliterazione della necessità di un qualche riferimento oggettivo per la propaganda razzista.

Il diritto a chiedere asilo e a essere messo nelle condizioni perché la valutazione dei presupposti di tale diritto - peraltro fondato su ben tre fonti gerarchicamente sovraordinate rispetto alla legge ordinaria (art. 10 Cost., Convenzione di Ginevra e Direttive UE sulla protezione internazionale) - sia effettivo è stato così degradato ad opzione possibile di un programma politico.

Un tale approccio, inconsapevolmente autolesionista, forse non a caso è stato propugnato dal medesimo Governo che ha portato avanti la gestione hotspot, anche quando si era andata costituendo una rete di associazioni e cooperative disponibili a realizzare forme di accoglienza diffusa. ${ }^{6}$

La mancanza di un progetto alternativo alla accoglienza condizionata e subalterna degli immigrati non poteva convincere nemmeno gli amministratori dello stesso partito di maggioranza al governo e così il sistema di accoglienza SPRAR da ordinario ha finito per essere riservato a pochi fortunati, relegando tutti gli altri richiedenti - cioè la stragrande maggioranza - a strutture di accoglienza che sarebbero dovute essere straordinarie e transitorie (CAS, Centri di Accoglienza Straordinaria), e che invece sono diventate ordinarie e stabili.

Il mero fatto che i richiedenti protezione internazionale siano 'accolti' nei CAS è già di per sé, a prescindere dalle concrete condizio-

5 «Naufragio canale di Sicilia. Renzi: 'La Ue non ci lasci soli'», Corriere della Sera, 19 aprile 2015. URL https://bit.ly/2VIm6K4 (2019-10-14).

6 È significativo che si sia fatta largo nella prassi l'abitudine dei richiedenti asilo di chiamare 'campo' qualunque struttura di accoglienza, anche quando si tratta di appartamenti, a dimostrazione del fatto che il paradigma dell'accoglienza è quello del 'campo' tipico del grande hotspot, nel quale quasi tutti i richiedenti asilo hanno trascorso un periodo più o meno lungo. 
ni di accoglienza, foriero di violazioni di diritti umani, oltre che illecito sul piano degli obblighi europei.

Il Sistema Uniforme Europeo dell'Asilo (CEAS), infatti, si è evoluto attraverso una prima fase di armonizzazione di standard minimi, ${ }^{7}$ una successiva fase di verifica dei risultati, ${ }^{8}$ e una fase di costruzione di un sistema uniforme di definizione degli status, di procedure di garanzia della possibilità di riconoscere tali status agli aventi diritto e di misure di accoglienza volte a rendere effettive tali procedure. ${ }^{9}$

\section{L'Accoglienza dignitosa imposta dalla Direttiva UE 33/2013, in particolare con riferimento ai soggetti vulnerabili}

La costruzione di un sistema di criteri uniformi di garanzia delle procedure di esame delle domande d'asilo, così come l'elaborazione di standard comuni di accoglienza, oltre a voler evitare una sorta di effetto dumping nell'attrattività dei sistemi nazionali di accoglienza, è soprattutto finalizzata a mettere i richiedenti protezione internazionale nelle condizioni di poter esperire il proprio diritto fondamentale individuale di essere riconosciuti quali soggetti esposti ad un fondato timore di persecuzione o ad un grave danno alla propria incolumità in caso di rimpatrio nel Paese d'origine.

Non è difficile comprendere che prevedere la possibilità di sottoporre alle autorità una richiesta d'asilo senza predisporre una serie di 'servizi' accessori (quali interpretariato, mediazione culturale, supporto psicologico, accompagnamento sanitario, informazione legale, e così via) rende tale possibilità meramente astratta.

È soltanto grazie ad un sistema integrato di servizi di supporto, infatti, che i richiedenti protezione internazionale possono affrontare adeguatamente l'audizione in commissione o davanti al giudice, considerato che sovente sono poco scolarizzati, provengono da aree

7 Reg. (CE) 2735/2000 Eurodac 1 e Reg. (CE) 407/2002; Reg. (CE) 343/2003 Dublino (Reg. app. 1560/03); Dir. 2001/55/CE Prot. Temporanea; Dir. 2003/9/CE Accoglienza; Dir. 2004/83/CE Qualifiche; Dir. 2005/85/CE Procedure.

8 Nov. 2004 Programma dell'Aja, che impone alla Commissione UE una valutazione del CEAS entro il 2007; Adozione di nuovi strumenti da sottoporre al Parlamento entro il 2010; Com. 301/2007 Libro Verde sul futuro del CEAS; Com. 360/2008 Piano Strategico sull'Asilo (Proposte di modifica Reg. e Dir. e Reg. 439/2010 EASO); 1/1/2009 Trattato di Lisbona: TFUE Tit. IV art. 78 (già artt. 63 e 64 TCE): che prevede che la politica sull'Asilo è 'comune', che il principio di non-refoulment e quelli contenuti nella Conv. Ginevra del 1951 sono 'vincolanti'.

9 Reg. (CE) 603/2013 Eurodac 2; Reg. (CE) 604/2013 Dublino 3 (gen. 2014); Dir. 2011/51/ CE (mag. 2013); Dir. 2011/95/CE Qualifiche New (lug. 2014) d.lgs. 251/2007, modificato dal d.lgs. 18/2014; Dir. 2013/32/CE Procedure New (lug. 2015) d.lgs. 25/2008, modificato dal d.lgs. 140/2015 e D.P.R. 21/2015; Dir. 2013/33/CE Accoglienza New (lug. 2015) d.lgs. 140/2015. 
rurali, quasi sempre risultano traumatizzati dalle vicende occorse loro nei Paesi d'origine, dalle estreme difficoltà del viaggio, dai mesi o anni di detenzione brutale nelle carceri libiche ed infine dall'aver assistito a decessi di connazionali o persino familiari nella traversata via mare.

E anche in virtù della consapevolezza circa il background e i probabili trascorsi traumatici dei richiedenti asilo che l'art. 17 della Direttiva UE nr. 33/2013, c.d. Direttiva Accoglienza, ${ }^{10}$ impone agli stati membri di provvedere «a che le condizioni materiali di accoglienza assicurino un'adeguata qualità di vita che garantisca il sostentamento dei richiedenti e ne tuteli la salute fisica e mentale».

È altrettanto evidente (o dovrebbe esserlo) che l'accoglienza di persone particolarmente vulnerabili (minori, disabili, anziani, donne in stato di gravidanza, genitori singoli con figli minori, persone per le quali è stato accertato che hanno subito torture, stupri o altre forme gravi di violenza psicologica, fisica o sessuale) richiede servizi aggiuntivi e talvolta particolari accorgimenti organizzativi: si pensi ai richiedenti asilo minorenni, per i quali è prevista la collocazione in strutture che non debbono comportare promiscuità tra i richiedenti asilo minorenni e i maggiorenni. ${ }^{11}$

In base ai dati raccolti da UNICEF, IOM e UNHCR sui trend migratori relativi agli ultimi tre anni, un quinto del totale dei migranti che giungono sulle coste italiane è minorenne, e di questi meno di uno su dieci è accompagnato da un adulto. ${ }^{12}$ Solo nel 2016 i minorenni richiedenti asilo arrivati in Italia sono stati 33.800, di cui 25.846 sono minori stranieri non accompagnati (nel prosieguo, MSNA). Sebbene leggermente inferiore al picco del 2016, il numero dei minori e tra questi dei MSNA, che sono la grande maggioranza, rimane comunque in netto aumento rispetto agli anni precedenti. ${ }^{13}$

La predisposizione di un sistema di accoglienza e tutela che abbia un'attenzione peculiare e specifica per i minori (e, nello specifi-

10 https://eur-lex.europa.eu/legal-content/it/TXT/?uri=CELEX:32013L๑๑33 (2019-10-14).

11 «Gli Stati membri provvedono a che la qualità di vita sia adeguata alla specifica situazione delle persone vulnerabili, ai sensi dell'art. 21, nonché alla situazione delle persone che si trovano in stato di trattenimento» (Direttiva UE nr. 33/2013).

12 Cf. dati tratti da https://www.savethechildren.it/press/minori-stranierinon-accompagnati-tra-2012-e-2016-triplicato-il-numero-di-under-14-e (201910-14). Si noti, peraltro, che benché l'81\% dei minori accolti abbia tra i 16 e i 18 anni, si è assistito a un aumento anche della presenza di pre-adolescenti e bambini nella fascia 0-14 anni, passati da 698 nel 2012 a 2.050 nel 2016. Si noti ulteriormente che, del totale dei MSNA giunti in Italia nel 2016, appena 166 minori hanno usufruito dell'istituto della c.d. relocation.

13 Si vedano i dati forniti dal Ministero dell'Interno: http://www.libertaciviliimmigrazione.dlci.interno.gov.it/it/documentazione/statistica/i-numeri-dellasilo (2019-10-14). 
co, per i minori non accompagnati), così come per i richiedenti protezione internazionale, non risponde, come detto, solo a istanze di tipo morale e altruistiche, ma corrisponde altresì a uno specifico obbligo di matrice nazionale e internazionale gravante sugli Stati: quello di garantire il rispetto del principio del c.d. best interest of the child. Tale principio, la cui origine viene spesso ricondotta alla Convenzione Internazionale sui diritti dell'infanzia e dell'adolescenza del 1989, ha in realtà fatto ingresso in Italia già nella vigenza del Codice Civile del 1865, quando veniva sovente utilizzato dai giudici nelle decisioni vertenti sull'affidamento del figlio minore in caso di separazione legale dei genitori (Long 2006, 251), ed è oggi garantito nell'ordinamento giuridico italiano agli artt. 2, 3 e 31 della Costituzione, i quali, infatti, sono stati costantemente interpretati dalla Corte Costituzionale nel senso di garantire il preminente interesse del minore (Orlandi 2006,43), oltre che dalla normativa ordinaria.

A livello internazionale il principio del superiore interesse del minore è affermato e tutelato da una serie di atti internazionali tra i quali spiccano la Convenzione europea sull'adozione dei minori del 1967, la Carta dei diritti fondamentali dell'Unione europea (Carta di $\mathrm{Nizza}^{14}$ ) e, soprattutto, la Convenzione Internazionale sui diritti dell'infanzia e dell'adolescenza, che tratta del best interest of the child all'art. $3 .{ }^{15} \mathrm{La}$ Commissione per i diritti del fanciullo, in effetti, considera questo criterio come uno dei principi portanti dell'intera Convenzione, tale da modellare e sostenere tutte le altre previsioni ivi contenute (Freeman 2008). ${ }^{16}$

14 Ci si riferisce, in particolare, all'art. 24, comma 2, secondo cui «in tutti gli atti relativi ai bambini, siano essi compiuti da autorità pubbliche o da istituzioni private, l'interesse superiore del bambino deve essere considerato preminente». La Carta di Niz$\mathrm{za}$, come è noto, «ha lo stesso valore dei trattati» (art. 6, comma 1, TUE).

15 Il quale recita: «1. In tutte le decisioni relative ai fanciulli, di competenza delle istituzioni pubbliche o private di assistenza sociale, dei tribunali, delle autorità amministrative o degli organi legislativi, l'interesse superiore del fanciullo deve essere una considerazione preminente. 2. Gli Stati parti si impegnano ad assicurare al fanciullo la protezione e le cure necessarie al suo benessere, in considerazione dei diritti e dei doveri dei suoi genitori, dei suoi tutori o di altre persone che hanno la sua responsabilità legale, e a tal fine essi adottano tutti i provvedimenti legislativi e amministrativi appropriati. 3. Gli Stati parti vigilano affinché il funzionamento delle istituzioni, servizi e istituti che hanno la responsabilità dei fanciulli e che provvedono alla loro protezione sia conforme alle norme stabilite dalle autorità competenti in particolare nell'ambito della sicurezza e della salute e per quanto riguarda il numero e la competenza del loro personale nonché l'esistenza di un adeguato controllo».

16 Il principio del supremo interesse del bambino, infatti, si può ritrovare in diversi articoli della convenzione, quali: l'art. 9 (diritto a non essere separati dai genitori contro la propria volontà e a mantenere contatti con loro, salvo che la separazione stessa o la cessazione di ogni contatto corrisponda al miglior interesse del bambino); il 18 (il quale sancisce che la preoccupazione primaria dei genitori nei confronti dei figli deve essere quella di garantire il loro superiore interesse); il 20 (che attribuisce il diritto alla protezione e all'assistenza statale nei confronti del minore il cui migliore interes- 
Ė possibile apprezzare la pregnanza degli obblighi di adeguata accoglienza gravanti su ciascuno Stato membro, se si considera che essi gravano anche nelle more della determinazione dello Stato membro competente per l'esame della domanda di asilo del richiedente, in applicazione del regolamento di Dublino (Reg. CE nr. 343/2003), che consente allo Stato di accoglienza di indirizzare una richiesta di presa in carico o di ripresa in carico ad un altro Stato membro in quanto Stato membro competente. ${ }^{17}$

Tanto premesso al livello di principi generali, il d.lgs. 142 del 2015, che ha attuato la direttiva 2013/33/UE recante norme relative all'accoglienza dei richiedenti protezione internazionale, nonché la direttiva 2013/32/UE, recante procedure comuni ai fini del riconoscimento e della revoca dello status di protezione internazionale, definisce il minore straniero non accompagnato quale «lo straniero di età inferiore agli anni diciotto, che si trova, per qualsiasi causa, nel territorio nazionale, privo di assistenza e rappresentanza legale». ${ }^{18}$ Una definizione più ampia ma sostanzialmente identica è contenuta all'art. 2 della legge 47 del 2017, ${ }^{19}$ c.d. Legge Zampa, recante disposizioni in materia di protezione dei minori stranieri non accompagnati. ${ }^{20}$ Tale legge disciplina gli aspetti fondamentali per la vita dei minori migranti che arrivano in Italia senza genitori: dalle procedure per l'identificazione e l'accertamento dell'età agli standard dell'accoglienza, alla promozione dell'affido familiare alla figura del tutore, alle cure sanitarie, all'accesso all'istruzione.

La volontà politica alla base della elaborazione di una disciplina specificamente dedicata ai MSNA era quella di approntare maggiori tutele nell'accoglienza e nel trattamento degli stessi ed esprimeva la necessità di dare rilievo alla condizione di vulnerabilità che li carat-

se sia nel senso di venire allontanato dall'ambiente familiare); il 21 (relativo alla necessità di considerare il miglior interesse del bambino nel contesto di un'adozione); il 37 (che vieta che i minori privati della loro libertà vengano separati dagli adulti se ciò non corrisponde al loro migliore interesse); e il 40 (che impone, nel contesto di un processo penale a carico del minore, di tenere conto di alcuni dati personali che lo riguardano, a meno che ciò non sia contrario al suo miglior interesse).

17 Sent. Corte Giust. C-179/11; Cf. TAR Friuli Venezia Giulia, Sezione III, nr. 156 del 11 marzo 2011: «nelle more della determinazione dello Stato competente per l'esame di una richiesta di asilo, lo straniero è da considerarsi richiedente asilo a tutti gli effetti; in particolare, si applicano tutte le disposizioni relative all'assistenza».

18 Cf. art. 2, co. 1, lett. e) d.lgs. 142 del 2015.

19 Ex art. 2 di tale legge «per minore straniero non accompagnato presente nel territorio dello Stato si intende il minorenne non avente cittadinanza italiana o dell'Unione europea che si trova per qualsiasi causa nel territorio dello Stato o che è altrimenti sottoposto alla giurisdizione italiana, privo di assistenza e di rappresentanza da parte dei genitori o di altri adulti per lui legalmente responsabili in base alle leggi vigenti nell'ordinamento italiano».

20 Legge 47/2017, entrata in vigore il 6 maggio 2017. 
terizza, sia rispetto all'inserimento in un contesto estraneo e potenzialmente pericoloso per il minorenne, sia rispetto alle conseguenze sulla salute psico-fisica derivanti dal vissuto antecedente l'abbandono del Paese di origine.

Anche alla luce della normativa introdotta e implementata relativamente ai minori non accompagnati, la loro massiccia e non occasionale presenza tra le fila dei richiedenti asilo sarebbe stata tale da giustificare la necessità di provvedere in modo coerente e adeguato alla predisposizione di un sistema di accoglienza dedicato specificamente ai minori, che tenesse conto della condizione di vulnerabilità degli stessi.

Le strutture di prima accoglienza dove vengono indirizzati i MSNA, infatti, dovrebbero essere a loro specificamente dedicate e i minori non vi potrebbero sostare per più di trenta giorni. Ciò significa, quindi, che non solo le strutture di accoglienza 'ordinarie' devono essere specificamente e unicamente dedicate ai minori, ma la stessa previsione è estesa anche ai centri di prima accoglienza di cui all'art. 19, co. 1, del d.lgs. 142/2015.

Il successivo comma dello stesso articolo prevede poi che, dopo la fase di permanenza nei centri di prima accoglienza, «i minori non accompagnati sono accolti nell'ambito del Sistema di protezione per richiedenti asilo, rifugiati e minori stranieri non accompagnati [...] e in particolare nei progetti specificamente destinati a tale categoria di soggetti vulnerabili».

È evidente che l'introduzione di questa norma avrebbe dovuto spingere l'apparato amministrativo statale a predisporre all'interno del Sistema SPRAR un numero di posti riservati ai minori che fosse sufficiente a far fronte al numero di ingressi: si consideri invece che, ad oggi, si stima che i posti disponibili per i minori siano appena $2.127,{ }^{21}$ il che esclude il $90 \%$ dei minori richiedenti asilo da una accoglienza adeguata, considerato che la attuale collocazione presso i CAS insieme a maggiorenni evidentemente non garantisce.

La strutturale e persistente 'sottovalutazione' della condizione dei minori non accompagnati, in quanto soggetti vulnerabili ex le$g e$ - tanto da essere inespellibili indipendentemente dal fatto che presentino o meno la domanda di protezione internazionale, in ossequio alle previsioni della Convenzione di New York a suo tempo ratificata dall'Italia $-{ }^{22}$ costituisce l'indice più macroscopico e sintomatico della cifra delle politiche migratorie attuate dagli ultimi tre governi.

21 Cf. http://www.lastampa.it/2017/08/29/italia/cronache/ragazzi-fantasma-mila-minori-migranti-scomparsi-in-italia-nellultimo-anno-a7eqlTIaCPCCe2Uvuv@TQI/pagina.html (2019-10-14).

22 Convenzione di New York sui diritti dell'Infanzia del 20 novembre 1989, ratificata dall'Italia il 27 maggio 1991 con la l. 176. Nel Testo Unico Immigrazione (d.lgs. 286/98) l'inespellibilità dei minori stranieri non accompagnati è prevista dall'art. 19, comma 1 . 
I servizi speciali che andrebbero predisposti in considerazione delle esigenze particolari delle persone vulnerabili annoverano: a) l'alloggiamento in strutture che garantiscano, nei limiti del possibile, la tutela della vita familiare e dell'integrità del nucleo familiare; b) l'esercizio di regolari controlli per accertare la qualità dei servizi erogati dal centro di accoglienza, a carico e a cura delle Prefetture con l'ausilio dei servizi sociali del comune; c) una formazione degli operatori adeguata alla natura e alla complessità dei profili di vulnerabilità che si presentano di caso in caso; d) presa in carico da parte dell'ente gestore del centro di accoglienza (ordinario - SPRAR - o 'straordinario' - CAS - non importa) dell'iscrizione al servizio sanitario nazionale dei richiedenti asilo e dei loro familiari inseriti nei servizi di accoglienza; e) ammissione nei centri di accoglienza degli avvocati e dei rappresentanti dell'ACNUR e delle associazioni o degli enti di tutela autorizzato; f) assoggettamento all'obbligo scolastico dei minorenni ospitati a cura degli enti gestori.

Quando si ha a che fare con minori, è di fondamentale importanza accertarne correttamente l'età, la quale incide non solo sul loro grado di maturità ma anche sulla loro capacità di affrontare tutte le difficoltà, gli stenti e, sovente, i soprusi che sono costretti a vivere durante i terribili viaggi che li conducono fino alle coste italiane e europee; tanto che una certa giurisprudenza, nel concedere una delle possibili forme di protezione internazionale, dà rilievo proprio al fatto che il richiedente si sia allontanato dal Paese d'origine prima del compimento della maggiore età. ${ }^{23}$

Nel clima politico e culturale attuale, tuttavia, perfino quando l'accertamento della minore età del richiedente abbia dato esito positivo, al minore potrebbero non essere riconosciuti i propri diritti se non appare 'abbastanza minore'. Nel sistema di accoglienza extra-ordinem italiano dei minori richiedenti asilo, in cui il superiore interesse del fanciullo viene sistematicamente sacrificato ad altri interessi sulla base di valutazioni di ordine statistico o meramente empirico, infatti, i cosiddetti 'grandi minori', ovvero quelli prossimi al compimento della maggiore età - che sono la maggioranza -, vengono visti alla stregua di fruitori strumentali del sistema di accoglienza. In questa prospettiva tutta soggettivistica e moralistica, il minore de-

23 Cf., per un esempio recente, Trib. Palermo, ord. 30 giugno 2017, disponibile in http://www.meltingpot.org/IMG/pdf/ordinanza_trib_palermo_30062017.pdf (2019-10-14), in cui è stata riconosciuta la protezione sussidiaria a un richiedente gambiano fuggito dal Paese da minorenne. In tale ordinanza, infatti, si legge che «le circostanza di fatto del contesto di origine del richiedente, quelle inerenti alle ragioni di allontanamento dal suo paese [...], nonché, con estrema attenzione, l'età dello stesso al momento dell'allontanamento dalla sua terra d'origine e di approdo nel territorio di questa repubblica, debbono indurre l'interprete giudiziario a non pretendere un estremo rigore nella produzione e nel confezionamento della prova di quanto dal richiedente la tutela abbia potuto effettuare». 
ve dimostrarsi meritevole delle misure di accoglienza, pena il venire considerato un 'quasi adulto', in quanto tale non meritevole della tutela che gli sarebbe giuridicamente dovuta.

\section{I diritti umani sistematicamente violati nel sistema extra-ordinem dell'accoglienza dei minori richiedenti asilo: artt. 3, 8 e 13 CEDU}

Nel sistema di accoglienza dei minori richiedenti asilo, che vede la maggioranza di essi collocati presso enormi CARA o hotspot, oppure nei cosiddetti CAS, entrambi sistemi gestiti da enti appaltatori delle Prefetture con esclusione del coinvolgimento dei comuni, la prima macroscopica violazione di diritti fondamentali riguarda le modalità di accertamento dell'età. In effetti, il mancato ovvero l'erroneo accertamento dell'età del minorenne è già di per sé la prima e principale violazione del suo diritto ad essere tutelato non solo in quanto richiedente asilo bensì innanzitutto quale persona minorenne.

Nella maggior parte dei casi di c.d. grandi minori, infatti, l'autorità di pubblica sicurezza preposta a identificare il minore li considera maggiorenni alla luce delle risultanze di un esame radiologico del polso, illegittimamente imposto a tutti i richiedenti dei quali non sia certa l'età.

L'errata attribuzione dell'età determina tanto il mancato trattamento dei minori come tali e, di conseguenza, la loro mancata protezione così come prevista dall'ordinamento, quanto il loro collocamento e permanenza in centri di accoglienza per adulti in cui sono destinatari di trattamenti inadeguati a soggetti vulnerabili, quali essi sono, e che pertanto li espongono a situazioni di promiscuità, sovraffollamento e mancanza di una specifica assistenza da parte di personale specializzato, complessivamente configurandosi trattamenti potenzialmente inumani e degradanti, in violazione congiunta degli artt. 3 e 8 della Convenzione europea per la salvaguardia dei diritti dell'uomo e delle libertà fondamentali (CEDU).

Il metodo di accertamento all'età normalmente utilizzato non soddisfa i criteri raccomandati dall'UNHCR in particolare sotto tre profili. ${ }^{24}$ In primo luogo, la radiografia del distretto polso-mano viene normalmente valutata sulla base del metodo di Greulich-Pyle anziché dell'assai più recente e affidabile metodo TW3. È noto infatti tra gli esperti della materia che il metodo di Greulich-Pyle si basa su standard definiti su popolazioni (cittadini statunitensi di origine nordeu-

24 In tema di accertamento dell'età si invita a consultare il documento di UNHCR, L'accertamento dell'età dei minori stranieri non accompagnati e separati in Italia, disponibile al link https://www.unhcr.it/wp-content/uploads/2016/01/accertamento.pdf (2019-10-14). 
ropea nati tra le due guerre), ben differenti rispetto a quelle di appartenenza dei soggetti valutati.

In secondo luogo, gli esiti degli esami compiuti sui minori non riportano il margine di errore di +/- 2 anni insito nella variabilità biologica e nelle metodiche utilizzate, oppure, quando lo riportano, tale margine di errore viene utilizzato 'contro' il presunto minore (ad esempio se può avere tra i 16 e i 20 anni viene presunta la maggiore età, in aperto contrasto con la disciplina legale applicabile che impone di presumere la minore età fino a prova contraria ${ }^{25}$ ). Fino all'entrata in vigore della Legge Zampa, non esisteva nessuna norma che regolasse la procedura di accertamento dell'età dei MSNA, pertanto la necessità di indicare il margine di errore era stata desunta da una interpretazione analogica dell'obbligo contenuto all'art. 5, c. 3, del d.p.c.m. 234/2016 relativo alla determinazione dell'età dei minori non accompagnati vittime di tratta.

L'art. 19-bis del d.lgs. 142 del 2015, come modificato dalla legge Zampa, prevede ora che «nella relazione finale deve essere sempre indicato il margine di errore. Qualora, anche dopo l'accertamento socio-sanitario, permangano dubbi sulla minore età, questa si presume ad ogni effetto di legge».

Ma ciò che più conta è che nella stragrande maggioranza dei casi i richiedenti asilo che si dichiarano minorenni vengono sottoposti all'esame della maturazione ossea del distretto polso-mano, quale unico accertamento, anziché essere esaminati sulla base di un approccio multidisciplinare. La fonte dell'obbligo di utilizzare un approccio multidisciplinare è il co. 6 del citato art. 19-bis, il quale ha generalizzato l'obbligo già previsto per i minori vittime di tratta dal d.p.c.m. 234 del $2016 .^{26}$

La genericità della previsione della norma, che nulla dice circa quali debbano essere le caratteristiche di un esame multidisciplinare, legittimano l'interprete a riferirsi ancora una volta in via analogica a quanto previsto nel d.p.c.m. 234 del 2016, che specifica che l'esame multidisciplinare «consiste nello svolgimento di un colloquio sociale, vertente anche sulle pregresse esperienze di vita rilevanti per l'accertamento, di una visita pediatrica auxologica e di una valutazione psicologica o neuro-psichiatrica, alla presenza, se necessario, di un mediatore culturale o di un interprete». ${ }^{27}$

25 L'art. 4 d.lgs. 24 del 2014 già prevedeva che «la minore età dello straniero è, altresì, presunta nel caso in cui la procedura multidisciplinare svolta non consenta di stabilire con certezza l'età dello stesso». Tale previsione è stata introdotta dalla Legge Zampa anche in materia di protezione internazionale all'art. 19-bis del d.lgs. 142 del 2015.

26 Il comma 6 dell'art. 19-bis, d.lgs. 142 del 2015, prevede esplicitamente che «l'accertamento socio-sanitario dell'età deve essere svolto in un ambiente idoneo con un approccio multidisciplinare da professionisti adeguatamente formati e, ove necessario, in presenza di un mediatore culturale».

27 Così recita l'art. 5, co. 2, d.p.c.m. 234 del 2016. 
Quanto poi alle condizioni di vita all'interno delle strutture di accoglienza, l'art. 3 della CEDU (che vieta trattamenti inumani e degradanti) ${ }^{28}$ viene in rilievo tutte le volte che le strutture di accoglienza sono interessate da situazioni di sovraffollamento. ${ }^{29}$

Casi di violazione dell'art. 3 a causa delle condizioni inumane e degradanti cui vengono sottoposti i richiedenti asilo nei Paesi di accoglienza non sono d'altra parte ignoti alla giurisprudenza della Corte EDU (Corte europea dei diritti dell'uomo) che, al contrario, negli ultimi anni si è sovente pronunciata sul punto. La decisione forse più nota è quella relativa al caso M.S.S. c. Belgio e Grecia del 2011, ${ }^{30}$ laddove i giudici di Strasburgo hanno condannato sia la Grecia, responsabile per aver sottoposto M.S.S. a condizioni di vita degradanti durante il periodo di detenzione in un centro per richiedenti e dopo il rilascio da tale centro, quanto il Belgio. Le autorità belghe infatti, anche se consapevoli delle carenze del sistema greco in materia di richiedenti asilo, avevano proceduto comunque all'espulsione di M.S.S. in ottemperanza alle norme sulla competenza giurisdizionale di cui al Regolamento cd. Dublino II, limitandosi a un'applicazione automatica del Regolamento stesso senza procedere a una verifica effettiva circa l'applicazione delle garanzie e degli standard di protezione per i richiedenti asilo garantiti in Grecia.

Con riferimento specifico alle condizioni di accoglienza presenti in Italia, invece, si ricorda la decisione della Corte di Strasburgo

28 Tale articolo prevede che «nessuno può essere sottoposto a tortura né a pene o trattamenti inumani o degradanti».

29 Si pensi al 'Campo' di Cona in provincia di Venezia, il quale, a fronte della una capienza massima di 542 posti prevista all'art. 2, cpv. 1 dell'avviso di gara della Prefettura di Venezia del 18-05-2016, nel gennaio del 2017 contava ben 1.400 persone ospitate, tanto che i posti letto attribuiti ai ricorrenti si trovavano in una tensiostruttura delle dimensioni di circa $360 \mathrm{mq}$. dove si stima alloggiassero 250 persone adulte, di sesso maschile, tra i quali una sessantina di minorenni, in letti a castello fittamente affiancati e senza alcuno spazio personale. Il riscaldamento all'interno del tendone, inoltre, non risultava essere in funzione, né i bagni erano forniti di acqua calda. Nella struttura circolavano armi bianche, alcool e stupefacenti, e si verificavano non di rado episodi di prostituzione; era difficile accedere alla mensa a causa delle lunghe file che si formavano all'aperto e non vi erano tavoli per la consumazione dei pasti; i ricorrenti della Causa Darboe e Camara contro Italia, in corso di decisione alla Corte di Strasburgo, hanno riferito di non essere mai stati sottoposti a una visita medica nel 'campo' e di aver ricevuto i medicinali in un piccolo ambulatorio, gestito non da personale medico ma da uno degli ospiti, promosso a mediatore culturale; gli spazi dedicati all'insegnamento della lingua italiana erano inadeguati allo scopo e gli spazi ricreativi del tutto inesistenti; la presenza di operatori era troppo esigua per assicurare un controllo di quanto accadeva all'interno della struttura, con conseguenti prevaricazioni nei confronti delle persone più vulnerabili e rischi di violenze e abusi sessuali, anche in considerazione dell'assenza di operatori durante la notte; mancava l'assistenza psicologica e l'informazione legale.

30 Corte EDU, sentenza M.S.S. c. Belgio e Grecia [GC], N. 30696/09, 21 gennaio 2011. Si tratta della prima sentenza relativa all'applicazione del Regolamento cd. 'Dublino II'. 
nel caso Tarakhel c. Svizzera del 2014. ${ }^{31}$ In tale circostanza la Corte ha dichiarato che il rinvio verso l'Italia di richiedenti asilo particolarmente vulnerabili, quali un nucleo familiare con minori, è suscettibile, in mancanza di adeguate garanzie, di violare il divieto di trattamenti inumani o degradanti. Nell'assumere tale determinazione i giudici hanno fatto riferimento in particolare a tre aspetti critici che caratterizzano il sistema di accoglienza italiano: la lentezza delle procedure di identificazione; la limitatezza dei posti a disposizione nei centri di accoglienza; le generali condizioni di accoglienza nelle strutture disponibile. ${ }^{32}$

Circa un caso di violazione dell'art. 3 della CEDU riferita a un MSNA, invece, il riferimento va alla decisione Rahimi c. Grecia del $2008,^{33}$ in cui la Corte, oltre a criticare nuovamente le generali condizioni di accoglienza che caratterizzano il sistema greco, rileva altresì che il fatto che il ricorrente minorenne si trovi senza assistenza da parte di adulti concorre a definire una situazione di vulnerabilità che giustifica un'applicazione particolarmente attenta dei criteri di giudizio, a cominciare dalla valutazione sull'allegata violazione dell'art. 3 CEDU (Bubula 2011).

Poiché la Legge Zampa, come accennato, reca disposizioni che riguardano specificamente la materia dell'accoglienza di minori stranieri non accompagnati, essa non si occupa della definizione delle condizioni minime generali di accoglienza dei richiedenti asilo. A questo proposito, l'art. 19, comma 2-bis, del d. lgs. 142 del 2015, dispone che «le strutture nelle quali vengono accolti i minori stranieri non accompagnati devono soddisfare, nel rispetto dell'art. 117, secondo comma, lettera $\mathrm{m}$ ), della Costituzione, gli standard minimi dei servizi e dell'assistenza forniti dalle strutture residenziali per minorenni ed essere autorizzate o accreditate ai sensi della normativa nazionale e regionale in materia». Ciononostante, in molti casi i minori sono collocati in CAS consistenti in appartamenti insieme a richiedenti maggiorenni, con l'unica 'accortezza' - la quale basta da sola ad evidenziare l'improvvisazione e l'inadeguatezza del sistema di accoglienza - di collocare i minori insieme agli adulti con i quali sono giunti in Italia, esponendo così i minori alla convivenza forzata con potenziali trafficanti o comunque con estranei al novero dei familiari, sui quali raramente viene effettuata una verifica attenta.

31 Corte EDU, sentenza Tarakhel c. Svizzera [GC], N. 29217/12, 4 novembre 2014.

32 Si noti, a onor del vero, che la Corte stessa ammette che le condizioni di accoglienza nelle strutture italiane non sono tanto deficitarie quanto quelle greche; allo stesso tempo, tuttavia, esse non sono nemmeno tali da poter considerare come manifestamente infondato il rischio che un numero significativo di richiedenti asilo siano lasciati senza sistemazione o siano accolti in centri sovraffollati, senza privacy o addirittura in condizioni insalubri o di violenza.

33 Corte EDU, sentenza Rahimi c. Grecia [GC], N. 8687/08, 5 luglio 2011. 
Ciò conduce alla violazione dell'art. 8 della CEDU, relativo al diritto al rispetto della vita privata e familiare, ${ }^{34}$ tutte le volte in cui lo Stato italiano omette di vegliare sul corretto sviluppo e sulla realizzazione della personalità dei ricorrenti. L’art. 8 mira infatti a tutelare non solo l'integrità fisica dei soggetti, ma anche la loro integrità morale, estendendosi quindi anche ad aspetti relativi all'identità personale quali, per esempio, la loro identificazione nominativa o la loro identità sessuale. Non essendo agevole fornire una definizione precisa di cosa si debba intendere con le espressioni 'vita privata' e 'vita familiare', la Corte ha sempre deliberato utilizzando un approccio casistico, attento alle circostanze e ai fatti del caso concreto, e, allo stesso tempo, evolutivo, cercando di adeguare le proprie decisioni ai mutamenti sociali e della sensibilità collettiva (Bartole, Conforti, Raimondi 2001, 308).

Questa declinazione dell'art. 8 quale garanzia del corretto sviluppo della crescita e maturazione del minore al fine di tutelarne l'integrità morale, pur aderendo alla elaborazione teorica e dottrinale che è stata costruita su tale norma, rappresenta in realtà una novità rispetto alla giurisprudenza della Corte. L'art. 8 riferito ai MSNA, infatti, è stato generalmente richiamato nella giurisprudenza dei giudici di Strasburgo con riferimento a una tematica diversa, ossia quella dell'espulsione e respingimento del minore richiedente asilo. È questo l'ambito, pertanto, in cui più spesso è stata invocata e riconosciuta la violazione della vita privata e familiare dei minori. ${ }^{35}$

Si è rilevato, tuttavia, come la mancata adozione degli strumenti di tutela specificamente previsti per i minori (nomina di un tutore, assistenza psicologica, collocazione in un centro specificamente dedicato ai minori e così via) avesse avuto l'effetto di privarli della loro identità di minori, così ledendo, come accennato, la loro integrità morale.

Il d.lgs. 142/2015 sancisce espressamente che «l'assistenza affettiva e psicologica dei minori stranieri non accompagnati è assicura-

34 L'articolo così recita: «1. Ogni persona ha diritto al rispetto della propria vita privata e familiare, del proprio domicilio e della propria corrispondenza. 2. Non può esservi ingerenza di una autorità pubblica nell'esercizio di tale diritto a meno che tale ingerenza sia prevista dalla legge e costituisca una misura che, in una società democratica, è necessaria alla sicurezza nazionale, alla pubblica sicurezza, al benessere economico del paese, alla difesa dell'ordine e alla prevenzione dei reati, alla protezione della salute o della morale, o alla protezione dei diritti e delle libertà altrui». La formulazione dell'art. 8 non prescrive espressamente né un divieto, né la garanzia di uno specifico diritto; al contrario, tutta la costruzione gira attorno al concetto fondamentale di 'rispetto'. Questa nozione comporta l'imposizione agli Stati contraenti di un'obbligazione dal duplice contenuto: uno negativo, relativo al divieto di porre in essere interventi che possano, in qualsivoglia modo, ledere i diritti tutelati, e uno positivo, che impone loro di adoperarsi attivamente affinché le garanzie previste nell'articolo trovino attuazione concreta. 35 Si vedano, per esempio, le decisioni della Corte EDU nei casi Maslov c. Austria, N. 1638/03, 23 giugno 2008; Boultif c. Svizzera, N. 54273/00, 2 agosto 2001; Üner c. Paesi Bassi [GC], N. 46410/99, 18 ottobre 2006. 
ta, in ogni stato e grado del procedimento dalla presenza di persone idonee indicate dal minore, nonché di gruppi, fondazioni, associazioni od organizzazioni non governative». ${ }^{36}$ Allo stesso articolo, inoltre, è stato inserito un nuovo comma che sancisce il diritto del MSNA a partecipare ai procedimenti che lo riguardano e essere ascoltati nel merito, alla presenza di un mediatore culturale. Il nuovo art. 19-bis, infine, prevede che una volta entrato in contatto con il MSNA il personale della struttura di prima accoglienza debba subito svolgere un colloquio per approfondire la storia personale e familiare del minore richiedente, anche ai fini della sua domanda di protezione, alla presenza di un mediatore culturale.

Tutte queste previsioni sono state finora largamente disattese, tanto è vero che alcuni dei grandi centri di accoglienza CAS di tipo hotspot sono stati interessati da procedimenti giudiziari. ${ }^{37}$

L'art. 13 della CEDU, infine, stabilisce che ogni persona i cui diritti e le cui libertà riconosciuti nella Convenzione siano stati violati ha diritto a un ricorso effettivo davanti a un'istanza nazionale. La giurisprudenza della Corte Europea ha chiarito che il diritto a un ricorso effettivo è funzionale a due diversi obiettivi (Reneman 2016, 82): da un lato, quello di assicurare agli individui una tutela effettiva dei diritti previsti nella Convezione; ${ }^{38}$ dall'altro, quello di preservare la natura sussidiaria del sistema della Convenzione, dando espressione all'obbligo degli Stati di proteggere i diritti fondamentali dell'uomo anzitutto e precipuamente facendo uso del proprio sistema giurisdizionale interno. ${ }^{39}$

Nell'ordinamento giuridico italiano non esiste un mezzo di ricorso giudiziario o amministrativo che consenta in tempi accettabili di ottenere una concreta attuazione, per il minore richiedente la protezione internazionale, del diritto a condizioni alloggiative e di vita che non siano disumane e degradanti. Quanto agli esiti della procedura di accertamento dell'età, poi, essi non vengono comunicati ai ricorrenti, né viene loro notificato il provvedimento di attribuzione dell'età, privando così i richiedenti della possibilità di dare corso a qualsivoglia rimedio effettivo contro l'errata identificazione degli stessi come maggiorenni. Nel causa davanti alla Corte EDU, Darboe e Ca-

36 Nuovo art. 18, co. 2-bis del d.lgs. 142 del 2015, introdotto dalla Legge Zampa.

37 I più noti di questi processi sono certamente quello 'apripista' denominato Mafia Capitale nei confronti di Massimo Carminati e altre 36 persone, già concluso in appello e quello nei confronti dei titolari della Cooperativa Edeco (Ex Ecofficina) e del personale degli uffici prefettizi di Venezia e Padova, attualmente in fase di indagine.

38 Cf. Corte EDU, Kudla c. Polonia, N. 30210/96, 26 ottobre 2000.

39 Cf. Corte EDU, Conka c. Belgio, N. 51564/99, 5 febbraio 2002. 
mara vs. Italia, ${ }^{40}$ per esempio, i minori ricorrenti non avevano ricevuto alcuna informazione relativa ai loro diritti in quanto minorenni, né era stata loro fornita alcuna indicazione in merito ai diritti di accesso alla procedura di riconoscimento della protezione internazionale, né era stato nominato loro il tutore, impedendo ai medesimi la possibilità di presentare un riscorso giurisdizionale a tutela della propria condizione di minori ospitati nel Campo di Cona. ${ }^{41}$

In questo caso paradigmatico, che ha riguardato specificamente dei minori stranieri non accompagnati richiedenti asilo ma che si ripete, continuamente e con modalità analoghe, in innumerevoli occasioni, anche con riferimento a maggiorenni, si sono verificate tutte le più frequenti violazioni di diritti fondamentali che interessano i richiedenti asilo inseriti nei sistemi di accoglienza, quali: violazione del divieto di trattamenti inumani e degradanti (art. 3 CEDU); violazione della vita privata e familiare (art. 8 CEDU); violazione del diritto a un ricorso effettivo (art. 13 CEDU).

\section{Bibliografia}

Baracchi, Anna; Guariso, Alberto (2017). «I cittadini extra UE e acceso alle prestazioni di assistenza e sicurezza sociale». Morozzo della Rocca, Paolo (a cura di), Immigrazione, asilo e cittadinanza. Sant'Arcangelo di Romagna: Maggioli Editore, 435 e ss.

Bartole, Sergio; Conforti, Benedetto; Raimondi, Guido (2001). Commentario alla Convenzione europea per la tutela dei diritti dell'uomo e delle libertà fondamentali. Padova: Cedam.

Bubula, Fabio Augusto (2011). «L'“importanza di essere un minore non accompagnato"... oppure no? Nota a Corte EDU, Prima Sezione, sent. 5 aprile 2011, Pres. Vajić, ric. n. 8687/08, Rahimi c. Grecia». Diritto Penale Contemporaneo. URL https://www. penalecontemporaneo.it/d/1097-l--importanzadi-essere-un-minore-non-accompagnato-oppure-no (2019-10-15).

Ferrero, Marco; Perocco, Fabio (2011). «Razzismo, lavoro, discriminazioni, diritto». Ferrero, Marco; Perocco, Fabio (a cura di), Razzismo al lavoro. Il sistema

40 Ricorso nr. 5797/17, Darboe e Camara contro Italia, pubblicato in Hudoc, il sito ufficiale della Corte di Strasburgo: https://hudoc.echr.coe.int/eng\#\{ "fulltext":["dar boe"], "documentcollectionid2": ["JUDGMENTS", "DECISIONS", "COMMUNICATEDCASES" ], "itemid":[“००1-172034"]\} (2019-10-14).

41 Ai sensi dell'art. 35 della CEDU, infatti, è possibile adire la Corte solo successivamente all'infruttuoso esperimento delle vie di ricorso interne. L'esaurimento dei ricorsi interni rappresenta, pertanto, una condizione di ammissibilità del ricorso, superabile in un unico caso: qualora, ai sensi dell'art. 13, l'ordinamento interno non offra al ricorrente alcun rimedio effettivo per lamentare la violazione di propri diritti. In forza delle norme dell'ordinamento italiano relative all'acquisto della capacità di agire, i due ricorrenti non avrebbero potuto in alcun modo azionare giudizialmente i propri diritti se non per il tramite di un tutore che, ex art. 357 c.c., li rappresentasse in tutti gli atti civili. Tale tutore, tuttavia, non era mai stato nominato. 
Marco Ferrero

La vulnerabilità degli immigrati nel sistema di accoglienza extra-ordinem dei richiedenti asilo

della discriminazione sul lavoro, la cornice giuridica e gli strumenti di tutela. Milano: Franco Angeli, 7-62.

Freeman, Michael (2007). A Commentary on the United Nations Convention on the Rights of the Child, Article 3: The Best Interests of the Child. Leiden-Boston: Brill-Nijhof.

Long, Joelle (2006). «ll ruolo del principio del superiore interesse del minore nella disciplina dell'immigrazione». Minorigiustizia, 1, 251-9.

Orlandi, Maurizio (2006). Le adozioni internazionali in Italia: realtà e disciplina giuridica. Milano: Giuffré.

Reneman, Marcelle (2016). EU Asylum Procedures and the Right to an Effective Remedy. Oxford; Portland: Hart Publishing. 


\section{Parte III}

Condizioni di salute, disagio psichico, tutela assistenziale 



\title{
Migration and Torture: Building a Map of Knowledge
}

Pau Pérez-Sales

Hospital Universitario La Paz, Madrid, España

\begin{abstract}
The article presents a systematic literature review on migration, torture and health.

Keywords Migration. Torture. Literature review. Health. Asylum seekers.

Summary 1 The "Refugee Crisis". - 2 Research on Migration and Torture. - 3 Where Next? Concluding Remarks.
\end{abstract}

\section{The 'Refugee Crisis'}

We already have more than twenty-five years of academic research on migration and torture; the field has developed into an increasingly complex one since the first descriptive and epidemiological studies.

The recent war in Syria, added to previous conflicts in Afghanistan, Iran, Iraq, has led to mass displacement, especially since 2015, to neighbouring countries and Europe, in what has come to be known as the refugee crisis. The concept crisis as applied to Europe is a relative one. According to 2018 UNCHR figures, ${ }^{1}$ while there are 22.5 million refugees in the world, the top hosting countries are Turkey (2.9 M), Pakistan (1.4 M), Lebanon (1 M), Iran $(979,000)$, Uganda $(940,000)$ and Ethiopia $(791,000)$. While $30 \%$ of those living in Lebanon (a country with a very unstable religious and political equilibrium)

Published in Torture, 28(2), 2018 (courtesy).

1 Resettlement data finder accesed in http://rsq.unhcr.org/en (2019-10-16). 
are refugees, ${ }^{2}$ in Europe the proportion of refugees is marginal in demographic terms, even including the increase in the last two years.

Several episodes have marked the European political confrontation around the 'refugee crisis' in this period. These include, (a) Germany's decision to both open its doors in 2015 and 2016 and accept more than one million refugees, and then subsequently restrict entry in 2017 after the political environment altered due to various factors, including two Islamist attacks attributed to newly arrived refugees. (b) The EU decision in May 2017 to transfer 160,000 asylum seekers that were stuck in Greece and Italy to other European member states was met with widespread resistance. The European Union was not able to fully act on the decision and the transfer could only partially take place. As a result, around 65,000 refugees remained in both countries, and especially on the Greek Islands in precarious conditions. (c) In March 2016, in exchange for political and financial benefits, the EU signed an agreement with Turkey (recognised as $a$ safe country) to accept people being sent back from Greece. In spite of that, only around 2,000 persons ${ }^{3}$ were sent back due to resistance of the Greek courts to apply the agreement (Roman et al. 2016). Those who were returned faced detention in overcrowded cells and deportation (Ulusoy, Battjes 2017).

Europe is now trying similarly unacceptable arrangements with Libya, Egypt, Sudan, and Nigeria, among others, countries which can never be considered as safe countries for refugees to be sent back to.

The true refugee crisis is of course that around 4,600 persons are estimated to have died trying to cross the Mediterranean in the 2015-18 period, ${ }^{4}$ the sufferings of hundreds of migrants exploited, victims of extortion, tortured and abused on their way north, pushed back at borders violating non-refoulement principles or abandoned to their fate on the sea.

\section{Research on Migration and Torture}

It is worthwhile attempting a structural map of knowledge of where we are currently with respect to research. Figure 1 is not meant to be exhaustive, but illustrative.

\footnotetext{
2 https://ec.europa.eu/echo/files/aid/countries/factsheets/lebanon_syrian_crisis_en.pdf (2019-10-16).

3 http://www.dw.com/en/the-eu-turkey-refugee-agreement-areview/a-43028295 (2019-10-16).

4 https://www.opensocietyfoundations.org/explain-ers/understanding-migration-and-asylum-euro-pean-union (2019-09-01).
} 


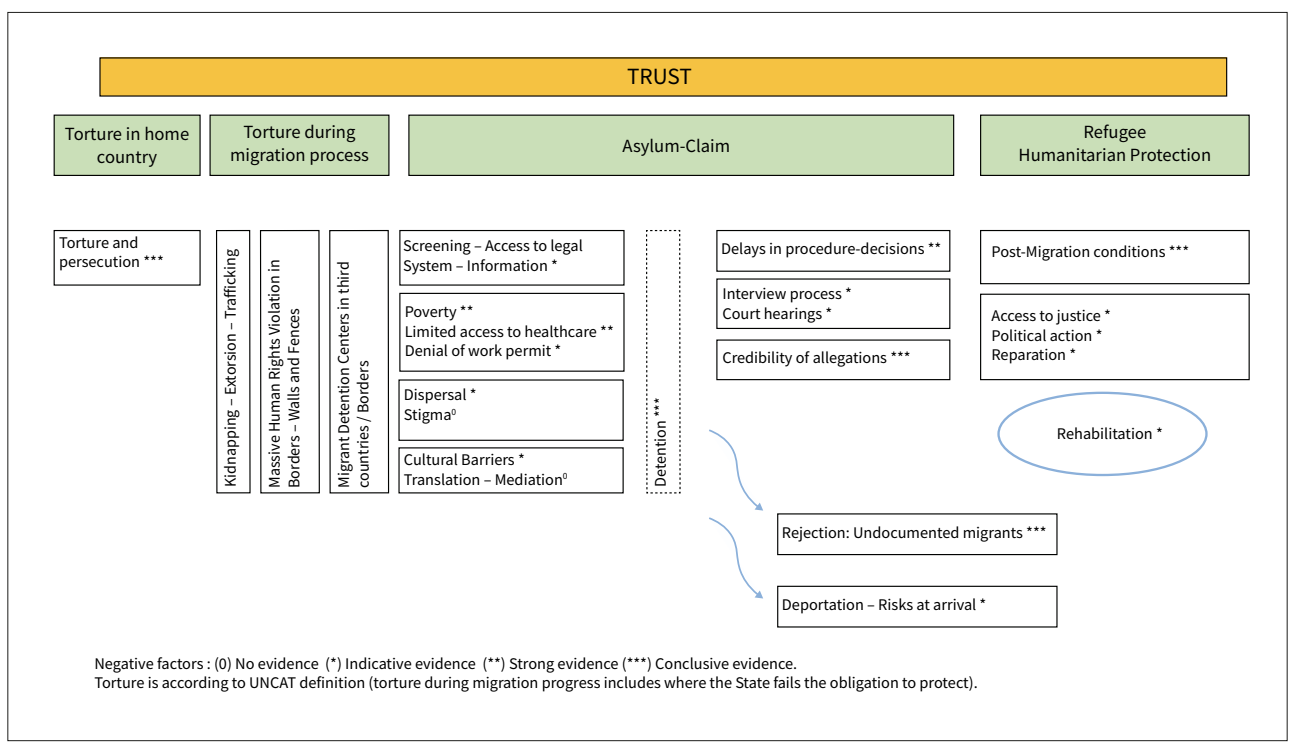

Figure 1 Migration and torture. Psychosocial determinations of health and well-being and right to rehabilitation

\subsection{Overarching Issues}

The Mental Health Impact of Torture

Different reviews since the 1990s have provided strong evidence of the mental health impact of persecution and torture (Johnson, Thompson 2008; Momartin et al. 2003; Steel et al. 2009). Just to mention one, a meta-analysis of 161 articles reporting results from a sample of 81,866 refugees from 40 countries showed that torture emerges as the strongest pre-migration factor associated with PTSD and depression, followed by cumulative exposure to potentially traumatic events (Steel et al. 2009).

\section{Distrust as a Cross-Cutting Element}

The impact of hardship and torture is not only measurable in clinical terms though. As academic research has shown, if one psychological element illustrates the migrant's experience and provides a framework of understanding of his or her inner experience, it is that of trust. The decision to flee for torture survivors is part of a complex process. Voutira and Harrell-Bond (1995) showed, among oth- 
ers, how adaptive distrust shaped the experience of survivors of war and torture. There is a mixture of individual, community, institutional and social mistrust shaped by the context of violence and menace behind the decision to flee (Lyytinen 2017). Key decisions during the route that could mean the difference between being dead or alive depended on trusting decisions. For years, mistrust is the norm (Daniel, Knudsen 1995). Particularly as refugees are often used as a bargaining chip in political disputes amongst countries, traders and local authorities, who often have their own hidden agenda, and NGO's and iNGOs can be unwilling to provide support for an extended period of time (Stedman, Tanner 2003).

Country of destination is rarely a decision of the asylum-seeker. Studies show that, at the beginning, the main concern is to find a safe place. Final destination, however, depends on having funds and very circumstantial decisions made in the heat of the moment, as well as being directed by smugglers, police, the military, governments, and/or agencies to particular countries with little choice (Robinson, Segrott 2002). Dispersal within a country or between countries can destabilise precarious social networks as well as disrupt the fragile bonds of trust of early psychological care (Griffiths 2012; Ní Raghallaigh 2014).

\section{Crossing Borders: When Torture Happens During Flight}

Borders have become places of very serious human rights violations, as the Special Rapporteur against Torture (2018) has noted in his latest thematic report. To give an example, just on the border between Guatemala and Mexico, according to official figures and reports of local organizations, ${ }^{5}$ an estimated 20,000 people were reported missing (desaparecidos) in the period 2015-17 at the hands of organised crime and trafficking with the necessary cooperation of the State and local police forces. This is to be added to the general situation in the Mexican-United States border itself, where there has been an estimated 800 cases of missing people in the last two years. According to independent reports, the US is maintaining a policy of illegal detention of asylum-seekers including extreme conditions in cells, indefinite separation of minors from their parents, lack of information and access to legal counsel, victimisation and other forms of coercion to accept returning to Mexico, in what human rights groups and the rapporteur himself have considered as amounting to systemic State torture (Hope Border Institute 2018; Human Rights First 2017). Eu-

5 Centro Fray Matias de Córdoba: http://cdhfray-matias.org/web. Movimiento Migrante Mesoamericano: https://movimientomigrantemesoameri-cano.org/inicio (2019-10-16). 
rope pursues similar policies by funding detention centres and coercive actions in North Africa where the systematic violations of human rights including torture and summary executions that take place have been denounced. ${ }^{6}$

There is a special need to address the challenges of migrants who, along their migration route, have suffered violence (including torture by non-State actors and sexual violence). They should be offered similar protection against further abuses and exploitation and to ensure access to basic human rights (to health, housing, education, rehabilitation, etc).

\section{Detention Centres}

The Global Detention Project (GDT) monitors detention of immigrants in 'host' countries, ${ }^{7}$ and has a global map and detailed data of around 2,000 immigration-related detention sites across the globe. Within the United States, which is the most dramatic example, there has been a sustained expansion with between 430,000-470,000 individuals being recently subject to some form of immigration detention annually compared to numbers as low as 6,000 in 1995 and 16,000 in 1998. Europe has also experienced a rapid expansion of detention, including outsourcing detention to border countries.

There is a large body of literature that analyses the health impact of detention on victims fleeing torture and violence in their countries and indicates that detention substantially worsens the health of asylum-seekers (Fazel, Silove 2006; Keller et al. 2003; Robjant et al. 2009; Sobhanian et al. 2006; Storm, Engberg 2013). Data show symptoms of depression, anxiety, exacerbation of PTSD, marked increase in reported negative mood states, suicidal ideation and selfdestructive thoughts. In all the studies the impacts are directly related to length of detention. Prolonged or indefinite detention per se produces learned helplessness and powerlessness (Storm, Engberg 2013). The transnational DEVAS study showed in 23 EU countries that almost half of the detainees in migration centres inside Europe did not understand the reason for their detention and equate their detention centre with that of a prison. Approximately one third referred to clear physical consequences and half described a negative impact on mental health. There was a general sense of indignity among detainees (Jesuit Refugee Service - Europe 2010). This is specially so in the subgroup of torture survivors (Filges et al. 2018; Storm, Engberg

6 See reports: http://ddhhfronterasur2017.org/es. See reports and maps by Migreurop: http://www.migreurop.org (2019-10-16).

7 https://www.globaldetentionproject.org (2019-10-16). 
2013). According to recommendations from the United Nations' High Commissioner for Refugees (UNHCR 2012) and the Special Rapporteur on Torture (2018), torture survivors and other vulnerable groups should generally not be detained. Health professionals should actively oppose this measure based on ethical and deontological principles (Brooker et al. 2016; Pearman, Olinga-Shannon 2017).

\section{Accessing the System of Protection for Torture Survivors}

There is an important concern in the anti-torture sector that torture survivors who suffer trauma-related mental disorders are being refused protection by countries in which they seek asylum. A pioneering study (Silove et al. 2006) followed a consecutive sample $(n=73)$ of recently arrived asylum seekers attending immigration agents in Sydney, Australia. Participants were followed up to assess the outcomes of their refugee applications. Although the participants reported high rates of torture (51\%), and this group is of course at the highest risk of suffering a combination of post-traumatic stress disorder (PTSD) and major depression, neither past torture nor current psychiatric disorder predicted the outcomes of refugee applications. Although the Asylum Procedures Directive 32/2013 (European Council 2013) establishes special measures to detect and properly document torture survivors, ${ }^{8}$ there are serious concerns regarding its proper application (IRCT 2016).

Faced with the increasing numbers of asylum seekers, a number of initial screening tools - up to 20 documented in academic journals and grey literature - have been proposed, the majority of large institutions having their own. There are suggestions and formats from national and international bodies including guidelines from the European Union itself (PROTECT Project 2016) (see also Mewes et al. 2018). The scene is variegated and requires revision as most are non-validated instruments that can be classified into two broad categories: (a) short clinical measures based on abbreviated diagnoses of posttraumatic stress or general psychological distress that can be applied by administrative staff (i.e. Hollifield et al. 2013); and, (b) general indicators of vulnerability (i.e. UNHCR et al. 2016). It should be remembered that there is a low to moderate correlation between experiences of torture and psychiatric disorders, and that PTSD is nei-

8 The Asylum Procedures Directive (recast) was adopted by the European Parliament and the Council in 2013 and was to be transposed into Member States' national legislations by July 2015. The Commission presented in July 2016 a Proposal for a new Asylum Procedure Regulation. Point 31 states that "National measures dealing with identification and documentation of symptoms and signs of torture or other serious acts of physical or psychological violence, including acts of sexual violence, in procedures covered by this Directive may, inter alia, be based on the [...] Istanbul Protocol" (European Council 2013). 
ther the only nor the most likely consequence of torture in the long term. Issues of transcultural validity of screening tools are also relevant. A review of validated measures and more theoretical debate and consensus is needed (Gadeberg, Norredam 2016; McColl et al. 2008).

\subsection{Post-Migration without Status}

Silove and colleagues have been providing sustained evidence of the negative impact on mental health of conditions of reception for asylum seekers in Australia, showing that its impact was even greater than torture and persecution in country of origin (Silove 2000; Steel, Silove 2001; Steel et al. 2009). A Norwegian case-control study in an in-patient psychiatric ward found highly significant differences in PTSD prevalence between asylum seekers, living in centres $(n=53,43.3 \%)$, and refugees $(n=45,11 \%)$, associated to the stresses of life in reception centres and the risk of being expelled from the country more than the experiences in countries of origin (Iversen, Morken 2004). Other studies have expanded these results to medical conditions (Porter 2007). In a recent systematic review (Kalt et al. 2013), combining data from 23 peer-reviewed studies among asylumseekers (30\% torture survivors), it was concluded that highly stressful asylum-seeking processes produced adverse mental and somatic health effects, associated to specific forms of exclusion linked to social conditions and hostile policy environments.

Whilst these conditions vary from country to country, there are some salient themes:

\section{Poverty}

Asylum seekers face economic hardship through an increasingly short and limited system of State social support and assistance even where there is one (Allsopp et al. 2014). While waiting for a determination decision, they often receive basic temporary benefits, well below the minimum of the country, usually aggravated by the denial of permission to work. This situation can extend for a long period of time. As an example, in a 2013 study for Freedom for Torture, Pettitt (2013) reveals that more than half of a sample of 84 torture survivors in the UK asylum system reported that they could never or not often afford to buy enough food of sufficient quality and variety to meet their needs for a nutritionally balanced diet; 34 were never able to buy enough food of any quality to avoid hunger; 53 could not buy adequate winter clothing. It is hard to imagine the situation of asylum seekers in countries with even lower levels of assistance. 


\section{Access to Healthcare}

According to the HealthQUEST study (European Commission 2008), most European countries limit the access of migrants and asylum seekers to health care, usually reducing it to basic care and emergencies. The system of free specialised healthcare is usually banned. The study shows that in Europe providing comprehensive adequate care (including mental health) would, paradoxically, save costs. In the United States where there is no national health system, the situation is so precarious that Asgary et al. (2013) showed in a sample of sub-Saharan asylum seekers (most of them torture survivors) that they had better access to social and health services in their home African countries than in the US.

\section{Stigma}

There is growing evidence that perceived discrimination carries a psychological toll. A wide study following a participatory action research process in Scotland showed how this was linked to mental health problems, especially in VoT (victims of torture) (Quinn 2014).

\section{Cultural Barriers}

Language and culture have been documented as central sources of stress, particularly in the long term (Montgomery 2011). The role of cultural mediators is crucial, undoubtedly another insufficiently researched topic.

\section{Access to Justice and Providing Meaning to the Experience of Torture}

Arriving in a host country is, for many survivors, part of a process of social and political commitment which cannot be easily continued. Giving testimony, being part of an ideological or political movement, helping those who remain in their country, and pursuing justice can be essential elements to providing meaning to the experience of torture and to have a sense of continuity in life. The asylum system too often victimises survivors and keeps them in a vulnerable legal position that precludes any possibility of activism or empowerment and the impacts of this are not well researched (EATIP et al. 2002; Tay, Silove 2017). 


\subsection{Facing Assessment}

\section{Abbreviated Procedures}

McColl et al. (2008) have shown that processes that are too fast-track can preclude proper medical documentation of allegations of torture or persecution.

\section{Delay of Decision}

In a series of focus group with survivors of torture in UK, the three biggest problems described were uncertainty, lack of perspective and a shortened future associated with endless waiting for a decision (Haoussou 2017). Although the European Asylum Procedures Directive 32/2013 (European Council 2013) envisages a maximum of six months for an asylum determination, the decision normally takes much longer, sometimes years. At the American border, asylum claimants often spend many years waiting for the adjudication of their cases creating a limbo situation (Haas 2017). A cross-sectional survey with Iraqi refugees whose determinations were pending showed that survivors waiting for a decision generally felt socially isolated and lacking in control over their life circumstances with a strong sense of injustice (Johnston et al. 2009).

\section{Stress of the Interview and Court Hearings}

After initial acceptance, and after a long waiting process, survivors of torture must prepare and undergo an in-depth interview (and sometimes a court hearing) where their fate will be decided. This is not a neutral process. A recent study in Berlin suggested that the asylum interview might decrease posttraumatic avoidance but trigger posttraumatic intrusions (Schock et al. 2015). Due to this stress, the interview might have a negative result. Similar studies in other countries have found less conclusive results (Hocking et al. 2015).

\section{Medical Reports}

Some studies show the importance of medical reports for proper documentation of torture. In a sample of close to 2,000 asylum-seekers in the US, $89 \%$ of those with a medical report from Physicians for $\mathrm{Hu}$ man Rights (PHR) were granted asylum, compared to the national average of $37.5 \%$ (Lustig et al. 2008). We need more data on which as- 
pects of forensic assessment in general and the Istanbul Protocol in particular are relevant for an administrative body or court to make a final decision on a protection claim, a much needed demand in the anti-torture sector (Freedom for Torture 2016; Pérez-Sales et al. 2017).

\section{Credibility}

It is probably the assessment of the credibility of allegations of torture that is the one of the most complex issues and on which, paradoxically, there is less academic research (Jubany 2017). On the one hand, there is a debate on whether health professional should make judgments of credibility (Good 2004). The debate often mistakes the credibility of the victim with the credibility of the victim's account. There is arguably an ethical duty to have a forensic report provided, especially in contexts in which the victim of torture lacks any other evidentiary element, has fled without any documentation, and there are no physical injuries or witnesses that can support her allegations (Pérez-Sales 2017a, 2017b). This is particularly the case when considering the crude reality that torture survivors are being refused protection in all likelihood due to the difficulties in giving a proper account of the facts (Silove et al. 2006; Masinda 2004).

There are numerous guidelines for credibility assessment of the different institutions and bodies working within the framework of asylum (Gyulai et al. 2013; Home Office 2015; Kane 2008; Mackey, Barnes 2013; Mind 2010), with very different perspectives and approaches and sometimes conflicting criteria. While in some cases the victim's account is said to be the weakest piece of evidence, in others it is the opposite that is emphasised and guidelines are worked out for the analysis of the narrative and its relationship with sources of corroboration or triangulation. None of the available guides to best practice have been validated and they are in any case only recommendations from experts. We also lack data for comparing credibility in this area with credibility in other fields and the standards of proof required (Freedom for Torture 2016).

It unfortunately remains the case that the asylum determination process relies heavily on remembering and narrating traumatising stories in a convincing way and without contradictions, despite mental health issues.

\section{Deportation}

In 2016 alone, the EU allocated a total of 806 million Euros to activities related to the deportation of migrants, including the expulsion of 113,835 people to the 15 countries with which Europe has 
signed a repatriation partnership agreement and the financing of migrant centres in countries such as Pakistan or Lybia. This figure would have increased noticeably if the agreement with Turkey had not been a failure ${ }^{9}$ and if transfer among EU members in application of the Dublin procedure were included..$^{10}$ During President Obama's administration, a record 2.5-3 million immigrants were deported in his eight years in office. In 2016, immigrant detention and deportation machinery alone in the US cost 3.3 billion dollars (Baker 2017). What happens with asylum-claimers who have been rejected and deported? Although some organizations try to keep track of them (Amnesty International 2017), there is scarce data on their fate. There are some ethnographic studies on the hardships of reintegration after deportation for economic migrants (e.g. Khosravi 2018) but literature is scarce on rejected asylum claimants.

The organisation Justice First followed in 2011 a sample of Congolese people deported from France and found out that all failed asylum seekers had been imprisoned, tortured, forced to pay a ransom, raped or subjected to sexual harassment upon their return (Ramos 2011). Reports from Freedom for Torture (2012) and Human Rights Watch (2012) have documented the systematic detention and torture of Tamils who were rejected asylum claimants and deported to Sri Lanka. Similar data have been reported for deportees to Eritrea, Malta, Libya including summary executions of deportees in Sudan (Alpes et al. 2017). There are documented cases of detention and torture of Ugandan citizens that demanded asylum due to being a member of the Lgtbq community (Onyoin 2017), and already mentioned is the fate of people deported to Turkey in application of the EU-Turkey agreement. This data should not be a surprise. In many countries, the deported person is handed to the national authorities on arrival. Having claimed asylum is viewed as suspicious and the person is often immediately detained and interrogated.

All together, these studies suggest that there is a real danger for deported people and this must be the responsibility of deporting authorities that do not have a post-deportation follow-up system (Stefanovska 2016). Additionally, a Rights Disability International campaign claims that deporting people with severe mental disorders or disabilities to countries where they will have no access to proper care or treatment or be secluded in institutions with conditions that can

9 http://www.publico.es/internacional/union-euro-pea-agencia-deportacion-masiva-migrantes.html (2019-10-16).

10 In January 2011, the European Court of Human Rights (EchRT) declared that the transfer of one person from Belgium to Greece in application of the Dublin rules violated Article 3 (torture and inhuman or degrading treatment or punishment) and Article 13 (effective remedy) of the European Chart of Human Rights. Following this decision, most of the member States of the European Union stopped Dublin transfers to Greece. 
amount to torture should be enough to stop deportation and humanitarian protection should always be granted (Kanter, Rosenthal 2018). ${ }^{11}$

\section{The Top of the Vulnerability Pyramid: Undocumented Migrants}

The alternative for survivors whose application has been rejected is to stay undocumented in the host country in even worst conditions than before. If asylum seekers and refugees suffer poverty, stigma, lack of health services or a work permit, this is to be added to having to hide from police, being defenceless from crime and violence and working in the underground economy. Overwhelming data show that this is the group with the highest risk of severe mental health disorders. One study, among many, in Zurich (Switzerland) showed that more than $80 \%$ had at least one clinically significant symptom, and more than $50 \%$ fulfilled the criteria for PTSD. This should come as no surprise as more than $60 \%$ had suffered imprisonment and $30 \%$ torture. The prevalence of torture was slightly lower than those of asylum seekers, but the prevalence of mental health problems was higher. The study showed, again, not only that refugee and humanitarian decision-making procedures may be failing but also that undocumented migrants are probably the most vulnerable and affected of populations due to an aggravation of pre-migration symptoms and the impossibility of access to treatment according to their right to rehabilitation (Mueller et al. 2011).

\section{Once Status is Granted: "El Dorado"}

The long journey finally ends for an estimated 30-40\% of torture survivors that ask for international protection obtaining it (Silove et al. 2006; Mueller et al. 2011; UNHCR 2017). The majority is, thus, undetected or rejected. There is a large body of literature showing that the refugee population, even with protection status, has very high levels of psychological suffering resulting from their pre-migration experiences, but particularly from life in the new host country (Porter 2007; Porter, Haslam 2005). A decent standard of living is not guaranteed.

\section{Right to Rehabilitation}

Torture survivors have a right to rehabilitation, as set out in the Convention against Torture and General Comment No. 3. Programs for

11 https://www.driadvocacy.org (2019-10-16). 
VoT who move about are a challenge. There is a need for short-term interventions that follow the do-no-harm principles in contexts where it might not be the time to talk. This creates a special set of conditions for rehabilitation programs that deserve special research, including long-term follow-up and non-clinical measures.

\section{Where Next? Concluding Remarks}

The over twenty-five years of research in the field appears to provide conclusive evidence regarding the negative impact on torture survivors of human right violations taking place throughout the migration continuum.

This idea of a migration continuum deserves special attention. Migrants who along their migration route have been suffering violence (including torture by non-State actors, sexual violence, and being unable to access basic conditions that respect human dignity) should be also offered protection and access to basic human rights (to health, housing, education, rehabilitation etc).

Figure 1 is intended to show a summary of the relationship between mental health and well-being and pre- and post-migration factors and the amount and strength of available evidence. Although we need more studies on psychosocial determinants during the asylum claim process, there is strong evidence on the impact of poverty and limitations of access to health care. Most of the research highlighted here is in relatively well-off host countries which reflects the absence of literature in more complex situations (e.g. Lebanon and Greece, not to mention other neighbouring countries to refugee-producing countries in the rest of the world). More research is especially needed regarding the migration process itself and the impact of massive human rights violations at borders, migrant detention centres in third countries, and by State and non-State actors on victims of persecution and torture fleeing from their country. This also applies to research on what happens to people being denied protection and deported to their countries of origin. Although there are indicative data, more research is also needed on the screening process and appropriateness of detention of torture survivors and vulnerable populations, the impact of the asylum process and interviews, on the role of translators and cultural mediators, and the effect of policies of dispersal and delays in procedure decisions. With respect to proper identification procedures, the need is poignant and urgent. Finally, we still lack more research and stronger evidence on the efficacy of rehabilitation programs for migrant torture survivors. 


\section{References}

Allsopp, Jennifer et al. (2014). Poverty Among Refugees and Asylum Seekers in the Uk. An Evidence and Policy Review. IRiS Working Paper Series No. 1. Birmingham: University of Birmingham. URL https://www.birmingham.ac.uk/ Documents/college-social-sciences/social-policy/iris/2014/ working-paper-series/IRIS-WP-1-2014.pdf (2019-10-16).

Alpes, Jill et al. (2017). "Post-Deportation Risks for Failed Asylum Seekers". Forced Migration Review, 54, 76-8. URL https://www.fmreview.org/ resettlement/alpes-blondel-preiss-sayosmonras (2019-10-16).

Amnesty International (2017). Human Rights in the Context of Forced Returns. Amsterdam: Amnesty International. URL https://bit.ly/2qdNHqy (2019-10-16).

Asgary, Ramin et al. (2013). "Socio-Medical Challenges of Asylum Seekers Prior and After Coming to the US". Journal of Immigrant and Minority Health, 15(5), 961-8. DOI https://doi.org/10.1007/s10903-012-9687-2.

Baker, Beth (2017). "The Hidden Costs of Mass Deportation”. Anthropology News, 58(5), e319-e323. DOI https://doi.org/10.1111/AN.632.

Ben Farhat, Jihane et al. (2018). "Syrian Refugees in Greece: Experience with Violence, Mental Health Status, and Access to Information During the Journey and While in Greece". BMC Medicine, 16, 40, 1-12. DOI https://doi. org/10.1186/s12916-018-1028-4.

Brooker, Stephen et al. (2016). Challenges to Providing Mental Health Care in Immigration Detention. Global Detention Project Working Paper No. 19. URL https://www.globaldetentionproject.org/wp-content/uploads/2016/12/Brooker-et-al-GDP-paper-2016.pdf (2019-10-16).

Crisp, Jeff (2009). "Refugees, Persons of Concern, and People on the Move: The Broadening Boundaries of UNHCR". Refuge, 26(1), 73-6.

Daniel, Valentine; Knudsen, John (1995). Mistrusting Refugees. Berkeley: University of California Press.

EATIP et al. (2002). Paisajes del Dolor, Senderos de esperanza. Salud mental y derechos humanos en el Cono Sur. Buenos Aires. URL http://www. cintras. org/textos/libros/libropaisajes.pdf (2019-10-16).

European Commission (2008). Quality in and Equality of Access to Healthcare Services. Bruxelles: European Comission. URL https://www.euro.centre.org/downloads/detail/882 (2019-10-16).

European Council (2013). L. 180/60 - Common Procedures for Granting and Withdrawing International Protection. Official Journal of the European Union, URL https://www.easo.europa.eu/sites/default/files/public/ Dve-2013-32-Asylum-Procedures.pdf (2019-10-16).

Fazel, Mina; Silove, Derrick (2006). "Detention of refugees”. British Medical Journal,332, 7536, 251-2. Dol https://doi.org/10.1136/bmj.332.7536.251.

Filges, Trine et al. (2018). "The Impact of Detention on the Health of Asylum Seekers: A Systematic Review”. Research on Social Work Practice, 28(4), 399-414. DOI https://doi.org/10.1177/1049731516630384.

Freedom for Torture (2012). "Sri Lankan Tamils Tortured on Return from the UK”. Briefing, 13 September 2012. URL https://www. refworld.org/pdfid/505321402.pdf (2019-10-16).

Freedom for Torture (2016). Proving Torture. Demanding the Impossible. Home Office Mistreatment of Expert Medical Evidence. London: Freedom for Torture. URL https://www. asylumineurope.org/sites/default/files/ resources/proving_torture.pdf (2019-10-16). 
Gadeberg, Anne Kristine; Norredam, Marie (2016). "Urgent Need for Validated Trauma and Mental Health Screening Tools for Refugee Children and Youth". European Child \& Adolescent Psychiatry, 25(8), 929-31. DOI https://doi. org/10.1007/s@0787-016-0837-2.

Good, Anthony (2004). "Undoubtedly an Expert? Anthropologists in British Asylum Courts". Journal of The Royal Anthropology Institute, 10(1), 113-33. DOI https://doi.org/10.1111/j.1467-9655.2004.00182.x.

Griffiths, Melanie (2012). "Vile Liars and Truth Distorters". Anthropology Today, 28(5), 8-12. DOl https://doi.org/10.1111/j.1467-8322.2012.00896.x.

Gyulai, Gábor et al. (2013). Credibility Assessment in Asylum Procedures. A Multidisciplinary Training Manual, vol. 1. Budapest: Hungarian Helsinki Committee. URL https://helsinki.hu/wp-content/uploads/Credibility-Assessment-in-Asylum-Procedures-CREDO-manual.pdf (2019-10-16).

Haas, Bridget (2017). "Citizens-in-Waiting, Deportees-in-Waiting: Power, Temporality, and Suffering in the U.S. Asylum System". Ethos, 45(1), 75-97. DOI https://doi.org/10.1111/etho.12150.

Haoussou, Kolbassia (2017). "Perspective - The Long Journey to Rehabilitation for Torture Survivors". Torture, 27(1), 66-74.

Hocking, Debbie et al. (2015). "Mental Disorders in Asylum Seekers: The Role of the Refugee Determination Process and Employment". Journal of Nervous and Mental Disease, 203(1), 28-32. DOI https://doi.org/10.1097/ NMD. 00000000000000230.

Hollifield, Michael et al. (2013). "The Refugee Health Screener-15 (RHS-15): Development and Validation of an Instrument for Anxiety, Depression, and PTSD in Refugees". General Hospital Psychiatry, 35(2), 202-9. DOI https:// doi.org/10.1016/j.genhosppsych.2012.12.002.

Home Office (2015). Asylum Policy Instruction: Assessing Credibility and Refugee Status. Version 9.0. URL https://assets.publishing.service. gov.uk/government/uploads/system/uploads/attachment_da$\mathrm{ta} / \mathrm{file}$ /397778/ASSESSING_CREDIBILITY_AND_REFUGEE_STATUS_ V9_0.pdf (2019-10-16).

Hope Border Institute (2018). Sealing the Border. The Criminalization of Asylum Seekers in the Trump Era. El Paso. URL https://docs.wixstatic.com/ ugd/e07ba9_909b9230ae734e179cda4574ef4b6dbb.pdf (2019-10-16).

Human Rights First (2017). Violations at the Border. The El Paso Sector. URL http://www.humanrightsfirst.org/sites/default/files/hrfviolations-at-el-paso-border-rep.pdf (2019-10-16).

Human Rights Watch (2012). UK: Halt Deportations of Tamils to Sri Lanka. URL https://www.hrw.org/news/2012/02/24/uk-halt-deportationstamils-sri-lanka (2019-10-16).

IRCT, International Rehabilitation Council for Torture Victims (2016). Falling Through the Cracks. Asylum Procedures and Reception Conditions for Torture Victims in the European Union. Copenhagen: IRCT. URL https://irct. org/assets/uploads/pdf_20161120142453.pdf (2019-10-16).

Iversen, Valentina; Morken, Gunnar (2004). "Differences in Acute Psychiatric Admissions Between Asylum Seekers and Refugees". Nordic Journal of Psychiatry, 58(6), 465-70. DOI https://doi.org/10.1080/08039480410011696.

Jesuit Refugee Service - Europe (2010). Becoming Vulnerable in Detention. Civil Society Report on the Detention of Vulnerable Asylum Seekers and Irregular Migrants in the European Union. URL http://www.europarl .europa. 
eu/document/activities/cont/201110/20111014ATT29338/20111 014ATT29338EN.pdf (2019-10-16).

Johnson, Howard; Thompson, Andrew (2008). "The Development and Maintenance of Post-traumatic Stress Disorder (PTSD) in Civilian Adult Survivors of War Trauma and Torture: A Review". Clinical Psychology Review, 28, 36-47. DOI https://doi.org/10.1016/j.cpr.2007.01.017.

Johnston, Vanessa et al. (2009). "Measuring the Health Impact of Human Rights Violations Related to Australian Asylum Policies and Practices: A Mixed Methods Study". BMC International Health and Human Rights, 9, 1. URL https://www.ncbi.nlm.nih.gov/pubmed/19192307 (2019-10-16).

Jubany, Olga (2017). Screening Asylum in a Culture of Disbelief. Truths, Denials and Skeptical Borders. London: Palgrave McMillan.

Kalt, Anne et al. (2013). "Asylum Seekers, Violence and Health: A Systematic Review of Research in High-Income Host Countries". American Journal of Public Health, 103(3), e30-e42. DOI https://doi.org/10.2105/ AJPH. 2012.301136.

Kane, John (2008). Judging Credibility. New York: American Bar Association.

Kanter, Arlene; Rosenthal, Eric (2018). "The Right of People with Disabilities to Asylum and Protection from Deportation on the Grounds of Persecution or Torture Related to their Disability", Human Rights Brief, April. DOI http:// dx.doi.org/10.2139/ssrn.3171691.

Keller, Allen et al. (2003). "Mental Health of Detained Asylum Seekers". Lancet, 362(9397), 1721-23. DOI https://doi.org/10.1016/S01406736(03)14846-5.

Khosravi, Sharam (ed.) (2018). After Deportation. Ethographic Perspectives. London: Palgrave McMillan.

Lustig, Stuart et al. (2008). "Asylum Grant Rates Following Medical Evaluations of Maltreatment Among Political Asylum Applicants in the United States". Journal of Immigrant and Minority Health/Center for Minority Public Health, 10, 7-15. DOI https://doi.org/10.1007/s10903-007-9056-8.

Lyytinen, Evelina (2017). "Refugees' 'Journeys of Trust': Creating an Analytical Framework to Examine Refugees' Exilic Journeys with a Focus on Trust". Journal of Refugee Studies, 30(4), 489-510. DOI https://doi. org/10.1093/jrs/few035.

Mackey, Allan; Barnes, John (2013). Assessment of Credibility in Refugee and Subsidiary Protection claims under the EU Qualification Directive Judicial criteria and standards Prepared by Allan Mackey and John Barnes for the International Association of Refugee. Haarlem: International Association of Refugee and Migration Judges. DOI https://www. refworld.org/docid/557028564.html (2019-10-16).

Masinda, Mambo (2004). "Quality of Memory: Impact on Refugee Hearing Decisions". Traumatology, 10(2), 131-9. DOI https://doi. org/10.1177/153476560401000205.

McColl, Helen et al. (2008). "Mental Healthcare of Asylum-Seekers and Refugees". Advances in Psychiatric Treatment, 14, 452-9. DOI https://doi. org/10.1192/apt.bp.107.005041.

Mewes, Ricarda et al. (2018). "Validation of the Protect Questionnaire: A Tool to Detect Mental Health Problems in Asylum Seekers by Non-Health Professionals". Torture, 28(2), 56-71.

Mind (2010). Achieving Justice for Victims and Witnesses with Mental Distress. A Mental Health Toolkit for Prosecutors and Advocates. London: Mind. 
URL https://www.mind.org.uk/media/207147/Prosecutors_toolkit.pdf (2019-10-16).

Momartin, Shakeh et al. (2003). "Dimensions of Trauma Associated with Posttraumatic Stress Disorder (PTSD) Caseness, Severity and Functional Impairment: A Study of Bosnian Refugees Resettled in Australia". Social Science \& Medicine, 57(5), 775-81. DOI https://doi.org/10.1016/SO2779536(02) $00452-5$.

Montgomery, Edith (2011). "Trauma, Exile and Mental Health in Young Refugees”. Acta Psychiatrica Scandinavica, 124, suppl. 440, 1-46. DOI https:// doi.org/10.1111/j.1600-๑447.2011.01740.x.

Mueller, Julia et al. (2011). "Mental Health of Failed Asylum Seekers as Compared with Pending and Temporarily Accepted Asylum Seekers". European Journal of Public Health, 21(2), 184-9. DOI https://doi.org/10.1093/ eurpub/ckq016.

Ní Raghallaigh, Muireann (2014). "The Causes of Mistrust Amongst Asylum Seekers and Refugees: Insights from Research with Unaccompanied Asylum-seeking Minors Living in the Republic of Ireland". Journal of Refugee Studies, 27(1), 82-100. DOI https://doi.org/10.1093/jrs/fet@०6.

Onyoin, Charity Ahumuza (2017). "A Grim Return: Post-Deportation Risks in Uganda". Forced Migration Review, 54, 81-3.

Pearman, April; Olinga-Shannon, Stephanie (2017). "Australian Immigration Detention and the Silencing of Practitioners". Torture, 27(3), 90-3.

Pérez-Sales, Pau (2017a). Psychological Torture: Definition, Evaluation and Measurement. London-New York: Routledge.

Pérez-Sales, Pau (2017b). "Psychotherapy for Torture Survivors: Suggested Pathways for Research". Torture, 27(1), 1-12.

Pérez-Sales, Pau et al. (2017). "Rehabilitation of Torture Survivors and Prevention of Torture: Priorities for Research Through a Modified Delphi Study". Torture, 27(3), 3-48.

Pettitt, Jo (2013). The Poverty Barrier: The Right to Rehabilitation for Survivors of Torture in the UK. Freedom for Torture. URL https://www. freedomfromtorture.org/sites/default/files/2019-04/Poverty\%20report\%20FINAL\%20a4\%20web.pdf (2019-10-16).

Porter, Matthew (2007). "Global Evidence for a Biopsychosocial Understanding of Refugee Adaptation”. Transcultural Psychiatry, 44(3), 418-39. DOI https://doi.org/10.1177/1363461507081642.

Porter, Matthew; Haslam, Nick (2005). "Predisplacement and Postdisplacement Factors Associated with Mental Health of Refugees and Internally Displaced Persons: a Meta-Analysis". Journal of the American Medical Association, 294(5), 602-12. DOI https://doi.org/10.1001/jama.294.5.602.

PROTECT Project (2016). PROTECT. Process of Recognition and Orientation of Torture Victims in European Countries to Facilitate Care and Treatment. Brussels. URL http://protect-able.eu/wp-content/uploads/2013/01/ protect-global-eng.pdf (2019-10-16).

Quinn, Neil (2014). "Participatory Action Research with Asylum Seekers and Refugees Experiencing Stigma and Discrimination: The Experience from Scotland". Disability and Society, 29(1), 58-70. DOI https://doi.org/10. 1080/09687599.2013.769863.

Ramos, Catherine (2011). Unsafe Return: Refoulement of Congolese Asylum Seekers. Report. URL http://justicefirst.org.uk/wp-content/uploads/ UNSAFE-RETURN-DECEMBER-5TH-2011.pdf (2019-10-16). 
Robinson, Vaughan; Segrott, Jeremy (2002). Understanding the Decision-Making of Asylum Seekers. Home Office Research Study 243. URL https://bit. ly/2BjtTo7 (2019-10-16).

Robjant, Katy et al. (2009). "Mental Health Implications of Detaining Asylum Seekers: Systematic Review”. British Journal of Psychiatry, 194(4), 306-12. DOI https://doi.org/10.1192/bjp.bp.108.053223.

Roman, Emanuela et al. (2016). "Why Turkey is Not a 'Safe Country'”. Statewatch Analysis, February. URL https://www.statewatch.org/analyses/no-283-why-turkey-is-not-a-safe-country.pdf (2019-10-17).

Schock, Katrin et al. (2015). "Impact of Asylum Interviews on the Mental Health of Traumatized Asylum Seekers". European Journal of Psychotraumatology, 6, 262-86. DOI https://doi.org/10.3402/ejpt.v6.26286.

Silove, Derrick (2000). "Policies of Deterrence and the Mental Health of Asylum Seekers". JAMA, 284(5), 604-11. DOI https://doi.org/10.1001/jama.284.5.604.

Silove, Derrick (2013). "The ADAPT Model: A Conceptual Framework for Mental Health and Psychosocial Programming in Post Conflict Settings". Intervention, 11(3), 237-48.

Silove, Derrick et al. (2006). "Torture, Mental Health Status and the Outcomes of Refugee Applications among Recently Arrived Asylum Seekers in Australia". International Journal of Migration, Health and Social Care, 1(2), 4-14. DOI https://doi.org/10.1108/17479894200600002.

Sobhanian, Farahnaz et al. (2006). "Living in the Australian Community Psychological Status of Former Refugee Detainees From the Woomera Detention Centre Now Living in the Australian Community". Psychiatry, Psychology and Law, 13(2), 37-41.

Special Rapporteur against Torture (2018). Migration-Related Torture and Ill-treatment (a/Hrc/37/50). United Nations. URL https://documentsdds-ny.un.org/doc/UNDOC/GEN/G18/347/27/PDF/G1834727. pdf?OpenElement (2019-10-16).

Stedman, Stephen John; Tanner, Fred (2003). Refugee Manipulation. War, Politics and the Abuse of Human Suffering. Washington: Brookings Institution Press.

Steel, Zachary et al. (2009). "Association of Torture and Other Potentially Traumatic Events with Mental Health Outcomes Among Populations Exposed to Mass Conflict and Displacement: A Systematic Review and Meta-Analysis". JAMA, 302(5), 537-49. DOI https://doi.org/10.1001/jama.2009.1132.

Steel, Zachary; Silove, Derrick (2001). "The Mental Health Implications of Detaining Asylum Seekers". Medical Journal of Australia, 175, 596-9.

Stefanovska, Vesna (2016). "Diplomatic Assurances and the State's Responsibility When Considering Extraditing a Person Whose Human Rights May Be Violated". Varstvoslovje - Journal of Criminal Justice and Security, 2, 167-82.

Storm, Tania; Engberg, Marianne (2013). "The Impact of Immigration Detention on the Mental Health of Torture Survivors is Poorly Documented. A Systematic Review". Danish Medical Journal, 60(11), 1-7.

Tay, Alvin; Silove, Derrick (2017). "The ADAPT Model: Bridging the Gap Between Psychosocial and Individual Responses to Mass Violence and Refugee Trauma". Epidemiology and Psychiatric Sciences, 26(2), 142-5. Dol https://doi. org/10.1017/S2045796016000925.

Ulusoy, Orçun; Battjes, Hemme (2017). Situation of Readmitted Migrants and Refugees from Greece to Turkey under the EU-Turkey Statement. Amsterdam: Vrije Universiteit Amsterdam. VU Migration Law Series 15. URL https:// 
rechten.vu.nl/en/Images/UlusoyBattjes_Migration_Law_Series_No_15_tcm248-861076.pdf (2019-10-16).

UNHCR (2012). Detention Guidelines. Geneva: UNHCR. URL https://www. unhcr.org/publications/legal/505b10ee9/unhcr-detentionguidelines.html (2019-10-16).

UNHCR (2017). Global Trends. Forced Displacement in 2016. Geneva: UNHCR. URL https://www.unhcr.org/5943e8a34.pdf (2019-10-16).

UNHCR et al. (2016). Vulnerability Screening Tool. Identifying and Addressing Vulnerability: a Tool for Asylum and Migration Systems. Geneva. URL https:// www. refworld.org/pdfid/57f21f6b4.pdf (2019-10-16).

Viñar, Marcelo (2007). "Civilization and Torture: Beyond the Medical and Psychiatric Approach". International Review of the Red Cross, 89(867), 619-33.

Voutira, Eftihia; Harrell-Bond, Barbara (1995). "In Search of the Locus of Trust: The Social World of the Refugee Camp". Daniel, Valentine; Knudsen, John (eds), Mistrusting refugees. Berkeley: University of California Press, 207-25. 



\title{
Immigrazione e salute mentale Violenza, psicotraumatologia, politiche per la salute e tutela assistenziale
}

\author{
Marco Mazzetti \\ psichiatra, analista transazionale didatta, Milano - Area Sanitaria Caritas Roma, \\ Società Italiana di Medicina delle Migrazioni, Italia
}

\section{Salvatore Geraci}

Caritas Italiana, Roma, Italia

\begin{abstract}
This chapter discusses psychopathological issues related to migration with particular attention to the so-called "forced migrants", migrants who are forced to flee their countries to save themselves due to violence. As a consequence, they are more at risk for mental health issues due to the lack of a migration project (which is a resilience factor in migrants) and to the psychotraumatic experiences they suffered. We will describe the psychopathological reactions in traumatized persons, the effect of re-traumatization they suffer in transit countries, the role of Post-Migration Living Difficulties occurring in Italy in worsening mental health of asylum seekers. Finally, we will discuss present difficulties of the Italian National Health Service to adequately cure them, and the indication given by the Italian National Guidelines to create services dedicated to these issues.
\end{abstract}

Keywords Asylum seekers. Refugees. Post-Traumatic Stress Disorder (PTSD). Psychotraumatology. Psychopathology.

Sommario 1 Introduzione. - 2 Traumatizzazione e ritraumatizzazione. - 3 Esiti di tor ture e violenze sulla salute mentale. - 4 Accogliere il paziente traumatizzato. - 5 Politiche per la salute e linee guida per la tutela assistenziale. - 6 Conclusioni. 


\section{Introduzione}

Lo straniero separato dai suoi concittadini
e dalla sua famiglia
dovrebbe ricevere un amore maggiore
da parte degli uomini e degli dei

(Platone)

La Heimweh, questa malattia così spesso mortale, non è stata sinora descritta dai medici... Il nome tedesco indica il dolore di coloro che si trovano lontani dalla Patria, e che temono di non rivedere più la terra natale. I francesi, osservando gli svizzeri colpiti in Francia da tale sventura, hanno coniato la definizione di mal du pays (malattia della patria), e, poiché essa non ha alcun nome in latino, così ho pensato di chiamarla, dal greco, nostalgia, da nòstos, il ritorno in patria, e àlgos, dolore e sofferenza.

Così il medico svizzero Johannes Hofer descriveva, nella sua tesi di dottorato del 1688, una malattia che osservava tra i mercenari svizzeri, presso i quali svolgeva la sua opera di medico: non solo ha coniato un termine che da allora descrive con efficacia quel particolare sentimento di chi lascia un ambiente caro, ma ha anche dato quella che probabilmente è la prima codificazione psichiatrica di una patologia dell'emigrazione. Egli, inoltre, come terapia di un quadro clinico psichiatrico proponeva un intervento di tipo sociale: il trasloco del paziente nella sua terra. Nella Dissertatio Medica de Nostalgia di Hofer ci sono già in nuce gli elementi riconosciuti come essenziali nella moderna psichiatria dell'immigrazione: un ruolo decisivo è giocato dall'esperienza stessa di migrare, e dalle condizioni di vita in cui gli emigrati/immigrati si trovano a vivere; di conseguenza, anche gli interventi curativi e riabilitativi non possono prescindere, per essere efficaci, dalla dimensione sociale.

Fatto salvo il precedente di Hofer, è però dall'Ottocento che le osservazioni cliniche sulla salute mentale degli immigrati cominciano a trovare uno spazio regolare sulle pubblicazioni scientifiche; tuttavia per molti anni esse hanno presentato caratteristiche essenzialmente aneddotiche, e fino alla metà del Novecento sembrano soprattutto aver contribuito a creare e a mantenere stereotipi e pregiudizi contro gli immigrati, presentati non di rado come soggetti con una certa tendenza all'instabilità e alla vulnerabilità psichica (Ranney 1850; Foville 1875; Ødegaard 1932). Queste idee contrastano, prima ancora che con le successive evidenze scientifiche, con il buon senso, se consideriamo che nella storia dell'umanità, lo stile di vita stanziale è da considerarsi l'eccezione, piuttosto che la regola, dato che la specie homo sapiens sapiens è stata, nel corso dei circa 130.000 anni della sua storia, soprattutto nomade. 
In anni recenti, la letteratura scientifica si è piuttosto focalizzata sull'identificazione di quei fattori in grado di promuovere, o viceversa di mettere in pericolo, la salute psichica degli immigrati. ${ }^{1}$ Per quanto riguarda l'Italia, terra di immigrazione privilegiata per millenni a causa delle condizioni di vita particolarmente favorevoli (clima, fertilità della terra, centralità politica, amministrativa e geografica), divenuta per circa un secolo e mezzo terra di emigrazione (tra Ottocento e Novecento) e poi da un trentennio tornata alla sua vocazione storica di luogo di attrazione per le popolazioni in movimento, le osservazioni cliniche e la ricerca scientifica sono relativamente recenti, proprio per l'intervallo storico in cui l'Italia ha dimenticato la propria connotazione immigratoria per diventare Paese di emigrazione, per un periodo breve nella storia dell'umanità ma lungo se paragonato alla vita media di noi esseri umani, tanto da far dimenticare una realtà storica più che millenaria.

Le osservazioni hanno quindi cominciato a svilupparsi a partire dagli anni Novanta del secolo passato: i dati raccolti nel corso di numerose indagini epidemiologiche, hanno consentito di definire quello che è stato chiamato «effetto migrante sano» (Costa 1993; Geraci 1995). Cioè: gli immigrati partono sani dal proprio Paese (cosa abbastanza ovvia, se si considera quanto impegnativo sia, in genere, il percorso migratorio, e come richieda buona salute per essere affrontato), e sani di norma arrivano nel Paese ospite. Queste osservazioni, che si riferiscono a studi dei primi anni Novanta, sono stati regolarmente confermati in seguito (WHO 2018): non esistono in misura significativa patologie da importazione tra gli immigrati nel nostro Paese. Questi dati, riferiti all'epidemiologia complessiva dell'immigrazione in Italia, si sono rivelati validi anche in ambito psichiatrico: i tassi di ospedalizzazione per diagnosi psichiche tra gli immigrati sono stati particolarmente bassi nonostante quanto ci si sarebbe potuto attendere per la presenza di fattori di rischio legati all'esperienza migratoria.

Per quanto riguarda la salute in generale, le osservazioni clinicoepidemiologiche hanno semmai consentito di rilevare, in base alle caratteristiche dei ricoveri ospedalieri della popolazione immigrata confrontata con quella italiana, che a incidere maggiormente sulla salute sono piuttosto le condizioni di impianto nel Paese d'arrivo. In altre parole, gli immigrati si ammalano in Italia a causa delle condizioni di vita in cui si trovano a vivere: ad esempio, mostrano tassi di

1 Mentre, parallelamente, si è anche andato diffondendo un atteggiamento di attenzione alle differenti sensibilità culturali, che è andato sotto il nome di 'etnopsichiatria'. Con questo termine ci si riferisce alla cura della sofferenza psichica contestualizzata nei riferimenti culturali del paziente: è una definizione che noi non amiamo, perché riteniamo che nessuna buona psichiatria possa trascurare la cultura del paziente, e il termine etnopsichiatria rischia quindi di diventare una tautologia. 
ospedalizzazione per infortunistica sensibilmente più elevati rispetto agli italiani, perché si concentrano maggiormente nelle attività lavorative più pericolose (Geraci 2001; Osservatorio Nazionale sulla salute nelle regioni italiane 2005-18; INAIL 2012).

Anche in ambito psichiatrico si sono fatte osservazioni analoghe: le cosiddette Post-Migration Living Difficulties (PMLD: difficoltà di vita in terra di immigrazione) sembrano giocare un ruolo nella genesi e nel mantenimento delle reazioni post-traumatiche, da disadattamento e somatizzazione, indipendentemente da altri fattori di rischio, inclusi i traumi subiti in patria prima della partenza (in specie per quanto riguarda i migranti forzati) o quelli sofferti durante il percorso migratorio (Aragona et al. 2011, 2012a, 2013, 2014). Queste osservazioni hanno suggerito ai ricercatori di concentrarsi soprattutto sulla comprensione delle dinamiche migratorie, e su come queste influenzino la salute psichica degli immigrati, per identificare i fattori protettivi e quelli di rischio in grado di influirvi.

Queste considerazioni, che hanno mantenuto una sostanziale validità per oltre un ventennio, sembrano però avere la necessità di qualche aggiustamento legato ai fenomeni avvenuti nel corso degli ultimi anni, durante i quali abbiamo osservato un cambiamento sia di tipo socio-demografico, sia legato al percorso migratorio dei nuovi arrivati.

Si è passati, infatti, a partire dalla cosiddetta 'Emergenza Nord Africa' nel 2011, da una migrazione ordinaria di tipo economico a un'altra essenzialmente di profughi, che presentano percorsi migratori prolungati e spesso estremamente duri. Chi opera nel settore ha inoltre visto crescere il numero di assistiti di basso livello di istruzione, spesso analfabeti, e con storie non solo di psicotraumatologia ma anche talora di emarginazione sociale precedente l'emigrazione. Le ragioni di questo fenomeno non sono ancora del tutto chiare, e ci mancano anche dati ufficiali in grado di confrontare con precisione, e sui grandi numeri, ad esempio, i livelli di istruzione dei nuovi arrivati con la precedente immigrazione in Italia, che mostrava una popolazione con livelli di istruzione medio-alti (Coccia, Pittau 2016).

Siamo francamente in difficoltà nel commentare questa nuova realtà, proprio per l'assenza di dati che ci consentano ragionamenti basati su osservazioni scientifiche ragionevolmente affidabili, e l'ultima cosa che vogliamo è aggiungere la nostra voce al coro di chi parla dell'immigrazione seguendo impressioni personali e per sentito dire. Tuttavia, ci troviamo a dover scegliere se tacere completamente questa che ci appare a tutti gli effetti come una nuova realtà, o parlarne, sia pure con tutte le prudenze e le riserve del caso. Abbiamo scelto questa seconda opzione perché prendere atto dei cambiamenti che appaiono essere in corso ha notevoli implicazioni sia per le possibili scelte socio-assistenziali da compiere, sia soprattutto per i bisogni sanitari che da essa stanno emergendo. E non solo in ambito psichiatrico, dato che la cosiddetta health literacy (che pos- 
siamo tradurre con 'alfabetizzazione sanitaria') è un fattore chiave nella protezione della salute ed è strettamente collegata ai livelli di istruzione generale.

Pur in assenza di dati numerici, infatti, la realtà riportata dagli operatori impegnati nell'assistenza ai profughi e richiedenti asilo, sia nello SPRAR (Sistema di Protezione per Richiedenti Asilo e Rifugiati) sia negli altri sistemi di accoglienza, è costantemente coerente con l'osservazione del mutamento socio-demografico di cui si è detto. Negli stessi anni, inoltre, è stato possibile registrare (Baglio et al. 2018) un cambiamento nei tassi di ricovero per problemi psichiatrici negli ospedali psichiatrici italiani, che merita qualche commento. In generale, infatti, in Italia i tassi di ricovero per patologie psichiatriche tra gli stranieri erano tradizionalmente più bassi di quelli tra gli italiani. Questa dinamica resta invariata anche negli ultimi anni per quanto riguarda gli immigrati in generale; tuttavia, se si seleziona la popolazione dei giovani maschi provenienti dai Paesi da cui attualmente arrivano i migranti forzati richiedenti asilo, si vede che successivamente al 2011 la curva dei tassi di questo sottogruppo si impenna superando di molto sia quella degli altri immigrati, sia quella degli italiani. In sostanza, è come se in questa popolazione vi fosse una vulnerabilità maggiore che porta più spesso le persone a ricoverarsi, e ciò coincide temporalmente con la disgregazione dello stato libico, con il conseguente caos che ha portato a un aumento delle partenze (anche di persone che non avevano preventivato di venire in Europa) e a un aumento delle gravi esperienze traumatiche in quel Paese (incarcerazioni, percosse, lavoro forzato, malnutrizione e disidratazione, abusi e violenze fisici e sessuali, torture).

La fonte ministeriale da cui lo studio di Baglio et al. (2018) prende i dati non consente un'analisi più fine in base alla scolarità, all'essere o meno un effettivo richiedente asilo/rifugiato, etc. Tuttavia, i dati sono sufficienti a suggerire chiaramente che in quella popolazione qualcosa di nuovo sta avvenendo, che il profilo di salute mentale non è più buono come quello degli immigrati arrivati nei decenni passati, e che questo pone una sfida al sistema sanitario nazionale. Suggerimento, questo, confermato anche da un'indagine qualitativa condotta su un campione di operatori sociali e psichiatrici in Italia: «Dalle interviste effettuate sia con il privato sociale con lunga esperienza di trattamento di psicopatologie tra i migranti, sia di psichiatri operanti in strutture pubbliche, è emersa una diversa tipologia del migrante attuale che spesso si presenta con un substrato psichico già compromesso, con una capacità di resilienza ridotta e in assenza di un progetto migratorio chiaro» (Medici Senza Frontiere 2016, 11). 


\section{Traumatizzazione e ritraumatizzazione}

La migrazione di per sé è un evento complesso che può essere vissuto in maniera traumatica. Tuttavia in psicotraumatologia quando si parla di traumi, o meglio di eventi potenzialmente traumatici, è d'uso riferirsi a quegli eventi che la persona subisce direttamente o di cui è testimone, o di cui viene a conoscenza se riguarda persone della sua stretta cerchia, caratterizzati da «morte reale o minaccia di morte, grave lesione, oppure violenza sessuale» (American Psychiatric Association 2013). Questa definizione copre un amplissimo spettro di possibili eventi: catastrofi naturali, incidenti, episodi avvenuti in battaglia, aggressioni, violenza pubblica e privata, etc. In questi casi la migrazione, specie se avviene nelle condizioni difficili cui abbiamo accennato in precedenza, può costituire, come vedremo, un evento ritraumatizzante.

Oggi sempre di più, a causa degli stravolgimenti geopolitici in seno a molti Paesi africani e asiatici, per i richiedenti protezione internazionale ognuno di questi eventi può essere occorso, ed in più vi è spesso una specificità legata al fatto che nei casi di esposizione a situazioni di violenza subita (e che ha spinto la persona a scappare dal proprio Paese, la cosiddetta 'migrazione forzata') essa è di tipo intenzionale. Ciò vuol dire che qualcuno ha perpetrato atti brutali volti a infliggere dolore e/o morte per scopi definiti, verso qualcun altro, in modo volontario e consapevole. Questo comporta una maggior peculiarità del trauma, definito anche 'trauma estremo': violenze interpersonali ripetute, praticate volontariamente da una persona e/o da un gruppo, in una situazione di privazione della libertà.

L'esempio simbolo di tale tipologia di esperienza è quello della tortura, definita dall'ONU:

qualunque atto che per mezzo di gravi dolori o sofferenze, o fisiche o mentali, sia intenzionalmente inflitto a una persona per scopi quali ottenere da questa o da terzi informazioni o una confessione, punirla per un atto che essa o una terza persona ha commesso o si sospetta che abbia commesso, o intimidire o costringere essa o una terza persona, per una ragione qualsiasi basata su una discriminazione di qualunque tipo, quando tale dolore o sofferenza è inflitta da o su istigazione di o con il consenso o il tacito consenso di un pubblico ufficiale o di altra persona che agisce nell'ambito delle sue funzioni ufficiali. ${ }^{2}$

In generale, oltre alla tortura, si parla di 'trattamenti inumani e degradanti' per tutte quelle violenze intenzionali che minano profonda-

2 https://treaties.un.org/pages/ViewDetails.aspx?src=TREATY\&mtdsg_no=IV9\&chapter=4\&clang=_en $(2019-10-15)$. 
mente la dignità della persona (per esempio, la riduzione in schiavitù o la tratta), anche se non perpetrate da pubblici ufficiali. Molti emigranti che giungono in Italia con gli sbarchi e la quasi totalità dei richiedenti protezione internazionale denunciano di aver subito eventi di questo tipo, soprattutto prima della partenza dal proprio Paese (in questo caso il trauma ha agito come push factor), così come durante il passaggio in Paesi di transito (violenze dei passeurs, sequestri, detenzioni, stupri).

Questi eventi sono 'potenzialmente' traumatici, poiché si è riscontrata un'importante differenza tra esposizione a eventi psicotraumatici ed effettiva prevalenza del Disturbo da Stress Post-Traumatico (Post-Traumatic Stress Disorder, PTSD). Ciò vuol dire che se da un lato l'evento-trauma in sé provoca un complesso corteo di reazioni psicologiche, dall'altro lato non c'è una relazione di necessità tra trauma e PTSD. In altre parole, non c'è una relazione causa-effetto diretta, ma interviene un'altra serie di fattori tra i quali le caratteristiche della persona esposta al trauma, le sue difese psicologiche (la resilienza e la capacità di coping), le sue appartenenze culturali, familiari e religiose, il modo in cui ha integrato l'evento nella propria esperienza soggettiva e culturale, da cui deriva poi il vissuto di tale esperienza, e il senso che gli ha dato.

Inoltre, la deriva psicopatologica dell'esperienza verso un eventuale vero e proprio PTSD dipende anche dal contesto nel quale essa è avvenuta e dagli eventi che sono succeduti al trauma: alcuni ne possono mitigare gli aspetti traumatizzanti, mentre altri invece costituiscono una catena drammatica, che ne complica ulteriormente gli esiti. Infatti, gli studi concordano nel suggerire che vi sia un effetto quantitativo legato all'esordio della sindrome, per cui maggiore è il numero di traumi subiti, maggiore è il rischio relativo di sviluppare un PTSD (Aragona et al. 2014). La natura di tali traumi, legata alla ferocia (poiché perpetrati da un altro individuo) e alla volontarietà delle azioni di violenza, provoca effetti più gravi e rende le vittime più vulnerabili alle già citate 'difficoltà vitali post-migratorie'.

Questo ci introduce al concetto di traumatizzazione secondaria, con il quale si intende la riattivazione dell'esperienza traumatica attraverso nuovi eventi. In effetti, questo è un dato ricorrente nell'esperienza degli immigrati traumatizzati: essi non solo hanno dovuto subire esperienze traumatiche gravi prima della partenza e/o durante il viaggio, ma dopo l'arrivo in Europa la loro condizione di vulnerabilità li espone a ulteriori ritraumatizzazioni, sia per la non sufficiente tutela di alcune parti del sistema di accoglienza (si pensi ad esempio agli emigranti ospitati in centri sovraffollati dove è più probabile che avvengano frizioni tra gruppi di ospiti), sia per le situazioni traumatiche legate alle nuove barriere interne (si pensi agli emigranti, spesso famiglie con anziani e bambini, che ai confini balcanici si sono visti sparare contro pallottole di gomma e lacrimoge- 
ni), sia perché una parte delle persone esce dal circuito dell'accoglienza (ad esempio, i transitanti che si allontanano prima di venire registrati e, in questo momento, i titolari di protezione umanitaria che rischiano di essere allontanati dai centri per effetto della legge 132/2018, finendo per strada e innescando fenomeni di marginalizzazione sociale che rende più probabile l'esposizione a nuovi soprusi ed esperienze traumatizzanti).

Questi eventi, oltre ad avere un possibile effetto psicotraumatico intrinseco per la loro gravità (si pensi alle violenze sulle donne vittime di tratta), spesso sono patogeni perché riattivano il vissuto traumatico originario al quale vengono associati (ad esempio la riattivazione traumatica del torturato che ha un iperarousal - ovvero l'iperattivazione del sistema nervoso simpatico e di conseguenza di tutta una serie di ormoni dello stress - quando deve andare in questura, perché i poliziotti in uniforme gli ricordano i perpetratori del trauma). A queste esperienze potenzialmente ritraumatizzanti, si associano poi altre difficoltà vitali post-migratorie (noia, discriminazione, scarso accesso ai servizi, lungaggini burocratiche, preoccupazioni per la propria vita e per quella dei familiari, paura dell'espulsione, povertà) che nell'insieme vanno a completare il quadro delle PMLD.

Le ricerche dimostrano che le PMLD hanno un ruolo patogeno definito. In particolare, si è visto (Aragona et al. 2014) che nei rifugiati e richiedenti asilo avere più esperienze di questo tipo comporta un aumentato rischio di sviluppare un PTSD, con un aumento di gravità dei sintomi, una resistenza al processo terapeutico e una maggior difficoltà nel processo di integrazione sociale.

In generale, l'aver subito traumi nel Paese di origine, unitamente a condizioni di disagio nel Paese ospite, tende a peggiorare il livello di sofferenza psicopatologica. E occorre sottolineare che se ridurre il rischio di incorrere in esperienze traumatiche pre-migratorie è complesso, e necessita un lungo lavoro a livello internazionale contro le diseguaglianze e la conflittualità in molte zone del mondo, i tempi sono decisamente più brevi per intervenire sulle difficoltà di vita post-migratorie. Vista la loro alta frequenza e l'impatto esercitato dalle stesse sulla salute mentale degli immigrati (e di conseguenza sulle possibilità di integrazione sociale e sui comportamenti a rischio), è da sottolineare l'importanza di incidere subito sulle politiche di accoglienza, per diminuire i fattori di rischio e la possibile traumatizzazione secondaria.

\section{Esiti di torture e violenze sulla salute mentale}

Le violenze intenzionali di cui si è trattato nel precedente paragrafo hanno, come si diceva, un effetto psicotraumatico intrinseco e specifico. Attraverso la nostra esperienza sappiamo che le vittime di tortura difficilmente approdano ai servizi di salute mentale; accedono 
più facilmente all'assistenza medica come portatori di un disagio che assume le forme più varie: malesseri poco chiari, cefalee resistenti, disturbi somatici senza riscontro medico, insonnie, irritabilità. Raramente emergono i quadri più gravi: depressioni, fenomeni dissociativi come i flashback, disturbi della memoria (amnesie, difficoltà a memorizzare esperienze nuove), abuso di sostanze, comportamenti autolesionistici, tentati suicidi.

Il quadro clinico che la psichiatria identifica come specifico delle vittime esposte a un trauma è, come abbiamo visto, il Disturbo da Stress Post-Traumatico. Secondo il Manuale diagnostico e statistico dei disturbi mentali DSM-5 (American Psychiatric Association 2013) la reazione psicotraumatica è caratterizzata da un certo numero (prefissato nel manuale) dei seguenti sintomi:

- Ricordi dolorosi, intrusivi, involontari e ricorrenti dell'evento traumatico. La persona può apparire a volte come assorta, mentre al suo interno vi è una lotta tra pensieri e ricordi disturbanti e lo sforzo attivo e spesso inefficace di respingerli.

- Alterazione della coscienza, con vere e proprie reazioni dissociative (come i flashback) in cui l'individuo sente o agisce come se l'evento traumatico si stesse ripetendo. Dal punto di vista di chi osserva, in casi di questo tipo si vede la persona che si blocca, come se non fosse più in contatto con l'ambiente circostante, occupato da immagini o suoni, vividi o sfumati, in cui il vissuto traumatico si ripropone in tutta la sua drammaticità e con il corrispondente vissuto affettivo.

- Incapacità di ricordare aspetti importanti dell'evento traumatico (fenomeno che rientra anch'esso nelle alterazioni dissociative dello stato di coscienza, in questo caso per un'amnesia dissociativa).

- Sofferenza fisica e/o psicologica dinanzi a stimoli che simbolizzano o richiamano l'evento traumatico o un suo aspetto. È spesso fonte di ritraumatizzazioni secondarie. Ciò fa sì che la persona cerchi di sottrarsi a questa sofferenza sforzandosi di evitare quegli elementi esterni (persone, posti, conversazioni, attività, oggetti, situazioni) che possano rievocare e attivare ricordi, pensieri o sentimenti associati all'evento traumatico. Ad esempio, per una persona torturata dai militari in centri di detenzione uno stimolo scatenante può essere il trovarsi di fronte a forze dell'ordine o a personale in divisa, in situazioni in cui la persona deve rispondere a domande o a un interrogatorio, il che la riporta a rivivere esperienze del passato. Ciò comporta a volte difficoltà impreviste nel normale espletamento di pratiche necessarie per un richiedente protezione: persone che non riescono ad andare nella stazione di polizia (dove c'è l'ufficio preposto) per avviare la pratica; altre che si bloccano durante l'audizione in commissione perché si sentono sotto interrogatorio. 
- Disturbo del sonno, con difficoltà a iniziarlo o a mantenerlo, o quasi totale assenza di sonno, a causa di rimuginio o pensieri intrusivi, senza riuscire a rilassarsi. Quando alla fine, stremati, ci riescono, dopo poco arrivano incubi ricorrenti. Gli incubi post-traumatici sono caratteristici, perché il contenuto e/o l'affetto del sogno sono direttamente legati all'evento traumatico. Alla fine questi disturbi del sonno riducono le energie della persona, con importanti ricadute nel funzionamento diurno.

- Credenze o aspettative negative circa se stessi, gli altri o il mondo (per es. «Io sono cattivo», «Non mi posso fidare di nessuno», «Il mondo è un posto del tutto pericoloso», «Il mio sistema nervoso è rovinato per sempre»). A volte questi pensieri distorti riguardano le cause e le conseguenze dell'evento traumatico e conducono la persona a incolpare se stesso o gli altri (ad esempio i pazienti possono sentirsi in colpa di essere gli unici sopravvissuti, oppure per essere stati la causa, magari con il proprio impegno politico, delle sofferenze occorse ai propri familiari).

- Stati emotivi persistentemente negativi (per esempio: orrore, rabbia, colpa, vergogna); marcata diminuzione nell'interesse o nel piacere di fare le cose; sentimenti di distacco o di estraneità nei confronti degli altri; persistente incapacità a sperimentare emozioni positive.

- Iperarousal, che fa parte di una reazione normale ed evolutivamente sana in situazioni di pericolo reale, e che diventa patologica nel momento in cui il sistema continua ad essere attivato nonostante la situazione di pericolo non sia più presente. In un più generale quadro di iperattivazione si inscrivono vari sintomi post-traumatici come il comportamento irritabile e gli scoppi d'ira, alcuni comportamenti a rischio o autodistruttivi, la continua ipervigilanza con eccessive risposte di allarme (le persone che 'scattano' improvvisamente a un banale rumore). Occorre notare che gli scoppi d'ira apparentemente immotivati sono spesso una manifestazione che provoca allarme e che spesso non viene riconosciuta come possibile espressione di una sofferenza post-traumatica.

- Difficoltà di concentrazione: un sintomo molto importante perché influenza in modo negativo il percorso di integrazione, dovuta sia alla sonnolenza conseguente all'insonnia di cui si diceva sopra, sia al fatto che i pensieri intrusivi disturbano i processi cognitivi.

Non bisogna però ritenere che il PTSD esaurisca la psicotraumatologia clinica. Esistono infatti altre modalità con cui la sofferenza si può manifestare, con sintomatologie parziali, non tali cioè da configurare l'intero quadro clinico del PTSD, o con sintomi che possono apparire isolati, ad esempio atteggiamenti di ritiro sociale e isola- 
mento; crisi di paura e di ansia, o di aggressività e/o autoaggressività; disturbi della concentrazione, del pensiero o della memoria (che possono essere sospettati ad esempio in presenza di una difficoltà ad apprendere la lingua della terra d'asilo); sindromi depressive che a volte culminano in tentativi di suicidio; disturbi somatoformi, cioè sintomi fisici aspecifici e migranti nell'organismo; atteggiamenti paranoidei. La Herman (1992) ha anche descritto un quadro clinico particolarmente grave che ha definito PTSD Complesso, che si osserva in particolare in presenza di violenza intenzionale e in condizioni di prolungata coercizione, e che è caratterizzato da una molteplicità di sintomi di tipo somatico, cognitivo, affettivo, comportamentale e relazionale. In questo caso si osservano disturbi somatoformi particolarmente intensi, fenomeni dissociativi marcati, reazioni depressive intense e prolungate, sfiducia e sospettosità nelle relazioni interpersonali e scompaginamento dell'identità personale («Tutte le strutture del Sé - l'immagine corporea, l'immagine internalizzata degli altri, e i valori e gli ideali che danno un senso di coerenza e uno scopo - sono invalidate e sistematicamente danneggiate», Herman 1992, 385).

Rispetto all'espressione somatica della sofferenza mentale, ricerche italiane (Aragona et al. 2008, 2011, 2012b) mostrano che:

a. Almeno un quarto dei pazienti $(25,6 \%)$ visitati in servizi di medicina di base dedicati agli immigrati presenta una sindrome da somatizzazione, con conseguenti, importanti ricadute per la terapia. Ad esempio, scambiare una somatizzazione per un dolore infiammatorio comporta la prescrizione inappropriata di antinfiammatori, con rischi di cronicizzazione e di possibili danni iatrogeni.

b. Non tutti i gruppi di immigrati somatizzano allo stesso modo, essendovi una maggiore frequenza tra sud- e centroamericani e tra gli africani.

c. Diversamente dalle aspettative (per cui si potrebbe pensare che chi ha una bassa scolarità ha una maggiore difficoltà a trovare le parole per esprimere la sofferenza sul piano psicologico, e dunque tende con più facilità a esprimerla attraverso il corpo), negli immigrati non è stata riscontrata una correlazione significativa tra scolarità e somatizzazioni.

d. Le donne somatizzano più degli uomini, ma ciò non sembra essere legato a differenze biologiche perché questa differenza non è presente in tutti i gruppi studiati: ciò accade solo nei caucasici e nei sud- e centroamericani, mentre questa differenza di genere non appare rilevabile in africani e asiatici. Dunque non è l'essere donna in sé, ma l'essere donna in un certo gruppo geografico/etnico/culturale che influenza la frequenza e il tipo di somatizzazioni.

e. I pazienti non si dividono in modo netto tra chi somatizza e chi invece esprime il disagio con sintomi mentali (somatizers 
versus psychologizers), perché i dati indicano che vi è un'alta correlazione tra somatizzazioni, ansia e depressione, che dunque tendono a coesistere.

f. Infine, ed è il dato più rilevante in questa sede, gli immigrati che somatizzano hanno maggior probabilità di avere un PTSD, quasi tutti i sintomi di PTSD sono più frequenti nei somatizzatori, e all'aumentare del numero di sintomi post-traumatici aumenta significativamente il rischio di avere una sindrome da somatizzazione.

Le somatizzazioni possono quindi essere un sintomo sentinella di una possibile patologia post-traumatica nascosta: la persona traumatizzata può non parlare della sua sofferenza post-traumatica (a volte semplicemente perché non sa che può essere curata, altre volte per vergogna, o per i fenomeni di evitamento di cui abbiamo parlato), la quale rimane sostanzialmente invisibile, mentre può richiedere un aiuto per i sintomi che esperisce nel corpo.

È allora importante che i medici, così come gli operatori che si occupano di accoglienza e assistenza, siano preparati a cogliere nelle somatizzazioni la spia di una possibile violenza subita.

Come le somatizzazioni, così l'abuso di alcool e di sostanze può essere connesso a un malessere profondo, esito di violenza estrema: in effetti, tanti emigranti ci hanno raccontato che l'alcol è come un auto-terapia per silenziare, nello stordimento dovuto all'ebbrezza, il rimuginio del pensiero o l'iperarousal, di cui si è parlato in precedenza.

\section{$4 \quad$ Accogliere il paziente traumatizzato}

Un'accoglienza che metta al centro la relazione creando un ambiente favorevole all'incontro ha già valore terapeutico, perché consente alla persona di recuperare la sua dignità riscoprendosi capace di rapporti interpersonali.

Le strutture che ospitano le persone traumatizzate, sia come sedi abitative, sia provvisoriamente come luoghi di assistenza e cura, devono essere tranquille e accoglienti, che suggeriscano una dimensione domestica. In particolare le attività di prima accoglienza dovrebbero essere svolte da persone senza divisa, vestite in abiti civili; questa scelta può costituire per i nostri pazienti un primo momento di rassicurazione perché alcuni di loro, che hanno subito maltrattamenti o violenze da forze di polizia o militari, possono avere reazioni emotivamente intense a causa delle esperienze traumatiche e dell' $i$ perarousal a queste conseguente.

Il luogo dove avvengono le visite mediche, i trattamenti fisioterapeutici o psicoterapeutici, le sale d'attesa e gli altri spazi devono essere puliti, ben arredati, accoglienti, rassicuranti, con un'atmosfera 
domestica e familiare. È necessario che i pazienti abbiano la sensazione di trovarsi in un ambiente che possono controllare e quindi è utile che le porte siano aperte/apribili e, in specie al primo incontro, che siano mostrati loro gli spazi dell'ambiente, l'accesso alla toilette, luoghi dove rinfrescarsi oppure bere qualcosa. Per tutti, ma soprattutto per coloro che hanno vissuto un'esperienza traumatica di reclusione forzata o comunque di coercizione, è decisivo potersi sentire liberi di muoversi a proprio agio.

Luoghi con stimoli rumorosi irritano facilmente i pazienti, a causa dell'elevato stato di arousal in cui si trovano, e in particolare lo possono fare rumori che rievochino momenti del trauma vissuto: ad esempio il rumore di una porta che si apre all'improvviso con energia può richiamare quello dell'apertura della cella del carcere, che preludeva a una sessione di tortura. Di notte sono frequenti risvegli ripetuti o crisi d'ansia, in specie se i pazienti vivono in ambienti sovraffollati e rumorosi.

Ė utile che i servizi siano 'a bassa soglia', cioè di facile accesso, con formalità e passaggi burocratici ridotti al minimo.

È necessario che non solo l'ambiente ma anche la relazione con chi si prende cura delle persone traumatizzate sia affidabile, con un rigoroso rispetto degli orari e degli impegni presi, chiedendo il consenso prima di ogni azione (ad esempio, manovre semeiologiche nel corso di una visita medica, una telefonata, etc).

Il personale deve essere efficacemente formato per gestire anche stimoli relazionali sgradevoli, come atteggiamenti aggressivi o crisi di rabbia: la relazione con il paziente deve quindi essere 'resiliente', capace di tollerare attacchi e frustrazioni, e allo stesso tempo accogliente, comprensiva, empatica, delicata e con disponibilità di tempo. Occorre offrire supporto e incoraggiamento al paziente, senza paternalismi, ma in un rapporto paritario.

Ci diverrà più facile un atteggiamento simile se terremo a mente che, nonostante il loro aspetto magari dimesso, i nostri pazienti sono persone che sono state capaci di superare prove straordinarie, $e$ che meritano soprattutto la nostra ammirazione per il loro coraggio, la loro determinazione, la loro tenacia. I richiedenti asilo e i rifugiati, infatti, hanno in genere fronteggiato con successo vicissitudini e difficoltà fuori dal comune, che hanno richiesto coraggio e straordinaria forza d'animo.

La comunicazione può essere difficoltosa sia perché le vittime di violenza e torture possono avere capacità di concentrazione e di attenzione compromesse, sia per la conoscenza limitata della lingua locale, con un comprensibile elevato rischio di fraintendimenti. Anche per questo è necessario offrire ampia disponibilità di tempo alla conversazione, spiegando ogni passaggio, dando informazioni anche non richieste ma che potrebbero essere utili, verificando la comprensione dell'interlocutore, offrendo attivamente spazio per domande e chiarimenti. 
$\mathrm{Al}$ momento di compilare la cartella clinica è necessario informare i pazienti sul segreto professionale e sulla riservatezza di quanto sarà detto, che sono liberi di rispondere o no alle domande, che sono invitati a chiedere spiegazioni, possono alzarsi e anche abbandonare il colloquio in ogni momento. Devono avere la sensazione di sentirsi liberi, ed esserlo davvero. Questa iniziale procedura richiede senz'altro un poco di tempo aggiuntivo, ma mette a proprio agio i nostri interlocutori e favorisce una relazione di fiducia.

La prescrizione dei farmaci richiede spesso cautela, perché le vittime di violenza intenzionale (a causa delle difficoltà di concentrazione e memoria di cui si è detto) possono presentare irregolarità nell'assunzione dei farmaci, per dimenticanze o cattiva comprensione della posologia. A volte sono necessarie soluzioni creative da parte dei clinici, come scrivere di proprio pugno sulle confezioni la posologia dei farmaci, o preparare piccole confezioni monodose con la data e l'orario di ogni assunzione.

Vogliamo sottolineare come l'accoglienza iniziale costituisca non solo un momento delicato della relazione tra servizio e utenti ma anche e forse soprattutto un'opportunità: al primo approccio con l'équipe che si prenderà cura di lui, il paziente potrà sentirsi accolto e sentire di aver trovato il luogo giusto dove esprimere le proprie difficoltà e trovarvi sollievo. Un'accoglienza calda e rispettosa è il segnale di un atteggiamento ospitale e aperto, non solo capace di favorire una presa in carico efficace, ma anche una funzione riabilitativa, perché significa riconoscere e incontrare la persona come essere prezioso e unico, nella sua storia e nella sua dignità.

\section{Politiche per la salute e linee guida per la tutela assistenziale}

Seppur in ritardo rispetto ad altri Paesi europei (in Italia fino al 1990 c'era una 'riserva geografica', cioè non tutti coloro che giungevano nel nostro Paese potevano chiedere protezione e asilo ma solo quelli che arrivavano da alcune nazioni), l'Italia ha nel tempo normato e strutturato un sistema di protezione per coloro che ne fanno richiesta di grande significato politico e sociale. Anche dal punto di vista sanitario l'Italia ha una tradizione importante nell'ambito della tutela degli immigrati e profughi. Dal 1995 politiche e norme hanno tenuto conto della popolazione straniera, anche in condizione di fragilità sociale e debolezza giuridica, definendo un corpo giuridico altamente inclusivo (Marceca et al. 2012). In ambito programmatico alcuni documenti hanno supportato questa impostazione. Nell'ultimo anno tale attenzione, sull'accoglienza, la protezione e la tutela, ha però subito un grave ridimensionamento (Geraci 2018). 
A seguito dell'incremento degli sbarchi e all'evidenza di numeri significativi di persone traumatizzate psichicamente, dopo un intenso lavoro preparatorio, il 24 aprile 2017 è stato pubblicato sulla Gazzetta Ufficiale il decreto del Ministero della Salute su «Linee guida per la programmazione degli interventi di assistenza e riabilitazione nonché per il trattamento dei disturbi psichici dei titolari dello status di rifugiato e dello status di protezione sussidiaria che hanno subito torture, stupri o altre forme gravi di violenza psicologica, fisica o sessuale» con i relativi schemi per i vari interventi sanitari da compiere (Ministero della Salute 2017). Le linee guida, come descritto al momento dell'Intesa Stato Regioni e P.A. che ha preceduto il decreto ministeriale, hanno come obiettivo quello di tutelare chi richiede protezione internazionale in condizioni di particolare vulnerabilità in qualunque fase del suo percorso di riconoscimento della protezione e ovunque sia ospitato, creando le condizioni perché le vittime di eventi traumatici possano effettivamente accedere alle procedure previste dalla norma e la loro condizione possa essere adeguatamente tutelata.

Individuazione precoce delle vittime di violenza intenzionale e presa in carico chi, dove, come

\begin{tabular}{|c|c|c|}
\hline $\begin{array}{l}\text { Operatori sociali } \\
\text { delle strutture } \\
\text { d'accoglienza }\end{array}$ & $\begin{array}{l}\text { Medici e psicologi } \\
\text { delle strutture d'accoglienza } \\
\text { o del SSR }\end{array}$ & $\begin{array}{l}\text { Servizi specialistici } \\
\text { del DSM o altre strutture } \\
\text { riconosciute dal SSR }\end{array}$ \\
\hline $\begin{array}{l}\text { Nella quotidianità } \\
\text { Osservazione di ‘sintomi } \\
\text { sentinella' previa formazione } \\
\text { specifica }\end{array}$ & $\begin{array}{l}\text { In un setting adeguato } \\
\text { Colloquio/i eventualmente } \\
\text { con il supporto di strumenti } \\
\text { specifici }\end{array}$ & $\begin{array}{l}\text { Percorsi multidisciplinari integrati } \\
\text { Conferma diagnostica e presa } \\
\text { in carico terapeutica }\end{array}$ \\
\hline
\end{tabular}

Le ragioni delle linee guida sono spiegate nella loro premessa e sottolineano che i richiedenti e titolari di protezione internazionale e umanitaria sono una popolazione a elevato rischio di sviluppare sindromi psicopatologiche a causa della frequente incidenza di esperienze stressanti o propriamente traumatiche. Sono persone costrette ad abbandonare il proprio Paese generalmente per sottrarsi a persecuzioni o al rischio concreto di subirne. Possono anche fuggire da contesti di violenza generalizzata determinati da guerre o conflitti civili nel proprio Paese di origine. Inoltre, durante il percorso migratorio, sono sovente esposti a incidenti e traumi aggiuntivi determinati dalla pericolosità di questi viaggi che si possono concretizzare in situazioni di sfruttamento, violenze e aggressioni di varia natura compresa quella sessuale, la malnutrizione, l'impossibilità di essere curati, l'umiliazione psicofisica, la detenzione e i respingimenti. Gli eventi traumatici che li colpiscono determinano gravi conseguenze sulla loro salute fisica e psichica con ripercussioni sul benessere individua- 
le e sociale dei familiari e della collettività. Secondo le linee guida, per fornire una risposta adeguata è pertanto urgente riorientare il sistema sanitario italiano verso l'attenzione ai bisogni emergenti, la prossimità ai gruppi a rischio di marginalità, l'equità dell'offerta per assicurare un'assistenza sanitaria in linea con le loro necessità e nel rispetto dei principi costituzionali. Ė necessario, quindi, avviare la programmazione di strumenti operativi adeguati ad assistere questa nuova e utenza multiculturale, eterogenea, segnata in modo consistente dai traumi subiti. Certamente un'accoglienza adeguata alla complessità dei bisogni e alla tutela dei diritti di cui questi soggetti sono portatori richiede una riorganizzazione dei servizi sanitari, con definizione di procedure, di competenze e attività formativa del personale, resa difficile anche dal pesante ostacolo rappresentato dalle limitate risorse disponibili (una criticità infatti è la mancanza di previsione di risorse economiche dedicate).

Rispetto alle problematiche di salute mentale, la succitata linea guida ministeriale per la presa in carico dei soggetti con disturbi psichici e che hanno subito torture o altre forme gravi di violenza psicologica, fisica o sessuale, ha individuato interventi sanitari da compiere nelle diverse fasi del percorso di riconoscimento della protezione. Il documento sottolinea alcuni aspetti chiave dell'accoglienza e della presa in carico, quali l'emersione precoce dei bisogni di salute mentale, il riconoscimento e l'accessibilità dei diritti, l'adeguatezza del setting e lo sviluppo di competenze mediante attività formative rivolte al personale.

Le Regioni dovranno implementare, contestualizzare e rendere operative le indicazioni ricevute e per questo sono stati proposti alcuni progetti ministeriali. Ad oggi la sola Regione Lazio con la Delibera di Giunta Regionale nr. 590 del 16 ottobre 2018, «Indicazioni e procedure per l'accoglienza e la tutela sanitaria dei richiedenti protezione internazionale», ha ripreso il documento nazionale, seguendone l'impostazione, richiamandone i principi e adattandone le indicazioni al contesto organizzativo regionale. Ogni Azienda sanitaria coinvolta dovrà monitorare l'attuazione dei percorsi multidisciplinari, elaborando un rapporto quali-quantitativo annuale sulle attività svolte $e$ sulle principali problematiche riscontrate a livello clinico, organizzativo e di bisogni formativi, da trasmettere alla Direzione Salute e Integrazione Socio Sanitaria della Regione, con particolare riferimento al tema della salute delle vittime di violenza intenzionale e di tortura.

\section{Conclusioni}

Lo scenario attuale della salute mentale tra i profughi richiede complessità di analisi e di intervento. Le caratteristiche demografiche dei movimenti migratori in entrata in Italia sembrano essersi modificate negli ultimi anni, e questo inevitabilmente richiede dati e analisi di 
cui al momento non siamo ancora del tutto in possesso per comprendere nei dettagli che cosa stia avvenendo, come queste variazioni abbiano effetto sulla salute mentale dei richiedenti protezione e quali possano essere gli interventi di tutela più efficaci.

I primi indicatori mostrano che la richiesta di cure psichiatriche da parte di questa utenza è in aumento, anche se le caratteristiche qualitative del fenomeno appaiono ancora non del tutto definite. I fattori riconosciuti come in grado di aumentare il rischio di sofferenza psichica tra gli immigrati sembrano tuttavia essere in crescita, secondo quanto è possibile sapere sui nuovi arrivi.

Per quanto riguarda l'offerta di assistenza, essa vede al momento i servizi psichiatrici in difficoltà, per ragioni organizzative e clinico-operative.

Un primo problema è che le caratteristiche dell'utenza fanno sì che la necessità di traduttori competenti si sia fatta particolarmente elevata e difficile da soddisfare, perché servono con grande frequenza mediatori in lingue africane poco diffuse e conosciute. Inoltre, nel setting psichiatrico e psicologico il mediatore ha un ruolo chiave molto particolare, per cui servono competenze che vanno al di là dell'abilità linguistica, per le quali i mediatori devono ricevere adeguata formazione. Infine, in molte aziende sanitarie ancora mancano convenzioni chiare che rendano la chiamata del mediatore un atto fluido, per cui a volte i clinici si sentono impotenti di fronte alle barriere linguistico-culturali. Occorre sottolineare che spesso la mancanza dei mediatori ha conseguenze gravi sulle modalità di assistenza, a parità di patologia e gravità del caso (Tarsitani et al. 2013), per cui è inevitabile che queste resistenze debbano essere superate.

Un secondo problema è che i servizi psichiatrici, già in molte aree del Paese sotto pressione per la scarsità di personale, oltre a essere sottodimensionati soffrono a volte della mancanza di competenze specifiche, sia per quanto riguarda la psicotraumatologia da violenza intenzionale (un settore della psicopatologia relativamente recente), sia per la capacità di intervenire in modo culturalmente sensibile. La pubblicazione da parte del Ministero della Salute delle «Linee guida per la programmazione degli interventi di assistenza e riabilitazione nonché per il trattamento dei disturbi psichici dei titolari dello status di rifugiato e dello status di protezione sussidiaria che hanno subito torture, stupri o altre forme gravi di violenza psicologica, fisica o sessuale» dovrebbe colmare questo vuoto a livello organizzativo, sostenendo le aziende sanitarie nella creazione di percorsi specifici, mentre corsi specifici vengono organizzati da vari enti per formare gli operatori, anche con il supporto di fondi ad hoc. Tuttavia il fatto che il legislatore abbia ritenuto che l'implementazione delle linee guida debba farsi «isorisorse», cioè senza specifici finanziamenti ma nell'ambito dei finanziamenti ordinari, già a loro volta carenti, sta complicando il percorso, visto il numero progressivamente 
decrescente degli operatori della salute mentale in servizio nei Dipartimenti di Salute Mentale in molte zone d'Italia.

Eppure, per quanto la situazione possa apparire complessa, e anche francamente difficile, la fermezza nel guardare con realismo alla situazione attuale non potrà che accrescere le competenze del sistema di accoglienza nel nostro Paese. E, come in genere accade, affrontare difficoltà si rivela spesso un'opportunità da cogliere per la crescita e la maturazione complessiva di tutti i servizi assistenziali e sanitari coinvolti, con potenziali ricadute positive su tutto il sistema, di cui potrà, auspicabilmente, avvantaggiarsi il Paese nel suo complesso.

\section{Bibliografia}

American Psychiatric Association (2013). Diagnostic and Statistical Manual of Mental Disorders (DSM-5). Washington, DC: American Psychiatric Publishing.

Aragona, Massimiliano et al. (2008). «Effect of Gender And Marital Status on Somatization Symptoms of Immigrants From Various Ethnic Groups Attending a Primary Care Service». German Journal of Psychiatry, 11, 64-72.

Aragona, Massimiliano et al. (2011). «The Role of Post-migration Living Difficulties on Somatization Among First-Generation Immigrants Visited in a Primary Care Service». Annali dell'Istituto Superiore di Sanità, 47, 207-13.

Aragona, Massimiliano et al. (2012a). «Post-Migration Living Difficulties as a Significant Risk Factor for PTSD in Immigrants: A Primary Care Study». Italian Journal of Public Health, 9(3), 67-74. DOI https://doi.org/10.2427/7525.

Aragona, Massimiliano et al. (2012b). «Somatization in a Primary Care Service for Immigrants». Ethnicity \& Health, 17, 477-91.

Aragona, Massimiliano et al. (2013). «Traumatic Events, Post-Migration Living Difficulties and Post-Traumatic Symptoms in First Generation Immigrants: a Primary Care Study». Annali dell'Istituto Superiore di Sanità, 49, 169-75.

Aragona, Massimiliano et al. (2014). Quando le ferite sono invisibili. Vittime di tortura e di violenza: strategie di cura. Bologna: Edizioni Pendragon.

Baglio, Giovanni et al. (2018). «La salute psichica dei migranti e il fenomeno degli sbarchi: un'analisi delle dimissioni ospedaliere da reparti psichiatrici in Italia (2008-2015)». Bonciani, Manila et al. (a cura di), Dinamiche di salute e immigrazione, tra continuità e bisogni. Bologna: Edizioni Pendragon, 255.

Caritas Migrantes (2010). Dossier Statistico Immigrazione. Roma: Idos.

Caritas Migrantes (2012). Dossier Statistico Immigrazione. Roma: Idos.

Coccia, Benedetto; Pittau, Franco (2016). Le migrazioni qualificate in Italia. Ricerche, statistiche, prospettive. Roma: Idos.

Costa, Giuseppe (1993). «Immigrati extracomunitari: un profilo epidemiologico». Epidemiologia \& Prevenzione, 17, 234-8.

Da Silva, Estela et al. (2016). «Variazioni demografiche e impatto sulla salute psichica dei richiedenti asilo in Italia». Affronti, Mario (a cura di), Persone e popoli in movimento. Promuovere dignità, diritti e salute. Bologna: Pendragon, 138.

Foville, Achille (1875). «Les aliénés voyageurs ou migrateurs. Étude clinique sur certains cas de lypémanie». Annales méd-psychol, 33(14), 5-45.

Geraci, Salvatore (1995). Argomentidi Medicina delle Migrazioni. Roma: Perì Tecnés. 
Geraci, Salvatore (2001). «ll profilo di salute dell'immigrato tra aree critiche e percorsi di tutela». Annali Italiani di Medicina Interna, 16 (suppl. 1), 167-71.

Geraci, Salvatore (2018). «Migrazione e salute. Sicurezza, una legge patogena». Scienzainrete, 28 novembre. URL https://www.scienzainrete.it/articolo/sicurezza-legge-patogena/salvatore-geraci/2018-11-28 (2019-10-15).

Herman, Judith Lewis (1992). Trauma and Recovery: The Aftermath of Violence. New York: Basic Books.

INAIL (2012). Rapporto Annuale 2011. Parte quarta/Statistiche Infortunie malattie professionali. Roma: INAIL.

Kinzie John D. (2006). «Immigrants and Refugees: The Psychiatric Perspective». Transcultural Psychiatry, 43, 577-91.

Marceca, Maurizio et al. (2012). «Immigrants' Health Protection: Political, Institutional and Social Perspectives at International and Italian Level». Italian Journal of Public Health, 9(3), 1-11.

Mazzetti, Marco (2008). «Trauma and Migration. A Transactional Analytic Approach Towards Refugees and Torture Victims». Transactional Analysis Journal, 38(4), 285-302.

Medici Senza Frontiere (2016). Traumi ignorati. Richiedenti asilo in Italia: un'indagine sul disagio mentale e l'accesso ai servizi sanitari territoriali. Roma: Medici Senza Frontiere.

Ministero della Salute (2017). «Linee guida per la programmazione degli interventi di assistenza e riabilitazione nonché per il trattamento dei disturbi psichici dei titolari dello status di rifugiato e dello status di protezione sussidiaria che hanno subito torture, stupri o altre forme gravi di violenza psicologica, fisica o sessuale». Gazzetta Ufficiale della Repubblica Italiana, 95, 29-118. URL http://www.salute.gov.it/imgs/C_17_pubblicazioni_2599_ allegato.pdf (2019-10-15).

Osservatorio Nazionale sulla salute nelle regioni italiane (2005-18). Rapporto Osservasalute dell'Osservatorio nazionale sulla salute nelle regioni italiane. Milano: Prex. (Rapporti usciti annualmente).

Ødegaard, Ørnulv (1932). «Emigration and Insanity. Study on Mental Diseases among Norvegian Born Population of Minnesota». Acta Psychiatrica Neurologica Scandinavica, 7 (Suppl. 4), 1-206.

Ranney, M.H. (1850). «On Insane Foreigners». American Journal of Insanity, 7, 53-63.

Tarsitani, Lorenzo et al. (2013). «Acute Psychiatric Treatment and the Use of Physical Restraint in First-Generation Immigrants in Italy: A Prospective Concurrent Study». International Journal of Social Psychiatry, 59, 613-18.

WHO, World Health Organization Regional Office for Europe (2018). Report on the Health of Refugees and Migrants in the WHO European Region. No Public Health Without Refugee and Migrant Health. URL https://bit.ly/2Sm6mNw (2019-03-03). 


\section{SCHEDA}

\section{Il Progetto Ferite invisibili della Caritas di Roma}

Con l'aumento dei richiedenti protezione internazionale, sono cresciuti in Italia i cittadini stranieri che hanno subito varie forme di tortura ed oppressione. I monitoraggi effettuati finora suggeriscono che il numero di sopravvissuti a torture, a gravi violazioni dei diritti umani o comunque a eventi psicotraumatici è di una certa consistenza. La delicatezza dei casi e la complessità del tema richiedono un approccio clinico e riabilitativo nei confronti di queste persone basato non solo su una particolare attenzione, ma anche, soprattutto, su specifiche competenze e alta professionalità.

Il progetto Ferite Invisibili della Caritas di Roma nasce dalla necessità di riconoscere, accogliere e curare queste individui, dando loro la possibilità di riscoprirsi come persone. Nella valorizzazione dell'incontro, anche terapeutico, si riconosce nell'altro dignità, valore e si garantisce rispetto e relazione reciproca.

\section{Un progetto di servizio}

Per queste ragioni, alla fine del 2005 è stato avviato, presso il Poliambulatorio Caritas per immigrati (in via Marsala, sotto la stazione Termini), un servizio, in collegamento con quanti già a vario titolo si impegnano in questo ambito, mirato specificatamente alla riabilitazione psicologica di queste persone che si trovano in condizione di fragilità sociale. Il progetto dal nome Ferite invisibili ha visto una fase di studio ed approfondimento con la revisione della letteratura internazionale sull'argomento (report per la conferenza internazionale One billion del dicembre 2004), l'integrazione della stessa con le esperienze maturate nel corso degli ultimi 15 anni dall'équipe del progetto, e la realizzazione di un tavolo permanente di collegamento tra le realtà che a Roma si occupano di richiedenti asilo e rifugiati (aspetti legati all'accoglienza ed alla sanità: Gruppo regionale Immigrazione e Salute GrIS - richiedenti e rifugiati). Alla luce di queste esperienze si è avviata una progettazione di interventi mirati alla riabilitazione psicologica e fisica di chi ha subito violenza, tortura ed in genere traumi legati alla mancanza di accoglienza ed all'ingiustizia sociale: formazione, ricerca, screening della popolazione a rischio, attività clinica. 


\section{L’attività clinica}

L'equipe è formata da psicoterapeuti, psichiatri, mediatori culturali, infermieri, operatori del sociale, medici; offre un servizio di ascolto, di psicoterapia transculturale e, più recentemente, di medicina legale.

L'aiuto, attraverso un attento lavoro di equipe, consiste innanzitutto nel far riconoscere l'orrore vissuto e le 'ferite' psichiche indotte, affinché queste persone possano riappropriarsi della dignità di esseri umani, dare un significato alla loro esperienza e riprogettare un futuro per la loro esistenza. Nel contempo cerca anche di costituire una fitta rete socio-assistenziale per sostenere percorsi legali, informativi e formativi (accoglienza protetta, insegnamento della lingua italiana, formazione professionale, inserimento lavorativo...). Il setting terapeutico, aperto e flessibile, si avvale di molte risorse e favorisce modi diversi di concepire le relazioni e gli affetti, la salute e la malattia, di interpretare gli eventi e la realtà circostante; e soprattutto è un luogo di appartenenza in cui sentirsi protetti e sostenuti.

Nel febbraio 2012, l'Ufficio delle Nazioni Unite dell'Alto Commissario per i Diritti Umani ha riconosciuto il servizio all'interno della rete sovranazionale di sostegno e cura alle vittime di tortura.

\section{Qualche dato sull'attività clinica}

In poco più di 13 anni di progetto (fine 2005-aprile 2019) sono stati presi in carico 366 pazienti (288 uomini e 78 donne) e sono stati effettuati 5.545 colloqui psicoterapeutici con una media di 15 visite/ paziente a sottolineare la complessità e la delicatezza dell'approccio terapeutico.

Negli ultimi 16 mesi abbiamo seguito 37 pazienti, di cui 20 nuovi. Sono state prese in carico 36 persone, mentre una, dopo aver fatto il colloquio di valutazione, è stata orientata ad altri servizi territoriali. Finora sono state effettuate 499 sedute terapeutiche.

Fino al 2010 i pazienti provenivano soprattutto dall'Afghanistan, seguiti dalla Guinea, Nigeria e Eritrea. Dal 2011 al 2013 sono stati prevalenti coloro che provenivano da Costa D'Avorio, seguiti da Afghanistan, Camerun e Senegal. Tra il 2014 e 2015 si nota un aumento graduale e significativo da quelli provenienti dal Gambia. Attualmente prevalgono le persone provenienti dalla Nigeria, Gambia e Senegal. Negli anni sono stati seguiti oltre 110 minori stranieri non accompagnati (MSNA). 


\section{La sostenibilità}

Progetti specifici di assistenza, completamente gratuiti per i beneficiari, sono spesso inficiati dalla sostenibilità economica che ne condiziona la prosecuzione nel tempo. Per evitare questo la Caritas di Roma ha attivato molte collaborazioni con enti diversi, chiedendo impegni e risorse diversificate e nel contempo definendo un'organizzazione molto elastica ed integrata con gli altri servizi e strutture, in particolare dell'Area sanitaria. Sostegni parziali del progetto, in varia natura, sono stati garantiti nel tempo dall'8×1000 della Chiesa Cattolica e da offerte di sostenitori, dalla Regione Lazio, dall'Istituto Tata Giovanni e dall'United Nations Voluntary Fund for Victims of Torture (UNVFVT). 


\title{
Tortura e disagio psichico tra i rifugiati e i richiedenti asilo Presa in carico e intervento psico-sociale
}

\author{
Michela Nosè \\ Università degli Studi di Verona, Italia
}

\begin{abstract}
In recent years there has been a progressive rise in the number of asylum seekers and refugees displaced from their country of origin, with significant social, economic, humanitarian and public health implications. In comparison with the general population, refugees and asylum seekers have been shown to experience higher prevalence rates of a range of disorders, including Post-Traumatic Stress Disorder (PTSD). Although there is substantial variability in prevalence rates, PTSD is frequent and is much more likely in refugees and asylum seekers compared to host populations. In terms of psychosocial interventions, cognitive behavioral interventions and narrative exposure therapy are supported by some evidence of efficacy that would suggest the possibility of decreasing PTSD symptoms in this vulnerable group. Overall, psychosocial interventions for asylum seekers and refugees with PTSD were found to provide significant benefits in reducing PTSD symptoms. Therefore these data provide an evidence base to inform decisions in clinical practice and in policy making, considering that an appropriate treatment is a right of refugees and a way to better adaptation and integration in hosted country.
\end{abstract}

Keywords Refugees. Asylum seekers. Mental health. Psychological distress. Posttraumatic stress disorder.

Sommario 1 Introduzione. - 2 Disagio psicologico e patologia psichiatrica nei richiedenti asilo e rifugiati. - 2.1 II Disturbo Post-Traumatico da Stress (PTSD) nei rifugiati e richiedenti asilo. - 2.2 Il trauma e il racconto della storia. - 3 Interventi psico-sociali e presa in carico psicologica per i richiedenti asilo e rifugiati. - 4 Conclusioni. 


\section{Introduzione}

L'immigrazione in Italia è un fenomeno strutturale ed è importante tutelare l'accesso alla protezione dei richiedenti asilo che arrivano nel nostro Paese e favorirne l'integrazione (UNHCR 2017). La tutela della salute dei richiedenti protezione internazionale e dei rifugiati è di estrema importanza per tutti i Paesi accoglienti, in particolare la tutela della salute mentale se si considera la forte esposizione a esperienze traumatiche sia prima che durante e dopo il viaggio migratorio.

I dati della letteratura sono concordi nell'affermare una maggiore prevalenza di disagio psicologico e patologia psichiatrica nella popolazione di rifugiati e nelle vittime di tortura, nonostante l'eterogeneità dei dati legata, anche, alle caratteristiche di questa popolazione e delle realtà che la accolgono. Emerge come necessaria una valutazione precoce e attuata in maniera routinaria delle condizioni psicologiche, con particolare attenzione alla valutazione di presenza di sintomatologia legata al Disturbo Post-Traumatico da Stress (PTSD) e alla sintomatologia di tipo depressivo, che sono i disturbi psichici con una prevalenza in questa popolazione superiore rispetto alla popolazione generale. Conseguentemente, il modello di accoglienza di questa popolazione dovrebbe prevedere una presa in carico sanitaria specialistica, qualora emerga un disagio psicologico e un disturbo psichico.

Nella letteratura internazionale rimane aperto un importante dibattito sui possibili interventi terapeutici che abbiano evidenze di efficacia in questa popolazione. È infatti necessario capire se i trattamenti raccomandati per il Disturbo Post-Traumatico da Stress, e per gli altri disturbi psichiatrici, nella popolazione generale possano essere ugualmente applicati ed essere efficaci nei rifugiati. Recenti revisioni della letteratura sottolineano che vi sono numerosi studi che valutano l'efficacia degli interventi psicosociali in questa popolazione di pazienti, se pur con limiti metodologici. Tali interventi psicosociali hanno caratteristiche diverse e i risultati ottenuti nei singoli studi non sono sempre generalizzabili ad altri contesti e ad altre popolazioni. Per poter affrontare tale complesso ambito, è importante conoscere il panorama di riferimento della letteratura internazionale e le evidenze attualmente disponibili.

\section{Disagio psicologico e patologia psichiatrica nei richiedenti asilo e rifugiati}

La letteratura internazionale è concorde nell'affermare che la popolazione dei rifugiati e richiedenti asilo sperimenta una condizione di salute fisica e mentale significativamente peggiore rispetto alla popolazione generale. Infatti, la violenza intenzionale a cui questa po- 
polazione è generalmente sottoposta determina gravi conseguenze sia in ambito fisico e neurobiologico, che psicologico, sociale ed economico con effetti a breve e a lungo termine (Porter, Haslam 2005; Fazel, Wheeler, Danesh 2005; Steel et al. 2009).

Particolare attenzione merita l'ambito della salute mentale: i rifugiati si trovano a vivere in condizioni altamente stressanti e traumatiche, sono vittime di tortura fisica e psichica con conseguenze emotive spesso più distruttive dei danni fisici. Gli eventi traumatici a cui questa popolazione è esposta in fase pre-migratoria, migratoria e post-migratoria rappresentano un fattore di rischio ben noto per le patologie psichiatriche in generale (Priebe, Giacco, El-Nagib 2016). $\grave{E}$, quindi, facilmente intuibile che in questa popolazione si possa riscontrare con maggiore frequenza una sofferenza psicologica, che può peggiorare anche in veri e propri disturbi psichiatrici.

I quadri clinici che più frequentemente si osservano, pur con alcune criticità associate alla validità trans-culturale delle diagnosi, sono riconducibili prevalentemente al Disturbo Post-Traumatico da Stress e ai disturbi di tipo depressivo. Tuttavia, anche altre patologie presentano una prevalenza aumentata in tale popolazione e stanno diventando sempre di più oggetto di studi: disturbo d'ansia generalizzata, disturbo da attacchi di panico, disturbi di somatizzazione etc. (Silove 2012; Turrini et al. 2017)

Le principali revisioni della letteratura sulla frequenza dei disturbi psichici nei richiedenti asilo e rifugiati sono concordi nell'affermare una maggiore prevalenza di disagio psicologico e di alcuni disturbi psichici in questa popolazione.

Una delle prime revisioni sistematica della letteratura su tale argomento (Fazel, Wheeler, Danesh 2005) ha stimato che, nei diversi gruppi culturali, i tassi di prevalenza del PTSD siano di circa il 9\%, di depressione del 5\% e di disturbo d'ansia generalizzato del 4\%. Successivamente altre revisioni hanno evidenziato tassi molto più alti, intorno al 30\% (Steel et al. 2009; van Wyk et al. 2012), e in altri lavori addirittura dell'80\% (Mollica et al. 1998; Bogic, Njoku, Priebe 2015). Questa variabilità è chiaramente comprensibile se si considerano l'eterogeneità delle realtà che accolgono queste persone (a seconda che si trovino per esempio in Paesi ad alto reddito o in Paesi in via di sviluppo, del tipo di strutture di accoglienza o dell'assenza di queste, delle tempistiche delle pratiche amministrative, ecc.) e delle popolazioni considerate (si pensi ad esempio alle differenze di Paese di provenienza, d'età, di religione, di status giuridico, ecc.) (Silove 2012). Una recente revisione (Turrini et al. 2017) ha inoltre sottolineato che i dati di prevalenza dei disturbi psichici nella popolazione dei rifugiati rispetto alla popolazione generale sono più alti non solo per il PTSD, ma anche per i disturbi ansioso e depressivi, con tassi fino al $40 \%$.

Per quanto riguarda i fattori associati a tale aumentata prevalen$\mathrm{za}$, in letteratura emerge che le esperienze traumatiche sono l'ele- 
mento che più fortemente si associa al rischio di disagio psicologico e disturbi psichici, con una sorta di effetto cumulativo, in cui il rischio aumenta tanto più una persona è esposta a traumi. I fattori socio-demografici, soprattutto quelli legati alla condizione pre-migratoria, come il livello sociale o di istruzione, non sembrano, invece, avere una forte correlazione (Nosè et al. 2018). In generale, la letteratura internazionale fino ad oggi si è occupata prevalentemente dell'influenza dei fattori pre-migratori sulla salute mentale dei richiedenti asilo e rifugiati, mentre gli studi più recenti si stanno invece focalizzando maggiormente sugli effetti degli eventi post-migratori (Li, Liddell, Nickerson 2016; Schock et al. 2016). Queste ultime ricerche sottolineano che fattori post-migratori, quali le condizioni socio-economiche, interpersonali e gli eventi correlati al percorso per l'ottenimento della protezione internazionale, influenzano direttamente il benessere psicologico dei richiedenti asilo. In particolare, viene evidenziato il ruolo cruciale giocato dalla durata delle procedure per la richiesta di protezione internazionale e la percentuale di diniego della stessa sul benessere psico-fisico dei richiedenti asilo (Müller et al. 2018).

È chiaro, comunque, che una precoce valutazione delle condizioni psichiche nella popolazione dei rifugiati sia necessaria e debba essere fatta in maniera routinaria (Hollifield et al. 2002), con particolare attenzione alla valutazione di presenza di sintomatologia depressiva e traumatica.

A livello italiano in letteratura si trovano pochi studi epidemiologici e i dati a disposizione sono molto scarsi, nonostante la grande presenza di richiedenti asilo nel territorio.

Due studi sono stati condotti in Sicilia. Il primo (Firenze et al. 2016) riguarda una popolazione di richiedenti asilo accolta presso un CARA (Centro di Accoglienza per Richiedenti Asilo) e segnala una prevalenza di PTSD del 17,4\% e di Depressione Maggiore del 7,3\%. Il secondo studio (Crepet et al. 2017), condotto da Medici Senza Frontiere (MSF) sempre in Sicilia, riporta una prevalenza di PTSD del 31\%, di Depressione Maggiore del 20\% (severa nel 12\% dei casi) e di Disturbi d'Ansia dell'11\%. Gli autori segnalano inoltre un'alta presenza di comorbilità tra più di una diagnosi psichiatrica (nel 67\% dei casi). Un altro studio epidemiologico è stato recentemente condotto a Verona (Nosè et al. 2018): obiettivo dello studio era la valutazione sulla frequenza del disagio psicologico e patologie psichiatriche in tutta la popolazione adulta di richiedenti asilo e rifugiati afferenti al percorso SPRAR a Verona durante il periodo di un anno e mezzo. A tutto il campione identificato è stata offerta una valutazione psicologico-psichiatrica con l'obiettivo di riconoscere, descrivere e misurare la frequenza del disagio psicologico e psichiatrico. È emersa un'alta frequenza di eventi traumatici; più di un terzo dei soggetti presentava un disagio psicologico clinicamente significativo, e circa un quarto soddisfava i criteri per una diagnosi psichiatrica, principal- 
mente di PTSD e di disturbo depressivo. I dati emersi sono risultati in linea con la letteratura internazionale e appaiono particolarmente significativi se si pensa alla realtà italiana, in cui vi è sempre più la necessità di definire un modello di assistenza con il riconoscimento precoce del disagio psicologico e l'eventuale presa in carico o invio accompagnato ai servizi specialistici.

Come già esposto, il disturbo maggiormente riscontrabile in questa popolazione è il Disturbo Post-Traumatico da Stress (PTSD), che mostra delle specificità nella popolazione dei rifugiati e richiedenti asilo.

\subsection{Il Disturbo Post-Traumatico da Stress (PTSD) nei rifugiati e richiedenti asilo}

Le persone esposte a gravi eventi traumatici, vittime o testimoni, presentano una maggior suscettibilità allo sviluppo di reazioni psicotraumatiche fortemente invalidanti; il principale disturbo in questa categoria è il Disturbo Post-Traumatico da Stress.

Recentemente, nella revisione del sistema classificatore del DSMV (American Psychiatric Association 2014), sono stati modificati alcuni dei criteri diagnostici per tale disturbo, in particolare è stata ampliata la definizione relativamente alla tipologia di stress alla base del disturbo, sono state introdotte delle alterazioni 'negative' a livello cognitivo e comportamentale, ed è stata aggiunta la specificazione relativa alla presenza di sintomi dissociativi. La tabella 1 riporta nel dettaglio i criteri diagnostici del PTSD nel DSM-V.

Tabella 1 Criteri diagnostici del PTSD secondo il DSM-V (APA 2014)

A. Esposizione a morte reale o minaccia di morte, grave lesione, violenza sessuale, in uno o più dei seguenti modi:

1. Fare esperienza diretta dell'evento/i traumatico/i.

2. Assistere direttamente a un evento/i traumatico/i accaduto ad altri.

3. Venire a conoscenza di un evento/i traumatico/i accaduto a un membro della famiglia oppure ad un amico/a stretto/a. In caso di morte reale o di minaccia di morte di un membro della famiglia o di un amico/a, l'evento/i deve essere stato violento o accidentale.

4. Fare esperienza di una ripetuta o estrema esposizione a dettagli crudi dell'evento/i traumatico/i (esempio: i primi soccorritori che raccolgono resti umani; agenti di polizia ripetutamente esposti a dettagli di abuso su minori). 
B. Presenza di uno o più dei seguenti sintomi intrusivi associati all'evento/i traumatico/i, che hanno inizio successivamente all'evento/i traumatico/i:

1. Ricorrenti, involontari e intrusivi ricordi spiacevoli dell'evento/i traumatico/i.

2. Ricorrenti sogni spiacevoli in cui il contenuto e/o le emozioni del sogno sono collegati all'evento/i traumatico/i.

3. Reazioni dissociative (esempio: flashback) in cui il soggetto sente o agisce come se l'evento/i traumatico/i si stesse ripresentando (tali reazioni possono verificarsi lungo un continuum in cui l'espressione estrema è la completa perdita di consapevolezza dell'ambiente circostante).

4. Intensa o prolungata sofferenza psicologica all'esposizione a fattori scatenanti interni o esterni che simboleggiano o assomigliano a qualche aspetto dell'evento/i traumatico/i.

5. Marcate reazioni fisiologiche a fattori scatenanti interni o esterni che simboleggiano o assomigliano a qualche aspetto dell'evento/i traumatico/i.

C. Evitamento persistente degli stimoli associati all'evento/i traumatico/i, iniziato dopo l'evento/i traumatico/i, come evidenziato da uno o più dei seguenti criteri:

1. Evitamento o tentativi di evitare ricordi spiacevoli, pensieri o sentimenti relativi o strettamente associati all'evento/i traumatico/i.

2. Evitamento o tentativi di evitare fattori esterni (persone, luoghi, conversazioni, attività, oggetti, situazioni) che suscitano ricordi spiacevoli, pensieri o sentimenti relativi o strettamente associati all'evento/i traumatico/i.

D. Alterazioni negative di pensieri ed emozioni associati all'evento/i traumatico/i, iniziate o peggiorate dopo l'evento/i traumatico/i, come evidenziato da due o più dei seguenti criteri:

1. Incapacità di ricordare qualche aspetto importante dell'evento/i traumatico/i (dovuta tipicamente ad amnesia dissociativa e non ad altri fattori come trauma cranico, alcool, droghe).

2. Persistenti ed esagerate convinzioni o aspettative negative relative a se stessi, ad altri, o al mondo (esempio: «io sono cattivo», «non ci si può fidare di nessuno», «il mondo è assolutamente pericoloso», «il mio sistema nervoso è definitivamente rovinato»).

3. Persistenti, distorti pensieri relativi alla causa o alle conseguenze dell'evento/i traumatico/i che portano l'individuo a dare la colpa a se stesso oppure agli altri.

4. Persistente stato emotivo negativo (esempio: paura, orrore, rabbia, colpa, vergogna).

5. Marcata riduzione di interesse o partecipazione ad attività significative.

6. Sentimenti di distacco o di estraneità verso gli altri.

7. Persistente incapacità di provare emozioni positive (esempio: incapacità di provare felicità, soddisfazione o sentimenti di amore). 
E. Marcate alterazioni dell'arousal e della reattività associati all'evento/i traumatico/i, iniziate o peggiorate dopo l'evento/i traumatico/i, come evidenziato da due o più dei seguenti criteri:

1. Comportamento irritabile ed esplosioni di rabbia (con minima o nessuna provocazione) tipicamente espressi nella forma di aggressione verbale o fisica nei confronti di persone o oggetti.

2. Comportamento spericolato o autodistruttivo.

3. Ipervigilanza.

4. Esagerate risposte di allarme.

5. Problemi di concentrazione.

6. Difficoltà relative al sonno (esempio: difficoltà nell'addormentarsi o nel rimanere addormentati, oppure sonno non ristoratore).

F. La durata delle alterazioni dei criteri B, C, D, E è superiore a un mese.

G. L'alterazione provoca disagio clinicamente significativo o compromissione del funzionamento in ambito sociale, lavorativo o in altre aree importanti.

H. L'alterazione non è attribuibile agli effetti fisiologici di una sostanza o a un'altra condizione medica.

\section{Specificare se: Con sintomi dissociativi.}

i sintomi dell'individuo soddisfano i criteri per un disturbo da stress post-traumatico e, inoltre, in risposta all'evento stressante l'individuo fa esperienza di sintomi persistenti o ricorrenti di uno dei due seguenti criteri:

1. Depersonalizzazione: persistenti o ricorrenti esperienze di sentirsi staccato dai, e come se fosse un osservatore esterno dei, propri processi mentali o del proprio corpo (per es., sensazione di essere in un sogno; sensazione di irrealtà di se stessi o del proprio corpo o del lento scorrere del tempo)

2. Derealizzazione: persistenti o ricorrenti esperienze di irrealtà dell'ambiente circostante (per es., il mondo esterno all'individuo viene da lui vissuto come irreale, onirico, distante o distorto)

\section{Specificare se: Con espressione ritardata.}

Se i criteri diagnostici non sono soddisfatti appieno entro i sei mesi dall'evento (ancorché l'insorgenza e l'espressione di alcuni sintomi possano essere immediate).

Nella popolazione dei rifugiati le manifestazioni sintomatologiche del PTSD, al di là dei criteri diagnostici del sistema nosografico di riferimento, possono essere peculiari e spesso culturalmente mediate. La cultura di provenienza ha un'influenza non irrilevante sull'espressione sintomatica dello stress da trauma. È importante, infatti, tenere in considerazione tale aspetto al fine di ridurre la possibilità di effettuare errori diagnostici.

Il quadro sintomatologico più frequentemente riscontrato è caratterizzato in prevalenza da intrusioni notturne e diurne, con associate reazioni emotive e fisiche, disturbi del sonno, della memoria, dell'attenzione e della concentrazione. Tali aspetti spesso si accompagnano a sintomi depressivi, sospettosità, facile irritabilità, labilità emotiva, episodi dissociativi, condotte di evitamento, ideazione suicidaria, abuso di sostanze, sintomi somatoformi tra cui dolore cronico (Teodorescu et al. 2015). È importante ricordare che alcuni sintomi cognitivi, come deficit dell'attenzione e della memoria (spesso i rifu- 
giati non ricordano, anche per molto tempo, i dettagli del trauma), possono essere responsabili delle apparenti contraddizioni nella narrazione della propria storia traumatica, che potrebbero mettere in difficoltà i membri delle commissioni territoriali preposti a vagliare le domande di protezione. L'ipermnesia, l'iperreattività agli stimoli e il rivivere l'esperienza traumatica, coesistono con l'intorpidimento psichico, l'evitamento, l'amnesia e l'anedonia. Alcuni autori sottolineano come spesso le persone traumatizzate tentino di compensare la propria iperreattività cronica con una chiusura totale a livello comportamentale, evitando gli stimoli che ricordano il trauma, mediante un intorpidimento emozionale sia nei confronti dell'esperienza legata al trauma, sia dell'esperienza quotidiana. Nelle vittime di situazioni traumatiche, specie se estreme e ripetute nel tempo, può persistere, nel corso degli anni, una sorta di cronica iposensibilità nei confronti dell'ambiente esterno, alternata da iperreattività fisiologica intermittente in risposta a stimoli di origine emotiva e a specifiche sollecitazioni che possono indurre il ricordo dell'evento traumatico (Hollifield et al. 2002; Klarić et al. 2007; Norris, Alegria 2005).

Vengono inoltre descritti dei quadri sintomatologici culturalmente specifici per determinate popolazioni. È chiaro che l'eterogeneità dei quadri con cui si può venire a contatto rende necessarie delle cornici culturali e terapeutiche mirate.

Le complicanze di tale disturbo hanno delle implicazioni importanti e invalidanti per la salute e il funzionamento del soggetto, con ripercussioni in termini di riduzione della qualità della vita. Nei rifugiati ciò appare particolarmente significativo perché la sofferenza psichica si associa spesso ad una disabilità elevata, legata, oltre che alla sintomatologia spesso molto pervasiva, anche alle molteplici problematiche sociali che sono connesse allo status di rifugiato (Palic et al. 2014; Tay et al. 2015). È necessario quindi tener presente che le complicanze di un PTSD cronico o non trattato possono avere anche delle conseguenze a livello comunitario e sociale, con difficoltà di integrazione e aumento di sacche di disagio e vulnerabilità sociale.

Abbiamo già potuto sottolineare che il PTSD può essere considerato come l'unico disturbo mentale per il quale sia identificabile chiaramente una causa esterna nella forma di un evento riconducibile al trauma, o agli eventi traumatici, sperimentati dalla persona. La raccolta delle esperienze traumatiche, quindi, diventa un elemento fondamentale della relazione terapeutica. 


\subsection{Il trauma e il racconto della storia}

Il trauma è una causa che agendo violentemente su un organismo vi induce delle modificazioni. Una persona traumatizzata è innanzitutto una persona che è stata spaventata, ovvero che ha sperimentato un'intensa ed inaspettata paura, seguita da un'intensa risposta fisica e da un brusco cambiamento nel proprio equilibrio. L'impressione che si ha ascoltando la narrazione di chi ha vissuto un trauma è allo stesso tempo di perdita e di invasione. Lo 'spavento' frantuma la persona, distruggendo il mondo abituale noto. L'aiuto che può essere dato a queste persone passa per la costruzione di un nuovo mondo, che deve essere calato nella prospettiva peculiare di una determinata cultura.

È utile osservare il trauma da una prospettiva multidimensionale [tab. 2] al fine di comprendere al meglio il complesso quadro clinico che caratterizza la popolazione dei rifugiati. Infatti, questa popolazione non sperimenta solamente il trauma (o più spesso i traumi) nel Paese di origine, che già di per sé rappresenta un evento estremamente drammatico (Steel et al. 2009; Van Ommeren et al. 2001). Vi sono, anche, il trauma migratorio e quello post-migratorio, che determinano spesso una prosecuzione della sofferenza, piuttosto che rappresentare una salvifica via di fuga da essa (Silove 2004; Steel et al. 2006). In particolare, una volta giunti nel nuovo Paese, spesso i rifugiati si trovano in un contesto definito da norme culturali, politiche e sociali differenti, a contatto con stili di vita nuovi e spesso associati alla precarietà, in un clima di continua incertezza rispetto al futuro e caratterizzato da un'integrazione spesso difficile, da marginalizzazione ed esclusione sociale. Spesso, ai problemi di salute mentale, si sommano problemi di salute aspecifici legati al degrado e alla povertà, che gettano i rifugiati in un circolo vizioso di sofferenza continua. Abbiamo già sottolineato che i fattori post-migratori sono fortemente associati al disagio psicologico e al rischio di sviluppare patologie psichiatriche. 
Tabella 2 Il trauma prolungato multidimensionale

\begin{tabular}{|c|c|c|}
\hline $\begin{array}{l}\text { Il trauma } \\
\text { premigratorio: }\end{array}$ & $\begin{array}{l}\text { Il contesto del } \\
\text { Paese di origine }\end{array}$ & $\begin{array}{l}\text { - Violenza correlata con la guerra } \\
\text { - Disastri ambientali, carestie, epidemie } \\
\text { - Violenze, minacce, terrore } \\
\text { - Persecuzione, abusi sessuali } \\
\text { - Reclusioni forzate, tortura } \\
\text { - Deprivazioni e costrizioni } \\
\text { - Testimonianza di violenze } \\
\text { - Scomparsa o morte di persone care } \\
\text { - Perdita di affetti, posizione economica e ruolo sociale } \\
\text { - Insicurezza, sospettosità, paura } \\
\text { - Fuga }\end{array}$ \\
\hline $\begin{array}{l}\text { Il trauma } \\
\text { migratorio: }\end{array}$ & La fuga e il viaggio & $\begin{array}{l}\text { - Partenza forzata, improvvisa, con impossibilità } \\
\text { di avvisare le persone care } \\
\text { - } \quad \text { Permanenza prolungata in campi profughi } \\
\text { - } \quad \text { Viaggi drammatici, malnutrizione, malattie non curate, } \\
\text { aggressione, talvolta morte dei compagni di viaggi } \\
\text { - } \quad \text { Sfruttamento e violenze, anche sessuali } \\
\text { - } \\
\text { - }\end{array}$ \\
\hline \multirow[t]{3}{*}{$\begin{array}{l}\text { Il trauma } \\
\text { postmigratorio: }\end{array}$} & $\begin{array}{l}\text { Politiche } \\
\text { migratorie e fattori } \\
\text { di rischio per la } \\
\text { salute mentale }\end{array}$ & 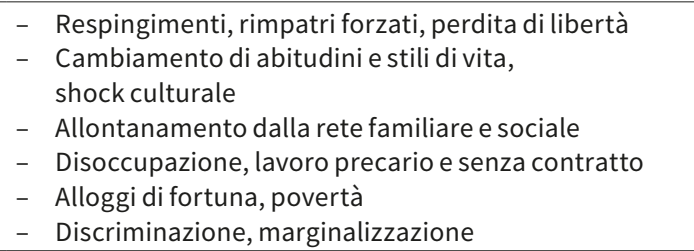 \\
\hline & $\begin{array}{l}\text { Diritto negato } \\
\text { o ritardo } \\
\text { nell'accesso } \\
\text { ai Servizi Sanitari }\end{array}$ & $\begin{array}{l}\text { - Barriere all'accessibilità alle informazioni e ai diritti } \\
\text { - } \text { Barriere all'accessibilità e fruibilità dei Servizi } \\
\text { - } \\
\text { - }\end{array}$ \\
\hline & $\begin{array}{l}\text { Le procedure } \\
\text { d'asilo: } \\
\text { diritto negato } \\
\text { o riconosciuto } \\
\text { tardivamente }\end{array}$ & $\begin{array}{l}\text { - } \text { Respingimenti, rimpatri } \\
\text { - Politiche migratorie, ostacoli nell'accesso } \\
\text { - } \text { Complessità della procedura, durata eccessiva } \\
\text { - Esito incerto } \\
\text { - }\end{array}$ \\
\hline
\end{tabular}

È inoltre utile soffermarsi su un altro aspetto legato al trauma, che appare particolarmente rilevante nel lavoro con i rifugiati. Come già evidenziato, la relazione terapeutica si articola molto spesso sul racconto delle storie migratorie, che possono diventare un lungo e drammatico elenco di eventi traumatici. Ma nel lavoro di presa in carico della sofferenza di queste persone la domanda che ci dobbiamo porre dovrebbe essere quale possa essere il significato da dare a questo tipo di traumi e come vadano collocati nel continuum emotivo e della memoria di queste persone. Come ha sottolineato Sabatini Scalmati in un suo lavoro di alcuni anni fa, la sensazione è che 
tali eventi traumatici abbiano fatto irruzione nella psiche, causando nella mente la formazione di aree scisse, di aree fissate al trauma, simili agli stigmi e alle cicatrici di ferite concrete che si possono osservare sul corpo di queste persone. I traumi di cui stiamo parlando, infatti, sembrano aver provocato

un'improvvisa modificazione dello status quo, strutturando nella psiche un nucleo scisso, fissato all'evento a cui l'Io reagisce con un contro-investimento più o meno massiccio, che lo indebolisce e gli sottrae materiale rappresentativo ed affettivo. La pressione che si determina in questo campo di forze genera un'alterazione della funzione e della struttura della memoria che, per così dire, si congela attorno ai fatti traumatici per poi irradiare il suo cono di ombra sullo spazio/tempo precedente gli eventi lesivi. L'esperienza dell'orrore marchia lo psichismo ed altera il lavoro della memoria. (Sabatini Scalmati 2000, 177)

La memoria delle violenze di cui si è stati vittime e testimoni sembra incistarsi all'interno del polo di fissazione che si è coagulato attorno alla brutalità di quelle esperienze. La mente non è in grado di integrare, legare ed elaborare le reazioni emotive ed affettive che tali traumi suscitano e di conseguenza tali reazioni rimangono in qualche maniera congelate. Escono, quindi, dal normale flusso della memoria, non possono essere ri-trascritte e categorizzate con delle finalità adattative. Non entrano nelle comuni reti associative, non possono venire integrate, né integrare altri fatti che possano ampliare gli aspetti esperienziali, rinnovandoli e creando dei nuovi significati. La sensazione che ne deriva è che questi racconti traumatici sembrano non frutto di memoria, quindi associati ad un periodo del passato, ma che siano quasi ripresentazioni allucinatorie e che, perciò, vengano rivissuti come eventi nuovamente in atto. In questi racconti si ha la sensazione che il passato si sovrapponga al presente, e il presente sia percepito identico a quanto accaduto nel passato. L'attenzione alla memoria muta, cambia e si trasforma diventando una sorta di memoria patologica, fissa e rigida. Questa memoria patologica ha un potente effetto di azzeramento anche sul racconto del passato, che sembra essere senza affetti o legami di valore o senso. Gli aspetti emotivi della vita pre-traumatica sembrano scomparire e ridursi a risposte frettolose e impersonali. Il racconto di anni di vita è sintetizzato in poche frasi, come se in realtà non fosse possibile accedere ad alcun ricordo, come se quelle aree congelate avessero creato intorno il vuoto ed il vissuto traumatico abbia creato un deserto emotivo (Nosè 2016).

Queste considerazioni sembrano assumere una particolare importanza nel momento in cui viene richiesto un intervento terapeutico e una presa in carico. In un ascolto attento, empatico e rispettoso, as- 
sume fondamentale importanza per il rifugiato la possibilità di raccontare i traumi subiti, la propria storia di migrazione e l'arrivo nel Paese ospitante (Crosby 2013). L'essere ascoltati diventa un mezzo per restituire dignità alle persone che attraversano questi percorsi di vita e permette l'emergere di una sintomatologia psichica che deve essere valutata per poter ricevere, poi, l’aiuto adeguato.

\section{Interventi psico-sociali e presa in carico psicologica per i richiedenti asilo e rifugiati}

In generale, da quanto emerge dalla letteratura internazionale, esistono vari tipi di interventi psicosociali con evidenze scientifiche di efficacia nel PTSD nella popolazione dei rifugiati, ma quale sia l'intervento terapeutico più indicato nei rifugiati e richiedenti asilo con disturbi psichiatrici correlati al trauma è ancora oggetto di dibattito.

Una delle questioni più discusse è se i trattamenti raccomandati per il PTSD, e per gli altri disturbi psichiatrici, nella popolazione generale possano essere ugualmente applicati ai rifugiati (Silove 2012). Le linee guida per il trattamento del PTSD negli adulti consigliano vari tipi di interventi psicoterapeutici quali: trauma-focused Cognitive Behaviour Therapy (CBT) individuale o di gruppo, EMDR e stress management. Indicano, inoltre, che i farmaci antidepressivi ed ipnotici non andrebbero usati in prima linea ma solo nel momento in cui le psicoterapie risultino inefficaci o siano presenti concomitanti sintomi depressivi o insonnia (Tol et al. 2013; Shalev, Liberzon, Marmar 2017).

Esistono numerose linee guida internazionali sull'argomento e in tutte viene sottolineata l'importanza di una presa in carico articolata, con interventi individualizzati in base alla gravità e persistenza della patologia e alla tipologia di pazienti, e di una precocità per il rischio delle complicanze che si potrebbero instaurare a lungo termine. Il trattamento del PTSD viene considerato prioritario su altri quadri (ad esempio quello depressivo) che potrebbero appunto essere secondari ad esso. Le linee guida NICE raccomandano nei casi meno gravi e di recente insorgenza un'iniziale osservazione, con follow-up entro un mese. Nei casi più gravi o che non vanno incontro a risoluzione spontanea si consiglia innanzitutto psicoterapia di tipo cognitivo comportamentale, la TF-CBT (CBT centrata sul trauma) con un numero di sedute che dipende dalla cronicità del quadro. Se questa non dovesse risultare efficace si consiglia di prendere in considerazione un altro tipo di trattamento psicologico comunque centrato sul trauma, o l'augmentation con la farmacoterapia. Le linee guida dell'APA consigliano di valutare lo specifico paziente per scegliere il trattamento più adeguato, sottolineando l'importanza di interventi di tipo supportivo, la psicoeducazione e il case manage- 
ment. In generale, tutte concordano sull'utilità della farmacoterapia non in maniera preventiva ma in tutti quei casi che non presentano una risoluzione del quadro in breve tempo, o che presentino una sintomatologia invalidante.

Rimane aperta la questione relativa alla possibilità e opportunità di applicare interventi studiati su persone che soffrono di PTSD correlato ad un unico evento traumatico, ad una popolazione peculiare come quella dei rifugiati, vista la sua complessità e la multidimensionalità dei suoi bisogni. A tale proposito in varie linee guida viene sottolineata l'importanza di un'attenzione agli aspetti culturali sia per quanto concerne la sintomatologia che il trattamento. Nelle linee guida NICE viene, per esempio, consigliato specificatamente uno screening della popolazione dei rifugiati e dei richiedenti asilo, data l'elevata prevalenza del PTSD.

Rispetto al dibattito sugli interventi terapeutici nei richiedenti asilo con PTSD, è necessario segnalare che i pochi studi presenti in letteratura sono caratterizzati, anche in questo caso, da un'ampia variabilità dal punto di vista metodologico, del tipo di intervento e della popolazione presa in esame (McFarlane, Kaplan 2012). Esistono diverse revisioni della letteratura che hanno indagato principalmente l'efficacia di interventi psicosociali nella popolazione di rifugiati che soffrono di disagio psichico in generale e di PTSD in particolare.

In una delle prime revisioni sistematiche sui trial clinici randomizzati (RCT) riguardanti il trattamento del PTSD nei rifugiati e richiedenti asilo condotta nel 2010 (Crumlish, O’Rourke 2010), gli autori sottolineano che nessuno degli interventi psicologici considerati nei 10 trial inclusi presenta solide basi scientifiche di efficacia nel ridurre la sintomatologia del PTSD, pur sottolineando risultati favorevoli soprattutto per la Narrative Exposure Therapy (NET) e per alcuni interventi basati sulla Cognitive Behaviour Therapy (CBT). In questa revisione non vi sono studi che analizzino degli approcci integrati o multidisciplinari. Negli ultimi anni, ulteriori revisioni della letteratura hanno aumentato le conoscenze in questo ambito. McFarlane e Kaplan hanno pubblicato nel 2012 una revisione sistematica sugli interventi psicologici per gli adulti sopravvissuti a torture e traumi. Vengono considerati 40 studi effettuati negli ultimi 30 anni, di cui 11 RCT, 8 studi di confronto non randomizzati e 21 studi di coorte. I risultati vengono presentati suddivisi per tipologia di studio considerato e in secondo luogo per popolazione destinataria dell'intervento (rifugiati, richiedenti asilo, sfollati, e persone che hanno subito persecuzione e gravi violazioni dei diritti umani nel Paese d'origine). Anche questo lavoro prende in considerazione una varietà di differenti approcci terapeutico-riabilitativi, e rileva che, al di là dell'elevata variabilità, nella maggior parte degli studi emergono significativi miglioramenti negli outcome considerati (anch'essi differenti nei vari studi: principalmente riduzione dei sintomi del PTSD o depressivi, ma anche so- 
matici, ansiosi e miglioramento del funzionamento). Interessante notare gli effetti a lungo termine che vengono generalmente mantenuti nei 3-18 mesi successivi, ma che si affievoliscono nei 2-3 anni a seguire, probabilmente in ragione della persistenza del trauma post-migratorio, con le insicurezze ad esso connesse (McFarlane, Kaplan 2012).

Nel 2014 è stata pubblicata una revisione della Cochrane (Patel, Kellezi, Williams 2014), che valuta gli RCT relativi ai trattamenti per le vittime di tortura con disturbi psicologici. Non è diretta specificatamente al trattamento del PTSD, ma, di fatto, buona parte dei lavori in essa analizzati si occupa di popolazioni con questo disturbo. Emerge che i 9 RCT inclusi riguardano esclusivamente trattamenti psicologici, fra cui: 4 utilizzano la NET, uno la CBT e gli altri 4 usano tecniche miste per la sintomatologia traumatica. Gli autori sottolineano la bassa qualità degli studi e la difficile generalizzazione alla luce di interventi molto differenti fra loro. Non vengono rilevati benefici immediati agli outcome primari (distress-depressione, sintomi o casi di PTSD, qualità della vita). In alcuni casi si rileva un moderato beneficio rispetto ai controlli al follow-up in tutti gli outcome considerati. Gli autori concludono sottolineando la difficoltà di generalizzare tali risultati e la necessità di ulteriori studi in tal senso. Più recentemente una revisione sistematica e meta-analisi (Nosè et al. 2017) si è focalizzata sugli interventi psicosociali nella popolazione di richiedenti asilo con PTSD accolti nei Paesi ad alto reddito. Sono stati inclusi 12 studi nella meta-analisi, in cui si è evidenziata una sostanziale efficacia nel ridurre la sintomatologia del PTSD rispetto al gruppo di controllo. L'intervento con maggiori evidenze scientifiche è risultato essere la NET, nonostante gli autori abbiano confermato la bassa qualità metodologica degli studi. Tali dati sono stati confermati anche in un'altra revisione (Turrini el al. 2019) che prendeva in considerazione anche gli studi fatti sulla popolazione dei rifugiati accolti nei Paesi in via di sviluppo.

Di seguito una breve descrizione dei principali trattamenti utilizzati nel PTSD:

\section{- Cognitive Behaviour Therapy (CBT)}

La CBT è un'ampia categoria di terapie basate sui principi dell'apprendimento, del condizionamento e su teorie cognitive, sviluppata per trattare i disturbi psicologici, e include componenti sia della terapia cognitiva che comportamentale. Nella CBT, componenti come l'esposizione, la ristrutturazione cognitiva e varie strategie di coping vengono utilizzate da sole o in associazione (Cusack et al. 2016).

Per il trattamento delle condizioni post-traumatiche, la CBT si serve principalmente di alcune delle sue componenti come l'esposizione ripetuta e la ristrutturazione cognitiva. Nel trattamento del PTSD sono stati sviluppati degli approcci più specifici come la CBT centrata sul trauma (TF-CBT): tale intervento è strutturato e orientato al- 
la comprensione e al cambiamento dei pensieri disfunzionali che determinano le emozioni negative, sintomi somatici e comportamenti non adattivi. Il modello teorico cognitivo di Ehlers e Clark (2000) riporta che la reazione post-traumatica perdura quando gli individui percepiscono l'evento traumatico come una minaccia grave ed imminente. Questa percezione è la conseguenza di un'interpretazione negativamente eccessiva del trauma e di una mancata elaborazione ed integrazione nella memoria autobiografica. Nel caso specifico del TF-CBT l'enfasi è posta sull'esposizione immaginativa o in vivo allo stimolo, associato ad una ristrutturazione cognitiva della percezione dell'evento e dell'impatto nella vita della persona.

\section{- Narrative Exposure Therapy (NET)}

La NET è un trattamento breve per la cura dei disturbi trauma-correlati, descritto come una variante standardizzata e manualizzata della Testimony Therapy, ed include diversi elementi che sono stati adattati dalla CBT (Neuner et al. 2002). Inizialmente sviluppato per l'utilizzo in Paesi a basso reddito, attualmente è utilizzato per il trattamento di rifugiati e richiedenti asilo in contesti ad alto reddito. La NET si basa sulla teoria della rappresentazione duplice dei ricordi traumatici (Schauer, Neuner, Elbert 2005), con un focus sulla concettualizzazione di elementi che sono associati al network della paura di matrice sensoriale, affettiva e cognitiva. Tali associazioni dovrebbero facilitare l'elaborazione delle memorie traumatiche nella cornice della vita dell'individuo. Il paziente nella NET costruisce, con l'aiuto del terapeuta, una narrazione ordinata cronologicamente che ha l'obiettivo di lavorare sulle esperienze traumatiche dove i racconti frammentati di tali eventi si trasformano in una narrazione coerente. La comprensione empatica, l'ascolto attivo, la congruenza sono componenti chiave del comportamento del terapeuta. Per quanto riguarda le esperienze traumatiche, il terapeuta chiede un resoconto dettagliato circa le emozioni, le cognizioni e le sensazioni, nonché le risposte fisiologiche, e cerca di elicitare osservazioni reciproche su tali vissuti. Il paziente è incoraggiato a far emergere queste emozioni mentre racconta senza perdere il contatto con il presente: usando aspetti rievocanti il trauma sia emotivi sia fisiologici, il paziente collega le esperienze a fatti episodici, come il tempo e lo spazio. In questo modo si facilita la rielaborazione, la costruzione del significato e l'integrazione dell'evento traumatico.

\section{- Eye Movement Desensitization and Reprocessing (EMDR)}

L'EMDR è nata come terapia elettiva del PTSD e ha modellato la struttura fondante della propria procedura di intervento (nonché le proprie basi teoriche) su questa patologia. È generalmente considerata 
come una combinazione di elementi della CBT, della terapia di esposizione e una specifica attenzione ai movimenti oculari.

La procedura-tipo o algoritmo di intervento consta di otto fasi specifiche, composte di elementi 'non specifici' (relazione terapeutica, elementi psico-educazionali, ecc.), di elementi genuinamente specifici (movimenti oculari o altri tipi di stimolazione adeguata, sequenzialità degli interventi effettuati) ed elementi mutuati da altre tradizioni di ricerca (assessment cognitivo e ristrutturazione, esposizione graduale, abreazione, assecondamento delle libere associazioni, valorizzazione di immagini a contenuto simbolico, manipolazione delle immagini mentali, tecniche provenienti dalla tradizione della terapia ipnotica ed altro ancora).

La fase della desensibilizzazione consiste nell'applicazione della stimolazione alternata più adeguata al paziente che ha avuto accesso al materiale traumatico. All'interno di questa fase si deve includere ogni forma di risposta che il paziente esibisce nei confronti dell'intervento; il compito del terapeuta è di stimolare la rielaborazione spontanea del trauma. Quando il coinvolgimento emotivo è notevolmente ridotto o eliminato si interviene agevolando la ristrutturazione cognitiva delle cognizioni disfunzionali (o inadeguate rispetto al tempo presente) che usualmente sono associate ad un trauma. Attraverso la stimolazione alternata si intende integrare il materiale mnestico originario con nuove informazioni che precedentemente erano inaccessibili emozionalmente e/o cognitivamente (Lipke 2000). Il protocollo standard di intervento per il PTSD prevede, inoltre, che sia clinicamente necessario rielaborare gli eventi traumatici passati, ma intervenire anche sulle situazioni contingenti attuali che fungono da triggers nei confronti degli eventi traumatici e, infine, sulle ipotetiche situazioni future che si presumano possano riattivare i comportamenti disfunzionali connessi alle originarie esperienze traumatiche. Di conseguenza, è teoreticamente corretto e clinicamente efficace considerare come target d'intervento non tanto il singolo evento che il paziente riferisce come traumatico, quanto piuttosto il ricordo in questione all'interno di una specifica rete mnestica, con connessioni al passato, al presente ed al futuro (Giannantonio 2001).

Per quanto riguarda la realtà italiana, sono state pubblicate recentemente sulla Gazzetta Ufficiale le «Linee guida per la programmazione degli interventi di assistenza e riabilitazione nonché per il trattamento dei disturbi psichici dei titolari dello status di rifugiato e dello status di protezione sussidiaria che hanno subito torture, stupri o altre forme gravi di violenza psicologica, fisica o sessuale» (Ministero della Salute 2017). Questo documento appare di notevole importanza per vari aspetti. Prima di tutto pone l'attenzione su una problematica grave e urgente nel nostro Paese. Propone, inoltre, una linea di intervento chiara e sistematica che fa riferimento non solo alle evidenze di 
letteratura, ma anche alla realtà territoriale. Infine fornisce delle indicazioni per diverse professionalità per poter essere implementate in diversi ambiti. Gli interventi consigliati in questo documento sono, infatti, strutturati secondo un modello a più livelli: in prima istanza coinvolgono il personale non sanitario delle strutture di accoglienza, salendo di livello aumenta il grado di specializzazione del personale e dei servizi interessati. Gli interventi riguardano l'individuazione precoce, la prevenzione e il trattamento delle problematiche psicopatologiche. Tutti gli interventi indicati dovrebbero essere messi in atto da team multidisciplinari che applichino un approccio il più possibile olistico e transculturale. Vengono fornite, inoltre, indicazioni in merito a come produrre la certificazione medica, all'importanza degli interventi di mediazione culturale, alla tutela della salute degli operatori coinvolti e alla loro formazione. Le linee guida sottolineano che l'identificazione delle vittime di tortura è già di base complessa per le difficoltà del racconto delle esperienze passate e che:

a tale difficoltà bisogna aggiungere le diversità linguistiche e culturali: esprimere pensieri, sensazioni, ricordi, già di per sé difficili da elaborare, in una lingua diversa dalla propria aggrava il disagio delle vittime. Il ricorso ad una visita medica specialistica con clinico esperto in ambito di cura psicofisica delle vittime di tortura, alla presenza di un mediatore culturale adeguatamente formato, risulta spesso indispensabile. L'attenzione rivolta a far emergere queste sofferenze, spesso trascurate o tenute nascoste, si deve tradurre in interventi integrati medico-psicologici e quindi in valutazioni e procedure specifiche in campo giuridico. Fondamentale la possibilità di intervento in tempi adeguatamente brevi e la predisposizione di strutture di prima accoglienza dotate di personale formato, anche in materia di educazione ai diritti umani, in grado di individuare eventuali segnali di vissuti traumatici. (Ministero della Salute 2017, 30)

Per concludere, possiamo sottolineare che esistono interventi specifici per i richiedenti asilo e i rifugiati che manifestano un disagio psicologico o un disturbo psichiatrico, in particolare modo il PTSD. Tuttavia, come assunto di base, per delle persone con delle ferite così gravi e profonde, appare sostanziale un'atmosfera di fiducia e di affidabilità degli operatori, gli individui devono sentirsi accolti, accettati e compresi, liberi di poter parlare dei sintomi e del malessere senza il rischio di interpretazioni o paragoni. L'effetto di una relazione operatore-utente aiuta in ogni momento la ricostruzione dell'identità lesa e l'atmosfera di fiducia, l'empatia e l'affidabilità del terapeuta e dell'operatore permettono alla vittima di elaborare il proprio vissuto, integrarlo costruttivamente nella propria identità e superare il danno (Santone 2010). Essere vittima di un evento psico-traumatico 
comporta l'attivazione del sistema motivazionale dell'attaccamento; un attaccamento sicuro rappresenta già un primo atto terapeutico (Santone 2010). Tale aspetto diventa l'elemento cardine di qualunque intervento terapeutico, al di là della specificità dello stesso.

\section{Conclusioni}

La salute mentale dei rifugiati, e più in generale delle persone vittime di tortura, rappresenta una realtà da affrontare con soluzioni strutturali, non più basate sull'emergenza, che tenga in considerazione la cultura, la storia migratoria e gli eventi traumatici a cui questa popolazione è sottoposta. Questa popolazione ha un'alta frequenza di sofferenza psicologica e un'elevata possibilità che tale sofferenza si trasformi in disturbi psichici trauma correlati. Il sistema di accoglienza non può prescindere, quindi, dalla necessità di una valutazione precoce dello stato psicologico dell'individuo, all'interno di un modello integrato con la presenza di equipe multidisciplinari, che prendano in considerazione tutte le dimensioni coinvolte: sociosanitaria, legale, economica.

Un'adeguata presa in carico del disagio psicologico e un intervento precoce ed efficace dei disturbi psichici correlati agli aspetti traumatici sono un diritto e un dovere nei confronti di questa popolazione, anche per permettere una migliore integrazione lavorativa e sociale e di funzionamento generale nel Paese.

\section{Bibliografia}

APA, American Psychiatric Association (2014). DSM-5: Manuale diagnostico e statistico dei disturbi mentali. Milano: Raffaello Cortina.

Bogic, Marija; Njoku, Anthony; Priebe, Stefan (2015). «Long-Term Mental Health of War-Refugees: a Systematic Literature Review». BMC International Health and Human Rights, 15, 29. DOI https://doi.org/10.1186/s12914-015๑๑64-9.

Crepet, Anna et al. (2017). «Mental Health and Trauma in Asylum Seekers Landing in Sicily in 2015: A Descriptive Study of Neglected Invisible Wounds». Conflict and Health, 11, 1. DOI https://doi.org/10.1186/s13031-0170103-3.

Crosby, Sondra (2013). «Primary Care Management of Non-English Speaking Refugees Who Have Experienced Trauma». JAMA, 310(5), 519-28. DOI https://doi.org/10.1001/jama.2013.8788.

Crumlish, Niall; O'Rourke, Kevin (2010). «A Systematic Review of Treatments for Post-Traumatic Stress Disorder Among Refugees and Asylum Seekers». The Journal of Nervous and Mental Disease, 198(4), 237-51. DOI https://

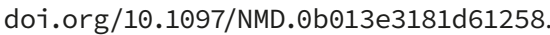

Cusack, Karen et al. (2016). «Psychological Treatments for Adults with PostTraumatic Stress Disorder: A Systematic Review and Meta-Analysis». Clin- 
ical Psychology Review, 43, 128-41. DOI https://doi.org/10.1016/j. cpr.2015.10.003.

Ehlers, Anke; Clark, David (2000). «A Cognitive Model of Posttraumatic Stress Disorder». Behavioural Research and Therapy, 38(4), 319-45. DOI https://

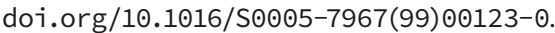

Fazel, Mina; Wheeler, Jeremy; Danesh, John (2005). «Prevalence of Serious Mental Disorder in 7000 Refugees Resettled in Western Countries: A Systematic Review». The Lancet, 365(9467), 1309-14.

Firenze, Alberto et al. (2016). «The Occurrence of Diseases and Related Factors in a Center for Asylum Seekers in Italy». Slovenian Journal of Public Health, 55(1), 21-28. DOI https://doi.org/10.1515/sjph-2016-๑๑๑3.

Giannantonio, Michele (2001). «Eye Movement Desensitization and Reprocessing (EMDR) e psicoterapia del Disturbo Post-traumatico da Stress: considerazioni critiche e linee di tendenza». Rivista di Psicoterapia Cognitiva e Comportamentale, 1, 5-23.

Hollifield Michael et al. (2002). «Measuring Trauma and Health Status in Refugees: A Critical Review». JAMA, 288(5), 611-21. DOI https://doi. org/10.1001/jama.288.5.611.

Klarić, Miro et al. (2007). «Psychological Consequences of War Trauma and Postwar Social Stressors in Women in Bosnia and Herzegovina». Croatian Medical Journal, 48(2), 167-76. URL https://www.ncbi.nlm.nih.gov/ pmc/articles/PMC2080525/ (2019-10-15).

Li, Susan; Liddell, Belinda; Nickerson, Angela (2016). «The Relationship Between Post-Migration Stress and Psychological Disorders in Refugees and Asylum Seekers». Current Psychiatry Reports, 18(9). DOI https://doi. org/10.1007/s11920-๑16-@723-๑.

Lipke, Howard (2000). EMDR and Psychoterapy Integration. Theoretical and Clinical Suggestions with Focus on Traumatic Stress. Boca Raton: CRC Press.

McFarlane, Colleen; Kaplan, Ida (2012). «Evidence-Based Psychological Interventions for Adult Survivors of Torture and Trauma: A 30-Year Review». Transcultural Psychiatry, 49(3-4), 539-67. DOI https://doi. org/10.1177/1363461512447608.

Ministero della Salute (2017). «Linee guida per la programmazione degli interventi di assistenza e riabilitazione nonché per il trattamento dei disturbi psichici dei titolari dello status di rifugiato e dello status di protezione sussidiaria che hanno subito torture, stupri o altre forme gravi di violenza psicologica, fisica o sessuale». Gazzetta Ufficiale della Repubblica Italiana. URL http://www.salute.gov.it/imgs/C_17_pubblicazioni_2599_ allegato.pdf (2019-10-15).

Mollica, Richard et al. (1998). «Assessing Symptom Change in Southeast Asian Refugee Survivors of Mass Violence and Torture». The American Journal of Psychiatry, 147, 83-8.

Müller, Matthias et al. (2018). «The Negative Impact of an Uncertain Residence Status: Analysis of Migration-Related Stressors in Outpatients with Turkish Migration Background and Psychiatric Disorders in Germany Over a 10-Year Period». J Immigr Minor Health, 20(2), 317-26.

Neuner, Frank et al. (2002). «A Narrative Exposure Treatment as Intervention in a Refugee Camp: A Case Report». Behavioural and Cognitive Psychotherapy, 30, 211-15. URL http://nbn-resolving.de/urn:nbn:de:bsz:352opus-43573 (2019-10-20). 
Norris, Fran; Alegria, Margarita (2005). «Mental Health Care for Ethnic Minority Individuals and Communities in the Aftermath of Disasters and Mass Violence». CNS spectrums, 10(2), 132-40. DOI https://doi.org/10.1017/ S1092852900019477.

Nosè, Michela (2016). «Racconti e percorsi in migrazione». Sul bordo di una cascata. Trasformazioni nel mondo e nella psicoanalisi. Pisa: ETS, 253-22.

Nosè, Michela et al. (2017). "Psychosocial Interventions for Post-Traumatic Stress Disorder in Refugees and Asylum Seekers Resettled in High-Income Countries: Systematic Review and Meta-Analysis». PLOS ONE, 12(2). DOI https://doi.org/10.1371/journal.pone.0171030.

Nosè, Michela et al. (2018). «Prevalence and Correlates of Psychological Distress and Psychiatric Disorders in Asylum Seekers and Refugees Resettled in an Italian Catchment Area». Journal of Immigrant and Minority Health, 20(2), 263-70. DOI https://doi.org/10.1007/s10903-017-0629-x.

Palic, Sabina et al. (2014). «Comparison of Psychiatric Disability on the Health of Nation Outcome Scales (HoNOS) in Resettled Traumatized Refugee Outpatients and Danish Inpatients». BMC Psychiatry, 14, 330. DOI https:// doi.org/10.1186/s12888-014-0330-8.

Patel, Nimisha; Kellezi, Blerina; Williams, Amanda (2014). «Psychological, Social and Welfare Interventions for Psychological Health and Well-Being of Torture Survivors». The Cochrane Database of Systematic Reviews, 11. DOI https://doi.org/10.1002/14651858.CD009317.pub2.

Porter, Matthew; Haslam, Nick (2005). «Predisplacement and Postdisplacement Factors Associated With Mental Health of Refugees and Internally Displaced Persons: A Meta-Analysis». JAMA, 294(5), 602-12.

Priebe, Stefan; Giacco, Domenico; El-Nagib, Rawda (2016). Public Health Aspects of Mental Health Among Migrants and Refugees: A Review of the Evidence on Mental Health Care for Refugees, Asylum Seekers and Irregular Migrants in the Who European Region. Copenhagen: WHO Regional Office for Europe. Health Evidence Network synthesis report 47. URL http://www. euro.who.int/__data/assets/pdf_file/0003/317622/HEN-synthesis-report-47.pdf (2019-10-15).

Sabatini Scalmati, Anna (1999). «Memorie congelate, memorie evitate: a proposito della relazione terapeutica con le vittime di torture». Richard \& Piggle. Studi psicanalitici del bambino e dell'adolescente, 8(2), 172-80.

Santone, Giancarlo (2010). «La salute mentale dei rifugiati. Una visione di insieme del fenomeno». Caldarozzi, Alessandra (a cura di), Le dimensioni del disagio mentale nei richiedenti asilo e rifugiati. Nepi: Varigrafica Altolazio, 13-74. URL https://www.sprar.it/wp-content/uploads/2016/06/Le_dimensioni_del_disagio_mentale_-_Cittalia_2010.pdf (2019-10-15).

Schauer, Maggie; Neuner, Frank; Elbert, Thomas (2005) Narrative Exposure Therapy: A Short-Term Intervention for Traumatic Stress Disorders after War, Terror, or Torture. Ashland: Hogrefe \& Huber.

Schock, Katrin et al. (2016). «Impact of New Traumatic or Stressful Life Events on Pre-Existing PTSD in Traumatized Refugees: Results of a Longitudinal Study». European Journal of Psychotraumatology, 7(1). DOI https://doi. org/10.3402/ejpt.v7.32106.

Shalev, Arieh; Liberzon, Israel; Marmar, Charles (2017). «Post-Traumatic Stress Disorder». New England Journal of Medicine, 376(25), 2459-69. DOI https:// doi.org/10.1056/NEJMra1612499. 
Silove, Derrick (2004). «The Challenges Facing Mental Health Programs for PostConflict and Refugee Communities». Journal of the World Association for Emergency and Disaster Medicine, 19, 90-6.

Silove, Derrick (2012). «Treatment of Refugees at the Crossroads: The Need for an Evidence Base». Australian and New Zealand Journal of Psychiatry, 46(10), 921-23.

Steel, Zachary et al. (2006). «Impact of Immigration Detention and Temporary Protection on the Mental Health of Refugees». The British Journal of Psychiatry, 188, 58-64. DOI https://doi.org/10.1192/bjp.bp.104.007864.

Steel, Zachary et al. (2009). «Association of Torture and Other Potentially Traumatic Events with Mental Health Outcomes Among Populations Exposed to Mass Conflict and Displacement». JAMA, 302(5), 537-49. DOI https://doi. org/10.1001/jama.2009.1132.

Tay, Alvin et al. (2015). «The structure of Post-Traumatic Stress Disorder and Complex Post-Traumatic Stress Disorder Amongst West Papuan Refugees». BMC Psychiatry, 15, 111. DOI https://doi.org/10.1186/s12888-0150480-3.

Teodorescu, Dinu-Stefan et al. (2015). «Chronic Pain in Multi-Traumatized Outpatients with a Refugee Background Resettled in Norway: a Cross-sectional Study». BMC Psychology, 3, 7. DOI https://doi.org/10.1186/s40359๑15-0064-5.

Tol, Wietse et al. (2013). «Management of Acute Stress, PTSD, and Bereavement: WHO Recommendations». JAMA, 310(5), 477-8. DOI https://doi. org/10.1001/jama.2013.166723.

Turrini, Giulia et al. (2017). «Common Mental Disorders in Asylum Seekers and Refugees: Umbrella Review of Prevalence and Intervention Studies». International Journal of Mental Health Systems, 11, 51. DOI https://doi.

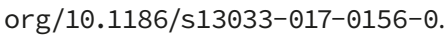

Turrini, Giulia et al. (2019). «Efficacy and Acceptability of Psychosocial Interventions in Asylum Seekers and Refugees: Systematic Review and Meta-Analysis». Epidemiol Psychiatr Sci, 28(4), 376-88. DOI https://doi. org/10.1017/S2045796019000027.

UNHCR (2017). Global Trends. Forced Displacement in 2017. URL https://www. unhcr.org/globaltrends2017/ (2019-10-15).

Van Ommeren, Mark et al. (2001). «Psychiatric disorders among tortured Bhutanese refugees in Nepal». Archives of General Psychiatry, 58, 475-482.

Van Wyk, Sierra et al. (2012). «A Longitudinal Study of Mental Health in Refugees from Burma: The Impact of Therapeutic Interventions». Australian and New Zealand Journal of Psychiatry, 46, 995-1003. 



\title{
Da vittima a testimone: il percorso di rinascita del sopravvissuto alla tortura
}

\author{
Carlo Bracci \\ Associazione Umanitaria Medici contro la Tortura, Roma, Italia \\ Angelo Coppola \\ Associazione Umanitaria Medici contro la Tortura, Roma, Italia
}

\begin{abstract}
Starting from the experience of the Association Doctors against torture, the essay focuses on the path of re-birth, from victim to witness, of the person who has suffered torture.
\end{abstract}

Keywords Torture. Rebirth. Witness. Association Doctors against torture.

Nell'esilio camminai guardando solo il presente. Il futuro mi apparve come un vortice terribilmente sconosciuto.

Giorno dopo giorno camminai incerta trascinandomi il passato.

Scelsi la notte per il passato e il giorno per il presente.

Ma solo nelle effimera ora aurorale

il passato e il presente si unirono per generare in ogni incontro un pezzettino di futuro.

Questi versi sono apposti all'inizio di un romanzo ispirato dalle vicende dell'autrice, figlia di un emigrante italiano in Uruguay, sequestrata da militari per alcuni giorni al tempo della dittatura e poi fuggita in Italia (Milazzo 2012).

L'autrice chiese di incontrarci dopo aver visitato il sito della nostra Associazione Medici contro la tortura. Dopo venti e più anni di vita serena e atti- 
va in Italia, ha subito un trauma apparentemente banale e ha iniziato ad essere invasa da schegge di ricordi terribili; è venuta con una domanda precisa: voi che avete esperienza di persone che sono sopravvissute alla tortura, dovete dirmi se sto impazzendo. La risposta è stata chiara: quello che lei viveva drammaticamente era il riaffiorare di ricordi cancellati e bisognava affrontare i suoi problemi partendo da quelli.

Quello è stato uno degli incontri che l'Associazione Medici contro la tortura, fondata da un gruppo di medici aderenti ad Amnesty International, ha avuto a partire dagli anni Ottanta con cittadini italiani perseguitati dai regimi dittatoriali dell'America Latina.

È un'esperienza che merita di essere ricordata perché si rivolgeva a perseguitati che, in quanto cittadini italiani, avevano pieno diritto a risiedere nel nostro Paese, senza i problemi burocratici che ostacolano oggi il percorso dei richiedenti protezione internazionale; avevano una cultura vicina a quella italiana e sono stati accolti in Italia con grande solidarietà.

Il libro da cui è tratta la poesia è il frutto di un lungo lavoro che la nostra amica ha potuto svolgere con la collaborazione della responsabile culturale di Amnesty International e costituisce un importante momento del suo percorso.

L'autrice ha riallacciato i rapporti con l'emigrazione uruguagia in Europa, è riuscita a tornare a Montevideo a cercare le persone che avevano sofferto con lei. Tutto è proceduto verso l'acquisizione di un nuovo equilibrio, percepibile anche dal suo aspetto; alla presentazione del volume in una grande libreria era quasi irriconoscibile: sembrava più alta, era elegante e sicura di sé, tuttavia il suo percorso di rinascita non si è svolto in modo lineare. In Italia si è svolto il processo contro alcuni militari uruguaiani imputati di violenze e uccisioni di cittadini italiani emigrati nel Paese latino-americano e l'autrice è andata ad assistere a qualche udienza. È stata un'esperienza molto pesante che le ha provocato la riaccensione della condizione psicologica che l'aveva portata a cercare il primo incontro con la nostra Associazione.

Tutta la nostra esperienza dimostra quanto sia lungo e difficile il percorso che noi chiamiamo di rinascita e non di guarigione. Negli anni successivi abbiamo incontrato tante persone diverse: curdi che fuggivano dall'Iraq e dalla Turchia, militanti politici venuti in Italia per chiedere protezione secondo le procedure per il riconoscimento dello status di rifugiato; uomini, donne e minori fuggiti dall'Afghanistan, dal Corno d'Africa, dall'Africa subsahariana. Differenti tra loro per i motivi della persecuzione, cultura, religione, abitudine all'introspezione, e che si trovano a vivere in un Paese, l'Italia, che a volte li accoglie a volte li respinge.

Queste persone hanno la necessità di riferire la propria storia di persecuzione e di violenze subite nel Paese di origine o durante il 
viaggio secondo tempi che non sono quelli dettati dalle regole del percorso di rinascita. Spesso ci troviamo a dover raccogliere storie di sofferenze estreme da persone che in quel momento dovrebbero pensare solo al presente, iniziando a progettare il futuro, ma che invece sono spinte dalla necessità di dimostrare le violenze e le persecuzioni subite nell'audizione della Commissione per il riconoscimento della protezione internazionale.

Il nostro sistema giuridico tiene conto delle particolari condizioni in cui si trova chi richiede la protezione internazionale e stabilisce un indebolimento dell'onere della prova. Significative, a questo proposito, sono le affermazioni della Direttiva Qualifiche e una sentenza della Cassazione: «qualora taluni aspetti delle dichiarazioni del richiedente non siano suffragati da prove documentali o di altro tipo, la loro conferma non è comunque necessaria» purché il richiedente mostri di avere compiuto «sinceri sforzi per circostanziare la domanda», di aver fornito una spiegazione soddisfacente della mancanza di altri elementi o dichiarazioni «ritenute coerenti e plausibili», di essere «in generale attendibile». ${ }^{1}$

Nel procedimento per il riconoscimento della protezione internazionale il richiedente deve provare, quanto meno in via presuntiva, il concreto pericolo cui andrebbe incontro con il rimpatrio, precisando l'effettività e l'attualità di esso. Inoltre il richiedente deve dimostrare di essere credibile, assolvendo al relativo onere probatorio secondo le regole del nostro ordinamento. ${ }^{2}$

In mancanza di documenti, che difficilmente chi fugge riesce a portare con sé, la prova si basa necessariamente su quanto il richiedente protezione internazionale riferisce sulle persecuzioni e sulle violenze subite. Ma l'esperienza ci ha insegnato che i tempi di chi ha subito tortura sono diversi da persona a persona; possono essere brevi ma anche molto lunghi. Il riemergere di ricordi dolorosi rende il percorso discontinuo, fatto di passi in avanti e di passi indietro; essere costretti a non osservare i tempi imposti dalle condizioni del rifugiato rende difficile il lavoro di accompagnamento verso una nuova vita.

Se il principale elemento, se non l'unico, di prova è la testimonianza del sopravvissuto alla tortura, dobbiamo chiederci quali sono i fattori che rendono difficile il racconto delle persecuzioni subite; è utile, ad esempio, che esse siano certificate dai professionisti che accompagnano il richiedente protezione internazionale. Queste difficoltà, legate alla condizione del richiedente protezione internazionale, possono essere così indicate schematicamente:

1 Direttiva Qualifiche 2004/83/CE art. 4 § 5, recepita in Italia con d.lgs. 251/2007 (art. 3 §) e successive modificazioni.

2 Cassazione civile a sezioni unite, sentenza del 17 novembre 2008, nr. 27310. 
mancanza di fiducia, vergogna, paura di non essere creduti, memoria traumatica, ritraumatizzazione, frammentazione del pensiero, crisi di depersonalizzazione.

Un'importante illustrazione di questi quadri clinici e delle loro basi neurofisiologiche la troviamo nel volume Il Corpo accusa il colpo di Bessel van der Kolk, figlio di un deportato olandese nei campi nazisti, approdato negli Stati Uniti dove, come psicologo, ha incontrato generazioni di gravi traumatizzati a partire dai reduci della guerra in Vietnam (van der Kolk 2015).

Le condizioni di chi ha subito tortura sono rilevate non solo da medici e psicologi ma anche dagli operatori sociali dei centri di accoglienza e dagli insegnanti di italiano, dagli operatori giuridici che curano la preparazione al colloquio presso la Commissione; costoro possono osservare comportamenti anomali quali cambiamenti di umore, chiusura in sé stessi, difficoltà nello studio dell'italiano, disturbi del sonno.

Dalla nostra esperienza deriva un'indicazione importante per chi segue il percorso di cura dei sopravvissuti alla tortura: la necessità di un lavoro in comune di tutti coloro che interagiscono con i sopravvissuti alla tortura (accertati o presunti) con un continuo scambio di esperienze ed osservazioni.

Per chi deve certificare gli esiti delle violenze subite fornendo al richiedente protezione una documentazione utile a sostenere la sua domanda, è importante un'analisi dei pareri delle Commissioni e, per i casi ricorrenti in giudizio, delle sentenze della magistratura. ${ }^{3}$

Dobbiamo infine segnalare che le normative più recenti hanno reso particolarmente difficile il nostro lavoro.

Le modifiche introdotte dal d.1. 13/2017 (il cosiddetto decreto Minniti-Orlando) - che ha eliminato il secondo grado di giudizio e che ha dato al giudice la possibilità di esprimere il proprio giudizio sulla base degli atti raccolti dalle Commissioni per il riconoscimento della protezione internazionale senza interrogare il richiedente asilo e senza acquisire eventuale nuova documentazione - colpiscono soprattutto i più fragili, ovvero coloro che, come abbiamo visto, hanno difficoltà a ricostruire con la coerenza richiesta la propria storia traumatica.

Il recente d.l. 113/2018 (Sicurezza e immigrazione) rende più difficile il percorso soprattutto delle persone più fragili, quelle che non sono capaci di fornire alla Commissione racconti coerenti. La limitazione dell'accoglienza negli SPRAR a coloro che sono titolari di protezione internazionale priva i richiedenti asilo della possibilità di

3 Documentazione in questo senso è reperibile nei siti di Meltingpot, ASGI, Ciac di Parma https://www.meltingpot.org; https://www.asgi.it; http://www.ciaconlus. org (2019-10-16). 
avere un'assistenza adeguata, che non viene fornita nei Centri di accoglienza straordinaria - come illustrato da una indagine sui bandi per l'assegnazione dei CAS che ne evidenzia la carenza delle risorse a disposizione. ${ }^{4}$

Si apre quindi in questi mesi una fase nuova e difficile, con la consapevolezza che è in gioco non solo il destino degli assistiti ma anche il patrimonio culturale e giuridico di garanzie previste dalla Costituzione italiana.

\section{Bibliografia}

Milazzo, Anna (2012). Anahí del mare: la dittatura in Uruguay, la notte di un popolo. Formigine: Infinito editore.

van der Kolk, Bessel (2015). Il corpo accusa il colpo. Mente, corpo e cervello nell'elaborazione delle memorie traumatiche. Milano: Cortina. 


Forma estrema di rapporto sociale di sottomissione, la tortura è ancora diffusa ovunque e ciò si deve anche a molteplici processi tipici dell'era neo-liberista, a partire dalle politiche di blindatura securitaria della società. II volume indaga il fenomeno della tortura nei confronti degli immigrati focalizzandosi sulla situazione in diversi paesi, sugli aspetti relativi alla salute, e mettendone in luce il legame con il peggioramento delle condizioni della migrazione e la guerra agli immigrati.

As an extreme form of social relationship of submission, torture is still an ongoing and widespread practice; this is also due to several processes typical of the neo-liberal era, starting from the policies aimed at the security armoring of society. This volume investigates torture against immigrants, focusing on the situation in different countries and on aspects related to health, highlighting the link with the worsening conditions of migration and the war on migrants.

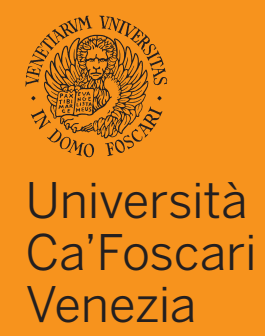

